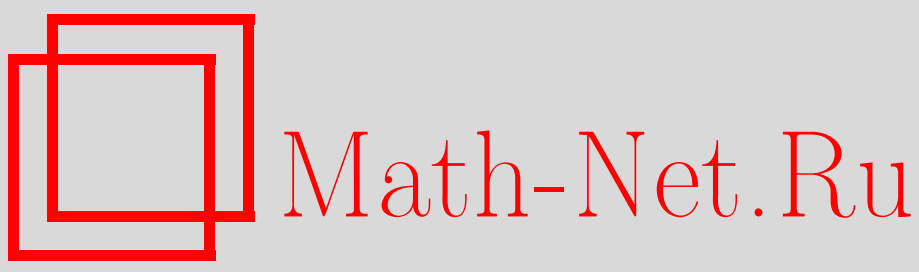

И. Г. Лысёнок, Бесконечные бернсайдовы группы четного периода, Изв. РАН. Сер. матем., 1996, том 60, выпуск 3, 3-224

DOI: https://doi.org/10.4213/im77

Использование Общероссийского математического портала Math-Net.Ru подразумевает, что вы прочитали и согласны с пользовательским соглашением

http://www . mathnet.ru/rus/agreement

Параметры загрузки:

IP : 3.89 .197 .203

26 апреля 2023 г., 13:01:06 


\title{
И.Г. Лысёнок
}

\section{Бесконечные бернсай,довы группы четного периода}

\begin{abstract}
Излагается модифицированный вариант теории Новикова-Адяна для свободных бернсайдовых групп периода $n=16 k \geqslant 8000$, на основе которого получено отрицательное решение проблемы Бернсайда для четных значений периода $n \geqslant 8000$ и доказан ряд утверждений о бернсайдовых группах периода $n=16 k \geqslant 8000$.

Библиография: 13 наименований.
\end{abstract}

\section{$\S 1$. Введение и формулировка основных результатов}

Свободная $m$-порожденная бернсайдова группа $\mathbf{B}(m, n)$ периода $n$ по определению есть группа с заданием

$$
\left.\left\langle a_{1}, a_{2}, \ldots, a_{m}\right| X^{n}=1 \text { для всех слов } X\right\rangle,
$$

или, другими словами, $\mathbf{B}(m, n)$ есть относительно свободная $m$-порожденная группа в многообразии всех групп периода $n$. Несмотря на простоту этого определения вопросы о строении и свойствах бернсайдовых групп $\mathbf{B}(m, n)$ оказались необычайно трудными. Среди них особое место занимает вопрос о конечности группы $\mathbf{B}(m, n)$ для данных $m, n \geqslant 2$, впервые сформулированный У. Бернсайдом [10] в 1902 г. и известный под названием проблемы Бернсайда о периодических группах. Другая естественная формулировка проблемы Бернсайда: верно ли, что любая $m$-порожденная группа периода $n$ конечна? Конечность групп $\mathbf{B}(m, n)$ установлена в случаях $n=2,3[10], n=4$ [8], $m=2[10]$ и $n=6$ [11] для любого количества порождаюших $m$. Отрицательное решение проблемы Бернсайда было получено в работе [5], в которой бесконечность $\mathbf{B}(m, n)$ доказана при любом $m \geqslant 2$ и нечетном $n \geqslant 4381$. Усовершенствованный вариант доказательства этого результата изложен в [1]. В той же работе [1] была понижена оценка для нечетного значения периода $n$ до $n \geqslant 665$.

Для изучения бернсайдовых групп достаточно большого нечетного периода в работах $[5],[1]$ была создана специальная теория преобразований слов. Ее характерной особенностью является построение для группы $\mathbf{B}(m, n)$ системы определяюших соотношений вида $X^{n}=1$, разбитой на подмножества, индексированные натуральным параметром, называемым рангом. При этом изучение групшы

Работа была выполнена при финансовой поддержке Международного научного фонда (грант № MUV000), Российского фонда фундаментальных исследований (проект № 93-011-16015), Американского математического общества (исследовательский грант для математиков из бывшего Советского Союза), а также Международного фонда "Культурная инициатива" и Академии естественных наук России. 
$\mathbf{B}(m, n)$ сводится к изучению последовательности групп $\mathbf{B}_{\alpha}(m, n)$, задаваемых всеми соотношениями ранга, не превосходяшего $\alpha$. Для анализа соотношений в группах $\mathbf{B}_{\alpha}(m, n)$ большое число утверждений доказывается одновременной индукцией по рангу $\alpha$. Позднее теория Новикова-Адяна и ее различные модификации нашли многочисленные применения для решения других задач теории групп, в том числе и не связанных непосредственно с проблемой Бернсайда (см., например, обзоры [2], [9]). Более короткое по сравнению с [1] доказательство теоремы Новикова-Адяна для нечетных значений периода $n>10^{10}$ было предложено А. Ю. Ольшанским [6] (это доказательство вошло впоследствии в монографию [7]). Хотя его технической основой служит геометрический язык диаграмм Линдона-ван Кампена, системы понятий и ряд ключевых утверждений в [1] и в [6], [7] имеют глубокую аналогию (см. [9]). Таким образом, в настоящее время под теорией Новикова-Адяна, по-видимому, следует понимать некоторый общий подход, допускающий различные технические реализации.

До недавнего времени сушественным ограничением применимости теории Новикова-Адяна являлось условие нечетности периода $n$. В частности, оставался открытым вопрос о существовании бесконечных бернсайдовых групп периода вида $2^{k}$. Заметим, что из теоремы Новикова-Адяна следует бесконечность бернсайдовых групп $\mathbf{B}(m, n)$ для периодов $n$, деляшихся на нечетное число $r \geqslant 665$, и, следовательно, из бесконечности бернсайдовых груп $\mathbf{B}\left(m, 2^{k}\right)$ при каком-нибудь $k$ вытекает отрицательное решение проблемы Бернсайда для почти всех значений периода $n$. Трудности при переходе от нечетных значений $n$ к четным связаны с тем, что при четном $n$ группа $\mathbf{B}(m, n)$ содержит инволюции, отсутствие которых использовалось в доказательстве одного из утверждений теории (см. [1, лемма II.5.21] и [7, лемму 18.9]). Как показывает более глубокий анализ, в случае четного $n$ возникает необходимость изучения строения конечных подгрупп групп $\mathbf{B}_{\alpha}(m, n)$.

Результат о бесконечности групп $\mathbf{B}(m, n)$ для четных значений периода $n$ был объявлен независимо С. В. Ивановым [12] для $n \geqslant 2^{48}$ и автором [4] для $n \geqslant 2^{13}$. Подробное доказательство результата Иванова опубликовано в статье [13], в которой фактически разработан вариант теории, применимый к бернсайдовым группам периода $n \geqslant 2^{48}$, делящегося на $2^{9}$.

Цель настоящей работы - подробное изложение варианта теории Новикова-Адяна для значений периода $n=16 k \geqslant 8000$, на основе которого доказывается результат, объявленный в [4].

Tеорема 1. При любых $m \geqslant 2$ и $n \geqslant 8000$ группа $\mathbf{B}(m, n)$ бесконечна.

Как и в случае нечетного периода, бесконечность группы $\mathbf{B}(m, n)$ по существу следует из утверждения о том, что любое непустое несократимое слово, равное 1 в $\mathbf{B}(m, n)$, должно содержать периодическое слово с достаточно большим числом периодов. Этот факт составляет содержание п. (i) следующей теоремы. Остальные утверждения теоремы также можно рассматривать как аналоги соответствуюших утверждений для нечетного $n$ (см. [1]).

TeOpema 2. Пусть $n=16 k \geqslant 8000 u m \geqslant 2$. 
(i) Если непустое несократимое в свободной әруппе слово $X$ равно 1 в әруппе $\mathbf{B}(m, n)$, то $X$ содержит непустое подслово вида $A^{n / 2-1240}$.

(ii) Группа $\mathbf{B}(m, n)$ имеет показательный рост.

(iii) Группа $\mathbf{B}(m, n)$ не мохет быть задана конечной системой определяющих соотношений.

(iiii) Любая конечная подгруппа группь $\mathbf{B}(m, n)$ изоморфно вкладывается в конечное прямое произведение $\mathbf{D}_{n} \times \mathbf{D}_{\bar{n}} \times \cdots \times \mathbf{D}_{\bar{n}}$, где $\mathbf{D}_{r}-$ диэдральная группа порядка $2 r$ и $\bar{n}$-наибольший делитель $n$ вида $2^{k}$.

ЗАмЕчАниЕ 1. Существуют (достаточно сложные) примеры соотношений в группах $\mathbf{B}(m, n)$ для значений периода $n=4 k+2, n=8 k+4$ и $n=16 k+8$, показываюшие, что такие группы существенно отличаются от остальных бернсайдовых групп. По-видимому, возникающие здесь трудности с применением теории Новикова-Адяна значительно превосходят те, которые были преодолены при переходе от нечетных значений $n$ к значениям вида $n=16 k$. В этом смысле требование, чтобы число $n$ делилось на 16, является существенным.

ЗАмечАниЕ 2. Можно доказать обращение п. (iii) теоремы 2: любое конечное прямое произведение вида $\mathbf{D}_{n} \times \mathbf{D}_{\bar{n}} \times \cdots \times \mathbf{D}_{\bar{n}}$ изоморфно вкладьвается в группу $\mathbf{B}(m, n)$. Доказательство этого результата, а также ряда других результатов о подгруппах групп $\mathbf{B}(m, n)$ при $n=16 k \geqslant 8000$ планируется опубликовать в последующих работах автора.

Мне приятно выразить благодарность С. И. Адяну за поддержку и А. А. Разборову за полезные обсуждения.

\section{§ 2. Основные обозначения и соглашения. Периодические слова}

На протяжении всей работы мы будем считать фиксированными значение периода $n=16 k \geqslant 8000$ и $m$-буквенный алфравит порождающих

$$
\mathcal{A}=\left\{a_{1}, a_{2}, \ldots, a_{m}\right\} .
$$

Все слова рассматриваются в групповом алфавите

$$
\mathcal{A}^{ \pm 1}=\left\{a_{1}, a_{2}, \ldots, a_{m}, a_{1}^{-1}, a_{2}^{-1}, \ldots, a_{m}^{-1}\right\} .
$$

Мы будем использовать следуюшие обозначения для числовых параметров:

$$
\begin{gathered}
p=240, \quad q=\frac{n}{2}-2 p-178, \quad h=2 p+128=608, \\
q_{0}=p+68=308, \quad q_{1}=q_{0}+2 h=1524, \quad q_{2}=q_{1}+2 h=2740, \\
q_{3}=q_{2}+9=2749, \quad q_{4}=q-2 p-68=\frac{n}{2}-1206, \\
\zeta=\frac{1}{50}, \quad \eta=\frac{2}{5} .
\end{gathered}
$$


Для обозначения слов будут использоваться буквы латинского алфавита $A$, $B, \ldots, Z, u, v, \ldots, z$, для обозначения групп - заглавные жирные буквы $\mathbf{G}, \mathbf{H}, \ldots$, для элементов групп - строчные жирные буквы $\mathbf{g}, \mathbf{h}, \ldots$ Буквами $\mathrm{U}, \mathrm{V}, \ldots, \mathbf{Z}$ будут обозначаться вхождения в слова. Для обозначения геометрических объектоввершин, ребер, путей и т.п., будут использоваться рубленые латинские буквы А, $\mathrm{B}, \ldots, \mathrm{Z}, \mathrm{a}, \mathrm{b}, \ldots, \mathrm{z}$. Запись $X \rightleftharpoons Y$ обозначает " $X$ по определению равно $Y$ ”, а выражение "при $X:=Y$ " является сокрашением выражения "при подстановке $Y$ вместо $X$ ".

Через $\lfloor x\rfloor$ и $\lceil x\rceil$ будем обозначать соответственно целую часть числа $x$ и наименьшее целое $y \geqslant x$.

Длина слова $X$ обозначается через $|X|$. Отношение графического равенства слов $X$ и $Y$ обозначается через $X$ 으 $Y$, а отношение " $Y$ есть циклический сдвиг слова $X$ " - через $X \approx Y$. Для обозначения пустого слова и единичного элемента групшы будем использовать символ 1 . Запись $P * E * Q$ обозначает данное вхождение слова $E$ в слово $P E Q$. Говоря о подслове $E$ данного слова $X$, мы обычно будем подразумевать некоторое фиксированное вхождение $E$ в $X$ (какое - ясно из контекста).

Мы будем использовать стандартные теоретико-групповые обозначения $[\mathbf{x}, \mathbf{y}]$ для коммутатора $\mathbf{x}^{-1} \mathbf{y}^{-1} \mathbf{x y}$ и $\mathbf{N}_{\mathbf{G}}(\mathbf{H})$ для нормализатора подгруппы $\mathbf{H}$ в группе $\mathbf{G}$. Записи $\mathbf{H} \subseteq \mathbf{G}$ и $\mathbf{H} \unlhd \mathbf{G}$ означают соответственно, что $\mathbf{H}$ - подгруппа $\mathbf{G}$ и $\mathbf{H}$ - нормальная подгруппа $\mathbf{G}$. Если $\mathbf{X}$ - некоторое множество элементов группы $\mathbf{G}$ и $t \in \mathbb{Z}$, то по определению $\mathbf{X}^{t} \rightleftharpoons\left\{\mathbf{x}^{t} \mid \mathbf{x} \in \mathbf{X}\right\}$.

На протяжении всей основной части работы (§ 5-17) рассматривается последовательность групп - фрагментов группы $\mathbf{B}(m, n)$, обозначаемых через $\mathbf{B}_{\alpha}$ и определяемых заданием вида

$$
\mathbf{B}_{\alpha} \rightleftharpoons\left\langle\mathcal{A} \mid A^{n}=1 \quad\left(A \in \mathcal{E}_{1} \cup \mathcal{E}_{2} \cup \cdots \cup \mathcal{E}_{\alpha}\right)\right\rangle,
$$

где, как и в [1], слова из множества $\mathcal{E}_{\alpha}$ называются әлементарными периодами ранга $\alpha$ (определение будет дано в $\S 5$ ). При этом $\mathbf{B}_{0}$ по определению есть свободная группа с базисом $\mathcal{A}$. Равенство слов $X$ и $Y$ в группе $\mathbf{B}_{\alpha}$ обозначается через $X \stackrel{\alpha}{=} Y$. Мы будем использовать специальное обозначение $[X]_{\alpha}$ для элемента группы $\mathbf{B}_{\alpha}$, представленного словом $X$. Таким образом, равенство $X \stackrel{\alpha}{=} Y$ обозначает то же самое, что и равенство $[X]_{\alpha}=[Y]_{\alpha}$. Для любых рангов $\alpha$ и $\beta$ при $\beta \leqslant \alpha$ определен естественный эпиморфизм

$$
\pi_{\beta, \alpha}: \mathbf{B}_{\beta} \rightarrow \mathbf{B}_{\alpha}, \quad[X]_{\beta} \mapsto[X]_{\alpha}
$$

Пусть $A$ - непустое слово. Слово $P$ назьвается периодическим словом с периодом $A$, если $P$ входит в некоторое слово $A^{t}$ при $t>0$. Множество всех периодических слов с периодом $A$ будем обозначать через Пер $(A)$. Множество периодических слов $P$ с периодом $A$, удовлетворяюших условию $|P| \geqslant t|A|$, обозначим через Пер $(A, t)$. Очевидно, Пер $(A)=\Pi е p\left(A^{\prime}\right)$ и Пер $(A, t)=\Pi е p\left(A^{\prime}, t\right)$, если $A \approx A^{\prime}$, а множества Пер $\left(A^{-1}\right)$ и Пер $\left(A^{-1}, t\right)$ состоят в точности из всех слов, обратных 
к словам из множеств Пер $(A)$ и Пер $(A, t)$. Как правило, в качестве периодов $A$ будут рассматриваться простые слова, т.е. непредставимые в виде $A$ 을 $B^{r}$ при $r \geqslant 2$.

Нам понадобится следующая лемма, первые два утверждения которой представляют собой известные свойства периодических слов (см., например, $[1$, леммы I.2.2 и I.2.3]), а третье - легкое упражнение.

2.1. Лемма. (i) Ecли $A$ - простое слово и $A$ 드 $X Y$ 드 $Y X$, mo хотя бы одно из слов $X$ или $Y$ nусто.

(ii) Если $A, B$ - простые слова и Пер $(A, 2) \cap \Pi е р(B, 2) \neq \varnothing$, mо $A \approx B$.

(iii) Eсли для некоторого $t>0$ виполнено $A^{t} \approx X Y \approx X Z u|X| \geqslant|A|$, то $Y$ 드 $Z$.

\section{§ 3. Граф Кэли группы}

Пусть $\mathbf{G}$ - некоторая группа и $\mathcal{A}$ - алфавит порождающих для $\mathbf{G}$. Тем самым мы подразумеваем, что задан эпиморфизм свободной группы $\mathbf{F}_{\mathcal{A}}$ с базисом $\mathcal{A}$ на $\mathbf{G}$, coпоставляющий каждому слову $X$ в групповом алфавите $\mathcal{A}^{ \pm 1}$ элемент $[X]_{\mathbf{G}} \in \mathbf{G}$. Граф Кәли $\Gamma(\mathbf{G}, \mathcal{A})$ группы $\mathbf{G}$ относительно $\mathcal{A}$ есть ориентированный граф с множеством вершин $\mathbf{G}$ и множеством ориентированных ребер $\mathbf{G} \times \mathcal{A}^{ \pm 1}$, где по определению ребро $\left(\mathbf{g}, a^{\epsilon}\right)$ соединяет вершины $\mathbf{g}$ и $\mathbf{g}\left[a^{\epsilon}\right]_{\mathbf{G}}$. При этом ориентированные ребра $\left(\mathbf{g}, a^{\epsilon}\right)$ и $\left(\mathbf{g}\left[a^{\epsilon}\right]_{\mathbf{G}}, a^{-\epsilon}\right)$ считаются взаимно обратными.

Буква $a^{\epsilon}$ группового алфавита $\mathcal{A}^{ \pm 1}$ называется меткой ребра е $=\left(\mathbf{g}, a^{\epsilon}\right)$ и будет обозначаться через $\mathcal{L}(\mathrm{e})$. Если $\mathrm{X}=\mathrm{e}_{1} \mathrm{e}_{2} \ldots \mathrm{e}_{r}$ - непустой путь на графе $\Gamma(\mathbf{G}, \mathcal{A})$, то слово $\mathcal{L}(\mathrm{X}) \rightleftharpoons \mathcal{L}\left(\mathrm{e}_{1}\right) \mathcal{L}\left(\mathrm{e}_{2}\right) \ldots \mathcal{L}\left(\mathrm{e}_{r}\right)$ называется меткой пути $\mathrm{X}$, а путь $\mathrm{X}^{-1} \rightleftharpoons \mathrm{e}_{r}^{-1} \mathrm{e}_{r-1}^{-1} \ldots \mathrm{e}_{1}^{-1}-$ обратным $\kappa \mathrm{X}$. Начальную вершину и конечную вершину пути $\mathbf{X}$ на графе $\Gamma(\mathbf{G}, \mathcal{A})$ будем обозначать через $\iota(\mathbf{X})$ и $\tau(\mathbf{X})$ соответственно. Очевидно, имеем $\left(\mathrm{X}^{-1}\right)^{-1}=\mathrm{X}, \iota\left(\mathrm{X}^{-1}\right)=\tau(\mathrm{X}), \tau\left(\mathrm{X}^{-1}\right)=\iota(\mathrm{X})$ и $\mathcal{L}\left(\mathbf{X}^{-1}\right)$ 으 $(\mathcal{L}(\mathbf{X}))^{-1}$. В качестве путей на $\Gamma(\mathbf{G}, \mathcal{A})$ мы будем рассматривать также nyстые nути, состояшие из одной вершины $\mathbf{g}$ графа $\Gamma(\mathbf{G}, \mathcal{A})$. При этом по определению $\iota(\mathbf{g})=\tau(\mathbf{g})=\mathbf{g}$ и $\mathcal{L}(\mathbf{g})$ 으 1 . Обратным к пустому пути $\mathbf{X}$ будем считать сам путь $\mathrm{X}$. Через $|\mathrm{X}|$ будет обозначаться длина пути $\mathrm{X}$, которая, очевидно, равна длине слова $\mathcal{L}(\mathrm{X})$.

На множестве пар путей $\mathrm{X}$ и $\mathrm{Y}$ на $\Gamma(\mathbf{G}, \mathcal{A})$ с условием $\tau(\mathrm{X})=\iota(\mathrm{Y})$ естественным образом определена операция произведения $\mathrm{XY}$ путей $\mathrm{X} u \mathrm{Y}$, для которой пустые пути играют роль единиц. При этом, очевидно, $\mathcal{L}(\mathrm{XY})$ 으 $\mathcal{L}(\mathrm{X}) \mathcal{L}(\mathrm{Y})$. Путь $\mathrm{Y}$ есть подпуть пути $\mathrm{X}$, если $\mathrm{X}=\mathrm{UY}$ для некоторых путей $\mathrm{U}$ и $\mathrm{V}$. Если при этом $\mathrm{U}$ или $\mathrm{V}$ - пустой путь, то будем говорить, что $\mathrm{Y}$ есть начало или соответственно конеи пути $\mathrm{X}$. Если $\mathrm{Y}$ есть подпуть пути $\mathrm{X}$, то будем также говорить, что $\mathrm{Y}$ coдержится в $\mathbf{X}$. Вершина $\mathbf{g}$ графа $\Gamma(\mathbf{G}, \mathcal{A})$ лежит на пути $\mathrm{X}$, если либо $\mathrm{X}$ - пустой путь и $\mathbf{g}=\mathbf{X}$, либо $\mathbf{g}=\iota(\mathrm{e})$ или $\mathbf{g}=\tau(\mathrm{e})$ для некоторого ребра е, входящего в $\mathbf{X}$. Путь $\mathrm{X}$ назьвается простылм, если на $\mathrm{X}$ лежит ровно $|\mathrm{X}|+1$ вершин, т.е. $\mathbf{X}$ не проходит ни через одну вершину дважды.

Так как для любой вершины $\mathbf{g} \in \mathbf{G}$ граффа $\Gamma(\mathbf{G}, \mathcal{A})$ и любой буквы $a^{\epsilon} \in \mathcal{A}^{ \pm 1}$ из $\mathbf{g}$ выходит ровно одно ребро с меткой $a^{\epsilon}$, то для любой вершины $\mathbf{g} \in \mathbf{G}$ и лю- 
бого слова $X$ существует единственный путь $\mathrm{X}$ на $\Gamma(\mathbf{G}, \mathcal{A})$, для которого $\iota(\mathrm{X})=\mathrm{g}$ и $\mathcal{L}(\mathrm{X})$ 으 $X$. Неформально говоря, путь $\mathrm{X}$ можно рассматривать как "геометрическое изображение" слова $X$, при этом начало пути $\mathrm{X}$ можно поместить в любую заданную наперед вершину $\mathbf{g}$ графа $\Gamma(\mathbf{G}, \mathcal{A})$. Если при этом $\mathbf{X}$ - простой путь, то имеется естественное взаимно однозначное соответствие между непустыми подпутями пути $\mathrm{X}$ и подсловами слова $X$ : если $\mathrm{X}=\mathrm{USV}$, то подпути $\mathrm{S}$ соответствует вхождение $\mathcal{L}(\mathrm{U}) * \mathcal{L}(\mathrm{S}) * \mathcal{L}(\mathrm{V})$ слова $\mathcal{L}(\mathrm{S})$ в слово $X^{1}$. Если $\mathrm{U}$ - вхождение некоторого слова в слово $X$, то через $\psi_{\mathbf{X}}(\mathrm{U})$ будем обозначать соответствуюший подпуть пути $\mathrm{X}$. Очевидно, вхождения $\mathrm{U}$ и $\mathrm{V}$ в слово $X$ не пересекаются тогда и только тогда, когда пути $\psi_{\mathbf{X}}(\mathrm{U})$ и $\psi_{\mathbf{X}}(\mathrm{V})$ не имеют обших ребер.

На множестве непустых подпутей простого пути $X$ будем рассматривать два отношения: $\mathrm{S}<\mathrm{x} T$ и $\mathrm{S} \ll \mathrm{x} T$, соответствуюшие использованным в [1] отношениям "вхождение U расположено левее вхождения V" и "вхождение U расположено строго левее вхождения V". Если $\mathrm{X}=\mathrm{USV}=\mathrm{WTZ}$, то по определению $\mathrm{S}<\mathrm{x}$ T тогда и только тогда, когда $|\mathrm{U}|<|\mathrm{W}|$ и $|\mathrm{V}|>|\mathrm{Z}|$, и $\mathrm{S} \ll \mathrm{x}$ Т тогда и только тогда, когда $|\mathrm{US}| \leqslant|\mathrm{W}|$. Если ни один из подпутей $\mathrm{S}$ и $\mathrm{T}$ пути $\mathrm{X}$ не содержится в другом, то, очевидно, истинно ровно одно из отношений $\mathrm{S}<\mathrm{x} T$ или $\mathrm{T}<\mathrm{x} S$, а если при этом $\mathrm{S}$ и $T$ не имеют общих ребер, то имеем либо $S \ll x \mathrm{~T}$, либо $\mathrm{T} \ll \mathrm{x} S$.

Очевидно, если $\mathrm{X}$ - подпуть простого пути $\mathrm{Y}$ и $\mathrm{S}, \mathrm{T}$ - подпути $\mathrm{X}$, то $\mathrm{S}<\mathrm{x} \mathrm{T} \Leftrightarrow$ $\mathrm{S}<_{\mathrm{Y}} \mathrm{T}$ и $\mathrm{S} \ll \mathrm{X} \mathrm{T} \Leftrightarrow \mathrm{S} \ll \mathrm{Y} T$. Если $\mathrm{S}, \mathrm{T}, \mathrm{U}, \mathrm{X}$ - подпути $\mathrm{Y}$, пути $\mathrm{S}, \mathrm{T}$ содержатся в $\mathrm{X}$ и $\mathrm{S}<_{\mathrm{Y}} \mathrm{U}<_{\mathrm{Y}} \mathrm{T}$, то и $\mathrm{U}$ содержится в $\mathrm{X}$.

Если $S, T$ - непустые подпути простого пути $X$, то через $S \cup_{X} T$ будем обозначать подпуть пути $X$ наименьшей длины, содержаший $S$ и $T$ в качестве подпутей (это соответствует операции объединения двух вхождений в данное слово $\mathrm{X}$ ).

Легко видеть, что для любого пути $\mathrm{X}$ на графе $\Gamma(\mathbf{G}, \mathcal{A})$ имеет место равенство

$$
\tau(\mathbf{X})=\iota(\mathbf{X})[\mathcal{L}(\mathbf{X})]_{\mathbf{G}}
$$

В частности, отсюда следует, что элемент $[\mathcal{L}(\mathbf{X})]_{\mathbf{G}}$ группы $\mathbf{G}$, представленный словом $\mathcal{L}(\mathrm{X})$, определяется только начальной и конечной вершинами пути $\mathrm{X}$. В качестве еще одного следствия формулы (3.1) получаем, что путь $X$ на графе $\Gamma$ является замкнутым тогда и только тогда, когда $[\mathcal{L}(\mathrm{X})]_{\mathbf{G}}=1$, и простым тогда и только тогда, когда $[Y]_{\mathbf{G}} \neq 1$ для любого непустого подслова $Y$ слова $\mathcal{L}(\mathrm{X})$.

Так как $\mathcal{A}$ есть множество порождающих для $\mathbf{G}$, то из (3.1) следует, что граф $\Gamma(\mathbf{G}, \mathcal{A})$ связен, т.е. любые две его вершины $\mathbf{g}$ и $\mathbf{h}$ могут быть соединены некоторым путем.

Еще один способ перехода от языка слов к языку путей на графе $\Gamma(\mathbf{G}, \mathcal{A})$ основан на том, что слова, равные 1 в групе $\mathbf{G}$, изображаются замкнутыми путями на $\Gamma(\mathbf{G}, \mathcal{A})$. Например, пусть в группе $\mathbf{G}$ имеют место соотношения $X_{1}=X_{2} X_{3} X_{4}$ и $X_{5}=X_{4}^{-1} X_{6} X_{7}$. Тогда сушествуют пути $\mathrm{X}_{i}(i=1,2, \ldots, 7)$ на графе $\Gamma(\mathbf{G}, \mathcal{A})$

\footnotetext{
${ }^{1}$ На самом деле требование простоты пути $X$ здесь несущественно. Можно было бы определить понятие подпути $\mathrm{Y}$ пути $\mathrm{X}$ как "вхождения" $\mathrm{Y}$ в $\mathrm{X}$. Однако в этом случае возникают неудобства технического характера, например, если $Y$ - подпуть пути $X, Z$ - подпуть пути $Y$, то формально $Z$ не есть подпуть пути $X$ и т.п.
} 
с $\mathcal{L}\left(\mathrm{X}_{i}\right)$ 으 $X_{i}$, расположенные как на рис. 1 . Здесь формально подразумевается, что $\iota\left(\mathrm{X}_{1}\right)=\iota\left(\mathrm{X}_{2}\right), \tau\left(\mathrm{X}_{2}\right)=\iota\left(\mathrm{X}_{3}\right), \tau\left(\mathrm{X}_{3}\right)=\iota\left(\mathrm{X}_{4}\right)=\iota\left(\mathrm{X}_{6}\right), \tau\left(\mathrm{X}_{1}\right)=\tau\left(\mathrm{X}_{4}\right)=\iota\left(\mathrm{X}_{5}\right)$, $\tau\left(\mathrm{X}_{6}\right)=\iota\left(\mathrm{X}_{7}\right)$ и $\tau\left(\mathrm{X}_{5}\right)=\tau\left(\mathrm{X}_{7}\right)$ (но не обязательно пути $\mathrm{X}_{i}$ не имеют обших вершин, отличных от указанных). Заметим, что какая-нибудь одна из вершин $\iota\left(\mathrm{X}_{i}\right)$ или $\tau\left(\mathbf{X}_{i}\right)$ может быть помешена в любой заданной наперед вершине графа $\Gamma(\mathbf{G}, \mathcal{A})$. Такой способ изображения соотношений в группе $\mathbf{G}$ с помошью путей на графе $\Gamma(\mathbf{G}, \mathcal{A})$ будет широко использоваться.

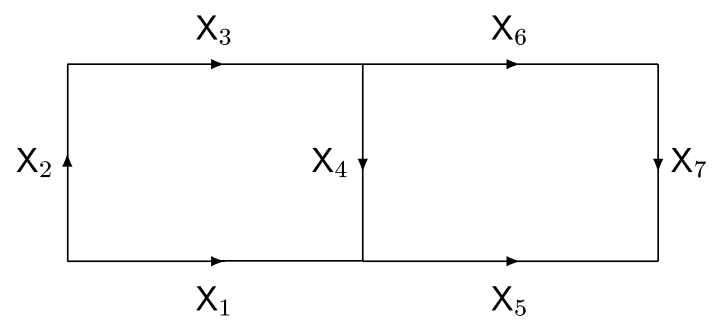

Рис. 1

В дальнейшем мы будем свободно переходить от языка слов и вхождений к языку путей на графе $\Gamma(\mathbf{G}, \mathcal{A})$ и обратно.

На графе $\Gamma(\mathbf{G}, \mathcal{A})$ определено естественным образом действие группы $\mathbf{G}$ левыми сдвигами: если е $=\left(\mathbf{g}, a^{\epsilon}\right)$ - ребро графа $\Gamma(\mathbf{G}, \mathcal{A})$, то по определению $\mathbf{h e} \rightleftharpoons\left(\mathbf{h g}, a^{\epsilon}\right)$. Это действие распространяется на множество всех путей на $\Gamma(\mathbf{G}, \mathcal{A})$, при этом сохраняются отображения $\iota, \tau,{ }^{-1}, \mathcal{L}$ и операция произведения путей: для любых путей $\mathbf{X}, \mathbf{Y}$ и любого элемента $\mathbf{h} \in \mathbf{G}$ имеем $\iota(\mathbf{h X})=\mathbf{h} \iota(\mathbf{X})$, $\tau(\mathbf{h X})=\mathbf{h} \tau(\mathbf{X}),(\mathbf{h X})^{-1}=\mathbf{h}\left(\mathbf{X}^{-1}\right), \quad \mathcal{L}(\mathbf{h X})$ 드 $\mathcal{L}(\mathbf{X})$ и $\mathbf{h}(\mathrm{XY})=\mathbf{h} \mathbf{X} \cdot \mathbf{h} \mathbf{Y}$, если $\iota(\mathbf{Y})=\tau(\mathbf{X})$. В частности, из равенства $\iota(\mathbf{h} \mathbf{X})=\mathbf{h} \iota(\mathbf{X})$ следует, что $\mathbf{G}$ действует транзитивно и свободно (т.е. с тривиальными стабилизаторами) на множестве путей на $\Gamma(\mathbf{G}, \mathcal{A})$, имеющих одну и ту же метку. Очевидно, Ү есть подпуть, начало или конец пути $\mathbf{X}$ тогда и только тогда, когда $\mathbf{h} \mathbf{Y}$ есть соответственно подпуть, начало или конец пути $\mathbf{h} \mathbf{X}$.

Если $\mathbf{G}$ и $\mathbf{H}$ - две группы с одним и тем же множеством порождающих $\mathcal{A}$ и отображение $\pi:[X]_{\mathbf{G}} \mapsto[X]_{\mathbf{H}}$ является эпиморфизмом $\mathbf{G}$ на $\mathbf{H}$, то $\pi$ естественным образом продолжается до отображения графа $\Gamma(\mathbf{G}, \mathcal{A})$ в $\Gamma(\mathbf{H}, \mathcal{A})$ (которое является накрытием графов при естественной геометрической интерпретации). Это отображение индуцирует отображение множества путей на графе $\Gamma(\mathbf{G}, \mathcal{A})$ на множество путей на графе $\Gamma(\mathbf{H}, \mathcal{A})$, которое будет обозначаться тем же символом $\pi$. Очевидно, $\pi(\mathbf{g X})=\pi(\mathbf{g}) \pi(\mathrm{X})$, и, следовательно, $\pi(\mathrm{X})=\pi(\mathrm{Y})$ в том и только том случае, если $\mathbf{X}=\mathbf{g Y}$ для некоторого $\mathbf{g} \in \operatorname{ker} \pi$. Если $\mathrm{Y}$ есть подпуть (начало, конец) пути $\mathrm{X}$, то $\pi(\mathrm{Y})$ есть подпуть (соответственно, начало, конец) пути $\pi(\mathrm{X})$. Обратно, если $\pi(\mathrm{X})=\widetilde{\mathrm{X}}$ и $\widetilde{\mathrm{X}}=\widetilde{\mathrm{U}} \widetilde{\mathrm{Z}}$, то, положив $\mathrm{X}=\mathrm{UYV}$, где $\mathcal{L}(\widetilde{\mathrm{U}})$ 으 $\mathcal{L}(\mathrm{U}), \mathcal{L}(\widetilde{\mathrm{Y}})$ 으 $\mathcal{L}(\mathrm{Y})$, $\mathcal{L}(\widetilde{\mathrm{V}})$ 으 $\mathcal{L}(\mathrm{V})$, получим подпуть $\mathrm{Y}$ пути $\mathrm{X}$, для которого $\pi(\mathrm{Y})=\widetilde{\mathrm{Y}}$. Ясно, что если $\widetilde{\mathrm{X}}$-простой путь, то подпуть $\mathrm{Y}$ пути $\mathrm{X}$ однозначно определяется условием $\pi(\mathrm{Y})=\widetilde{\mathrm{Y}}$. 
Путь $\mathrm{P}$ на графе $\Gamma(\mathbf{G}, \mathcal{A})$ с $\mathcal{L}(\mathrm{P}) \in \Pi$ Пер $(A)$ будем называть периодическим отрезком $с$ периодом $A$. Множество периодических отрезков с периодом $A$ на графе $\Gamma \rightleftharpoons \Gamma(\mathbf{G}, \mathcal{A})$ обозначим через Пер $(\Gamma, A)$. Для множества периодических отрезков $\mathrm{P} \in$ Пер $(\Gamma, A)$ с условием $|\mathrm{P}| \geqslant t|A|$ (т.е. $\mathcal{L}(\mathrm{P}) \in \Pi$ Пер $(A, t)$ ) будем использовать обозначение Пер $(\Gamma, A, t)$. Если $\mathrm{P}$ - подпуть пути $\mathrm{Q}$, где $\mathrm{P}, \mathrm{Q} \in$ Пер $(\Gamma, A, 2)$ для некоторого $A$, то $\mathrm{Q}$ будем называть периодическим продолжением пути $\mathrm{P}$.

3.1. Лемма. (i) Если $\mathrm{Q}$ есть периодическое продолжение $\mathrm{P}$, mo $^{-1}$ есть периодическое продолжение $\mathrm{P}^{-1}$.

(ii) Если Q есть периодическое продолжение $\mathrm{P}$, то для любого $\mathbf{h} \in \mathbf{G}$ путь $\mathbf{h Q}$ есть периодическое продолжение $\mathbf{h P}$.

(iii) Если $\mathrm{Q}$ есть периодическое продолжение $\mathrm{P}$ и $\mathrm{R}$ есть периодическое продолэсение $\mathrm{Q}$, то $\mathrm{R}$ есть периодическое продолжсение $\mathrm{P}$.

(iv) Любые два периодических продолжения одного пути имеют общее периодическое продолжение.

(v) $E c л и \mathrm{P}, \mathrm{Q} \in \Pi$ Пер $(\Gamma, A, 2)$, то найдется әлемент $\mathbf{h} \in \mathbf{G}$ такой, что пути $\mathbf{h P} u \mathrm{Q}$ имеют общее периодическое продолжение.

ДоКАЗАТЕЛЬСТво. Утверждения (i) и (ii) очевидны. Утверждение (iii) вытекает из леммы 2.1, (ii). Докажем (iv). Пусть $\mathrm{P} \in \Pi$ ер $\left(\Gamma, A_{1}, 2\right) \cap \Pi е р\left(\Gamma, A_{2}, 2\right)$ и $\mathrm{Q}=\mathrm{U}_{1} \mathrm{PV}_{1} \in \Pi е \mathrm{p}\left(\Gamma, A_{1}, 2\right), \mathrm{R}=\mathrm{U}_{2} \mathrm{PV}_{2} \in \Pi е \mathrm{p}\left(\Gamma, A_{2}, 2\right)$ - периодические продолжения Р. Без ограничения общности можно считать, что $A_{i}$ - простые слова. По лемме 2.1 , (ii) имеем $A_{1} \approx A_{2}$, откуда легко следует, что одно из слов $\mathcal{L}\left(\mathrm{U}_{1}\right), \mathcal{L}\left(\mathrm{U}_{2}\right)$ есть конец другого, а одно из слов $\mathcal{L}\left(\mathrm{V}_{1}\right), \mathcal{L}\left(\mathrm{V}_{2}\right)$ есть начало другого. Тогда один из путей $\mathrm{U}_{1}, \mathrm{U}_{2}$ - конец другого, один из путей $\mathrm{V}_{1}, \mathrm{~V}_{2}$ - начало другого, и в качестве общего периодического продолжения путей $Q$ и $\mathrm{R}$ можно взять UPV, где каждый из путей $\mathrm{U}$ и $\mathrm{V}$ есть один из путей $\mathrm{U}_{i}$ или соответственно $\mathrm{V}_{i}$, имеющий максимальную длину. Для доказательства (v) нужно рассмотреть периодические продолжения $\bar{P}$, $\overline{\mathrm{Q}}$ для $\mathrm{P}$ и $\mathrm{Q}$ такие, что $\mathcal{L}(\overline{\mathrm{P}})=\mathcal{L}(\overline{\mathrm{Q}})$, и положить $\mathbf{h} \rightleftharpoons \iota(\overline{\mathrm{Q}})(\iota(\overline{\mathrm{P}}))^{-1}$.

Пусть $\mathrm{P} \in$ Пер $(\Gamma, A, 2)$, и пусть $\mathrm{P}=\mathrm{UXV}$, где $|\mathrm{V}| \geqslant|A|$. Тогда по аналогии с вхождениями слов определен результат сдвига пути $\mathrm{X}$ вдоль $\mathrm{P}$ на период $A$, т.е. такой подпуть $\mathrm{X}_{1}$ пути $\mathrm{P}$, что $\mathrm{P}=\mathrm{U}_{1} \mathrm{X}_{1} \mathrm{~V}_{1}$, где $\left|\mathrm{U}_{1}\right|=|\mathrm{U}|+|A|$ и $\left|\mathrm{V}_{1}\right|=|\mathrm{V}|-|A|$. При этом, очевидно, имеем $\mathcal{L}\left(\mathrm{X}_{1}\right)$ 을 $\mathcal{L}(\mathrm{X})$. Используя формулу (3.1), получаем

$$
\iota\left(\mathrm{X}_{1}\right)(\iota(\mathrm{X}))^{-1}=\iota(\mathrm{P})\left[\mathcal{L}\left(\mathrm{U}_{1}\right)\right]_{\mathbf{G}}[\mathcal{L}(\mathrm{U})]_{\mathbf{G}}^{-1}(\iota(\mathrm{P}))^{-1} .
$$

Так как $\mathcal{L}\left(\mathrm{U}_{1}\right)$ 으 $A_{1} \mathcal{L}(\mathrm{U})$, где $A_{1}$ - начало слова $\mathcal{L}\left(\mathrm{U}_{1}\right)$ длины $\left|A_{1}\right|=|A|$, то отсюда следует, что $\mathrm{X}_{1}$ получается из $\mathrm{X}$ умножением слева на элемент групाы $\mathbf{G}$

$$
\mathbf{s}_{A, \mathrm{P}} \rightleftharpoons \iota(\mathrm{P})\left[A_{1}\right]_{\mathbf{G}}(\iota(\mathrm{P}))^{-1},
$$

где $A_{1}$ - циклический сдвиг $A$, с которого начинается $\mathcal{L}(\mathrm{P})$.

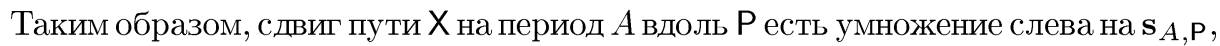
и тем самым мы можем рассматривать эту операцию для произвольного пути $\mathrm{X}$ на графе Г. Сдвиг пути $\mathrm{X}$ на $t$ периодов $A$ вдоль $\mathrm{P}$ (это соответствует сдвигу подслова 
периодического слова $P \in \Pi е p(A, 2)$ на $t$ периодов $A$ вправо) есть умножение слева на элемент $\mathbf{s}_{A, \mathrm{p}}^{t}$. Следуюшая лемма легко вытекает из определений и формул (3.1), (3.2) (для доказательства п. (vii) нужно воспользоваться еще леммой 2.1, (i)) и будет часто использоваться в дальнейшем.

3.2. Лемма. Пусть $\mathrm{P} \in \Pi$ Пер $(\Gamma, A, 2)$, где $\Gamma \rightleftharpoons \Gamma(\mathbf{G}, \mathcal{A})$. Тогда:

(i) если $\mathrm{S}, \mathbf{s}_{A, \mathrm{P}} \mathrm{S}-$ подпути $\mathrm{P}$, то $\mathrm{S}<\mathrm{P} \mathbf{s}_{A, \mathrm{P}} \mathrm{S}$, а если при әтом $|\mathrm{S}| \leqslant|A|$, то $\mathrm{S} \ll \mathrm{P} \mathbf{s}_{A, \mathrm{P}} \mathrm{S}$

(ii) если вериина $\mathbf{g}$ графа $\Gamma$ лежит на $\mathrm{P}$, то для любого $t \in \mathbb{Z}$ вершина $\mathbf{s}_{A, \mathrm{P}}^{t} \mathbf{g}$ лежит на некотором периодическом продолжении $\mathrm{P} ;$ если $\mathrm{S}$ - подпуть пути $\mathrm{P}$, то для любого $t \in \mathbb{Z}$ путь $\mathbf{s}_{A, \mathrm{P}}^{t} \mathrm{~S}$ содержится в некотором периодическом продолжении $\mathrm{P}$;

(iii) если $\mathrm{P}$ и $\mathrm{Q}$ имеют общее периодическое продолжение, то $\mathbf{s}_{A, \mathrm{P}}=\mathbf{s}_{A, \mathrm{Q}}$;

(iv) $\mathbf{s}_{A, \mathbf{g P}}=\mathbf{g s}_{A, \mathbf{P}} \mathbf{g}^{-1}$;

(v) $\mathbf{s}_{A^{-1}, \mathrm{P}^{-1}}=\left(\mathbf{s}_{A, \mathrm{P}}\right)^{-1}$;

(vi) если $\pi:[X]_{\mathbf{G}} \mapsto[X]_{\mathbf{H}}-$ эпиморфизм $\mathbf{G} н а \mathbf{H}, \mathbf{m o}_{A, \pi(\mathrm{P})}=\pi\left(\mathbf{s}_{A, \mathrm{P}}\right)$;

(vii) если $A$ - простое слово и пути $\mathrm{P}$, gP имеют общее периодическое продолжение, $\operatorname{mo} \mathbf{g}=\mathbf{s}_{A, \mathrm{P}}^{t}$ для некоторого $t \in \mathbb{Z}$.

3.3. ОПРЕДЕЛЕНИЕ. Периодические отрезки $\mathrm{P} \in$ Пер $(\Gamma, A, 2)$ и $\mathrm{Q} \in$ Пер $(\Gamma, B, 2)$ назовем согласованнылми, если для некоторого $t>0$ выполнено $\mathbf{s}_{A, \mathbf{P}}^{t}=\mathbf{s}_{B, \mathbf{Q}}^{t}$, или, эквивалентно, $[A]_{\mathbf{G}}^{t}=\mathbf{x}[B]_{\mathbf{G}}^{t} \mathbf{x}^{-1}$, где $\mathbf{x} \rightleftharpoons(\iota(\mathrm{P}))^{-1} \iota(\mathrm{Q})$. Отношение согласованности периодических отрезков $\mathrm{P}$ и $\mathrm{Q}$ обозначим через $\operatorname{Cozл}(\mathrm{P}, \mathrm{Q})$.

Вообще говоря, отношение $C о г л(\mathrm{P}, \mathrm{Q})$ зависит от выбора периодов $A$ и $B$, для которых $\mathrm{P} \in$ Пер $(\Gamma, A, 2)$ и $\mathrm{Q} \in \Pi_{\text {- }}(\Gamma, B, 2)$. В дальнейшем мы будем предполагать, что $A$ и $B$ - простые слова и, следовательно, определены с точностью до циклического сдвига. В этом случае истинность отношения Согл $(\mathrm{P}, \mathrm{Q})$ зависит только от Р и $\mathrm{Q}$.

Следуюшие свойства этого отношения легко вытекают из определения и леммы 3.2 .

3.4. Лемма. (i) Согл( $\mathrm{P}, \mathrm{Q})$ есть отношение әквивалентности на множестве $\bigcup_{A}$ Пер $(\Gamma, A, 2)$.

(ii) Для любого $\mathbf{g} \in \mathbf{G}$ имеем Согл(P, Q) $\Leftrightarrow \operatorname{Cогл(~}(\mathbf{g P}, \mathbf{g Q})$.

(iii) $\operatorname{Cozл}(\mathrm{P}, \mathrm{Q}) \Leftrightarrow \operatorname{Cozл}\left(\mathrm{P}^{-1}, \mathrm{Q}^{-1}\right)$.

(iv) Если $\mathrm{P} u \mathrm{Q}$ имеют общее периодическое продолжение, то Согл(Р, $\mathrm{Q})$.

3.5. Лемма. Пусть $\mathbf{G}-$ свободная группа с базисом $\mathcal{A}$. Если $A, B-u и к-$ лически несократимые слова, $\mathrm{P} \in \Pi \operatorname{\mathrm {p}}(\Gamma, A, 2), \mathrm{Q} \in \Pi \operatorname{ep}(\Gamma, B, 2)$ и $\mathbf{s}_{A, \mathrm{P}}^{r}=\mathbf{s}_{B, \mathrm{Q}}^{t}$ для некоторых $r, t>0$, то $\mathrm{P} u \mathrm{Q}$ имеют общее периодическое продолжение.

ДокаЗАТЕльСтво. Так как для любых $C$ и $\mathrm{R} \in \Pi е р(\Gamma, C, 2)$ из $C$ 으 $D^{k}$ при $k>0$ в силу (3.2) следует $\mathbf{s}_{C, \mathrm{R}}=\mathbf{s}_{D, \mathrm{R}}^{k}$, то достаточно ограничиться рассмотрением случая, когда $A, B$-простые слова. Выберем путь $\mathrm{X}$, соединяющий начальные вершины путей $\mathrm{P}$ и $\mathrm{Q}$, метка которого является несократимым в свободной группе $\mathbf{G}$ словом. Можно считать, что слова $\mathcal{L}(\mathrm{P})$ и $\mathcal{L}(\mathrm{Q})$ начинаются соответственно 
с $A$ и $B$. Согласно $(3.2)$ имеем $\iota(\mathrm{P})[A]_{\mathbf{G}}^{r}(\iota(\mathrm{P}))^{-1}=\iota(\mathrm{Q})[B]_{\mathbf{G}}^{t}(\iota(\mathrm{Q}))^{-1}$, что в силу формулы (3.1) можно переписать в виде

$$
A^{r}=\mathcal{L}(\mathrm{X}) B^{t}(\mathcal{L}(\mathrm{X}))^{-1}
$$

Так как слова $A$ и $B$ циклически несократимы, то из (3.3) в силу хорошо известных свойств свободных групп следует $r=t$ и $A \approx B$. Заменяя $\mathrm{P}$ и $\mathrm{Q}$ на некоторые их периодические продолжения в силу леммы 3.4 , (iv), можно считать $A$ 으 $B$ и $\mathcal{L}(\mathrm{P})$ 으 $\mathcal{L}(\mathrm{Q})$. Так как централизатор элемента $[A]_{\mathbf{G}}^{r}$ в $\mathbf{G}$ есть циклическая подгруппа, порожденная $[A]_{\mathbf{G}}$, то в этом случае из $(3.3)$ вытекает $\mathcal{L}(\mathbf{X})=A^{k}$ в $\mathbf{G}$ для некоторого $k \in \mathbb{Z}$. В силу несократимости слова $\mathcal{L}(\mathrm{X})$ имеем $\mathcal{L}(\mathrm{X})$ 드 $A^{k}$. Тогда в зависимости от знака $k$ один из путей $\mathrm{X}^{-1} \mathrm{P}$ или $\mathrm{XQ}$ есть общее периодическое продолжение путей $\mathrm{P}$ и $\mathrm{Q}$.

\section{§ 4. Карты и диаграммы}

Картой мы называем конечньй двумерный топологический клеточньй комплекс, гомеоморфно вложенньй в плоскость $\mathbb{R}^{2}$. Эквивалентное определение состоит в том, что карта $\Delta$ представляет собой набор попарно непересекающихся ограниченных подмножеств $\mathbb{R}^{2}$, разбитых на три типа - вершины, ребра и области карты $\Delta$ - и удовлетворяюших следуюшим условиям: вершины являются одноточечными множествами; ребра и области гомеоморфны соответственно открытому интервалу $(0,1)$ и открытому 2 -диску; топологическое замыкание каждого ребра е карты $\Delta$ есть объединение е и одной или двух вершин карты $\Delta$, а топологическое замыкание каждой области $\mathrm{A}$ есть объединение $\mathrm{A}$ и конечного числа вершин и ребер карты $\Delta$. Множества вершин, ребер и областей карты $\Delta$ будем будем обозначать через $\mathbf{V}(\Delta), \mathbf{E}(\Delta)$ и $\mathbf{R}(\Delta)$ соответственно. Обозначение $\Delta$ будем использовать также для теоретико-множественного объединения всех вершин, ребер и областей карты $\Delta$. Карта $\Delta$ называется связной, если она связна как подмножество $\mathbb{R}^{2}$, и односвязной, если дополнение $\mathbb{R}^{2} \backslash \Delta$ связно. В дальнейшем, если не оговаривается обратное, все карты предполагаются связными. Ребро е карты $\Delta$ назовем граничным, если е принадлежит границе $\partial \Delta$ карты $\Delta$, и внутренним в противном случае.

Ребро карты $\Delta$ вместе с фиксированным для него одним из двух возможных направлений движения вдоль этого ребра называется ориентированным ребром карты $\Delta$. Множество всех ориентированных ребер карты $\Delta$ обозначим через $\overrightarrow{\mathbf{E}}(\Delta)$. Если е $\in \overrightarrow{\mathbf{E}}(\Delta)$, то через $\mathrm{e}^{-1}$ будем обозначать противоположно ориентированное ребро, а через $\iota(\mathrm{e})$ и $\tau(\mathrm{e})$ - соответственно начальную вериинуи конечную вериину ориентированного ребра е. Понятие $n y m u$ (пустого или непустого) на карте $\Delta$, начальной вершины $\iota(\mathrm{X})$ пути $\mathrm{X}$, конечной вершины $\tau(\mathrm{X})$ пути $\mathrm{X}$, обратного $\kappa \mathrm{X}$ пути $\mathrm{X}^{-1}$, произведения $\mathrm{XY}$ путей $\mathrm{X}$ и $\mathrm{Y}$ на $\Delta$, простого пути и подпути пути $\mathrm{X}$ на карте $\Delta$ определяются точно так же, как и для графа $\Gamma(\mathbf{G}, \mathcal{A})$. Простой замкнутый путь на карте $\Delta$ - путь $\mathrm{X}$ на $\Delta$, не проходящий ни через одну вершину дважды, за исключением вершины $\iota(\mathbf{X})=\tau(\mathbf{X})$. Если $\mathbf{X}$ и $\mathbf{Y}$ - замкнутые пути 
на карте $\Delta$ и $\mathrm{X}$ есть некоторый циклический сдвиг $\mathrm{Y}$ (т.е. $\mathrm{Y}$ получается некоторой циклической перестановкой ориентированных ребер, образуюших путь $X)$, то в этом случае пишем $\mathrm{X} \approx \mathrm{Y}$. Для любого пути $\mathrm{X}$ на карте $\Delta$ через $\operatorname{supp} \mathrm{X}$ обозначим подмножество плоскости $\mathbb{R}^{2}$, покрываемое $X$, т.е. теоретико-множественное объединение всех ребер, входящих в X, и всех вершин, лежаших на X. Если X-простой (замкнутый) путь, то supp $\mathrm{X}$ есть отрезок кривой (соответственно замкнутая кривая) без самопересечений.

Мы предполагаем фиксированной некоторую ориентацию плоскости $\mathbb{R}^{2}$. Тем самым для каждой области карты $\Delta$ и каждой связной компоненты дополнения $\mathbb{R}^{2} \backslash \Delta$ определено положительное направление обхода их границы. При обходе границы области А карты $\Delta$ в положительном направлении последовательность проходимых ориентированных ребер карты $\Delta$ образует замкнутый путь на карте $\Delta$, который мы будем называть граничным ииклом области А и обозначать $\delta$ А. При обходе границы связных компонент дополнения $\mathbb{R}^{2} \backslash \Delta$ получается конечное число замкнутых путей на $\Delta$, которые будем называть граничны.ми ииклами кар$m b$. Граничные циклы карты $\Delta$ и ее областей определены с точностью до циклического сдвига замкнутого пути. Граничный цикл карты $\Delta$, образуемый при обходе неограниченной связной компоненты дополнения $\mathbb{R}^{2} \backslash \Delta$, будем называть внешним граничным циклом карты $\Delta$, а остальные граничные циклы карты $\Delta-$ внутренними (в силу предположения о связности карты $\Delta$ она имеет ровно один внешний граничный цикл). Если карта $\Delta$ односвязна, то она имеет единственный (c точностью до циклического сдвига) граничный цикл, который мы обозначаем $\delta \Delta$. При изображении на рисунках положительным направлением обхода будет считаться то, при котором внешний граничньй цикл карты обходится против часовой стрелки, а внутренние граничные циклы и граничные циклы областей - по часовой стрелке.

Если $\mathrm{X}, \mathrm{Y}$ - пути на карте $\Delta$ и $\operatorname{supp} \mathrm{X} \cap \operatorname{supp} \mathrm{Y}=\varnothing$, то будем говорить, что $\mathrm{X}$ и $\mathrm{Y}$ не пересекаются. Карту $\Delta$ назовем неособой, если ее граничные циклы - попарно непересекаюшиеся простые замкнутые пути. В этом случае как подмножество $\mathbb{R}^{2}$ карта $\Delta$ гомеоморфна диску с $r-1$ "дырками", где $r$ - число связных компонент границы (или число граничных циклов) карты $\Delta$.

Неособую односвязную карту будем называть дисковой, а неособую карту с двумя граничными циклами - кольцевой.

Эйлерова характеристика $\chi(\Delta)$ (не обязательно связной) карты $\Delta$ определяется равенством

$$
\chi(\Delta) \rightleftharpoons 2 \cdot \text { число связных компонент } \Delta-\text { число граничных циклов } \Delta .
$$

Очевидно, для любой связной карты $\Delta$ выполнено $\chi(\Delta) \leqslant 1$, а если $\Delta-$ неособая связная карта, то число $\chi(\Delta)$ однозначно определяет топологический тип $\Delta$ (как подмножества $\mathbb{R}^{2}$ ). В частности, для неособой связной карты $\Delta$ имеем $\chi(\Delta)=1$ тогда и только тогда, когда $\Delta$ - дисковая карта, и $\chi(\Delta)=0$ тогда и только тогда, когда $\Delta$ - кольцевая карта.

Подкартой карты $\Delta$ называется карта $\Theta$, для которой $\mathbf{V}(\Theta) \subseteq \mathbf{V}(\Delta), \mathbf{E}(\Theta) \subseteq$ $\mathbf{E}(\Delta)$ и $\mathbf{R}(\Theta) \subseteq \mathbf{R}(\Delta)$. Если $\Theta_{1}$ и $\Theta_{2}-$ подкарты карты $\Delta$, то подкарту $\Theta$ карты $\Delta$, 
для которой $\mathbf{R}(\Theta)=\mathbf{R}\left(\Theta_{1}\right) \cup \mathbf{R}\left(\Theta_{2}\right), \mathbf{E}(\Theta)=\mathbf{E}\left(\Theta_{1}\right) \cup \mathbf{E}\left(\Theta_{2}\right)$ и $\mathbf{V}(\Theta)=\mathbf{V}\left(\Theta_{1}\right) \cup$ $\mathbf{V}\left(\Theta_{2}\right)$, будем называть объединением подкарт $\Theta_{1}$ и $\Theta_{2}$ и обозначать $\Theta_{1} \cup \Theta_{2}$. Если $\Theta$ - подкарта карты $\Delta$, то не обязательно связную подкарту карты $\Delta$, состоящую из вершин, ребер и областей карты $\Delta$, содержашихся в топологическом замыкании дополнения $\Delta \backslash \Theta$, будем называть результатом удаления подкарты $\Theta$ из карты $\Delta$ и обозначать $\Delta-\Theta$. Если вместо $\Delta \backslash \Theta$ рассмотрим $\Delta \backslash \operatorname{int} \Theta$, где int $X$ обозначает внутренность подмножества $X \subseteq \mathbb{R}^{2}$, то соответствующую подкарту карты $\Delta$ будем называть результатом удаления внутренности подкарты $\Theta$ из карты $\Delta$ и обозначать $\Delta-\operatorname{int} \Theta$. Подкарты $\Delta-\Theta$ и $\Delta-\operatorname{int} \Theta$ различаются только в случае, когда $\Delta$ и $\Theta$ имеют обшие граничные ребра. В этом случае если е - такое ребро, то е принадлежит карте $\Delta-\operatorname{int} \Theta$, но не принадлежит карте $\Delta-\Theta$. Заметим, что граничные циклы карты $\Delta-\operatorname{int} \Theta$ состоят из граничных циклов карты $\Delta$ и замкнутых путей, обратных к граничным циклам карты $\Theta$.

Пути $\mathrm{X}, \mathrm{Y}$ на карте $\Delta$ называются гомотопныци конечной (возможно, пустой) последовательностью преобразований следуюших двух видов:

(i) вставка или удаление подпутей вида $\mathrm{ee}^{-1}$, где е $\in \overrightarrow{\mathbf{E}}(\Delta)$;

(ii) вставка или удаление граничных циклов областей карты $\Delta$.

Иногда, чтобы указать явно карту $\Delta$, на которой рассматривается отношение гомотопии, мы будем говорить о гомотопных на $\Delta$ путях $\mathrm{X}$ и $\mathrm{Y}$. Ясно, что пути, гомотопные на подкарте $\Theta$ карты $\Delta$, гомотопны также и на $\Delta$. Замкнутый путь $\mathrm{X}$ на $\Delta$ называется стягиваемым, если $\mathrm{X}$ гомотопен пустому пути. Так как преобразования (i) и (ii) не изменяют начальной и конечной вершин пути, то для гомотопных путей $\mathrm{X}_{\text {и }} \mathrm{Y}$ имеем $\iota(\mathrm{X})=\iota(\mathrm{Y})$ и $\tau(\mathrm{X})=\tau(\mathrm{Y})$. Из определения легко следует, что пути $\mathrm{X}$ и $\mathrm{Y}$ на карте $\Delta$ гомотопны тогда и только тогда, ког да путь $X \mathrm{Y}^{-1}$ стягиваем. Известно, что простой непустой замкнутый путь $X$ на карте $\Delta$ стягиваем тогда и только тогда, когда $X$ или $X^{-1}$ есть граничный цикл некоторой дисковой подкарты карты $\Delta$. Класс эквивалентности гомотопных путей на карте $\Delta$, содержаший путь $\mathrm{X}$, мы обозначаем через $[\mathrm{X}]_{\Delta}$. Множество классов гомотопных замкнутых путей на карте $\Delta$ с фиксированной начальной и конечной вершиной а $\in \mathbf{V}(\Delta)$ с операцией умножения $[\mathrm{X}]_{\Delta} \cdot[\mathrm{Y}]_{\Delta} \rightleftharpoons[\mathrm{XY}]_{\Delta}$ образует (комбинаторную) фундаментальную группу $\pi_{1}\left(\Delta\right.$, a) карты $\Delta$ в вершине а. Группа $\pi_{1}(\Delta, a)$ есть свободная группа ранга $r-1$, где $r$ - число граничных циклов карты $\Delta$. Если $\Delta$ - односвязная карта, то из тривиальности групшы $\pi_{1}(\Delta$, a) следует, что любой замкнутый путь на $\Delta$ стягиваем и любые два пути на $\Delta$ с общими начальными и конечными вершинами гомотопны.

Пусть $\Delta$ - некоторая карта. Отображение $\mathcal{L}: \overrightarrow{\mathbf{E}}(\Delta) \rightarrow \mathcal{A}^{ \pm 1} \cup\{1\} \quad$ (1 обозначает пустое слово) будем назьвать размечающей функиией на карте $\Delta$ над алфавитом $\mathcal{A}$, если для любого ориентированного ребра е $\in \overrightarrow{\mathbf{E}}(\Delta)$ выполнено $\mathcal{L}\left(\mathrm{e}^{-1}\right)$ 으 $(\mathcal{L}(\mathrm{e}))^{-1}$. Размечающая функция $\mathcal{L}$ на карте $\Delta$ естественным образом распространяется на множество путей на карте $\Delta$, принимая значения в множестве слов в групповом алфавите $\mathcal{A}^{ \pm 1}$. Очевидно, функция $\mathcal{L}$ согласуется с операциями взятия обратного пути и произведения путей: для любого пути $\mathrm{X}$ на $\Delta$ выполнено $\mathcal{L}\left(\mathrm{X}^{-1}\right)$ 으 $(\mathcal{L}(\mathrm{X}))^{-1}$ и для любых путей $\mathrm{X}, \mathrm{Y}$ при $\tau(\mathrm{X})=\iota(\mathrm{Y})$ выполнено 
$\mathcal{L}(\mathrm{XY})$ 으 $\mathcal{L}(\mathrm{X}) \mathcal{L}(\mathrm{Y})$. Значение функции $\mathcal{L}(\mathrm{X})$ называется меткой пути $\mathrm{X}$.

Пусть

$$
\mathcal{P}=\langle\mathcal{A} \mid R=1 \quad(R \in \mathcal{D})\rangle
$$

- задание некоторой группы G. Карта $\Delta$ называется диаграммой над задани$e \mathcal{M} \mathcal{P}$, если на $\Delta$ задана размечающая функция $\mathcal{L}_{\Delta}$ над алфавитом $\mathcal{A}$ такая, что для любой области А карты $\Delta$ слово $\mathcal{L}_{\Delta}(\delta \mathrm{A})$ либо пусто, либо имеет вид $a^{\epsilon} a^{-\epsilon}$ для некоторой буквы $a^{\epsilon} \in \mathcal{A}^{ \pm 1}$, либо есть циклический сдвиг слова вида $R^{ \pm 1}$, где $R \in \mathcal{D}$. (это один из вариантов определения диаграммы Линдона-ван Кампена, по-видимому, впервые использованный в [7]). В дальнейшем индекс $\Delta$ в записи $\mathcal{L}_{\Delta}$ будет обычно опускаться.

Следующее известное утверждение устанавливает связь между соотношениями равенства и сопряженности в группе $\mathbf{G}$ и диаграммами над заданием $\mathcal{P}$ (см. $[3$, гл. V, теорема 1.1, лемма 5.2], [7, леммы 11.1 и 11.2]).

4.1. ПРЕДЛОЖЕНИЕ. (i) Для любого слова $X$, равного 1 в группе $\mathbf{G}$, существует дисковая диаграмма $\Delta$ над заданием $\mathcal{P}$ с $\mathcal{L}(\delta \Delta) \approx X$.

(ii) Если слова X и $Y$ сопряжены в $\mathbf{G}$, то существует кольцевая диаграмма над заданием $\mathcal{P}$ с внешним граничным ииклом $\mathrm{C}$ и внутренним граничнымм чиклом $\mathrm{D}$ такая, ито $\mathcal{L}(\mathrm{C}) \approx X u(\mathcal{L}(\mathrm{D}))^{-1} \approx Y$.

Из определения гомотопных путей на карте непосредственно вытекает

4.2. Лемма. Если $\mathrm{X} u \mathrm{Y}$-гомотопные пути на диаграмме $\Delta$ над заданием $\mathcal{P}$, то слова $\mathcal{L}(\mathrm{X})$ и $\mathcal{L}(\mathrm{Y})$ равны в группе $\mathbf{G}$.

Из леммы 4.2 вытекает следуюшее обрашение п. (i) предложения 4.1: для любой односвязной (в частности, дисковой) диаграммы $\Delta$ в $\mathbf{G}$ имеет место равенство $\mathcal{L}(\delta \Delta)=1$. Нам понадобится также следующее обрашение п. (ii) этого предложения.

4.3. ЛЕмма. Если $\Delta$ - диаграмма над заданием $\mathcal{P}$ с двумя граничными циклами, $\mathrm{C}$ и $\mathrm{D}$, то слова $\mathcal{L}(\mathrm{C})$ и $(\mathcal{L}(\mathrm{D}))^{-1}$ сопряжены в группе $\mathbf{G}$. Более того, для любого пути $\mathrm{T}$ на $\Delta$, соединяющего вершинь $\iota(\mathrm{C})$ и $\iota(\mathrm{D}), \varepsilon \mathbf{G}$ истинно равенство $\mathcal{L}(\mathrm{C})=\mathcal{L}(\mathrm{T})(\mathcal{L}(\mathrm{D}))^{-1}(\mathcal{L}(\mathrm{T}))^{-1}$.

ДокАЗАТЕльство. Пусть $\mathrm{S}$ - путь на $\Delta$ наименьшей длины (т.е. содержаший наименьшее возможное число ориентированных ребер), соединяюший некоторую вершину, лежашую на C, с некоторой вершиной, лежашей на D. Ясно, что S - простой путь, не содержащий отличных от $\iota(\mathrm{S})$ и $\tau(\mathrm{S})$ вершин в пересечении с гранищей диаграммы $\Delta$.

Пусть $\mathrm{C}=\mathrm{C}_{1} \mathrm{C}_{2}$ и $\mathrm{D}=\mathrm{D}_{1} \mathrm{D}_{2}$, где $\tau\left(\mathrm{C}_{1}\right)=\iota(\mathrm{S})$ и $\tau\left(\mathrm{D}_{1}\right)=\tau(\mathrm{S})$ (рис. 2). Разрезав диаграмму $\Delta$ вдоль $\mathrm{S}$, получим односвязную диаграмму $\Delta^{*}$ с граничным циклом $\mathrm{C}_{2}^{*} \mathrm{C}_{1}^{*} \mathrm{~S}^{*} \mathrm{D}_{2}^{*} \mathrm{D}_{1}^{*} \mathrm{~S}^{\prime-1}$, где метки путей $\mathrm{C}_{i}^{*}, \mathrm{D}_{i}^{*}, \mathrm{~S}^{*}$ и $\mathrm{S}^{\prime}$ равны меткам соответствующих путей $\mathrm{C}_{i}, \mathrm{D}_{i}$ и $\mathrm{S}$. Тогда по предложению 4.1 , (i) в $\mathbf{G}$ имеет место равенство

$$
\mathcal{L}\left(\mathrm{C}_{2} \mathrm{C}_{1} \mathrm{SD}_{2} \mathrm{D}_{1} \mathrm{~S}^{-1}\right)=1
$$




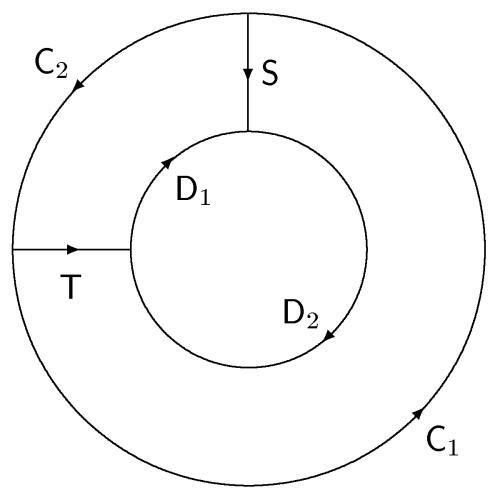

Рис. 2

откуда, обозначив $\mathrm{R} \rightleftharpoons \mathrm{C}_{1} \mathrm{SD}_{2}^{-1}$, выводим

$$
\mathcal{L}(\mathrm{C})=\mathcal{L}(\mathrm{R})(\mathcal{L}(\mathrm{D}))^{-1}(\mathcal{L}(\mathrm{R}))^{-1} \text { в } \mathbf{G},
$$

что и доказывает первое утверждение.

Пусть теперь $\mathrm{T}$ - произвольный путь, соединяюший вершины $\iota(\mathrm{C})$ и $\iota(\mathrm{D})$. Так как $\pi_{1}\left(\Delta, \iota\left(\mathrm{C}_{1}\right)\right)$ - циклическая группа, порожденная классом $[\mathrm{C}]_{\Delta}$, то путь $\mathrm{TR}^{-1}$ гомотопен пути $\mathrm{C}^{t}$ при некотором $t$. Следовательно, с учетом леммы 4.2 имеем

$$
\mathcal{L}(\mathbf{T})=(\mathcal{L}(\mathrm{C}))^{t} \mathcal{L}(\mathrm{R}) \text { в } \mathbf{G},
$$

что вместе с (4.2) влечет искомое равенство

$$
\mathcal{L}(\mathrm{C})=\mathcal{L}(\mathbf{T})(\mathcal{L}(\mathrm{D}))^{-1}(\mathcal{L}(\mathrm{T}))^{-1} \text { в } \mathbf{G} .
$$

Диаграмма $\Theta$ называется поддиаграммой диаграммы $\Delta$, если карта $\Theta$ есть подкарта карты $\Delta$ и $\mathcal{L}_{\Theta}=\left.\mathcal{L}_{\Delta}\right|_{\overrightarrow{\mathbf{E}}(\Theta)}$. Если $\Theta$ и $\Theta^{\prime}-$ поддиаграммы диаграммы $\Delta$, то карты $\Theta \cup \Theta^{\prime}, \Delta-\Theta$ и $\Delta-$ int $\Theta$ естественным образом можно рассматривать как поддиаграммы диаграммы $\Delta$.

Пусть $\Delta$ - диаграмма над заданием $\mathcal{P}$. Опишем два типа преобразований, переводящих $\Delta$ в некоторую новую диаграмму $\Delta^{\prime}$ над заданием $\mathcal{P}$, которые будем называть әлементарными измельчениями диаграммы $\Delta$. Если е - неориентированное (т.е. геометрическое) ребро диаграммы, то через ё временно условимся обозначать соответствуюшее ориентированное ребро (при необходимости направление ё будет явно указьваться).
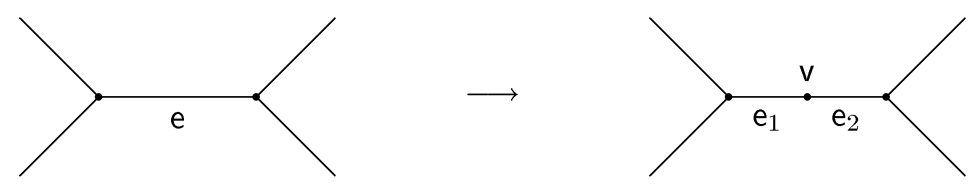

Рис. 3 
(i) Пусть е - ребро диаграммы $\Delta$. Пусть диаграмма $\Delta^{\prime}$ получена из $\Delta$ добавлением новой вершины $v \subset$ е и заменой е на два новых ребра, $e_{1}$ и $e_{2}$, на которые e разбивается вершиной v (см. рис. 3), причем для соответствуюших одинаково направленных ориентированных ребер $\overline{\mathrm{e}}, \overline{\mathrm{e}}_{1}$ и $\overline{\mathrm{e}}_{2}$ выполнено

$$
\mathcal{L}_{\Delta^{\prime}}\left(\overline{\mathrm{e}}_{1}\right) \mathcal{L}_{\Delta^{\prime}}\left(\overline{\mathrm{e}}_{2}\right) \text { 으 } \mathcal{L}_{\Delta}(\overline{\mathrm{e}})
$$

(отсюда следует, в частности, что метка по крайней мере одного из ориентированных ребер $\bar{e}_{i}$ есть пустое слово). Диаграмму $\Delta^{\prime}$ будем называть результатом добавления вериинь $\vee \kappa$ диаграмме $\Delta$.

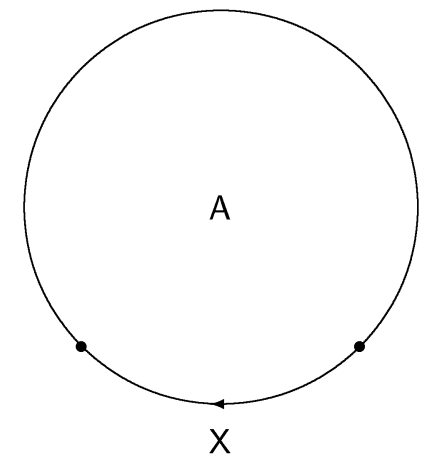

(ii) Пусть А - область диаграммы $\Delta$ и $\mathrm{X}$ - простой непустой путь на $\Delta$, входящий в качестве подпути в граничный цикл области $\mathrm{A}$. Предположим, $\mathcal{L}(\mathbf{X})$ есть буква алфавита $\mathcal{A}^{ \pm 1}$ или пустое слово. Рассмотрим карту $\Delta^{\prime}$, полученную из $\Delta$ добавлением нового ребра е $\subseteq \mathrm{A}$, соединяюшего начальную и конечную вершины пути $\mathrm{X}$, и заменой А на две новых образовавшихся области, $\mathrm{A}_{1}$ и $\mathrm{A}_{2}$ (см. рис. 4). Превратим $\Delta^{\prime}$ в диаграмму над заданием $\mathcal{P}$, полагая $\mathcal{L}_{\Delta^{\prime}}(\overline{\mathrm{e}}) \rightleftharpoons \mathcal{L}_{\Delta}(\mathrm{X})$, где ориентация ребра ё та же, что и у пути $\mathrm{X}$, т.е. $\iota(\overline{\mathrm{e}})=\iota(\mathrm{X})$ и $\tau(\overline{\mathrm{e}})=\tau(\mathrm{X})$. Диаграмму $\Delta^{\prime}$ будем называть результатом добавления ребра е $к$ диаграмме $\Delta$ вдоль пути $\mathrm{X}$.

Если диаграмма $\Delta^{\prime}$ получена из $\Delta$ некоторой (возможно, пустой) последовательностью элементарных измельчений, то будем говорить, что $\Delta^{\prime}$ есть результат измельчения диаграммы $\Delta$.

Отметим ряд свойств этого понятия, легко вытекаюших из определения.

Если диаграмма $\Delta^{\prime}$ есть результат измельчения диаграммы $\Delta$, то $\Delta$ и $\Delta^{\prime}$ совпадают как подмножества $\mathbb{R}^{2}$.

Каждому пути $\mathrm{X}$ на диаграмме $\Delta$ естественным образом соответствует путь $\mathrm{X}^{\prime}$ на диаграмме $\Delta^{\prime}$, который будем называть образом на диаграмме $\Delta^{\prime}$ пути $\mathrm{X}$. Для этого в случае, когда $\Delta^{\prime}$ получена из $\Delta$ добавлением ребра, положим $\mathrm{X}^{\prime} \rightleftharpoons \mathrm{X}, \mathrm{a}$ в случае, когда $\Delta^{\prime}$ получена из $\Delta$ добавлением вершины, в качестве $X^{\prime}$ возьмем результат замены в $X$ всех вхождений ориентированного ребра $\overline{\mathrm{e}}^{ \pm 1}$ на $\left(\overline{\mathrm{e}}_{1} \overline{\mathrm{e}}_{2}\right)^{ \pm 1}$ (в обозначениях из (i)). В общем случае, когда $\Delta^{\prime}$ получена из $\Delta$ некоторой последовательностью элементарных измельчений, понятие образа пути определяется естественным образом по индукции. Очевидно, если путь $\mathrm{X}^{\prime}$ есть образ на диаграмме $\Delta^{\prime}$ 


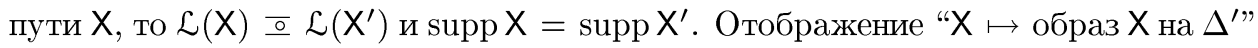
сохраняет начальную и конечную вершины пути, операции произведения путей и взятия обратного пути и свойство пути быть граничным циклом диаграммы $\Delta$. Легко проверить также, что пути $\mathrm{X}$ и $\mathrm{Y}$ на $\Delta$ гомотопны тогда и только тогда, когда гомотопны их образы на $\Delta^{\prime}$, и что указанное соответствие путей на диаграммах $\Delta$ и $\Delta^{\prime}$ индуцирует изоморфизм фундаментальных групा $\pi_{1}\left(\Delta\right.$, a) $\rightarrow \pi_{1}\left(\Delta^{\prime}\right.$, a).

Каждой поддиаграмме $\Theta$ диаграммы $\Delta$ соответствует подлиаграмма $\Theta^{\prime}$ диаграммы $\Delta^{\prime}$, которая совпадает с $\Theta$ как подмножество $\mathbb{R}^{2}$. Легко видеть, что диаграмма $\Theta^{\prime}$ есть результат измельчения диаграммы $\Theta$, причем граничные циклы диаграммы $\Theta^{\prime}$ являются образами на диаграмме $\Delta^{\prime}$ соответствуюших граничных циклов диаграммы $\Theta$.

Наконец, отметим, что между областями диаграмм $\Delta$ и $\Delta^{\prime}$, имеюшими нетривиальную метку (т.е. не равную 1 в свободной групе), существует взаимно однозначное соответствие, сохраняющее метку граничного цикла области.

В дальнейшем измельчения диаграмм будут играть роль "несущественных" преобразований диаграмм, так как все рассматриваемые ниже свойства диаграмм, их поддиаграмм и путей на диаграммах будут сохраняться при измельчениях.

Нам понадобится следующее наблюдение.

4.4. ЛЕмма. Пусть диаграммы $\Delta^{\prime}$ и $\Delta^{\prime \prime}$ получены измельчением диаграммы $\Delta$. Тогда с точностью до изотопной деформачии $\Delta^{\prime} u \Delta^{\prime \prime}$ обладают общим измельчением $\Delta^{*}$.

"С точностью до изотопной деформации" здесь означает, что при выполнении операций әлементарного измельчения, переводящих $\Delta$ в $\Delta^{\prime}$ и в $\Delta^{\prime \prime}$, добавляемые вершины могут сдвигаться вдоль содержащих их ребер, а добавляемые ребра могут перемещаться с закрепленными концами внутри содержащих их областей.

ДокАЗАТЕЛЬСтво. Ввиду очевидной индукции достаточно рассмотреть случай, когда $\Delta^{\prime}$ получена из $\Delta$ применением одной операции элементарного измельчения. Пусть

$$
\Delta=\Delta_{0} \rightarrow \Delta_{1} \rightarrow \cdots \rightarrow \Delta_{t}=\Delta^{\prime \prime}
$$

- последовательность элементарных измельчений, переводящая $\Delta$ в $\Delta^{\prime \prime}$.

Предположим сначала, что диаграмма $\Delta^{\prime}$ получена из $\Delta$ добавлением вершины $\mathrm{v}$, лежащей на ребре е диаграммы $\Delta$. Пусть $\overline{\mathrm{e}}_{1} \overline{\mathrm{e}}_{2}-$ образ ориентированного ребра $\overline{\mathrm{e}}$ на $\Delta^{\prime}$ и $\overline{\mathrm{d}}_{1} \overline{\mathrm{d}}_{2} \ldots \overline{\mathrm{d}}_{r}$ - образ е̄ на $\Delta^{\prime \prime}$. В зависимости от того, метка какого из ребер, $\overline{\mathrm{e}}_{1}$ или $\overline{\mathrm{e}}_{2}$, есть пустое слово, сдвинем вершину $\mathrm{v}$ вдоль е так, чтобы она попала соответственно на $\mathrm{d}_{1}$ или на $\mathrm{d}_{r}$. Пусть диаграмма $\Delta_{i}^{\prime}$ получена из $\Delta_{i}$ добавлением вершины v, где соответственно $\mathcal{L}_{\Delta_{i}^{\prime}}\left(\overline{\mathrm{e}}_{1}\right)$ 으 1 или $\mathcal{L}_{\Delta_{i}^{\prime}}\left(\overline{\mathrm{e}}_{2}\right)$ 으 1 . Тогда, очевидно, $\Delta^{\prime}=\Delta_{0}^{\prime} \rightarrow \Delta_{1}^{\prime} \rightarrow \cdots \rightarrow \Delta_{t}^{\prime}$ - последовательность элементарных измельчений диаграммы $\Delta^{\prime}$ (где каждое элементарное измельчение имеет тот же тип (i) или (ii), что и в последовательности (4.3)), и в качестве $\Delta^{*}$ можно взять $\Delta_{t}^{\prime}$.

Пусть теперь диаграмма $\Delta^{\prime}$ получена из $\Delta$ добавлением ребра е, расположенного внутри области А диаграммы $\Delta$. Как и в предыдушем случае, мы построим последовательность $\Delta^{\prime}=\Delta_{0}^{\prime} \rightarrow \Delta_{1}^{\prime} \rightarrow \cdots \rightarrow \Delta_{t}^{\prime}$ измельчений диаграммы $\Delta^{\prime}$, 
где $\Delta_{i+1}^{\prime}$ есть результат измельчения диаграммы $\Delta_{i}^{\prime}$ (но теперь измельчения, вообще говоря, не будут элементарными). Если $\Delta_{1}$ получена из $\Delta_{0}$ добавлением некоторой вершины $\mathrm{v}$, то, как мы видели выше, передвинув вершину $\mathrm{v}$ и добавив $\mathrm{v}$ к диаграмме $\Delta^{\prime}=\Delta_{0}^{\prime}$, получим диаграмму $\Delta_{1}^{\prime}$, которая одновременно является результатом добавления ребра к $\Delta_{1}$. В этом случае работает индукция по длине последовательности (4.3).

Если $\Delta_{1}$ получена из $\Delta_{0}$ добавлением ребра $\mathrm{d}$ и $\mathrm{e} \cap \mathrm{d}=\varnothing$, то $\mathrm{d}$ можно добавить к $\Delta_{0}^{\prime}$, и в этом случае получается диаграмма $\Delta_{1}^{\prime}$, которая одновременно является результатом добавления ребра е к $\Delta_{1}$. Тогда снова работает индукция по длине последовательности (4.3).

Пусть $\Delta_{1}$ получена из $\Delta_{0}$ добавлением ребра $\mathrm{d}$, где е $\cap \mathrm{d} \neq \varnothing$. В частности, $\mathrm{d} \subseteq \mathrm{A}$. Продеформировав е, можно считать, что е и $\mathrm{d}$ пересекаются в одной точке (иначе, продеформировав е, можно добиться, чтобы кривые е и $\mathrm{d}$ не пересекались, и тогда этот случай сводится к предыдущему). Пусть $\mathrm{w} \rightleftharpoons\{x\}=\mathrm{e} \cap \mathrm{d}$. Пусть $\mathrm{P}$ и $\mathrm{Q}$ - пути, входящие в граничный цикл области $\mathrm{A}$, вдоль которых добавляются ребра е и $\mathrm{d}$ соответственно. Тогда либо $\mathrm{P}=\mathrm{RS}, \mathrm{Q}=\mathrm{ST}$, либо $\mathrm{P}=\mathrm{ST}, \mathrm{Q}=\mathrm{RS}$, где $\mathrm{R}, \mathrm{S}$ и $\mathrm{T}$ - непустые пути. В силу полной аналогии ограничимся рассмотрением случая, когда $\mathrm{P}=\mathrm{RS}$ и $\mathrm{Q}=\mathrm{ST}$. Пусть диаграмма $\Delta_{1}^{\prime}$ получена из $\Delta$ добавлением вершины ш и четырех ребер, $\mathrm{e}_{1}, \mathrm{e}_{2}, \mathrm{~d}_{1}$ и $\mathrm{d}_{2}$, где $\mathcal{L}\left(\overline{\mathrm{e}}_{1}\right)$ 드 $\mathcal{L}(\mathrm{R}), \mathcal{L}\left(\overline{\mathrm{e}}_{2}\right)$ 드 $\mathcal{L}(\mathrm{S})$, $\mathcal{L}\left(\overline{\mathrm{d}}_{1}\right)$ 으 1 и $\mathcal{L}\left(\overline{\mathrm{d}}_{2}\right)$ 으 $\mathcal{L}(\mathrm{ST})$ (см. рис. 5 , где показана ориентация ребер $\overline{\mathrm{e}}_{i}$ и $\left.\overline{\mathrm{d}}_{i}\right)$. Тогда $\Delta_{1}^{\prime}$ есть результат добавления вершины $\mathbf{w}$ и двух ребер к каждой из диаграмм $\Delta_{1}$ и $\Delta^{\prime}: \mathrm{e}_{1}, \mathrm{e}_{2}$ для $\Delta_{1}$ и $\mathrm{d}_{1}, \mathrm{~d}_{2}$ для $\Delta^{\prime}$.

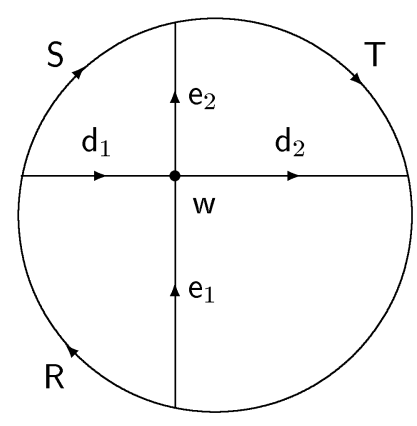

Рис. 5

Если $t \geqslant 2$ и диаграмма $\Delta_{2}$ есть результат добавления вершины к $\Delta_{1}$, то построение диаграммы $\Delta_{2}^{\prime}$ сводится к уже рассмотренному вьше случаю, когда $\Delta^{\prime}$ есть результат добавления вершины к $\Delta$ (нужно положить $\Delta:=\Delta_{1}, \Delta^{\prime}:=\Delta_{2}$ и $\left.\Delta^{\prime \prime}:=\Delta_{1}^{\prime}\right)$. Если $\Delta_{2}$ есть результат добавления ребра $\mathrm{f}_{\mathrm{k}} \Delta_{1}$, то $\mathrm{f}$ может пересекаться только с одним из ребер $\mathrm{e}_{1}$ или $\mathrm{e}_{2}$. Пусть, скажем, $\mathrm{f} \cap \mathrm{e}_{1}=\varnothing$. Так как $\mathrm{e}_{1}$ и е $\mathrm{e}_{2}$ расположены внутри двух различных областей диаграммы $\Delta_{1}$, то для того, чтобы получить диаграмму $\Delta_{2}^{\prime}$, нужно сначала добавить ш и $\mathrm{e}_{1} \mathrm{\kappa} \Delta_{1}$, а затем применить предыдущее рассуждение при е $:=e_{2}$ и $\mathrm{d}:=\mathrm{d}$. 
В общем случае диаграмма $\Delta_{i}^{\prime}$ есть результат добавления к $\Delta_{i}$ некоторого множества вершин и ребер $\mathrm{e}_{1}, \mathrm{e}_{2}, \ldots, \mathrm{e}_{k}$, где $\mathrm{e}_{i}$ расположены внутри попарно различных областей диаграммы $\Delta_{i}$, и диаграмма $\Delta_{i}^{\prime}$ строится аналогичным образом.

Опишем несколько ситуаций, в которых будут использоваться измельчения диаграмм.

4.5. Разложение пути на непустые сомножители. Пусть $X$ - непустой путь на диаграмме $\Delta$ и задано произвольное разбиение слова $\mathcal{L}(\mathrm{X})$ в произведение подслов $\mathcal{L}(\mathrm{X})$ 을 $Y_{1} Y_{2} \ldots Y_{k}$. Тогда $\mathrm{X}$ можно представить в виде $\mathrm{X}=\mathrm{Y}_{1} \mathrm{Y}_{2} \ldots \mathrm{Y}_{k}$, где $\mathcal{L}\left(\mathrm{Y}_{i}\right)$ ㅇ $Y_{i}$ при всех $i$. В дальнейшем нам понадобится такое разложение пути $\mathrm{X}$ с дополнительным условием, чтобы все пути $\mathrm{Y}_{i}$ были непусты (в общем случае, если $Y_{i}$ - пустое слово, то путь $\mathrm{Y}_{i}$ может оказаться пустым). Покажем, как добиться вьполнения этого условия с помощью операции измельчения. Предположим, один из сомножителей $Y_{i}-$ пустой путь. Выберем $Y_{i}$ так, чтобы один из соседних сомножителей, $Y_{i-1}$ или $Y_{i+1}$, был непуст. Пусть, например, это $Y_{i-1}$. Пусть $\mathrm{Y}_{i-1}=\mathrm{Y}_{i-1}^{\prime}$ е, где е - ориентированное ребро. Добавим к $\Delta$ новую вершину таким образом, чтобы ребро е заменилось на произведение $\mathrm{fg}$ двух ребер $\mathrm{f}$ и $\mathrm{g}$, где $\mathcal{L}(\mathrm{f})$ 으 $\mathcal{L}(\mathrm{e})$ и $\mathcal{L}(\mathrm{g})$ 으 1 . Заменяя $\mathrm{Y}_{i-1}$ и $\mathrm{Y}_{i}$ соответственно на $\mathrm{Y}_{i-1}^{\prime} \mathrm{f}$ и $\mathrm{g} \mathrm{Y}_{i}$, получим произведение $\mathrm{Y}_{1} \mathrm{Y}_{2} \ldots \mathrm{Y}_{k}$, в котором метки путей $\mathrm{Y}_{j}$ не изменились, но путь $\mathrm{Y}_{i}$ стал непустым. Продолжая аналогичным образом, придем к диаграмме $\Delta^{\prime}$, полученной измельчением из $\Delta$, у которой образ пути $\mathrm{X}$ имеет вид $\mathrm{Y}_{1} \mathrm{Y}_{2} \ldots \mathrm{Y}_{k}$, где $\mathcal{L}\left(\mathrm{Y}_{i}\right)$ 으 $Y_{i}$ при всех $i$ и все пути $\mathrm{Y}_{i}$ непусты.

4.6. Склейка участков границы диаграммы. Пусть $\Delta$ - неособая диаграмма над заданием $\mathcal{P}$ и некоторый граничный цикл $\Delta$ имеет вид $\mathrm{RXSY}^{-1}$, где $\mathrm{X}$ и $\mathrm{Y}$ - непустые пути и $\mathcal{L}(\mathrm{X})$ 으 $\mathcal{L}(\mathrm{Y})$. Добавляя новые вершины к диаграмме $\Delta$, можно получить диаграмму $\Delta^{\prime}$ такую, что образы $\mathrm{X}^{\prime}$ и $\mathrm{Y}^{\prime}$ на $\Delta^{\prime}$ путей $\mathrm{X}$ и $\mathrm{Y}$ имеют вид $\mathrm{X}^{\prime}=\mathrm{e}_{1} \mathrm{e}_{2} \ldots \mathrm{e}_{r}$ и $\mathrm{Y}^{\prime}=\mathrm{d}_{1} \mathrm{~d}_{2} \ldots \mathrm{d}_{r}$, где $\mathrm{e}_{1}, \mathrm{~d}_{i}$ - ориентированнье ребра с $\mathcal{L}\left(\mathrm{e}_{i}\right)$ 으 $\mathcal{L}\left(\mathrm{d}_{i}\right)$ для каждого $i$. Склеивая участки границы диаграммы $\Delta^{\prime}$ вдоль путей $\mathrm{X}, \mathrm{Y}$ и отождествляя при этом соответствуюшие ориентированные ребра $\mathrm{e}_{i}$

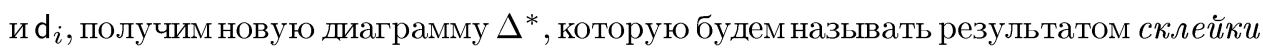
участков границы диаграммы $\Delta$ вдоль путей $\mathrm{X} u \mathrm{Y}$.

4.7. Замена поддиаграммы. Пусть теперь $\Delta$ - произвольная диаграмма над заданием $\mathcal{P}$ и $\Theta$ - неособая (как карта) поддиаграмма диаграммы $\Delta$. Обозначим через $C_{1}$ и $C_{2}, \ldots, C_{t}$ соответственно внешний и внутренние граничные циклы диаграммы $\Theta$. Пусть $\Theta^{*}$ - какая-нибудь другая неособая диаграмма над заданием $\mathcal{P}$ того же топологического типа, что и $\Theta$, имеющая внешний граничный цикл $\mathrm{D}_{1}$ и внутренние граничные циклы $\mathrm{D}_{2}, \ldots, \mathrm{D}_{t}$ такие, что каждая пара соответствующих граничных циклов $\mathrm{C}_{i}$ и $\mathrm{D}_{i}$ имеет вид $\mathrm{C}_{i}^{\prime} \approx \mathrm{e}_{1}^{i} \mathrm{e}_{2}^{i} \ldots \mathrm{e}_{r}^{i}$ и $\mathrm{D}_{i}^{\prime} \approx \mathrm{d}_{1}^{i} \mathrm{~d}_{2}^{i} \ldots \mathrm{d}_{r}^{i}$, где $\mathrm{e}_{j}^{i}$ и $\mathrm{d}_{j}^{i}-$ ориентированные ребра с одинаковыми метками. Мы можем удалить поддиаграмму $\Theta$ из диаграммы $\Delta$ и вклеить вместо нее копию диаграммы $\Theta^{*}$, отождествляя ориентированные ребра $\mathrm{e}_{j}^{i}$ с копиями ориентированных ребер $\mathrm{d}_{j}^{i}$. Полученную в результате диаграмму $\Delta^{*}$ будем называть результатом замены поддиаграм⿻ы $\Theta$ диаграммы $\Delta$ на диаграмму $\Theta^{*}$. Замена подлиаграммы возможна и в более об- 
щей ситуации, когда вместо совпадения меток ориентированных ребер, составляюших граничные циклы поддиаграммы $\Theta$ диаграммы $\Delta$ и диаграммы $\Theta^{*}$, потребовать лиш совпадение меток соответствуюших граничных циклов $C_{i}$ и $\mathrm{D}_{i}$ диаграмм $\Theta$ и $\Theta^{*}$. В этом случае нужно предварительно добавить к диаграммам $\Delta$ и $\Theta^{*}$ новые вершины так, чтобы $\mathrm{C}_{i}$ и $\mathrm{D}_{i}$ получили необходимьй вид.

4.8. Окаймление границы области. Пусть A - произвольная область диаграммы $\Delta$. Рассмотрим последовательность элементарньх измельчений диаграммы $\Delta$, изображенную на рис. 6 , где метки ребер, нарисованных более толстыми линиями, являются пустыми словами, а метки остальных ребер совпадают с метками соответствующих ребер, входящих в граничный цикл области А (каких - ясно из рис. 6).

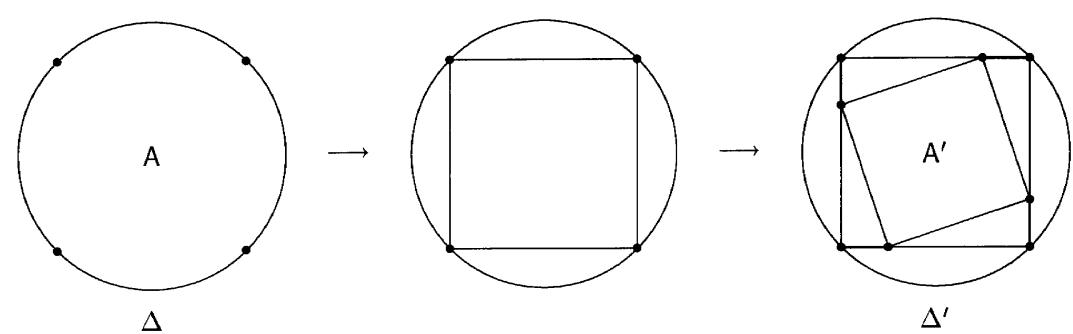

Рис. 6

Если A - область с нетривиальной меткой граничного цикла, то ей соответствует область $A^{\prime}$ на полученной диаграмме $\Delta^{\prime}$, граничный цикл которой - простой замкнутый путь, не пересекающийся с граничными циклами диаграммы $\Delta$ и с граничными циклами остальных областей диаграммы $\Delta$ с нетривиальной меткой граничного цикла. Указанную операцию будем называть окаймлением гранииь области А диаграммы $\Delta$.

4.9. Раздвоение пути. Пусть RXS - простой путь на неособой диаграмме $\Delta$, состоящий из подпутей $\mathrm{R}$ и $\mathrm{S}$, входящих в граничные циклы диаграммы $\Delta$, и непустого пути $\mathrm{X}$, не имеющего отличных от $\iota(\mathrm{X})$ и $\tau(\mathrm{X})$ вершин в пересечении с границей диаграммы $\Delta$. Тогда можно выполнить измельчение диаграммы $\Delta$ таким образом, чтобы на полученной диаграмме $\Delta^{\prime}$ сушествовал путь $\mathrm{Y}$, гомотопный пути RXS, для которого $\mathcal{L}(\mathrm{Y})$ 으 $\mathcal{L}(\mathrm{RXS})$ и $\operatorname{supp} \mathrm{Y} \cap \operatorname{supp} \mathrm{RXS}=\operatorname{supp} \mathrm{Y} \cap \partial \Delta^{\prime}=\iota(\mathrm{R}) \cup \tau(\mathrm{S})$. Из рис. 7 видно, каким образом можно получить диаграмму $\Delta^{\prime}$ (здесь, как и на рис. 6 , метки ребер, нарисованных более толстыми линиями, являются пустыми словами). Указанную операцию будем называть раздвоением пути RSX.

Операции измельчения и замены поддиаграммы будут использоваться при рассмотрении диаграмм над заданиями групп $\mathbf{B}_{\alpha}$ в $\S 9,10$. Нам понадобятся два вспомогательных утверждения о сохранении некоторых свойств диаграмм при выполнении этих операций. 

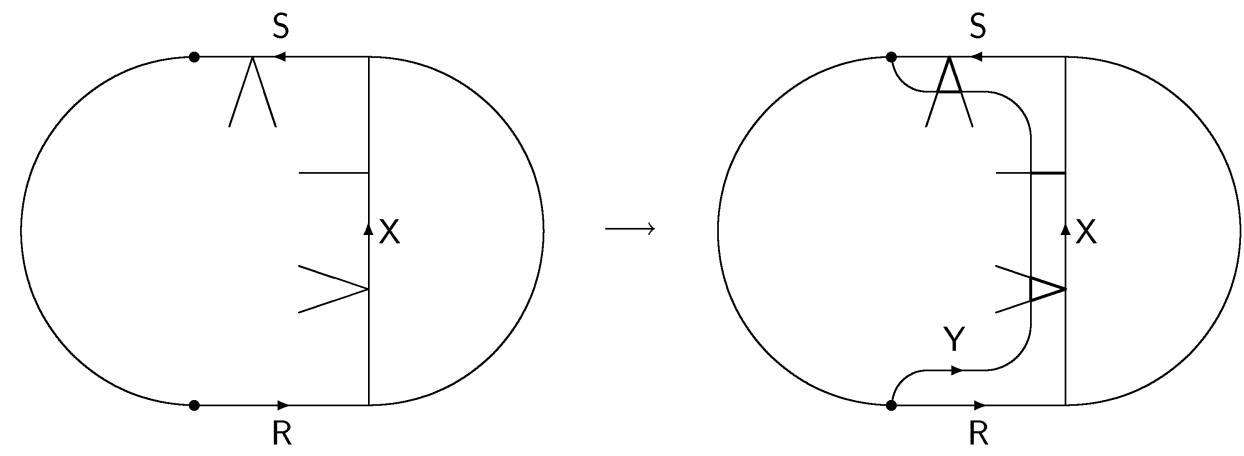

Рис. 7

4.10. Лемма. Пусть $\Delta, \Delta^{\prime}-$ диаграммы над заданием $\mathcal{P} и \Delta^{\prime}$ получена из $\Delta$ некоторой последовательностью измельчений и замен дисковых поддиаграмм. Тогда для любого пути Х на $\Delta$, соединяющего две граничные вериины диаграммы $\Delta$, существует путь $\mathrm{X}^{\prime}$ на диаграмме $\Delta^{\prime}$ с теми же начальной и конечной вериинами, что и у пути $\mathrm{X}$, такой, что $\mathcal{L}(\mathrm{X})=\mathcal{L}\left(\mathrm{X}^{\prime}\right)$ в группе $\mathbf{G}$.

ДокАЗАТЕЛЬСтво. Достаточно рассмотреть случай, когда диаграмма $\Delta^{\prime}$ получена из диаграммы $\Delta$ заменой дисковой поддиаграммы $\Theta$ на некоторую дисковую диаграмму $\Theta^{\prime}$. Пусть $\mathrm{X}$ - путь на $\Delta$, соединяюший две граничные вершины диаграммы $\Delta$. Представим $\mathrm{X}$ в виде произведения $\mathrm{X}=\mathrm{Y}_{0} \mathrm{Z}_{1} \mathrm{Y}_{1} \ldots \mathrm{Z}_{k} \mathrm{Y}_{k}$, где пути $Z_{i}$ состоят только из внутренних ребер диаграммы $\Theta$, а пути $Y_{i}$ не содержат таких ребер. Тогда начальная и конечная вершины каждого пути $Z_{i}$ принадлежат границе $\Theta$, и так как $\Theta$ - дисковая диаграмма, то путь $Z_{i}$ гомотопен на $\Theta$ (а значит, и на $\Delta$ ) некоторому пути $Z_{i}^{\prime}$, входящему в граничный цикл диаграммы $\Theta$. В качестве $\mathrm{X}^{\prime}$ возьмем путь $\mathrm{Y}_{0} \mathrm{Z}_{1}^{\prime} \mathrm{Y}_{1} \ldots \mathrm{Z}_{k}^{\prime} \mathrm{Y}_{k}$ на поддиаграмме $\Delta-\operatorname{int} \Theta$ диаграммы $\Delta$, являющейся одновременно и поддиаграммой диаграммы $\Delta^{\prime}$. Так как пути $\mathrm{X}$ и $\mathbf{X}^{\prime}$ гомотопны на $\Delta$, то по лемме 4.2 в $\mathbf{G}$ истинно равенство $\mathcal{L}(\mathbf{X})=\mathcal{L}\left(\mathbf{X}^{\prime}\right)$, что и требовалось доказать.

Из леммы 4.2 следует, что отображение, сопоставляющее каждому пути $\mathrm{X}$ на диаграмме $\Delta$ его метку, индуцирует отображение множества классов гомотопных путей на диаграмме $\Delta$ в групп $\mathbf{G}$, сопоставляюшее каждому классу $[\mathrm{X}]_{\Delta}$ элемент $[\mathcal{L}(\mathbf{X})]_{\mathbf{G}}$. Мы будем обозначать это отображение через $[\mathcal{L}]_{\mathbf{G}}$. Заметим, что ограничение $[\mathcal{L}]_{\mathbf{G}}$ на фундаментальную группу $\pi_{1}(\Delta, a)$ является гомоморфизмом групп.

4.11. ЛЕмма. Пусть $\Delta, \Delta^{\prime}-$ диаграммы с тремя граничными ииклами над заданием $\mathcal{P}$ и $\Delta^{\prime}$ получена из $\Delta$ некоторой последовательностью измельчений и замен дисковых или кольцевых поддиаграмм. Пусть C - некоторый граничныцй иикл диаграммы $\Delta u$ а $\rightleftharpoons \iota(\mathrm{C})=\tau(\mathrm{C})$. Тогда $[\mathcal{L}]_{\mathbf{G}}\left(\pi_{1}\left(\Delta^{\prime}, \mathrm{a}\right)\right)=\mathbf{g}\left([\mathcal{L}]_{\mathbf{G}}\left(\pi_{1}(\Delta, \mathrm{a})\right)\right) \mathbf{g}^{-1}$ для некоторого әлемента $\mathbf{g} \in \mathbf{G}$, комммутирующего $c[\mathcal{L}(\mathrm{C})]_{\mathbf{G}}$. 
ДокАЗАТЕЛЬСтво. Достаточно рассмотреть случай, когда диаграмма $\Delta^{\prime}$ получена из диаграммы $\Delta$ заменой дисковой или кольцевой поддиаграммы $\Theta$ на некоторую (соответственно дисковую или кольцевую) диаграмму $\Theta^{\prime}$. Если $\Theta$ - дисковая диаграмма, то, как мы видели в доказательстве предыдущей леммы, для любого пути $\mathbf{X}$ на $\Delta$ с $\iota(\mathbf{X})=\tau(\mathbf{X})=$ а найдется гомотопный пути $\mathbf{X}$ путь $\mathbf{X}^{\prime}$ на диаграмме $\Delta-\operatorname{int} \Theta$. Отсюда следует, что вложение $\Delta-\operatorname{int} \Theta \subseteq \Delta$ индуцирует эпиморфизм $\pi_{1}\left(\Delta-\operatorname{int} \Theta\right.$, a) $\rightarrow \pi_{1}\left(\Delta\right.$, a). Так как $[\mathcal{L}]_{\mathbf{G}}\left(\pi_{1}(\Delta-\operatorname{int} \Theta\right.$, a) $) \rightarrow$ $[\mathcal{L}]_{\mathbf{G}}\left(\pi_{1}(\Delta, \mathrm{a})\right)$ - вложение, то имеем $[\mathcal{L}]_{\mathbf{G}}\left(\pi_{1}(\Delta-\operatorname{int} \Theta, \mathrm{a})\right)=[\mathcal{L}]_{\mathbf{G}}\left(\pi_{1}(\Delta, \mathrm{a})\right)$. Но диаграмма $\Delta-\operatorname{int} \Theta$ является также и поддиаграммой диаграммы $\Delta^{\prime}$, следовательно, имеем $[\mathcal{L}]_{\mathbf{G}}\left(\pi_{1}(\Delta-\operatorname{int} \Theta\right.$, a) $)=[\mathcal{L}]_{\mathbf{G}}\left(\pi_{1}\left(\Delta^{\prime}, \mathrm{a}\right)\right)$ и, таким образом, $[\mathcal{L}]_{\mathbf{G}}\left(\pi_{1}(\Delta, a)\right)=[\mathcal{L}]_{\mathbf{G}}\left(\pi_{1}\left(\Delta^{\prime}, \mathrm{a}\right)\right)$. Пусть $\Theta-$ кольцевая диаграмма. Тогда диаграмма $\Delta-\operatorname{int} \Theta$ состоит из двух связных компонент $\Delta_{1}$ и $\Delta_{2}$. Можно считать, что $\mathrm{C}$ - граничный цикл диаграммы $\Delta_{1}$. Рассмотрим три случая.
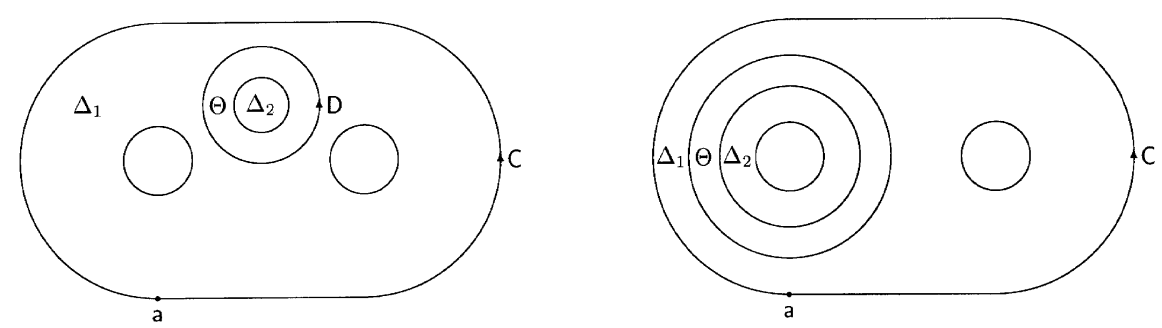

Рис. 8

Случай 1: $\Delta_{2}$ не содержит в качестве граничных циклов ни одного граничного цикла диаграммы $\Delta$, т.е. $\Delta_{2}$ - односвязная диаграмма (см. рис. 8 , слева). Тогда $\Delta^{\prime}$ есть результат замены дисковой поддиаграммы $\Theta \cup \Delta_{2}$ диаграммы $\Delta$ на диаграмму $\Theta^{\prime} \cup \Delta_{2}^{\prime}$, где $\Delta_{2}^{\prime}$ есть копия диаграммы $\Delta_{2}$, приклеенная к $\Theta^{\prime}$. В этом случае по доказанному $[\mathcal{L}]_{\mathbf{G}}\left(\pi_{1}(\Delta, \mathrm{a})\right)=[\mathcal{L}]_{\mathbf{G}}\left(\pi_{1}\left(\Delta^{\prime}, \mathrm{a}\right)\right)$.

Случай 2: $\Delta_{2}$ содержит в качестве граничного цикла ровно один граничный цикл диаграммы $\Delta$ (см. рис. 8 , справа). Пусть $\mathrm{D}$ - граничный цикл кольцевой диаграммы $\Sigma \rightleftharpoons \Theta \cup \Delta_{2}$ такой, что $\mathrm{D}^{-1}$ является граничным циклом диаграммы $\Delta_{1}$. Так как $\pi_{1}(\Sigma, \iota(\mathrm{D}))$ - циклическая группа, порожденная классом $[\mathrm{D}]_{\Sigma}$, то любой путь на $\Sigma$, соединяюший две вершины, лежашие на $D$, гомотопен некоторому пути, состоящему только из ребер, входящих в D. Тогда, рассуждая аналогично доказательству леммы 4.10 , получаем, что любой путь $\mathrm{X}$ на диаграмме $\Delta$ с начальной и конечной вершиной а гомотопен некоторому пути на поддиаграмме $\Delta_{1}$. Отсюда следует, что вложение $\Delta_{1} \subseteq \Delta$ индуцирует эпиморфизм $\pi_{1}\left(\Delta_{1}\right.$, a) $\rightarrow \pi_{1}(\Delta$, a), и, как и в случае дисковой диаграммы $\Theta$, приходим к равенству $[\mathcal{L}]_{\mathbf{G}}\left(\pi_{1}(\Delta, \mathrm{a})\right)=[\mathcal{L}]_{\mathbf{G}}\left(\pi_{1}\left(\Delta^{\prime}, \mathrm{a}\right)\right)$.

Случай 3: $\Delta_{2}$ содержит в качестве граничных циклов два граничных цикла диаграммы $\Delta$ (рис. 9). Пусть $\mathrm{D}$ - граничный цикл диаграммы $\Delta_{2}$ такой, что $\mathrm{D}^{-1}$ является граничныт циклом диаграммы $\Theta$. Обозначим $\mathrm{b} \rightleftharpoons \iota(\mathrm{D})$, и пусть $\mathrm{T}-$ произвольный путь на поддиаграмме $\Delta_{1} \cup \Theta$ диаграммы $\Delta$, соединяюший вершины а 


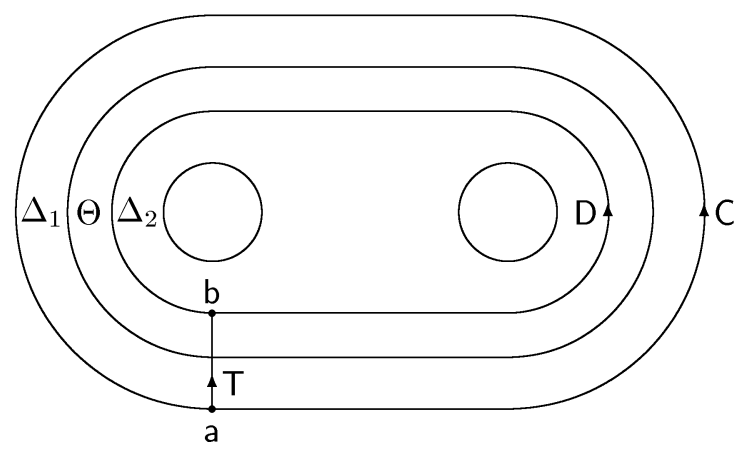

Рис. 9

и b. Рассуждая аналогично доказательству леммы 4.10 , получаем, что любой путь на $\Delta$ c начальной и конечной вершиной ь гомотопен некоторому пути на $\Delta_{2}$. Следовательно, вложение $\Delta_{2} \subseteq \Delta$ индуцирует эпиморфизм $\pi_{1}\left(\Delta_{2}, \mathrm{~b}\right) \rightarrow \pi_{1}(\Delta, \mathrm{b})$, и поэтому имеем $[\mathcal{L}]_{\mathbf{G}}\left(\pi_{1}(\Delta, \mathrm{b})\right)=[\mathcal{L}]_{\mathbf{G}}\left(\pi_{1}\left(\Delta_{2}, \mathrm{~b}\right)\right)$. С другой стороны, по формуле замены начальной вершины для фундаментальной группы имеем

$$
\pi_{1}(\Delta, \mathrm{a})=[\mathrm{T}]_{\Delta} \pi_{1}(\Delta, \mathrm{b})[\mathrm{T}]_{\Delta}^{-1}
$$

Следовательно,

$$
[\mathcal{L}]_{\mathbf{G}}\left(\pi_{1}(\Delta, \mathrm{a})\right)=[\mathcal{L}(\mathbf{T})]_{\mathbf{G}}\left([\mathcal{L}]_{\mathbf{G}}\left(\pi_{1}\left(\Delta_{2}, \mathrm{~b}\right)\right)\right)[\mathcal{L}(\mathbf{T})]_{\mathbf{G}}^{-1} .
$$

Выбрав путь $\mathrm{T}^{\prime}$ на поддиаграмме $\Delta_{1} \cup \Theta^{*}$ диаграммы $\Delta^{\prime}$, где $\Theta^{*}$ есть копия диаграммы $\Theta^{\prime}$, вклеенная вместо $\Theta$, аналогично выводим

$$
[\mathcal{L}]_{\mathbf{G}}\left(\pi_{1}\left(\Delta^{\prime}, \mathrm{a}\right)\right)=\left[\mathcal{L}\left(\mathbf{T}^{\prime}\right)\right]_{\mathbf{G}}\left([\mathcal{L}]_{\mathbf{G}}\left(\pi_{1}\left(\Delta_{2}, \mathrm{~b}\right)\right)\right)\left[\mathcal{L}\left(\mathbf{T}^{\prime}\right)\right]_{\mathbf{G}}^{-1} .
$$

Из двух полученных равенств вытекает

$$
[\mathcal{L}]_{\mathbf{G}}\left(\pi_{1}\left(\Delta^{\prime}, \mathrm{a}\right)\right)=\mathbf{g}\left([\mathcal{L}]_{\mathbf{G}}\left(\pi_{1}(\Delta, \mathrm{a})\right)\right) \mathbf{g}^{-1},
$$

где

$$
\mathbf{g} \rightleftharpoons\left[\mathcal{L}\left(\mathbf{T}^{\prime}\right)\right]_{\mathbf{G}}[\mathcal{L}(\mathrm{T})]_{\mathbf{G}}^{-1} .
$$

Воспользовавшись два раза леммой 4.3 при $\Delta:=\Delta_{1} \cup \Theta$ и $\Delta:=\Delta_{1} \cup \Theta^{*}$, получаем, что в $\mathbf{G}$ истинны равенства

$$
\mathcal{L}(\mathrm{C})=\mathcal{L}(\mathrm{T}) \mathcal{L}(\mathrm{D})\left(\mathcal{L}(\mathrm{T})^{-1}, \quad \mathcal{L}(\mathrm{C})=\mathcal{L}\left(\mathrm{T}^{\prime}\right) \mathcal{L}(\mathrm{D})\left(\mathcal{L}\left(\mathrm{T}^{\prime}\right)^{-1}\right.\right.
$$

откуда вытекает, что $\mathbf{g}$ коммутирует с $[\mathcal{L}(\mathbf{C})]_{\mathbf{G}}$.

4.12. В заключение этого параграфа укажем связь между диаграммами над заданием $\mathcal{P}$ группы $\mathbf{G}$ и графом Кэли $\Gamma=\Gamma(\mathbf{G}, \mathcal{A})$ (где множество порождаюших $\mathcal{A}$ 
то же, что и в $\mathcal{P})$. Пусть $\Delta$ - некоторая дисковая диаграмма над заданием $\mathcal{P}$. Зафиксируем произвольным образом вершину $\mathbf{v}_{0}$ диаграммы $\Delta$ и вершину $\mathbf{g}_{0} \in \mathbf{G}$ графа Г. Для любой вершины v диаграммы $\Delta$ положим $\omega(\mathrm{v}) \rightleftharpoons \mathrm{g}_{0}[\mathcal{L}(\mathrm{P})]_{\mathrm{G}}$, где $\mathrm{P}-$ произвольный путь, соединяюший вершины $\mathrm{v}_{0}$ и v. Так как в силу односвязности $\Delta$ и леммы 4.2 для любых путей $\mathrm{P}, \mathrm{P}^{\prime}$ на $\Delta$ с одинаковыми начальными и конечными вершинами выполнено $[\mathcal{L}(\mathrm{P})]_{\mathbf{G}}=\left[\mathcal{L}\left(\mathrm{P}^{\prime}\right)\right]_{\mathbf{G}}$, то $\omega$-корректно определенное отображение множества вершин диаграммы $\Delta$ во множество вершин графа $\Gamma$.

Определим $\omega$ на ориентированных ребрах диаграммы $\Delta$ следуюшим образом. Если е - ориентированное ребро с $\mathcal{L}(\mathrm{e})$ 으 1 , то положим $\omega(\mathrm{e})=\omega(\iota(\mathrm{e}))=\omega(\tau(\mathrm{e}))$. Иначе, если $\mathcal{L}(\mathrm{e})$ 으 $a^{\epsilon} \in \mathcal{A}^{ \pm 1}$, то, как легко видеть, вершины $\omega(\iota(\mathrm{e}))$ и $\omega(\tau(\mathrm{e}))$ графа $\Gamma$ соединены ребром с меткой $a^{\epsilon}$, которое мы и возьмем в качестве $\omega(\mathrm{e})$. Отображение $\omega$ естественным образом распространяется на множество путей на

диаграмме $\Delta$. Очевидно, $\omega$ сохраняет начальную и конечную вершину пути, его метку, а также операции произведения путей и взятия обратного пути.

\section{§ 5. Основные определения}

Определения 5.1-5.9 даются одновременной индукцией по рангу $\alpha$.

\section{1. База индукиии.}

Группа $\mathbf{B}_{0}$ по определению есть свободная группа с $m$-элементным базисом $\left\{a_{1}, a_{2}, \ldots, a_{m}\right\}$.

Множество $\mathcal{R}_{0}$ слов, приведенных в ранге 0 , состоит из слов, несократимых в свободной группе $\mathbf{B}_{0}$.

Множество $\mathcal{H}_{0}$ по определению состоит из одного пустого слова.

Слова $X$ и $Y$ назовем близкими в ранге 0 , если $X=Y$ в группе $\mathbf{B}_{0}$.

Пусть теперь $\alpha \geqslant 1$ и определены группа $\mathbf{B}_{\alpha-1}$, множества слов $\mathcal{R}_{\alpha-1}, \mathcal{H}_{\alpha-1}$ и отношение “слова $X$ и $Y$ близки в ранге $\alpha-1$ ”.

5.2. Периодом ранга $\alpha$ назовем непустое слово $A$, для которого Пер $(A) \subseteq$ $\mathcal{R}_{\alpha-1}$.

5.3. Период $A$ ранга $\alpha$ минимален, если не сушествует чисел $r \geqslant 1, t \geqslant 2$ и слова $B$ таких, что $A^{r}$ и $B^{t r}$ сопряжены в группе $\mathbf{B}_{\alpha-1}$. Множество всех минимальных периодов ранга $\alpha$ обозначим через $\mathcal{M}_{\alpha}$.

5.4. Минимальные периоды $A, B \in \mathcal{M}_{\alpha}$ назовем родственны.ми в ранге $\alpha$ (обозначаем $\operatorname{Poд}_{\alpha}(A, B)$ ), если для некоторого $t>0$ слова $A^{t}$ и $B^{t}$ сопряжены в $\mathbf{B}_{\alpha-1}$.

5.5. Индукцией по $i$ определим последовательность подмножеств $\mathcal{N}_{\alpha, i} \subseteq \mathcal{M}_{\alpha}$ $(i \geqslant 0)$ :

$$
\begin{gathered}
\mathcal{N}_{\alpha, 0} \rightleftharpoons\left\{A \in \mathcal{M}_{\alpha} \mid \text { существуют близкие в ранге } \alpha-1\right. \text { слова } \\
\left.P \in \text { Пер }(A) \text { и } Q \in \text { Пер }(B, p) \text { при } B \in \mathcal{M}_{\alpha} \text { и } \neg P \text { оод } \alpha(A, B)\right\}, \\
\mathcal{N}_{\alpha, i+1} \rightleftharpoons\left\{A \in \mathcal{M}_{\alpha} \mid \text { существуют близкие в ранге } \alpha-1\right. \text { слова } \\
\left.P \in \Pi е p(A) \text { и } Q \in \Pi е p(B, 10) \text { при } B \in \mathcal{N}_{\alpha, i} \text { и } \neg P \text { оод } \alpha(A, B)\right\},
\end{gathered}
$$


5.6. По определению положим

$$
\mathcal{E}_{\alpha} \rightleftharpoons \mathcal{M}_{\alpha} \backslash \bigcup_{i \geqslant 0} \mathcal{N}_{\alpha, i}
$$

Слова из $\mathcal{E}_{\alpha}$ назовем әлементарнымми периодами ранга $\alpha$. Положим

$$
\mathbf{B}_{\alpha} \rightleftharpoons\left\langle\mathcal{A} \mid A^{n}=1, \quad A \in \bigcup_{\beta=1}^{\alpha} \varepsilon_{\alpha}\right\rangle
$$

5.7. Слово $X$ назовем приведенным в ранге $\alpha$, если $X$ приведено в ранге $\alpha-1$ и не сушествует близких в ранге $\alpha-1$ подслова слова $X$ и слова $P \in \Pi e p(A, n-q)$ при $A \in \mathcal{E}_{\alpha}$. Множество всех приведенных в ранге $\alpha$ слов обозначим через $\mathcal{R}_{\alpha}$.

Слово $X$ назовем чиклически приведенным в ранге $\alpha$, если любой циклический сдвиг слова $X$ есть приведенное в ранге $\alpha$ слово.

5.8. Положим

$$
\mathcal{H}_{\alpha} \rightleftharpoons\left\{u P v \mid u, v \in \mathcal{H}_{\alpha-1}, \quad P-\text { подслово слова } A^{n} \text { при } A \in \mathcal{E}_{\alpha}\right\} .
$$

5.9. Наконец, слова $X$ и $Y$ назовем близкими в ранге $\alpha$, если $X \stackrel{\alpha}{=} u Y v$ для некоторых $u, v \in \mathcal{H}_{\alpha}$ (напомним, что символом $\stackrel{\alpha}{=}$ обозначается равенство слов в группе $\mathbf{B}_{\alpha}$ ).

Индуктивные определения 5.1-5.9 завершены.

Отметим некоторые свойства введеных понятий, которые легко получаются из данных выше определений. Прежде всего, заметим, что все эти понятия инвариантны относительно замены слов на обратные. Это означает, что множества периодов ранга $\alpha$ и множества $\mathcal{M}_{\alpha}, \mathcal{N}_{\alpha, i}, \mathcal{E}_{\alpha}, \mathcal{R}_{\alpha}, \mathcal{H}_{\alpha}$ вместе с каждым словом содержат обратное слово, а отношения “слова $X$ и $Y$ близки в ранге $\alpha$ " и $\operatorname{Poд}_{\alpha}(A, B)$ сохраняются при замене слов на обратные. Этот факт непосредственно вытекает из определений 5.1-5.9 по индукции. Забегая вперед, отметим, что все относящиеся к словам понятия, которые будут вводиться ниже, также инвариантны относительно замены слов на обратные, а все понятия, относящиеся к путям на графах Кэли групп $\mathbf{B}_{\alpha}$, будут инвариантны относительно замены путей на обратные. Как и в рассмотренном выше случае, этот факт будет непосредственно следовать из соответствующих определений (см. также леммы 3.1, (i), 3.2, (v) и 3.4, (iii)), и мы будем постоянно пользоваться им в рассуждениях (ср. приниип симметричности в [1, I.5.3]).

Множества периодов ранга $\alpha, \mathcal{M}_{\alpha}, \mathcal{N}_{\alpha, i}$ и $\mathcal{E}_{\alpha}$ обладают еще одним свойством симметрии: вместе с каждым словом они содержат любой его циклический сдвиг. Это также легко вытекает из определений $5.2,5.3,5.5$ и 5.6 , для множества $\mathcal{N}_{\alpha, i}$ нужно лишь заметить, что в случае, если $A_{1}$ есть циклический сдвиг слова $A$, имеет место эквивалентность $\operatorname{Poд}_{\alpha}(A, B) \Leftrightarrow \operatorname{Poд}_{\alpha}\left(A_{1}, B\right)$.

Отметим еще один факт, непосредственно вытекающий из определения 5.7, который будет постоянно использоваться в дальнейшем: любое подслово приведенного в ранге $\alpha$ слова само является приведенным в ранге $\alpha$ словом. 
5.10. ЛЕмма. Если $\alpha \geqslant 2$, то любой период ранга а является периодом ранга $\alpha-1$ и любой минимальный период ранга $\alpha$ является минимальным периодом ранга $\alpha-1$.

ДокАЗАТЕЛЬСТВо. Для периодов ранга $\alpha$ это следует непосредственно из определения 5.2 и включения $\mathcal{R}_{\alpha} \subseteq \mathcal{R}_{\alpha-1}$. Для минимальных периодов ранга $\alpha$ утверждение вытекает из определения 5.3 , предыдущего утверждения и факта о том, что группа $\mathbf{B}_{\alpha}$ есть гомоморфный образ группы $\mathbf{B}_{\alpha-1}$.

5.11. ЛЕмма. Любой минимальный период ранга $\alpha$ есть простое слово.

ДокАЗАТЕЛЬСТво. Если $A$ - период ранга $\alpha$ и $A$ 으 $B^{t}$ при $t \geqslant 2$, то согласно определению 5.2 слово $B$ есть период ранга $\alpha$, и тогда в силу определения 5.3 имеем $A \notin \mathcal{M}_{\alpha}$.

5.12. Лемма. Для любого $\alpha \geqslant 1$ выполнено $\mathcal{H}_{\alpha-1} \subseteq \mathcal{H}_{\alpha}$.

ДоКАЗАТЕЛЬСТво. Если $u \in \mathcal{H}_{\alpha-1}$, то в качестве $P$ и $v$ в определении 5.8 можно взять пустые слова.

Из леммы 5.12 вытекает, в частности, что при любом $\alpha \geqslant 0$ множество $\mathcal{H}_{\alpha}$ содержит пустое слово, и, следовательно, отношение "слова $X$ и $Y$ близки в ранге $\alpha$ " рефлексивно. Так как из $X \stackrel{\alpha}{=} u Y v$ следует $Y \stackrel{\alpha}{=} u^{-1} X v^{-1}$, то это отношение также и симметрично.

5.13. ЛЕмма. При любом $\alpha \geqslant 1$ выполнено $\mathcal{M}_{\alpha+1} \subseteq \bigcup_{i \geqslant 0} \mathcal{N}_{\alpha, i}$.

ДокаЗАтельСтво. Для любого $A \in \mathcal{E}_{\alpha}$ имеем $A^{n} \notin \mathcal{R}_{\alpha}$ в силу определения 5.7. Тогда в силу определения $5.2 \mathcal{E}_{\alpha} \cap \mathcal{M}_{\alpha+1}=\varnothing$, откуда в силу определения 5.6 и леммы 5.10 выводим $\mathcal{M}_{\alpha+1} \subseteq \bigcup_{i \geqslant 0} \mathcal{N}_{\alpha, i}$.

В системе индуктивных определений 5.14-5.20 будет сушественно использоваться язык графа Кэли $\Gamma\left(\mathbf{B}_{\alpha}, \mathcal{A}\right)$ группы $\mathbf{B}_{\alpha}$ относительно множества порождающих $\mathcal{A}$. Начиная с этого момента, нам будет удобно использовать специальное обозначение

$$
\Gamma_{\alpha} \rightleftharpoons \Gamma\left(\mathbf{B}_{\alpha}, \mathcal{A}\right) .
$$

Следуюшие понятия будут предполагаться известными при переходе от меньших значений ранга $\alpha$ к бо́льшим.

- Множество әлементарных слов ранга $\alpha$ и для любого $r \geqslant 8$ множество элементарных $r$-степеней ранга $\alpha$. Для любого $\beta \geqslant \alpha$ через Эл $\alpha\left(\Gamma_{\beta}\right)$ и Эл $\alpha\left(\Gamma_{\beta}, r\right)$ будут обозначаться множества путей $\mathrm{E}$ на графе $\Gamma_{\beta}$, для которых слово $\mathcal{L}(\mathrm{E})$ есть элементарное слово ранга $\alpha$, или соответственно элементарная $r$-степень ранга $\alpha$. Путь $\mathrm{E} \in \ni{ }_{\alpha}\left(\Gamma_{\beta}\right)$ будем называть әлементарным отрезком ранга а на графе $\Gamma_{\beta}$.

- Отношение ЭСогл $(\mathrm{E}, \mathrm{F})$ согласованности әлементарных отрезков $\mathrm{E}$ $u \mathrm{~F}$ ранга $\alpha$ на графе $\Gamma_{\beta}$ (формально для каждого $\beta$ имеется свое отношение ЭСог, $\Omega_{\alpha}(\mathrm{E}, \mathrm{F})$, значение $\beta$ всегда будет ясным из контекста). 
5.14. Элементарным словом ранга 0 назовем всякое однобуквенное слово. Соответственно әлементарный отрезок ранга 0 на графе $\Gamma_{\beta}$ есть любой путь длины 1 на $\Gamma_{\beta}$. Любое элементарное слово ранга 0 является әлементарной $r$-степенью ранга 0 при любом $r$. Таким образом, Эл 0 ( $\left.\Gamma_{\beta}, r\right)=\ni_{0}\left(\Gamma_{\beta}\right)$.

По определению для любых $\mathrm{E}, \mathrm{F} \in$ Эл $_{0}\left(\Gamma_{\beta}\right)$ отношение $Э С о г \Omega_{0}(\mathrm{E}, \mathrm{F})$ истинно тогда и только тогда, когда $\mathrm{E}=\mathrm{F}$.

Теперь пусть $\alpha \geqslant 1$.

5.15. Число $\beta \leqslant \alpha-1$ назовем рангом активности минимального периода $A \in \mathcal{M}_{\alpha}$, если никакое слово $P \in \Pi$ П $(B)$ не содержит в качестве подслов элементарных $q_{3}$-степеней ранга $\gamma$ при $\beta<\gamma \leqslant \alpha-1$, но некоторое слово $P \in \Pi е p(A)$ содержит элементарную $q_{3}$-степень ранга $\beta$. Ранг активности данного периода $A \in \mathcal{M}_{\alpha}$ обозначим через $\rho(A)$. Так как согласно определению 5.2 минимальный период ранга $\alpha$ есть непустое слово, а согласно определению 5.14 однобуквенное слово является элементарной $q_{3}$-степенью ранга 0 , то ранг активности данного минимального периода $A$ ранга корректно определен.

5.16. Путь $\mathrm{E}$ на графе $\Gamma_{\alpha-1}$ назовем почти периодическим отрезком ранга $\alpha$, порожденным периодом $A \in \mathcal{M}_{\alpha}$, если $\mathcal{L}(\mathrm{E}) \in \mathcal{R}_{\alpha-1}$ и для некоторого $\mathrm{P} \in$ Пер $\left(\Gamma_{\alpha-1}, A, 2\right)$ существуют начало $\mathrm{K}$ и конец $\mathrm{L}$ пути $\mathrm{P}$, начало $\mathrm{K}^{\prime}$ и конец $\mathrm{L}^{\prime}$ пути $\mathrm{E}$

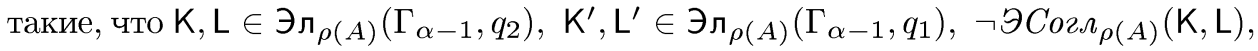
$\vartheta \operatorname{Coz}_{\rho(A)}\left(\mathrm{K}, \mathrm{K}^{\prime}\right)$ и $Э \operatorname{Coz}_{\rho(A)}\left(\mathrm{L}, \mathrm{L}^{\prime}\right)$.

Будем путь $\mathrm{P}$ называть периодической основой для Е. Множество почти периодических отрезков ранга $\alpha$, порожденных данным периодом $A \in \mathcal{M}_{\alpha}$, обозначим через ППер $\alpha$ (A).

Слово $E$ будем называть почти периодическим словом ранга $\alpha$, порожденнымм периодом $A$, если $\mathcal{L}(\mathrm{E})$ 으 $E$ для некоторого $\mathrm{E} \in \Pi_{\text {Пер }}(A)$ (это понятие соответствует понятию целого слова ранга $\alpha$ в [1]). Множество почти периодических слов ранга $\alpha$, порожденных периодом $A$, будем обозначать через ППер $\alpha(A)$.

5.17. Укажем некоторый способ измерения "количества периодов" $A$, содержащихся в почти периодическом слове или почти периодическом отрезке ранга $\alpha$, порожденном $A$. Пусть $A \in \mathcal{M}_{\alpha}$ и $\mathrm{E} \in \Pi_{\text {Пер }}(A)$. Через $\ell_{\alpha, A}(\mathrm{E})$ обозначим наибольшее число $r$ такое, что найдется периодическая основа $\mathrm{P} \in \Pi_{\operatorname{ep}}\left(\Gamma_{\alpha-1}, A, 2\right)$ для $\mathrm{E}$, содержашая в качестве подпутей пути $\mathrm{K}, \mathbf{s}_{A, \mathrm{P}} \mathrm{K}, \ldots, \mathbf{s}_{A, \mathrm{P}}^{r} \mathrm{~K}$, где $\mathrm{K} \in$ $Э_{\rho(A)}\left(\Gamma_{\alpha-1}, q_{2}\right)$.

Если $E \in \Pi \Pi е p_{\alpha}(A)$, то по определению

$$
\ell_{\alpha, A}(E) \rightleftharpoons \ell_{\alpha, A}(\mathrm{E}), \quad \text { где } \mathcal{L}(\mathrm{E}) \text { 드 } E .
$$

Обозначим

$$
\begin{aligned}
& \Pi \Pi е p_{\alpha}(A, r) \rightleftharpoons\left\{\mathrm{E} \in \Pi \Pi e p_{\alpha}(A) \mid \ell_{\alpha, A}(\mathrm{E}) \geqslant r\right\}, \\
& \Pi \Pi e p_{\alpha}(A, r) \rightleftharpoons\left\{E \in \Pi \Pi e p_{\alpha}(A) \mid \ell_{\alpha, A}(E) \geqslant r\right\}=\left\{\mathcal{L}(\mathrm{E}) \mid \mathrm{E} \in \Pi \Pi \operatorname{ep}_{\alpha}(A, r)\right\} .
\end{aligned}
$$

Слово $E \in \Pi$ Пер $\alpha(A, r)$ будем называть почти периодической $r$-степенью ранга $\alpha$, порожденной периодом $A$. 
5.18. Почти периодические отрезки $\mathrm{E} \in$ ППер ${ }_{\alpha}(A)$ и $\mathrm{F} \in \Pi_{\text {Пер }}(B)$ ранга $\alpha$ назовем согласованнылми (обозначаем ПСогл $\Omega_{\alpha}(\mathrm{E}, \mathrm{F})$ ), если согласованы их некоторые периодические основы $\mathrm{P} \in \Pi_{\mathrm{p}}\left(\Gamma_{\alpha-1}, A, 2\right)$ и $\mathrm{Q} \in \Pi_{\mathrm{ep}}\left(\Gamma_{\alpha-1}, B, 2\right)$.

5.19. Элементарным словом ранга $\alpha$ и әлементарной $r$-степенью ранга $\alpha$ при $r \geqslant 8$ назовем любое слово $E \in \Pi$ Пер $\alpha(A, 8)$ или соответственно $E \in$ ППер $\alpha(A, r)$, где $A \in \mathcal{E}_{\alpha}$. Тем самым для любого $\beta \geqslant \alpha$ определены множества Эл $\alpha\left(\Gamma_{\beta}\right)$ и Эл $\alpha\left(\Gamma_{\beta}, r\right)$. Для любого элементарного слова $E$ ранга $\alpha$ и любого пути $\mathrm{E} \in$ Эл $_{\alpha}\left(\Gamma_{\beta}\right)$ положим

$$
\ell_{\alpha}(E) \rightleftharpoons \max _{A \in \mathcal{E}_{\alpha}} \ell_{\alpha, A}(E), \quad \ell_{\alpha}(\mathrm{E}) \rightleftharpoons \ell_{\alpha}(\mathcal{L}(\mathrm{E}))
$$

Очевидно, $E$ есть элементарная $r$-степень ранга $\alpha$ тогда и только тогда, когда $\ell_{\alpha}(E) \geqslant r$.

5.20. Элементарные отрезки $\mathrm{E}, \mathrm{F}$ ранга $\alpha$ на графе $\Gamma_{\beta}$ при $\beta \geqslant \alpha$ назовем согласованными, если для некоторых $A, B \in \mathcal{E}_{\alpha}$ сушествуют их прообразы $\overline{\mathrm{E}} \in \Pi$ Пер $(A, 8), \overline{\mathrm{F}} \in \Pi$ Пер $(B, 8)$ на графе $\Gamma_{\alpha-1}$ относительно отображения $\pi_{\alpha-1, \beta}$, (см. $\S 2,3)$ такие, что ПСогл $\alpha(\overline{\mathrm{E}}, \overline{\mathrm{F}})$ или $\Pi$ Согл $\alpha\left(\overline{\mathrm{E}}, \overline{\mathrm{F}}^{-1}\right)$.

Определения совместной индукцией завершены.

Как уже отмечалось вьше, из определений непосредственно вытекает, что все введенные понятия инвариантны относительно замены слов и путей на графах $\Gamma_{\alpha}$ на обратные. Отметим еще одно важное свойство понятий, связанных с путями на графах $\Gamma_{\alpha}$ : все введенные в пп. 5.14-5.20 множества путей на граффах $\Gamma_{\alpha}$, функции и отношения на них инвариантны относительно действия групшы $\mathbf{B}_{\alpha}$ на $\boldsymbol{\Gamma}_{\alpha}$ левыми сдвигами. Это вытекает из соответствуюших определений и инвариантности всех функций и отношений на графах $\Gamma_{\alpha}$ относительно действия групшы $\mathbf{B}_{\alpha}$, которые эти определения используют (см. $\S 3$, в частности лемму 3.4 , (ii)). Например, из определения 5.16 следует, что если $\mathrm{E} \in$ ППер $\alpha(A)$ и $\mathrm{P} \in$ Пер $\left(\Gamma_{\alpha-1}, A, 2\right)$ - периодическая основа для $\mathrm{E}$, то для любого $\mathbf{g} \in \mathbf{B}_{\alpha-1}$ имеем $\mathbf{g E} \in$ ППер $\alpha(A)$ и $\mathbf{g P}$ - периодическая основа для $\mathbf{g E}$, а из определения 5.17 следует, что $\ell_{\alpha, A}(\mathrm{E})=\ell_{\alpha, A}(\mathrm{gE})$ для любых $\mathrm{E} \in$ ППер $\alpha(A)$ и $\mathbf{g} \in \mathbf{B}_{\alpha-1}$. В качестве следствия отметим, что в (5.1) число $\ell_{\alpha, A}(E)$ не зависит от выбора пути $\mathrm{E} \in \Pi$ Пер $\alpha(A)$, для которого $\mathcal{L}(\mathrm{E})$ 으 $E$.

Так как действие группы $\mathbf{B}_{\alpha-1}$ на графе $\Gamma_{\alpha-1}$ транзитивно на множестве путей с одинаковой меткой, то из определения 5.19 вытекает

5.21. Лемма. Если $\mathrm{E}$ - путь на $\Gamma_{\alpha-1}$, метка которого есть әлементарное слово (әлементарная $r$-степень) ранга $\alpha$, то $\mathrm{E} \in \Pi$ Пер $\alpha(A, 8)$ (соответственно $\mathrm{E} \in \Pi$ Пер $\alpha(A, r))$ для некоторого $A \in \mathcal{E}_{\alpha}$.

\section{$\S 6$. Строго близкие периодические отрезки на графе $\Gamma_{\alpha-1}$}

Начиная с этого параграфа и до конца 116 доказательство всех утвержсдений, формулировка которых зависит от ранга $\alpha$, проводится совместной 
индукцией по $\alpha$. На протяжении $\S 6-16$ мы будем считать фиксированным значение ранга $\alpha$. Все утверждения, за исключением небольшого числа, формулируются и доказываются для значения ранга $\alpha \geqslant 1$. В этих исключительных случаях область значений для $\alpha$ будет указываться сразу перед формулировкой утверждения.

Ввиду совместной индукции все участвующие в ней утверждения в $\S 6-16$ формально равноценны. Тем не менее одна часть утверждений формулируется в виде предложений, а другая - в виде лемм. Это деление условно и отражает лишь авторскую оценку роли того или иного утверждения в общей схеме рассуждений: предложения составляют "каркас" доказательства и в меньшей степени зависимы от конкретной технической реализации (для многих из них можно указать соответствуюшие аналоги в [1] и [7]).

В этом параграфе мы докажем ряд утверждений о взаимном расположении периодических отрезков на графе $\Gamma_{\alpha-1}$ с минимальными в ранге $\alpha$ периодами (предложения 6.10-6.12). В частности, предложения 6.11 и 6.12 дают достаточные условия согласованности двух периодических отрезков на графе $\Gamma_{\alpha-1}$. Результаты этого параграфа играют ту же роль, что и так называемая периодизачия в [1], и в некотором смысле могут рассматриваться как обобщения леммы о наложении периодических слов. При этом вместо периодических слов рассматриваются два периодических отрезка на графе $\Gamma_{\alpha-1}$, а вместо условия графического равенства слов рассматривается некоторое условие "геометрической близости" путей (условие строгой близости путей в ранге $\beta \leqslant \alpha-1$, определение 6.5). Отметим, что в [1] эту роль играют порождающие вхождения ранга $\alpha$ и условие их взаимной нормированности в ранге $\alpha-1$.

В качестве основного инструмента при рассмотрении периодических отрезков на графе $\Gamma_{\alpha-1}$ будут использоваться содержащиеся в них элементарные отрезки ранга $\beta \leqslant \alpha-1$ и отношение согласованности ЭСогл $\beta$, которое в силу индуктивного предположения 8.7 является отношением эквивалентности на множестве $\ni_{\beta}\left(\Gamma_{\alpha-1}\right)$. Мы будем использовать специальное обозначение Эл $\beta(\mathrm{X}, r)$ для множества подпутей $\mathrm{K} \in$ Эл $\left(\Gamma_{\gamma}, r\right)$ данного пути $\mathrm{X}$ на графе $\Gamma_{\gamma}$, т.е. подпутей $\mathrm{K}$ пути $\mathrm{X}$, метка которых есть элементарная $r$-степень ранга $\beta$. Заметим, что в силу индуктивного предположения 11.4 любой путь на графе $\Gamma_{\alpha-1}$ с $\mathcal{L}(\mathrm{X}) \in \mathcal{R}_{\alpha-1}$ является простым. В этом случае на множестве непустых подпутей пути $X$ корректно определены отношения $<\mathrm{x}$ и $\ll \mathrm{x}($ см. $\S 3)$. В частности, в силу определения 5.2 это верно для любого пути $\mathrm{X} \in$ Пер $\left(\Gamma_{\alpha-1}, A\right)$, где $A$ - период ранга $\alpha$.

6.1. ЛЕмма. Если $\mathbf{H}$ - конечная подгруппа группь $\mathbf{G}, \mathbf{g}, \mathbf{h} \in \mathbf{G} u$ иля некоторого $r$ выполнено $\mathbf{h}^{-i-r} \mathbf{g h}^{i} \in \mathbf{H}$ при всех $i \in \mathbb{Z}$, то $\mathbf{g}^{\ell}=\mathbf{h}^{\ell r} \partial$ ля некоторого $\ell>0$.

ДоКАЗАТЕЛЬСТво. При Любом $j>0$ имеем

$$
\mathbf{h}^{-j r} \mathbf{g}^{j}=\mathbf{h}^{-j r} \mathbf{g h}^{(j-1) r} \cdot \mathbf{h}^{-(j-1) r} \mathbf{g h}^{(j-2) r} \cdot \ldots \cdot \mathbf{h}^{-r} \mathbf{g} \in \mathbf{H} .
$$

Так как подгруппа $\mathbf{H}$ конечна, то отсюда следует $\mathbf{h}^{-j r} \mathbf{g}^{j}=\mathbf{h}^{-k r} \mathbf{g}^{k}$ для некоторых $j \neq k$. Тогда имеем $\mathbf{g}^{|j-k|}=\mathbf{h}^{|j-k| r}$. 
6.2. Лемма. Пусть некоторая группа $\mathbf{G}$ действует (слева) на множестве $X$. Пусть $\mathbf{g}, \mathbf{h} \in \mathbf{G}, x_{0}, x_{1}, \ldots, x_{t} \in X$ и для некоторых $r, s \geqslant 0$ выполнено

$$
\mathbf{g} x_{i}=x_{i+r} \quad(i=0,1, \ldots, t-r), \quad \mathbf{h} x_{i}=x_{i+s} \quad(i=0,1, \ldots, t-s) .
$$

Пусть, далее, стабилизатор $\mathbf{H}$ әлемента $x_{0}$ в группе $\mathbf{G}$ конечен и для некоторого $i_{0} \geqslant 0$ удовлетворяет следующему условию: если әлементы $\mathbf{x}, \mathbf{w} \in \mathbf{G}$ таковы, что $\mathbf{x}^{-i} \mathbf{w} \mathbf{x}^{i} \in \mathbf{H}$ при $i=0,1, \ldots, i_{0}$, mо $\mathbf{x}^{-i} \mathbf{w} \mathbf{x}^{i} \in \mathbf{H}$ при любом $i \in \mathbb{Z}$.

Тогда если $t \geqslant r+s+i_{0}-1$ и НОД $(r, s)=1$, то существует әлемент $\mathbf{d} \in \mathbf{G}$ такой, что $\mathbf{g}^{\ell}=\mathbf{d}^{\ell r} u \mathbf{h}^{\ell}=\mathbf{d}^{\ell s}$ для некоторого $\ell>0$.

ДокАЗАТЕЛЬСтво. Ввиду леммы 6.1 достаточно указать элемент $\mathbf{d} \in \mathbf{G}$ такой, что $\mathbf{d}^{-i-r} \mathbf{g} \mathbf{d}^{i} \in \mathbf{H}$ и $\mathbf{d}^{-i-s} \mathbf{h} \mathbf{d}^{i} \in \mathbf{H}$ при любом $i$. Индукцией по $r+s$ докажем утверждение, которое получается заменой условия " $\mathrm{g}^{\ell}=\mathbf{d}^{\ell r}$ и $\mathbf{h}^{\ell}=\mathbf{d}^{\ell s}$ для некоторого $\ell>0$ " в формулировке леммы 6.2 на "gd $\mathbf{d}^{i} \in \mathbf{d}^{i+r} \mathbf{H}$ и $\mathbf{h} \mathbf{d}^{i} \in \mathbf{d}^{i+s} \mathbf{H}$ при любом $i \in \mathbb{Z}$ ". Так как утверждение леммы симметрично относительно перестановки $\mathbf{g}$ и $\mathbf{h}$, то можно считать $r \leqslant s$.

Если $r=0$, то из НОД $(r, s)=1$ следует $s=1$, из $t \geqslant r+s+i_{0}-1$ следует $t \geqslant i_{0}$, а из (6.1) следует $\mathbf{h}^{-i} \mathbf{g h} \mathbf{h}^{i} \in \mathbf{H}$ при $0 \leqslant i \leqslant t$. Тогда в силу условия на $\mathbf{H}$ получаем $\mathbf{h}^{-i} \mathbf{g h}^{i} \in \mathbf{H}$ при любом $i \in \mathbb{Z}$, и можно положить $\mathbf{d} \rightleftharpoons \mathbf{h}$.

Пусть $r \geqslant 1$. Так как из (6.1) следует $\mathbf{g}^{-1} \mathbf{h} x_{i}=x_{i+s-r}(i=0,1, \ldots, t-s)$, то по индуктивному предположению при $\mathbf{g}:=\mathbf{g}, \mathbf{h}=\mathbf{g}^{-1} \mathbf{h}, r:=r, s:=s-r$ и $t:=t-r$ найдется $\mathbf{d} \in \mathbf{G}$ такой, что при любом $i \in \mathbb{Z}$ выполнено $\mathbf{g d}^{i} \in \mathbf{d}^{i+r} \mathbf{H}$ и $\mathbf{g}^{-1} \mathbf{h} \mathbf{d}^{i} \in \mathbf{d}^{i+s-r} \mathbf{H}$. Тогда имеем $\mathbf{h} \mathbf{d}^{i}=\mathbf{g} \cdot \mathbf{g}^{-1} \mathbf{h} \mathbf{d}^{i} \in \mathbf{d}^{i+s} \mathbf{H}$, что и требовалось доказать.

6.3. ЛЕмма. Пусть $\beta \leqslant \alpha-1$, әлементы $\mathbf{g}, \mathbf{h} \in \mathbf{B}_{\alpha-1}$, числа $r, s \geqslant 0 u$ пути $\mathrm{M}_{0}, \mathrm{M}_{1}, \ldots, \mathrm{M}_{t} \in$ Эл $_{\beta}\left(\Gamma_{\alpha-1}\right)$ таковы, что истинны соотношения

$$
\begin{aligned}
& { } \operatorname{Coz}_{\beta}\left(\mathrm{gM}_{i}, \mathrm{M}_{i+r}\right) \quad(i=0,1, \ldots, t-r) \text {, } \\
& \text { ЭСогл }_{\beta}\left(\mathbf{h M}_{i}, \mathbf{M}_{i+s}\right) \quad(i=0,1, \ldots, t-s) \text {. }
\end{aligned}
$$

Если $t \geqslant r+s+4$ и НОД $(r, s)=1$, то существует әлемент $\mathbf{d} \in \mathbf{B}_{\alpha-1}$ такой, что $\mathbf{g}^{\ell}=\mathbf{d}^{\ell r} u \mathbf{h}^{\ell}=\mathbf{d}^{\ell s}$ для некоторого $\ell>0$.

ДОКАЗАТЕЛЬСТВО будет состоять в применении леммы 6.2. В качестве группы $\mathbf{G}$ рассмотрим группу $\mathbf{B}_{\alpha-1}$, а в качестве элементов множества $X-$ классы эквивалентности множества Эл $\beta\left(\Gamma_{\alpha-1}\right)$ по отношению ЭСогл $\beta$. Заметим, что на $X$ корректно определено действие групшы $\mathbf{B}_{\alpha-1}$ ввиду инвариантности отношения $\ni$ Согл $\beta$ относительно действия $\mathbf{B}_{\alpha-1}$. По индуктивному предположению 8.8 стабилизатор класса, содержащего $\mathbf{M}_{0}$, т.е. подгруппа $\mathbf{H}$, состоящая из элементов $\mathbf{x} \in \mathbf{B}_{\alpha-1}$, для которых $Э \operatorname{Cozл}_{\beta}\left(\mathrm{M}_{0}, \mathbf{x} \mathrm{M}_{0}\right)$, конечен. По индуктивному предположению 16.1 подгруппа $\mathbf{H}$ удовлетворяет условию из формулировки леммы 6.2 при $i_{0}=5$. Таким образом, выполнены все условия этой леммы, которая и утверждает сушествование искомого элемента $\mathbf{d}$. 
6.4. ЛЕмма. Пусть $S_{0}, S_{1}, \ldots, S_{k}(k \geqslant 1)$ - произвольные множества, удовлетворяющие следующим условиям:

(i) на $\bigcup_{i} S_{i}$ задано некоторое отношение әквивалентности $a \sim b$;

(ii) для каждого $i$ на $S_{i}$ задано транзитивное отношение $a \prec_{i} b$ такое, что для любых $a, b \in S_{i}$ истинно ровно одно из отношений $a \prec_{i} b, a \sim b$ или $b \prec_{i} a, u$ для любых $a, a^{\prime}, b, b^{\prime} \in S_{i}$, если $a \sim a^{\prime} u b \sim b^{\prime}$, выполнено $a \prec_{i} b \Leftrightarrow a^{\prime} \prec_{i} b^{\prime} ;$ другими словами, отношение $a \prec_{i} b$ индуцирует линейный порядок на классах эквивалентности множсества $S_{i}$ по отношению $a \sim b$;

(iii) еслu $a, b \in S_{i}, a^{\prime}, b^{\prime} \in S_{i+1}, \quad a \sim a^{\prime}, \quad b \sim b^{\prime}$, mo $a \prec_{i} b \Leftrightarrow a^{\prime} \prec_{i+1} b^{\prime}$;

(iv) для каждого $i$ выделено некоторое подмножество устойчивых әлементов множсества $S_{i}$, обладающих следующим свойством: если $a, b, c \in S_{i}$, $a^{\prime}, c^{\prime} \in S_{j}, \quad a \prec_{i} b \prec_{i} c, a \sim a^{\prime}, c \sim c^{\prime}, a^{\prime} \prec_{j} c^{\prime} u b-$ устойчивый әлемент, то существует $b^{\prime} \in S_{j}$ такой, что $b \sim b^{\prime} u a^{\prime} \prec_{j} b^{\prime} \prec_{j} c^{\prime}$.

Пусть әлементы $a_{i}, b_{i} \in S_{i}, a_{i+1}^{\prime}, b_{i+1}^{\prime} \in S_{i+1} \quad(i=0,1, \ldots, k-1)$ устойчивыл, $a_{i} \sim a_{i+1}^{\prime}, \quad b_{i} \sim b_{i+1}^{\prime} u a_{i} \prec_{i} b_{i}$ при всех $i$.

Пусть, наконец, $c_{i} \in S_{i}(i=0,1, \ldots, k-1), \quad c_{0} \sim c_{1} \sim \cdots \sim c_{k-1}$, $a_{i} \preceq_{i} c_{i} \preceq_{i} b_{i}$ при $i=0,1, \ldots, k-1$ (әде $a \preceq_{i} b$ обозначает $a \prec_{i} b$ или $\left.a \sim b\right) u$ $c_{0}-$ устойчивый әлемент.

Тогда существует $c_{k} \in S_{k}$ такой, что $c_{k-1} \sim c_{k}$.

ДОКАЗАТЕЛЬСТВО. Индукция по $k$. Если $k=1$, то по условию имеем $a_{0} \sim a_{1}^{\prime}, \quad b_{0} \sim b_{1}^{\prime}, \quad a_{0} \preceq_{0} c_{0} \preceq_{0} \quad b_{0}, \quad c_{0}-$ устойчивьй элемент, и тогда утверждение вытекает из (iii) и (iv). Пусть $k>1$. В силу (iii), (iv) и устойчивости $c_{0}$ достаточно указать элементы $d_{0}, d_{1}, \ldots, d_{k}, e_{0}, e_{1}, \ldots$, $e_{k}\left(d_{i}, e_{i} \in S_{i}\right)$ такие, что $d_{0} \sim d_{1} \sim \ldots \sim d_{k}, e_{0} \sim e_{1} \sim \ldots$ $\cdots \sim e_{k}$ и $d_{0} \preceq_{0} \quad c_{0} \preceq_{0} e_{0}$. Докажем сушествование элементов $d_{i} \in S_{i}$ с $d_{0} \sim d_{1} \sim \cdots \sim d_{k}$ и $d_{0} \preceq_{0} c_{0}$ (существование элементов $e_{i} \in S_{i}$ с $e_{0} \sim e_{1} \sim \cdots \sim e_{k}$ и $c_{0} \preceq_{0} e_{0}$ доказывается аналогично). Так как по условию $a_{i} \preceq_{i} c_{i}$ при всех $i$, то в силу (iii) достаточно указать цепочку $d_{0} \sim d_{1} \sim \cdots \sim d_{k}$ $\left(d_{i} \in S_{i}\right)$, содержащую некоторьй элемент $a_{i}$. Сделаем это с помощью индукции по $r(r=1,2 \ldots, k)$, на каждом шаге строя последовательность $\left(d_{i}\right)_{i=0}^{r}$, где $d_{i} \in S_{i}, d_{0} \sim d_{1} \sim \cdots \sim d_{r}, d_{i_{0}}=a_{i_{0}}$ для некоторого $i_{0} \leqslant r-1$ и $a_{i} \preceq d_{i}$ для всех $0 \leqslant i \leqslant r-1$. При $r=1$ можно положить $d_{0} \rightleftharpoons a_{0}$ и $d_{1} \rightleftharpoons a_{1}^{\prime}$. Предположим, при некотором $1 \leqslant r \leqslant k-1$ последовательность $\left(d_{i}\right)_{i=0}^{r}$ уже построена. В силу (iі) имеем либо $d_{r} \prec_{r} a_{r}$, либо $a_{r} \preceq_{r} d_{r}$. Если $d_{r} \prec a_{r}$, то, учитывая, что $a_{r} \preceq c_{r}$, и используя (iv) в случае $a_{r} \prec c_{r}$, найдем $d_{i}^{\prime} \in S_{i}(i=0,1, \ldots, r-1)$ такие, что $d_{0}^{\prime} \sim d_{1}^{\prime} \sim \cdots \sim d_{r-1}^{\prime} \sim a_{r}$ и $d_{i} \prec_{i} d_{i}^{\prime} \preceq_{i} c_{i}$. В этом случае в качестве новой последовательности $\left(d_{i}\right)_{i=0}^{r+1}$ можно взять $\left(d_{0}^{\prime}, d_{1}^{\prime}, \ldots, d_{r-1}^{\prime}, a_{r}, a_{r+1}^{\prime}\right)$. В случае $d_{r} \succeq_{r} a_{r}$ по индуктивному предположению леммы при $\left(S_{i}\right)_{i=0}^{k}:=\left(S_{i}\right)_{i=i_{0}+1}^{r+1}$, $\left(a_{i}\right)_{i=0}^{k-1}:=\left(a_{i}\right)_{i=i_{0}+1}^{r}, \quad\left(b_{i}\right)_{i=0}^{k-1}:=\left(b_{i}\right)_{i=i_{0}+1}^{r}$ и $\left(c_{i}\right)_{i=0}^{k-1}:=\left(a_{i_{0}+1}^{\prime}, d_{i_{0}+2}, \ldots, d_{r}\right)$ найдется $d_{r+1} \in S_{r+1}$ такой, что $d_{r} \sim d_{r+1}$. Тогда $\left(d_{i}\right)_{i=0}^{r+1}-$ искомая последовательность.

6.5. ОПРЕДЕЛЕНИЕ. Пусть $0 \leqslant \beta \leqslant \alpha-1$. Будем говорить, что пути $\mathrm{X}$ и $\mathrm{Y}$ на графе $\Gamma_{\alpha-1}$ строго близки в ранге $\beta$, если найдутся начало $\mathrm{K}$ и конец $\mathrm{L}$ пути $\mathrm{X}$, на- 
чало $\mathrm{K}^{\prime}$ и конец $\mathrm{L}^{\prime}$ пути $\mathrm{Y}$ такие, что $\mathrm{K}, \mathrm{L} \in$ Эл $_{\beta}\left(\Gamma_{\alpha-1}, q_{1}\right), \mathrm{K}^{\prime}, \mathrm{L}^{\prime} \in$ Эл $_{\beta}\left(\Gamma_{\alpha-1}, q_{1}\right)$, $\neg \vartheta \operatorname{Coгл}_{\beta}(\mathrm{K}, \mathrm{L}), \quad Э \operatorname{Coгл}_{\beta}\left(\mathrm{K}, \mathrm{K}^{\prime}\right)$ и $Э \operatorname{Coгл}_{\beta}\left(\mathrm{L}, \mathrm{L}^{\prime}\right)$.

6.6. Лемма. Пусть $0 \leqslant \beta \leqslant \alpha-1, k \geqslant 1, \mathrm{Z}_{0}, \mathrm{Z}_{1}, \ldots, \mathrm{Z}_{k}$ - пути на графе $\Gamma_{\alpha-1}$ c $\mathcal{L}\left(Z_{i}\right) \in \mathcal{R}_{\alpha-1} \quad$ и Эл ${ }_{\gamma}\left(Z_{i}, q_{4}\right)=\varnothing$ для всех $\gamma$ при $\beta+1 \leqslant \gamma \leqslant \alpha-1$. Пусть для каждого $i=0,1, \ldots, k-1$ nодпуть $\mathrm{X}_{i}$ nути $\mathrm{Z}_{i}$ u nодпуть $\mathrm{Y}_{i}$ пути $\mathrm{Z}_{i+1}$ строго близки в ранге $\beta$. Пусть, далее, $\mathrm{H}_{i} \in \mathrm{Z}_{\beta}\left(\mathrm{X}_{i}, q_{0}+h\right)$

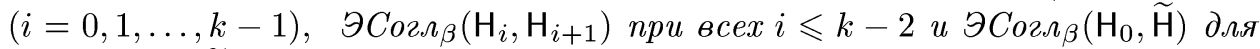
некоторого $\widetilde{\mathrm{H}} \in$ Эл $_{\beta}\left(\mathrm{Z}_{0}, q_{1}\right)$. Тогда существует подпуть $\mathrm{H}_{k} \in$ Эл $_{\beta}\left(\mathrm{Z}_{k}, q_{0}+h\right)$ пути $\mathrm{Z}_{k}$ такой, что $Э \operatorname{Cozл}_{\beta}\left(\mathrm{H}_{k-1}, \mathrm{H}_{k}\right)$.

ДОКАЗАТЕЛЬСТВО состоит в применении леммы 6.4. Положим

$$
\begin{aligned}
S_{i} & \rightleftharpoons \text { Эл }_{\beta}\left(\mathrm{Z}_{i}, q_{0}+h\right), \\
\mathrm{M} \sim \mathrm{N} & \rightleftharpoons \text { ЭСол }(\mathrm{M}, \mathrm{N}), \\
\mathrm{M} \prec_{i} \mathrm{~N} & \rightleftharpoons \mathrm{M}<\mathrm{z}_{i} \mathrm{~N} \& \neg \text { ЭСогл } \beta(\mathrm{M}, \mathrm{N}), \\
\mathrm{M} \mathrm{устойчив} & \rightleftharpoons \mathrm{M} \in \text { Эл }_{\beta}\left(\mathrm{Z}_{i}, q_{1}\right) .
\end{aligned}
$$

Тогда условие (i) леммы 6.4 выполнено в силу индуктивного предположения 8.7, условие (ii) - в силу индуктивных предположений 8.40 и 11.25 , условие (iii) - в силу определения 6.5 и индуктивного предположения 11.27 и условие (iv) - в силу индуктивного предположения 11.23 и равенства $q_{1}=q_{0}+2 h$.

Пусть $\mathrm{K}_{i}, \mathrm{~L}_{i} \in$ Эл $_{\beta}\left(\mathrm{X}_{i}, q_{1}\right)$ - начало и конец $\mathrm{X}_{i}, \mathrm{~K}_{i}^{\prime}, \mathrm{L}_{i}^{\prime} \in$ Эл $_{\beta}\left(\mathrm{Y}_{i}, q_{1}\right)$ - начало и конец $\mathrm{Y}_{i}$, где $\neg$ ЭСогл ${ }_{\beta}\left(\mathrm{K}_{i}, \mathrm{~L}_{i}\right)$, ЭСогл $\beta\left(\mathrm{K}_{i}, \mathrm{~K}_{i}^{\prime}\right)$ и $Э$ Согл $\beta\left(\mathrm{L}_{i}, \mathrm{~L}_{i}^{\prime}\right)$. В силу индуктивного предположения 8.40 имеем $\mathrm{K}_{i} \prec_{i} \mathrm{~L}_{i}$ и $\mathrm{K}_{i} \preceq_{i} \mathrm{H}_{i} \preceq_{i} \mathrm{~L}_{i}(0 \leqslant i \leqslant k-1)$. Так как $Э$ Согл $_{\beta}\left(\mathrm{H}_{0}, \widetilde{\mathrm{H}}\right)$, то в силу индуктивного предположения 11.25 имеем также $\mathrm{K}_{0} \preceq_{0} \widetilde{\mathrm{H}} \preceq_{0} \mathrm{~L}_{0}$. Тем самым мы проверили все условия посылки леммы 6.4 при $a_{i}:=\mathrm{K}_{i}, b_{i}:=\mathrm{L}_{i}, a_{i+1}^{\prime}:=\mathrm{K}_{i}^{\prime}, b_{i+1}^{\prime}:=\mathrm{L}_{i}^{\prime}, c_{0}:=\widetilde{\mathrm{H}}$ и $c_{i}:=\mathrm{H}_{i}(1 \leqslant i \leqslant k-1)$, и в силу этой леммы найдется $\mathrm{H}_{k} \in$ Эл $\beta\left(\mathrm{Z}_{k}, q_{0}+h\right)$ такой, что $Э С о г \Omega_{\beta}\left(\mathrm{H}_{k-1}, \mathrm{H}_{k}\right)$.

Нам будет удобно в дальнейшем использовать следуюшие обозначения, по существу введенные в доказательстве леммы 6.6. Пусть $\mathrm{X}$ - путь на графе $\Gamma_{\alpha-1} \mathrm{c}$ $\mathcal{L}(\mathrm{X}) \in \mathcal{R}_{\alpha-1}$. Для любых $\mathrm{K}, \mathrm{L} \in$ Эл $_{\beta}\left(\mathrm{X}, q_{0}\right)$ положим

$$
\begin{aligned}
& \mathrm{K} \prec \mathrm{x} \mathrm{L} \rightleftharpoons \mathrm{K}<\mathrm{x} L \& \neg \operatorname{COол}_{\beta}(\mathrm{K}, \mathrm{L}), \\
& \mathrm{K} \preceq \mathrm{x} \mathrm{L} \rightleftharpoons \mathrm{K}<\mathrm{xL} \text { или } Э \operatorname{Cолл~}_{\beta}(\mathrm{K}, \mathrm{L}) \text {. }
\end{aligned}
$$

Заметим, что в силу индуктивных предположений 8.7 и 11.25 отношения $\mathrm{K} \prec \mathrm{x} \mathrm{L}$ и $\mathrm{K} \preceq \mathrm{x} \mathrm{L}$ транзитивны и для любых $\mathrm{K}, \mathrm{L} \in$ Эл $_{\beta}\left(\mathrm{X}, q_{0}\right)$ имеет место ровно одно из отношений $\mathrm{K} \prec \mathrm{x} \mathrm{L}$, ЭСогл $\beta(\mathrm{K}, \mathrm{L})$ или $\mathrm{L} \prec \mathrm{x} \mathrm{K}$ (эти два факта уже использовались в доказательстве леммы 6.6).

6.7. Лемма. Если $A$ - период ранга $\alpha$, то әлемент $[A]_{\alpha-1}$ имеет бесконечныи порядок и для любых $\mathrm{P} \in \Pi$ Пер $\left(\Gamma_{\alpha-1}, A, 2\right), \mathrm{K} \in \ni{ }_{\beta}\left(\Gamma_{\alpha-1}\right)$ при $\beta \leqslant \alpha-1$ $u k \neq 0$ выполнено $\neg Э \operatorname{Согл}_{\beta}\left(\mathrm{K}, \mathrm{s}_{A, \mathrm{P}}^{k} \mathrm{~K}\right)$. 
ДокАЗАТЕЛЬСТвО. Первое утверждение вытекает из определения 5.2 и индуктивного предположения 11.4. Второе следует из первого в силу формулы (3.2) и индуктивного предположения 8.8 .

На протяжении оставшейся части параграфа мы будем считать фиксированным некоторое значение ранга $\beta \leqslant \alpha-1$.

6.8. ОПРЕДЕЛЕНИЕ. Пусть $A \in \mathcal{M}_{\alpha}$ и $\mathrm{R}, \mathrm{S} \in$ Пер $\left(\Gamma_{\alpha-1}, A, 2\right)$. На множестве Эл $\beta\left(\Gamma_{\alpha-1}\right)$ введем бинарное отношение

$$
\begin{aligned}
\operatorname{Coоm}_{A, \mathrm{R}, \mathrm{S}}(\mathrm{M}, \mathrm{N}) \rightleftharpoons & \exists \mathbf{g} \in \mathbf{B}_{\alpha-1}\left(\text { ЭСогл } \beta_{\beta}(\mathrm{gM}, \mathrm{N})\right. \text { и } \\
& \mathrm{gR}, \mathrm{S} \text { имеют общее периодическое продолжение }) .
\end{aligned}
$$

(Обозначение связано с тем, что в случае, когда $\mathrm{M}$ содержится в $\mathrm{R}$ и $\mathrm{N}$ содержится в S, истинность $\operatorname{Coom}_{A, \mathrm{R}, \mathrm{S}}(\mathrm{M}, \mathrm{N})$ можно понимать как соответствие по фазе относительно периода $A$ подпути $\mathrm{M}$ пути $\mathrm{R}$ и подпути $\mathrm{N}$ пути $\mathrm{S}$, с точностью до согласованности, см. [1, I.2.5]).

6.9. Лемма. (i) Отношение $\operatorname{Coоm}_{A, \mathrm{R}, \mathrm{S}}(\mathrm{M}, \mathrm{N})$ рефлексивно, симметрично и транзитивно на парах $(\mathrm{R}, \mathrm{M})$ и $(\mathrm{S}, \mathrm{N})$, т.е. имеют место соотношения

$$
\begin{gathered}
\operatorname{Coom}_{A, \mathrm{R}, \mathrm{R}}(\mathrm{M}, \mathrm{M}) \\
\operatorname{Coom}_{A, \mathrm{R}, \mathrm{S}}(\mathrm{M}, \mathrm{N}) \Rightarrow \operatorname{Coom}_{A, \mathrm{~S}, \mathrm{R}}(\mathrm{N}, \mathrm{M}) \\
\operatorname{Coom}_{A, \mathrm{R}, \mathrm{S}}(\mathrm{M}, \mathrm{N}) \& \operatorname{Coom}_{A, \mathrm{~S}, \mathrm{~T}}(\mathrm{~N}, \mathrm{~L}) \Rightarrow \operatorname{Coom}_{A, \mathrm{R}, \mathrm{T}}(\mathrm{M}, \mathrm{L})
\end{gathered}
$$

(ii) Для любых $\mathrm{M}, \mathrm{N}, \mathrm{M}^{\prime}, \mathrm{N}^{\prime} \in \ni_{\beta}\left(\Gamma_{\alpha-1}\right)$ если $Э \operatorname{Coгл}_{\beta}\left(\mathrm{M}, \mathrm{M}^{\prime}\right)$ и $Э \operatorname{Coгл}_{\beta}\left(\mathrm{N}, \mathrm{N}^{\prime}\right)$, mo $\operatorname{Coom}_{A, \mathrm{R}, \mathrm{S}}(\mathrm{M}, \mathrm{N}) \Leftrightarrow \operatorname{Coom}_{A, \mathrm{R}, \mathrm{S}}\left(\mathrm{M}^{\prime}, \mathrm{N}^{\prime}\right)$.

(iii) $\operatorname{Coom}_{A, \mathrm{R}, \mathrm{R}}(\mathrm{M}, \mathrm{N}) \Leftrightarrow \exists i \in \mathbb{Z} \quad \exists \operatorname{Coz}_{\beta}\left(\mathbf{s}_{A, \mathrm{R}}^{i} \mathrm{M}, \mathrm{N}\right)$.

(iv) $E c л и ~ r \geqslant q_{0}$, то для любых $\mathrm{R}, \mathrm{S} \in \prod_{\mathrm{ep}}\left(\Gamma_{\alpha-1}, A, 2\right), \quad \mathrm{M} \in$ Эл $_{\beta}(\mathrm{R}, r) u$ $\mathrm{N} \in$ Эл $_{\beta}(\mathrm{S}, r)$, если $\mathrm{s}_{A, \mathrm{R}} \mathrm{M}$ содержится в $\mathrm{R}$, то найдется $\mathrm{N}^{\prime} \in \ni \boldsymbol{л}_{\beta}(\mathrm{R}, r)$ такой, umo $\operatorname{Coom}_{A, \mathrm{~S}, \mathrm{R}}\left(\mathrm{N}, \mathrm{N}^{\prime}\right) u \mathrm{M} \preceq_{\mathrm{R}} \mathrm{N}^{\prime} \prec_{\mathrm{R}} \mathbf{s}_{A, \mathrm{R}} \mathrm{M}$.

Доказательство. (i) Для доказательства соотношения $\operatorname{Coom}_{A, \mathrm{R}, \mathrm{R}}(\mathrm{M}, \mathrm{M})$ достаточно положить $\mathrm{g}=1$ в определении 6.8. Если $\mathrm{T}$ - общее периодическое продолжение $\mathbf{g R}$ и $\mathrm{S}$, то $\mathbf{g}^{-1} \mathbf{T}$ - общее периодическое продолжение $\mathrm{R}$ и $\mathbf{g}^{-1} \mathrm{~S}$, а из $Э С о г \Omega_{\beta}(\mathrm{gM}, \mathrm{N})$ вытекает $Э С о г \Omega_{\beta}\left(\mathrm{g}^{-1} \mathrm{~N}, \mathrm{M}\right)$. Следовательно, верна импликация $\operatorname{Coom}_{A, \mathrm{R}, \mathrm{S}}(\mathrm{M}, \mathrm{N}) \Rightarrow \operatorname{Coom}_{A, \mathrm{~S}, \mathrm{R}}(\mathrm{N}, \mathrm{M})$. Пусть, далее, истинны отношения $\operatorname{Coom}_{A, \mathrm{R}, \mathrm{S}}(\mathrm{M}, \mathrm{N})$ и $\operatorname{Coom}_{A, \mathrm{~S}, \mathrm{~T}}(\mathrm{~N}, \mathrm{~L})$. Пусть $\mathbf{g}, \mathbf{h} \in \mathbf{B}_{\alpha-1}$ таковы, что $Э \operatorname{Coгл}_{\beta}(\mathrm{gM}, \mathrm{N}), \quad$ ЭСогл $\beta(\mathbf{h N}, \mathrm{L}), \quad \mathrm{gR}$ и $\mathrm{S}$ имеют общее периодическое продолжение $\mathbf{U}, \mathbf{h S}$ и $\mathrm{T}$ имеют общее периодическое продолжение $\mathrm{V}$. C учетом индуктивного предположения 8.7 имеем ЭСогл $\beta(\mathbf{h g M}, \mathrm{L})$, и так как hU и V периодические продолжения $\mathbf{h S}$, то по лемме 3.1 , (iv) $\mathbf{h} \mathbf{U}$ и $\mathbf{V}$ имеют общее периодическое продолжение W. Тогда в силу леммы 3.1 , (iii) W есть общее периодическое продолжение $\mathbf{h g R}$ и $\mathrm{T}$ и, таким образом, истинно отношение $\operatorname{Coom}_{A, \mathrm{R}, \mathrm{T}}(\mathrm{M}, \mathrm{L})$. 
(ii) вытекает непосредственно из определения 6.8 и индуктивного предположения 8.7.

(iii) Если $Э$ Согл $\beta_{\beta}\left(\mathbf{s}_{A, \mathrm{R}}^{i} \mathrm{M}, \mathrm{N}\right)$ для некоторого $i$, то, полагая $\mathbf{g} \rightleftharpoons \mathbf{s}_{A, \mathrm{R}}^{i}$ в определении 6.8, получаем $\operatorname{Coom}_{A, \mathrm{R}, \mathrm{R}}(\mathrm{M}, \mathrm{N})$. Обратно, если периодические отрезки $\mathrm{gR}$ и $\mathrm{R}$ имеют общее периодическое продолжение, то по лемме 3.2 , (vii) имеем $\mathbf{g}=\mathbf{s}_{A, \mathrm{R}}^{i}$ для некоторого $i$ и, следовательно, верна импликация $\operatorname{Coom}_{A, \mathrm{R}, \mathrm{R}}(\mathrm{M}, \mathrm{N}) \Rightarrow \exists i$ $\left(\right.$ ЭСогл $\left.\Omega_{\beta}\left(\mathbf{s}_{A, \mathrm{R}}^{i} \mathrm{M}, \mathrm{N}\right)\right)$.

(iv) Пусть $r \geqslant q_{0}, \mathrm{R}, \mathrm{S} \in \Pi$ Пер $\left(\Gamma_{\alpha-1}, A, 2\right), \mathrm{M} \in$ Эл $_{\beta}(\mathrm{R}, r), \mathrm{N} \in$ Эл $_{\beta}(\mathrm{S}, r)$ и $\mathbf{s}_{A, \mathrm{R}} \mathrm{M}$ содержится в R. По лемме 3.1 , (v) выберем элемент $\mathbf{g} \in \mathbf{B}_{\alpha-1}$ такой, что $\mathbf{g} \mathbf{S}$ и $\mathrm{R}$ имеют обшее периодическое продолжение Т. Имеем $\operatorname{Coom}_{A, \mathrm{~S}, \mathrm{R}}(\mathrm{N}, \mathrm{gN})$, и, следовательно, ввиду (i) и (iii) нам достаточно найти $\mathrm{N}^{\prime} \in$ Эл $_{\beta}(\mathrm{R}, r)$ такой, что $\mathrm{M} \preceq_{\mathrm{R}} \mathrm{N}^{\prime} \prec_{\mathrm{R}}$ $\mathbf{s}_{A, \mathrm{R}} \mathrm{M}$ и $Э$ Согл $\beta\left(\mathbf{s}_{A, \mathrm{R}}^{i} \mathbf{g N}, \mathrm{N}^{\prime}\right)$ для некоторого $i$. Если $Э$ Согл $\beta_{\beta}\left(\mathbf{s}_{A, \mathrm{R}}^{i} \mathbf{g N}, \mathrm{M}\right)$ для некоторого $i$, то можно взять $\mathrm{N}^{\prime} \rightleftharpoons \mathrm{M}$. Пусть $\neg$ ЭСогл $\beta\left(\mathrm{s}_{A, \mathrm{R}}^{i} \mathrm{gN}, \mathrm{M}\right)$ ни для какого $i$. Так как пути $\mathrm{M}, \mathbf{s}_{A, \mathrm{R}} \mathrm{M}$ и $\mathbf{g N}$ содержатся в $\mathrm{T}$, то с учетом лемм 6.7 и 3.2 , (i) имеет место один из трех случаев $\mathrm{M} \prec_{\mathrm{T}} \mathbf{g N} \prec_{\mathrm{T}} \mathbf{s}_{A, \mathrm{R}} \mathrm{M}, \mathrm{gN} \prec_{\mathrm{T}} \mathrm{M}$ или $\mathbf{s}_{A, \mathrm{R}} \mathrm{M} \prec_{\mathrm{T}} \mathbf{g N}$. B первом случае можно положить $\mathrm{N}^{\prime} \rightleftharpoons \mathrm{gN}$. В случае $\mathbf{g N} \prec \mathbf{T}$, взяв максимальное $i$, для которого $\mathrm{s}_{A, \mathrm{R}} \mathrm{gN} \prec \mathrm{T} \mathrm{M}$ и, воспользовавшись леммой 3.2 , (ii), получим $\mathrm{M} \prec \mathbf{U} \mathbf{s}_{A, \mathrm{R}}^{i+1} \mathbf{g N} \prec_{\mathrm{U}} \mathbf{s}_{A, \mathrm{R}} \mathrm{M}$ для некоторого периодического продолжения $\mathrm{U}$ для $\mathrm{T}$, откуда вытекает, что $\mathbf{s}_{A, \mathrm{R}}^{i+1} \mathbf{g N}$ содержится в R и $\mathrm{M} \prec_{\mathrm{R}} \mathbf{s}_{A, \mathrm{R}}^{i+1} \mathbf{g N} \prec_{\mathrm{R}} \mathbf{s}_{A, \mathrm{R}} \mathrm{M}$. В случае $\mathbf{s}_{A, \mathrm{R}} \mathrm{M} \prec \mathbf{T} \mathbf{g N}$, взяв минимальное $i \leqslant 0$, для которого $\mathbf{s}_{A, \mathrm{R}} \mathrm{M} \prec \mathrm{T} \mathbf{s}_{A, \mathrm{R}}^{i} \mathbf{g N}$, аналогично получим $\mathrm{M} \prec_{\mathrm{R}} \mathbf{s}_{A, \mathrm{R}}^{i-1} \mathrm{gN} \prec_{\mathrm{R}} \mathbf{s}_{A, \mathrm{R}} \mathrm{M}$.

6.10. ПРЕДЛОЖЕНИЕ. Пусть $A \in \mathcal{M}_{\alpha}, \quad \rho(A) \leqslant \beta \leqslant \alpha-1$ и пути $\mathrm{P}, \mathrm{Q} \in$ Пер $\left(\Gamma_{\alpha-1}, A, 2\right)$ строго близки в ранге $\beta$. Пусть $\ell \geqslant 4 u \mathrm{~K}, \mathbf{s}_{A, \mathrm{P}} \mathrm{K}, \ldots, \mathbf{s}_{A, \mathrm{P}}^{\ell} \mathrm{K}$ Эл $\beta\left(\mathrm{P}, q_{1}\right)$. Тогда $|\mathrm{Q}| \geqslant 3|A|$ и существует әлемент $\mathbf{g} \in \mathbf{B}_{\alpha-1}$ такой, ито $\mathbf{Q}$ $u \mathrm{gP}$ имеют общее периодическое продолжение и ЭСол $\beta_{\beta}\left(\mathbf{s}_{A, \mathrm{P}}^{i} \mathrm{~K}, \mathbf{g s}_{A, \mathrm{P}}^{i} \mathrm{~K}\right) n p u$ $0 \leqslant i \leqslant \ell$.

ДокаЗАТЕльство. Определим два типа операций на парах вида $(R, M)$, где $\mathrm{R} \in\{\mathrm{P}, \mathrm{Q}\}$ и $\mathrm{M} \in$ Эл $_{\beta}\left(\mathrm{R}, q_{0}+h\right)$. По определению пара $(\mathrm{R}, \mathrm{M})$ переходит в пару $(\mathrm{S}, \mathrm{N})$ в следуюших двух случаях:

(*) $\operatorname{Coom}_{A, \mathrm{R}, \mathrm{S}}(\mathrm{M}, \mathrm{N})$ ( "операция сдвига");

(**) $\{\mathrm{R}, \mathrm{S}\}=\{\mathrm{P}, \mathrm{Q}\}$ и $Э \mathrm{Coгл}_{\beta}(\mathrm{M}, \mathrm{N})$ ("операция перехода").

Для $\mathrm{R} \in\{\mathrm{P}, \mathrm{Q}\}$ через $\mathcal{J}_{\mathrm{R}}$ обозначим множество всех подпутей $\mathrm{M}$ пути $\mathrm{R}$ таких, что пара $(\mathrm{R}, \mathrm{M})$ получается из пары $(\mathrm{P}, \mathrm{K})$ некоторой последовательностью операций $(*)$ и $(* *)$.

Используя лемму 6.6 , докажем, что

если $\mathrm{M} \in \mathcal{J}_{\mathrm{R}}$, то $к$ паре $(\mathrm{R}, \mathrm{M})$ применима опереция перехода $(* *)$, m.е. найдется $\mathrm{N} \in \mathcal{J}_{\mathrm{S}}$ такой, что $Э \operatorname{Coz}_{\beta}(\mathrm{M}, \mathrm{N})$, əде $\{\mathrm{R}, \mathrm{S}\}=\{\mathrm{P}, \mathrm{Q}\} .(6.3)$

Действительно, пусть пара ( $R, M)$ получается из пары $(\mathrm{P}, \mathrm{K})$ некоторой последовательностью операций сдвига и перехода. Заметим, что в силу леммы 6.9 , (i) не- 
сколько последовательных операций сдвига можно заменить одной, а тождественньй переход $(\mathrm{R}, \mathrm{M}) \rightarrow(\mathrm{R}, \mathrm{M})$ можно рассматривать как операцию сдвига. Поэтому без ограничения общности можно считать, что пара $(\mathrm{P}, \mathrm{K})$ переводится в $(\mathrm{R}, \mathrm{M})$ последовательностью следующего вида:

$$
\begin{aligned}
(\mathrm{P}, \mathrm{K})=\left(\mathrm{S}_{0}, \mathrm{~N}_{0}\right) \stackrel{(*)}{\longrightarrow}\left(\mathrm{R}_{1}, \mathrm{M}_{1}\right) \stackrel{(* *)}{\longrightarrow}\left(\mathrm{S}_{1}, \mathrm{~N}_{1}\right) \stackrel{(*)}{\longrightarrow}\left(\mathrm{R}_{2}, \mathrm{M}_{2}\right) \stackrel{(* *)}{\longrightarrow} \ldots \\
\stackrel{(* *)}{\longrightarrow}\left(\mathrm{S}_{k-1}, \mathrm{~N}_{k-1}\right) \stackrel{(*)}{\longrightarrow}\left(\mathrm{R}_{k}, \mathrm{M}_{k}\right)=(\mathrm{R}, \mathrm{M}) .
\end{aligned}
$$

В соответствии с условиями $(*)$ и $(* *)$ имеем

$$
\vartheta_{\operatorname{Coz} \Omega_{\beta}}\left(\mathrm{M}_{i}, \mathrm{~N}_{i}\right), \quad 1 \leqslant i \leqslant k-1
$$

и при $i=0,1, \ldots, k-1$ найдется элемент $\mathbf{g}_{i} \in \mathbf{B}_{\alpha-1}$ такой, что $\mathrm{S}_{i}$ и $\mathbf{g}_{i} \mathrm{R}_{i+1}$ имеют общее периодическое продолжение $\mathrm{T}_{i}$ и

$$
\vartheta^{-\operatorname{Coz}_{\beta}}\left(\mathrm{N}_{i}, \mathbf{g}_{i} \mathrm{M}_{i+1}\right), \quad 0 \leqslant i \leqslant k-1 .
$$

Положим

$$
\mathrm{H}_{i} \rightleftharpoons \mathbf{g}_{0} \mathbf{g}_{1} \ldots \mathbf{g}_{i} \mathrm{M}_{i+1} \quad(0 \leqslant i \leqslant k-1) \text { и } \tilde{\mathrm{H}} \rightleftharpoons \mathrm{K}=\mathrm{N}_{0} .
$$

Тогда из (6.4) и (6.5) вытекает

$$
\ni \operatorname{Coгл}_{\beta}\left(\mathrm{H}_{i}, \mathrm{H}_{i+1}\right) \quad(0 \leqslant i \leqslant k-2) \text { и } \vartheta_{\operatorname{Coг} \Omega_{\beta}}\left(\mathrm{H}_{0}, \widetilde{\mathrm{H}}\right) \text {. }
$$

Пусть, далее,

$$
\begin{array}{rlrl}
\mathrm{X}_{i} \rightleftharpoons \mathbf{g}_{0} \mathbf{g}_{1} \ldots \mathbf{g}_{i} \mathrm{R}_{i+1} & (0 \leqslant i \leqslant k-1), & \mathrm{Y}_{i} \rightleftharpoons \mathbf{g}_{0} \mathbf{g}_{1} \ldots \mathbf{g}_{i} \mathrm{~S}_{i+1} \quad(0 \leqslant i \leqslant k-2), \\
\mathrm{Z}_{i} \rightleftharpoons \mathbf{g}_{0} \mathbf{g}_{1} \ldots \mathbf{g}_{i-1} \mathrm{~T}_{i} \quad(0 \leqslant i \leqslant k-1), & \mathrm{Z}_{k} \rightleftharpoons \mathrm{Y}_{k-1} \rightleftharpoons \mathbf{g}_{0} \mathbf{g}_{1} \ldots \mathbf{g}_{k-1} \mathrm{~S} .
\end{array}
$$

Так как $\mathrm{Z}_{i} \in$ Пер $\left(\Gamma_{\alpha-1}, A\right)$, то $\mathcal{L}\left(\mathrm{Z}_{i}\right) \in \mathcal{R}_{\alpha-1}$ в силу определения 5.2. Кроме того, из неравенства $\rho(A) \leqslant \beta$ в силу определения 5.15 следует $Э л_{\gamma}\left(Z_{i}, q_{4}\right)=\varnothing$ при $\beta+1 \leqslant \gamma \leqslant \alpha-1$. Далее, так как $\left\{\mathrm{R}_{i}, \mathrm{~S}_{i}\right\}=\{\mathrm{P}, \mathrm{Q}\} \quad(1 \leqslant i \leqslant k-1)$, $\left\{\mathrm{R}_{k}, \mathrm{~S}\right\}=\{\mathrm{P}, \mathrm{Q}\}$ и по условию пути $\mathrm{P}$ и $\mathrm{Q}$ строго близки в ранге $\beta$, то пути $\mathrm{X}_{i}$ и $\mathrm{Y}_{i}$ строго близки в ранге $\beta$. Наконец, $\mathrm{X}_{i}$ содержится в $\mathrm{Z}_{i}$ и $\mathrm{Y}_{i}$ содержится в $\mathrm{Z}_{i+1}$. Таким образом, выполнены все условия посылки леммы 6.6 и согласно этой лемме найдется $\mathrm{H}_{k} \in \ni_{\beta}\left(\mathrm{Z}_{k}, q_{0}+h\right)$ такой, что $\ni$ Согл $\Omega_{\beta}\left(\mathrm{H}_{k-1}, \mathrm{H}_{k}\right)$. Тогда с учетом (6.6) получаем

$$
\ni \operatorname{Coz}_{\beta}\left(\mathrm{M}_{k},\left(\mathbf{g}_{0} \mathbf{g}_{1} \ldots \mathbf{g}_{k-1}\right)^{-1} \mathrm{H}_{k}\right)
$$

и, следовательно, в качестве искомого $\mathrm{N}$ можно взять $\left(\mathbf{g}_{0} \mathbf{g}_{1} \ldots \mathbf{g}_{k-1}\right)^{-1} \mathrm{H}_{k}$. Этим завершается доказательство (6.3).

Выберем теперь представители $\left\{\mathrm{M}_{i}\right\}_{i=0}^{t}$ для всех классов эквивалентности множества $\mathcal{J}_{\mathrm{P}}$ по отношению эквивалентности $Э$ Согл $\beta$. Можно считать, что

$$
M_{0} \prec_{\mathrm{P}} \mathrm{M}_{1} \prec_{\mathrm{P}} \mathrm{M}_{2} \prec_{\mathrm{P}} \ldots \prec_{\mathrm{P}} \mathrm{M}_{t}
$$


Согласно (6.3) для каждого $i$ найдется подпуть $\mathrm{N}_{i} \in \mathcal{J}_{\mathbf{Q}}$ пути $\mathrm{Q}$, для которого

$$
\operatorname{\vartheta Cozл}_{\beta}\left(\mathrm{M}_{i}, \mathrm{~N}_{i}\right)
$$

причем $\left\{\mathrm{N}_{i}\right\}_{i=0}^{t}$ есть множество представителей для $\mathcal{J}_{\mathrm{Q}}$ по отношению эквивалентности ЭСогл $\beta$. По индуктивному предположению 11.27 имеем

$$
\mathrm{N}_{0} \prec_{\mathrm{Q}} \mathrm{N}_{1} \prec_{\mathrm{Q}} \mathrm{N}_{2} \prec_{\mathrm{Q}} \ldots \prec_{\mathrm{Q}} \mathrm{N}_{t} .
$$

По условию предложения и в силу определения $\mathcal{J}_{\mathrm{P}}$ имеем

$$
\mathbf{s}_{A, \mathrm{P}}^{i} \mathrm{~K} \in \mathcal{J}_{\mathrm{P}}, \quad 0 \leqslant i \leqslant \ell .
$$

Так как $\mathrm{M}_{0} \preceq_{\mathrm{P}} \mathrm{K}$, то в силу лемм 3.2, (i) и 6.7 имеем $\mathbf{s}_{A, \mathrm{P}} \mathrm{M}_{0} \prec_{\mathrm{R}} \mathbf{s}_{A, \mathrm{P}}^{2} \mathrm{~K}$, где $\mathrm{R}-$ некоторое периодическое продолжение $\mathrm{P}$. Следовательно, $\mathbf{s}_{A, \mathrm{P}} \mathrm{M}_{0}$ содержится в $\mathrm{P}$. По лемме 6.9 , (iv) найдется $\mathrm{N}^{\prime} \in \ni\left(\mathrm{P}, q_{1}\right)$ такой, что $\mathrm{M}_{0} \preceq_{\mathrm{P}} \mathrm{N}^{\prime} \prec_{\mathrm{P}} \mathbf{s}_{A, \mathrm{P}} \mathrm{M}_{0}$ и $\operatorname{Coom}_{A, \mathrm{P}, \mathrm{Q}}\left(\mathrm{N}^{\prime}, \mathrm{N}_{0}\right)$, т.е. для некоторого $\mathbf{g} \in \mathbf{B}_{\alpha-1}$ выполнено $Э \operatorname{Coгл}_{\beta}\left(\mathbf{g N}^{\prime}, \mathrm{N}_{0}\right)$ и периодические отрезки $\mathrm{Q}, \mathrm{gP}$ имеют общее периодическое продолжение $\mathrm{T}$. Согласно определению $\mathcal{J}_{\mathrm{P}}$ и $\mathcal{J}_{\mathbf{Q}}$ имеем $\mathrm{N}^{\prime} \in \mathcal{J}_{\mathrm{P}}$, и, значит, для некоторого $s \geqslant 0$ выполнено $\ni \operatorname{\vartheta ozл}_{\beta}\left(\mathrm{N}_{0}, \mathrm{gM}_{s}\right)$. Индукцией по $i$ докажем, что

$$
{ } \operatorname{Coгл}_{\beta}\left(\mathrm{N}_{i}, \mathrm{gM}_{i+s}\right) \text { при всех } i=0,1, \ldots, t-s .
$$

Предположим, это доказано для некоторого значения $i \leqslant t-s-1$. Допустим, $\neg$ ЭСогл $\beta\left(\mathrm{N}_{i+1}, \mathbf{g M}_{i+s+1}\right)$. Тогда либо $\mathrm{N}_{i+1} \prec \mathbf{T} \mathbf{g M}_{i+s+1}$, либо $\mathbf{g M}_{i+s+1} \prec \mathbf{T}$ $\mathrm{N}_{i+1}$. Если $\mathrm{N}_{i+1} \prec \mathbf{T} \mathbf{g} \mathbf{M}_{i+s+1}$, то $\mathbf{g}^{-1} \mathbf{N}_{i} \prec \mathbf{g}^{-1} \mathbf{T} \mathbf{g}^{-1} \mathbf{N}_{i+1} \prec \mathbf{g}^{-1} \mathbf{T} \mathbf{M}_{i+s+1}$, откуда, используя соотношение $Э С о г \Omega_{\beta}\left(\mathrm{N}_{i}, \mathrm{gM}_{i+s}\right)$ и индуктивное предположение 11.25 , выводим $\mathrm{M}_{i+s} \prec_{\mathbf{g}^{-1} \mathbf{T}} \mathbf{g}^{-1} \mathrm{~N}_{i+1} \prec_{\mathbf{g}^{-1} \mathbf{T}} \mathrm{M}_{i+s+1}$. Отсюда следует, что $\mathbf{g}^{-1} \mathrm{~N}_{i+1}$ содержится в $\mathrm{P}$ и $\neg$ ЭСол $\Omega_{\beta}\left(\mathrm{g}^{-1} \mathrm{~N}_{i+1}, \mathrm{M}_{j}\right)$ при всех $j$. Так как $\operatorname{Coom}_{A, \mathrm{Q}, \mathrm{P}}\left(\mathrm{N}_{i+1}, \mathbf{g}^{-1} \mathrm{~N}_{i+1}\right)$, то $\mathbf{g}^{-1} \mathrm{~N}_{i+1} \in \mathcal{J}_{\mathrm{P}}$. Но это невозможно в силу выбора представителей $\mathrm{M}_{i}$. В случае, если $\mathbf{g M}_{i+s+1} \prec \mathbf{T} \mathrm{N}_{i+1}$, используя соотношения $\mathbf{g M}_{i+s} \prec \mathbf{T} \mathbf{g M}_{i+s+1}$ и $Э$ Согл $_{\beta}\left(\mathrm{N}_{i}, \mathbf{g M}_{i+s}\right)$, получаем, что $\mathbf{g M}_{i+s+1}$ содержится в $\mathrm{Q}$ и $\neg ⿹^{\operatorname{Coz}_{\beta}}\left(\mathrm{gM}_{i+s+1}, \mathrm{~N}_{j}\right)$ при всех $j$. Так как $\operatorname{Coom}_{A, \mathrm{P}, \mathrm{Q}}\left(\mathrm{M}_{i+s+1}, \mathbf{g M}_{i+s+1}\right)$, то $\mathbf{g M}_{i+s+1} \in \mathcal{J}_{\mathbf{Q}}$, и мы получаем противоречие с тем, что $\mathrm{N}_{i}$ образуют множество представителей для $\mathcal{J}_{Q}$ по отношению эквивалентности $Э С о г л_{\beta}$. Тем самым мы доказали соотношение $Э \operatorname{Coz}_{\beta}\left(\mathrm{N}_{i+1}, \mathbf{g M}_{i+s+1}\right)$ и, таким образом, (6.9) установлено для всех значений $i \leqslant t-s$.

Так как $\mathbf{s}_{A, \mathrm{P}} \mathrm{M}_{0} \in \mathcal{J}_{\mathrm{P}}$, то для некоторого $r \geqslant 0$ имеем $Э \operatorname{Cozл}_{\beta}\left(\mathbf{s}_{A, \mathrm{P}} \mathrm{M}_{0}, \mathrm{M}_{r}\right)$. С помощью рассуждений, аналогичных использованньг в доказательстве (6.9), получаем

$$
\vartheta \operatorname{Coz}_{\beta}\left(\mathbf{s}_{A, \mathrm{P}} \mathrm{M}_{i}, \mathrm{M}_{i+r}\right) \quad(i=0,1, \ldots, t-r) .
$$

Из $\mathrm{N}^{\prime} \prec \mathrm{P} \mathbf{s}_{A, \mathrm{P}} \mathrm{M}_{0}$ и $Э С о г \Omega_{\beta}\left(\mathrm{N}^{\prime}, \mathrm{M}_{s}\right)$ ввиду (6.10) следует $s<r$. Из (6.8) и (6.10) следует $t \geqslant \ell r \geqslant 4 r$. Так как $\mathbf{g P}$ и $\mathrm{Q}$ имеют общее периодическое продолжение, 
то по лемме 3.2 , (iii), (iv) имеем $\mathbf{s}_{A, \mathbf{Q}}=\mathbf{g s}_{A, \mathrm{P}} \mathbf{g}^{-1}$. Обозначив через $\sim$ отношение эквивалентности ЭСогл $\beta$ и используя (6.9), (6.10), выводим

$$
\mathbf{s}_{A, \mathrm{Q}} \mathrm{N}_{0} \sim \mathbf{s}_{A, \mathrm{Q}} \mathbf{g M}_{s} \sim \mathbf{g s}_{A, \mathrm{P}} \mathrm{M}_{s} \sim \mathbf{g M}_{r+s} \sim \mathrm{N}_{r}
$$

Тогда, вновь рассуждая, как в доказательстве соотношений (6.9), получаем

$$
\ni \operatorname{Coz}_{\beta}\left(\mathbf{s}_{A, \mathrm{Q}} \mathrm{N}_{i}, \mathrm{~N}_{i+r}\right), \quad i=0,1, \ldots, t-r .
$$

Отсюда следует $Э$ Согл $\beta_{\beta}\left(\mathbf{s}_{A, \mathbf{Q}}^{\ell} \mathrm{N}_{0}, \mathrm{~N}_{\ell r}\right)$. Так как в силу лемм 3.2 , (i) и 6.7 имеем $\mathbf{s}_{A, \mathbf{Q}}^{\ell-1} \mathrm{~N}_{0} \prec \mathrm{Q} \mathrm{N}_{\ell r}$, то $\mathbf{s}_{A, \mathbf{Q}}^{\ell-1} \mathrm{~N}_{0}$ содержится в $\mathbf{Q}$. Следовательно, $|\mathrm{Q}| \geqslant(\ell-1)|A| \geqslant 3|A|$. Тем самым доказана первая часть искомого утверждения.

Если $s=0$, то из (6.7) и (6.9) следует ЭСогл $\beta\left(\mathrm{M}_{i}, \mathbf{g M}_{i}\right)(0 \leqslant i \leqslant t)$, и тогда с учетом (6.8) получаем ЭСогл $\beta\left(\mathbf{s}_{A, \mathrm{P}} \mathrm{K}_{,} \mathbf{g s}_{A, \mathrm{P}}{ }_{A, \mathrm{~K}}\right)$ при всех $0 \leqslant i \leqslant \ell$, что в точности совпадает со второй частью заключения предложения 6.10. Нам остается доказать невозможность случая $s \neq 0$. Допустим, $s \geqslant 1$. Идея рассуждений состоит в применении леммы 6.3 , после чего легко получается противоречие с минимальностью периода $A$.

Так как $r>s \geqslant 1$, то $t \geqslant 4 r \geqslant r+s+4$. Из всех условий посылки леммы 6.3 остается проверить только равенство НОД $(r, s)=1$. Докажем его.

По выбору представителей $\mathrm{M}_{i}, \mathrm{~N}_{i}$ для каждого $\mathrm{M} \in \mathcal{J}_{\mathrm{P}} \cup \mathcal{J}_{\mathrm{Q}}$ существует единственный индекс $i(\mathrm{M})$ такой, что $Э \operatorname{Coz}_{\beta}\left(\mathrm{M}_{i(\mathrm{M})}, \mathrm{M}\right)$. Если $\mathrm{M} \in \mathcal{J}_{\mathrm{R}}, \mathrm{N} \in \mathrm{J}_{\mathrm{S}}$ и паpa $(\mathrm{S}, \mathrm{N})$ получается из пары $(\mathrm{R}, \mathrm{M})$ операцией перехода, то $i(\mathrm{M})=i(\mathrm{~N})$. Поэтому достаточно проверить, что в случае, когда пара $(\mathrm{S}, \mathrm{N})$ получается из пары $(\mathrm{R}, \mathrm{M})$ операцией сдвига, разность $i(\mathrm{M})-i(\mathrm{~N})$ есть целочисленная линейная комбинация $r$ и $s$. Так как каждая из пар $\left(\mathrm{P}, \mathrm{M}_{0}\right)$ и $\left(\mathrm{P}, \mathrm{M}_{1}\right)$ получается из пары $(\mathrm{P}, \mathrm{K})$ некоторой последовательностью операций сдвига и перехода, то отсюда будет следовать, что 1 есть целочисленная линейная комбинация $r$ и $s$, т.е. НОД $(r, s)=1$.

Итак, пусть $\mathrm{M} \in \mathcal{J}_{\mathrm{R}}, \mathrm{N} \in \mathcal{J}_{\mathrm{S}}$ и $(\mathrm{S}, \mathrm{N})$ получается из $(\mathrm{R}, \mathrm{M})$ применением операции сдвига, т.е. истинно отношение $\operatorname{Coom}_{A, \mathrm{R}, \mathrm{S}}(\mathrm{M}, \mathrm{N})$. Если $\mathrm{R}=\mathrm{S}=\mathrm{P}$ или $\mathrm{R}=\mathrm{S}=\mathrm{Q}$, то $i(\mathrm{M})-i(\mathrm{~N}) \equiv 0(\bmod r)$ ввиду леммы 6.9 , (iii) и $(6.10)$ или соответственно (6.11), (6.7). Пусть $\{\mathrm{R}, \mathrm{S}\}=\{\mathrm{P}, \mathrm{Q}\}$. С точностью до перестановки М и N можно считать $\mathrm{R}=\mathrm{P}$ и $\mathrm{S}=\mathrm{Q}$. В силу (6.10) и леммы 6.9 , (iii) имеем $\operatorname{Coom}_{A, \mathrm{P}, \mathrm{P}}\left(\mathrm{M}, \mathrm{M}_{i}\right)$, где $r \leqslant i<2 r$ и $i \equiv i(\mathrm{M})(\bmod r)$. В силу $(6.9)$ имеем $\operatorname{Coom}_{A, \mathrm{P}, \mathrm{Q}}\left(\mathrm{M}_{i}, \mathrm{~N}_{i-s}\right)$. Тогда из $\operatorname{Coom}_{A, \mathrm{P}, \mathrm{Q}}(\mathrm{M}, \mathrm{N})$ с учетом леммы 6.9, (i) следует $\operatorname{Coom}_{A, \mathrm{Q}, \mathrm{Q}}\left(\mathrm{N}_{i-s}, \mathrm{~N}\right)$, откуда, используя лемму 6.9 , (iii) и $(6.11)$, выводим $i-s \equiv i(\mathrm{~N})(\bmod r)$ и, таким образом, получаем наконец $i(\mathrm{M})-i(\mathrm{~N}) \equiv s(\bmod r)$.

Тем самым мы завершили проверку выполнения посылки леммы 6.3, и по этой лемме найдется элемент $\mathbf{d} \in \mathbf{B}_{\alpha-1}$ такой, что $\mathbf{s}_{A, \mathrm{P}}^{k}=\mathbf{d}^{r k}$ для некоторого $k>0$. Так как в силу (3.2) элементы $\mathbf{s}_{A, \mathrm{P}}$ и $[A]_{\alpha-1}$ сопряжены, то для некоторого $D$ слова $A^{k}$ и $D^{r k}$ сопряжены в группе $\mathbf{B}_{\alpha-1}$. Но это противоречит минимальности периода $A$ в ранге $\alpha$ (определение 5.3 ), ибо $r \geqslant s+1 \geqslant 2$. Полученное противоречие завершает доказательство предложения 6.10 . 
6.11. ПРЕДЛОЖЕНИЕ. Пусть $A \in \mathcal{M}_{\alpha}, \quad \rho(A) \leqslant \beta \leqslant \alpha-1 \quad u \quad$ nутu $\mathrm{P}, \mathrm{Q} \in \Pi_{\mathrm{ep}}\left(\Gamma_{\alpha-1}, A\right)$ строго близки в ранге $\beta$. Тогда если для некоторого $\mathrm{K} \in$ Эл $_{\beta}\left(\Gamma_{\alpha-1}, q_{1}\right)$ пути $\mathrm{K}, \mathrm{s}_{A, \mathrm{P}} \mathrm{K}, \ldots, \mathbf{s}_{A, \mathrm{P}}^{5} \mathrm{~K}$ входят в $\mathrm{P}$, то $|\mathrm{Q}| \geqslant 3|A| u$ $\operatorname{Corл}(\mathrm{P}, \mathrm{Q})$.

ДоКАЗАТЕЛЬСТВО. По предложению 6.10 имеет место неравенство $|\mathrm{Q}| \geqslant 3|A|$ и существует $\mathbf{g} \in \mathbf{B}_{\alpha-1}$ такой, что $\mathbf{g P}, \mathbf{Q}$ имеют общее периодическое продолжение и $\vartheta^{-} \operatorname{Coz}_{\beta}\left(\mathbf{s}_{A, \mathrm{P}}^{i} \mathrm{~K}, \mathbf{g s}_{A, \mathrm{P}}^{i} \mathrm{~K}\right)(0 \leqslant i \leqslant 5)$. По индуктивному предположению 8.8 имеем $\mathbf{s}_{A, \mathrm{P}}^{-i} \mathbf{g s}{ }_{A, \mathrm{P}}^{i} \in \mathbf{H}(0 \leqslant i \leqslant 5)$, где $\mathbf{H}-$ некоторая конечная подгруппа группы $\mathbf{B}_{\alpha-1}$. По индуктивному предположению 16.1 включение $\mathbf{s}_{A, \mathrm{P}}^{-i} \mathbf{g s} \mathbf{s}_{A, \mathrm{P}}^{i} \in \mathbf{H}$ имеет место при любом $i$. Тогда $\mathbf{s}_{A, \mathrm{P}}^{-t} \mathbf{g s}_{A, \mathrm{P}}^{t}=\mathbf{g}$ для некоторого $t>0$. Так как $\mathbf{s}_{A, \mathrm{Q}}=\mathbf{g s}_{A, \mathrm{P}} \mathbf{g}^{-1}$ в силу леммы 3.2 , (iii), (iv), то отсюда следует $\mathbf{s}_{A, \mathrm{Q}}^{t}=\mathbf{s}_{A, \mathrm{P}}^{t}$, т.е. $С$ огл $(\mathrm{P}, \mathrm{Q})$.

6.12. ПрЕДЛОЖЕНИЕ. Пусть $A, B \in \mathcal{M}_{\alpha}, \max \{\rho(A), \rho(B)\} \leqslant \beta \leqslant \alpha-1 u$ nути $\mathrm{P} \in \Pi_{\mathrm{p}}\left(\Gamma_{\alpha-1}, A, 2\right)$ u $\mathrm{Q} \in \Pi_{\mathrm{ep}}\left(\Gamma_{\alpha-1}, B, 2\right)$ строго близки в ранге $\beta$. Тогда если для некоторых $\mathrm{K}, \mathrm{L} \in \ni\left(\Gamma_{\alpha-1}, q_{1}\right)$ пути $\mathrm{K}, \mathrm{s}_{A, \mathrm{P}} \mathrm{K}, \ldots, \mathrm{s}_{A, \mathrm{P}}^{6} \mathrm{~K}$ входят в $\mathrm{P}$

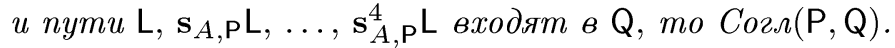

ДокАЗАТЕльство. Согласно определению 6.5 найдутся начало $\mathrm{K}^{\prime} \in$ Эл $\beta\left(\mathrm{P}, q_{1}\right)$ пути $\mathrm{P}$ и начало $\mathrm{L}^{\prime} \in \ni\left(\mathrm{Q}, q_{1}\right)$ пути $\mathrm{Q}$ такие, что $Э \operatorname{Coгл}_{\beta}\left(\mathrm{K}^{\prime}, \mathrm{L}^{\prime}\right)$. Заменяя $\mathrm{K}$ на $\mathrm{K}^{\prime}$ в случае $\mathrm{K}^{\prime}<\mathrm{p} \mathrm{K}$, можно считать, что $\mathrm{K}$ содержится в $\mathrm{K}^{\prime}$. Тогда по индуктивному предположению 8.40 имеем $Э \operatorname{Coгл}_{\beta}\left(\mathrm{K}, \mathrm{K}^{\prime}\right)$. Аналогично, можно считать, что ЭСогл $\beta\left(\mathrm{L}, \mathrm{L}^{\prime}\right)$. Тогда получаем $Э \operatorname{Coгл}_{\beta}(\mathrm{K}, \mathrm{L})$.

Дальнейшие рассуждения фактически повторяют доказательство предложения 6.10. Пусть множества $\mathcal{J}_{\mathrm{P}} \subseteq$ Эл $_{\beta}\left(\mathrm{P}, q_{0}+h\right)$ и $\mathcal{J}_{\mathrm{Q}} \subseteq$ Эл $_{\beta}\left(\mathrm{Q}, q_{0}+h\right)$ определены точно так же, как и в доказательстве предложения 6.10 , за исключением операции сдвига $(*)$, определение которой модифищируется следуюшим образом:

$(*)(\mathrm{R}, \mathrm{M}) \rightarrow(\mathrm{S}, \mathrm{N})$, если либо $\mathrm{R}=\mathrm{S}=\mathrm{P}$ и $\operatorname{Coоm}_{A, \mathrm{P}, \mathrm{P}}(\mathrm{M}, \mathrm{N})$, либо $\mathrm{R}=\mathrm{S}=\mathrm{Q}$ и $\operatorname{Coom}_{B, \mathrm{Q}, \mathrm{Q}}(\mathrm{M}, \mathrm{N})$.

(Эта модификация связана с тем, что периоды $A$ и $B$, вообще говоря, различны.) Дословно повторяя рассуждения из доказательства предложения 6.10 , выберем множества $\left\{\mathrm{M}_{i}\right\}_{i=0}^{t}$ и $\left\{\mathrm{N}_{i}\right\}_{i=0}^{t}$ представителей классов эквивалентности множеств $\mathcal{J}_{\mathrm{P}}$ и $\mathcal{J}_{\mathrm{Q}}$ по отношению $Э С о г \Omega_{\beta}$ таким образом, что выполнены соотношения

$$
M_{0} \prec_{\mathrm{P}} \mathrm{M}_{1} \prec_{\mathrm{P}} \mathrm{M}_{2} \prec_{\mathrm{P}} \ldots \prec_{\mathrm{P}} \mathrm{M}_{t}, \quad \mathrm{~N}_{0} \prec_{\mathrm{Q}} \mathrm{N}_{1} \prec_{\mathrm{Q}} \mathrm{N}_{2} \prec_{\mathrm{Q}} \ldots \prec_{\mathrm{Q}} \mathrm{N}_{t}
$$

и

$$
{ } \operatorname{Coz}_{\beta}\left(\mathrm{M}_{i}, \mathrm{~N}_{i}\right), \quad i=0,1, \ldots, t .
$$

Продолжая рассуждения из доказательства предложения 6.10 , получаем, что для некоторых взаимно простых чисел $r$ и $s$ выполнено

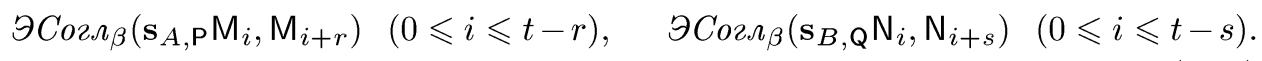

Из определения множеств $\mathcal{J}_{\mathrm{P}}$ и $\mathcal{J}_{\mathrm{Q}}$ следует $\mathbf{s}_{A, \mathrm{P}}^{i} \mathrm{~K} \in \mathcal{J}_{\mathrm{P}}$ при $i=0,1, \ldots, 6$ и $\mathbf{s}_{B, \mathrm{Q}}^{i} \mathrm{~L} \in$ $J_{\mathrm{Q}}$ при $i=0,1, \ldots, 4$. Тогда с учетом (6.13) получаем $t \geqslant 6 r$ и $t \geqslant 4 s$. По лемме 6.7 
имеем $r, s \geqslant 1$, откуда и из предыдуших неравенств легко вытекает $t \geqslant r+s+4$. Кроме того, в силу (6.12) в соотношениях (6.13) $\mathrm{N}_{j}$ можно заменить на $\mathrm{M}_{j}$. Тем самым мы проверили все условия посылки леммы 6.3. В силу этой леммы найдется элемент $\mathbf{d} \in \mathbf{B}_{\alpha-1}$ такой, что $\mathbf{s}_{A, \mathrm{P}}^{\ell}=\mathbf{d}^{r \ell}$ и $\mathbf{s}_{B, \mathrm{Q}}^{\ell}=\mathbf{d}^{s \ell}$ для некоторого $\ell>0$. Из минимальности периодов $A, B$ вытекает $s=r=1$. Следовательно, $\mathbf{s}_{A, \mathrm{P}}^{\ell}=\mathbf{s}_{A, \mathrm{Q}}^{\ell}$, т.е. $\operatorname{Coz} \Omega(\mathrm{P}, \mathrm{Q})$.

\section{$\S 7$. Согласованные периодические отрезки на графе $\Gamma_{\alpha-1}$ и связанные с ними подгруппы группы $\mathbf{B}_{\alpha-1}$}

7.1. ОПРЕДЕЛЕНИЕ. Пусть $A \in \mathcal{M}_{\alpha}$. Положим

$$
\begin{aligned}
\mathbf{N}_{\alpha-1}^{+}(A) & \rightleftharpoons\left\{\mathbf{g} \in \mathbf{B}_{\alpha-1} \mid \mathbf{g}^{-1}[A]_{\alpha-1}^{t} \mathbf{g}=[A]_{\alpha-1}^{t} \text { для некоторого } t \neq 0\right\}, \\
\mathbf{N}_{\alpha-1}^{-}(A) & \rightleftharpoons\left\{\mathbf{g} \in \mathbf{B}_{\alpha-1} \mid \mathbf{g}^{-1}[A]_{\alpha-1}^{t} \mathbf{g}=[A]_{\alpha-1}^{-t} \text { для некоторого } t \neq 0\right\}, \\
\mathbf{N}_{\alpha-1}(A) & \rightleftharpoons \mathbf{N}_{\alpha-1}^{+}(A) \cup \mathbf{N}_{\alpha-1}^{-}(A)= \\
& =\left\{\mathbf{g} \in \mathbf{B}_{\alpha-1} \mid \mathbf{g}^{-1}[A]_{\alpha-1}^{t} \mathbf{g}=[A]_{\alpha-1}^{\epsilon t} \text { для некоторых } t \neq 0 \text { и } \epsilon= \pm 1\right\} .
\end{aligned}
$$

Так как из $\mathbf{g}^{-1}[A]_{\alpha-1}^{t} \mathbf{g}=[A]_{\alpha-1}^{\epsilon t}$ и $\mathbf{h}^{-1}[A]_{\alpha-1}^{r} \mathbf{h}=[A]_{\alpha-1}^{\delta r}$ следует $(\mathbf{g h})^{-1}[A]_{\alpha-1}^{t r} \mathbf{g h}=[A]_{\alpha-1}^{\epsilon \delta t r}$, то $\mathbf{N}_{\alpha-1}^{+}(A)$ и $\mathbf{N}_{\alpha-1}(A)-$ подгруппы группы $\mathbf{B}_{\alpha-1}$.

7.2. Лемма. (i) $\mathbf{N}_{0}^{-}(A)=\varnothing u \mathbf{N}_{0}(A)=\mathbf{N}_{0}^{+}(A)=\left\langle[A]_{0}\right\rangle$ для любого $A \in \mathcal{M}_{1}$. (ii) Для любого $A \in \mathcal{M}_{\alpha}$ либо $\mathbf{N}_{\alpha-1}^{-}(A)=\varnothing u \mathbf{N}_{\alpha-1}(A)=\mathbf{N}_{\alpha-1}^{+}(A)$, либо $\mathbf{N}_{\alpha-1}^{+}(A)$ имеет индекс 2 в $\mathbf{N}_{\alpha-1}(A)$ и в этом слуиае $\mathbf{N}_{\alpha-1}^{+}(A)$ и $\mathbf{N}_{\alpha-1}^{-}(A)$ смежние классы группь $\mathbf{N}_{\alpha-1}(A)$ по подгруппе $\mathbf{N}_{\alpha-1}^{+}(A)$.

(iii) Eсли $A, B \in \mathcal{M}_{\alpha} u \mathbf{h}^{-1}[A]_{\alpha-1}^{r} \mathbf{h}=[B]_{\alpha-1}^{\epsilon r}$ для некоторых $r \neq 0 u \epsilon= \pm 1$, $m o \mathbf{h}^{-1} \mathbf{N}_{\alpha-1}^{+}(A) \mathbf{h}=\mathbf{N}_{\alpha-1}^{+}(B) u \mathbf{h}^{-1} \mathbf{N}_{\alpha-1}(A) \mathbf{h}=\mathbf{N}_{\alpha-1}(B)$.

ДокАЗАТЕЛЬСтво. Лемма легко вытекает из определений, для доказательства (ii) нужно лишь заметить, что $\mathbf{N}_{\alpha-1}^{+}(A) \cap \mathbf{N}_{\alpha-1}^{-}(A)=\varnothing$ ввиду бесконечности порядка элемента $[A]_{\alpha-1}$ (лемма 6.7).

7.3. ПРЕДЛОЖЕНИЕ. Пусть $A \in \mathcal{M}_{\alpha}, \mathrm{P}, \mathrm{Q} \in \Pi_{\mathrm{ep}}\left(\Gamma_{\alpha-1}, A, 2\right)$ и слова $\mathcal{L}(\mathrm{P})$, $\mathcal{L}(\mathrm{Q})$ начинаются $с$ A. Тогда

$$
\begin{aligned}
\operatorname{Co\imath л}(\mathrm{P}, \mathrm{Q}) & \Leftrightarrow(\iota(\mathrm{P}))^{-1} \iota(\mathrm{Q}) \in \mathrm{N}_{\alpha-1}^{+}(A), \\
\operatorname{Co\imath л}\left(\mathrm{P}, \mathrm{Q}^{-1}\right) & \Leftrightarrow(\iota(\mathrm{P}))^{-1} \iota(\mathrm{Q}) \in \mathbf{N}_{\alpha-1}^{-}(A) .
\end{aligned}
$$

ДокАЗАТЕЛЬСтво. Непосредственно вытекает из определения 3.3, формулы (3.2) и леммы $3.2,(\mathrm{v})$.

Заметим, что в условиях предложения 7.3 элемент $(\iota(\mathrm{P}))^{-1} \iota(\mathrm{Q})$ равен $[\mathcal{L}(\mathrm{X})]_{\alpha-1}$, где $\mathrm{X}$ - произвольный путь, соединяюший начальные вершины путей $\mathrm{P}$ и $\mathrm{Q}$. Мы будем часто использовать этот факт вместе с предложением 7.3. 
7.4. ОПРЕДЕЛЕНИЕ. Будем говорить, что вершины $\mathbf{g}, \mathbf{h} \in \mathbf{B}_{\gamma}$ граффа $\Gamma_{\gamma}$ близки в ранге $\beta$, если $\mathbf{g}^{-1} \mathbf{h}=[u]_{\gamma}$ для некоторого слова $u \in \mathcal{H}_{\beta}$, или, эквивалентно, $\mathbf{g}$ и $\mathbf{h}$ можно соединить некоторым путем u c $\mathcal{L}(\mathbf{u}) \in \mathcal{H}_{\beta}$.

Пути $\mathrm{X}_{\text {и }} \mathrm{Y}$ на графе $\Gamma_{\gamma}$ назовем близкими в ранге $\beta$, если их начальные вершины близки в ранге $\beta$ и их конечные вершины близки в ранге $\beta$.

Из определения вытекает, что слова $X$ и $Y$ близки в ранге $\beta$ тогда и только тогда, когда существуют близкие в ранге $\beta$ пути $\mathrm{X}$ и $\mathrm{Y}$ на графе $\Gamma_{\beta}$ с $\mathcal{L}(\mathrm{X})$ 으 $X$ и $\mathcal{L}(\mathrm{Y})$ 으 $Y$.

Кроме того, заметим, что если $\mathrm{X}$ и $\mathrm{Y}$ - близкие в ранге $\beta$ пути на графе $\Gamma_{\gamma}$, то $\mathrm{X}$ и $\mathrm{Y}$ близки в любом ранге $\beta_{1}>\beta$. Этот факт непосредственно вытекает из леммы 5.12 .

7.5. Лемма. Пусть $A \in \mathcal{M}_{\alpha}, B$ - период ранга $\alpha, \mathrm{P} \in \Pi$ Пер $\left(\Gamma_{\alpha-1}, A, 2\right)$, $\mathrm{Q} \in$ Пер $\left(\Gamma_{\alpha-1}, B, 2\right) u \mathbf{s}_{A, \mathrm{P}}^{k t}=\mathbf{s}_{B, \mathrm{Q}}^{t}$ для некоторых $k, t>0$. Тогда существуют близкие в ранге $\rho(A)$ периодические продолжения периодических отрезков $\mathrm{P}$ $u \mathrm{Q}$.

ДоКАЗАТЕЛЬСТВО. При $\alpha=1$ это вытекает из леммы 3.5. Пусть $\alpha \geqslant 2$. Без ограничения обшности можно считать, что слова $\mathcal{L}(\mathrm{P})$ и $\mathcal{L}(\mathrm{Q})$ начинаются с $A$ и с $B$ соответственно. Выберем произвольное слово $T$ такое, что

$$
(\iota(\mathrm{P}))^{-1} \iota(\mathrm{Q})=[T]_{\alpha-1} .
$$

В силу (3.2) равенство $\mathbf{s}_{A, \mathrm{P}}^{k t}=\mathbf{s}_{B, \mathrm{Q}}^{t}$ можно переписать в виде

$$
T^{-1} A^{k t} T \stackrel{\alpha=1}{=} B^{t} .
$$

В силу индуктивного предположения 11.32 найдутся начала $R$ и $S$ слов $A^{t}$ и $B^{t}$ соответственно, слово $u \in \mathcal{H}_{\rho(A)}$ и число $r \in \mathbb{Z}$ такие, что

$$
T^{\alpha=1} A^{k t r} R u S^{-1} .
$$

Положим $\mathbf{g} \rightleftharpoons \iota(\mathrm{P})\left[A^{k t r} R\right]_{\alpha-1}$ и $\mathbf{h} \rightleftharpoons \iota(\mathrm{Q})[S]_{\alpha-1}$. Из равенств $(7.1)$ и (7.2) вытекает $\mathbf{g}^{-1} \mathbf{h}=[u]_{\alpha-1}$, следовательно, вершины $\mathbf{g}$ и $\mathbf{h}$ графа $\Gamma_{\alpha-1}$ близки в ранге $\rho(A)$. Очевидно, $\mathbf{h}$ лежит на $\mathbf{Q}$ и $\mathbf{g}$ лежит на некотором периодическом продолжении Р. Тогда в силу леммы 3.2 , (ii) при любом $i \in \mathbb{Z}$ вершины $\mathbf{s}_{A, \mathrm{P}}^{i} \mathbf{g}$ и $\mathbf{s}_{B, \mathrm{Q}}^{i} \mathbf{h}$ лежат на некоторых периодических продолжениях для $\mathrm{P}$ и $\mathrm{Q}$ соответственно. Кроме того, из равенства $\mathbf{s}_{A, \mathrm{P}}^{k t}=\mathbf{s}_{B, \mathrm{Q}}^{t}$ следует, что в случае, если $i$ кратно $t$, вершины $\mathbf{s}_{A, \mathrm{P}}^{i k} \mathbf{g}$ и $\mathbf{s}_{B, \mathbf{Q}}^{i} \mathbf{h}$ близки в ранге $\rho(A)$. Выберем достаточно длинные периодические продолжения $\overline{\mathrm{P}}=\mathrm{UPV}$ и $\overline{\mathrm{Q}}=\mathrm{WQZ}$ для $\mathrm{P}$ и $\mathrm{Q}$ так, чтобы для некоторых $i, j \in \mathbb{Z}$ на путях $\mathrm{U}, \mathrm{V}, \mathrm{W}$ и Z лежали вершины $\mathbf{s}_{A, \mathrm{P}}^{i k t} \mathbf{g}, \mathbf{s}_{A, \mathrm{P}}^{j k t} \mathbf{g}, \mathbf{s}_{B, \mathrm{Q}}^{i t} \mathbf{h}$ и $\mathbf{s}_{B, \mathrm{Q}}^{j t} \mathbf{h}$ соответственно. Тогда в качестве искомых периодических продолжений для $\mathrm{P}$ и $\mathrm{Q}$ можно взять подпуть пути $\overline{\mathrm{P}}$, соединяюший вершины $\mathbf{s}_{A, \mathrm{P}}^{i k t} \mathbf{g}$ и $\mathbf{s}_{A, \mathrm{P}}^{j k t} \mathbf{g}$, и подпуть пути $\overline{\mathrm{Q}}$, соединяющий вершины $\mathbf{s}_{B, \mathrm{Q}}^{i t} \mathbf{h}$ и $\mathbf{s}_{B, \mathrm{Q}}^{j t} \mathbf{h}$. 
7.6. Лемма. Пусть $A-$ период ранга $\alpha, \mathrm{P} \in \Pi_{\mathrm{pp}}\left(\Gamma_{\alpha-1}, A, 2\right), \quad \beta \leqslant \alpha-1$ $u \mathrm{~K} \in$ Эл $\beta_{\beta}(\mathrm{P}, r)$, где $r \geqslant 19$. Тогда $|\mathrm{K}|<2|A|$ и для некоторых начала $\mathrm{K}^{\prime}$ и конца $\mathrm{K}^{\prime \prime}$ пути $\mathrm{K}$ выполнено $\left|\mathrm{K}^{\prime}\right|,\left|\mathrm{K}^{\prime \prime}\right| \leqslant|A|, \mathrm{K}^{\prime}, \mathrm{K}^{\prime \prime} \in$ эл $_{\beta}\left(\Gamma_{\alpha-1}, r-9\right)$, $\ni \operatorname{Coz}_{\beta}\left(\mathrm{K}, \mathrm{K}^{\prime}\right)$ u $Э \operatorname{Coz}_{\beta}\left(\mathrm{K}, \mathrm{K}^{\prime \prime}\right)$.

ДоКАЗАТЕЛЬСТВо. В силу симметрии ограничимся доказательством утверждения о сушествовании начала $\mathrm{K}^{\prime} \in$ Эл $_{\beta}\left(\Gamma_{\alpha-1}, r-9\right)$ пути $\mathrm{K}$, такого, что $\left|\mathrm{K}^{\prime}\right| \leqslant|A|$ и $Э \operatorname{Coгл}_{\beta}\left(\mathrm{K}, \mathrm{K}^{\prime}\right)$. При $\beta=0$ слово $\mathcal{L}(\mathrm{K})$ является буквой алфавита $\mathcal{A}^{ \pm 1}$, поэтому неравенство $|\mathrm{K}|<2|A|$ имеет место в силу непустоты слова $A$, а в качестве $\mathrm{K}^{\prime}$ можно взять сам путь $\mathrm{K}$. Пусть $\beta \geqslant 1$. Удлинив $\mathrm{P}$, можно считать, что путь $\mathbf{s}_{A, \mathrm{P}} \mathrm{K}$ содержится в $\mathrm{P}$. По лемме $6.7 \neg Э \operatorname{Co\imath }_{\beta}\left(\mathrm{K}, \mathrm{s}_{A, \mathrm{P}} \mathrm{K}\right)$. Тогда по индуктивному предположению 8.41 имеем $\mathrm{K}=\mathrm{K}^{\prime} \mathrm{K}^{*}$, где $\mathrm{K}^{\prime} \in$ Эл $_{\beta}\left(\Gamma_{\alpha-1}, r-9\right), \quad$ ЭСол $\beta\left(\mathrm{K}, \mathrm{K}^{\prime}\right)$ и $\mathrm{K}^{\prime} \ll \mathbf{p}_{A, \mathrm{P}} \mathrm{K}$. Так как $\mathbf{s}_{A, \mathrm{P}} \mathrm{K}^{\prime}$ есть начало $\mathbf{s}_{A, \mathrm{P}} \mathrm{K}$, то отсюда следует $\left|\mathrm{K}^{\prime}\right| \leqslant|A|$. Если бы выполнялось неравенство $\left|\mathrm{K}^{*}\right| \geqslant|A|$, то путь $\mathbf{s}_{A, \mathrm{P}^{\prime}}$ содержался бы в $\mathrm{K}$, что невозможно в силу индуктивного предположения 8.40. Следовательно, $|\mathrm{K}|<2|A|$.

7.7. ПРЕДЛОЖЕНИЕ. Пусть $A \in \mathcal{M}_{\alpha}, B-$ период ранга $\alpha, \quad \mathrm{P} \in$ Пер $\left(\Gamma_{\alpha-1}, A, 2\right), \quad \mathrm{Q} \in \Pi_{\mathrm{ep}}\left(\Gamma_{\alpha-1}, B, 2\right) u \mathbf{s}_{A, \mathrm{P}}^{k t}=\mathbf{s}_{A, \mathrm{Q}}^{t}$ для некоторых $k, t>0$. Тогда существуют строго близкие в ранге $\rho(A)$ периодические продолжения $\overline{\mathrm{P}}$ для $\mathrm{P} u \overline{\mathrm{Q}}$ для $\mathrm{Q}$.

Более того, $\overline{\mathrm{P}}$ и $\overline{\mathrm{Q}}$ могут быть выбраны таким образом, ито $\overline{\mathrm{P}}=\mathrm{K} \cup_{\overline{\mathrm{P}}} \mathbf{s}_{A, \mathrm{P}} \mathrm{K}$ $u \overline{\mathrm{Q}}=\mathrm{L} \cup_{\overline{\mathrm{Q}}} \mathrm{L}^{\prime}$ для некоторых $\mathrm{K} \in$ Эл $_{\rho(A)}\left(\overline{\mathrm{P}}, q_{3}\right), \mathrm{L}, \mathrm{L}^{\prime} \in \ni_{\rho(A)}\left(\overline{\mathrm{Q}}, q_{1}\right) u r \geqslant 1$, где ЭСогл $\Omega_{\rho(A)}(\mathrm{K}, \mathrm{L})$ u $Э \operatorname{Coz}_{\rho(A)}\left(\mathbf{s}_{A, \mathrm{P}}^{r} \mathrm{~K}, \mathrm{~L}^{\prime}\right)$.

ДоКАЗАТЕЛЬСТВо. В силу леммы 7.5 достаточно рассмотреть случай, когда $\mathrm{P}$ и $\mathrm{Q}$ близки в ранге $\rho(A)$. Выбрав периодическое продолжение $\mathrm{T}_{1} \mathrm{PT}_{2}$ для $\mathrm{P}$ с $\left|\mathrm{T}_{1}\right|,\left|\mathrm{T}_{2}\right| \geqslant 5|A|$ и применив лемму 7.5 еще раз, найдем близкие в ранге $\rho(A)$ периодические продолжения $\widetilde{\mathrm{P}}=\mathrm{U}_{1} \mathrm{PU}_{2}$ и $\widetilde{\mathrm{Q}}=\mathrm{V}_{1} \mathrm{QV} \mathrm{V}_{2}$ для $\mathrm{P}$ и $\mathrm{Q}$, где $\left|\mathrm{U}_{1}\right|,\left|\mathrm{U}_{2}\right| \geqslant 5|A|$. Тогда пути $\mathrm{U}_{i}$ и $\mathrm{V}_{i}$ также близки в ранге $\rho(A)$. В силу определения 5.15 и леммы 7.6 каждое периодическое слово $R \in \Pi е p(A, 3)$ содержит в качестве подслова некоторую элементарную $q_{3}$-степень $E$ ранга $\rho(A)$. Отсюда следует, что $\mathbf{s}_{A, \mathrm{P}}^{-1} \mathrm{~K}, \mathrm{~K}, \mathbf{s}_{A, \mathrm{P}} \mathrm{K} \in \ni_{\rho(A)}\left(\mathrm{U}_{1}, q_{3}\right)$ и $\mathbf{s}_{A, \mathrm{P}}^{r-1} \mathrm{~K}, \mathbf{s}_{A, \mathrm{P}}^{r} \mathrm{~K}, \mathbf{s}_{A, \mathrm{P}}^{r+1} \mathrm{~K} \in$ Эл $_{\rho(A)}\left(\mathrm{U}_{2}, q_{3}\right)$ для некоторых $\mathrm{K}$ и $r \geqslant 1$, где $\mathcal{L}(\mathrm{K})$ 을 $E$. В силу индуктивного предположения 11.22 найдутся $\mathrm{L} \in$ Эл $_{\rho(A)}\left(\mathrm{V}_{1}, q_{3}-h\right)$ и $\mathrm{L}^{\prime} \in \ni_{\rho(A)}\left(\mathrm{V}_{2}, q_{3}-h\right)$ такие, что ЭСог $\Omega_{\rho(A)}(\mathrm{K}, \mathrm{L})$ и $Э \operatorname{Coгл}_{\rho(A)}\left(\mathbf{s}_{A, \mathrm{P}}^{r} \mathrm{~K}, \mathrm{~L}^{\prime}\right)$. Так как $q_{3}-h>q_{1}$ и из леммы 6.7 следует $\neg$ ЭСогл $\rho(A)\left(\mathrm{K}, \mathbf{s}_{A, \mathrm{P}}^{r} \mathrm{~K}\right)$, то $\overline{\mathrm{P}} \rightleftharpoons \mathrm{K} \cup_{\widetilde{\mathrm{P}}} \mathbf{s}_{A, \mathrm{P}}^{r} \mathrm{~K}$ и $\overline{\mathrm{Q}} \rightleftharpoons \mathrm{L} \cup_{\widetilde{\mathrm{Q}}} \mathrm{L}^{\prime}-$ искомые строго близкие в ранге $\rho(A)$ периодические продолжения для $\mathrm{P}$ и $\mathrm{Q}$.

7.8. ПРЕДЛОЖЕНИЕ. Пусть $A \in \mathcal{M}_{\alpha}, \mathrm{P} \in \Pi_{\mathrm{ep}}\left(\Gamma_{\alpha-1}, A, 2\right), \mathbf{g} \in \mathbf{B}_{\alpha-1} u$ Согл $(\mathrm{P}, \mathbf{g P})$. Тогда для любого $\mathrm{K} \in$ Эл $_{\rho(A)}\left(\mathrm{P}, q_{1}\right)$ существует число $t \in \mathbb{Z}$ maкое, что ЭСогл $\Omega_{\rho(A)}\left(\mathbf{s}_{A, \mathrm{P}}^{i} \mathrm{~K}, \mathbf{g s}_{A, \mathrm{P}}^{i-t} \mathrm{~K}\right)$ nри всех $i \in \mathbb{Z}$.

ДокАЗАТЕльство. Выберем произвольное $k>2$ и периодическое продолжение $\mathrm{R}$ для $\mathrm{P}$, содержащее в качестве подпутей пути $\mathbf{s}_{A, \mathrm{P}} \mathrm{K}$ при $-k \leqslant i \leqslant k$. Используя предложение 7.7 , удлинив при этом $\mathrm{R}$, можно считать, что $\mathrm{R}$ и некоторое периодическое продолжение $\mathrm{S}$ для $\mathbf{g} \mathrm{P}$ строго близки в ранге $\rho(A)$. Тогда по предложению 6.10 сушествует элемент $\mathbf{h} \in \mathbf{B}_{\alpha-1}$ такой, что $Э \operatorname{Coг}_{\rho(A)}\left(\mathbf{s}_{A, \mathrm{P}}^{i} \mathrm{~K}, \mathbf{h} \mathbf{s}_{A, \mathrm{P}}^{i} \mathrm{~K}\right)$ 
при $-k \leqslant i \leqslant k$ и $\mathbf{S}, \mathbf{h} \mathbf{R}$ имеют общее периодическое продолжение $\mathrm{T}$. Так как $\mathrm{T}$ есть общее периодическое продолжение для $\mathbf{h P}$ и $\mathbf{g P}$, то по лемме 3.2 , (vii) для некоторого $t$ имеем $\mathbf{g}=\mathbf{s}_{A, \mathrm{P}}^{t} \mathbf{h}$. Следовательно,

$$
\vartheta \operatorname{Coz}_{\rho(A)}\left(\mathbf{s}_{A, \mathrm{P}}^{i} \mathrm{~K}, \mathbf{g s}_{A, \mathrm{P}}^{i-t} \mathrm{~K}\right), \quad-k \leqslant i \leqslant k .
$$

Так как $k$ произвольно, а из леммы 6.7 следует, что $t$ не зависит от выбора $k$, то соотношение (7.3) верно при любом $i \in \mathbb{Z}$.

7.9. ОПРЕДЕЛЕНИЕ. Пусть $A \in \mathcal{M}_{\alpha}$ и $\mathbf{g} \in \mathbf{N}_{\alpha-1}^{+}(A)$. Выберем периодический отрезок $\mathrm{P} \in$ Пер $\left(\Gamma_{\alpha-1}, A, 2\right)$ с начальной вершиной $\iota(\mathrm{P})=1$, метка которого начинается с $A$, так, чтобы $\mathrm{P}$ содержал некоторый подпуть $\mathrm{K} \in \ni$ л $_{(A)}\left(\mathrm{P}, q_{1}\right)$ (это можно сделать в силу определения 5.15). Согласно предложению 7.3 имеем Согл $(\mathrm{P}, \mathrm{gP})$ и, следовательно, мы находимся в условиях предложения 7.8. Число $t$, сушествование которого утверждается в предложении 7.8 , будем называть показателем степени периода $A$ в $\mathbf{g}$ и обозначать $\operatorname{ind}_{A}(\mathrm{~g})$.

7.10. ПреДЛОЖЕНИЕ. Для любого $A \in \mathcal{M}_{\alpha}$ верны следующие утверждения:

(i) для любого $\mathbf{g} \in \mathbf{N}_{\alpha-1}^{+}(A)$ существует число $r>0$ такое, что $\mathbf{g}^{r}=$ $[A]_{\alpha-1}^{r \operatorname{ind}_{A}(\mathbf{g})}$

(ii) число $\operatorname{ind}_{A}(\mathrm{~g})$ не зависит от выбора $\mathrm{P} u \mathrm{~K}$ в определении 7.9. Следовательно, для данного $A$ корректно определено отображсене $\operatorname{ind}_{A}: \mathbf{N}_{\alpha-1}^{+}(A) \rightarrow$ $\mathbb{Z}$;

(iii) $\operatorname{ind}_{A}\left([A]_{\alpha-1}^{k}\right)=k$ для любого $k \in \mathbb{Z}$;

(iv) $\operatorname{ind}_{A}-$ эпиморфизм группь $\mathbf{N}_{\alpha-1}^{+}(A)$ на $\mathbb{Z}$.

ДокАЗАТЕЛЬСТво. (i) Пусть $\mathrm{P}$ и $\mathrm{K}$ таковы, как указано в определении 7.9. По индуктивному предположению 8.8

$$
\mathbf{H} \rightleftharpoons\left\{\mathbf{h} \in \mathbf{B}_{\alpha-1} \mid \mathcal{Э}^{\operatorname{Coz} \Omega_{\rho(A)}}(\mathrm{K}, \mathbf{h K})\right\}
$$

- конечная подгруппа группы $\mathbf{B}_{\alpha-1}$. Заметим, что $\mathbf{s}_{A, \mathrm{P}}=[A]_{\alpha-1}$ согласно формуле (3.2). Так как согласно определению 7.9 имеем $Э \operatorname{Coz}_{\rho(A)}\left(\mathbf{s}_{A, \mathrm{P}}^{i} \mathrm{~K}, \mathbf{g s}_{A, \mathrm{P}}^{i-\mathrm{ind}_{A}(\mathbf{g})} \mathrm{K}\right)$ при любом $i$, то

$$
[A]_{\alpha-1}^{-i} \mathbf{g}[A]_{\alpha-1}^{i-\operatorname{ind}_{A}(\mathbf{g})}=\mathbf{s}_{A, \mathrm{P}}^{-i} \mathbf{g} \mathbf{s}_{A, \mathrm{P}}^{i-\operatorname{ind}_{A}(\mathbf{g})} \in \mathbf{H} \quad(i \in \mathbb{Z}) .
$$

Тогда по лемме 6.1 для некоторого $r>0$ выполнено $\mathbf{g}^{r}=[A]_{\alpha-1}^{r \text { ind }_{A}(\mathbf{g})}$.

(ii) Из $\mathbf{g}^{r_{1}}=[A]_{\alpha-1}^{r_{1} t_{1}}$ и $\mathbf{g}^{r_{2}}=[A]_{\alpha-1}^{r_{2} t_{2}}$ следует $[A]_{\alpha-1}^{\left(t_{1}-t_{2}\right) r_{1} r_{2}}=1$, откуда с учетом леммы 6.7 выводим $t_{1}=t_{2}$. Таким образом, (ii) вытекает из (i).

(iii) Утверждение непосредственно следует из (i), (ii) и леммы 6.7 .

(iv) Ввиду (iii) достаточно проверить, что отображение $\operatorname{ind}_{A}: \mathbf{N}_{\alpha-1}^{+}(A) \rightarrow \mathbb{Z}$ является гомоморфизмом групп. Соотношение ind ${ }_{A}\left(\mathrm{~g}^{-1}\right)=-\operatorname{ind}_{A}(\mathbf{g})$ непосредственно вытекает из (i) и бесконечности порядка элемента $[A]_{\alpha-1}$. Далее, из соотношений (7.5) при $\mathbf{g}:=\mathbf{g}$ и $\mathbf{g}:=\mathbf{h}$ следует

$$
\mathbf{g h}[A]_{\alpha-1}^{-\operatorname{ind}_{A}(\mathbf{g})-\operatorname{ind}_{A}(\mathbf{h})}=\mathbf{g}[A]_{\alpha-1}^{-\operatorname{ind}_{A}(\mathbf{g})} \cdot[A]_{\alpha-1}^{\operatorname{ind}_{A}(\mathbf{g})} \mathbf{h}[A]_{\alpha-1}^{-\operatorname{ind}_{A}(\mathbf{g})-\operatorname{ind}_{A}(\mathbf{h})} \in \mathbf{H},
$$


а полагая в (7.5) $\mathbf{g}:=\mathbf{g h}$, выводим

$$
\mathbf{g h}[A]_{\alpha-1}^{-\operatorname{ind}_{A}(\mathbf{g h})} \in \mathbf{H}
$$

Следовательно, $[A]_{\alpha-1}^{\operatorname{ind}_{A}(\mathbf{g h})-\operatorname{ind}_{A}(\mathbf{g})-\operatorname{ind}_{A}(\mathbf{h})} \in \mathbf{H}$, и ввиду бесконечности порядка элемента $[A]_{\alpha-1} \operatorname{ind}{ }_{A}(\mathbf{g h})=\operatorname{ind}_{A}(\mathbf{g})+\operatorname{ind}_{A}(\mathbf{h})$ для любых $\mathbf{g}, \mathbf{h} \in \mathbf{N}_{\alpha-1}^{+}(A)$.

7.11. СЛЕДСТВИЕ. В условиях предложения 7.8 число $t$ не зависит от выбора $\mathrm{K}$ и равно $\operatorname{ind}_{A_{1}}\left((\iota(\mathrm{P}))^{-1} \mathrm{~g} \iota(\mathrm{P})\right)$, где $A_{1}-$ ииклический сдвиг слова $A$, с которого начинается слово $\mathcal{L}(\mathrm{P})$.

ДоКАЗАТЕЛЬСТво. Предположим, верны посылка и для некоторого $t$ заключение предложения 7.8. Положим $\mathrm{P}_{1} \rightleftharpoons(\iota(\mathrm{P}))^{-1} \mathrm{P}, \mathrm{K}_{1} \rightleftharpoons(\iota(\mathrm{P}))^{-1} \mathrm{~K}$ и $\mathbf{g}_{1} \rightleftharpoons$ $(\iota(\mathrm{P}))^{-1} \mathbf{g} \iota(\mathrm{P})$. Из Согл $(\mathrm{P}, \mathbf{g P})$ следует $\operatorname{Coгл~}\left(\mathrm{P}_{1}, \mathrm{~g}_{1} \mathrm{P}_{1}\right)$. Так как в силу леммы 3.2 , (iv) имеем $\mathbf{s}_{A, \mathrm{P}}=\iota(\mathrm{P}) \mathbf{s}_{A, \mathrm{P}_{1}}(\iota(\mathrm{P}))^{-1}$, то из $Э$ Согл $\rho(A)_{\rho}\left(\mathbf{s}_{A, \mathrm{P}}^{i} \mathrm{~K}, \mathbf{g s}_{A, \mathrm{P}}^{i-t} \mathrm{~K}\right)$ следует ЭСогл $\rho(A)_{\rho}\left(\mathbf{s}_{A, \mathrm{P}_{1}}^{i} \mathrm{~K}_{1}, \mathbf{g}_{1} \mathbf{s}_{A, \mathrm{P}_{1}}^{i-t} \mathrm{~K}_{1}\right)$. Так как $A_{1}, \mathbf{g}_{1}, \mathrm{P}_{1}$ и $\mathrm{K}_{1}$ в качестве $A, \mathbf{g}, \mathrm{P}$ и $\mathrm{K}$ удовлетворяют условиям определения 7.9, то по предложению 7.10 , (ii) получаем $t=\operatorname{ind}_{A_{1}}\left(\mathbf{g}_{1}\right)$, что и требовалось.

7.12. ОПРЕДЕЛЕниЕ. $\mathbf{N}_{\alpha-1}^{*}(A) \rightleftharpoons \operatorname{ker}\left(\operatorname{ind}_{A}\right)$.

7.13. ОПРеДЕЛЕНИЕ. Для любых $A \in \mathcal{E}_{\alpha}$ и $\beta \geqslant \alpha$ положим

$$
\begin{array}{ll}
\mathbf{S}_{\beta}^{+}(A) \rightleftharpoons \pi_{\alpha-1, \beta}\left(\mathbf{N}_{\alpha-1}^{+}(A)\right), & \mathbf{S}_{\beta}^{-}(A) \rightleftharpoons \pi_{\alpha-1, \beta}\left(\mathbf{N}_{\alpha-1}^{-}(A)\right), \\
\mathbf{S}_{\beta}(A) \rightleftharpoons \pi_{\alpha-1, \beta}\left(\mathbf{N}_{\alpha-1}(A)\right), & \mathbf{S}_{\beta}^{*}(A) \rightleftharpoons \pi_{\alpha-1, \beta}\left(\mathbf{N}_{\alpha-1}^{*}(A)\right) .
\end{array}
$$

7.14. ПРЕДЛОЖЕНИЕ. (i) Пусть $A \in \mathcal{M}_{\alpha}$. Если $\rho(A)=0$, mo $\mathbf{N}_{\alpha-1}^{*}(A)=1$. Eсли $\rho(A)>0$, то $\mathbf{N}_{\alpha-1}^{*}(A) \subseteq \mathbf{x S}_{\alpha-1}(B) \mathbf{x}^{-1}$ для некоторьх $\mathbf{x} \in \mathbf{B}_{\alpha-1} u$ $B \in \mathcal{E}_{\rho(A)}$.

(ii) Для любого $A \in \mathcal{M}_{\alpha}$ группа $\mathbf{N}_{\alpha-1}^{*}(A)$ конечна.

(iii) Для любых $A \in \mathcal{E}_{\alpha}$ u $\beta \geqslant \alpha$ группа $\mathbf{S}_{\beta}(A)$ конечна.

ДокАЗАТЕЛЬСтво. (i), (ii): Из соотношения (7.5), полученного в доказательстве предложения $7.10,(\mathrm{i})$, следует $\mathbf{N}_{\alpha-1}^{*}(A) \subseteq \mathbf{H}$, где $\mathbf{H}$ - конечная подгруппа группы $\mathbf{B}_{\alpha-1}$, определенная равенством (7.4) для некоторого $\mathrm{K} \in \ni{ }_{\rho(A)}\left(\Gamma_{\alpha-1}\right)$. Если $\rho(A)=0$, то $\mathbf{H}=1$ в силу определения 5.14. Если $\rho(A)>0$, то индуктивному предположению $8.8 \mathbf{H} \subseteq \mathbf{x S}_{\alpha-1}(B) \mathbf{x}^{-1}$ для некоторых $\mathbf{x} \in \mathbf{B}_{\alpha-1}$ и $B \in \mathcal{E}_{\rho(A)}$.

(iii) В силу леммы 7.2 , (ii) имеем $\left|\mathbf{S}_{\beta}(A): \mathbf{S}_{\beta}^{+}(A)\right| \leqslant 2$, так что достаточно доказать конечность $\mathbf{S}_{\beta}^{+}(A)$. В силу определений $7.13,7.12$ и предложения 7.10 , (iii), (iv) факторгруппа $\mathbf{S}_{\beta}^{+}(A) / \mathbf{S}_{\beta}^{*}(A)$ есть гомоморфный образ бесконечной циклической группы $\mathbf{N}_{\alpha-1}^{+}(A) / \mathbf{N}_{\alpha-1}^{*}(A)=\left\langle[A]_{\alpha-1} \mathbf{N}_{\alpha-1}^{*}(A)\right\rangle$ при гомоморфизме, индуцированном $\pi_{\alpha-1, \beta}$. Так как $[A]_{\beta}^{n}=1$ в силу определения 5.6 , то $\mathbf{S}_{\beta}^{+}(A) / \mathbf{S}_{\beta}^{*}(A)-$ конечная циклическая группа. Остается заметить, что группа $\mathbf{S}_{\beta}^{*}(A)$ конечна в силу определения 7.13 и (ii). 
7.15. Лемма. (i) Для любых $A \in \mathcal{M}_{\alpha} u \mathbf{g} \in \mathbf{N}_{\alpha-1}^{+}(A)$ выполнено

$$
\operatorname{ind}_{A^{-1}}(\mathbf{g})=-\operatorname{ind}_{A}(\mathbf{g}) \text {. }
$$

(ii) Ecли $A, B \in \mathcal{M}_{\alpha} u \mathbf{h}^{-1}[A]_{\alpha-1}^{t} \mathbf{h}=[B]_{\alpha-1}^{\epsilon t}$ для некоторых $t \neq 0 u \epsilon= \pm 1$, то для любого $\mathbf{g} \in \mathbf{N}_{\alpha-1}^{+}(A)$ выполнено

$$
\operatorname{ind}_{A}(\mathbf{g})=\operatorname{ind}_{B^{\epsilon}}\left(\mathbf{h}^{-1} \mathbf{g h}\right) .
$$

В частности, с учетом предложения 7.2, (iii) получаем

$$
\mathbf{h}^{-1} \mathbf{N}_{\alpha-1}^{*}(A) \mathbf{h}=\mathbf{N}_{\alpha-1}^{*}(B) .
$$

ДоКАЗАТЕЛЬСТВО. Оба утверждения легко вытекают из предложения 7.10, (i).

7.16. СлЕДСТВИЕ. $\mathbf{N}_{\alpha-1}^{*}(A)$ есть нормальная подгруппа группы $\mathbf{N}_{\alpha-1}(A)$.

ДоКАЗАТЕЛЬСТВО. Действительно, из определений 7.1, 7.12 и леммы 7.15 следует, что для любых $\mathbf{g} \in \mathbf{N}_{\alpha-1}^{*}(A)$ и $\mathbf{h} \in \mathbf{N}_{\alpha-1}^{-}(A)$ выполнено $\mathbf{h}^{-1} \mathbf{g h} \in \mathbf{N}_{\alpha-1}^{*}(A)$. Так как подгруппа $\mathbf{N}_{\alpha-1}^{*}(A)$ нормальна в $\mathbf{N}_{\alpha-1}^{+}(A)$, то $\mathbf{N}_{\alpha-1}^{*}(A)$ нормальна и в $\mathbf{N}_{\alpha-1}(A)$.

7.17. СлЕДСТВИЕ. Для любого $\mathbf{g} \in \mathbf{N}_{\alpha-1}^{-}(A)$ выполнено $\mathbf{g}^{2} \in \mathbf{N}_{\alpha-1}^{*}(A)$.

ДоКАЗАТЕЛЬСТво. Используя пп. (i) и (ii) леммы 7.15 , с учетом леммы 7.2, (ii) получаем

$$
\operatorname{ind}_{A}\left(\mathbf{g}^{2}\right)=\operatorname{ind}_{A}\left(\mathbf{g}^{-1} \cdot \mathbf{g}^{2} \cdot \mathbf{g}\right)=\operatorname{ind}_{A^{-1}}\left(\mathbf{g}^{2}\right)=-\operatorname{ind}_{A}\left(\mathbf{g}^{2}\right)
$$

и, следовательно, $\operatorname{ind}_{A}\left(\mathbf{g}^{2}\right)=0$.

7.18. ПРЕДЛОЖЕНИЕ. Для любого $A \in \mathcal{M}_{\alpha}$ имеют место следующие соотношения в группе $\mathbf{B}_{\alpha-1}$ :

(i) $\mathbf{g}^{n}=[A]_{\alpha-1}^{n \operatorname{ind}_{A}(\mathbf{g})} \partial$ ля любого $\mathbf{g} \in \mathbf{N}_{\alpha-1}^{+}(A)$;

(ii) $\mathbf{g}^{-1}[A]_{\alpha-1}^{n / 2} \mathbf{g}=[A]_{\alpha-1}^{n / 2}$ для любого $\mathbf{g} \in \mathbf{N}_{\alpha-1}^{+}(A)$;

(iii) $\mathbf{g}^{-1}[A]_{\alpha-1}^{n / 2} \mathbf{g}=[A]_{\alpha-1}^{-n / 2}$ для любого $\mathbf{g} \in \mathbf{N}_{\alpha-1}^{-}(A)$.

ДокАЗАТЕЛЬСТВО. При $\alpha=1$ утверждение легко вытекает из определения 7.1. При $\alpha \geqslant 2$ вьполнение соотношений (i) и (ii) вытекает из предложения 7.14, (ii), следствия 7.16 и индуктивного предположения 15.11 , (i) при $\mathbf{G}:=\mathbf{N}_{\alpha-1}^{*}(A)$ и, соответственно, $\mathbf{x}:=[A]_{\alpha-1}^{\operatorname{ind}_{A}(\mathbf{g})}, \mathbf{g}:=[A]_{\alpha-1}^{-\operatorname{ind}_{A}(\mathbf{g})} \mathbf{g}$ или $\mathbf{x}:=[A]_{\alpha-1}, \mathbf{g}:=$ $[A]_{\alpha-1}^{-\operatorname{ind}_{A}(\mathbf{g})} \mathbf{g}$. Соотношение (iii) истинно в силу предложения 7.14 , (ii), следствий $7.16,7.17$ и индуктивного предположения 15.11 , (ii) при $\mathbf{x}:=[A]_{\alpha-1}$ и $\mathbf{h}:=\mathbf{g}$.

7.19. СлЕДСтвИЕ. Для любого $A \in \mathcal{E}_{\beta}$ при $\beta \leqslant \alpha$ әлемент $[A]_{\alpha}^{n / 2}$ принадлежит чентру группы $\mathbf{S}_{\alpha}(A)$. 
7.20. ЛЕмма. Если $A, B \in \mathcal{E}_{\beta}, \quad \beta \leqslant \alpha u \mathbf{h}^{-1}[A]_{\beta-1}^{r} \mathbf{h}=[B]_{\beta-1}^{\epsilon r} \partial \Omega я н$ некоторых $r \neq 0$ u $\epsilon= \pm 1$, mо $\tilde{\mathbf{h}}^{-1} \mathbf{S}_{\alpha}(A) \tilde{\mathbf{h}}=\mathbf{S}_{\alpha}(B), \quad \tilde{\mathbf{h}}^{-1} \mathbf{S}_{\alpha}^{+}(A) \tilde{\mathbf{h}}=\mathbf{S}_{\alpha}^{+}(B) u$ $\tilde{\mathbf{h}}^{-1} \mathbf{S}_{\alpha}^{*}(A) \tilde{\mathbf{h}}=\mathbf{S}_{\alpha}^{*}(B)$, əде $\tilde{\mathbf{h}} \rightleftharpoons \pi_{\beta-1, \alpha}(\mathbf{h})$.

ДокАЗАТЕЛЬСТво вытекает из определения 7.13 и лемм 7.2, (iii), 7.15, (ii).

7.21. Лемма. Пусть $A \in \mathcal{M}_{\alpha}, B$ - период ранга $\alpha, \quad \mathrm{P} \in \Pi \operatorname{pe}\left(\Gamma_{\alpha-1}, A, 2\right)$, $\mathrm{Q} \in \Pi_{\mathrm{ep}}\left(\Gamma_{\alpha-1}, B, 2\right) \quad u \mathbf{s}_{A, \mathrm{P}}^{k t}=\mathbf{s}_{B, \mathrm{Q}}^{t}$ для некоторых $k, t>0$. Пусть $\mathrm{K} \in$

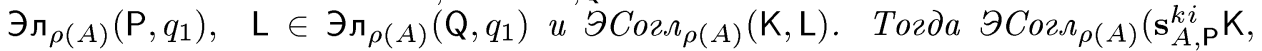
$\left.\mathbf{s}_{B, \mathrm{Q}}^{i} \mathrm{~L}\right) \partial \Omega_{\text {s }}$ любого $i \in \mathbb{Z}$.

$B$ частности, если $A, B \in \mathcal{M}_{\alpha}, \mathrm{P} \in \Pi_{\mathrm{ep}}\left(\Gamma_{\alpha-1}, A, 2\right), \quad \mathrm{Q} \in \Pi_{\text {ep }}\left(\Gamma_{\alpha-1}, B, 2\right)$

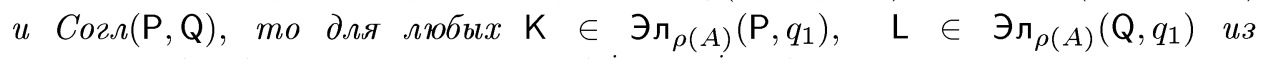

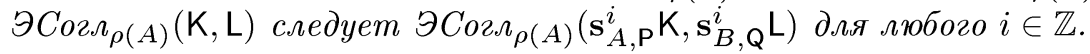

ДОКАЗАТЕЛЬСТВО. Без ограничения общности можно считать, что слово $\mathcal{L}(\mathrm{P})$ начинается с $A$. Обозначив $\mathrm{P}_{1} \rightleftharpoons \mathrm{s}_{B, \mathrm{Q}} \mathrm{P}$, по лемме 3.2 , (iv) получаем $\mathbf{s}_{A, \mathrm{P}_{1}}^{k t}=$ $\mathbf{s}_{B, \mathrm{Q}}^{-1} \mathbf{s}_{A, \mathrm{P}}^{k t} \mathbf{s}_{B, \mathrm{Q}}=\mathbf{s}_{A, \mathrm{P}}^{k t}$ и, следовательно, Согл( $\left.\mathrm{P}, \mathbf{s}_{B, \mathrm{Q}} \mathrm{P}\right)$. По предложению 7.3

$$
(\iota(\mathrm{P}))^{-1} \mathbf{s}_{B, \mathrm{Q}} \iota(\mathrm{P})=(\iota(\mathrm{P}))^{-1} \iota\left(\mathbf{s}_{B, \mathrm{Q}} \mathrm{P}\right) \in \mathbf{N}_{\alpha-1}^{+}(A) .
$$

Так как в силу п. (iii) предложения 7.10

$$
\operatorname{ind}_{A}\left((\iota(\mathrm{P}))^{-1} \mathbf{s}_{B, \mathrm{Q}}^{t} \iota(\mathrm{P})\right)=\operatorname{ind}_{A}\left((\iota(\mathrm{P}))^{-1} \mathbf{s}_{A, \mathrm{P}}^{k t} \iota(\mathrm{P})\right)=\operatorname{ind}_{A}\left([A]_{\alpha-1}^{k t}\right)=k t,
$$

то в силу п. (iv) предложения 7.10 для любого $i \in \mathbb{Z}$ имеем

$$
\operatorname{ind}_{A}\left((\iota(\mathrm{P}))^{-1} \mathbf{s}_{B, \mathrm{Q}}^{i} \iota(\mathrm{P})\right)=k i
$$

Используя предложение 7.8 и следствие 7.11 при $\mathbf{g}:=\mathbf{s}_{B, \mathrm{Q}}^{i}$ и $i:=k i$, получаем $Э \operatorname{Coгл}_{\rho(A)}\left(\mathbf{s}_{A, \mathrm{P}}^{k i} \mathrm{~K}, \mathbf{s}_{B, \mathrm{Q}}^{i} \mathrm{~K}\right)$, что с учетом $\vartheta^{C o z \Omega_{\rho(A)}}(\mathrm{K}, \mathrm{L})$ влечет $\vartheta \operatorname{Coz}_{\rho(A)}\left(\mathbf{s}_{A, \mathrm{P}}^{k i} \mathrm{~K}, \mathbf{s}_{B, \mathrm{Q}}^{i} \mathrm{~L}\right)$.

7.22. Лемма. Если $A, B \in \mathcal{M}_{\alpha}, \mathrm{P} \in \Pi \operatorname{\Pi e}\left(\Gamma_{\alpha-1}, A, 2\right), \quad \mathrm{Q} \in \Pi \operatorname{ep}\left(\Gamma_{\alpha-1}, B, 2\right)$ и Согл(P, Q), то Род $(A, B)$. Обратно, если Род $\alpha(A, B)$, то существуют согласованные периодические отрезки $\mathrm{P} \in \Pi_{\mathrm{ep}}\left(\Gamma_{\alpha-1}, A, 2\right) u \mathrm{Q} \in \Pi_{\mathrm{ep}}\left(\Gamma_{\alpha-1}, B, 2\right)$.

ДокАЗАТЕЛЬСтво. Первое утверждение непосредственно вытекает из определений $3.3,5.4$ и формулы (3.2). Докажем второе утверждение. Пусть $P_{o} \partial_{\alpha}(A, B)$, т.е. $[A]_{\alpha-1}^{t}=\mathbf{g}[B]_{\alpha-1}^{t} \mathbf{g}^{-1}$ для некоторых $t>0$ и $\mathbf{g} \in \mathbf{B}_{\alpha-1}$. Рассмотрим пути $\mathrm{P} \in \Pi_{\mathrm{ep}}\left(\Gamma_{\alpha-1}, A, 2\right)$ и $\mathrm{Q} \in \Pi_{\mathrm{ep}}\left(\Gamma_{\alpha-1}, B, 2\right)$ с начальньми вершинами $\iota(\mathrm{P})=1$, $\iota(\mathrm{Q})=\mathbf{g}$ и метками $\mathcal{L}(\mathrm{P})$ 으 $A^{2}$ и $\mathcal{L}(\mathrm{Q})$ 으 $B^{2}$. Так как по формуле $(3.2)$ имеем $\mathbf{s}_{A, \mathrm{P}}=[A]_{\alpha-1}$ и $\mathbf{s}_{B, \mathrm{Q}}=\mathbf{g}[B]_{\alpha-1} \mathbf{g}^{-1}$, то $\mathbf{s}_{A, \mathrm{P}}^{t}=\mathbf{s}_{B, \mathrm{Q}}^{t}$, т.е. $\operatorname{Coгл}(\mathrm{P}, \mathrm{Q})$. 


\section{§ 8. Почти периодические, элементарные слова и отрезки}

8.1. Лемма. Если $\mathrm{E} \in \Pi_{\text {- }}(A)$ u $\mathrm{P} \in \Pi_{\alpha}\left(\Gamma_{\alpha-1}, A, 2\right)$ - периодическая основа для $\mathrm{E}$, то пути $\mathrm{P} u \mathrm{E}$ строго близки в ранге $\rho(A)$.

Утверждение непосредственно вытекает из определений 5.16 и 6.5.

8.2. ЛЕмма. Пусть $\beta \leqslant \alpha-1$.

(i) Отношение "пути $\mathrm{X} u \mathrm{Y}$ строго близки в ранге $\beta$ " на множестве путей на графе $\Gamma_{\alpha-1}$ транзитивно.

(ii) Если пути $\mathrm{X}$ и $\mathrm{Y}$ на графе $\Gamma_{\alpha-1}$ строго близки в ранге $\beta$, то $\mathrm{X} u \mathrm{Y}$ близки в ранге $\beta$. В частности, если $\mathrm{E} \in \Pi_{\text {Пер }}(A)$ u $\mathrm{P} \in \Pi_{\mathrm{ep}}\left(\Gamma_{\alpha}, A, 2\right)-$ периодическая основа для $\mathrm{E}$, то пути $\mathrm{E} u \mathrm{P}$ близки в ранге $\rho(A)$.

(iii) Пусть $\mathrm{X} u \mathrm{Y}$ - строго близкие в ранге $\beta$ пути на графе $\Gamma_{\alpha-1}$ $c \mathcal{L}(\mathrm{X}), \mathcal{L}(\mathrm{Y}) \in \mathcal{R}_{\alpha-1}$. Предположим, Эл $\left(\mathrm{X}, q_{4}\right)=\varnothing$ или Эл $\gamma\left(\mathrm{Y}, q_{4}\right)=\varnothing$ для всех $\gamma=\beta+1, \beta+2, \ldots, \alpha-1$. Пусть $r \geqslant q_{1}+h, \mathrm{M}_{1}, \mathrm{M}_{2}, \ldots, \mathrm{M}_{t} \in$ Эл $_{\beta}(\mathrm{X}, r)$, $\mathrm{M}_{1}<\mathrm{x} \mathrm{M}_{2}<\mathrm{x} \cdots<\mathrm{x} \mathrm{M}_{t} u \neg \vartheta$ Согл $\beta\left(\mathrm{M}_{i}, \mathrm{M}_{j}\right)$ при $i \neq j$. Тогда существуют $\mathrm{N}_{1}, \mathrm{~N}_{t} \in \ni_{\beta}\left(\mathrm{Y}, q_{1}\right), \mathrm{N}_{2}, \ldots, \mathrm{N}_{t-1} \in$ Эл $_{\beta}(\mathrm{Y}, r-h)$ maкие, что $\ni \operatorname{Coгл}_{\beta}\left(\mathrm{M}_{i}, \mathrm{~N}_{i}\right)$ для каждого $і$ и $\mathrm{N}_{1}<_{\mathrm{Y}} \mathrm{N}_{2}<_{\mathrm{Y}} \cdots<_{\mathrm{Y}} \mathrm{N}_{t}$.

ДОКАЗАТЕЛЬСТВО. Из индуктивного предположения 8.40 следует, что если пути $\mathrm{K}, \mathrm{K}^{\prime} \in$ Эл $\beta_{\beta}\left(\Gamma_{\alpha-1}, q_{1}\right)$ являются началами или конщами некоторого пути $\mathrm{X}$,

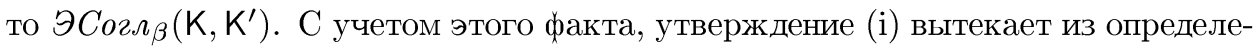
ния 6.5 и индуктивного предположения 8.7 .

Первое утверждение (ii) при $\beta=0$ непосредственно следует из определений 6.5 , 5.14 и 7.4. При $\beta \geqslant 1$ нужно сослаться на индуктивное предположение 8.45. Второе утверждение (ii) вытекает из первого в силу леммы 8.1.

Пусть истинна посылка (iii). Пусть $\mathrm{K}, \mathrm{L} \in$ Эл $_{\beta}\left(\mathrm{X}, q_{1}\right)$ - начало и конец $\mathrm{X}$ и $\mathrm{K}^{\prime}, \mathrm{L}^{\prime} \in$ Эл $_{\beta}\left(\mathrm{Y}, q_{1}\right)$ - начало и конец $\mathrm{Y}$, где $Э \operatorname{Coгл}_{\beta}\left(\mathrm{K}, \mathrm{K}^{\prime}\right), \quad$ ЭСол $\Omega_{\beta}\left(\mathrm{L}, \mathrm{L}^{\prime}\right)$ и $\neg$ ЭСогл $\beta(\mathrm{K}, \mathrm{L})$. Если $Э \operatorname{Cогл}_{\beta}\left(\mathrm{K}, \mathrm{M}_{1}\right)$ или $Э \operatorname{Cогл}_{\beta}\left(\mathrm{L}, \mathrm{M}_{1}\right)$, то в качестве $\mathrm{N}_{1}$ возьмем соответственно $\mathrm{K}^{\prime}$ или $\mathrm{L}^{\prime} . \mathrm{B}$ случае $\neg$ ЭСогл $\beta\left(\mathrm{K}, \mathrm{M}_{1}\right)$ и $\neg$ ЭСогл $\beta\left(\mathrm{L}, \mathrm{M}_{1}\right)$ по индуктивному предположению 8.40 имеем $\mathrm{K}<_{\mathrm{x}} \mathrm{M}_{1}<_{\mathrm{x}} \mathrm{L}, \mathrm{K}^{\prime}<_{\mathrm{Y}} \mathrm{L}^{\prime}$, и по индуктивному предположению 11.23 найдется $\mathrm{N}_{1} \in \ni л\left(\mathrm{Y}, q_{1}\right)$ такой, что $Э \operatorname{Coгл}_{\beta}\left(\mathrm{M}_{1}, \mathrm{~N}_{1}\right)$. Если $t=1$, то доказывать больше нечего. Пусть $t>1$. Аналогично предыдущему для некоторого $\mathrm{N}_{t} \in \ni л\left(\mathrm{Y}, q_{1}\right)$ имеем $Э \operatorname{Coг}_{\beta}\left(\mathrm{M}_{t}, \mathrm{~N}_{t}\right)$. По индуктивному предположению $11.27 \mathrm{~N}_{1}<_{\mathrm{\gamma}} \mathrm{N}_{t}$. Тогда по индуктивному предположению 11.23 при $\left(\mathrm{K}, \mathrm{L}, \mathrm{M}, \mathrm{K}^{\prime}, \mathrm{L}^{\prime}\right):=\left(\mathrm{M}_{1}, \mathrm{M}_{t}, \mathrm{M}_{i}, \mathrm{~N}_{1}, \mathrm{~N}_{t}\right)$ для каждого $2 \leqslant i \leqslant t-1$ найдется $\mathrm{N}_{i} \in$ Эл $(\mathrm{Y}, r-h)$ такой, что $Э \operatorname{Coz}_{\beta}\left(\mathrm{M}_{i}, \mathrm{~N}_{i}\right)$. Вновь используя индуктивное предположение 11.27 , получаем $\mathrm{N}_{1}<_{\mathrm{Y}} \mathrm{N}_{2}<_{\mathrm{Y}} \cdots<_{\mathrm{Y}} \mathrm{N}_{t}$.

8.3. ЛЕмма. Если $\mathrm{E} \in \Pi$ Пер $\alpha(A, 5) u \mathrm{P}, \mathrm{Q} \in \Pi_{\mathrm{ep}}\left(\Gamma_{\alpha-1}, A, 2\right)$ - периодические основы для $\mathrm{E}$, то $\operatorname{Cогл~}(\mathrm{P}, \mathrm{Q})$.

ДоКАЗАТЕЛЬСТВо. В силу определения 5.17 и транзитивности отношения Согл(P,Q) достаточно рассмотреть случай, когда $\mathrm{K}, \mathrm{s}_{A, \mathrm{P}} \mathrm{K} \ldots \mathrm{s}_{A, \mathrm{P}}^{5} \mathrm{~K} \in$ $\exists_{\rho(A)}\left(\mathrm{P}, q_{2}\right)$ для некоторого К. В этом случае утверждение следует из лемм 8.1, $8.2,(\mathrm{i})$ и предложения 6.11. 
8.4. Лемма. Если $A$ - период ранга $\alpha$ и в некоторое слово $P \in \Pi е р(A)$ входит әлементарная $r$-степень ранга $\beta \leqslant \alpha-1$ при $r \geqslant 19$, то некоторый ииклический сдвиг $A$ содержит әлементарную $(r-9)$-степень ранга $\beta, u$, следовательно, любой периодический отрезок $\mathrm{P} \in \Pi_{\mathrm{p}}\left(\Gamma_{\alpha-1}, A, t\right)$ содержит в качестве подпутей $t-1$ nутей $\mathrm{K}, \mathbf{s}_{A, \mathrm{P}} \mathrm{K}, \mathbf{s}_{A, \mathrm{P}}^{2} \mathrm{~K}, \ldots, \mathbf{s}_{A, \mathrm{P}}^{t-2} \mathrm{~K}$, где $\mathrm{K} \in \ni{ }_{\beta}\left(\Gamma_{\alpha-1}\right.$, $r-9)$.

В частности, если $A \in \mathcal{M}_{\alpha} u \mathrm{P} \in \Pi_{\text {ep }}\left(\Gamma_{\alpha-1}, A, t\right)$, то для некоторого $\mathrm{K}$ имеем $\mathrm{K}, \mathrm{s}_{A, \mathrm{P}} \mathrm{K}, \ldots, \mathrm{s}_{A, \mathrm{P}}^{t-2} \mathrm{~K} \in \ni_{\rho(A)}\left(\mathrm{P}, q_{2}\right)$.

ДоКАЗАТЕЛЬСТвО вытекает из леммы 7.6 , определения 5.15 и равенства $q_{3}=$ $q_{2}+9$

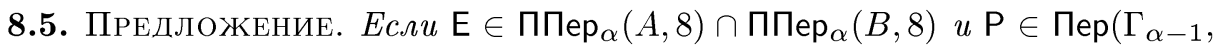
$A, 2), \quad \mathrm{Q} \in \Pi_{\mathrm{ep}}\left(\Gamma_{\alpha-1}, B, 2\right)$ - периодические основы для $\mathrm{E}$, то Согл(Р, $\left.\mathrm{Q}\right)$.

ДоКАЗАТЕЛЬСТВо. В силу леммы 8.3 и определения 5.17 достаточно рассмотреть случай, когда $\mathrm{M}, \mathbf{s}_{A, \mathrm{P}} \mathrm{M}, \ldots, \mathbf{s}_{A, \mathrm{P}}^{8} \mathrm{M} \in$ Эл $_{\rho(A)}\left(\mathrm{P}, q_{2}\right)$ и $\mathrm{N}, \mathbf{s}_{B, \mathrm{Q}} \mathrm{N}, \ldots, \mathbf{s}_{B, \mathrm{Q}}^{8} \mathrm{~N} \in$ $\ni_{\rho(B)}\left(\mathrm{Q}, q_{2}\right)$ для некоторых $\mathrm{M}$ и $\mathrm{N}$. Если $\rho(B)=\rho(A)$, то утверждение вытекает из лемм $8.1,8.2$, (i) и предложения 6.12. Пусть $\rho(A) \neq \rho(B)$. Без ограничения общности можно считать $\rho(A)>\rho(B)$. Пусть $\mathrm{K}, \mathrm{L} \in \ni(A)\left(\mathrm{P}, q_{2}\right)-$ начало и конец $\mathrm{P}$,

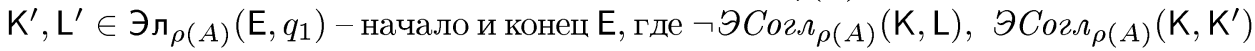
и $Э \operatorname{Coz}_{\rho(A)}\left(\mathrm{L}, \mathrm{L}^{\prime}\right)$. По лемме 8.2 , (ii) пути $\mathrm{E}$ и $\mathrm{Q}$ близки в ранге $\rho(B)$. Тогда по индуктивному предположению 11.21 найдутся $\mathrm{K}^{*}, \mathrm{~L}^{*} \in \ni_{\rho(A)}\left(\mathrm{Q}, q_{2}-h\right)$ такие, что $Э \operatorname{Coг}_{\rho(A)}\left(\mathrm{K}, \mathrm{K}^{*}\right)$ и $Э \operatorname{Coг}_{\rho(A)}\left(\mathrm{L}, \mathrm{L}^{*}\right)$. По индуктивному предположению 11.27 имеем $\mathrm{K}^{*}<{ }_{\mathrm{Q}} \mathrm{L}^{*}$. Пусть $\mathrm{Q}^{*} \rightleftharpoons \mathrm{K}^{*} \cup_{\mathrm{Q}} \mathrm{L}^{*}$ и $\mathrm{Q}=\mathrm{UQ} \mathrm{Q}^{*} \mathrm{~V}$. Если $|\mathrm{U}| \geqslant|B|, \operatorname{to~}_{B, \mathrm{Q}}^{-1} \mathrm{~K}^{*}$ содержится в $\mathrm{Q}$ и тогда в силу леммы 6.7 и индуктивных предположений $11.21,11.27$

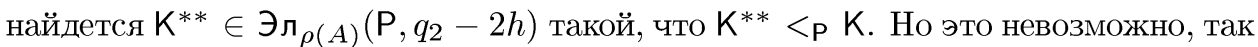
как $\mathrm{K}$ есть начало $\mathrm{P}$. Следовательно, $|\mathrm{U}|<|B|$. Аналогично доказывается, что $|\mathrm{V}|<|B|$. Так как $|\mathrm{Q}| \geqslant 8|B|$, то отсюда следует $\left|\mathrm{Q}^{*}\right|>6|B|$. В силу леммы 8.4 для некоторого $\mathrm{N}^{\prime}$ имеем $\mathrm{N}^{\prime}, \mathbf{s}_{B, \mathrm{Q}} \mathrm{N}^{\prime}, \ldots, \mathbf{s}_{B, \mathrm{Q}}^{4} \mathrm{~N}^{\prime} \in$ Эл $_{\rho(A)}\left(\mathrm{Q}^{*}, q_{2}-h-9\right)$. Так как $q_{2}-h-9>q_{1}$, то по предложению 6.12 получаем $\operatorname{Cozл}\left(\mathrm{P}, \mathrm{Q}^{*}\right)$ и, следовательно, $\operatorname{Cozл}(\mathrm{P}, \mathrm{Q})$.

8.6. СлЕДСтвиЕ. (i) Если $\mathrm{E}, \mathrm{F} \in \bigcup_{A}$ ППер $(A, 8)$, $\Pi$ Согл $\alpha(\mathrm{E}, \mathrm{F}), \quad \mathrm{P} u \mathrm{Q}-$ любие периодические основы для $\mathrm{E}$ и $\mathrm{F}$ соответственно, то Согл( $\mathrm{P}, \mathrm{Q})$.

(ii) Отношение ПСогл $(\mathrm{E}, \mathrm{F})$ на множестве $\bigcup_{A}$ ППер $\alpha(A, 8)$ является отношением эквивалентности.

ДоКАЗАТЕЛЬСТВО вытекает из определения 5.18, предложения 8.5 и леммы 3.4, (i).

8.7. СлЕДСТВИЕ $(\alpha \geqslant 0)$. Для любого $\beta \geqslant \alpha$ отношение $Э С о г \Omega_{\alpha}(\mathrm{E}, \mathrm{F})$ на множестве Эл $\alpha\left(\Gamma_{\beta}\right)$ является отношением әквивалентности.

ДокАЗАТЕЛЬСТво. При $\alpha=0$ доказывать нечего (см. определение 5.14). Пусть $\alpha \geqslant 1$. Рефлексивность и симметричность отношения ЭСогл $\alpha(\mathrm{E}, \mathrm{F})$ вытекает из определения 5.20 , рефлексивности и симметричности отношения 
ПСогл $\alpha(\overline{\mathrm{E}}, \overline{\mathrm{F}})$ для $\overline{\mathrm{E}}, \overline{\mathrm{F}} \in \bigcup_{A}$ ППер $\alpha(A, 8)$ и инвариантности этого отношения относительно замены путей на обратные. Пусть $\mathrm{E}, \mathrm{F}, \mathrm{G} \in \ni \boldsymbol{л}_{\alpha}\left(\Gamma_{\beta}\right), Э \mathrm{Coz}_{\alpha}(\mathrm{E}, \mathrm{F})$ и $Э С о г \Omega_{\alpha}(\mathrm{F}, \mathrm{G})$. Согласно определению 5.20 для некоторых прообразов $\overline{\mathrm{E}}, \overline{\mathrm{F}}$, $\bar{F}^{\prime}$ и $\bar{G}$ на графе $\Gamma_{\alpha-1}$ путей $\mathrm{E}, \mathrm{F}$ и $\mathrm{G}$ относительно отображения $\pi_{\alpha-1, \beta}$ имеем ПСогл $\Omega_{\alpha}\left(\overline{\mathrm{E}}, \overline{\mathrm{F}}^{\epsilon}\right)$ и ПСогл $\Omega_{\alpha}\left(\overline{\mathrm{F}}^{\prime}, \overline{\mathrm{G}}^{\delta}\right)$, где $\epsilon, \delta= \pm 1$. Так как $\pi_{\alpha-1, \beta}(\overline{\mathrm{F}})=\pi_{\alpha-1, \beta}\left(\overline{\mathrm{F}}^{\prime}\right)=$ $\mathrm{F}$, то $\overline{\mathrm{F}}^{\prime}=\mathrm{g} \overline{\mathrm{F}}$ для некоторого $\mathrm{g} \in \operatorname{ker} \pi_{\alpha-1, \beta}$. Из ПСогл $\Omega_{\alpha}\left(\overline{\mathrm{F}}^{\prime}, \overline{\mathrm{G}}^{\delta}\right)$ умножением на $\mathbf{g}^{-1}$ получаем ПСогл $\Omega_{\alpha}\left(\overline{\mathrm{F}}, \mathbf{g}^{-1} \overline{\mathrm{G}}^{\delta}\right)$, что вместе с ПСогл $\alpha\left(\overline{\mathrm{E}}, \overline{\mathrm{F}}^{\epsilon}\right)$ в силу следствия 8.6, (ii) дает ПСогл $\Omega_{\alpha}\left(\overline{\mathrm{E}}, \mathbf{g}^{-1} \overline{\mathrm{G}}^{\epsilon \delta}\right)$. Тогда согласно определению 5.20 $\exists \operatorname{Coz} \Omega_{\alpha}(\mathrm{E}, \mathrm{G})$.

8.8. СлЕДСТВИЕ $(\alpha \geqslant 0)$. Для любых $\beta \geqslant \alpha u \mathrm{E} \in \ni_{\alpha}\left(\Gamma_{\beta}\right)$ множсество $\mathbf{H} \rightleftharpoons\left\{\mathbf{g} \in \mathbf{B}_{\beta} \mid\right.$ ЭСогл $\left.\alpha(\mathrm{E}, \mathbf{g E})\right\}$ есть конечная подгруппа группь $\mathbf{B}_{\beta}$. Более того, $\mathbf{H}=1$ при $\alpha=0 u \mathbf{H} \subseteq \mathbf{x S}_{\beta}(A) \mathbf{x}^{-1}$ для некоторых $\mathbf{x} \in \mathbf{B}_{\beta} u A \in \mathcal{E}_{\alpha} n р и$ $\alpha>0$.

ДокАЗАТельство. Утверждение тривиально при $\alpha=0$. Пусть $\alpha \geqslant 1$. Тот факт, что $\mathbf{H}$ - подгруппа группы $\mathbf{B}_{\beta}$, вытекает из инвариантности отношения $\vartheta$ Согл $_{\alpha}(\mathrm{F}, \mathrm{G})$ относительно действия групшы $\mathbf{B}_{\beta}$ на графе $\Gamma_{\beta}$ и следствия 8.7. Выберем произвольный прообраз $\overline{\mathrm{E}}$ на графе $\Gamma_{\alpha-1}$ пути $\mathrm{E}$. Согласно лемме 5.21 имеем $\overline{\mathrm{E}} \in \Pi_{\text {Пер }}(A, 8)$ для некоторого $A \in \mathcal{E}_{\alpha}$. Пусть $\mathrm{P} \in \Pi$ Пер $\left(\Gamma_{\alpha-1}, A, 2\right)$ - периодическая основа для $\overline{\mathrm{E}}$. Заменяя слово $A$ на его циклический сдвиг, можно считать, что слово $\mathcal{L}(\mathrm{P})$ начинается с $A$. Пусть $\mathbf{g} \in \mathbf{H}-$ произвольный элемент. Согласно определению 5.20 для некоторых $\overline{\mathrm{E}}^{\prime}$ и $\overline{\mathrm{E}}^{\prime \prime}$ имеем $\pi_{\alpha-1, \beta}\left(\overline{\mathrm{E}}^{\prime}\right)=\mathrm{E}, \pi_{\alpha-1, \beta}\left(\overline{\mathrm{E}}^{\prime \prime}\right)=\mathbf{g E}$ и ПСогл $\Omega_{\alpha}\left(\overline{\mathrm{E}}^{\prime}, \overline{\mathrm{E}}^{\prime \prime}\right)$ или ПСогл $\Omega_{\alpha}\left(\overline{\mathrm{E}}^{\prime}, \overline{\mathrm{E}}^{\prime \prime}-1\right)$. Умножая $\overline{\mathrm{E}}^{\prime}$ и $\overline{\mathrm{E}}^{\prime \prime}$ слева на $\iota(\overline{\mathrm{E}})\left(\iota\left(\overline{\mathrm{E}}^{\prime}\right)\right)^{-1}$, можно считать $\overline{\mathrm{E}}=\overline{\mathrm{E}}^{\prime}$. Имеем $\overline{\mathrm{E}}^{\prime \prime}=\overline{\mathbf{g}} \overline{\mathrm{E}}$, где $\pi_{\alpha-1, \beta}(\overline{\mathbf{g}})=\mathbf{g}$, и так как $\overline{\mathbf{g}} \mathrm{P}$ - периодическая основа для $\overline{\mathrm{E}}^{\prime \prime}$, то в силу следствия 8.6, (i) и предложения $7.3(\iota(\mathrm{P}))^{-1} \overline{\mathbf{g}} \iota(\mathrm{P}) \in$ $\mathbf{N}_{\alpha-1}(A)$. Следовательно, $\mathbf{H} \subseteq \mathbf{x S}_{\beta}(A) \mathbf{x}^{-1}$, где $\mathbf{x} \rightleftharpoons \pi_{\alpha-1, \beta}(\iota(\mathrm{P}))$. Остается заметить, что в силу предложения 7.14 , (iii) группа $\mathbf{S}_{\beta}(A)$ конечна.

8.9. ПрЕДЛОЖЕНИЕ. Если $\mathrm{E} \in \Pi \Pi е p_{\alpha}(A, 8), \mathrm{F} \in \Pi_{\text {Mep }}(A) u \mathrm{E}$ содержится ${ }_{\varepsilon} \mathrm{F},{\operatorname{mo~} \Pi \operatorname{Coz} \Omega_{\alpha}}(\mathrm{E}, \mathrm{F})$.

ДокаЗАТЕЛЬСтво. Пусть $\mathrm{P}, \mathrm{Q} \in$ Пер $\left(\Gamma_{\alpha-1}, A, 2\right)$ - периодические основы для Е и $\mathrm{F}$ соответственно. В силу леммы 8.3 можно считать, что $\mathrm{M}, \mathrm{s}_{A, \mathrm{P}} \mathrm{M}, \ldots, \mathrm{s}_{A, \mathrm{P}}^{8} \mathrm{M} \in$ $\ni_{\rho(A)}\left(\mathrm{P}, q_{2}\right)$ для некоторого М. Так как $q_{2}=q_{1}+2 h$, то, применив два раза лемму 8.2, (iii) вначале при $\mathrm{X}:=\mathrm{P}$ и $\mathrm{Y}:=\mathrm{E}$, а затем при $\mathrm{X}:=\mathrm{F}$ и $\mathrm{Y}:=\mathrm{Q}$, найдем

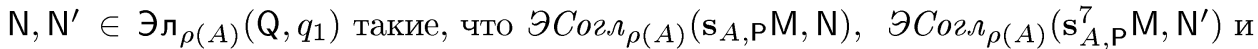
$\mathrm{N}<_{\mathrm{Q}} \mathrm{N}^{\prime}$. Тогда по предложению 6.11 при $\mathrm{P}:=\mathbf{s}_{A, \mathrm{P}} \mathrm{M} \cup \cup_{\mathrm{P}} \mathbf{s}_{A, \mathrm{P}}^{7} \mathrm{M}$ и $\mathrm{Q}:=\mathrm{N} \cup_{\mathrm{Q}} \mathrm{N}^{\prime}$

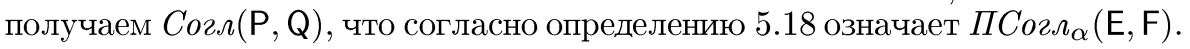

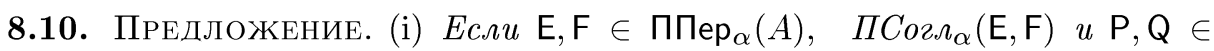
Пер $\left(\Gamma_{\alpha-1}, A, 2\right)$ - периодические основы для $\mathrm{E}$ и $\mathrm{F}$ соответственно, то существует периодическая основа $\widetilde{\mathrm{Q}}$ для $\mathrm{F}$ такая, что $\mathrm{P} u \widetilde{\mathrm{Q}}$ имеют общее периодическое продолжение и $\mathcal{L}(\widetilde{\mathrm{Q}})$ 으 $\mathcal{L}(\mathrm{Q})$.

(ii) Ecли $\mathrm{E}, \mathrm{F} \in \Pi$ Пер $\alpha(A, 8), \mathrm{E}$ содержится в $\mathrm{F} u \mathrm{P} \in \Pi$ ер $\left(\Gamma_{\alpha-1}, A, 2\right)$ - neриодическая основа для $\mathrm{E}$, то в качестве периодической основы для $\mathrm{F}$ можно взять некоторое периодическое продолжение $\mathrm{P}$. 
ДокаЗАтеЛЬСтво. Пусть $\mathrm{E}, \mathrm{F} \in \Pi^{-} \in \operatorname{pop}_{\alpha}(A), \quad$ ПСогл $\alpha(\mathrm{E}, \mathrm{F})$ и $\mathrm{P}, \mathrm{Q} \in$ Пер $\left(\Gamma_{\alpha-1}, A, 2\right)$ - периодические основы для $\mathrm{E}$ и $\mathrm{F}$ соответственно. Пусть $\mathrm{K}, \mathrm{L} \in$

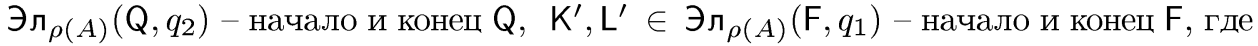
$\neg \vartheta \operatorname{Coz}_{\rho(A)}(\mathrm{K}, \mathrm{L}), Э \operatorname{Coz}_{\rho(A)}\left(\mathrm{K}, \mathrm{K}^{\prime}\right)$ и $Э \operatorname{Coz}_{\rho(A)}\left(\mathrm{L}, \mathrm{L}^{\prime}\right)$. По лемме $3.1,(\mathrm{v})$ выберем элемент $\mathbf{g} \in \mathbf{B}_{\alpha-1}$ такой, что $\mathbf{g Q}$ и $\mathrm{P}$ имеют общее периодическое продолжение $\mathrm{R}$.

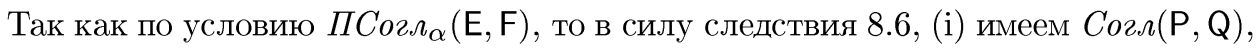
откуда и из $\operatorname{Cozл}(\mathrm{P}, \mathbf{g Q})$ следует $\operatorname{Cozл(P,~} \mathrm{gP})$. В силу предложения 7.8 и следствия 7.11 для некоторого $t$ имеем $Э \operatorname{Cozл}_{\rho(A)}\left(\mathrm{K}, \mathbf{g s}_{A, \mathrm{Q}}^{t} \mathrm{~K}\right)$ и $\vartheta^{-C_{0} \Omega_{\rho(A)}}\left(\mathrm{L}, \mathbf{g s}_{A, \mathrm{Q}}^{t} \mathrm{~L}\right)$. Так как пути $\mathrm{K}^{*} \rightleftharpoons \mathbf{g s}_{A, Q}^{t} \mathrm{~K}_{\text {и }} \mathrm{L}^{*} \rightleftharpoons \mathbf{g s}_{A, Q}^{t} \mathrm{~L}$ содержатся в некотором периодическом продолжении $g Q$, то, удлинив $R$, можно считать, что $\mathrm{K}^{*}$ и $\mathrm{L}^{*}$ - подпути $\mathrm{R}$. Из $\neg$ ЭСогл $\rho_{(A)}(\mathrm{K}, \mathrm{L})$ и $\mathrm{K}<<_{\mathrm{Q}} \mathrm{L}$ следует $\neg$ ЭСогл $\rho(A)\left(\mathrm{K}^{*}, \mathrm{~L}^{*}\right)$ и $\mathrm{K}^{*}<{ }_{\mathrm{R}} \mathrm{L}^{*}$ и, таким образом, $\widetilde{\mathrm{Q}} \rightleftharpoons \mathrm{K}^{*} \cup_{\mathrm{R}} \mathrm{L}^{*}$ - периодическая основа для $\mathrm{F}$. Так как, очевидно, $\widetilde{\mathrm{Q}}=\mathbf{g s}_{A, \mathrm{Q}}^{t} \mathrm{Q}$, то $\mathcal{L}(\widetilde{Q})$ 으 $\mathcal{L}(\mathrm{Q})$. Тем самым доказано (i). Пусть выполнена посылка (ii). В силу предложения 8.9 мы находимся в условиях п.(i), и тогда в силу (i) найдется периодическая основа $\widetilde{Q}$ для $F$ такая, что $P$ и $\widetilde{Q}$ имеют общее периодическое продолжение R. В этом случае, используя индуктивное предположение 11.25 , легко видеть, что $\widetilde{Q} \cup_{R} P$ - также периодическая основа для $F$, которую и можно взять в качестве искомой.

8.11. ПРЕДЛОЖЕНИЕ. Пусть $\mathrm{X}-$ nуть на графе $\Gamma_{\alpha-1} c \mathcal{L}(\mathrm{X}) \in \mathcal{R}_{\alpha-1}$. Пусть

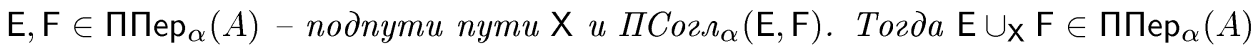
и $\ell_{\alpha, A}(\mathrm{E} \cup \mathrm{X} F) \geqslant \max \left\{\ell_{\alpha, A}(\mathrm{E}), \ell_{\alpha, A}(\mathrm{~F})\right\}$, а если пути $\mathrm{E} u \mathrm{~F}$ не имеют общих ребер, mo $\ell_{\alpha, A}(\mathrm{E} \cup \mathrm{x} F) \geqslant \ell_{\alpha, A}(\mathrm{E})+\ell_{\alpha, A}(\mathrm{~F})-1$.

ДоКАЗАТЕЛЬСТво. Если $E$ содержится в $F$ или $F$ содержится в $E$, то доказывать нечего. Пусть ни один из путей $E, F$ не содержится в другом, т.е. $\mathrm{E}<\mathrm{x} \quad \mathrm{F}$ или $\mathrm{F}<\mathrm{x}$ E. Без ограничения общности можно считать $\mathrm{E}<\mathrm{x}$ F. Пусть $\mathrm{P}, \mathrm{Q} \in$ Пер $\left(\Gamma_{\alpha-1}, A, 2\right)$ - периодические основы для $\mathrm{E}$ и $\mathrm{F}$ соответственно. В силу определения 5.17 и предложения 8.10, (i) можно считать, что $\mathrm{P}$ и $\mathrm{Q}$ имеют обшее периодическое продолжение $\mathrm{R}$, причем $\mathrm{M}$, $\mathbf{s}_{A, \mathrm{R}} \mathrm{M}, \ldots, \mathbf{s}_{A, \mathrm{R}}^{r} \mathrm{M} \in$ Эл $_{\rho(A)}\left(\mathrm{P}, q_{2}\right)$ и $\mathrm{N}, \mathbf{s}_{A, \mathrm{R}} \mathrm{N}, \ldots, \mathbf{s}_{A, \mathrm{R}}^{t} \mathrm{~N} \in$ Эл $_{\rho(A)}\left(\mathrm{Q}, q_{2}\right)$ для некоторых $\mathrm{M}$ и N, где $r \rightleftharpoons \ell_{\alpha, A}(\mathrm{E})$ и $t \rightleftharpoons \ell_{\alpha, A}(\mathrm{~F})$. Пусть $\mathrm{K}, \mathrm{L} \in$ Эл $\rho(A)\left(\mathrm{P}, q_{2}\right)-$ начало и конец $\mathrm{P}, \mathrm{K}^{\prime}, \mathrm{L}^{\prime} \in$ Эл $_{\rho(A)}\left(\mathrm{E}, q_{1}\right)$ - начало и конец $\mathrm{E}$, где $\neg$ ЭСогл $\Omega_{\rho(A)}(\mathrm{K}, \mathrm{L})$, $\ni \operatorname{Cогл}_{\rho(A)}\left(\mathrm{K}, \mathrm{K}^{\prime}\right)$ и $Э$ Согл $_{\rho(A)}\left(\mathrm{L}, \mathrm{L}^{\prime}\right)$. Пусть $\widetilde{\mathrm{K}}, \tilde{\mathrm{L}} \in \ni_{\rho(A)}\left(\mathrm{Q}, q_{2}\right)$ - начало и конец $\mathrm{Q}, \widetilde{\mathrm{K}}^{\prime}, \widetilde{\mathrm{L}}^{\prime} \in \ni_{\rho(A)}\left(\mathrm{F}, q_{1}\right)$ - начало и конец $\mathrm{F}$, где $\neg Э \operatorname{Coгл}_{\rho(A)}(\widetilde{\mathrm{K}}, \widetilde{\mathrm{L}})$, ЭСогл $\Omega_{(A)}\left(\widetilde{\mathrm{K}}, \widetilde{\mathrm{K}}^{\prime}\right)$ и $Э С о г \Omega_{\rho(A)}\left(\widetilde{\mathrm{L}}, \widetilde{\mathrm{L}}^{\prime}\right)$. Из $\mathrm{E}<\mathrm{x} F$ в силу индуктивного предположения 11.27 следует $\mathrm{K} \preceq_{\mathrm{R}} \widetilde{\mathrm{K}}$ и $\mathrm{L} \preceq_{\mathrm{R}} \widetilde{\mathrm{L}}$ (в обозначениях (6.2)). Тогда, очевидно, $E \cup_{X} F$ есть почти периодический отрезок ранга $\alpha$ c периодической основой $\mathrm{P} \cup_{\mathrm{R}} \mathrm{Q}$ и $\ell_{\alpha, A}(\mathrm{E} \cup \mathrm{X} F) \geqslant \max \left\{\ell_{\alpha, A}(\mathrm{E}), \ell_{\alpha, A}(\mathrm{~F})\right\}$.

Предположим теперь, что $E$ и $F$ не имеют общих ребер. Тогда в силу индуктивного предположения 11.27 имеем $\mathrm{L} \preceq_{\mathrm{R}} \widetilde{\mathrm{K}}$, а в силу индуктивного предположения 8.40 имеем $\mathbf{s}_{A, \mathrm{R}}^{r} \mathrm{M} \preceq_{\mathrm{R}} \mathrm{L}$ и $\widetilde{\mathrm{K}} \preceq_{\mathrm{R}} \mathrm{N}$. В силу транзитивности отношения $\preceq_{\mathrm{R}}$ получаем $\mathbf{s}_{A, \mathrm{R}}^{r} \mathrm{M} \preceq_{\mathrm{R}} \mathrm{N}$, откуда, используя лемму 6.7 , легко выводим, что $\mathbf{s}_{A, \mathrm{R}}^{r+t-1} \mathrm{M} \prec_{\mathrm{R}}$ $\mathbf{s}_{A, \mathrm{R}}^{t} \mathrm{~N}$. Последнее означает, что пути $\mathbf{s}_{A, \mathrm{R}}^{i} \mathrm{M}$ содержатся в $\mathrm{P} \cup_{\mathrm{R}} \mathrm{Q}$ при $i=0,1, \ldots$ 
$\ldots, r+t-1$, и, таким образом, $\ell_{\alpha, A}(\mathrm{E} \cup \mathrm{x} F) \geqslant \ell_{\alpha, A}(\mathrm{E})+\ell_{\alpha, A}(\mathrm{~F})-1$.

8.12. Лемма. Если $\mathrm{E}, \mathrm{F} \in \Pi_{\text {Пер }}(A)$ - строго близкие в ранге $\rho(A)$ пути, то любая периодическая основа $\mathrm{P} \in \Pi_{\mathrm{ep}}\left(\Gamma_{\alpha-1}, A, 2\right)$ для $\mathrm{E}$ является периодической основой для $\mathrm{F}$ и обратно. В частности, ПСогл $\Omega_{\alpha}(\mathrm{E}, \mathrm{F})$ и $\ell_{\alpha, A}(\mathrm{E})=$ $\ell_{\alpha, A}(\mathrm{~F})$.

ДокАЗАТЕЛЬСТво. Утверждение легко следует из определений 5.16, 6.5 и индуктивного

предположения 8.40.

8.13. ОпРЕДЕЛЕНИЕ. В п. 5.17 мы определили числовую функцию $\ell_{\alpha}, A$ на множестве почти периодических слов ранга $\alpha$, порожденных периодом $A$. Теперь распространим эту функцию на подслова почти периодических слов ранга $\alpha$, порожденных периодом $A$. По определению $\ell_{\alpha, A}(E)=r$, где $r$ - максимальное число, для которого в $E$ входит слово $E^{\prime} \in \Pi$ ППер $\alpha(A, r)$. Заметим, что формально значение $\ell_{\alpha, A}(E)$ определно для любого слова $E$. Как легко следует из предложения 8.10 , (ii), для любого $E \in \Pi$ Пер $\alpha(A, 8)$ значения функции $\ell_{\alpha, A}(E)$ в старом и в новом смысле совпадают.

8.14. ПрЕДЛОЖЕНИЕ. (i) Пусть $E$ 으 $E_{1} E_{2} \in \Pi$ Пер $p_{\alpha}(A, r) . \quad$ Eсли $t \rightleftharpoons$ $\ell_{\alpha, A}\left(E_{1}\right) \leqslant r-3$, то существует конеи, $E_{2}^{\prime}$ слова $E_{2}$ такой, что $E_{2}^{\prime} \in$ ППер ${ }_{\alpha}(A, r-t-2)$. Если $t \rightleftharpoons \ell_{\alpha, A}\left(E_{2}\right) \leqslant r-3$, то существует начало $E_{1}^{\prime}$ слова $E_{1}$ такое, что $E_{1}^{\prime} \in \Pi$ Пер $\alpha(A, r-t-2)$.

(ii) $E с л и E^{\prime}-$ подслово некоторого слова $E \in \Pi$ Пер $\alpha$ (A) u $E^{\prime}$ 으 $E_{1} E_{2} \ldots E_{k}$ nрu $k \geqslant 1$, mo $\sum_{i=1}^{k} \ell_{\alpha, A}\left(E_{i}\right) \geqslant \ell_{\alpha, A}\left(E^{\prime}\right)-2 k+2$.

ДокаЗАТЕЛЬСтво. Утверждение (ii) вытекает из (i). Докажем (i). В силу симметрии ограничимся доказательством первого утверждения. Пусть $E$ 으 $E_{1} E_{2} \in \Pi \Pi е p_{\alpha}(A, r)$ и $t \rightleftharpoons \ell_{\alpha, A}\left(E_{1}\right) \leqslant r-3$. Рассмотрим путь $\mathrm{E} \in \Pi_{\text {तер }}(A, r)$ на графе $\Gamma_{\alpha-1}$ с $\mathcal{L}(\mathrm{E})$ 으 $E$. Пусть $\mathrm{P}$ - периодическая основа для $\mathrm{E}$, и

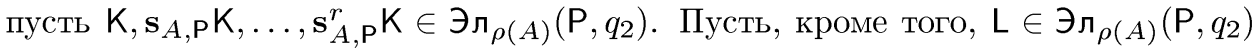
и $\mathrm{L}^{\prime} \in \ni л_{\rho(A)}\left(\mathrm{E}, q_{1}\right)$ - концы соответственно $\mathrm{P}$ и $\mathrm{E}$, где $Э \operatorname{Coz}_{\rho(A)}\left(\mathrm{L}, \mathrm{L}^{\prime}\right)$. По лемме 8.2, (iii) (с учетом леммы 6.7) сушествуют $\mathrm{M}_{0}, \mathrm{M}_{r} \in \ni_{\rho}(A)\left(\mathrm{E}, q_{1}\right)$, $\mathrm{M}_{1}, \ldots, \mathrm{M}_{r-1} \in Э \boldsymbol{л}_{(A)}\left(\mathrm{E}, q_{2}-h\right)$ такие, что Согл $\rho(A)_{\rho}\left(\mathbf{s}_{A, \mathrm{P}}^{i} \mathrm{~K}, \mathrm{M}_{i}\right)$ для каждого $i$ и $\mathrm{M}_{0}<\mathrm{E} \mathrm{M}_{1}<\mathrm{E} \cdots<_{\mathrm{E}} \mathrm{M}_{r}$. Так как $2 p+70<h$, то, укоротив $\mathrm{M}_{i}$ при $1 \leqslant i \leqslant r-1$ в силу индуктивного предположения 8.41 и считая $\mathrm{M}_{i} \in$ Эл $_{\rho(A)}\left(\mathrm{E}, q_{1}\right)$ вместо $\mathrm{M}_{i} \in$ Эл $\rho_{(A)}\left(\mathrm{E}, q_{2}-h\right)$, можно предполагать, что $\mathrm{M}_{0} \ll_{\mathrm{E}} \mathrm{M}_{1} \ll_{\mathrm{E}} \cdots \ll_{\mathrm{E}} \mathrm{M}_{r-1} \ll_{\mathrm{E}} \mathrm{M}_{r}$. Если бы путь $\mathrm{M}_{t+1}$ содержался в $\mathrm{E}_{1}$, мы имели бы $\mathrm{M}_{0} \cup_{\mathrm{E}} \mathrm{M}_{t+1} \in \Pi$ Пер $\alpha(A, t+1)$ вопреки равенству $\ell_{\alpha, A}\left(E_{1}\right)=t$. Следовательно, $\mathrm{M}_{t+1}$ не содержится в $\mathrm{E}_{1}$ и тогда $\mathrm{M}_{t+2}$ содержится в $\mathrm{E}_{2}$. Так как $\mathrm{E}_{2}^{\prime} \rightleftharpoons \mathrm{M}_{t+2} \cup_{\mathrm{E}} \mathrm{L}^{\prime} \in \Pi$ Пер $\alpha(A, r-t-2)$, то в качестве $E_{2}^{\prime}$ можно взять $\mathcal{L}\left(\mathrm{E}_{2}^{\prime}\right)$.

8.15. Лемма $(\alpha \geqslant 2)$. Если $A \in \mathcal{M}_{\alpha}, B \in \mathcal{E}_{\alpha-1}$ и слова $P \in \Pi е р(A) u$ $Q \in \Pi$ Пер(B) близки в ранге $\alpha-2$, то $|P|<10|A|$. 
ДокаЗАтельство. Допустим противное: слова $P \in \Pi е p(A, 10)$ и $Q \in \Pi е p(B)$ близки в ранге $\alpha-2$, где $A \in \mathcal{M}_{\alpha}$ и $B \in \mathcal{E}_{\alpha-1}$. По лемме 5.13 имеем $A \in \mathcal{N}_{\alpha-1, i}$ для некоторого $i$. Так как порядок $[A]_{\alpha-1}$ бесконечен, а порядок $[B]_{\alpha-1}$ делит $n$, то никакие нетривиальные степени $A$ и $B$ на могут быть сопряжены в $\mathbf{B}_{\alpha-1}$, а значит, и в $\mathbf{B}_{\alpha-2}$. Тогда согласно определению 5.4 периоды $A$ и $B$ не родственны в ранге $\alpha-1$ и согласно определению $5.5 B \in \mathcal{N}_{\alpha-1, i+1}$. Но это противоречит включению $B \in \mathcal{E}_{\alpha-1}$ и определению 5.6.

8.16. Лемма $(\alpha \geqslant 2)$. Пусть $E-$ произвольное слово $и r \rightleftharpoons \ell_{\alpha, A}(E) \geqslant 2$. Тогда E имеет вид $E$ 드 $U_{0} M_{1} U_{1} \ldots M_{r-1} U_{r-1}$, где каждое слово $M_{i}$ есть әлементарная 118-степень ранга $\alpha-1$ и никакое слово $M_{k} U_{k} \ldots M_{k+9} U_{k+9} M_{k+10}$ не входит в слово $X \in \mathcal{R}_{\alpha-2}$, близкое в ранге $\alpha-2$ $\kappa$ слову $R \in \Pi е р(C)$ при $C \in \mathcal{E}_{\alpha-1}$.

ДокАЗАТЕЛЬСтво. Очевидно, достаточно рассмотреть случай, когда $E \in$ ППер $\alpha(A, r)$. Выберем путь $\mathrm{E} \in \Pi$ Пер $\alpha(A, r)$ с $\mathcal{L}(\mathrm{E})$ 으 $E$, пусть $\mathrm{P} \in$ Пер $\left(\Gamma_{\alpha-1}, A, 2\right)$ - периодическая основа для $\mathrm{E}$, и пусть $\mathrm{K}, \mathbf{s}_{A, \mathrm{P}} \mathrm{K}, \ldots, \mathbf{s}_{A, \mathrm{P}}^{r} \mathrm{~K} \in$ Эл $\rho(A)\left(\mathrm{P}, q_{2}\right)$. Рассмотрим два случая.

Случай 1: $\rho(A)=\alpha-1$. Используя леммы $6.7,8.2$, (iii) и индуктивное предположение 8.41 , как в доказательстве предложения 8.14 , найдем $\mathrm{M}_{1}, \mathrm{M}_{2}, \ldots, \mathrm{M}_{r-1} \in$ Эл ${ }_{\alpha-1}\left(\mathrm{E}, q_{1}\right)$ такие, что $Э$ Сол $\Omega_{\alpha-1}\left(\mathbf{s}_{A, \mathrm{P}}^{i-1} \mathrm{~K}, \mathrm{M}_{i}\right)(i=1,2, \ldots, r-1)$ и $\mathrm{M}_{1} \ll_{\mathrm{E}} \mathrm{M}_{2} \ll_{\mathrm{E}} \cdots$ $\ldots \ll \mathrm{E} \mathrm{M}_{r-1}$, т.е. Е имеет вид $\mathrm{U}_{0} \mathrm{M}_{1} \mathrm{U}_{1} \ldots \mathrm{M}_{r-1} \mathrm{U}_{r-1}$. Тогда $E$ 으 $U_{0} M_{1} U_{1} \ldots$ $\ldots M_{r-1} U_{r-1}$, где $M_{i} \rightleftharpoons \mathcal{L}\left(\mathrm{M}_{i}\right)$ и $U_{i} \rightleftharpoons \mathcal{L}\left(\mathrm{U}_{i}\right)$.

Докажем, что даже никакое слово $M_{k} U_{k} M_{k+1}$ не входит в слово $X \in \mathcal{R}_{\alpha-2}$, близкое в ранге $\alpha-2$ к слову $R \in \Pi е p(C)$ при $C \in \mathcal{E}_{\alpha-1}$. Допустим, найдутся такие $k, X, R$ и $C$. Выберем прообраз $\overline{\mathrm{M}}_{k} \overline{\mathrm{U}}_{k} \overline{\mathrm{M}}_{k+1}$ пути $\mathrm{M}_{k} \mathrm{U}_{k} \mathrm{M}_{k+1}$ на графе $\Gamma_{\alpha-2}$, где $\mathcal{L}\left(\overline{\mathrm{M}}_{k}\right)$ 드 $M_{k}, \mathcal{L}\left(\overline{\mathrm{U}}_{k}\right)$ 으 $U_{k}$ и $\mathcal{L}\left(\overline{\mathrm{M}}_{k+1}\right)$ 으 $M_{k+1}$. Пусть $\mathrm{X}=\mathrm{VM}_{k} \overline{\mathrm{U}}_{k} \overline{\mathrm{M}}_{k+1} \mathrm{~W}$, где $\mathcal{L}(\mathrm{X})$ - $X$, и пусть $\mathrm{R}$ - близкий в ранге $\alpha-2$ к пути $\mathrm{X}$ путь на графе $\Gamma_{\alpha-2} \mathrm{c}$ $\mathcal{L}(\mathrm{R})$ 드 $R$. По лемме 5.21 имеем $\overline{\mathrm{M}}_{k} \in \Pi \Pi_{\mathrm{ep}}{ }_{\alpha-1}\left(B_{1}, q_{1}\right)$ и $\overline{\mathrm{M}}_{k+1} \in \Pi \Pi_{\mathrm{ep}} \mathrm{p}_{\alpha-1}\left(B_{2}, q_{1}\right)$ для некоторых $B_{1}, B_{2} \in \mathcal{E}_{\alpha-1}$. По индуктивному предположению 8.26 существуют подпути $\mathrm{N}_{i} \in$ ППер $\alpha-1\left(B_{i}, q_{1}-34\right)(i=1,2)$ пути $\mathrm{R}$ такие, что ПСогл $\Omega_{\alpha-1}\left(\overline{\mathrm{M}}_{k}, \mathrm{~N}_{1}\right)$ и ПСогл $\Omega_{\alpha-1}\left(\overline{\mathrm{M}}_{k+1}, \mathrm{~N}_{2}\right)$. Пусть $\mathrm{Q}_{i} \in \Pi_{\text {ер }}\left(\Gamma_{\alpha-2}, B_{i}, 2\right)$ - периодическая основа для $\mathrm{N}_{i}$. По индуктивному предположению 8.2 , (ii) пути $\mathrm{Q}_{i}$ и $\mathrm{N}_{i}$ близки в ранге $\alpha-2$. Пусть $\mathrm{R}^{*} \in$ Пер $\left(\Gamma_{\alpha-2}, C, 2\right)$ - произвольный путь, содержаший $\mathrm{R}$. По индуктивному предположению 8.36 имеем $\operatorname{Cozл}\left(\mathrm{Q}_{i}, \mathrm{R}^{*}\right)(i=1,2)$, и, следовательно, Согл $\left(\mathrm{Q}_{1}, \mathrm{Q}_{2}\right)$. Тогда согласно определению 5.18 имеем $\Pi$ Согл $\Omega_{\alpha-1}\left(\mathrm{~N}_{1}, \mathrm{~N}_{2}\right)$, откуда вытекает ПСогл $\Omega_{\alpha-1}\left(\overline{\mathrm{M}}_{k}, \overline{\mathrm{M}}_{k+1}\right)$. Согласно определению 5.20 получаем ЭСогл $\Omega_{\alpha-1}\left(\mathrm{M}_{k}, \mathrm{M}_{k+1}\right)$, и, следовательно, $Э$ Согл $\Omega_{\alpha-1}\left(\mathbf{s}_{A, \mathrm{P}}^{k} \mathrm{~K}, \mathbf{s}_{A, \mathrm{P}}^{k+1} \mathrm{~K}\right)$. Но это невозможно в силу леммы 6.7. Случай 1 рассмотрен.

Случай $2: \rho(A) \leqslant \alpha-2$. По лемме 8.2 , (ii) пути $\mathrm{P}$ и $\mathrm{E}$ близки в ранге $\alpha-2$. Так как в силу определения 5.15 имеем Эл $_{\alpha-1}\left(\mathrm{P}, q_{4}\right)=\varnothing$, то по индуктивному предположению 11.6 найдутся близкие в ранге $\alpha-2$ прообразы $\overline{\mathrm{P}}$ и $\overline{\mathrm{Q}}$ путей $\mathrm{P}$ и $\mathrm{Q}$ на графе $\Gamma_{\alpha-2}$. В силу леммы 5.13 при $\alpha:=\alpha-1$ и индуктивного предположения 8.34 в некоторый циклический сдвиг слова $|A|$ входит элементарная 152 -степень $G$ ранга $\alpha-1$. Так как $|\overline{\mathrm{P}}|>r|A|$, то путь $\overline{\mathrm{P}}$ содержит $r-1$ подпутей 
$\mathrm{K}, \mathrm{s}_{A, \overline{\mathrm{P}}} \mathrm{K}, \ldots, \mathbf{s}_{A, \overline{\mathrm{P}}}^{r-2} \mathrm{~K}$, где $\mathcal{L}(\mathrm{K})$ 으 $G$. По лемме 5.21 имеем $\mathrm{K} \in \Pi_{\text {Пер }}{ }_{\alpha-1}(B, 152)$ для некоторого $B \in \mathcal{E}_{\alpha-1}$. Используя индуктивные предположения 8.29 , (i) и 8.12 , найдем подпути $\mathrm{M}_{i} \in$ ППер ${ }_{\alpha-1}(B, 118) \quad(i=1,2, \ldots, r-1)$ пути $\overline{\mathrm{E}}$ такие, что ПСогл $\Omega_{\alpha-1}\left(\mathrm{~s}_{A, \overline{\mathrm{P}}}^{i-1} \mathrm{M}, \mathrm{M}_{i}\right)$ и $\mathrm{M}_{1} \ll_{\overline{\mathrm{E}}} \mathrm{M}_{2} \ll_{\overline{\mathrm{E}}} \cdots \ll_{\overline{\mathrm{E}}} \mathrm{M}_{r-1}$. Имеем $\overline{\mathrm{E}}=\mathrm{U}_{0} \mathrm{M}_{1} \mathrm{U}_{1} \ldots \mathrm{M}_{r-1} \mathrm{U}_{r-1}$, и, следовательно, $E$ 으 $U_{0} M_{1} U_{1} \ldots M_{r-1} U_{r-1}$, где $M_{i} \rightleftharpoons \mathcal{L}\left(\mathrm{M}_{i}\right)$ и $U_{i} \rightleftharpoons \mathcal{L}\left(\mathrm{U}_{i}\right)$.

Для доказательства леммы остается проверить, что ни при каком $k$ слово $M_{k} U_{k} \ldots M_{k+9} U_{n+9} M_{k+10}$ не входит в слово $X \in \mathcal{R}_{\alpha-2}$, близкое в ранге $\alpha-2$ к слову $R \in \Pi е p(C)$ при $C \in \mathcal{E}_{\alpha-1}$. Допустим, это неверно для некоторых $k$, $X, R$ и $C$. Тогда путь $\mathrm{M}_{k} \mathrm{U}_{k} \ldots \mathrm{M}_{k+9} \mathrm{U}_{n+9} \mathrm{M}_{k+10}$ входит в качестве подпути в путь $\mathrm{X}$, близкий в ранге $\alpha-2$ к некоторому пути $\mathrm{R} \in \Pi_{\mathrm{ep}}\left(\Gamma_{\alpha-2}, C\right)$. По индуктивному предположению 8.29 , (ii) существуют близкие в ранге $\alpha-2$ подпути $\mathrm{P}^{\prime} \in$ Пер $\left(\Gamma_{\alpha-1}, A, 10\right)$ и $\mathrm{R}^{\prime}$ путей $\overline{\mathrm{P}}$ и $\mathrm{R}$. Но это противоречит лемме 8.15.

Из леммы 8.16 непосредственно вытекает

8.17. ПрЕДЛОЖЕНИЕ $(\alpha \geqslant 2)$. Если слово $E$ входит в слово $X \in \mathcal{R}_{\alpha-2}$, близкое в ранге $\alpha-2$ к некоторому слову $R \in \Pi$ Пер $(C)$ при $C \in \mathcal{E}_{\alpha-1}$, то $\ell_{\alpha, A}(E) \leqslant 11$.

8.18. ПРЕДЛОЖЕНИЕ. Если $E$ - элементарное слово ранга $\alpha$, mо $|E| \geqslant 7 \times$ $117^{\alpha-1}$. В частности, E- непустое слово.

ДоКАЗАТЕЛЬСТВО. При $\alpha=1$ в силу определений 5.16 и 5.17 имеем ППер $p_{1}(A, r) \subseteq \Pi е p(A, r)$, и, следовательно, если $E \in \Pi$ Пер $p_{1}(A, r)$, то $|E| \geqslant r$. При $\alpha \geqslant 2$, применяя лемму 8.16 последовательно при $\alpha:=\alpha, \alpha-1, \ldots, 2$, получаем, что любое слово $E \in \Pi$ ППе $p_{\alpha}(A, 8)$ содержит $7 \cdot 117^{\alpha-2}$ попарно непересекающихся вхождений элементарных 118-степеней ранга 1.

8.19. ОПРЕДЕЛЕНИЕ. Покрытием ранга $\beta$ слова $X$ назовем конечный (возможно, пустой) набор $\Omega=\left\{\left(\mathrm{U}_{i}, \gamma_{i}\right)\right\}_{i=1}^{r}$ пар $\left(\mathrm{U}_{i}, \gamma_{i}\right)$, где $\mathrm{U}_{i}, \mathrm{U}_{2}, \ldots \mathrm{U}_{r}$ - вхождения слов $E_{1}, E_{2}, \ldots, E_{r}$ соответственно в слово $X$, в совокупности покрываюшие $X$, $1 \leqslant \gamma_{i} \leqslant \beta$ и для каждого $i$ слово $E_{i}$ входит в некоторое слово $\bar{E}_{i} \in \mathcal{R}_{\gamma_{i}-1}$, близкое в ранге $\gamma_{i}-1$ к слову $P_{i} \in \Pi е p\left(A_{i}\right)$ при $A_{i} \in \mathcal{E}_{\gamma_{i}}$. Каждой паре $\left(\mathrm{U}_{i}, \gamma_{i}\right)$ сопоставим вес $\zeta^{\beta-\gamma_{i}}$ и определим вес $\mathcal{W}_{\beta}(\Omega)$ покрытия $\Omega$ ранга $\beta$ как сумму $\sum_{i=1}^{r} \zeta^{\beta-\gamma_{i}}$ всех весов пар $\left(\mathrm{U}_{i}, \gamma_{i}\right)$.

По определению вес $\mathcal{W}_{\beta}(X)$ слова $X$ в ранге $\beta$ есть минимальный из всех весов $\mathcal{W}_{\beta}(\Omega)$ его покрытий ранга $\beta$. При этом естественно считать, что $\mathcal{W}_{\beta}(1)=0$ и $\mathcal{W}_{\beta}(X)=\infty$, если для $X$ не сушествует покрытия ранга $\beta$; в частности, $\mathcal{W}_{0}(X)$ может принимать лишь два значения: 0 , если слово $X$ пусто, и $\infty$, если $X-$ непустое слово.

Из определения непосредственно вытекают следуюшие свойства функции $\mathcal{W}_{\beta}(X)$.

8.20. Лемма. (i) Если слово $X$ и некоторое слово $P \in \Pi е p(A)$ при $A \in \mathcal{E}_{\beta}$ близки в ранге $\beta-1$, то $\mathcal{W}_{\beta}(X) \leqslant 1$;

(ii) если $\beta \geqslant 1$, mo $\mathcal{W}_{\beta}(X) \leqslant \zeta \mathcal{W}_{\beta-1}(X)$; 
(iii) $\mathcal{W}_{\beta}(X Y) \leqslant \mathcal{W}_{\beta}(X)+\mathcal{W}_{\beta}(Y)$;

(iv) если $Y$ есть подслово слова $X$, то $\mathcal{W}_{\beta}(Y) \leqslant \mathcal{W}_{\beta}(X)$.

8.21. Лемма $(\alpha \geqslant 2)$. Если слово $X \in \mathcal{R}_{\alpha-2}$ содержит $t$ попарно непересекающихся вхождений элементарных 64-степеней ранга $\alpha-1$, mо $_{\alpha-2}(X) \geqslant$ $\frac{50}{13} t$.

ДокАЗАТЕЛЬСТвО. Утверждение тривиально при $\alpha=2$. Пусть $\alpha \geqslant 3$. Пусть $\left\{\mathrm{V}_{i}\right\}_{i=1}^{t}$ - попарно непересекаюшиеся вхождения в слово $X$ элементарных 64-степеней ранга $\alpha-1$. Пусть $\Omega=\left\{\left(\mathrm{U}_{i}, \gamma_{i}\right)\right\}_{i=1}^{r}-$ произвольное покрытие ранга $\alpha-2$ слова $X$. Мы должны показать, что $\mathcal{W}_{\alpha-2}(\Omega) \geqslant \frac{50}{13} t$. Укоротив каждое вхождение $\mathrm{V}_{i}$ в силу индуктивных предположений 8.14, (i) и 8.17 (при этом вместо элементарных 64-степеней ранга $\alpha-1$ возникнут элементарные 51-степени ранга $\alpha-1$ ), можно считать, что для каждой пары $\left(\mathrm{U}_{k}, \gamma_{k}\right)$ при $\gamma_{k}=\alpha-2$ вхождение $\mathrm{U}_{k}$ пересекается не более чем с одним $\bigvee_{i}$. Согласно лемме 8.16 каждое вхождение $V_{i}$ содержит 50 попарно непересекаюшихся вхождений $W_{i j}(1 \leqslant j \leqslant 50)$ элементарных 64 -степеней ранга $\alpha-2$ таких, что для каждой пары $\left(\mathrm{U}_{k}, \gamma_{k}\right)$ при $k=\alpha-2$ вхождение $\mathrm{U}_{k}$ пересекается не более чем с 13 вхождениями $\mathrm{W}_{i j}$. Пусть $r_{1}$ - число пар $\left(\mathrm{U}_{k}, \gamma_{k}\right)$, для которых $\gamma_{k}=\alpha-2$, пусть $X_{1}, X_{2}, \ldots, X_{s}$ - непустые попарно непересекаюшиеся подслова слова $X$, составляюшие объединение всех вхождений $\bigcup_{k}$ при $\gamma_{k}<\alpha-2$, и пусть $\Omega_{i}$ есть покрытие ранга $\alpha-3$ слова $X_{i}$, индуцированное покрытием $\Omega$. Общее число вхождений $W_{i j}$, содержащихся в подсловах $X_{i}$, не меньше $50 t-13 r_{1}$. Тогда по индуктивному предположению имеем

$$
\sum_{i=1}^{s} \mathcal{W}_{\alpha-3}\left(\Omega_{i}\right) \geqslant \frac{50}{13}\left(50 t-13 r_{1}\right)=\frac{1}{\zeta}\left(\frac{50}{13} t-r_{1}\right) .
$$

Следовательно, с учетом определения 8.19

$$
\mathcal{W}_{\alpha-2}(\Omega)=r_{1}+\zeta \sum_{i=1}^{s} \mathcal{W}_{\alpha-3}\left(\Omega_{i}\right) \geqslant \frac{50}{13} t
$$

что и требовалось доказать.

8.22. ПРЕДЛОЖЕНИЕ. (i) Для любъх $A \in \mathcal{M}_{\alpha}, X \in \mathcal{R}_{\alpha-1}$ и любого подслова $E$ слова $X$ выполнено $\ell_{\alpha, A}(E) \leqslant 13 \mathcal{W}_{\alpha-1}(X)+1$.

(ii) Для любых $A \in \mathcal{M}_{\alpha}$ u $X \in \Pi$ Пер $(A)$ выполнено $|X| \leqslant\left(13 \mathcal{W}_{\alpha-1}(X)+2\right)|A|$.

ДоКАЗАТЕЛЬСТво. Утверждение тривиально при $\alpha=1$. Пусть $\alpha \geqslant 2$.

(i) Пусть $\ell_{\alpha, A}(E)=t+1$ и $\left\{\mathrm{V}_{i}\right\}_{i=1}^{t}-$ попарно непересекающиеся вхождения в слово $X$ элементарных 118-степеней ранга $\alpha-1$, полученные по лемме 8.16. Пусть $\Omega=\left\{\left(\mathrm{U}_{i}, \gamma_{i}\right)\right\}_{i=1}^{r}-$ произвольное покрытие ранга $\alpha-1$ слова $X$. Пусть $r_{1}-$ число пар $\left(\mathrm{U}_{i}, \gamma_{i}\right)$ с $\gamma_{i}=\alpha-1$, пусть $X_{1}, X_{2}, \ldots, X_{s}$ - попарно непересекающиеся подслова слова $X$, составляющие объединение всех вхождений $\mathrm{U}_{i}$ при $\gamma_{i} \leqslant \alpha-2$, и пусть $\Omega_{i}$ - индуцированное покрытие ранга $\alpha-2$ слова $X_{i}$. Используя лемму 8.21 
и тот факт, что каждое вхождение $\bigcup_{i}$ при $\gamma_{i}=\alpha-1$ пересекается не более чем с 13 вхождениями $\mathrm{V}_{j}$, получаем

$$
\mathcal{W}_{\alpha-1}(\Omega)=r_{1}+\zeta \sum_{i=1}^{s} \mathcal{W}_{\alpha-2}\left(\Omega_{i}\right) \geqslant r_{1}+\zeta \frac{50}{13}\left(t-13 r_{1}\right)=\frac{1}{13} t
$$

и, значит, верно искомое неравенство $\ell_{\alpha, A}(E) \leqslant 13 \mathcal{W}_{\alpha-1}(X)+1$.

(ii) Пусть $\lfloor|P| /|A|\rfloor=t+1$, где $\lfloor x\rfloor$ - целая часть числа $x$. Достаточно проверить, что $t \leqslant 13 \mathcal{W}_{\alpha-1}(X)$. В силу леммы 5.13 и индуктивного предположения 8.34 слово $X$ имеет вид $G^{\prime} E G E \ldots G E G^{\prime \prime}$, где $E$ - элементарная 152-степень ранга $\alpha-1, E G$ есть циклический сдвиг слова $A$ и число вхождений слова $E$ равно $t$. Пусть $\Omega=\left\{\left(\mathrm{U}_{i}, \gamma_{i}\right)\right\}_{i=1}^{r}-$ покрытие ранга $\alpha-1$ слова $X$. В силу леммы 8.15 каждое вхождение $\bigcup_{i}$ при $\gamma_{i}=\alpha-1$ пересекается менее чем с 13 вхождениями слова $E$. Далее рассуждения аналогичны доказательству п. (i).

8.23. ПРЕДЛОЖЕНИЕ. Пусть $\mathrm{P} \in \Pi_{\mathrm{ep}}\left(\Gamma_{\alpha-1}, A, r\right)$ u $\mathrm{X}$ - близкие в ранге $\alpha-1$ пути на графе $\Gamma_{\alpha-1}$, где $A \in \mathcal{M}_{\alpha} u \mathcal{L}(\mathrm{X}) \in \mathcal{R}_{\alpha-1}$. Тогда если $r \geqslant 37$, то существуют подпути $\overline{\mathrm{P}} u \mathrm{E}$ путей $\mathrm{P} u \mathrm{X}$ соответственно такие, что $\mathrm{E} \in \Pi$ Пер $\alpha(A), \quad \overline{\mathrm{P}}-$ периодическая основа для $\mathrm{E} u \ell_{\alpha, A}(\mathrm{E}) \geqslant r-36$.

ДокАЗАТЕЛЬСтво. По индуктивному предположению 11.9 имеем $\mathrm{P}=\mathrm{U}_{1} \mathrm{P}^{\prime} \mathrm{U}_{2}$, $\mathrm{X}=\mathrm{V}_{1} \mathrm{X}^{\prime} \mathrm{V}_{2}$, где $\mathcal{W}_{\alpha-1}\left(\mathcal{L}\left(\mathrm{U}_{i}\right)\right)<1+2 \zeta(i=1,2)$ и пути $\mathrm{P}^{\prime}$ и $\mathrm{X}^{\prime}$ близки в ранге $\rho(A)$. По предложению 8.22, (ii) имеем $\left|\mathcal{L}\left(\mathrm{U}_{i}\right)\right|<16|A|$ и, следовательно, $\left|\mathrm{P}^{\prime}\right|>(r-$ $32)|A|$. Тогда в силу леммы $8.4 \mathrm{P}^{\prime}=\mathrm{W}_{0} \mathrm{~K}_{1} \mathrm{~W}_{1} \ldots \mathrm{K}_{t} \mathrm{~W}_{t}$, где $\mathrm{K}_{i} \in$ Эл $\rho(A)\left(\Gamma_{\alpha-1}, q_{2}\right)$, $\mathrm{K}_{i}=\mathrm{s}_{A, \mathrm{P}}^{i-1} \mathrm{~K}_{1}$ и $t \geqslant r-33$. В силу леммы 6.7 и индуктивного предположения 11.23 найдутся $\mathrm{L}_{i} \in \ni_{\rho(A)}\left(\mathrm{X}^{\prime}, q_{2}-h\right)(i=2,3, \ldots, t-1)$ такие, что ЭCогл $\Omega_{\rho(A)}\left(\mathrm{K}_{i}, \mathrm{~L}_{i}\right)$. По индуктивному предположению 11.27 имеем $\mathrm{L}_{2}<\mathrm{X}^{\prime} \mathrm{L}_{3}<\mathrm{X}^{\prime} \ldots<\mathrm{X}^{\prime} \mathrm{L}_{t-1}$, и можно положить $\overline{\mathrm{P}} \rightleftharpoons \mathrm{K}_{2} \cup_{\mathrm{P}} \mathrm{K}_{t-1}$ и $\mathrm{E} \rightleftharpoons \mathrm{L}_{2} \cup_{\mathrm{X}^{\prime}} \mathrm{L}_{t-1}$.

8.24. СЛЕДСТВИЕ. Если слова $X \in \mathcal{R}_{\alpha-1} u P \in \Pi е p(A, r)$ nрu $A \in \mathcal{M}_{\alpha} u$ $r \geqslant 37$ близки в ранге $\alpha-1$, то в $X$ входит некоторое слово $E \in \Pi$ Пер $\alpha(A$, $r-36)$.

8.25. ЛЕммА. Для любого пути $\mathrm{E} \in \Pi_{\text {Пер }}(A, 35)$ существует подпуть $\overline{\mathrm{E}} \in$ ППер $(A)$ пути $\mathrm{E}$, обладающий следующими свойствами:

(i) $\Pi C \operatorname{Coz} \Omega_{\alpha}(\mathrm{E}, \overline{\mathrm{E}}), \quad \ell_{\alpha, A}(\overline{\mathrm{E}}) \geqslant \ell_{\alpha, A}(\mathrm{E})-34 u \mathrm{E}=\mathrm{E}_{1} \overline{\mathrm{E}}_{2}$, rдe $\ell_{\alpha, A}\left(\mathcal{L}\left(\mathrm{E}_{i}\right)\right) \geqslant 15$ $(i=1,2)$ (в смысле определения 8.13);

(ii) Если $\mathrm{E}$ - подпуть пути $\mathrm{X}$, пути $\mathrm{X} u \mathrm{Y}$ близки в ранге $\alpha-1 u \mathcal{L}(\mathrm{X}), \mathcal{L}(\mathrm{Y}) \in$ $\mathcal{R}_{\alpha-1}$, то существует подпуть $\mathrm{F} \in \Pi_{\text {пер }}(A)$ пути $\mathrm{Y}$, строго близкий в ранге $\rho(A) \kappa \overline{\mathrm{E}} . \quad B$ частности, в силу (i) и леммы 8.12 имеем ПСогл $\alpha(\mathrm{E}, \mathrm{F})$ и $\ell_{\alpha, A}(\mathrm{~F})=\ell_{\alpha, A}(\overline{\mathrm{E}}) \geqslant \ell_{\alpha, A}(\mathrm{E})-34$.

ДокаЗАтельство. Пусть $\mathrm{P} \in$ Пер $\left(\Gamma_{\alpha-1}, A, 2\right)$ - периодическая основа для $\mathrm{E}$ и $\mathrm{K}, \mathbf{s}_{A, \mathrm{P}} \mathrm{K}, \ldots, \mathbf{s}_{A, \mathrm{P}}^{r} \mathrm{~K} \in$ Эл $_{\rho(A)}\left(\mathrm{P}, q_{2}\right)$, где $r \rightleftharpoons \ell_{\alpha, A}(\mathrm{E}) \geqslant 35$. Используя леммы 6.7 и 8.2 , (iii), найдем $\mathrm{L}_{0}, \mathrm{~L}_{r} \in$ Эл $\rho(A)\left(\mathrm{E}, q_{1}\right), \mathrm{L}_{i} \in$ Эл $\rho(A)\left(\mathrm{E}, q_{2}-h\right)(i=1,2, \ldots, r-$ 1) такие, что ЭСогл $\Omega_{\rho(A)}\left(\mathbf{s}_{A, \mathrm{P}}^{i} \mathrm{~K}, \mathrm{~L}_{i}\right)$ при всех $i$ и $\mathrm{L}_{0}<\mathrm{E} \mathrm{L}_{1}<\mathrm{E} \cdots<_{\mathrm{E}} \mathrm{L}_{r}$. Пусть $\overline{\mathrm{E}} \rightleftharpoons \mathrm{L}_{17} \cup_{\mathrm{E}} \mathrm{L}_{r-17}$ и $\mathrm{E}=\mathrm{E}_{1} \overline{\mathrm{E}}_{2}$. Тогда имеем $\overline{\mathrm{E}} \in \Pi$ Пер $\alpha(A), \ell_{\alpha, A}(\overline{\mathrm{E}}) \geqslant \ell_{\alpha, A}(\mathrm{E})-34$ 
и по предложению 8.9 ПСогл $\alpha$ (E, $\overline{\mathrm{E}})$. Из $\mathrm{L}_{15}<\mathrm{E} \mathrm{L}_{16}<\mathrm{E} \mathrm{L}_{17}, \neg$ ЭСог $\Omega_{\rho(A)}\left(\mathrm{L}_{i}, \mathrm{~L}_{j}\right)$ при $i \neq j$ и индуктивного предположения 8.41 легко следует $\mathrm{L}_{15} \ll \mathrm{E} \mathrm{L}_{17}$, и так как $\mathrm{L}_{0} \cup_{\mathrm{E}} \mathrm{L}_{15} \in \Pi \Pi \mathrm{p}_{\alpha}(A, 15)$, то $\ell_{\alpha, A}\left(\mathcal{L}\left(\mathrm{E}_{1}\right)\right) \geqslant 15$. Аналогично доказывается, что $\ell_{\alpha, A}\left(\mathcal{L}\left(\mathrm{E}_{2}\right)\right) \geqslant 15$. Тем самым доказано утверждение (i).

Докажем (ii). Пусть даны близкие в ранге $\alpha-1$ пути $\mathrm{X}$ и $\mathrm{Y}$ на графе $\Gamma_{\alpha-1}$ такие, что $\mathcal{L}(\mathrm{X}), \mathcal{L}(\mathrm{Y}) \in \mathcal{R}_{\alpha-1}$ и $\mathrm{X}$ содержит $\mathrm{E}$. Заметим, что в силу леммы 8.2, (ii) и индуктивного предположения 11.3 слова $\mathcal{L}(\mathrm{P})$ и $\mathcal{L}(\mathrm{E})$ близки в ранге $\rho(A)$. Тогда в силу индуктивного предположения 8.27 слово $\mathcal{L}(\mathrm{E})$ не содержит элементарных $\left(q_{3}+34\right)$-степеней ранга $\beta$ при $\rho(A)+1 \leqslant \beta \leqslant \alpha-1$, т.е. имеем Эл $\beta\left(\mathrm{E}, q_{3}+34\right)=\varnothing$ при $\rho(A)+1 \leqslant \beta \leqslant \alpha-1$. Так как $q_{3}+34<q_{4}$, то по индуктивному предположению 11.9 имеем $\mathrm{E}=\mathrm{U}_{1} \mathrm{E}^{\prime} \mathrm{U}_{2}, \mathrm{Y}=\mathrm{V}_{1} \mathrm{Y}^{\prime} \mathrm{V}_{2}$, где пути $\mathrm{E}^{\prime}$ и $\mathrm{Y}$ близки в ранге $\rho(A)$ и $\mathcal{W}_{\alpha-1}\left(\mathcal{L}\left(\mathrm{U}_{i}\right)\right)<1+2 \zeta(i=1,2)$. Так как $\ell_{\alpha, A}\left(\mathrm{~L}_{0} \cup_{\mathrm{X}} \mathrm{L}_{15}\right) \geqslant 15$ и $\ell_{\alpha, A}\left(\mathrm{~L}_{r-15} \cup_{\mathrm{X}} \mathrm{L}_{r}\right) \geqslant 15$, то, используя предложение 8.22 , (i), получаем, что $\mathrm{L}_{15}$ не содержится в $\mathrm{U}_{1}$, a $\mathrm{L}_{r-15}$ не содержится в $\mathrm{U}_{2}$. Используя индуктивное предположение 8.41, найдем подпути $\overline{\mathrm{L}}_{16}, \overline{\mathrm{L}}_{r-16} \in$ Эл $\rho(A)\left(\mathrm{E}, q_{2}-h-p-35\right)$ путей $\mathrm{L}_{16}$ и $\mathrm{L}_{r-16}$ такие, что ЭСогл $\Omega_{\rho(A)}\left(\mathrm{L}_{16}, \overline{\mathrm{L}}_{16}\right)$, ЭСогл $\Omega_{\rho(A)}\left(\mathrm{L}_{r-16}, \overline{\mathrm{L}}_{r-16}\right)$ и $\mathrm{L}_{15} \ll_{\mathrm{E}} \overline{\mathrm{L}}_{16}<\mathrm{E}$ $\overline{\mathrm{L}}_{r-16} \ll \mathrm{E} \mathrm{L}_{r-15}$, т.е. $\overline{\mathrm{L}}_{16} \cup_{\mathrm{E}} \overline{\mathrm{L}}_{r-16}$ содержится в $\mathrm{E}^{\prime}$. Используя далее индуктивные предположения 11.23 и 11.27 и учитывая, что $q_{1}=q_{2}-2 h$, найдем под-

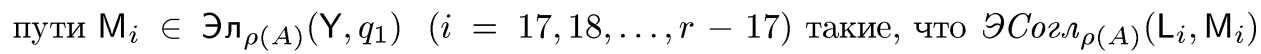
и $\mathrm{M}_{17}<\mathrm{Y} \mathrm{M}_{18}<_{\mathrm{Y}} \cdots<_{\mathrm{Y}} \mathrm{M}_{r-17}$. Тогда, очевидно, в качестве $\mathrm{F}$ можно взять $\mathrm{M}_{17} \cup_{\mathrm{Y}} \mathrm{M}_{r-17}$.

Непосредственным следствием леммы 8.25 являются следуюшие предложения 8.26 и 8.27.

8.26. ПРЕДЛОЖЕНИЕ. Пусть $\mathrm{X} u \mathrm{Y}$ - близкие в ранге $\alpha-1$ пути на граge $\Gamma_{\alpha-1}$. Пусть $\mathcal{L}(\mathrm{X}), \mathcal{L}(\mathrm{Y}) \in \mathcal{R}_{\alpha-1}, \quad r \geqslant 35 u \mathrm{E} \in \Pi \Pi_{\text {ep }}(A, r)-$ noдnymb nути $\mathrm{X}$. Тогда существует подпуть $\mathrm{F} \in \Pi_{\text {Пер }}(A, r-34)$ nути $\mathrm{Y}$ такой, что $\Pi \operatorname{Coz}_{\alpha}(\mathrm{E}, \mathrm{F})$.

8.27. ПРЕДЛОЖЕнИЕ. Если слова $X, Y \in \mathcal{R}_{\alpha-1}$ близки в ранге $\alpha-1, r \geqslant 35$ и в $X$ входит әлементарная $r$-степень ранга $\alpha$, то в $Y$ входит әлементарная $(r-34)$-степень ранга $\alpha$.

8.28. Назовем устойчивой частью пути $\mathrm{E} \in \Pi_{\text {Пер }}(A, 35)$ подпуть $\overline{\mathrm{E}}$ пути $\mathrm{E}$, построенный в доказательстве леммы 8.25.

Так как все понятия, относящиеся к путям на граффе $\Gamma_{\alpha-1}$, инвариантны относительно действия групшы $\mathbf{B}_{\alpha-1}$ на графе $\Gamma_{\alpha-1}$ левыми сдвигами, то для любого пути $\mathrm{E} \in$ ППер $\alpha(A, 35)$ и любого элемента $\mathbf{g} \in \mathbf{B}_{\alpha-1}$, если $\overline{\mathrm{E}}$ есть устойчивая часть $\mathrm{E}$, то $\mathbf{g} \overline{\mathrm{E}}$ есть устойчивая часть $\mathbf{g E}$.

Отметим еще один факт, легко вытекающий из доказательства леммы 8.25 и леммы 8.12. Если пути $\mathrm{E}, \mathrm{F} \in$ ППер $\alpha(A, 35)$ строго близки в ранге $\rho(A)$ и $\overline{\mathrm{E}}-$ устойчивая часть $\mathrm{E}$, то сушествует устойчивая часть $\overline{\mathrm{F}}$ для $\mathrm{F}$ такая, что $\overline{\mathrm{E}}$ и $\overline{\mathrm{F}}$ строго близки в ранге $\rho(A)$.

8.29. Лемма. Пусть $A \in \mathcal{M}_{\alpha}, \mathrm{P} \in \Pi \operatorname{ep}\left(\Gamma_{\alpha-1}, A, 2\right) u \mathrm{E}, \mathrm{s}_{A, \mathrm{P}} \mathrm{E}, \ldots, \mathrm{s}_{A, \mathrm{P}}^{k} \mathrm{E}-$ nодпути $\mathrm{P}$, где $\mathrm{E} \in \Pi$ Пер $\alpha(B, r)$ для некоторого $r \geqslant 35 u|\mathrm{E}| \leqslant|A|$. 
(i) Если $\mathrm{P} u \mathrm{X}$ - близкие в ранге $\alpha-1$ пути и $\mathcal{L}(\mathrm{X}) \in \mathcal{R}_{\alpha-1}$, то существуют nодnymь $\overline{\mathrm{E}} \in \Pi$ Пер $\alpha(B, r-34)$ nymu $\mathrm{E}$ u nодnymu $\mathrm{F}_{0}, \mathrm{~F}_{1}, \ldots, \mathrm{F}_{k} \in \Pi$ Пер $\alpha(B, r-$ 34) пути $\mathrm{X}$ такие, что для каждого $i$ пути $\mathrm{s}_{A, \mathrm{P}}^{i} \overline{\mathrm{E}}$ u $\mathrm{F}_{i}$ строго близки в ранzе $\rho(B) u \mathrm{~F}_{0} \ll \mathrm{Y} \mathrm{F}_{1} \ll \mathrm{Y} \cdots \ll \mathrm{F}_{k}$.

(ii) Ecли $r \geqslant 69$, $\mathrm{P} u \mathrm{X}-$ близкие в ранге $\alpha-1$ пути, $\mathrm{X}$ содержится в $\mathrm{X}^{*}$, $\mathrm{X}^{*}$ и $\mathrm{Y}$ - близкие в ранге $\alpha-1$ пути и $\mathcal{L}\left(\mathrm{X}^{*}\right), \mathcal{L}(\mathrm{Y}) \in \mathcal{R}_{\alpha-1}$, то некоторыи nодпуть $\mathrm{P}^{\prime}$ nути $\mathrm{P}$ и некоторьй подпуть $\mathrm{Y}^{\prime}$ пути $\mathrm{Y}$ близки в ранге $\alpha-1$, причем $\left|\mathrm{P}^{\prime}\right| \geqslant k|A|$.

ДоказАТЕЛЬСтво. (i) В качестве $\overline{\mathrm{E}}$ возьмем устойчивую часть пути Е. По лемме 8.25 имеем $\mathrm{P}=\mathrm{UE} \mathrm{E}, \mathrm{X}=\mathrm{YF}_{0} \mathrm{Z}$, где $\mathrm{F}_{0} \in$ ППер $\alpha(B, r-34)$ и пути $\overline{\mathrm{E}}_{\text {и }} \mathrm{F}_{0}$ строго близки в ранге $\rho(B)$. Так как по условию $|\mathrm{E}| \leqslant|A|$, то $\mathbf{s}_{A, \mathrm{P}} \mathrm{E}$ содержится в $\mathrm{Z}$. Так как по лемме 8.2 , (ii) при $\mathrm{X}:=\overline{\mathrm{E}}$ и $\mathrm{Y}:=\mathrm{F}_{0}$ пути $\mathrm{Z}$ и $\mathrm{V}$ близки в ранге $\alpha-1$, то в силу леммы 8.25 и замечания из 8.28 существует подпуть $\mathrm{F}_{1}$ пути $\mathrm{V}$ такой, что $\mathrm{F}_{1} \in \Pi_{\text {Пер }}(A, r-34)$ и $\mathbf{s}_{A, \mathrm{P}} \overline{\mathrm{E}}, \mathrm{F}_{1}$ строго близки в ранге $\rho(B)$. Далее рассуждения повторяются.

(ii) Пусть $\overline{\mathrm{E}}$ - устойчивая часть $\mathrm{E}$ и $\overline{\overline{\mathrm{E}}}$ - устойчивая часть $\overline{\mathrm{E}}$. Как было доказано в (i), существуют подпути $\mathrm{F}_{0}, \mathrm{~F}_{1}, \ldots, \mathrm{F}_{k} \in$ ППер $\alpha(B, r-34)$ пути $\mathrm{X}$ такие, что

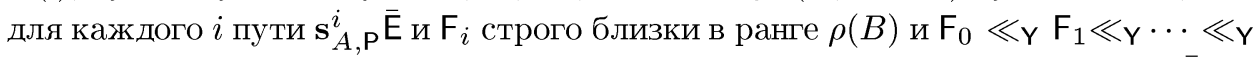
$\mathrm{F}_{k}$. В силу замечаний из 8.28 для каждого $i$ существует устойчивая часть $\overline{\mathrm{F}}_{i} \in$ ППер $\alpha(B, r-68)$ пути $\mathrm{F}_{i}$ такая, что $\mathbf{s}_{A, \mathrm{P}} \overline{\overline{\mathrm{E}}}_{\text {и }} \overline{\mathrm{F}}_{i}$ строго близки в ранге $\rho(B)$. Подобно тому, как в доказательстве (i), найдем подпути $\mathrm{G}, \mathrm{G}^{\prime} \in$ ППер $_{\alpha}(B, r-68)$ пути Y такие, что $\overline{\mathrm{F}}_{0}$ и $\mathrm{G}$ строго близки в ранге $\rho(B), \overline{\mathrm{F}}_{k}$ и $\mathrm{G}^{\prime}$ строго близки в ранге $\rho(B)$ и $\mathrm{G} \ll_{\mathrm{Y}} \mathrm{G}^{\prime}$. Так как в силу леммы 8.2, (i), (ii) пути $\overline{\overline{\mathrm{E}}} \cup_{\mathrm{P}} \mathbf{s}_{A, \mathrm{P}}^{r} \overline{\overline{\mathrm{E}}}$ и $\mathrm{G} \cup_{\mathrm{Y}} \mathrm{G}^{\prime}$ близки в ранге $\alpha-1$, то можно положить $\mathrm{P}^{\prime} \rightleftharpoons \overline{\overline{\mathrm{E}}} \cup_{\mathrm{P}} \mathbf{s}_{A, \mathrm{P}}^{r} \overline{\overline{\mathrm{E}}}$ и $\mathrm{Y}^{\prime} \rightleftharpoons \mathrm{G} \cup_{\mathrm{Y}} \mathrm{G}^{\prime}$.

8.30. ПРЕДЛОЖЕНИЕ $(\alpha \geqslant 2)$. Пусть слова $X, Y \in \mathcal{R}_{\alpha-1}$ близКи в ранге $\alpha-2$. Если в слово $X$ входит слово $E \in \Pi$ пер $\alpha(A, r)$ при $r \geqslant 11$, то в слово $Y$ входит некоторое слово $F \in \Pi$ Пер $\alpha(A, r-8)$.

ДокАЗАТЕЛЬСтво. Пусть $\overline{\mathrm{X}}$ и $\overline{\mathrm{Y}}$ - близкие в ранге $\alpha-2$ пути на графе $\Gamma_{\alpha-2}$ с $\mathcal{L}(\overline{\mathrm{X}})$ 으 $X, \mathcal{L}(\overline{\mathrm{Y}})$ 으 $Y$, и пусть $\overline{\mathrm{E}}-$ подпуть пути $\overline{\mathrm{X}}$ с $\mathcal{L}(\overline{\mathrm{E}})$ 으 $E$. Как в доказательстве леммы 8.25, получаем, что Эл $\beta\left(\overline{\mathrm{E}}, q_{4}\right)=\varnothing$ при $\rho(A) \leqslant \beta \leqslant \alpha-2$. Тогда по индуктивному предположению 11.9 имеем $\overline{\mathrm{E}}=\overline{\mathrm{U}}_{1} \overline{\mathrm{E}}^{\prime} \overline{\mathrm{U}}_{2}$ и $\overline{\mathrm{Y}}=\overline{\mathrm{V}}_{1} \overline{\mathrm{Y}}^{\prime} \overline{\mathrm{V}}_{2}$, где пути $\overline{\mathrm{E}}^{\prime}$ и $\overline{\mathrm{Y}}^{\prime}$ близки в ранге $\rho(A)$ и $\mathcal{W}_{\alpha-2}\left(\mathcal{L}\left(\overline{\mathrm{U}}_{i}\right)\right)<1+2 \zeta(i=1,2)$. Обозначим $\mathrm{E} \rightleftharpoons \pi_{\alpha-2, \alpha-1}(\overline{\mathrm{E}}), \mathrm{E}^{\prime} \rightleftharpoons \pi_{\alpha-2, \alpha-1}\left(\overline{\mathrm{E}}^{\prime}\right), \mathrm{U}_{i} \rightleftharpoons \pi_{\alpha-2, \alpha-1}\left(\overline{\mathrm{U}}_{i}\right)$ и $\mathrm{Y}^{\prime} \rightleftharpoons \pi_{\alpha-2, \alpha-1}\left(\overline{\mathrm{Y}}^{\prime}\right)$. Имеем $\mathrm{E} \in$ ППер $\alpha(A, r)$. Пусть $\mathrm{P} \in$ Пер $\left(\Gamma_{\alpha-1}, A, 2\right)$ - периодическая основа для $\mathrm{E}$, где $\mathrm{K}, \mathbf{s}_{A, \mathrm{P}} \mathrm{K}, \ldots, \mathbf{s}_{A, \mathrm{P}}^{r} \mathrm{~K} \in$ Эл $\rho(A)\left(\mathrm{P}, q_{2}\right)$ для некоторого $\mathrm{K}$. Используя леммy 8.2, (iii), найдем $\mathrm{L}_{0}, \mathrm{~L}_{r} \in$ Эл $\rho(A)\left(\mathrm{E}, q_{1}\right), \mathrm{L}_{i} \in \ni_{\rho(A)}\left(\mathrm{E}, q_{2}-h\right)(i=1,2, \ldots$ $\ldots, r-1)$ такие, что ЭСогл $\rho(A)\left(\mathbf{s}_{A, \mathrm{P}}^{i} \mathrm{~K}, \mathrm{~L}_{i}\right)$ при всех $i$ и $\mathrm{L}_{0}<_{\mathrm{E}} \mathrm{L}_{1}<_{\mathrm{E}} \cdots<_{\mathrm{E}} \mathrm{L}_{r}$. Так как $\ell_{\alpha, A}\left(\mathrm{~L}_{0} \cup_{\mathrm{E}} \mathrm{L}_{2}\right) \geqslant 2, \ell_{\alpha, A}\left(\mathrm{~L}_{r-2} \cup_{\mathrm{E}} \mathrm{L}_{r}\right) \geqslant 2$ и в силу леммы 8.20, (ii) имеем $\mathcal{W}_{\alpha-1}\left(\mathcal{L}\left(\mathrm{U}_{i}\right)\right)=\mathcal{W}_{\alpha-1}\left(\mathcal{L}\left(\overline{\mathbf{U}}_{i}\right)\right)<\zeta(1+2 \zeta)<\frac{1}{13}(i=1,2)$, то в силу предложения 8.22 , (i) $\mathrm{L}_{2}$ не содержится в $\mathrm{U}_{1}$ и $\mathrm{L}_{r-2}$ не содержится в $\mathrm{U}_{2}$. Далее повторяются рассуждения из доказательства утверждения (ii) леммы 8.25, где вместо $\mathrm{L}_{16}$ и $\mathrm{L}_{r-16}$ нужно взять $\mathrm{L}_{3}$ и $\mathrm{L}_{r-3}$ соответственно. 
8.31. Лемма. Если $A, B \in \mathcal{M}_{\alpha}$ u nути $\mathrm{P} \in \prod_{e p}\left(\Gamma_{\alpha-1}, A, 8\right)$ u $\mathrm{Q} \in$ Пер $\left(\Gamma_{\alpha-1}, B, 6\right)$ строго близки в некотором ранге $\beta \leqslant \alpha-1$, то Согл $(\mathrm{P}, \mathrm{Q})$.

ДокАЗАТЕЛЬСТво. Если $\beta \geqslant \gamma \rightleftharpoons \max \{\rho(A), \rho(B)\}$, то утверждение вытекает из предложения 6.12. Пусть $\beta<\gamma$. По лемме 8.2 , (ii) пути $\mathrm{P}$ и $\mathrm{Q}$ близки в ранге $\beta$. Используя лемму 8.4 и индуктивное предположение 11.21 , легко видеть, что найдутся $\mathrm{K} \in$ Эл $_{\gamma}\left(\mathrm{P}, q_{2}-h\right)$ и $\mathrm{L} \in$ Эл $_{\gamma}\left(\mathrm{Q}, q_{2}-h\right)$ такие, что $|\mathrm{K}| \leqslant|A|$ и $|\mathrm{L}| \leqslant|B|$. Можно считать, что пути $\mathrm{K}_{i} \rightleftharpoons \mathrm{s}_{A, \mathrm{P}}^{i} \mathrm{~K}$ при $i=0,1, \ldots, 7$ содержатся в $\mathrm{P}$, а пути $\mathrm{L}_{i} \rightleftharpoons \mathbf{s}_{B, \mathrm{Q}}^{i} \mathrm{~L}$ при $i=0,1, \ldots, 5$ содержатся в $\mathrm{Q}$. Используя индуктивное предположение 11.21 еше раз, найдем $\mathrm{K}_{i}^{\prime} \in \ni_{\gamma}\left(\mathrm{Q}, q_{1}\right)(i=0,1, \ldots, 6)$ и $\mathrm{L}_{i}^{\prime} \in$ Эл $_{\gamma}\left(\mathrm{P}, q_{1}\right)$ такие, что ЭСогл $\Omega_{\gamma}\left(\mathrm{K}_{i}, \mathrm{~K}_{i}^{\prime}\right)$ и $Э$ Согл $\Omega_{\gamma}\left(\mathrm{L}_{i}, \mathrm{~L}_{i}^{\prime}\right)$ при всех $i$. Пусть $\mathrm{M}$ и $\mathrm{N}$ - соответственно минимальный и максимальный относительно $\prec$ р элементы среди всех $\mathrm{K}_{i}$ и $\mathrm{L}_{i}^{\prime}$, a $\mathrm{M}^{\prime}$ и $\mathrm{N}^{\prime}$ - соответственно минимальный и максимальньй относительно $\prec Q$ элементы среди всех $\mathrm{K}_{i}^{\prime}$ и $\mathrm{L}_{i}$. Используя индуктивное предположение 11.27 , легко видеть, что $Э$ Согл $\Omega_{\gamma}\left(\mathrm{M}, \mathrm{M}^{\prime}\right)$ и $Э$ Согл $\left(\mathrm{N}, \mathrm{N}^{\prime}\right)$. Следовательно, положив $\mathrm{P}^{*} \rightleftharpoons \mathrm{M} \cup_{\mathrm{P}} \mathrm{N}$ и $\mathrm{Q}^{*} \rightleftharpoons \mathrm{M}^{\prime} \cup_{\mathrm{Q}} \mathrm{N}^{\prime}$, получим строго близкие в ранге $\gamma$ пути $\mathrm{P}^{*}$ и $\mathrm{Q}^{*}$. Тогда по предло-

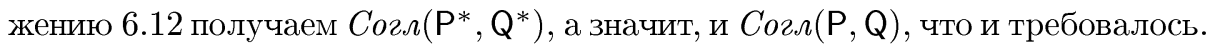

8.32. Лемма. Пусть $A, B \in \mathcal{M}_{\alpha}, \neg \operatorname{Pod}_{\alpha}(A, B), \quad \mathrm{P} \in \Pi_{\operatorname{pep}}\left(\Gamma_{\alpha-1}, A, 2\right), u$ путь $\mathrm{E} \in \Pi$ Пер $\alpha(B, 6)$ содержится в $\mathrm{P}$. Тогда $\neg \Pi$ жогл $\Omega_{\alpha}\left(\mathrm{E}, \mathrm{s}_{A, \mathrm{P}} \mathrm{E}\right)$.

ДоказАтельСтво. Допустим, ПСог $\Omega_{\alpha}\left(\mathrm{E}, \mathbf{s}_{A, \mathrm{P}} \mathrm{E}\right)$. Выбрав достаточно длинное периодическое продолжение $\mathrm{R}$ для $\mathrm{P}$ и воспользовавшись предложением 8.11, получим $\mathrm{F} \rightleftharpoons \mathrm{E} \cup_{\mathrm{R}} \mathbf{s}_{A, \mathrm{P}}^{8} \mathrm{E} \in$ ППер $\alpha(B, 6) \cap$ Пер $\left(\Gamma_{\alpha-1}, A, 8\right)$. Из определения 5.17 следует, что для $\mathbf{F}$ сушествует периодическая основа $\mathrm{S} \in \operatorname{\Pi еp}\left(\Gamma_{\alpha-1}, B\right) \mathrm{c}|\mathrm{S}| \geqslant$ $6|B|$. Так как пути $\mathrm{F}$ и $\mathrm{S}$ строго близки в ранге $\rho(B)$, то по лемме 8.31 имеем $\operatorname{Cozл}(\mathrm{F}, \mathrm{S})$. Тогда в силу леммы 7.22 получаем $\operatorname{Po}_{\alpha}(A, B)$ вопреки посылке леммы.

8.33. Лемма. Пусть $t \geqslant 1, A_{0}, A_{1}, \ldots, A_{t} \in \mathcal{M}_{\alpha}, \neg \operatorname{Pod}_{\alpha}\left(A_{i}, A_{i+1}\right) \quad(i=$ $0,1, \ldots, t-1)$ и для каждого $i=0,1, \ldots, t-1$ существуют близкие в ранге $\alpha-1$ слова $P_{i} \in \Pi$ Пер $\left(A_{i}\right)$ и $Q_{i} \in \Pi$ Пер $\left(A_{i+1}\right)$ такие, ито $\left|P_{0}\right| \geqslant p\left|A_{0}\right| u\left|P_{i}\right| \geqslant 10\left|A_{i}\right|$ при $i \geqslant 1$. Тогда в некоторый ииклический сдвиг слова $A_{t}$ входит слово $E \in \Pi \Pi e p_{\alpha}\left(A_{0}, 152\right)$.

\section{ДОКАЗАТЕЛЬСТВО (индукция по $t$ ).}

Случай 1: $t=1$ (база индукции). Рассмотрим близкие в ранге $\alpha-1$ пути $\mathrm{P}_{0}$ и $\mathrm{Q}_{0}$ на графе $\Gamma_{\alpha-1}$ с $\mathcal{L}\left(\mathrm{P}_{0}\right)$ 드 $P_{0}$ и $\mathcal{L}\left(\mathrm{Q}_{0}\right)$ 드 $Q_{0}$. Можно считать, что $\mathrm{Q}_{0}$ входит в качестве подпути в некоторый путь $\mathrm{Q} \in$ Пер $\left(\Gamma_{\alpha-1}, A_{1}, 2\right)$. Так как $p=240$, то по предложению 8.23 найдется подпуть $\mathrm{E}$ пути $\mathrm{Q}_{0}$ такой, что $\mathrm{E} \in \Pi_{\text {Пер }}\left(A_{0}, 204\right)$. По лемме 8.32 имеем $\neg \Pi$ Согл $\Omega_{\alpha}\left(\mathrm{E}, \mathrm{s}_{A_{1}, \mathrm{Q}} \mathrm{E}\right)$, следовательно, укоротив $\mathrm{E}$ в силу предложений 8.9 и $8.14,(\mathrm{i})$, получим подпуть $\widetilde{\mathrm{E}} \in$ ППер $\alpha\left(A_{0}, 195\right)$ пути $\mathrm{Q}$ такой, что $\widetilde{\mathrm{E}} \ll_{\mathrm{Q}} \mathbf{s}_{A, \mathrm{Q}} \mathrm{E}$, и, значит, $|\widetilde{\mathrm{E}}| \leqslant\left|A_{1}\right|$. Тогда в некоторый циклический сдвиг слова $A_{1}$ входит слово $\mathcal{L}(\widetilde{\mathrm{E}}) \in \Pi$ Пер $\alpha\left(A_{0}, 195\right)$.

Случай 2: $t>1$ (индуктивный шаг). Индукцией по убыванию $r$ при $r=t-$ 
$1, t-2, \ldots, 1$ докажем следуюшее утверждение:

существуют близкие в ранге $\alpha-1$ пути $\mathrm{P} \in \Pi_{\text {ер }}\left(\Gamma_{\alpha-1}, A_{r}\right)$

$u \quad \mathrm{Q} \in \operatorname{\Pi ер}\left(\Gamma_{\alpha-1}, A_{t}\right)$, әде $|\mathrm{P}| \geqslant 8\left|A_{r}\right| \quad u \quad|\mathrm{Q}|<6\left|A_{t}\right|$.

Рассмотрим близкие в ранге $\alpha-1$ пути $\mathrm{R} \in \prod_{\text {ep }}\left(\Gamma_{\alpha-1}, A_{r}, 10\right)$ и $\mathrm{S} \in$ Пер $\left(\Gamma_{\alpha-1}, A_{r+1}\right)$, существующие по условию леммы. По индуктивному предположению леммы найдется подпуть $\mathrm{E} \in$ ППер $\alpha\left(A_{0}, 152\right)$ пути $\mathrm{R}$ такой, что $|\mathrm{E}| \leqslant\left|A_{0}\right|$. Можно считать, что пути $\mathrm{s}_{A_{r}, \mathrm{R}} \mathrm{E}$ содержатся в $\mathrm{R}$ при $i=0,1, \ldots, 8$. По лемме 8.29, (i) найдутся подпуть $\overline{\mathrm{E}} \in$ ППер $\alpha\left(A_{0}, 118\right)$ пути $\mathrm{E}$ и подпути $\mathrm{F}_{0}, \mathrm{~F}_{8} \in$ ППер $\alpha\left(A_{0}, 118\right)$ пути $\mathrm{S}$ такие, что пути $\mathbf{s}_{A_{r}, \mathrm{R}} \overline{\mathrm{E}}$ и $\mathrm{F}_{i}$ строго близки в ранге $\rho\left(A_{0}\right)$ $(i=0,8)$ и $\mathrm{F}_{0} \ll s \mathrm{~F}_{8}$. Тогда пути $\mathrm{R}^{\prime} \rightleftharpoons \overline{\mathrm{E}} \cup_{\mathrm{R}} \mathbf{s}_{A_{r}, \mathrm{R}} \overline{\mathrm{E}}$ и $\mathrm{S}^{\prime} \rightleftharpoons \mathrm{F}_{0} \cup_{\mathrm{S}} \mathrm{F}_{8}$ строго близки в ранге $\rho\left(A_{0}\right)$ и так как $\left|\mathrm{R}^{\prime}\right| \geqslant 8\left|A_{r}\right|$, то из $\neg P о \partial_{\alpha}\left(A_{r}, A_{r+1}\right)$ в силу лемм $7.22,8.31$ следует $\left|\mathrm{S}^{\prime}\right|<6\left|A_{r+1}\right|$. По лемме 8.2, (ii) пути $\mathrm{R}^{\prime}$ и $\mathrm{S}^{\prime}$ близки в ранге $\alpha-1$. Следовательно, если $r=t-1$, то в качестве искомых $\mathrm{P}$ и $\mathrm{Q}$ можно взять соответственно $\mathrm{R}^{\prime}$ и $\mathrm{S}^{\prime}$. Пусть $r<t-1$. По индуктивному предположению (8.1) найдутся близкие в ранге $\alpha-1$ пути $\mathrm{P}^{*}$ и $\mathrm{Q}^{*}$ такие, что $\left|\mathrm{P}^{*}\right| \geqslant 8\left|A_{r+1}\right|$ и $\left|\mathrm{Q}^{*}\right|<6\left|A_{t}\right|$. Так как $\left|\mathrm{P}^{*}\right|>\left|\mathrm{S}^{\prime}\right|+2\left|A_{r+1}\right|$, то, действуя на $\mathrm{P}^{*}$ и $\mathrm{Q}^{*}$ подходящим элементом группы $\mathbf{B}_{\alpha-1}$, можно считать, что $\mathrm{S}^{\prime}$ содержится в $\mathrm{P}^{*}$. По лемме 8.29 , (ii) при $\mathrm{P}:=\mathrm{R}$, $\mathrm{X}:=\mathrm{S}, \mathrm{X}^{*}:=\mathrm{P}^{*}$ и $\mathrm{Y}:=\mathrm{Q}^{*}$ некоторьй подпуть $\mathrm{P}$ пути $\mathrm{P}^{*}$ и некоторый подпуть $\mathrm{Q}$ пути $\mathrm{Q}^{*}$ близки в ранге $\alpha-1$, где $|\mathrm{P}| \geqslant 8\left|A_{r}\right|$. Так как $\left|\mathrm{Q}^{*}\right|<6\left|A_{t}\right|$, то $|\mathrm{Q}|<6\left|A_{t}\right|$, что и завершает доказательство (8.1).

Завершим доказательство леммы 8.33. Рассмотрим близкие в ранге $\alpha-1$ пути $\mathrm{P} \in$ Пер $\left(\Gamma_{\alpha-1}, A_{1}, 8\right)$ и $\mathrm{Q} \in \Pi_{\mathrm{ep}}\left(\Gamma_{\alpha-1}, A_{t}\right)$, сушествуюшие в силу (8.1). Можно считать, что $\mathrm{Q}$ входит в качестве подпути в некоторьй путь $\mathrm{Q}^{\prime} \in$ Пер $\left(\Gamma_{\alpha-1}, A_{t}, 2\right)$. Как было установлено при рассмотрении случая $1, \mathrm{P}$ содержит некоторые $\mathrm{E}, \mathbf{s}_{A_{1}, \mathrm{P}} \mathrm{E} \in \Pi \operatorname{lep}_{\alpha}\left(A_{0}, 195\right)$, причем $\neg \Pi$ Сог $\Omega_{\alpha}\left(\mathrm{E}, \mathbf{s}_{A_{1}, \mathrm{P}} \mathrm{E}\right)$. Тогда по предложению 8.26 найдутся подпути $\mathrm{F}, \mathrm{F}^{\prime} \in$ ППер $\alpha\left(A_{0}, 161\right)$ пути $\mathrm{Q}$ такие, что $\neg \Pi$ Согл $\Omega_{\alpha}\left(\mathrm{F}, \mathrm{F}^{\prime}\right)$. Если бы выполнялось $П С о г \Omega_{\alpha}\left(\mathrm{F}, \mathbf{s}_{A_{t}, \mathrm{Q}} \mathrm{F}\right)$, то для любых $i, j \in \mathbb{Z}$ имели бы ПСогл $\Omega_{\alpha}\left(\mathbf{s}_{A_{t}, \mathrm{Q}}^{i} \mathrm{~F}, \mathbf{s}_{A_{t}, \mathrm{Q}}^{j} \mathrm{~F}\right)$, откуда в силу предложений 8.11 и 8.9 легко следовало бы ПСог $\Omega_{\alpha}\left(\mathrm{F}, \mathrm{F}^{\prime}\right)$. Поэтому $\neg \Pi$ Сог $\Omega_{\alpha}\left(\mathrm{F}, \mathbf{s}_{A_{t}, \mathrm{Q}} \mathrm{F}\right)$. Укоротив F c использованием предложений 8.9 и 8.14, (i), найдем подпуть $\mathrm{F}^{*}$ пути $\mathrm{Q}$ такой, что $\mathrm{F}^{*} \in \Pi$ Пер $\alpha\left(A_{0}, 152\right)$ и $\left|\mathrm{F}^{*}\right| \leqslant\left|A_{t}\right|$. Тогда слово $\mathcal{L}\left(\mathrm{F}^{*}\right) \in \Pi$ Пер $\alpha\left(A_{0}, 152\right)$ входит в некоторьй циклический сдвиг слова $A_{t}$, что и требовалось доказать.

8.34. ПРЕДЛОЖЕНИЕ. Для любого $A \in \bigcup_{i \geqslant 0} \mathcal{N}_{\alpha, i}$ некоторый чиклический сдвиг слова $A$ содержит әлементарную 152-степень ранга $\alpha$.

ДокАЗАТЕЛЬСтво. На множестве $\mathcal{M}_{\alpha}$ введем бинарное отношение:

$$
\begin{aligned}
A>B \rightleftharpoons & \text { для некоторого } t \geqslant 1 \text { сушествуют } A_{0}, A_{1}, \ldots, A_{t} \in \mathcal{M}_{\alpha}, \\
& \text { удовлетворяюшие посылке леммы } 8.33, \text { где } A_{0} \text { 으 } B \text { и } A_{t} \text { 드 } A .
\end{aligned}
$$


Непосредственно проверяется, что отношение $A>B$ транзитивно. Сравнивая определение 5.5 и формулировку леммы 8.33, видим, что включение $A \in \bigcup_{i} \mathcal{N}_{\alpha, i}$ истинно тогда и только тогда, когда $A>B$ для некоторого $B \in \mathcal{M}_{\alpha}$. Следовательно, в силу определения $5.6 A$ - элементарньй период ранга $\alpha$ тогда и только тогда, когда $A$ - минимальньй относительно > элемент множества $\mathcal{M}_{\alpha}$.

Докажем, что если $A>B$, то любая почти периодическая $r$-степень ранга $\alpha$, порожденная периодом $A$, содержит в качестве подслов $r-1$ попарно непересекающихся 118-степеней ранга $\alpha$, порожденных периодом $B$. Пусть $E \in \Pi$ Пе $p_{\alpha}(A, r)$. Рассмотрим путь $\mathrm{E} \in \Pi_{\text {Пер }}(A, r)$ с $\mathcal{L}(\mathrm{E})$ 으 $E$, и пусть $\mathrm{P}$ - периодическая основа для $\mathrm{E}$ с $|\mathrm{P}| \geqslant r|A|$, сушествуюшая в силу определения 5.17. По лемме 8.33 путь $\mathrm{P}$ содержит $r-1$ подпутей $\mathrm{F}, \mathrm{s}_{A, \mathrm{P}} \mathrm{F}, \ldots, \mathrm{s}_{A, \mathrm{P}}^{r-2} \mathrm{~F}$, где $\mathrm{F} \in \Pi_{\text {Пер }}(B, 152)$. Тогда в силу леммы 8.29 путь $\mathrm{E}$ содержит $r-1$ попарно не имеющих общих ребер подпутей $\mathrm{F}_{i} \in$ ППер $\alpha(B, 118)$, и, следовательно, слово $E$ содержит $r-1$ попарно непересекающихся подслов $\mathcal{L}\left(\mathrm{F}_{i}\right) \in \Pi$ Пер $\alpha(B, 118)$.

Из доказанного непосредственно вытекает, что отношение $A>B$ удовлетворяет условию обрьва убывающих цепей. Отсюда следует, что для любого слова $A \in \bigcup_{i} \mathcal{N}_{\alpha, i}$ сушествует слово $B \in \mathcal{E}_{\alpha}$ такое, что $A>B$. Тогда в силу леммы 8.33 и определения 5.19 некоторый циклический сдвиг $A$ содержит элементарную 152-степень ранга $\alpha$.

8.35. ПРЕДЛОЖЕНИЕ. Если $A, B \in \mathcal{M}_{\alpha} u \operatorname{Poд}_{\alpha}(A, B)$, то любой путь $\mathrm{E} \in$ ППер $\alpha(A, 37)$ содержит в качестве подпути путь $\mathrm{F} \in \Pi_{\text {пер }}(B)$ такой, что $\ell_{\alpha, B}(\mathrm{~F}) \geqslant \ell_{\alpha, A}(\mathrm{E})-36$ u $\Pi \operatorname{Coz}_{\alpha}(\mathrm{E}, \mathrm{F})$.

ДоказАТЕЛЬСтво. Обозначим $r \rightleftharpoons \ell_{\alpha, A}(\mathrm{E})$, и пусть $\mathrm{P} \in \Pi_{\text {ep }}\left(\Gamma_{\alpha-1}, A, 2\right)-$ периодическая основа для $\mathrm{E}$, где $\mathrm{K}, \mathbf{s}_{A, \mathrm{P}} \mathrm{K}, \ldots, \mathbf{s}_{A, \mathrm{P}}^{r} \mathrm{~K} \in$ Эл $_{\rho(A)}\left(\mathrm{P}, q_{2}\right)$ для некоторого К. Согласно лемме 7.22 найдутся $\mathrm{P}^{*} \in \Pi_{\text {ep }}\left(\Gamma_{\alpha-1}, A, 2\right)$ и $\mathrm{Q} \in \Pi_{\mathrm{ep}}\left(\Gamma_{\alpha-1}, B, 2\right)$ такие, что $\operatorname{Cozл}\left(\mathrm{P}^{*}, \mathrm{Q}\right)$. В силу леммы 7.7 можно считать, что $\mathrm{P}^{*}$ и $\mathrm{Q}$ строго близки в ранге $\rho(A)$, а действуя на $\mathbf{P}^{*}$ и $\mathbf{Q}$ подходящим элементом группы $\mathbf{B}_{\alpha-1}$, можно считать что $\mathrm{P}$ содержится в $\mathrm{P}^{*}$.

По лемме 8.2, (iii) найдем $\mathrm{L}, \mathrm{L}^{\prime} \in$ Эл $_{\rho(A)}\left(\mathrm{E}, q_{1}\right)$ и $\mathrm{M} \in$ Эл $_{\rho(A)}\left(\mathrm{Q}, q_{1}\right)$ такие, что ЭСогл $\Omega_{(A)}(\mathrm{K}, \mathrm{L}), \quad$ ЭСогл $\Omega_{\rho(A)}(\mathrm{K}, \mathrm{M}), \quad$ ЭСогл $\Omega_{\rho(A)}\left(\mathbf{s}_{A, \mathrm{P}}^{r} \mathrm{~K}, \mathrm{~L}^{\prime}\right)$. В силу леммы 7.21 имеем Иогл $\rho(A)_{\rho}\left(\mathbf{s}_{A, \mathrm{P}}^{r} \mathrm{~K}, \mathbf{s}_{B, \mathrm{Q}}^{r} \mathrm{M}\right)$, следовательно, положив $\mathrm{E}^{\prime} \rightleftharpoons \mathrm{L}_{\mathrm{E}} \mathrm{L}^{\prime}$ и $\mathrm{Q}^{\prime} \rightleftharpoons$ $\mathrm{M} \cup \mathrm{Q}^{*} \mathbf{s}_{B, \mathrm{Q}} \mathrm{M}$, где $\mathrm{Q}^{*}$ - некоторое периодическое продолжение $\mathrm{Q}$, получим строго близкие в ранге $\rho(A)$, а значит, и близкие в ранге $\alpha-1$ подпуть $\mathrm{E}^{\prime}$ пути $\mathrm{E}$ и путь $\mathrm{Q}^{\prime} \in \Pi_{\mathrm{ep}}\left(\Gamma_{\alpha-1}, B, r\right)$. Остается воспользоваться предложением 8.23.

8.36. ПРЕДЛОЖЕНИЕ. Пусть $A, B \in \mathcal{E}_{\alpha}, u$ nymb $\mathrm{P} \in \Pi_{\mathrm{ep}}\left(\Gamma_{\alpha-1}, A, p\right)$ близок в ранге $\alpha-1 \kappa$ некоторому подпути пути $\mathrm{Q} \in \Pi_{\mathrm{ep}}\left(\Gamma_{\alpha-1}, B, 2\right)$. Тогда $\operatorname{Cozл}(\mathrm{P}, \mathrm{Q})$.

ДоКАЗАтЕЛЬСтво. Пусть $\mathrm{Q}^{\prime}$ - подпуть пути $\mathrm{Q}$, близкий в ранге $\alpha-1$ к $\mathrm{P}$. Так как $B \notin \mathcal{N}_{\alpha, 1}$ согласно определению 5.6 и слова $\mathcal{L}(\mathrm{P}) \in \Pi$ Пер $(A, p)$ и $\mathcal{L}\left(\mathrm{Q}^{\prime}\right) \in \Pi$ Пер $(B)$ близки в ранге $\alpha-1$, то согласно определению 5.5 имеем $\operatorname{Poд}_{\alpha}(A, B)$. Тогда в силу предложений $8.23,8.35$ и следствия 8.6 , (i) найдутся подпуть $\mathrm{E} \in$ ППер $\alpha(B, p-72)$ пути $\mathrm{Q}^{\prime}$ и периодическая основа $\mathrm{R} \in \Pi_{\mathrm{ep}}\left(\Gamma_{\alpha-1}, B, 2\right)$ 
для $\mathrm{E}$ такие, что Согл $(\mathrm{P}, \mathrm{R})$. Поскольку $\ell_{\alpha, B}(\mathrm{E})>6$, то можно считать, что $\mathrm{M}, \mathrm{s}_{B, \mathrm{R}} \mathrm{M}, \ldots, \mathrm{s}_{B, \mathrm{R}}^{6} \mathrm{M} \in$ Эл $_{\rho(B)}\left(\mathrm{R}, q_{2}\right)$ для некоторого $\mathrm{M}$. Так как $\mathrm{R}$ и $\mathrm{E}$ строго близки в ранге $\rho(B)$ в силу леммы 8.1 и $\mathrm{E}$ содержится в $\mathrm{Q}$, то по предложению 6.11

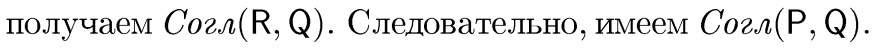

8.37. ПрЕДЛОЖЕнИЕ. Если $A, B \in \mathcal{E}_{\alpha}, P$ есть начало слова $A^{n}, Q$ есть начало слова $B^{n}, \quad u, v \in \mathcal{H}_{\alpha-1}, \quad P \stackrel{\alpha=1}{=} u Q v u|P| \geqslant p|A|$, mо $A^{n} \stackrel{\alpha=1}{=} u B^{n} u^{-1}$.

ДоказАТЕЛЬСТво. Рассмотрим пути $\mathrm{P}$ и uQv на графе $\Gamma_{\alpha-1}$ с общими начальной и конечной вершинами, где метки путей $\mathrm{P}, \mathrm{u}, \mathrm{Q}$ и $\mathrm{v}$ равны соответственно словам $P, u, Q$ и $v$. Можно считать, что $\iota(\mathrm{P})=1$ и $\mathrm{Q}$ является началом пути $\overline{\mathrm{Q}}$ с $\mathcal{L}(\overline{\mathrm{Q}})$ 으 $B^{n}$. Так как пути $\mathrm{P}$ и $\mathrm{Q}$ близки в ранге $\alpha-1$ и $|\mathrm{P}| \geqslant p|A|$, то по предложению 8.36 имеем $C о г л(\mathrm{P}, \overline{\mathrm{Q}})$, т.е. $\mathbf{s}_{A, \mathrm{P}}^{t}=\mathbf{s}_{B, \overline{\mathrm{Q}}}^{t}$ для некоторого $t>0$. Так как $\mathbf{s}_{A, \mathrm{P}}=$ $[A]_{\alpha-1}$ и $\mathbf{s}_{B, \overline{\mathbf{Q}}}=\left[u B u^{-1}\right]_{\alpha-1}$, то отсюда следует $A^{t} \stackrel{\alpha=1}{=} u B^{t} u^{-1}$. Тогда имеем $\left[u B u^{-1}\right]_{\alpha-1} \in \mathbf{N}_{\alpha-1}^{+}(A)$, и в силу предложения 7.10, (i) $\operatorname{ind}_{A}\left(\left[u B u^{-1}\right]_{\alpha-1}\right)=1$. В этом случае по предложению 7.18 , (i) $A^{n} \stackrel{\alpha=1}{=} u B^{n} u^{-1}$.

8.38. ПрЕДЛОЖЕНИЕ. (i) Пусть $A, B \in \mathcal{E}_{\alpha}, \quad \mathrm{E} \in \Pi$ Пер $\alpha(A, p+34) u \mathrm{P} \in$ Пер $\left(\Gamma_{\alpha-1}, A, 2\right)$ - периодическая основа для $\mathrm{E}$. Пусть $\mathrm{F}-$ путь на $\Gamma_{\alpha-1} c$ $\mathcal{L}(\mathrm{F}) \in \mathcal{R}_{\alpha-1}$, содержащий $\mathrm{E}, \quad \mathrm{Q} \in \Pi_{\mathrm{ep}}\left(\Gamma_{\alpha-1}, B, 2\right)$ и $\mathrm{F}$ и некоторый подпуть пути $\mathrm{Q}$ близки в ранге $\alpha-1$. Тогда Согл $(\mathrm{P}, \mathrm{Q})$.

(ii) Eсли $A, B \in \mathcal{E}_{\alpha}, \mathrm{E} \in \Pi \Pi \Pi_{\alpha}(A, p+34), \quad \mathrm{F} \in \Pi \Pi_{\text {ep }}(B) u \mathrm{E}$ coдержится в $\mathrm{F}, \operatorname{mo~} \Pi \operatorname{Coz} \Omega_{\alpha}(\mathrm{E}, \mathrm{F})$.

ДоКАЗАТЕЛЬСТво. (i) По предложению 8.26 для некоторого подпути $\mathrm{G}$ пути $\mathrm{Q}$ имеем $\mathrm{G} \in$ ППер $\alpha(A, p)$ и ПСогл $\Omega_{\alpha}(\mathrm{E}, \mathrm{G})$. Пусть $\mathrm{R} \in \Pi$ Пер $\left(\Gamma_{\alpha-1}, A, p\right)$ - периодическая основа для $\mathrm{G}$. В силу леммы 8.2, (i) и предложения 8.36 имеем $\operatorname{Coгл(R,~Q).~Так~}$ как в силу следствия 8.6, (i) Cогл(P, R), то отсюда следует $C$ огл(P, Q).

Утверждение (ii) вытекает из (i) с учетом леммы 8.2, (ii), при этом в качестве $Q$ нужно взять периодическую основу для $F$.

8.39. ПРЕДЛОЖЕНИЕ. Пусть $\mathrm{X}-n y т ь$ на графе $\Gamma_{\alpha-1} c \mathcal{L}(\mathrm{X}) \in \mathcal{R}_{\alpha-1}$. Пусть $\mathrm{E}_{i} \in \Pi$ Пер ${ }_{\alpha}\left(A_{i}, q_{0}\right) \quad(i=1,2,3,4)-$ подпути $\mathrm{X}$, где $A_{i} \in \mathcal{E}_{\alpha}$ при всех $i$. Если $\Pi$ Сог $\Omega_{\alpha}\left(\mathrm{E}_{1}, \mathrm{E}_{3}\right), \Pi$ Cогл $\Omega_{\alpha}\left(\mathrm{E}_{2}, \mathrm{E}_{4}\right) u \neg \Pi \operatorname{Coz} \Omega_{\alpha}\left(\mathrm{E}_{1}, \mathrm{E}_{2}\right)$, mo $\mathrm{E}_{1}<\mathrm{x} \mathrm{E}_{2} \Leftrightarrow \mathrm{E}_{3}<\mathrm{x} \mathrm{E}_{4}$.

ДокАЗАТЕЛЬСТво. Очевидно, достаточно рассмотреть случай, когда $\mathrm{E}_{1}=\mathrm{E}_{3}$. В силу предложения 8.38, (ii) истинно ровно одно из отношений $\mathrm{E}_{1}<\mathrm{x} \mathrm{E}_{2}$ или $\mathrm{E}_{1}>\mathrm{x}$ $\mathrm{E}_{2}$, и так как $\neg \Pi$ Согл $\Omega_{\alpha}\left(\mathrm{E}_{1}, \mathrm{E}_{4}\right)$, то истинно ровно одно из отношений $\mathrm{E}_{1}<\mathrm{x} \mathrm{E}_{4}$ или $\mathrm{E}_{1}>\mathrm{x} \mathrm{E}_{4}$. Допустим, $\mathrm{E}_{2}<\mathrm{x} \mathrm{E}_{1}<\mathrm{x} \mathrm{E}_{4}$. По предложению 8.38, (ii) путь $\mathrm{E}_{4}$ содержит а качестве подпути путь $\mathrm{F} \in \Pi$ Пер $\alpha\left(A_{2}, q_{0}-36\right)$ такой, что ПСогл $\Omega_{\alpha}\left(\mathrm{E}_{4}, \mathrm{~F}\right)$. B силу предложения 8.38, (ii) по-прежнему имеем $E_{1}<x F$. Тогда $E_{2} \cup_{X} F$ содержит $\mathrm{E}_{1}$ в качестве подпути, и, используя предложения 8.11 и 8.38 , (ii), получаем ПСогл $\alpha\left(\mathrm{E}_{1}, \mathrm{E}_{2}\right)$. Противоречие. Таким образом, случай $\mathrm{E}_{2}<\mathrm{x} \mathrm{E}_{1}<\mathrm{x} \mathrm{E}_{4}$ невозможен. Аналогично доказывается невозможность случая $\mathrm{E}_{4}<\mathrm{x} \mathrm{E}_{1}<\mathrm{x} \mathrm{E}_{2}$. Следовательно, $\mathrm{E}_{1}<\mathrm{x} \mathrm{E}_{2} \Leftrightarrow \mathrm{E}_{1}<\mathrm{x} \mathrm{E}_{4}$. 
8.40. ПРЕДЛОЖЕНИЕ $(\alpha \geqslant 0)$. Если $\beta \geqslant \alpha, \mathrm{E} \in \ni_{\alpha}\left(\Gamma_{\beta}, p+34\right), \mathrm{F} \in \ni{ }_{\alpha}\left(\Gamma_{\beta}\right)$ $u \mathrm{E}$ содержится в $\mathrm{F}$, то ЭСогл $\beta(\mathrm{E}, \mathrm{F})$. То же самое верно при $\alpha \geqslant 1$, если вместо $\mathrm{E} \in \ni л_{\alpha}\left(\Gamma_{\beta}, p+34\right)$ предполагать, ито $\mathrm{E} \in \exists_{\alpha}\left(\Gamma_{\beta}\right) u \mathrm{E} u \mathrm{~F}$ порождень одним әлементарным периодом $A \in \mathcal{E}_{\alpha}$ (см. определение 5.16).

В частности, если $\mathrm{X}-$ простой путь на графе $\Gamma_{\beta}, \mathrm{E}, \mathrm{F} \in \ni_{\alpha}(\mathrm{X}, p+34) u$ $\neg$ ЭСогл $\Omega_{\alpha}(\mathrm{E}, \mathrm{F})$, то либо $\mathrm{E}<\mathrm{x} F$, либо $\mathrm{F}<\mathrm{x} \mathrm{E}$.

ДоКАЗАТЕЛЬСТВО. При $\alpha=0$ доказывать нечего. При $\alpha \geqslant 1$ нужно выбрать прообразы $\widetilde{E}$ и $\widetilde{F}$ на графе $\Gamma_{\alpha-1}$ путей $E$ и $F$ такие, что $\widetilde{E}$ содержится в $\widetilde{F}$, и воспользоваться предложениями 8.38 , (ii) и 8.9 .

8.41. ПРЕДЛОЖЕНИЕ $(\alpha \geqslant 0)$. Пусть $\beta \geqslant \alpha, r \geqslant p+43, \mathbf{X}$ - простой путь на графе $\Gamma_{\beta}, \mathrm{E}, \mathrm{F} \in \ni л_{\alpha}(\mathrm{X}, r)$ u $\neg$ ЭСогл $\Omega_{\alpha}(\mathrm{E}, \mathrm{F})$. Если $\mathrm{E}<\mathrm{X} F$, то существует начало $\mathrm{E}^{\prime} \in \ni л_{\alpha}\left(\Gamma_{\beta}, r-p-35\right)$ nути $\mathrm{E}$ такое, что $Э С о г \Omega_{\alpha}\left(\mathrm{E}, \mathrm{E}^{\prime}\right) u \mathrm{E}^{\prime} \ll \mathrm{X} F$. Если $\mathrm{E}>\mathrm{x} \mathrm{F}$, то существует конеи $\mathrm{E}^{\prime} \in \ni{ }_{\alpha}\left(\Gamma_{\beta}, r-p-35\right)$ пути $\mathrm{E}$ такой, что ЭСогл $\Omega_{\alpha}\left(\mathrm{E}, \mathrm{E}^{\prime}\right)$ и $\mathrm{E}^{\prime} \gg \mathrm{x} \mathrm{F}$. При этом если $\alpha \geqslant 1$ и $\mathrm{E} u \mathrm{~F}$ порождены одним әлементарным периодом $A \in \mathcal{E}_{\alpha}$, то утверждение верно при $r \geqslant 19$ вместо $r \geqslant p+43$ и вместо $\mathrm{E}^{\prime} \in$ Эл $_{\alpha}\left(\Gamma_{\beta}, r-p-35\right)$ можно взять $\mathrm{E}^{\prime} \in$ Эл $_{\alpha}\left(\Gamma_{\beta}, r-9\right)$.

ДокаЗАТЕЛЬСтво. При $\alpha=0$ утверждение тривиально. При $\alpha \geqslant 1$ нужно перейти к прообразу пути $X$ на графе $\Gamma_{\alpha-1}$ и воспользоваться предложениями 8.38, (ii), 8.9 и 8.14, (i).

8.42. ЛЕмма. Для любого $\mathrm{E} \in$ Эл $_{\alpha}\left(\Gamma_{\beta}\right)$ при $\beta \geqslant \alpha \geqslant 1$ выполнено

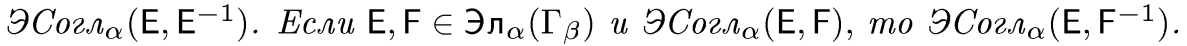

ДокАЗАТЕЛЬСтво. Первое утверждение вытекает из определения 5.20. Второе утверждение вытекает из первого в силу следствия 8.7 .

8.43. Лемма. Пусть $\mathrm{U}_{1} \mathrm{EU}_{2}$ u $\mathrm{P}-$ nути на графе $\Gamma_{\alpha-1}$, әде $\mathcal{L}\left(\mathrm{U}_{1} \mathrm{EU}_{2}\right) \in$ $\mathcal{R}_{\alpha-1}, \mathrm{E} \in \Pi$ Пер $\alpha(A)$ и $\mathrm{P} \in \Pi$ Пер $\left(\Gamma_{\alpha-1}, A, 2\right)$ - периодическая основа для $\mathrm{E}$.

(i) Пусть $\mathrm{P}_{1} \mathrm{~V}_{1} \in \Pi е р\left(\Gamma_{\alpha-1}, A, 2\right)$ - путь, имеющий общую начальную вершину с $\mathrm{P}$, т.е. один из путей $\mathrm{P}$ или $\mathrm{P}_{1} \mathrm{~V}_{1}$ является началом другого. Тогда если $\left|\mathrm{P}_{1}\right| \geqslant 20|A|$, то никакая вериина графа $\Gamma_{\alpha-1}$, лежащая на $\mathrm{U}_{1}$, не может быть близка в ранге $\alpha-1$ к вершине, лежсащей на $\mathrm{V}_{1}$.

(ii) Пусть $\mathrm{V}_{2} \mathrm{P}_{2} \in \Pi е р\left(\Gamma_{\alpha-1}, A, 2\right)$ - путь, имеющий общую конечную вершину с $\mathrm{P}$, т.е. один из путей $\mathrm{P}$ или $\mathrm{V}_{2} \mathrm{P}_{2}$ является концом другого. Тогда если $\left|\mathrm{P}_{2}\right| \geqslant 20|A|$, то никакая вершина графа $\Gamma_{\alpha-1}$, лежащая на $\mathrm{U}_{2}$, не может быть близка в ранге $\alpha-1$ к вершине, лежащей на $\mathrm{V}_{2}$.

ДоказАТЕЛЬСтво. Допустим, лемма неверна. Из двух симметричных случаев (i) и (ii) рассмотрим случай (i), когда некоторые вершины $\mathbf{g}$ и $\mathbf{h}$, лежашие соответственно на $\mathrm{U}_{1}$ и на $\mathrm{V}_{1}$, близки в ранге $\alpha-1$. Укоротив $\mathrm{U}_{1}$ и $\mathrm{V}_{1}$, можно считать $\mathbf{g}=\iota\left(\mathrm{U}_{1}\right)$ и $\mathbf{h}=\tau\left(\mathrm{V}_{1}\right)$. В силу леммы 8.2, (ii) вершины $\tau\left(\mathrm{U}_{1}\right)=\iota(\mathrm{E})$ и $\iota\left(\mathrm{P}_{1} \mathrm{~V}_{1}\right)=\iota(\mathrm{P})$ близки в ранге $\rho(A)$. Тогда по индуктивному предположению 11.9 при $\mathrm{X}:=\mathrm{U}_{1}^{-1}$ и $\mathrm{Y}:=\mathrm{P}_{1} \mathrm{~V}_{1}$ имеем $\mathrm{U}_{1}=\mathrm{W}_{1} \mathrm{U}^{\prime} \mathrm{W}_{2}$ и $\mathrm{P}_{1} \mathrm{~V}_{1}=\mathrm{Z}_{1} \mathrm{P}^{\prime} \mathrm{Z}_{2}$, где пути $\mathrm{U}^{\prime-1}$ и $\mathrm{P}^{\prime}$ близки в ранге $\rho(A)$ и $\mathcal{W}_{\alpha-1}\left(\mathcal{L}\left(\mathbf{Z}_{i}\right)\right)<1+2 \zeta(i=1,2)$. Так как $\tau\left(\mathrm{W}_{2}\right)$ и $\iota\left(\mathrm{Z}_{1}\right)$ 
близки в ранге $\rho(A)$, то пути $\left(\mathrm{U}^{\prime} \mathrm{W}_{2}\right)^{-1}$ и $\mathrm{Z}_{1} \mathrm{P}^{\prime}$ также близки в ранге $\rho(A)$. Следовательно, заменяя $\mathrm{U}^{\prime}$ и $\mathrm{P}^{\prime}$ на $\mathrm{U}^{\prime} \mathrm{W}_{2}$ и $\mathrm{Z}_{1} \mathrm{P}^{\prime}$, можем считать $\mathrm{U}_{1}=\mathrm{W}_{1} \mathrm{U}^{\prime}$ и $\mathrm{P}_{1} \mathrm{~V}_{1}=\mathrm{P}^{\prime} \mathrm{Z}_{2}$. По предложению 8.22, (ii) $\left|\mathrm{Z}_{2}\right|<16|A|$, следовательно, $\left|\mathrm{P}^{\prime}\right|>4|A|$. Для вывода искомого противоречия рассмотрим два случая.

Случай 1: $\rho(A)=0$. В силу индуктивного предположения 11.6 существуют близкие в ранге 0 прообразы на графе $\Gamma_{0}$ путей $\mathrm{U}^{\prime-1}$ и $\mathrm{P}^{\prime}$. Отсюда следует, что слова $\mathcal{L}\left(\mathrm{U}^{\prime-1}\right)$ и $\mathcal{L}\left(\mathrm{P}^{\prime}\right)$ близки в ранге 0 , т.е. $\left(\mathcal{L}\left(\mathrm{U}^{\prime}\right)\right)^{-1} \stackrel{0}{=} \mathcal{L}\left(\mathrm{P}^{\prime}\right)$. Так как $\mathcal{L}\left(\mathrm{U}^{\prime}\right), \mathcal{L}\left(\mathrm{P}^{\prime}\right) \in$ $\mathcal{R}_{\alpha-1} \subseteq \mathcal{R}_{0}$, то $\mathcal{L}\left(\mathrm{U}^{\prime}\right)$ и $\mathcal{L}\left(\mathrm{P}^{\prime}\right)$ - несократимые слова. Следовательно, $\left(\mathcal{L}\left(\mathrm{U}^{\prime}\right)\right)^{-1}$ 으 $\mathcal{L}\left(\mathrm{P}^{\prime}\right)$. Аналогично из близости путей $\mathrm{P}$ и $\mathrm{E}$ в ранге 0 с помощью индуктивного предположения 11.6 выводим $\mathcal{L}(\mathrm{P})$ 으 $\mathcal{L}(\mathrm{E})$. Пусть слово $\mathcal{L}(\mathrm{P})$ начинается с буквы $a^{\epsilon} \in \mathcal{A}^{ \pm 1}$. Так как одно из слов $\mathcal{L}\left(\mathrm{P}^{\prime}\right)$ или $\mathcal{L}(\mathrm{P})$ есть начало другого, то $\mathcal{L}\left(\mathrm{P}^{\prime}\right)$ также начинается с $a^{\epsilon}$, и, следовательно, слово $\mathcal{L}\left(\mathrm{U}^{\prime}\right)$ оканчивается на $a^{-\epsilon}$. Тогда $a^{-\epsilon} a^{\epsilon}$ входит в $\mathcal{L}\left(\mathrm{U}^{\prime} \mathrm{E}\right)$. Но это невозможно, так как $\mathcal{L}\left(\mathrm{U}^{\prime} \mathrm{E}\right)-$ подслово слова $\mathcal{L}\left(\mathrm{U}_{1} \mathrm{EU}_{2}\right) \in \mathcal{R}_{\alpha-1} \subseteq \mathcal{R}_{0}$.

Случай 2: $\rho(A)>0$. Пусть $\mathrm{P}$ и Е начинаются соответственно с $\mathrm{K} \in \ni{ }_{\rho(A)}\left(\mathrm{P}, q_{2}\right)$ и $\mathrm{L} \in$ Эл $\rho(A)\left(\mathrm{E}, q_{1}\right)$, где ЭСогл $\Omega_{(A)}(\mathrm{K}, \mathrm{L})$. В силу леммы 7.6 пути $\mathrm{K}, \mathbf{s}_{A, \mathrm{P}} \mathrm{K}$ и $\mathbf{s}_{A, \mathrm{P}}^{2} \mathrm{~K}$ содержатся в $\mathrm{P}^{\prime}$. Тогда в силу леммы 6.7 и индуктивного предположения 11.23 найдется $\mathrm{L}^{\prime} \in \ni_{\rho(A)}\left(\mathrm{U}^{\prime-1}, q_{2}-h\right)$ такой, что $Э \operatorname{Coz}_{\rho(A)}\left(\mathbf{s}_{A, \mathrm{P}} \mathrm{K}, \mathrm{L}^{\prime}\right)$. Так как ЭСогл $\rho(A)\left(\mathrm{L}^{\prime}, \mathrm{L}^{\prime-1}\right)$ в силу леммы 8.42 , то мы получили $Э \operatorname{Cozл}_{\rho(A)}\left(\mathbf{s}_{A, \mathrm{P}} \mathrm{K}, \mathrm{L}^{\prime-1}\right)$ и $\mathrm{L}^{\prime-1} \ll \mathrm{U}_{1} \mathrm{EU}_{2} \mathrm{~L}$. Но это невозможно в силу индуктивного предположения 11.27 (случай (i)), где в качестве $X$ и $Y$ нуно взять соответственно $U_{1} E_{2}$ и максимальный из путей $\mathrm{P}_{\text {и }} \mathrm{P}_{1} \mathrm{~V}_{1}$.

8.44. ПрЕДЛОЖЕНИЕ. (i) Если $A, B \in \mathcal{E}_{\alpha}$, слова $P \in \Pi е p(A, t) u Q \in \Pi е p(B)$ близки в ранге $\alpha-1$ и $t \geqslant p$, mо $|Q|>(t-44)|B|$.

(ii) Если $B \in \mathcal{E}_{\alpha}$, слова $X \in \mathcal{R}_{\alpha-1} u Q \in$ Пер(В) близки в ранге $\alpha-1$ и в слово $X$ входит әлементарная $t$-степень ранга $\alpha$ при $t \geqslant p+34$, то $|Q|>(t-78)|B|$.

ДокаЗАтельство. (i) Рассмотрим близкие в ранге $\alpha-1$ пути $\mathrm{P} \in$ Пер $\left(\Gamma_{\alpha-1}, A, t\right)$ и $\mathrm{Q} \in \Pi_{\mathrm{ep}}\left(\Gamma_{\alpha-1}, B\right)$ с $\mathcal{L}(\mathrm{P})$ 으 $P$ и $\mathcal{L}(\mathrm{Q})$ 드 $Q$. Допустим, $|Q| \leqslant(t-44)|B|$. Пусть $\overline{\mathrm{Q}} \in \Pi_{\mathrm{Nep}}\left(\Gamma_{\alpha-1}, B, 2\right)-$ произвольньй путь, содержащий $Q$ в качестве подпути. По предложению 8.36 имеем $\operatorname{Coгл}(\mathrm{P}, \overline{\mathrm{Q}})$. По лемме 8.4 имеем $\mathrm{K}, \mathbf{s}_{A, \mathrm{P}} \mathrm{K}, \ldots, \mathbf{s}_{A, \mathrm{P}}^{t-2} \mathrm{~K} \in$ Эл $_{\rho(A)}\left(\mathrm{P}, q_{2}\right)$ для некоторого $\mathrm{K}$, где $|\mathrm{K}| \leqslant|A|$. Используя предложение 7.7 и лем-

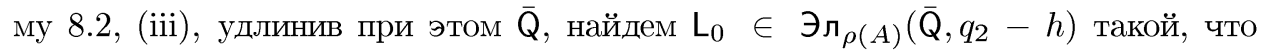
$\vartheta \operatorname{Coz}_{\rho(A)}\left(\mathbf{s}_{A, \mathrm{P}} \mathrm{K}, \mathrm{L}_{0}\right)$. По лемме 7.21 имеем $Э \operatorname{Coz}_{\rho(A)}\left(\mathbf{s}_{A, \mathrm{P}}^{i} \mathrm{~K}, \mathbf{s}_{B, \overline{\mathrm{Q}}}^{i} \mathrm{~L}\right)$ при всех $i$, где $\mathbf{L} \rightleftharpoons \mathbf{s}_{B, \bar{Q}}^{-1} \mathrm{~L}_{0}$. Снова удлинив $\overline{\mathrm{Q}}$, можно считать, что пути $\mathbf{s}_{B, \overline{\mathrm{Q}}}^{i} \mathbf{L}$ содержатся в $\overline{\mathrm{Q}}$ при $i=0,1, \ldots, t-2$, а укоротив $\mathrm{L}$ в силу леммы 7.6 , вместо $\mathrm{L} \in \ni \rho(A)\left(\overline{\mathrm{Q}}, q_{2}-h\right)$ получим $\mathrm{L} \in$ Эл $_{\rho(A)}\left(\overline{\mathrm{Q}}, q_{2}-h-9\right)$ и $|\mathrm{L}| \leqslant|B|$. Пусть $\mathrm{P}=\mathrm{U}_{0} \mathrm{P}_{1} \mathrm{U}_{1} \mathrm{P}_{2} \mathrm{U}_{2}$ и $\overline{\mathrm{Q}}=\mathrm{V}_{0} \mathrm{E}_{1} \mathrm{~V}_{1} \mathrm{E}_{2} \mathrm{~V}_{2}$, где $\mathrm{P}_{1} \rightleftharpoons \mathrm{K} \cup_{\mathrm{P}} \mathbf{s}_{A, \mathrm{P}}^{20} \mathrm{~K}, \mathrm{P}_{2} \rightleftharpoons \mathbf{s}_{A, \mathrm{P}}^{t-22} \mathrm{~K} \cup \mathrm{P} \mathbf{s}_{A, \mathrm{P}}^{t-2} \mathrm{~K}, \mathrm{E}_{1} \rightleftharpoons \mathrm{L} \cup_{\overline{\mathrm{Q}}} \mathbf{s}_{B, \overline{\mathrm{Q}}}^{20} \mathrm{~L}$ и $\mathrm{E}_{2} \rightleftharpoons \mathrm{s}_{B, \overline{\mathrm{Q}}}^{t-22} \mathrm{~L} \cup_{\overline{\mathrm{Q}}} \mathbf{s}_{B, \overline{\mathrm{Q}}}^{t-2} \mathrm{~L}$. Согласно определению $5.16 \mathrm{E}_{i} \in \Pi$ Пер $\alpha(A)$ и $\mathrm{P}_{i}-$ периодическая основа для $\mathrm{E}_{i}$. Так как $|\mathrm{Q}| \leqslant(t-44)|B|$ и $\left|\mathrm{V}_{1}\right| \geqslant\left|\mathbf{s}_{B, \bar{Q}}^{21} \mathrm{~L} \cup \mathrm{P} \mathbf{s}_{B, \overline{\mathrm{Q}}}^{t-23} \mathrm{~L}\right| \geqslant(t-$ $44)|B|$, то либо вершина $\iota(\mathrm{Q})$ лежит на пути $\mathrm{V}_{1} \mathrm{E}_{2} \mathrm{~V}_{2}$, либо вершина $\tau(\mathrm{Q})$ лежит 
на пути $\mathrm{V}_{0} \mathrm{E}_{1} \mathrm{~V}_{1}$. Но в каждом из этих случаев мы получаем противоречие с леммой 8.43 соответственно при $\left(\mathrm{U}_{1} \mathrm{EU}_{2}, \mathrm{E}, \mathrm{P}, \mathrm{P}_{2}, \mathrm{~V}_{2}\right):=\left(\mathrm{E}_{1} \mathrm{~V}_{1} \mathrm{E}_{2} \mathrm{~V}_{2}, \mathrm{E}_{1}, \mathrm{P}_{1}, \mathrm{P}_{1}, \mathrm{U}_{0}\right)$ и $\left(\mathrm{U}_{1} \mathrm{EU}_{2}, \mathrm{E}, \mathrm{P}, \mathrm{P}_{1}, \mathrm{~V}_{1}\right):=\left(\mathrm{V}_{0} \mathrm{E}_{1} \mathrm{~V}_{1} \mathrm{E}_{2}, \mathrm{E}_{2}, \mathrm{P}_{2}, \mathrm{P}_{2}, \mathrm{U}_{2}\right)$. Следовательно, $|Q|>(t-$ $44)|B|$.

(ii) Пусть $\mathrm{X}$ и $\mathrm{Q}$ - близкие в ранге $\alpha-1$ пути на графе $\Gamma_{\alpha-1}$ с $\mathcal{L}(\mathrm{X})$ 으 $X$ и $\mathcal{L}(\mathrm{Q})$ 으 $Q$. По условию $\mathrm{X}$ содержит в качестве подпути путь $\mathrm{E} \in \Pi$ Пер $\alpha(A, t)$, где $A \in \mathcal{E}_{\alpha}$ и $t \geqslant p+34$. В силу предложения 8.26 имеем $\mathrm{F} \in \Pi$ Пер $\alpha(A, t-34)$ для некоторого подпути пути $\mathrm{Q}$. Пусть $\mathrm{P} \in \Pi$ Пер $\left(\Gamma_{\alpha-1}, A, t-34\right)$ - периодическая основа для $\mathrm{F}$. Тогда в силу леммы 8.2 , (ii) и доказанного утверждения (i) $|Q| \geqslant|\mathrm{F}| \geqslant(t-$ 78) $|B|$.

8.45. ПРЕДЛОЖЕНИЕ $(\alpha \geqslant 0)$. Если $\beta \geqslant \alpha, \mathrm{E}, \mathrm{F} \in \ni \boldsymbol{л}_{\alpha}\left(\Gamma_{\beta}\right)$ u $Э \operatorname{Coгл}_{\alpha}(\mathrm{E}, \mathrm{F})$, то пути $\mathrm{E}$ и $\mathrm{F}$ близки в ранге $\alpha$.

ДоКАЗАТЕЛЬСТВО. При $\alpha=0$ утверждение вытекает из определений 5.14 и 7.4. Пусть $\alpha \geqslant 1$. В силу полной аналогии ограничимся доказательством утверждения о том, что начальные вершины $\mathbf{g} \rightleftharpoons \iota(\mathrm{E})$ и $\mathbf{h} \rightleftharpoons \iota(\mathrm{F})$ путей $\mathrm{E}$ и $\mathrm{F}$ близки в ранге $\alpha$. Согласно определению 5.20 сушествуют прообразы $\bar{E}$ и $\bar{F}$ на графе $\Gamma_{\alpha-1}$ путей $\mathrm{E}$ и $\mathrm{F}$ такие, что ПСогл $\alpha\left(\overline{\mathrm{E}}, \overline{\mathrm{F}}^{\epsilon}\right), \epsilon= \pm 1$. Тогда $\mathbf{g}=\pi_{\alpha-1, \beta}(\overline{\mathbf{g}})$ и $\mathbf{h}=\pi_{\alpha-1, \beta}(\overline{\mathbf{h}})$, где $\overline{\mathbf{g}} \rightleftharpoons \iota(\overline{\mathrm{E}})$ и $\overline{\mathbf{h}} \rightleftharpoons \iota(\overline{\mathrm{F}})$. Пусть $\mathrm{P} \in \Pi_{\text {ер }}\left(\Gamma_{\alpha-1}, A, 2\right)$ и $\mathrm{Q} \in \Pi_{\text {ер }}\left(\Gamma_{\alpha-1}, B, 2\right)-$ периодические основы для $\overline{\mathrm{E}}$ и $\overline{\mathrm{F}}$ соответственно, где $A, B \in \mathcal{E}_{\alpha}$ и $\operatorname{Coгл}\left(\mathrm{P}, \mathrm{Q}^{\epsilon}\right)$. В силу леммы 8.2 , (ii) вершины $\overline{\mathbf{g}}$ и $\iota(\mathrm{P})$ близки в ранге $\alpha-1$, т.е. соединены неко-

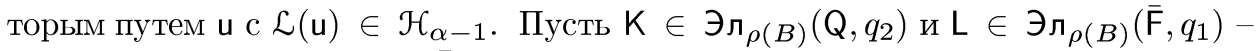
начала соответственно $\mathrm{Q}$ и $\overline{\mathrm{F}}$, где $Э \operatorname{\vartheta oz}_{\rho(B)}(\mathrm{K}, \mathrm{L})$. По предложению 7.7 найдем строго близкие в ранге $\rho(B)$ периодические продолжения $\widetilde{\mathrm{Q}}$ и $\widetilde{\mathrm{P}}$ для $\mathrm{Q}^{\epsilon}$ и $\mathrm{P} . \mathrm{B}$ силу леммы 8.2 , (iii) для некоторого $\mathrm{M} \in \ni_{\rho(B)}\left(\widetilde{\mathrm{P}}, q_{1}\right)$ выполнено $Э \operatorname{Coгл}_{\rho(B)}\left(\mathrm{K}^{\epsilon}, \mathrm{M}\right)$. Тогда имеем ЭСогл $\rho(B)\left(\mathrm{M}^{\epsilon}, \mathrm{L}\right)$, и по индуктивному предположению вершины $\iota\left(\mathrm{M}^{\epsilon}\right)$ и $\iota(\mathrm{L})=\overline{\mathbf{h}}$ соединены некоторым путем $\mathrm{v}$ c $\mathcal{L}(\mathrm{v}) \in \mathcal{H}_{\rho(B)} \subseteq \mathcal{H}_{\alpha-1}$. Так как вершины $\iota(\mathrm{P})$ и $\iota\left(\mathrm{M}^{\epsilon}\right)$ лежат на $\widetilde{\mathrm{P}}$, то $\iota(\mathrm{P})$ и $\iota\left(\mathrm{M}^{\epsilon}\right)$ соединены путем $\mathrm{R}$, входяшим в качестве подпути в $\widetilde{\mathrm{P}}$ или в $\widetilde{\mathrm{P}}^{-1}$. Мы имеем, таким образом, путь $\mathrm{X} \rightleftharpoons \mathrm{uRv}$, соединяющий $\overline{\mathbf{g}}$ и $\overline{\mathbf{h}}$, где $\mathcal{L}(\mathrm{u}), \mathcal{L}(\mathrm{v}) \in \mathcal{H}_{\alpha-1}$ и $\mathcal{L}(\mathrm{R}) \in \Pi е p(A) \cup \Pi е p\left(A^{-1}\right)$. Тогда путь $\pi_{\alpha-1, \beta}(\mathrm{X})$ соединяет вершины $\mathbf{g}$ и $\mathbf{h}$. Так как $A^{n} \stackrel{\beta}{=} 1$, то $\mathcal{L}(\mathrm{R}) \stackrel{\beta}{=} R$ для некоторого $R \in \Pi e p(A) \cup \Pi е p\left(A^{-1}\right)$ при $|R|<n|A|$. В таком случае $\mathbf{g}$ и $\mathbf{h}$ соединены также путем $\mathrm{X}^{\prime}$ с $\mathcal{L}\left(\mathrm{X}^{\prime}\right)$ 드 $\mathcal{L}(\mathrm{u}) R \mathcal{L}(\mathrm{v}) \in \mathcal{H}_{\alpha}$, что и требовалось доказать.

8.46. ПРЕДЛОЖЕНИЕ. Если $\mathrm{E} \in \Pi$ Пер $\alpha(A, 44) u \mathrm{~F} \in \Pi \Pi е p_{\alpha}(B, 44)-n о д n y т u$ nутu $\mathrm{X} c \mathcal{L}(\mathrm{X}) \in \mathcal{R}_{\alpha-1}$, mo $\neg \Pi C o л_{\alpha}\left(\mathrm{E}, \mathrm{F}^{-1}\right)$.

ДоКАЗАТЕЛЬСТво. Допустим, ПСогл $\Omega_{\alpha}\left(\mathrm{E}, \mathrm{F}^{-1}\right)$. Тогда в силу леммы 7.22 и предложения 8.35 найдется подпуть $\mathrm{G} \in$ ППер $\alpha\left(A^{-1}, 8\right)$ пути $\mathrm{F}$ такой, что $\Pi \operatorname{Coг}_{\alpha}(\mathrm{F}, \mathrm{G})$, и, следовательно, ПСогл $\alpha\left(\mathrm{E}, \mathrm{G}^{-1}\right)$. В силу предложения 8.10, (i) найдутся периодические основы $\mathrm{P} \in \Pi$ ер $\left(\Gamma_{\alpha-1}, A, 2\right)$ и $\mathrm{Q} \in \Pi_{\mathrm{ep}}\left(\Gamma_{\alpha-1}, A^{-1}, 2\right)$ для $\mathrm{E}$ и $\mathrm{F}$ такие, что $\mathrm{P}$ и $\mathrm{Q}^{-1}$ имеют обшее периодическое продолжение $\mathrm{R}$, и мы приходим к противоречию с определением 5.16 и индуктивным предположением 11.27 при $X:=X$ и $Y:=R$. 


\section{§ 9. Диаграммы с разбиением границы}

В этом параграфе мы рассмотрим некоторый специальный класс диаграмм ван Кампена над заданиями групп $\mathbf{B}_{\beta}$, а также введем ряд понятий, которые будут использоваться для анализа таких диаграмм в $\S 10$. Отметим, что утверждения этого параграфа носят обший характер и не используют совместную индукцию.

Напомним, что мы имеем дело с бесконечной последовательностью групп $\mathbf{B}_{\beta}$, определямых заданиями

$$
\mathcal{P}_{\beta} \rightleftharpoons\left\langle\mathcal{A} \mid A^{n}=1 \quad\left(A \in \mathcal{E}_{1} \cup \mathcal{E}_{2} \cup \ldots \cup \mathcal{E}_{\beta}\right)\right\rangle .
$$

Произвольную диаграмму над заданием $\mathcal{P}_{\beta}$ будем называть диаграммой ранга $\beta$.

Очевидно, всякая диаграмма ранга $\beta$ является диаграммой ранга $\gamma$ при любом $\gamma>\beta$.

Если А - область диаграммы $\Delta$ ранга $\beta$, то согласно определению из $\S 4$ имеем либо $\mathcal{L}(\delta \mathrm{A})$ 으 1 , либо $\mathcal{L}(\delta \mathrm{A})$ 으 $a^{\epsilon} a^{-\epsilon}$, где $a^{\epsilon}$ - буква алфавита $\mathcal{A}^{ \pm 1}$, либо $\mathcal{L}(\delta \mathrm{A}) \approx A^{n}$ для некоторого $A \in \mathcal{E}_{\gamma}$ при $\gamma \leqslant \beta$. В первых двух случаях А назовем областью ранга 0 , а в случае, если $\mathcal{L}(\delta \mathrm{A}) \approx A^{n}$ при $A \in \mathcal{E}_{\gamma},-$ областью ранга $\gamma$ диаграммы $\Delta$. Будем формально считать, что каждой области А диаграммы ранга $\beta$ приписано единственное значение ее ранга $\gamma$. (Из лемм 6.7 и 5.10 следует, что для любых $\beta_{1}<\beta_{2} \leqslant \alpha$ если $A \in \mathcal{E}_{\beta_{2}}$, то $[A]_{\beta_{1}}^{n} \neq 1$. Так как $[A]_{\beta_{1}}^{n}=1$ при $A \in \mathcal{E}_{\beta_{1}}$, то на самом деле ранг каждой области диаграммы ранга, не превосходящего $\alpha$, определяется единственным образом.) Множество всех областей ранга $\gamma$ диаграммы $\Delta$ обозначим через $\mathbf{R}_{\gamma}(\Delta)$.

Если $\Delta$ - диаграмма ранга $\beta \geqslant 1$, то через $\Delta^{\beta-1}$ будем обозначать поддиаграмму ранга $\beta-1$ диаграммы $\Delta$, полученную из $\Delta$ удалением всех областей ранга $\beta$. Очевидно, имеет место формула

$$
\chi\left(\Delta^{\beta-1}\right)=\chi(\Delta)-\# \mathbf{R}_{\beta}(\Delta) .
$$

Диаграмму $\Delta$ ранга $\beta$ будем называть неособой в ранге $\beta-1$, если $\Delta^{\beta-1}$ - неособая диаграмма. Очевидно, если $\Delta$ - неособая в ранге $\beta-1$ диаграмма ранга $\beta$, то сама диаграмма $\Delta$ является неособой.

Мы будем рассматривать диаграммы $\Delta$ ранга $\beta$, для каждого граничного цикла которых фиксировано разложение в виде циклического произведения $\mathrm{X}_{1} \cdot \mathrm{X}_{2} \cdot \ldots \cdot \mathrm{X}_{r}$ непустых путей $\mathrm{X}_{i}$, разбитых на несколько типов. Сомножители $\mathrm{X}_{i}$ будем называть участками границы диаграммы $\Delta$. В качестве участков границы допускаются и сами граничные циклы диаграммы $\Delta$, в этом случае будем говорить о ииклических участках границы диаграммы $\Delta$. Введем два специальных типа диаграмм ранга $\beta$ с разбиением границы указанного вида.

9.1. ОПРЕДЕЛЕНИЕ. Диаграммой ранга $\beta$ с разбиением граниџы типа $A$ (или, сокращенно, $A$-диаграммой ранга $\beta$ ) назовем неособую диаграмму $\Delta$ ранга $\beta$, участки гранищы которой разбиты на два непересекающихся подмножества $x_{\beta}(\Delta)$ и $y_{\beta}(\Delta)$ так, что выполнены следуюшие условия:

Серия математическая, №3 
(i) каждый граничный цикл С диаграммы $\Delta$ либо принадлежит $\mathcal{X}_{\beta}(\Delta)$ либо есть циклическое произведение чередующихся путей из множеств $X_{\beta}(\Delta)$ и $y_{\beta}(\Delta)$;

(ii) если $S \in X_{\beta}(\Delta)$, то слово $\mathcal{L}(\mathrm{S})$ приведено в ранге $\beta$ или циклически приведено в ранге $\beta$ соответственно в случаях, когда $S$ - нециклический или циклический участок границы диаграммы $\Delta$ (напомним, что согласно определению 5.7 слово $X$ циклически приведено в ранге $\beta$ тогда и только тогда, тогда любой циклический сдвиг слова $X$ есть приведенное в ранге $\beta$ слово);

(iii) для любого $\mathrm{u} \in \mathrm{y}_{\beta}(\Delta)$ выполнено $\mathcal{L}(\mathrm{u}) \in \mathcal{H}_{\beta}$.

9.2. ОПРЕДЕЛЕНИЕ. Диаграммой ранга $\beta \geqslant 1$ с разбиением границы типа $B$ (сокращенно, $B$-диаграммой ранга $\beta$ ) назовем неособую в ранге $\beta-1$ диаграмму $\Delta$ ранга $\beta$, участки гранищы которой разбиты на три непересекаюшихся подмножества $X_{\beta}(\Delta), y_{\beta-1}(\Delta)$ и $Z_{\beta}(\Delta)$ так, что выполнены условия:

(i) каждый граничный цикл $C$ диаграммы $\Delta$ либо принадлежит $X_{\beta}(\Delta)$, либо имеет вид $\mathrm{C} \approx \mathrm{S}_{1} \mathrm{u}_{1} \mathrm{~S}_{2} \mathrm{u}_{2} \ldots \mathrm{S}_{r} \mathrm{u}_{r}$, где $\mathrm{S}_{i} \in \mathcal{X}_{\beta}(\Delta) \cup z_{\beta}(\Delta), \mathrm{u}_{i} \in \mathrm{y}_{\beta-1}(\Delta)$ и никакие $\mathrm{S}_{i}, \mathrm{~S}_{i+1}(i \bmod r)$ не лежат одновременно в $z_{\beta}(\Delta)$;

(ii) если $S \in X_{\beta}(\Delta)$, то слово $\mathcal{L}(\mathrm{S})$ приведено в ранге $\beta$ или циклически приведено в ранге $\beta$ соответственно в случаях, когда $S$ - нециклический или циклический участок гранищы диаграммы $\Delta$;

(iii) для любого $\mathrm{u} \in \mathrm{y}_{\beta-1}(\Delta)$ выполнено $\mathcal{L}(\mathrm{u}) \in \mathcal{H}_{\beta-1}$;

(iv) для любого $S \in Z_{\beta}(\Delta)$ слово $\mathcal{L}(\mathrm{S})$ есть подслово слова $A^{n}$ при некотором $A \in \mathcal{E}_{\beta}$.

Участки гранищы $S \in X_{\beta}(\Delta)$ будем называть главными участками гранииы ранга $\beta A$-диаграммы или $B$-диаграммы $\Delta$ ранга $\beta$. Заметим, что только главные участки границы ранга $\beta A$-диаграммы или $B$-диаграммы $\Delta$ ранга $\beta$ могут быть циклическими участками ее границы.

Из определений 9.1 и 9.2 видно, что задание одного из множеств $\mathcal{X}_{\beta}(\Delta)$ или $y_{\beta}(\Delta)$ полностью определяет разбиение границы типа $A$ на диаграмме $\Delta$, а задание любых двух из множеств $X_{\beta}(\Delta), y_{\beta-1}(\Delta)$ или $z_{\beta}(\Delta)$ полностью определяет разбиение гранищы типа $B$.

В дальнейшем $A$-диаграммы и $B$-диаграммы ранга $\beta$ естественным образом будут возникать при рассмотрении соотношений в группе $\mathbf{B}_{\beta}$. Приведем типичный пример. Пусть $X, Y \in \mathcal{R}_{\beta}, u, v \in \mathcal{H}_{\beta}$, слово $Z$ циклически приведено в ранге $\beta$ и слова $X u Y v$ и $Z$ сопряжены в группе $\mathbf{B}_{\beta}$. По предложению 4.1 сушествует кольцевая диаграмма $\Delta$ ранга $\beta$ с граничными циклами $\mathrm{XuYv}$ и $Z^{-1}$, где метки слов $\mathrm{X}, \mathrm{u}$, $\mathrm{Y}, \mathrm{Y}$ и Z равны соответственно словам $X, u, Y, v$ и $Z$. В силу п. 4.5 можно считать, что пути $\mathrm{X}, \mathrm{u}, \mathrm{Y}$ и $\mathrm{Y}$ непусты. Тогда согласно определению $9.1 \Delta$ есть $A$-диаграмма ранга $\beta$, для которой $X_{\beta}(\Delta) \rightleftharpoons\left\{\mathrm{X}, \mathrm{Y}, \mathrm{Z}^{-1}\right\}$ и $\mathrm{y}_{\beta}(\Delta) \rightleftharpoons\{\mathrm{u}, \mathrm{v}\}$. Аналогичным образом будут вводиться в рассмотрение $B$-диаграммы ранга $\beta$, при этом нужно лишь дополнительно произвести окаймление границы каждой области ранга $\beta$, как описано в п. 4.8, чтобы диаграмма стала неособой в ранге $\beta-1$.

9.3. Каждую $B$-диаграмму ранга $\beta$ можно рассматривать как $A$-диаграмму ранга $\beta$ с тем же самым множеством $\chi_{\beta}(\Delta)$ и множеством $y_{\beta}(\Delta)$, состоящим из 
путей $\mathrm{u} \in y_{\beta-1}(\Delta)$, для которых соседние участки гранищы из $x_{\beta}(\Delta) \cup z_{\beta}(\Delta)$ лежат в $X_{\beta}(\Delta)$, и путей вида $\mathrm{vPw}$, где $\mathrm{v}, \mathrm{w} \in \mathcal{Y}_{\beta-1}(\Delta)$ и $\mathrm{P} \in z_{\beta}(\Delta)$ (тот факт, что метки этих путей лежат в $\mathcal{H}_{\beta}$, вытекает из включения $\mathcal{H}_{\beta-1} \subseteq \mathcal{H}_{\beta}$ и определения 5.8). В дальнейшем каждая $B$-диаграмма $\Delta$ ранга $\beta$ будет одновременно рассматриваться и как $A$-диаграмма ранга $\beta$ с указанным разбиением грани$u$ ub. В частности, обозначение $y_{\beta}(\Delta)$ теперь имеет смысл и в случае, когда $\Delta$ есть $B$-диаграмма ранга $\beta$.

Обратно, пусть $\Delta$ есть $A$-диаграмма ранга $\beta \geqslant 1$ и $y_{\beta}(\Delta)=\left\{\mathrm{u}_{1}, \mathrm{u}_{2}, \ldots, \mathrm{u}_{k}\right\}$. Предположим, для каждого слова $\mathcal{L}\left(\mathrm{u}_{i}\right)$ фиксировано некоторое разложение вида $\mathcal{L}\left(\mathbf{u}_{i}\right)$ 으 $v_{i} P_{i} w_{i}$, где $v_{i}, w_{i} \in \mathcal{H}_{\beta-1}$ и $\mathcal{L}\left(\mathrm{P}_{i}\right)$ есть подслово слова $A_{i}^{n}$ для некоторого $A_{i} \in \mathcal{E}_{\beta}$ (хотя бы одно такое разложение слова $\mathcal{L}\left(\mathrm{u}_{i}\right)$ существует в силу условия (iii) определения 9.1). Тогда, выполнив измельчение диаграммы $\Delta$ согласно п. 4.5, можно считать, что каждый путь $\mathrm{u}_{i}$ имеет вид $\mathrm{u}_{i}=\mathrm{v}_{i} \mathrm{P}_{i} \mathrm{w}_{i}$, где $\mathrm{v}_{i}, \mathrm{P}_{i}$ и $\mathrm{w}_{i}$ - непустые пути с метками соответственно $v_{i}, P_{i}$ и $w_{i}$. Кроме того, произведя окаймление границы каждой области ранга $\beta$ диаграммы $\Delta$, как описано в п. 4.8 , можно считать, что $\Delta-$ неособая в ранге $\beta-1$ диаграмма. Полагая $y_{\beta-1}(\Delta) \rightleftharpoons\left\{\mathrm{v}_{1}, \mathrm{w}_{1}, \ldots, \mathrm{v}_{k}, \mathrm{w}_{k}\right\}$ и $z_{\beta}(\Delta) \rightleftharpoons\left\{\mathrm{P}_{1}, \mathrm{P}_{2}, \ldots, \mathrm{P}_{k}\right\}$, мы удовлетворим всем условиям определения 9.2 , и, таким образом, $\Delta$ является $B$-диаграммой ранга $\beta$.

Так как Пер $(A) \subseteq \mathcal{R}_{\beta}$ при $A \in \mathcal{E}_{\beta}$ в силу определения 5.2, то для любой $B$-диаграммы $\Delta$ ранга $\beta$ ее поддиаграмму $\Delta^{\beta-1}$ можно рассматривать как $A$-диаграмму ранга $\beta-1$, для которой

$$
\begin{aligned}
& x_{\beta-1}\left(\Delta^{\beta-1}\right) \rightleftharpoons x_{\beta}(\Delta) \cup z_{\beta}(\Delta) \cup\left\{\delta \mathrm{A} \mid \mathrm{A} \in \mathbf{R}_{\beta}(\Delta)\right\}, \\
& y_{\beta-1}\left(\Delta^{\beta-1}\right) \rightleftharpoons y_{\beta-1}(\Delta) .
\end{aligned}
$$

В частности, имеем

$$
\# y_{\beta}(\Delta) \leqslant \# y_{\beta-1}\left(\Delta^{\beta-1}\right) \leqslant 2 \# y_{\beta}(\Delta) .
$$

9.4. ОПРЕДЕЛЕНИЕ. Пусть $\Delta$ и $\Delta^{\prime}$ - диаграммы ранга $\beta$ и $\gamma \leqslant \beta$. Будем говорить, что $\Delta^{\prime}$ получена из $\Delta$ внутренним преобразованием ранга $\gamma$, если имеет место один из следующих трех случаев:

(i) $\Delta^{\prime}$ есть результат измельчения диаграммы $\Delta$;

(ii) $\Delta^{\prime}$ есть результат замены дисковой поддиаграммы $\Theta$ ранга $\gamma$ диаграммы $\Delta$ на некоторую дисковую диаграмму $\Theta^{\prime}$ ранга $\gamma$;

(iii) $\Delta^{\prime}$ есть результат замены кольцевой поддиаграммы $\Theta$ ранга $\gamma$ диаграммы $\Delta$ на некоторую кольцевую диаграмму $\Theta^{\prime}$ ранга $\gamma$, где поддиаграмма $\Theta$ удовлетворяет следующему ограничению: для каждого из двух ее граничных циклов $C_{1}$ и $C_{2}$ элемент $\left[\mathcal{L}\left(\mathrm{C}_{i}\right)\right]_{\gamma}$ имеет конечный порядок.

Из определения непосредственно вытекает, что внутренние преобразования ранга $\gamma$ являются внутренними преобразованиями ранга $\gamma^{\prime}$ при $\gamma^{\prime}>\gamma$. 
При внутренних преобразованиях диаграмм естественным образом наследуется разбиение границы диаграммы (см. $\S 4)$. Таким образом, мы можем рассматривать внутренние преобразования ранга $\gamma A$-диаграмм или $B$-диаграмм ранга $\beta$.

Внутренние преобразования ранга $\gamma$ не изменяют набор слов, являюшихся метками участков границы $A$-диаграммы или $B$-диаграммы $\Delta$ ранга $\beta$. В большинстве случаев при рассмотрении $A$-диаграмм или $B$-диаграмм ранга $\beta$ нас будет интересовать только указанный набор слов, и в этих случаях от диаграммы $\Delta$ всегда можно перейти к диаграмме, полученной из нее некоторым внутренним преобразованием ранга $\beta$.

Заметим также, что при внутренних преобразованиях ранга $\gamma \leqslant \beta-1$ не изменяется число областей ранга $\beta$ диаграммы $\Delta$.

9.5. ЗАмЕЧАНИЕ. (i) Если $\Theta$ - подлиаграмма диаграммы $\Delta$, то любую последовательность $\Theta \rightarrow \cdots \rightarrow \Theta^{*}$ внутренних преобразований ранга $\gamma$ диаграммы $\Theta$ естественным образом можно рассматривать как последовательность $\Delta \rightarrow \cdots \rightarrow \Delta^{*}$ внутренних преобразований ранга $\gamma$ диаграммы $\Delta$. При этом, очевидно, диаграмма $\Delta^{*}-\Theta^{*}$ есть результат измельчения диаграммы $\Delta-\Theta$ (на самом деле $\Delta^{*}-\Theta^{*}$ получается из $\Delta-\Theta$ добавлением некоторого множества вершин, лежащих на границе $\Theta$ ).

(ii) Если $\Delta \rightarrow \cdots \rightarrow \Delta^{*}$ - последовательность внутренних преобразований ранга $\gamma$ и диаграмма $\Delta^{\prime}$ есть результат измельчения диаграммы $\Delta$, то, как легко следует из леммы 4.4 и 4.7 , с точностью до изотопной деформации диаграмм $\Delta^{*}$ и $\Delta^{\prime}$, оставляющей неподвижной границу диаграммы $\Delta$, сушествует диаграмма $\Delta^{* \prime}$, которая одновременно является результатом измельчения диаграммы $\Delta^{*}$ и результатом применения некоторой последовательности внутренних преобразований ранга $\gamma$ к диаграмме $\Delta^{\prime}$.

9.6. ОПрЕДЕЛЕНИЕ. Пусть $\Delta-B$-диаграмма ранга $\beta$, и пусть $S \in z_{\beta}(\Delta)$, где $\mathcal{L}(\mathrm{S})$ - подслово слова $A^{n}$ при $A \in \mathcal{E}_{\beta}$. Предположим, что $|\mathcal{L}(\mathrm{S})| \geqslant 2|A|$. Рассмотрим диаграмму $\Delta^{\prime}$ ранга $\beta$, полученную добавлением к $\Delta$ новой области А ранга $\beta$, для которой $\partial \mathrm{A} \cap \Delta=\operatorname{supp} \mathrm{S}$ и $\delta \mathrm{A} \approx \mathrm{SS}^{\prime-1}$, где слово $\mathcal{L}\left(\mathrm{S}^{\prime}\right)$ удовлетворяет равенству $\mathcal{L}(\mathrm{S})\left(\mathcal{L}\left(\mathrm{S}^{\prime}\right)\right)^{-1} \approx A^{n}$ (см. рис. 10$)$.

Определим на $\Delta^{\prime}$ разбиение гранищы типа $B$, полагая

$$
x_{\beta}\left(\Delta^{\prime}\right) \rightleftharpoons x_{\beta}(\Delta), \quad y_{\beta-1}\left(\Delta^{\prime}\right) \rightleftharpoons y_{\beta-1}(\Delta), \quad z_{\beta}\left(\Delta^{\prime}\right) \rightleftharpoons\left(z_{\beta}(\Delta) \backslash\{\mathrm{S}\}\right) \cup\left\{\mathrm{S}^{\prime}\right\} .
$$

$B$-диаграмму $\Delta^{\prime}$ ранга $\beta$ будем называть результатом добавления области ранга $\beta \kappa$ диаграмме $\Delta$ вдоль участка гранищь S.

Условимся считать, что эта операция неприменима, если для некоторого $A \in \mathcal{E}_{\beta}$ выполнено $\mathcal{L}(\mathrm{S}) \in \Pi$ ер $(A)$ и $|\mathcal{L}(\mathrm{S})|<2|A|$.

9.7. ЗАмЕчАНИЕ. В отличие от внутренних преобразований ранга $\gamma$, операция добавления области вдоль $\mathrm{S}$ изменяет слово $\mathcal{L}(\mathrm{S})$. При этом на новой $B$-диаграмме $\Delta^{\prime}$ ранга $\beta$ вместо $S$ возникает новый участок гранищы $S^{\prime}$ такой, что 


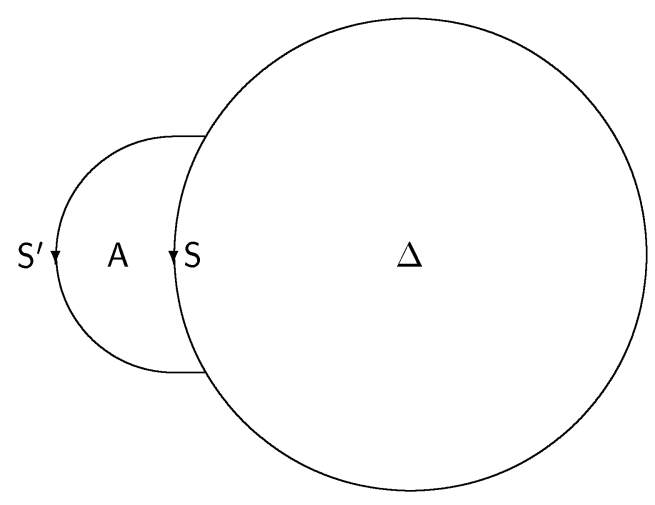

Рис. 10

$\mathcal{L}(\mathrm{S})\left(\mathcal{L}\left(\mathrm{S}^{\prime}\right)\right)^{-1} \approx A^{n}$, где $A \in \mathcal{E}_{\beta}$. Так как по условию определения 9.6 имеем $|\mathcal{L}(\mathrm{S})| \geqslant 2|A|$, то из леммы 2.1 , (ii), (iii) следует, что слово $\mathcal{L}\left(\mathrm{S}^{\prime}\right)$ определено единственным образом. Если теперь к $\Delta^{\prime}$ снова применима операция присоединения области вдоль $\mathrm{S}^{\prime}$ (для этого необходимо и достаточно, чтобы выполнялось неравенство $\left.\left|\mathcal{L}\left(\mathrm{S}^{\prime}\right)\right| \geqslant 2|A|\right)$, то легко видеть, что для нового участка границы $\mathrm{S}^{\prime \prime}$ новой диаграммы $\Delta^{\prime \prime}$ будем иметь $\mathcal{L}\left(\mathrm{S}^{\prime \prime}\right)$ 으 $\mathcal{L}(\mathrm{S})$. Отсюда следует, что если $B$-диаграмма $\Delta^{*}$ ранга $\beta$ получена из $B$-диаграммы $\Delta$ ранга $\beta$ некоторой последовательностью преобразований, состоящей из внутренних преобразований ранга $\beta$ и добавлений областей ранга $\beta$, то для каждого участка границы $\mathrm{S} \in \mathcal{Z}_{\beta}(\Delta)$ диаграммы $\Delta$ и соответствуюшего ему участка границы $\mathrm{S}^{*} \in \mathcal{Z}_{\beta}\left(\Delta^{*}\right)$ диаграммы $\Delta^{*}$ имеем либо $\mathcal{L}\left(\mathrm{S}^{*}\right)$ 으 $\mathcal{L}(\mathrm{S})$, либо $\mathcal{L}(\mathrm{S})\left(\mathcal{L}\left(\mathrm{S}^{*}\right)\right)^{-1} \approx A^{n}$, где $|\mathcal{L}(\mathrm{S})| \geqslant 2|A|$. В частности, если для некоторого $A \in \mathcal{E}_{\beta}$ выполнено $\mathcal{L}(\mathrm{S}) \in \Pi е p(A)$ и $|\mathcal{L}(\mathrm{S})|<2|A|$, то $\mathcal{L}\left(\mathrm{S}^{*}\right)$ 드 $\mathcal{L}(\mathrm{S})$.

9.8. ОПРЕДЕЛЕНИЕ. Пусть $\Delta-B$-диаграмма ранга $\beta$.

Будем говорить, что области $\mathrm{A}, \mathrm{B} \in \mathbf{R}_{\beta}(\Delta)$ образуют сократимую пару областей ранга $\beta$, если для некоторой дисковой поддиаграммы $\Theta$ диаграммы $\Delta$ выполнено $\mathbf{R}_{\beta}(\Theta)=\{\mathrm{A}, \mathrm{B}\}$ и $\mathcal{L}(\delta \Theta) \stackrel{\beta=1}{=} 1$.

Область $\mathrm{A} \in \mathbf{R}_{\beta}(\Delta)$ назовем свернутой областью ранга $\beta$, если существует кольцевая поддиаграмма $\Theta$ диаграммы $\Delta$ с граничными циклами $C_{1}$ и $C_{2}$ такая, что $\mathbf{R}_{\beta}(\Theta)=\{\mathrm{A}\}$, слова $\mathcal{L}\left(\mathrm{C}_{1}\right)$ и $\left(\mathcal{L}\left(\mathrm{C}_{2}\right)\right)^{-1}$ сопряжены в группе $\mathbf{B}_{\beta-1}$ и элементы $\left[\mathcal{L}\left(\mathrm{C}_{i}\right)\right]_{\beta-1}(i=1,2)$ имеют конечный порядок.

Пусть $\mathrm{A} \in \mathbf{R}_{\beta}(\Delta), \mathrm{S} \in \mathcal{Z}_{\beta}(\Delta)$ и $\mathcal{L}(\mathrm{S})$ - подслово слова $A^{n}$, где $A \in \mathcal{E}_{\beta}$. Будем говорить, что $\mathrm{A}$ и $\mathrm{S}$ образуют граничную сократимую пару ранга $\beta$, если существует дисковая поддиаграмма $\Theta$ диаграммы $\Delta$ такая, что $\mathbf{R}_{\beta}(\Theta)=\{\mathrm{A}\}, \delta \Theta \approx \mathrm{QR}$ для некоторого подпути $\mathrm{Q}$ пути $\mathrm{S},|\mathcal{L}(\mathrm{Q})| \geqslant 2|A|$ и $\mathcal{L}(\mathrm{R}) \stackrel{\beta=1}{=} X$, где слово $X$ определяется равенством $\mathcal{L}(\mathrm{Q}) X \approx A^{n}$ (см. рис. 11 ).

$B$-диаграмму $\Delta$ ранга $\beta$ назовем приведенной, если никакая $B$-диаграмма $\Delta^{\prime}$ ранга $\beta$, полученная из $\Delta$ последовательностью внутренних преобразований ранга $\beta-1$, не имеет сократимых пар областей ранга $\beta$, свернутых областей ранга $\beta$ 


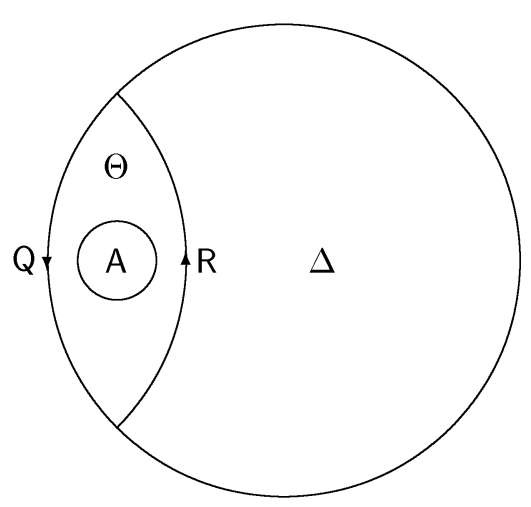

Рис. 11

и граничных сократимых пар ранга $\beta$.

9.9. ПРЕДЛОЖЕНИЕ. Любую В-диаграмму $\Delta$ ранга $\beta$ с помощью внутренних преобразований ранга $\beta$ и добавлений областей ранга $\beta$ можсно перевести в приведенную В-диаграмму $\Delta^{\prime}$ ранга $\beta$. При этом если сама $\Delta$ не является приведенной, то \# $\mathbf{R}_{\beta}\left(\Delta^{\prime}\right)<\# \mathbf{R}_{\beta}(\Delta)$.

ДокАЗАТЕЛЬСтво. Предположим, $\Delta$ не является приведенной $B$-диаграммой ранга $\beta$. В силу очевидной индукции диаграмму $\Delta$ с помощью преобразований указанного в предложении вида достаточно перевести в некоторую $B$-диаграмму $\Delta^{\prime}$ ранга $\beta$ с условием $\# \mathbf{R}_{\beta}\left(\Delta^{\prime}\right)<\# \mathbf{R}_{\beta}(\Delta)$. Применив к $\Delta$ последовательность внутренних преобразований ранга $\beta-1$, можно считать, что $\Delta$ имеет либо сократимую пару областей ранга $\beta$, либо свернутую область ранга $\beta$, либо граничную сократимую пару ранга $\beta$. Рассмотрим каждый из этих случаев отдельно.

Случай 1: $\Delta$ имеет сократимую пару областей ранга $\beta$, т.е. найдутся области $\mathrm{A}, \mathrm{B} \in \mathbf{R}_{\beta}(\Delta)$ и дисковая поддиаграмма $\Theta$ диаграммы $\Delta$ такие, что $\mathbf{R}_{\beta}(\Theta)=$ $\{\mathrm{A}, \mathrm{B}\}$ и $\mathcal{L}(\delta \Theta) \stackrel{\beta=1}{=} 1$. По предложению 4.1 , (i) существует дисковая диаграмма $\Theta^{\prime}$ ранга $\beta-1$, для которой $\mathcal{L}(\delta \Theta) \approx \mathcal{L}\left(\delta \Theta^{\prime}\right)$. Тогда, заменяя поддиаграмму $\Theta$ диаграммы $\Delta$ на $\Theta^{\prime}$, как описано в п. 4.7, получим новую $B$-диаграмму $\Delta^{\prime}$ ранга $\beta$, для которой \# $\mathbf{R}_{\beta}\left(\Delta^{\prime}\right)=\# \mathbf{R}_{\beta}(\Delta)-2$.

Случай 2: $\Delta$ содержит свернутую область А ранга $\beta$, т.е. найдется кольцевая поддиаграмма $\Theta$ диаграммы $\Delta$ с граничными циклами $C_{1}$ и $C_{2}$ такая, что $\mathbf{R}_{\beta}(\Theta)=\{\mathrm{A}\}$, слова $\mathcal{L}\left(\mathrm{C}_{1}\right)$ и $\left(\mathcal{L}\left(\mathrm{C}_{2}\right)\right)^{-1}$ сопряжены в группе $\mathbf{B}_{\beta-1}$ и элементы $\left[\mathcal{L}\left(C_{i}\right)\right]_{\beta-1}(i=1,2)$ имеют конечный порядок. Можно считать, что $C_{1}$ есть внешний граничный цикл подлиаграммы $\Theta$. По предложению 4.1, (ii) найдется кольцевая диаграмма $\Theta^{\prime}$ ранга $\beta-1$ с внешним граничным циклом $C_{1}^{\prime}$ и внутренним граничным циклом $\mathrm{C}_{2}^{\prime}$ такая, что $\mathcal{L}\left(\mathrm{C}_{i}\right) \approx \mathcal{L}\left(\mathrm{C}_{i}^{\prime}\right)(i=1,2)$. Тогда, заменяя поддиаграмму $\Theta$ диаграммы $\Delta$ на $\Theta^{\prime}$ аналогично случаю 1 , получим $B$-диаграмму $\Delta^{\prime}$ ранга $\beta$ с $\# \mathbf{R}_{\beta}\left(\Delta^{\prime}\right)=\# \mathbf{R}_{\beta}(\Delta)-1$.

Случай 3: $\Delta$ имеет граничную сократимую пару ранга $\beta$, т.е. найдутся область $\mathrm{A} \in \mathbf{R}_{\beta}(\Delta)$, участок границы $\mathrm{S} \in \mathcal{Z}_{\beta}(\Delta)$ и дисковая поддиаграмма $\Theta$ диаграммы $\Delta$ 
такие, что $\mathbf{R}_{\beta}(\Theta)=\{\mathrm{A}\}, \delta \Theta \approx \mathrm{QR}$ для некоторого подпути $\mathrm{Q}$ пути $\mathrm{S},|\mathcal{L}(\mathrm{Q})| \geqslant 2|A|$ и $\mathcal{L}(\mathrm{R}) \stackrel{\beta=1}{=} X$, где $\mathcal{L}(\mathrm{S})$ есть подслово слова $A^{n}, A \in \mathcal{E}_{\beta}$ и слово $X$ определяется равенством $\mathcal{L}(\mathrm{Q}) X \approx A^{n}$. Пусть $\Delta_{1}$ есть результат добавления к $\Delta$ области $\mathrm{B}$ ранга $\beta$ вдоль $\mathrm{S}$. Пусть $\mathrm{S}=\mathrm{UQV}$ и $\delta \mathrm{B} \approx \mathrm{SS}^{\prime-1}$ (см. рис. 12 ).

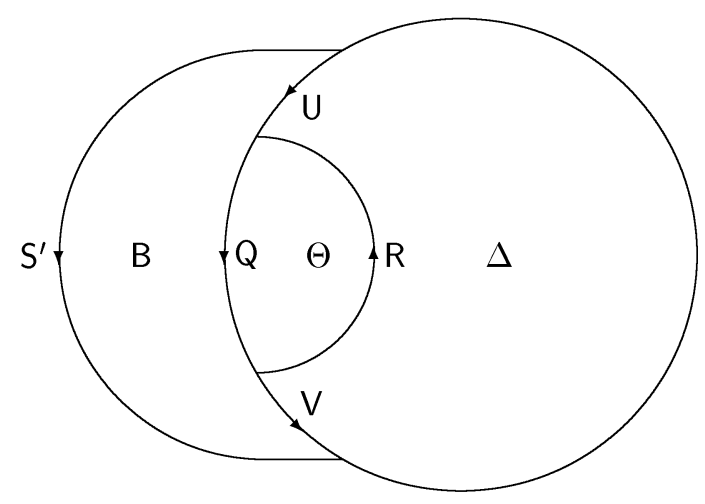

Рис. 12

Рассмотрим дисковую поддиаграмму $\Theta_{1}$ диаграммы $\Delta_{1}$ с граничным циклом $\delta \Theta_{1} \approx \mathrm{RU}^{-1} \mathrm{~S}^{\prime} \mathrm{V}^{-1}$, полученную объединением поддиаграммы $\Theta$ и области В. По лемме 2.1, (ii), (iii) имеем $\mathcal{L}\left(\mathrm{VS}^{\prime-1} \mathrm{U}\right)$ 으 $X \stackrel{\beta=1}{=} \mathcal{L}(\mathrm{R})$, следовательно, $\mathcal{L}\left(\delta \Theta_{1}\right) \stackrel{\beta=1}{=} 1$. Тогда $\mathrm{A}$ и В образуют сократимую пару областей ранга $\beta$ на $\Delta_{1}$. Повторяя далее рассуждения из случая 1 для $\Delta_{1}$ вместо $\Delta$, получим $B$-диаграмму $\Delta^{\prime}$ ранга $\beta$, которая является искомой так как $\# \mathbf{R}_{\beta}\left(\Delta^{\prime}\right)=\# \mathbf{R}_{\beta}\left(\Delta_{1}\right)-2=\# \mathbf{R}_{\beta}(\Delta)-1$.

9.10. ОПРЕДЕЛЕнИЕ (ср. [7]). Сөязкой ранга $\beta$ на $A$-диаграмме $\Delta$ ранга $\beta$ назовем путь х на $\Delta$, удовлетворяюший следуюшим условиям:

(i) $\mathrm{x}$ - простой непустой путь, имеюший ровно две вершины $\iota(\mathrm{x})$ и $\tau(\mathrm{x})$ в пересечении с гранищей диаграммы $\Delta$;

(ii) если a $\in\{\iota(\mathrm{x}), \tau(\mathrm{x})\}$, то а лежит на некотором главном участке $\mathrm{S}$ ранга $\beta$ диаграммы $\Delta$, причем если $\mathrm{S}$ - нециклический участок границы, то a $\neq \iota(\mathrm{S}), \tau(\mathrm{S})$;

(iii) $\mathcal{L}(\mathrm{x}) \in \mathcal{H}_{\beta}$.

9.11. ОПРедЕЛЕНИЕ. Связку х ранга $\beta$ на $A$-диаграмме $\Delta$ ранга $\beta$ назовем вырожденнойв следуюших двух случаях:

(i) путь $\mathrm{x}$ или $\mathrm{x}^{-1}$ гомотопен некоторому подпути участка границы $\mathrm{S} \in X_{\beta}(\Delta)$;

(ii) путь $\mathrm{x}$ или $\mathrm{x}^{-1}$ гомотопен пути вида $\mathrm{PuQ}$, где $\mathrm{P}$ есть конец некоторого пути $\mathrm{R} \in X_{\beta}(\Delta), \mathrm{Q}$ есть начало некоторого пути $\mathrm{S} \in \mathcal{X}_{\beta}(\Delta), \mathrm{u} \in y_{\beta}(\Delta)$ и $\mathcal{L}(\mathrm{P})$ 드 $\mathcal{L}(\mathrm{Q})$ 으 1 .

9.12. ОПРеДЕЛЕнИЕ. $A$-диаграмму $\Delta$ ранга $\beta$ назовем nростой, если никакая $A$-диаграмма $\Delta^{\prime}$ ранга $\beta$, полученная из $\Delta$ последовательностью внутренних преобразований ранга $\beta$, не имеет невырожденных связок ранга $\beta$. 
9.13. ОПРЕДЕлЕнИЕ (ср. [7]). Пусть $\Delta-A$-диаграмма ранга $\beta$. Поддиаграммой примыкания ранга $\beta$ главных участков $\mathrm{R}$ и $\mathrm{S}$ ранга $\beta$ диаграммы $\Delta$ назовем дисковую поддиаграмму П диаграммы $\Delta$ с граничным циклом вида PuQv, где пути $\mathrm{P}$ и $\mathrm{Q}$ непусты и входят в качестве подпутей соответственно в $\mathrm{R}$ и $\mathrm{S}$, а каждый из путей $\mathrm{u}, \mathrm{v}$ либо лежит в $y_{\beta}(\Delta)$, либо является невырожденной связкой ранга $\beta$ на $\Delta$. Пути $\mathrm{P}$ и $\mathrm{Q}$ будем называть участками примыкания поддиаграммы примыкания П.

Из определений 9.13, 5.9, условий (iii) определений 9.1, 9.10 и предложения 4.1, (i) непосредственно вытекает

9.14. ЛЕмма. Если $\mathrm{P}$ и $\mathrm{Q}$ - участки примыкания поддиаграммы примыкания П ранга $\beta$, то слова $\mathcal{L}(\mathrm{P})$ и $(\mathcal{L}(\mathrm{Q}))^{-1}$ близки в ранге $\beta$.

Пусть $\Delta-A$-диаграмма ранга $\alpha$ и $\mathcal{S}$ - некоторый набор ее попарно непересекающихся (как подмножеств $\mathbb{R}^{2}$ ) поддиаграмм примыкания ранга $\beta$. Пусть $\Theta$ - некоторая связная компонента диаграммы $\Delta-\bigcup_{\Pi \in \mathcal{S}}$ П. Из определений 9.10 и 9.13 следует, что $\Theta$ - неособая диаграмма и каждый ее граничный цикл либо является главным участком ранга $\beta$ диаграммы $\Delta$, либо имеет вид $\mathrm{S}_{1} \mathrm{u}_{1} \mathrm{~S}_{2} \mathrm{u}_{2} \ldots \mathrm{S}_{k} \mathrm{u}_{k}$, где $\mathrm{S}_{i}-$ непустые подпути главных участков ранга $\beta$ диаграммы $\Delta$ и для каждого $i$ либо $\mathrm{u}_{i} \in \mathrm{y}_{\beta}(\Delta)$, либо $\mathrm{u}_{i}^{-1}$ есть связка ранга $\beta$, входящая в граничньй цикл некоторой поддиаграммы примыкания $\Pi \in \mathcal{S}$. Так как подслово приведенного в ранге $\beta$ слова само является приведенным в ранге $\beta$ словом, то $\mathcal{L}\left(\mathrm{S}_{i}\right) \in \mathcal{R}_{\beta}$ при всех $i$. Далее, в силу определений 9.1 и 9.10 для каждого $i$ имеем $\mathcal{L}\left(\mathrm{u}_{i}\right) \in \mathcal{H}_{\beta}$. Следовательно, мы имеем разбиение границы типа $A$ на диаграмме $\Theta$, где $X_{\beta}(\Theta)$ состоит из принадлежаших $X_{\beta}(\Delta)$ граничных циклов диаграммы $\Delta$ и всех путей $S_{i}$ указанного вида, а $y_{\beta}(\Theta)$ состоит из всех путей $u_{i}$. Условимся рассматривать каждую связную компоненту $\Theta$ диаграммы $\Delta-\bigcup_{\Pi \in \mathcal{S}} \Pi$ как $A$-диаграмму ранга $\beta$ с указанным разбиением границы, а сами диаграммы $\Theta$ будем называть переходными поддиаграммами диаграммы $\Delta$ относительно $\mathcal{S}$. Множество всех переходных поддиаграмм диаграммы $\Delta$ относительно $\mathcal{S}$ обозначим через $\mathcal{T}(\Delta, \mathcal{S})$. Главные участки границы ранга $\beta$ переходных поддиаграмм диаграммы $\Delta$ относительно $\mathcal{S}$ будем называть переходныцми участками границы диаграммы $\Delta$ относительно $\mathcal{S}$. Легко видеть, что каждый главный участок $\mathrm{S}$ ранга $\beta$ диаграммы $\Delta$ либо является переходным участком гранищы диаграммы $\Delta$ относительно $\mathcal{S}$, либо представляется в виде произведения чередующихся участков примыкания поддиаграмм примыкания из $\mathcal{S}$ и переходных участков границы относительно $\mathcal{S}$.

9.15. Лемма. Пусть $\Delta-A$-диаграмма ранга $\beta$ и $\mathcal{S}$ - непустое конечное множество ее попарно непересекающихся поддиаграмм примыкания. Тогда

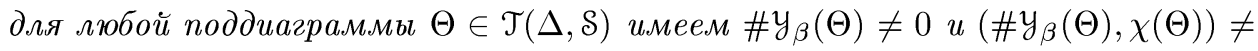
$(1,1)$.

ДоКАЗАТЕЛЬСТво. Заметим, что если $\Sigma$ - собственная поддиаграмма связной неособой диаграммы $\Delta$, то некоторый граничный цикл $\Sigma$ в качестве подпути содержит непустой путь, состояший только из внутренних ребер диаграммы $\Delta$. Так 
как в силу непустоты $\mathcal{S}$ имеем $\Theta \neq \Delta$, то отсюда следует, что некоторый граничный цикл $\Theta$ содержит в качестве подпути путь и, где $\mathbf{u}^{-1}$ - невырожденная связка ранга $\beta$ на $\Delta$. В частности, имеем $y_{\beta}(\Theta) \neq \varnothing$. Допустим, $\left(\# y_{\beta}(\Theta), \chi(\Theta)\right)=(1,1)$. Тогда граничный цикл $\Theta$ имеет вид $\mathrm{Pu}$, где $\mathrm{P} \in \mathcal{X}_{\beta}(\Theta)$ и u $\in y_{\beta}(\Theta)$. Так как пути $\mathrm{u}^{-1}$ и $\mathrm{P}$ гомотопны и $\mathrm{P}$ есть подпуть некоторого участка гранищы $\mathrm{S} \in X_{\beta}(\Delta)$, то в силу определения $9.11 \mathrm{u}^{-1}$ не может быть невырожденной связкой ранга $\beta$. Противоречие.

9.16. Лемма. Пусть $\Delta-A$-диаграмма ранга $\beta$ и $\mathcal{S}$ - конечное множество ее попарно непересекающихся поддиаграмм примыкания ранга $\beta$. Тогда если множество $\mathcal{T}(\Delta, \mathcal{S})$ непусто, то имеет место равенство

$$
\sum_{\Theta \in \mathcal{T}(\Delta, \delta)}\left(\# y_{\beta}(\Theta)-2 \chi(\Theta)\right)=\# y_{\beta}(\Delta)-2 \chi(\Delta) .
$$

ДокАЗАТЕЛЬСТво. Пусть $\Theta$ - произвольная (не обязательно связная) неособая карта и $\Pi$ - дисковая подкарта карты $\Theta$ такая, что пересечение $\partial \Theta \cap \partial П$ состоит из одного или двух отрезков кривых и не содержит вершин в качестве связных компонент. Из формулы (4.1) легко следует, что

$$
\chi(\Theta-\Pi)= \begin{cases}\chi(\Theta), & \text { если } \partial \Theta \cap \partial \Pi \text { состоит из одного отрезка кривой, } \\ \chi(\Theta)+1, & \text { если } \partial \Theta \cap \partial \Pi \text { состоит из двух отрезков кривых. }\end{cases}
$$

Если в граничньй цикл некоторой поддиаграммы П $\in \mathcal{S}$ входят в качестве подпути два участка границы из $y_{\beta}(\Delta)$, то $\Pi=\Delta$, и в этом случае $\mathcal{T}(\Delta, \mathcal{S})=\varnothing$. Поэтому можно считать, что граничный цикл каждой поддиаграммы $\Pi \in \mathcal{S}$ либо не содержит в качестве подпути ни одного участка гранищы из $y_{\beta}(\Delta)$, либо содержит ровно один такой участок. Заметим, что в этом случае $\partial \Delta \cap \partial \Pi$ состоит из двух или, соответственно, одного отрезка кривой. Тогда из (9.4) по индукции следует $\chi\left(\Delta-\bigcup_{\Pi \in \mathcal{S}} \Pi\right)=\chi(\Delta)+r$, где $r$ - число поддиаграмм $\Pi \in \mathcal{S}$, граничньй цикл которых не содержит ни одного подпути из $y_{\beta}(\Delta)$. Остается заметить, что $\chi\left(\Delta-\bigcup_{\Pi \in \delta} \Pi\right)=\sum_{\Theta \in \mathcal{T}(\Delta, \delta)} \chi(\Theta)$ и $\sum_{\Theta \in \mathcal{T}(\Delta, \delta)} \# y_{\beta}(\Theta)-\# y_{\beta}(\Delta)=2 r$.

9.17. Лемма. Пусть $\Delta-A$-диаграмма ранга $\beta, \mathcal{S}$ - конечное множество ее попарно непересекающихся поддиаграмм примыкания ранга $\beta$ и $\Theta \in \mathcal{T}(\Delta, \mathcal{S})$. Тогда любая невырожденная связка ранга $\beta$ на $\Theta$ является невырожденной связкой ранга $\beta$ на $\Delta$.

ДокАЗАТЕЛЬСТво. Из определения 9.10 следует, что любая связка ранга $\beta$ на $\Theta$ является связкой ранга $\beta$ на $\Delta$. Проверим, что из вырожденности связки х на $\Delta$ вытекает ее вырожденность на $\Theta$. В соответствии с определением 9.11 рассмотрим два случая.

Случай 1: Путь ${ }^{\epsilon}$ при некотором $\epsilon= \pm 1$ гомотопен пути $\mathrm{P}$, входящему в качестве подпути в некоторый участок границы $\mathrm{S} \in X_{\beta}(\Delta)$. Так как $\mathrm{Px}^{-\epsilon}-$ стягиваемый простой замкнутый путь, то $\mathrm{Px}^{-\epsilon} \approx \delta \Sigma$ для некоторой дисковой поддиаграммы $\Sigma$ диаграммы $\Delta$. Из определения 9.11 следует, что любая связка у ранга $\beta$ 
на диаграмме $\Delta$, содержащаяся в $\Sigma$ (т.е. для которой supp y $\subseteq \Sigma$ ), вырождена, а из определения 9.13 следует тогда, что $\Sigma$ не может содержать поддиаграмм примыкания $\Pi \in \mathcal{S}$, и потому содержится в одной из связных компонент диаграммы $\Delta-\bigcup_{\Pi \in \mathcal{S}} \Pi$ и $\mathbf{x}^{\epsilon}$. Так как $\Theta-$ связная компонента диаграммы $\Delta-\bigcup_{\Pi \in \mathcal{S}} \Pi$ и $\mathrm{x}^{\epsilon}$ содержится в $\Theta$, то $\Sigma$ содержится в $\Theta$. Тогда пути $\mathrm{P}$ и $\mathrm{x}^{-\epsilon}$ гомотопны на $\Theta$ и по определению $9.11 \mathrm{x}$ - вырожденная связка ранга $\beta$ на $\Theta$.

Cлучай 2: Путь $x^{\epsilon}$ при некотором $\epsilon= \pm 1$ гомотопен пути вида $\mathrm{PuQ}$, где $\mathrm{P}$-конец некоторого пути $\mathrm{R} \in \mathcal{X}_{\beta}(\Delta), \mathrm{Q}$ - начало некоторого пути $\mathrm{S} \in X_{\beta}(\Delta), \mathrm{u} \in \mathrm{y}_{\beta}(\Delta)$ и $\mathcal{L}(\mathrm{P})$ 드 $\mathcal{L}(\mathrm{Q})$ 으 1 . В этом случае проходят те же рассуждения, что и в случае $1, \mathrm{c}$ заменой $\mathrm{P}$ на $\mathrm{PuQ}$.

9.18. ОПрЕДЕЛЕНИЕ. Пусть $\Delta-A$-диаграмма ранга $\beta$. Конечное множество $\mathcal{S}$ ее попарно непересекаюшихся поддиаграмм примыкания ранга $\beta$ назовем картой примыканий ранга $\beta$ на $\Delta$, если выполнены следуюшие условия:

(i) для каждой поддиаграммы $\Theta \in \mathcal{T}(\Delta, \mathcal{S})$ выполнено $\left(\# y_{\beta}(\Theta), \chi(\Theta)\right) \neq(2,1)$, за исключением случая, когда $\Delta-$ кольцевая диаграмма, $\# y_{\beta}(\Delta)=0$ и \#S $=1$ (см. рис. 13);

(ii) каждая поддиаграмма $\Theta \in \mathcal{T}(\Delta, \mathcal{S})$ есть простая $A$-диаграмма ранга $\beta$.

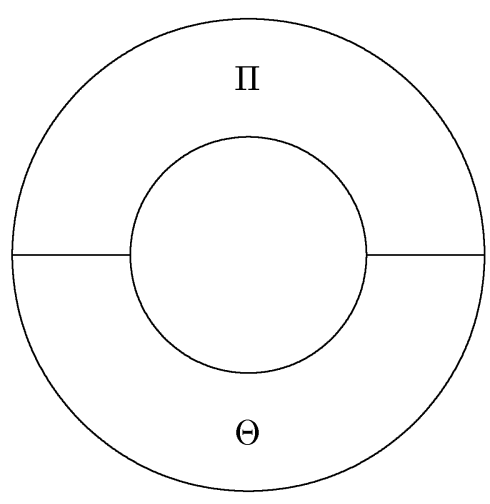

Рис. 13

9.19. ПреДЛОЖЕНИЕ. Любую А-диаграмму $\Delta$ ранга $\beta$ последовательностью внутренних преобразований ранга $\beta$ можно перевести в $A$-диаграмму $\Delta^{\prime}$ ранга $\beta$ такую, что существует некоторая карта примыканий $\mathcal{S}$ ранга $\beta$ на $\Delta^{\prime}$.

ДокАЗАТЕльСтво. Пусть $\Delta$ - произвольная $A$-диаграмма ранга $\beta$ и пусть $\mathcal{S}$ конечное множество ее попарно непересекаюшихся подлиаграмм примыкания ранга $\beta$. Опишем два типа преобразований, переводяших пару $(\Delta, \mathcal{S})$ в новую пару $\left(\Delta^{\prime}, \mathfrak{S}^{\prime}\right)$ указанного вида.

(i) Предположим, что некоторая поддиаграмма $\Theta \in \mathcal{T}(\Delta, \mathcal{S})$ является дисковой диаграммой и \# $y_{\beta}(\Theta)=2$. Пусть $y_{\beta}(\Theta)=\{u, v\}$. Предположим, кроме того, что либо один из путей u или $v$ принадлежит $y_{\beta}(\Delta)$, либо $\mathrm{u}^{-1}$ и $\mathrm{v}^{-1}-$ связки ранга $\beta$ 
на $\Delta$, входящие в граничные циклы двух различныхподдиаграмм примыкания из $\mathcal{S}$. $\mathrm{C}$ точностью до перестановки местами u и $\mathrm{v}$ возможны три случая:

(a) $\mathrm{u}, \mathrm{v} \in \mathrm{y}_{\beta}(\Delta)$

(b) $\mathrm{u} \in \mathrm{y}_{\beta}(\Delta), \mathrm{v} \notin \mathrm{y}_{\beta}(\Delta)$

(c) $\mathrm{u}, \mathrm{v} \notin \mathrm{y}_{\beta}(\Delta)$.

В каждом из этих случаев, не изменяя диаграммы $\Delta$, полагаем соответственно

$$
\mathcal{S}^{\prime} \rightleftharpoons\left\{\begin{array}{l}
\{\Theta\} \quad \text { в случае (a), } \\
(\mathcal{S} \backslash\{\Pi\}) \cup\{\Pi \cup \Theta\} \quad \text { в случае (b), } \\
\left(\mathcal{S} \backslash\left\{\Pi, \Pi^{\prime}\right\}\right) \cup\left\{\Pi \cup \Theta \cup \Pi^{\prime}\right\} \quad \text { в случае (c), }
\end{array}\right.
$$

где П, $\Pi^{\prime} \in \mathcal{S}$ - поддиаграммы примыкания, граничашие с $\Theta$ (см. рис. 14).

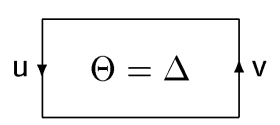

(a)

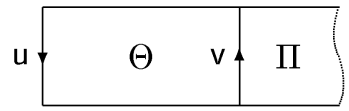

(b)

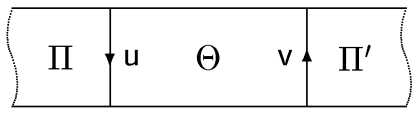

(c)

Рис. 14

(ii) Предположим, некоторая поддиаграмма $\Theta \in \mathcal{T}(\Delta, \mathcal{S})$ не является простой $A$-диаграммой ранга $\beta$. Согласно определению 9.12 диаграмму $\Theta$ некоторой последовательностью внутренних преобразований ранга $\beta$ можно перевести в $A$-диаграмму $\Theta^{*}$ ранга $\beta$, обладаюшую невырожденной связкой ранга $\beta$. Из определений 9.4 и 4.7 следует, что $\Theta^{*}$ есть поддиаграмма диаграммы $\Delta^{*}$, полученной из $\Delta$ соответствуюшей последовательностью внутренних преобразований ранга $\beta$. При этом, как легко видеть из определений, диаграмма $\Delta^{*}-\Theta^{*}$ есть результат измельчения диаграммы $\Delta-\Theta$ и образ $\Pi^{*}$ на $\Delta^{*}$ каждой поддиаграммы примыкания $\Pi \in \mathcal{S}$ является подлиаграммой примыкания ранга $\beta$ на $\Delta^{*}$. Пусть $\mathcal{S}^{*}-$ множество всех поддиаграмм $\Pi^{*}$. Очевидно, имеем $\Theta^{*} \in \mathcal{T}\left(\Delta^{*}, \mathcal{S}^{*}\right)$. Пусть $\mathrm{x}-$ невырожденная связка ранга $\beta$ на $\Theta^{*}$ и $\mathrm{R}, \mathrm{S}$ - главные участки ранга $\beta$ диаграммы $\Theta^{*}$, на которых лежат вершины $\iota(\mathrm{x})$ и $\tau(\mathrm{x})$ соответственно. Добавив к $\Delta^{*}$ две новые вершины, лежашие на $\mathrm{R}$ и на $\mathrm{S}$, можно считать, что $\iota(\mathrm{x})$ есть конечная вершина ребра $\mathrm{e}$ с $\mathcal{L}(\mathrm{e})$ 으 1 , входящего в $\mathrm{R}$, и $\tau(\mathrm{x})$ есть начальная вершина ребра $\mathrm{d}$ с $\mathcal{L}(\mathrm{d})$ 으 1 , входящего в $\mathrm{S}$, причем в случае, если $\mathrm{R}$ - нециклический участок гранищы диаграммы $\Theta^{*}$, начальная вершина ребра е отлична от $\iota(\mathrm{R})$, а в случае, если $\mathrm{S}-$ нециклический участок границы диаграммы $\Theta^{*}$, конечная вершина ребра $\mathrm{d}$ отлична от $\tau(\mathrm{S})$. Далее, произведя измельчение диаграммы $\Delta^{*}$, как описано в п. 4.9 , можно считать, что вершины $\iota(\mathrm{e})$ и $\tau(\mathrm{d})$ соединены путем $\mathrm{x}^{\prime} \mathrm{c} \mathcal{L}\left(\mathrm{x}^{\prime}\right)$ 드 $\mathcal{L}(\mathrm{exd})$ 으 $\mathcal{L}(\mathrm{x})$, гомотопным пути exd и не имеюшим обших вершин с х и отличных от $\iota(\mathrm{e}), \tau(\mathrm{d})$ вершин в пересечении с границей диаграммы $\Theta^{*}$ (см. рис. 15). Из определений 9.10 и 9.11 следует, что $\mathrm{x}^{\prime-1}$ есть невырожденная связка ранга $\beta$ на диаграмме $\Theta^{*}$. Тогда согласно лемме $9.17 \times$ и х ${ }^{\prime-1}$ - невырожденные связки ранга $\beta$ на диаграмме $\Delta$, и 


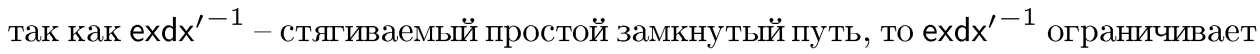
дисковую поддиаграмму П диаграммы $\Delta^{*}$. Согласно определению 9.13 П - поддиаграмма примыкания ранга $\beta$ на $\Delta^{*}$. Будем считать результатом применения преобразования (ii) к паре $(\Delta, \mathcal{S})$ пару $\left(\Delta^{*}, \mathcal{S}^{*} \cup\{\Pi\}\right)$.

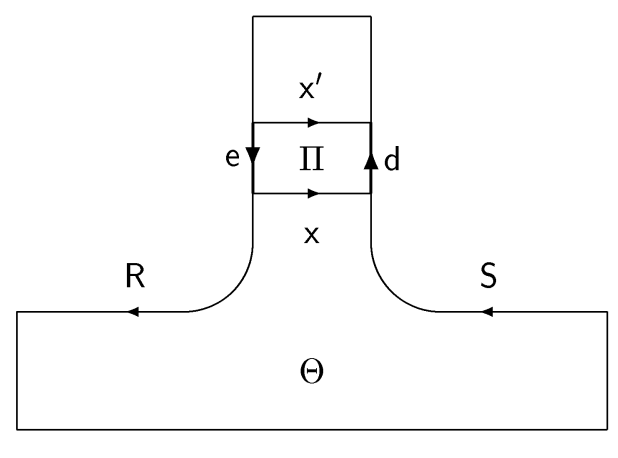

Рис. 15

Убедимся в том, что если к паре $(\Delta, \mathcal{S})$ неприменимо никакое преобразование (i) или (ii), то $\mathcal{S}$ есть карта примыканий ранга $\beta$ на $\Delta$. Действительно, невозможность применения к $(\Delta, \mathcal{S})$ преобразования вида (ii) равносильна выполнению условия (ii) определения 9.18. Невозможность применения преобразования вида (i) означает, что для любой поддиаграммы $\Theta \in \mathcal{T}(\Delta, \mathcal{S})$ с условием $\left(\# y_{\beta}(\Theta), \chi(\Theta)\right)=(2,1)$, если $y_{\beta}(\Theta)=\{\mathrm{u}, \mathrm{v}\}$, то $\mathrm{u}^{-1}$ и $^{-1}$ входят в граничный цикл некоторой одной поддиаграммы $\Pi \in \mathcal{S}$. Но если найдется такая поддиаграмма $\Theta$, то имеет место исключительный случай, описанньй в условии 9.18 (i) (рис. 13). Следовательно, невозможность применения к паре $(\Delta, \mathcal{S})$ преобразования (i) влечет выполнение условия (i) определения 9.18 .

Нам остается доказать, что сушествует конечная последовательность

$$
(\Delta, \mathcal{S})=\left(\Delta_{0}, \mathcal{S}_{0}\right) \rightarrow\left(\Delta_{1}, \mathcal{S}_{1}\right) \rightarrow \cdots \rightarrow\left(\Delta_{t}, \mathcal{S}_{t}\right)
$$

в которой пара $\left(\Delta_{i+1}, \mathcal{S}_{i+1}\right)$ получается из пары $\left(\Delta_{i}, \mathcal{S}_{i}\right)$ применением преобразования (i) или (ii) и к паре $\left(\Delta_{t}, \mathcal{S}_{t}\right)$ такое преобразование уже неприменимо (на самом деле несложная модификация приведенного ниже рассуждения показывает, что любая последовательность преобразований (i) и (iі) обрывается через конечное число шагов). Заметим, что если пара $\left(\Delta_{i+1}, \mathcal{S}_{i+1}\right)$ получена из пары $\left(\Delta_{i}, \mathcal{S}_{i}\right)$ применением преобразования (i), то $\mathcal{T}\left(\Delta_{i+1}, \mathcal{S}_{i+1}\right)=\mathcal{T}\left(\Delta_{i}, \mathcal{S}_{i}\right) \backslash\{\Theta\}$, где $\left(\# y_{\beta}(\Theta)\right.$, $\chi(\Theta))=(2,1)$. Отсюда следует, что, во-первых, любая последовательность преобразований (i) через конечное число шагов обрывается, и, во-вторых, если пара $\left(\Delta_{i}, \mathcal{S}_{i}\right)$ удовлетворяет условию 9.18 , (ii), и, следовательно, к ней неприменимо никакое преобразование (ii), то это верно и для пары $\left(\Delta_{i+1}, \mathcal{S}_{i+1}\right)$. Будем считать, что мы начинаем последовательность (9.5) с вьполнения преобразований (ii) до тех пор, пока это возможно, а затем выполняем преобразования (i). Тогда для доказательства предложения достаточно проверить, что процесс применения преобразований (ii) оборвется на конечном шаге. 
Итак, предположим, что пара $\left(\Delta_{i+1}, \mathcal{S}_{i+1}\right)$ получена из пары $\left(\Delta_{i}, \mathcal{S}_{i}\right)$ преобразованием (ii). Заметим, что это преобразование увеличивает число $\sum_{\Theta \in \mathcal{T}\left(\Delta_{i}, s_{i}\right)} \# y_{\beta}(\Theta)$ на 2 . Поэтому достаточно указать не зависяшую от $i$ верхнюю оченку для $\sum_{\Theta \in \mathcal{T}\left(\Delta_{i}, s_{i}\right)} \# y_{\beta}(\Theta)$. Без ограничения обшности можно считать $i \geqslant 1$. Так как $\# \mathcal{S}_{i+1}=\# \mathcal{S}_{i}+1$, то при $i \geqslant 1$ имеем $\mathcal{S}_{i} \neq \varnothing$. В этом случае из леммы 9.15 следует, что для любой поддиаграммы $\Theta \in \mathcal{T}(\Delta, \mathcal{S})$ выполнено $\# y_{\beta}(\Theta)-2 \chi(\Theta) \geqslant 0$, причем равенство $\# y_{\beta}(\Theta)-2 \chi(\Theta)=0$ возможно лишь в случае, если $\left(\# y_{\beta}(\Theta), \chi(\Theta)\right)=(2,1)$. Обозначим через $\mathcal{T}_{i}^{\prime}$ и $\mathcal{T}_{i}^{\prime \prime}$ соответственно множества поддиаграмм $\Theta \in \mathcal{T}\left(\Delta_{i}, \mathcal{S}_{i}\right)$, для которых $\left(\# y_{\beta}(\Theta), \chi(\Theta)\right)=(2,1)$ и $\left(\# y_{\beta}(\Theta), \chi(\Theta)\right) \neq(2,1)$. Оценим отдельно слагаемые $\sum_{\Theta \in \mathcal{T}_{i}^{\prime}} \# y_{\beta}(\Theta)$ и $\sum_{\Theta \in \mathcal{T}_{i}^{\prime \prime}} \# y_{\beta}(\Theta)$. Для оценки величины $\sum_{\Theta \in \mathcal{T}_{i}^{\prime \prime}} \# y_{\beta}(\Theta)$ воспользуемся леммой 9.16. Имеем

$$
\sum_{\Theta \in \mathcal{T}_{i}^{\prime \prime}}\left(\# y_{\beta}(\Theta)-2 \chi(\Theta)\right)=\# y_{\beta}\left(\Delta_{i}\right)-2 \chi\left(\Delta_{i}\right)=\# y_{\beta}(\Delta)-2 \chi(\Delta),
$$

откуда, учитывая, что для любой поддиаграммы $\Theta \in \mathcal{T}_{i}^{\prime \prime}$ из \# $y_{\beta}(\Theta)-2 \chi(\Theta) \geqslant 1$ и $\chi(\Theta) \leqslant 1$ следует \# $y_{\beta}(\Theta) \leqslant 3\left(\# y_{\beta}(\Theta)-2 \chi(\Theta)\right)$, получаем

$$
\sum_{\Theta \in \mathcal{T}_{i}^{\prime \prime}} \# y_{\beta}(\Theta) \leqslant 3\left(\# y_{\beta}(\Delta)-2 \chi(\Delta)\right) .
$$

Так как \#у $y_{\beta}(\Theta)=2$ для любой поддиаграммы $\Theta \in \mathcal{T}_{i}^{\prime}$, то остается оценить сверху число $\# \mathcal{T}_{i}^{\prime}$. Докажем, что для каждого $i$ существует не более одной под-

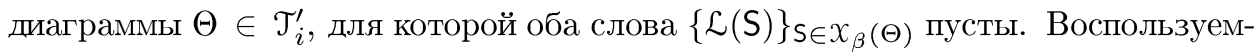
ся индукцией по $i$. Если $i=0$, то $\mathcal{T}\left(\Delta_{i}, \mathcal{S}_{i}\right)=\{\Delta\}$ и доказывать нечего. Пусть $i \geqslant 1$. Пусть $\left(\Delta_{i-1}, \mathcal{S}_{i-1}\right)=(\Delta, \mathcal{S})$ и $\left(\Delta_{i}, \mathcal{S}_{i}\right)=\left(\Delta^{*}, \mathcal{S}^{*} \cup\{\Pi\}\right)$ в обозначениях из (ii). Пусть $\left\{\Theta_{j}\right\}_{j=1}^{r}$, где $r=1$ или $r=2$, - одна или две связные компоненты поддиаграммы $\Theta^{*}-\Pi$ (на рис. 15 таких компонент две). Очевидно, имеем $\mathcal{T}\left(\Delta_{i}, \mathcal{S}_{i}\right)=\left(\mathcal{T}\left(\Delta_{i-1}, \mathcal{S}_{i-1}\right) \backslash\{\Theta\}\right) \cup\left\{\Theta_{j}\right\}_{j=1}^{r}$. Если бы для какой-нибудь из поддиаграмм $\Theta_{j}$ выполнялось $\left(\# y_{\beta}\left(\Theta_{j}\right), \chi\left(\Theta_{j}\right)\right)=(2,1)$ и оба слова $\{\mathcal{L}(\mathrm{S})\}_{\mathrm{s} \in \mathcal{X}_{\beta}(\Theta)}$ были бы пусты, то в силу условия (ii) определения 9.11 связка х на диаграмме $\Theta^{*}$ была бы вырожденной. Следовательно, если $\Theta_{j} \in \mathcal{T}_{i}^{\prime}$, то хотя бы одно из слов $\{\mathcal{L}(\mathrm{S})\}_{\mathrm{S} \in \mathcal{X}_{\beta}(\Theta)}$ непусто. Тогда из индуктивного предположения для $i:=i-1$ вытекает, что $\mathcal{T}^{\prime}\left(\Delta_{i}, \mathcal{S}_{i}\right)$ содержит не более одной поддиаграммы $\Theta$, для которой слова $\{\mathcal{L}(\mathrm{S})\}_{S \in X_{\beta}(\Theta)}$ пусты, что и требовалось. Из доказанного утверждения вытекает

$$
\# \mathcal{T}_{i}^{\prime} \leqslant \sum_{\mathrm{S} \in \mathcal{X}_{\beta}\left(\Delta_{i}\right)}|\mathcal{L}(\mathrm{S})|+1 \leqslant \sum_{\mathrm{S} \in \mathcal{X}_{\beta}(\Delta)}|\mathcal{L}(\mathrm{S})|+1 .
$$

Предложение 9.19 доказано.

9.20. ЛЕмма. Пусть $\Delta-A$-диаграмма ранга $\beta, \mathcal{S}$ - карта примыканий ранга $\beta$ на $\Delta$ и $\mathcal{S}_{1} \subseteq \mathcal{S}$. Если выполнено одно из условий $\# y_{\beta}(\Delta)-2 \chi(\Delta) \geqslant 1$ или $\# \mathcal{S}_{1} \geqslant 2$, то для любой поддиаграммы $\Theta \in \mathcal{T}\left(\Delta, \mathcal{S}_{1}\right)$ выполнено \#у ${ }_{\beta}(\Theta)$ $2 \chi(\Theta) \geqslant 1$. 
ДокАЗАТЕЛЬСТво. Пусть верна посылка леммы, и пусть $\Theta \in \mathcal{T}\left(\Delta, S_{1}\right)$. В случае $S_{1}=\varnothing$ имеем $\Theta=\Delta$, и тогда $\# y_{\beta}(\Theta)-2 \chi(\Theta) \geqslant 1$ по условию. Пусть $\mathcal{S}_{1} \neq \varnothing$. По лемме 9.15 невозможны случаи, когда пара $\left(y_{\beta}(\Theta), \chi(\Theta)\right)$ равна $(0,0)$, $(0,1)$ или $(1,1)$. Для доказательства неравенства \# $y_{\beta}(\Theta)-2 \chi(\Theta) \geqslant 1$ остается проверить, что $\left(y_{\beta}(\Theta), \chi(\Theta)\right) \neq(2,1)$. Допустим, $\left(y_{\beta}(\Theta), \chi(\Theta)\right)=(2,1)$. В силу условия 9.18 , (i) невозможен случай $\Theta \in \mathcal{T}(\Delta, \mathcal{S})$. Пусть $\Theta \notin \mathcal{T}(\Delta, \mathcal{S})$, т.е. некоторая поддиаграмма $\Pi \in \mathcal{S}$ содержится в $\Theta$. Из определений 9.13 и 9.11 следует, что возможны лишь три случая расположения П в $\Theta$, изображенные на рис. 16 , где главные участки ранга $\beta$ диаграммы $\Delta$ изображены более толстыми линиями. В последнем из этих случаев имеем $\Theta=\Delta$, что противоречит предположению $\mathcal{S}_{1} \neq \varnothing$. В остальных двух случаях для некоторой поддиаграммы $\Theta^{\prime} \in \mathcal{T}(\Delta, \mathcal{S})$, содержащейся в $\Theta$, выполнено $\left(y_{\beta}\left(\Theta^{\prime}\right), \chi\left(\Theta^{\prime}\right)\right)=(2,1)$. Но это противоречит условию 9.18 , (i).
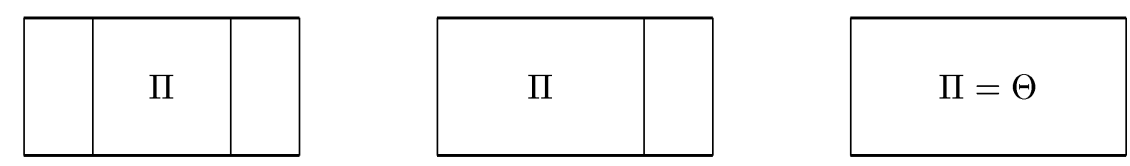

Рис. 16

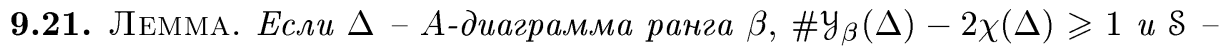
карта примыканий ранга $\beta$ на $\Delta$, то имеет место неравенство

$$
\# \mathcal{S} \leqslant \# y_{\beta}(\Delta)-3 \chi(\Delta) .
$$

ДоКАЗАТЕЛЬСТво. По лемме 9.20 для любой поддиаграммы $\Theta \in \mathcal{T}(\Delta, \mathcal{S})$ имеем \# $y_{\beta}(\Theta)-2 \chi(\Theta) \geqslant 1$, откуда с учетом неравенства $\chi(\Theta) \leqslant 1$ следует

$$
\frac{1}{2} \# y_{\beta}(\Theta) \leqslant \frac{3}{2}\left(\# y_{\beta}(\Theta)-2 \chi(\Theta)\right) .
$$

Просуммировав по всем поддиаграммам $\Theta \in \mathcal{T}(\Delta, \mathcal{S})$ и воспользовавшись леммой 9.16, получаем

$$
\frac{1}{2} \sum_{\Theta \in \mathcal{T}(\Delta, \delta)} \# y_{\beta}(\Theta) \leqslant \frac{3}{2}\left(\# y_{\beta}(\Delta)-2 \chi(\Delta)\right) .
$$

Так как множество $\bigcup_{\Theta \in \mathcal{T}(\Delta, \mathcal{s})} y_{\beta}(\Theta)$ состоит в точности из путей $\mathrm{x}$, для которых $\mathrm{x} \in \mathrm{y}_{\beta}(\Delta)$ либо $\mathrm{x}^{-1}$ - связка ранга $\beta$ на $\Delta$, входящая в граничный цикл поддиаграммы $\Pi \in \mathcal{S}$, то имеем

$$
\sum_{\Theta \in \mathcal{T}(\Delta, \delta)} \# y_{\beta}(\Theta)=\# y_{\beta}(\Delta)+2 \# \mathcal{S}
$$

откуда, подставив в предыдущее соотношение, получаем требуемое неравенство. 
9.22. ЛЕмма. Пусть $\mathcal{S}$ - карта примыканий ранга $\beta$ на А-диаграмме $\Delta$ ранга $\beta, \mathcal{S}_{1} \subseteq \mathcal{S} u \Theta \in \mathcal{T}\left(\Delta, \mathcal{S}_{1}\right)$. Тогда множество поддиаграмм $\Pi \in \mathcal{S}$, содержащихся в $\Theta$, образует карту примыканий ранга $\beta$ на $\Theta$.

ДокАЗАТЕЛЬство. Предположим, доказано, что каждая поддиаграмма П $\epsilon$ $\mathcal{S}_{1}$, содержащаяся в $\Theta$, является поддиаграммой примыкания ранга $\beta$ на $\Theta$. Тогда, очевидно, имеем $\mathcal{T}\left(\Theta, \mathcal{S}^{\prime}\right) \subseteq \mathcal{T}(\Delta, \mathcal{S})$, где $\mathcal{S}^{\prime}$ - множество поддиаграмм $\Pi \in \mathcal{S}$, содержашихся в $\Theta$. Легко видеть, что вьполнение условий (i) и (ii) определения 9.18 для $\Delta$ и $\mathcal{S}$ влечет выполнение этих условий для $\Theta$ и $\mathcal{S}^{\prime}$. Следовательно, $\mathcal{S}^{\prime}$ есть карта примыканий ранга $\beta$ на $\Theta$, и нам остается доказать, что каждая поддиаграмма $\Pi \in \mathcal{S}$, содержащаяся в $\Theta$, является поддиаграммой примыкания ранга $\beta$ на $\Theta$.

Пусть $\Pi \in \mathcal{S}$ и П содержится в $\Theta$. Пусть $\delta \Pi \approx \mathrm{PuQv}$, где $\mathrm{P}$ и $\mathrm{Q}$ - участки примыкания. Как видно из определения 9.13 , достаточно проверить, что каждый из путей u или $v$ либо принадлежит $y_{\beta}(\Theta)$, либо является невырожденной связкой ранга $\beta$ на $\Theta$. Докажем это для и. Если u $\in y_{\beta}(\Delta)$, то u $\in y_{\beta}(\Theta)$. Пусть u - невырожденная связка ранга $\beta$ на $\Delta$. Из определения 9.10 и того, что различные поддиаграммы из $\mathcal{S}$ не имеют обших вершин, следует, что и есть связка ранга $\beta$ на $\Theta$. Допустим, что $\mathbf{u}-$ вырожденная связка ранга $\beta$ на $\Theta$. Если путь и или и ${ }^{-1}$ гомотопен на $\Theta$ некоторому пути $\mathrm{R}$, входящему в качестве подпути в главный участок $\mathrm{R}^{\prime}$ ранга $\beta$ диаграммы $\Theta$, то $\mathrm{R}$ входит в главный участок $\mathrm{R}^{\prime \prime}$ ранга $\beta$ диаграммы $\Delta$, содержащий $\mathrm{R}^{\prime}$ в качестве подпути или совпадающий $\mathrm{c} \mathrm{R}^{\prime}$, и тогда $\mathrm{u}$ - вырожденная связка ранга $\beta$ на $\Delta$ вопреки предположению. Пусть путь $\mathbf{u}^{\epsilon}(\epsilon= \pm 1)$ гомотопен на $\Theta$ пути вида SwT, где $\mathrm{S}$ - конец пути из $X_{\beta}(\Theta), w \in y_{\beta}(\Theta), \mathrm{T}$ - начало пути из $X_{\beta}(\Theta)$ и $\mathcal{L}(\mathrm{S})$ 드 $\mathcal{L}(\mathrm{T})$ 드 1 . Если $\mathrm{w} \in \mathrm{y}_{\beta}(\Delta)$, то $\mathrm{S}$ и $\mathrm{T}$ - соответственно конец и начало некоторых путей из $X_{\beta}(\Delta)$, и тогда путь и снова являлся бы вырожденной связкой ранга $\beta$ на $\Delta$. Пусть $w \notin y_{\beta}(\Delta)$, т.е. $w^{-1}$ входит в граничный цикл некоторой поддиаграммы $\Pi^{\prime} \in \mathcal{S}_{1}$. Так как $\mathrm{SwTu}^{-\epsilon}$ - стягиваемый простой замкнутый путь на $\Theta$, то $\mathrm{SwTu}{ }^{-\epsilon} \approx \delta \Theta_{1}$ для некоторой дисковой поддиаграммы $\Theta_{1}$ диаграммы $\Theta$. Если $\epsilon=1$, то $\Theta_{1} \in \mathcal{T}\left(\Delta, S_{1} \cup\{\Pi\}\right)$, и так как $\left(\# y_{\beta}\left(\Theta_{1}\right), \chi\left(\Theta_{1}\right)\right)=(2,1)$ и $\#\left(\mathcal{S}_{1} \cup\{\Pi\}\right) \geqslant 2$, мы получаем противоречие с леммой 9.20. Пусть $\epsilon=-1$. Тогда $\mathrm{S}=\mathrm{QS}_{1}, \mathrm{~T}=\mathrm{T}_{1} \mathrm{P}$ (см. рис. 17$)$, и при этом $\mathcal{L}\left(\mathrm{S}_{1}\right)$ 드 $\mathcal{L}\left(\mathrm{T}_{1}\right)$ 으 1. Рассматривая $\mathrm{v}, \mathrm{S}_{1}$ и $\mathrm{T}_{1}$ вместо u, $\mathrm{S}$ и $\mathrm{T}$, мы, как и вьше, приходим к противоречию, получив, что либо $v$ - вырожденная связка ранга $\beta$ на диаграмме $\Delta$, либо для некоторой

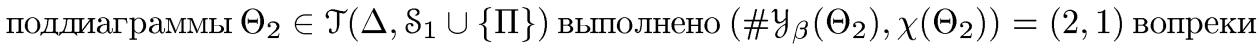
лемме 9.20 .

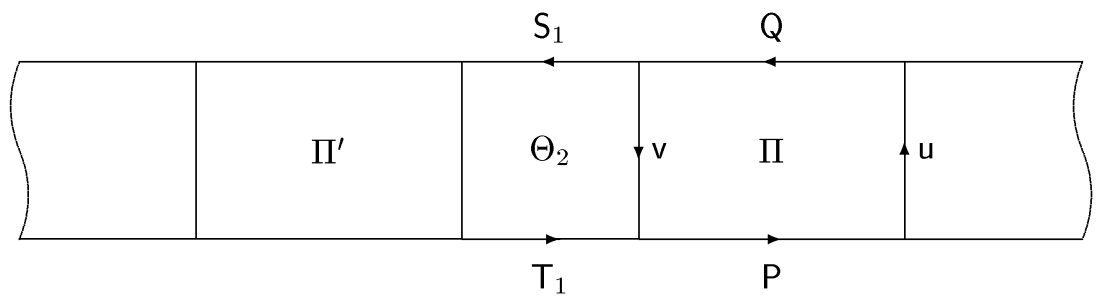

Рис. 17 
9.23. ЛЕмма. Пусть $\Delta-A$-диаграмма ранга $\beta, \mathcal{S}$ - карта примыканий ранга $\beta$ на $\Delta$ и $\mathrm{S} \in X_{\beta}(\Delta)$. Пусть $t(\mathrm{~S}, \mathrm{R})$ обозначает число поддиаграмм примыкания $\Pi \in \mathcal{S}$ участка гранищы $\mathrm{S} \kappa$ участку граничы $\mathrm{R} \in X_{\beta}(\Delta) u$ $r(\mathrm{~S}), s(\mathrm{~S})$ - соответственно число содержащихся в $\mathrm{S}$ участков примыкания поддиаграмм примыкания $\Pi \in \mathcal{S}$ и число содержащихся в $\mathrm{S}$ переходных участков диаграммы $\Delta$ относительно S. Тогда верны следующие утверждения.

(i) Если $\Delta-$ дисковая диаграмма, то $t(\mathrm{~S}, \mathrm{~S})=0, \quad t(\mathrm{~S}, \mathrm{R}) \leqslant 1$ для любого $\mathrm{R} \in X_{\beta}(\Delta)$ us $s(\mathrm{~S}) \leqslant \max \left\{1, \# y_{\beta}(\Delta)-2\right\}$.

(ii) Если $\Delta$ - кольчевая диаграмма и $\mathrm{S}$ - нециклический участок ее границы, то $t(\mathrm{~S}, \mathrm{~S}) \leqslant 1, \quad t(\mathrm{~S}, \mathrm{R}) \leqslant 1$, если $\mathrm{R}$ - чиклический участок границы, и $t(\mathrm{~S}, \mathrm{R}) \leqslant 2$, если $\mathrm{R}$ - нечиклический участок границы. При этом каждый из двух граничных ииклов диаграммы $\Delta$ содержит не более одного подпути $\mathrm{R} \in X_{\beta}(\Delta)$ c $t(\mathrm{~S}, \mathrm{R})=2$. Кроме того, $s(\mathrm{~S}) \leqslant \# y_{\beta}(\Delta)+4$.

(iii) Eсли $\Delta$ - кольцевая диаграмма и $\mathrm{S}$ - ее граничный цикл, то $t(\mathrm{~S}, \mathrm{~S})=0$, $t(\mathrm{~S}, \mathrm{R}) \leqslant 1$ для любого $\mathrm{R} \in \mathfrak{X}_{\beta}(\Delta), r(\mathrm{~S}) \leqslant \# y_{\beta}(\Delta)$ u $s(\mathrm{~S}) \leqslant \max \left\{1, \# y_{\beta}(\Delta)\right\}$.

(iv) Если $\Delta$ - диаграмма с тремя граничными ииклами и $\mathrm{S}$ - ее граничный цикл, то $t(\mathrm{~S}, \mathrm{~S}) \leqslant 1, \quad t(\mathrm{~S}, \mathrm{R}) \leqslant 1$, если $\mathrm{R}$ - ииклический участок, и $t(\mathrm{~S}, \mathrm{R}) \leqslant$ 2 , если $\mathrm{R}$ - нециклический участок. При этом каждый из двух отличных от $\mathrm{S}$ граничных ииклов диаграммы $\Delta$ содержит не более одного подпути $\mathrm{R} \in X_{\beta}(\Delta)$ ct $(\mathrm{S}, \mathrm{R})=2$. Кроме того, $r(\mathrm{~S}), s(\mathrm{~S}) \leqslant \# y_{\beta}(\Delta)+4$.

(v) Eсли $\Delta$ имеет четыре граничных цикла и все они принадлежат $X_{\beta}(\Delta)$, $\operatorname{mot}(\mathrm{S}, \mathrm{S}) \leqslant 3, t(\mathrm{~S}, \mathrm{R}) \leqslant 2$ для любого $\mathrm{R} \in X_{\beta}(\Delta) \backslash\{\mathrm{S}\}, \quad \sum_{\mathrm{R} \in X_{\beta}(\Delta) \backslash\{\mathrm{S}\}} t(\mathrm{~S}, \mathrm{R}) \leqslant 4$ $u r(\mathrm{~S}), s(\mathrm{~S}) \leqslant 10$.

ДоКАЗАТЕльСтво. Заметим, что оценка для $s(\mathrm{~S})$ в (iii) $-(\mathrm{v})$ вытекает из оценки для $r(\mathrm{~S})$, так как либо $\mathrm{S}$ является переходным участком диаграммы $\Delta$ относительно $\mathcal{S}$, и тогда $s(\mathrm{~S})=1$, либо $\mathrm{S}$ состоит из чередуюшихся переходных участков и участков примыкания поддиаграмм из $\mathcal{S}$, и в этом случае $s(\mathrm{~S})=r(\mathrm{~S})$.

(i) Допустим, $\Pi \in \mathcal{S}$ - поддиаграмма примыкания $\mathrm{S}$ к S. Пусть $\delta \Pi \approx \mathrm{PuQv}$, где $\mathrm{P}$ и $\mathrm{Q}$ - участки примыкания. Так как, очевидно, $\Pi \neq \Delta$, то один из путей u или $\mathrm{v}$, скажем $\mathrm{u},-$ невырожденная связка ранга $\beta$ на $\Delta$. Так как вершины $\iota(\mathrm{u})$ и $\tau(\mathrm{u})$ лежат на $\mathrm{S}$ и $\Delta$ - дисковая диаграмма, то путь и или и ${ }^{-1}$ гомотопен некоторому подпути участка границы S. Но это противоречит определению 9.11. Следовательно, $t(\mathrm{~S}, \mathrm{~S})=0$.

Допустим теперь, что для некоторого участка границы $\mathrm{R} \in \mathcal{X}_{\beta}(\Delta) \backslash\{\mathrm{S}\}$ имеются две различные поддиаграммы примыкания $\Pi_{1}, \Pi_{2} \in \mathcal{S}$ пути $S$ к пути $R$. Тогда, очевидно, для одной из трех поддиаграмм $\Theta \in \mathcal{T}\left(\Delta,\left\{\Pi_{1}, \Pi_{2}\right\}\right)$ граничный цикл $\Theta$ имеет вид $\mathrm{PuQv}$, где $\mathrm{P}$ входит в $\mathrm{S}, \mathrm{Q}$ входит в $\mathrm{R}, \mathrm{P}, \mathrm{Q} \in \mathfrak{X}_{\beta}(\Theta)$ и u, $v \in y_{\beta}(\Theta)$. Но это невозможно по лемме 9.20. Следовательно, $t(\mathrm{~S}, \mathrm{R}) \leqslant 1$ для любого $\mathrm{R} \in \mathcal{X}_{\beta}(\Delta)$.

Если $\# y_{\beta}(\Delta) \leqslant 1$, то по доказанному \#S $=0$ и, следовательно, $r(\mathrm{~S})=1$. Пусть $k \rightleftharpoons \# y_{\beta}(\Delta) \geqslant 2$. Пусть $\delta \Delta \approx \mathrm{Su}_{0} \mathrm{R}_{1} \mathrm{u}_{1} \ldots \mathrm{u}_{k-1} \mathrm{R}_{k} \mathrm{u}_{k}$, где $\mathrm{R}_{i} \in X_{\beta}(\Delta)$ и $\mathrm{u}_{i} \in$ $y_{\beta}(\Delta)$. Предположим, П $\in \mathcal{S}$ - поддиаграмма примыкания $\mathrm{S}$ к $\mathrm{R}_{1}$. Пусть $\delta \Pi \approx$ $\mathrm{PuQv}$, где $\mathrm{S}=\mathrm{S}^{\prime} \mathrm{PS}^{\prime \prime}$ и $\mathrm{R}_{1}=\mathrm{R}_{1}^{\prime} \mathrm{QR}_{1}^{\prime \prime}$. Если $\mathrm{u} \neq \mathrm{u}_{0}$, то для дисковой поддиаграммы $\Theta \in \mathcal{T}(\Delta,\{\Pi\})$ с граничным циклом $\delta \Theta \approx \mathrm{S}^{\prime \prime} \mathrm{u}_{0} \mathrm{R}_{1}^{\prime} \mathrm{u}^{-1}$ мы имели бы \# $\mathrm{y}_{\beta}(\Theta)=$ 


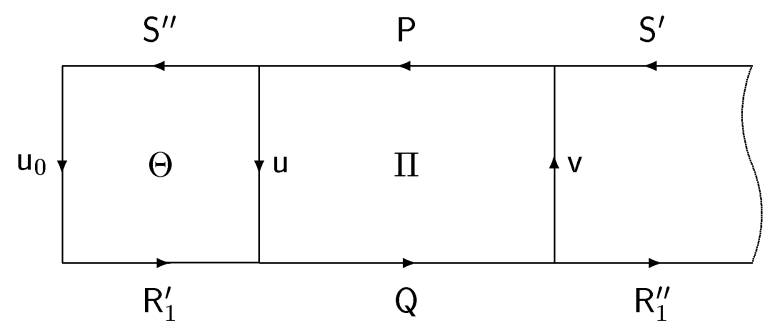

Рис. 18

2, что невозможно в силу леммы 9.20 (см. рис. 18). Следовательно, u $=\mathrm{u}_{0}$, и $\mathrm{P}$ есть конец S. Аналогичным образом замечаем, что если имеется поддиаграмма примыкания $\Pi \in \mathcal{S}$ пути $\mathcal{S}$ к пути $\mathrm{R}_{k}$, то соответствующий участок примыкания, входящий в $\mathrm{S}$, есть начало $\mathrm{S}$. Тогда из доказанного следует, что между первым и последним переходными участками диаграммы $\Delta$ относительно $\mathcal{S}$, входящими в $\mathrm{S}$, расположено не более $\max \left\{0, \# y_{\beta}(\Delta)-3\right\}$ участков примыкания. Следовательно, $s(\mathrm{~S}) \leqslant \max \left\{1, \# y_{\beta}(\Delta)-2\right\}$.

(ii) Пусть $\Delta$ - кольцевая диаграмма и $\mathrm{S} \in \mathcal{X}_{\beta}(\Delta)$ - нециклический участок. Пусть С и $\mathrm{D}$ - граничные циклы диаграммы $\Delta$, где $\mathrm{S}$ входит в $\mathrm{C}$.

Пусть $\mathcal{S}_{1} \subseteq \mathcal{S}$ - некоторое множество поддиаграмм примыкания участка гранищы $S$ к главным участкам ранга $\beta$ диаграммы $\Delta$. Заметим, что если среди $\mathcal{S}_{1}$ имеется хотя бы одна поддиаграмма примыкания $S$ к участку границы $\mathrm{R} \in X_{\beta}(\Delta)$, входящему в $\mathrm{D}$ или совпадающему с $\mathrm{D}$, то $\# \mathcal{T}\left(\Delta, \mathcal{S}_{1}\right)=\# \mathcal{S}_{1}$, причем каждая поддиаграмма $\Theta \in \mathcal{T}\left(\Delta, \mathcal{S}_{1}\right)$ является дисковой диаграммой. В противном случае, если

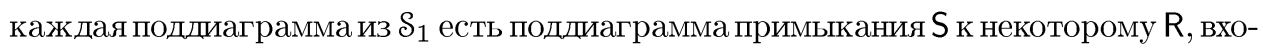
дящему в $C$, то $\# \mathcal{T}\left(\Delta, \mathcal{S}_{1}\right)=\# \mathcal{S}_{1}+1$, и в этом случае ровно одна из поддиаграмм $\Theta \in \mathcal{T}\left(\Delta, \mathcal{S}_{1}\right)$ является кольцевой, а остальные - дисковыми диаграммами.

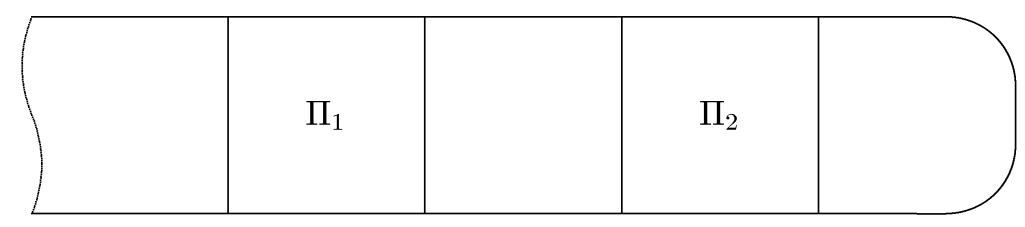

Рис. 19

Допустим, $t(\mathrm{~S}, \mathrm{~S}) \geqslant 2$, т.е. имеются две различные поддиаграммы примыкания $\Pi_{1}, \Pi_{2} \in \mathcal{S}$ пути $S$ к самому себе (см. рис. 19). Очевидно, по крайней мере для двух из трех поддиаграмм $\Theta \in \mathcal{T}\left(\Delta,\left\{\Pi_{1}, \Pi_{2}\right\}\right)$ верно утверждение:

один из граничных ииклов $\Theta$ имеет вид $\mathrm{PuQv}$ или $\mathrm{Pu}$, где $\mathrm{P} u \mathrm{Q}$ входят в $\mathrm{S}, \mathrm{P}, \mathrm{Q} \in \mathcal{X}_{\beta}(\Theta) u \mathrm{u}, \mathrm{v} \in \mathrm{y}_{\beta}(\Theta)$.

Одна из таких поддиаграмм $\Theta_{i}$ есть дисковая диаграмма. Но это противоречит лемме 9.20. Поэтому $t(\mathrm{~S}, \mathrm{~S}) \leqslant 1$. 
Допустим теперь, что $t(\mathrm{~S}, \mathrm{R}) \geqslant 2$ для некоторого циклического участка границы $\mathrm{R} \in \mathcal{X}_{\beta}(\Delta)$ или $t(\mathrm{~S}, \mathrm{R}) \geqslant 3$ для некоторого нециклического участка гранищы $\mathrm{R}$. Пусть $\mathcal{S}_{1} \rightleftharpoons\left\{\Pi_{i}\right\}_{i=1}^{k} \subseteq \mathcal{S}$ - множество поддиаграмм примыкания $\mathrm{S}$ к $\mathrm{R}$, где соответственно $k=2$ или $k=3$. Если $\mathrm{R}$ входит в $\mathrm{C}$ (и тогда $\mathrm{R}$ является нециклическим участком гранищы), то \# $\mathcal{T}\left(\Delta, \delta_{1}\right)=4$, причем по крайней мере для двух поддиаграмм $\Theta \in \mathcal{T}\left(\Delta, S_{1}\right)$ имеем

один из граничных ииклов $\Theta$ имеет вид $\mathrm{PuQv}$, где $\mathrm{P}$ входит в $\mathrm{S}$,

$\mathrm{Q}$ входит в $\mathrm{R}, \mathrm{P}, \mathrm{Q} \in X_{\beta}(\Theta) u \mathrm{u}, \mathrm{v} \in \mathrm{Y}_{\beta}(\Theta)$.

Тогда хотя бы одна из таких поддиаграмм $\Theta$ является дисковой диаграммой, и мы снова получаем противоречие с леммой 9.20 . Если $\mathrm{R}$ входит в $\mathrm{D}$ или совпадает $\mathrm{c} D$, то, как легко видеть, из соответствуюего предположения $k=3$ или $k=2$ следует, что хотя бы одна из поддиаграмм $\Theta \in \mathcal{T}\left(\Delta, \mathcal{S}_{1}\right)$ удовлетворяет условию (9.7), что опять противоречит лемме 9.20 . Следовательно, имеет место соответствуюшее неравенство $t(\mathrm{~S}, \mathrm{R}) \leqslant 1$ или $t(\mathrm{~S}, \mathrm{R}) \leqslant 2$.

Пусть, далее, один из граничных циклов диаграммы $\Delta$ содержит подпути $\mathrm{R}_{1}$, $\mathrm{R}_{2} \in X_{\beta}(\Delta)$ с $t\left(\mathrm{~S}, \mathrm{R}_{i}\right)=2(i=1,2)$. Пусть $\mathcal{S}_{1} \subseteq \mathcal{S}$ - множество всех поддиаграмм примыкания $\mathrm{S}_{\text {к }} \mathrm{R}_{1}$ или $\mathrm{R}_{2}$. Пусть $\mathrm{R}_{1}=\mathrm{U}_{0} \mathrm{P}_{1} \mathrm{U}_{1} \mathrm{P}_{2} \mathrm{U}_{2}$ и $\mathrm{R}_{2}=\mathrm{V}_{0} \mathrm{Q}_{1} \mathrm{~V}_{1} \mathrm{Q}_{2} \mathrm{~V}_{2}$, где $\mathrm{P}_{i}$, $\mathrm{Q}_{i}$ - участки примыкания поддиаграмм из $\mathcal{S}_{1}$, и пусть $\Theta_{1}, \Theta_{2} \in \mathcal{T}\left(\Delta, \mathcal{S}_{1}\right)$ - поддиаграммы, в граничные циклы которых входят соответственно пути $\mathrm{U}_{1}$ и $\mathrm{V}_{1}$. Если $\mathrm{R}_{1}$ и $\mathrm{R}_{2}$ входят в $\mathrm{C}$, то каждая из двух поддиаграмм $\Theta_{i}$ удовлетворяет условию $(9.7)$ соответственно при $\mathrm{R}:=\mathrm{R}_{1}$ и $\mathrm{R}:=\mathrm{R}_{2}$. Если $\mathrm{R}_{1}, \mathrm{R}_{2}$ входят в $\mathrm{D}$, то хотя бы одна $\Theta_{i}$ удовлетворяет условию (9.7). В любом случае для одной из поддиаграмм $\Theta_{i}$ имеем $\left(\chi\left(\Theta_{i}\right), \# y_{\beta}\left(\Theta_{i}\right)\right)=(1,2)$. Противоречие с леммой 9.20 .

Пусть $k_{i}(i=1,2)$ - число участков границы и $\in y_{\beta}(\Delta)$, входящих соответственно в $C$ и в $D$. Так как в $C$ входит ровно $k_{1}$ участков границы $\mathrm{R} \in X_{\beta}(\Delta)$, то по доказанному число поддиаграмм примыкания $\Pi \in \mathcal{S}$ участка границы $\mathrm{S}$ к участкам границы $\mathrm{R} \in \mathcal{X}_{\beta}(\Delta)$, входящим в $\mathrm{C}$ и отличным от $\mathrm{S}$, не превосходит $k_{1}$. Кроме того, в $\mathcal{S}$ имеется не более одной поддиаграммы примыкания $\mathrm{S}$ к $\mathrm{S}$. Далее, так как при $k_{2}=0$ имеем $\mathrm{D} \in X_{\beta}(\Delta)$, а при $k_{2}>0$ в $C$ входит ровно $k_{2}$ участков границы $\mathrm{R} \in X_{\beta}(\Delta)$, то по доказанному число поддиаграмм примыкания $\Pi \in \mathcal{S}$ участка границы $\mathrm{S}$ к участкам гранищы $\mathrm{R} \in \mathcal{X}_{\beta}(\Delta)$, входящим в $\mathrm{D}$, не превосходит $k_{2}+1$. Следовательно, в $\mathrm{S}$ входит не более $k_{1}+k_{2}+3=\# y_{\beta}(\Delta)+3$ участков примыкания поддиаграмм $\Pi \in \mathcal{S}$, и, таким образом, $r(\mathrm{~S}) \leqslant \# \mathrm{y}_{\beta}(\Delta)+4$. Утверждение (ii) доказано.

Утверждения (iii) и (iv) доказываются аналогично (ii): в случае, если не выполнена соответствующая оценка для $t(\mathrm{~S}, \mathrm{~S})$ или $t(\mathrm{~S}, \mathrm{R})$, легко видеть, что найдется дисковая поддиаграмма $\Theta \in \mathcal{T}\left(\Delta, \mathcal{S}_{1}\right), \mathcal{S}_{1} \subseteq \mathcal{S}$, удовлетворяюшая условию (9.6), и тогда мы приходим к противоречию с леммой 9.20. Оценка для $r(\mathrm{~S})$ вытекает из оценок для $t(\mathrm{~S}, \mathrm{~S})$ и $t(\mathrm{~S}, \mathrm{R})$.

(v) Допустим, $t(\mathrm{~S}, \mathrm{~S}) \geqslant 4$. Выберем подмножество $\mathcal{S}_{1}=\left\{\Pi_{i}\right\}_{i=1}^{4} \subseteq \mathcal{S}$ поддиаграмм примыкания $\mathrm{S}$ к $\mathrm{S}$. Пусть, для определенности, $\mathrm{S}$ есть внутренний граничный цикл диаграммы $\Delta$ и $\mathrm{D} \subseteq \mathbb{R}^{2}$ - диск, ограниченный кривой supp $\mathrm{S}$ (в случае, 
когда $\mathrm{S}$ есть внешний граничный цикл диаграммы $\Delta$, рассуждения аналогичны). Ясно, что если на плоскости $\mathbb{R}^{2}$ к диску D приклеено 4 "ручки" $\Pi_{i}(1 \leqslant i \leqslant 4)$, то не менее 4 -х связных компонент дополнения $\mathbb{R}^{2} \backslash\left(\mathrm{D} \cup \bigcup_{i=1}^{4} \Pi_{i}\right)$ граничат с $\mathrm{D}$, каждая не более чем по двум дугам. Отсюда следует, что по крайней мере 4 поддиаграммы $\Theta \in \mathcal{T}\left(\Delta, \mathcal{S}_{1}\right)$ удовлетворяют условию (9.6). Так как, помимо S, $\Delta$ содержит еще только три граничных цикла, вновь получаем противоречие с леммой 9.20.

Неравенство $t(\mathrm{~S}, \mathrm{R}) \leqslant 2$ при $\mathrm{R} \in X_{\beta}(\Delta) \backslash\{\mathrm{S}\}$ доказывается с помощью рассуждений, аналогичных использованным в доказательстве (ii).

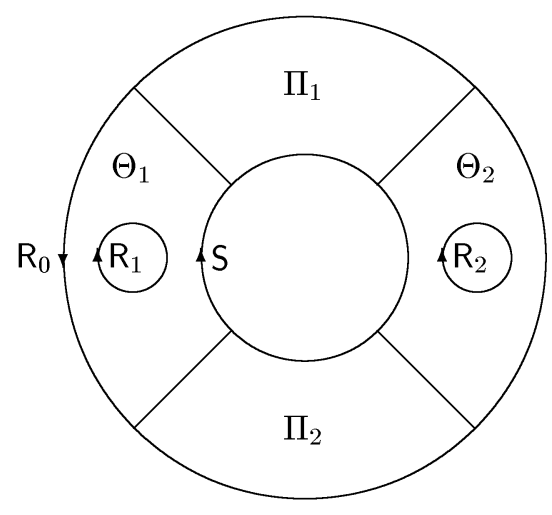

Рис. 20

Докажем, что $\sum_{\mathrm{R} \in X_{\beta}(\Delta) \backslash\{S\}} t(\mathrm{~S}, \mathrm{R}) \leqslant 4$. Если $t(\mathrm{~S}, \mathrm{R}) \leqslant 1$ для всех $\mathrm{R} \in X_{\beta}(\Delta) \backslash$ $\{\mathrm{S}\}$, то $\sum_{\mathrm{R} \in X_{\beta}(\Delta) \backslash\{\mathrm{S}\}} t(\mathrm{~S}, \mathrm{R}) \leqslant 3$. Пусть $\mathcal{X}_{\beta}(\Delta) \backslash\{\mathrm{S}\}=\left\{\mathrm{R}_{0}, \mathrm{R}_{1}, \mathrm{R}_{2}\right\}$ и $t\left(\mathrm{~S}, \mathrm{R}_{0}\right)=2$. Пусть $\Pi_{1}, \Pi_{2} \in \mathrm{S}$ - поддиаграммы примыкания $\mathrm{S}$ к $\mathrm{R}_{0}$. Каждая из двух поддиаграмм $\Theta_{1}, \Theta_{2} \in \mathcal{T}\left(\Delta,\left\{\Pi_{1}, \Pi_{2}\right\}\right)$ удовлетворяет условию (9.7) при $\mathrm{R}:=\mathrm{R}_{0}$, откуда по лемме 9.20 получаем, что $\mathrm{R}_{1}$ и $\mathrm{R}_{2}$ - граничные циклы различных поддиаграмм $\Theta_{1}$ и $\Theta_{2}$ (см. рис. 20). Тогда, воспользовавшись леммой 9.22 и доказанным утверждением (iii), получаем, что имеется не более одной поддиаграммы примыкания $\Pi \in \mathcal{S}$ граничного цикла $\mathrm{S}$ к каждому из граничных циклов $\mathrm{R}_{i}$. Следовательно, $\sum_{\mathrm{R} \in X_{\beta}(\Delta) \backslash\{\mathrm{S}\}} t(\mathrm{~S}, \mathrm{R}) \leqslant 4$, что и требовалось доказать.

Наконец, неравенство $r(\mathrm{~S}) \leqslant 10$ вытекает из неравенств $t(\mathrm{~S}, \mathrm{~S}) \leqslant 3$ и $\sum_{\mathrm{R} \in X_{\beta}(\Delta) \backslash\{S\}} t(\mathrm{~S}, \mathrm{R}) \leqslant 4$.

\section{$\S 10$. Оценки на диаграммах с разбиением границы}

В этом параграфе мы продолжим совместную индукцию по рангу $\alpha$, начатую в $\S 6-8$.

10.1. Лемма. Пусть $\Delta-B$-диаграмма ранга $\alpha u \Pi$ - поддиаграмма примыкания ранга $\alpha-1$ (рассматриваемая на $A$-диаграмме $\Delta^{\alpha-1}$ ранга $\left.\alpha-1\right)$ граничного иикла области $\mathrm{A} \in \mathbf{R}_{\alpha}(\Delta) \kappa$ некоторому главному участку $\mathrm{S}$ ранга $\alpha-1$ диаграммы $\Delta^{\alpha-1}$. Пусть $\mathcal{L}(\delta \mathrm{A}) \approx A^{n}$, әде $A \in \mathcal{E}_{\alpha}$, и пусть $\delta \Pi \approx \mathrm{PuQv}$, 
где $\mathrm{P}, \mathrm{Q}$ - участки примыкания, $\mathrm{P}$ входит в $\delta \mathrm{A} u \mathrm{Q}$ входит в $\mathrm{S}$. Тогда вернь следующие утверждения:

(i) $|\mathcal{L}(\mathrm{P})|<(n-q)|A|$, если $\mathrm{S} \in X_{\alpha}(\Delta)$;

(ii) если $\Delta$ - приведенная $B$-диаграмма ранга $\alpha$, mо $|\mathcal{L}(\mathrm{P})|<p|A|$ при $\mathrm{S} \notin$ $x_{\alpha}(\Delta)$, m.е. когда $\mathrm{S} \in Z_{\alpha}(\Delta)$ или $\mathrm{S}=\delta \mathrm{B}$ для некоторой области $\mathrm{B} \in \mathbf{R}_{\alpha}(\Delta)$.

ДокАЗАТЕЛЬСТво. Если $\mathrm{S} \in \mathcal{X}_{\alpha}(\Delta)$, то $(\mathcal{L}(\mathrm{Q}))^{-1}-$ подслово слова $(\mathcal{L}(\mathrm{S}))^{-1} \in$ $\mathcal{R}_{\alpha}$, и тогда неравенство $|\mathcal{L}(\mathrm{P})|<(n-q)|A|$ имеет место в силу определения 5.7 и леммы 9.14. Докажем утверждение (ii). Пусть $\Delta-$ приведенная $B$-диаграмма ранга $\alpha$. Допустим, что $|\mathcal{L}(\mathrm{P})| \geqslant p|A|$, и либо $\mathrm{S} \in \mathcal{Z}_{\alpha}(\Delta)$, либо $\mathrm{S}=\delta$ В для некоторой области $\mathrm{B} \in \mathbf{R}_{\alpha}(\Delta)$. Тогда, соответственно, для некоторого $B \in \mathcal{E}_{\alpha}$ либо $\mathcal{L}(\mathrm{S})$ есть подслово слова $B^{n}$, либо $\mathcal{L}(\mathrm{S}) \approx B^{n}$. Согласно определению 9.8 для вывода противоречия достаточно доказать, что $\Delta$ имеет либо сократимую пару областей ранга $\alpha$, либо граничную сократимую пару ранга $\alpha$, либо свернутую область ранга $\alpha$. Пусть $\delta \mathrm{A} \approx \mathrm{PR}$. Без ограничения обшности можно считать, что

$$
\mathcal{L}(\mathrm{PR}) \text { 드 } A^{n} \text { и } T \mathcal{L}(\mathrm{Q}) \text { 으 } B^{n} \text { для некоторого слова } T .
$$

По лемме 4.2 имеет место равенство

$$
\mathcal{L}(\mathrm{P}) \stackrel{\alpha=1}{=}(\mathcal{L}(\mathrm{v}))^{-1}(\mathcal{L}(\mathrm{Q}))^{-1}(\mathcal{L}(\mathrm{u}))^{-1}
$$

откуда в силу предложений $8.44,(\mathrm{i})$ и 8.37 следует $|\mathcal{L}(\mathrm{Q})|>2|B|$ и

$$
A^{n} \stackrel{\alpha=1}{=}(\mathcal{L}(\mathrm{v}))^{-1} B^{-n} \mathcal{L}(\mathrm{v})
$$

Рассмотрим два случая.

Случай 1: $\mathrm{S} \in \mathcal{Z}_{\alpha}(\Delta)$. Пусть $\Theta$ - дисковая поддиаграмма диаграммы $\Delta$, полученная объединением поддиаграммы П и области А (см. рис. 21).

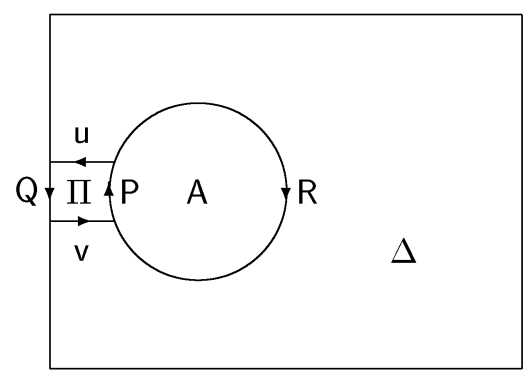

Рис. 21 
Из соотношений (10.1)-(10.3) выводим

$$
T \stackrel{\alpha=1}{=} B^{n}(\mathcal{L}(\mathrm{Q}))^{-1} \stackrel{\alpha=1}{=} \mathcal{L}(\mathrm{v})(\mathcal{L}(\mathrm{PR}))^{-1}(\mathcal{L}(\mathrm{v}))^{-1} \cdot \mathcal{L}(\mathrm{v}) \mathcal{L}(\mathrm{P}) \mathcal{L}(\mathrm{u}) \stackrel{\alpha=1}{=} \mathcal{L}\left(\mathrm{vR}^{-1} \mathrm{u}\right) .
$$

Тогда согласно определению 9.8 область А и участок границы $\mathrm{S}$ диаграммы $\Delta$ образуют граничную сократимую пару ранга $\alpha$.

Случай $2: \mathrm{S}=\delta \mathrm{B}$ для некоторой области $\mathrm{B} \in \mathbf{R}_{\alpha}(\Delta)$.

Рассмотрим два подслучая.

Случай $2 \mathrm{~A}: \mathrm{A} \neq \mathrm{B}$.

Пусть $\delta \mathrm{B} \approx \mathrm{QT}$. Тогда из соотношения $T \mathcal{L}(\mathrm{Q})$ 으 $B^{n}$ и неравенства $|\mathcal{L}(\mathrm{Q})|>$ $2|B|$ по лемме 2.1 , (ii), (iii) следует $T$ 으 $\mathcal{L}(\mathrm{T})$. Рассмотрим дисковую поддиаграмму $\Theta$ диаграммы $\Delta$, состоящую из областей $\mathrm{A}, \mathrm{B}$ и поддиаграммы П (см. рис. 22). Выше мы доказали равенство $T \stackrel{\alpha=1}{=} \mathcal{L}\left(\mathrm{vR}^{-1} \mathrm{u}\right)$, следовательно, $\mathcal{L}(\delta \Theta) \approx$ $\mathcal{L}\left(\mathrm{T}^{-1} \mathrm{vR}^{-1} \mathrm{u}\right) \stackrel{\alpha=1}{=} 1$. Тогда согласно определению 9.8 области $\mathrm{A}$ и $\mathrm{B}$ образуют сократимую пару областей ранга $\alpha$ на диаграмме $\Delta$.

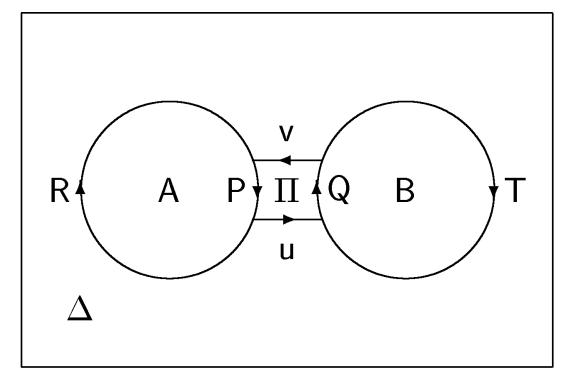

Рис. 22

Cлучай 2B: A = B.

Тогда $\mathrm{R}=\mathrm{MQN}$ для некоторых путей $\mathrm{M}$ и $\mathrm{N}$. Докажем, что $\mathrm{A}$ - свернутая область ранга $\alpha$. Рассмотрим кольцевую поддиаграмму $\Theta$ диаграммы $\Delta$, полученную объединением области А и поддиаграммы П (см. рис. 23). В силу определения 9.8 достаточно проверить, что слова $\mathcal{L}\left(\mathrm{N}^{-1} \mathrm{v}\right)$ и $\mathcal{L}\left(\mathrm{Mu}^{-1}\right)$ сопряжены в $\mathbf{B}_{\alpha-1}$ и $\left[\mathcal{L}\left(\mathrm{N}^{-1} \mathrm{v}\right)\right]_{\alpha-1}$ - элемент конечного порядка. В силу соотношений $(10.1)$ слово $B$ есть циклический сдвиг слова $A$ такой, что $B \mathcal{L}(\mathrm{N})$ 으 $\mathcal{L}(\mathrm{N}) A$. Тогда равенство (10.3) можно переписать в виде

$$
A^{n} \stackrel{\alpha=1}{=}\left(\mathcal{L}\left(\mathrm{N}^{-1} \mathbf{v}\right)\right)^{-1} A^{-n} \mathcal{L}\left(\mathrm{N}^{-1} \mathbf{v}\right) .
$$

Согласно определению $7.1\left[\mathcal{L}\left(\mathrm{N}^{-1} \mathrm{v}\right)\right]_{\alpha-1} \in \mathbf{N}_{\alpha-1}^{-}(A)$. В частности, в силу следствия 7.17 и предложения 7.14 , (ii) элемент $\left[\mathcal{L}\left(\mathrm{N}^{-1} \mathrm{v}\right)\right]_{\alpha-1}$ имеет конечный порядок. В силу предложения 7.18 , (iii) в группе $\mathbf{B}_{\alpha-1}$ имеет место равенство

$$
A^{n / 2} \stackrel{\alpha=1}{=}\left(\mathcal{L}\left(\mathrm{N}^{-1} \mathrm{v}\right)\right)^{-1} A^{-n / 2} \mathcal{L}\left(\mathrm{N}^{-1} \mathrm{v}\right),
$$


которое можно переписать в виде

$$
A^{n} \mathcal{L}\left(\mathrm{N}^{-1} \mathrm{v}\right) \stackrel{\alpha=1}{=} A^{n / 2} \mathcal{L}\left(\mathrm{N}^{-1} \mathrm{v}\right) A^{-n / 2}
$$

С другой стороны, используя равенство (10.2), видим, что

$$
\begin{aligned}
A^{n} \mathcal{L}\left(\mathrm{N}^{-1} \mathrm{v}\right) & \text { 으 } \mathcal{L}(\mathrm{PMQN}) \mathcal{L}\left(\mathrm{N}^{-1} \mathrm{v}\right) \stackrel{\alpha=1}{=} \\
& \stackrel{\alpha=1}{=} \mathcal{L}(\mathrm{P}) \mathcal{L}(\mathrm{M}) \mathcal{L}(\mathrm{QvP})(\mathcal{L}(\mathrm{P}))^{-1} \stackrel{\alpha=1}{=} \mathcal{L}(\mathrm{P}) \mathcal{L}\left(\mathrm{Mu}^{-1}\right)(\mathcal{L}(\mathrm{P}))^{-1} .
\end{aligned}
$$

Таким образом, слова $\mathcal{L}\left(\mathrm{N}^{-1} \mathrm{v}\right)$ и $\mathcal{L}\left(\mathrm{Mu}^{-1}\right)$ сопряжены в группе $\mathbf{B}_{\alpha-1}$ и, следовательно, А - свернутая область ранга $\alpha$. Лемма 10.1 доказана.

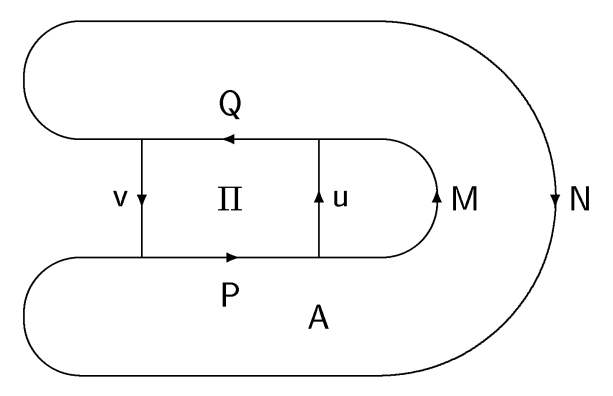

Рис. 23

10.2. ЛЕммА. Если $\Delta$ - простая А-диаграмма ранга 0 и $\left(\# y_{0}(\Delta), \chi(\Delta)\right) \neq$ $(2,1)$, то для кажсдого $\mathrm{S} \in \mathcal{X}_{0}(\Delta)$ слово $\mathcal{L}(\mathrm{S})$ пусто.

ДоказАтельство. Предположим, $\Delta$ - простая $A$-диаграмма ранга 0 и для некоторого $\mathrm{S} \in \mathcal{X}_{0}(\Delta)$ слово $\mathcal{L}(\mathrm{S})$ непусто. Докажем, что это возможно только в случае $\left(\# y_{0}(\Delta), \chi(\Delta)\right)=(2,1)$.

Пусть е е - ориентированное ребро с $\mathcal{L}\left(\mathrm{e}_{0}\right)$ 으 $a^{\epsilon} \in \mathcal{A}^{ \pm 1}$, входящее в S. Так как $\Delta$ - неособая диаграмма, то обратное ребро е ${ }_{0}^{-1}$ входит в граничный цикл ее некоторой области $\mathrm{A}_{0}$. Так как $\Delta$ - диаграмма ранга 0, то $\mathcal{L}\left(\delta \mathrm{A}_{0}\right) \approx a^{\epsilon} a^{-\epsilon}$, следовательно, $\delta \mathrm{A}_{0}$ имеет вид $\mathrm{M}_{0} \mathrm{e}_{0}^{-1} \mathrm{~N}_{0} \mathrm{e}_{1}$, где $\mathcal{L}\left(\mathrm{e}_{1}\right)$ 으 $a^{\epsilon}$ и $\mathcal{L}\left(\mathrm{M}_{0}\right)$ 으 $\mathcal{L}\left(\mathrm{N}_{0}\right)$ 으 1 . Если ориентированное ребро $\mathrm{e}_{1}^{-1}$ не входит ни в какой граничный цикл диаграммы $\Delta$, то для некоторой области $\mathrm{A}_{1} \in \mathbf{R}(\Delta)$ имеем $\delta \mathrm{A}_{1} \approx \mathrm{M}_{1} \mathrm{e}_{1}^{-1} \mathrm{~N}_{1} \mathrm{e}_{2}$, где $\mathcal{L}\left(\mathrm{e}_{2}\right)$ 으 $a^{\epsilon}$ и $\mathcal{L}\left(\mathrm{M}_{1}\right)$ 으 $\mathcal{L}\left(\mathrm{N}_{1}\right)$ 으 1 . Продолжая рассуждение, находим области $\mathrm{A}_{i}(0 \leqslant i \leqslant k)$ диаграммы $\Delta$ такие, что $\delta \mathrm{A}_{i} \approx \mathrm{M}_{i} \mathrm{e}_{i}^{-1} \mathrm{~N}_{i} \mathrm{e}_{i+1}, \mathcal{L}\left(\mathrm{e}_{i}\right)$ 으 $a^{\epsilon}, \mathcal{L}\left(\mathrm{M}_{i}\right)$ 으 $\mathcal{L}\left(\mathrm{N}_{i}\right)$ 으 1 для всех $i$ и $\mathrm{e}_{k+1}^{-1}$ входит в граничный цикл диаграммы $\Delta$. Так как согласно определениям 9.1 и 5.1 для любого и $\in y_{0}(\Delta)$ слово $\mathcal{L}(\mathrm{u})$ пусто, то е $k_{k+1}^{-1}$ входит в некоторый главный участок $\mathrm{R}$ ранга 0 диаграммы $\Delta$. Из построения легко видеть, что все ребра $\mathrm{e}_{i}$ и области $\mathrm{A}_{i}$ попарно различны. Произведем измельчение диаграммы $\Delta$, добавляя две новые вершины на каждом ребре $e_{i}$, так что при этом ребро е ${ }_{i}$ заменяется на произведение $\mathrm{d}_{i} \mathrm{e}_{i}^{\prime} \mathrm{f}_{i}$ трех peбер $\mathrm{d}_{i}, \mathrm{e}_{i}^{\prime}$ и $\mathrm{f}_{i} \mathrm{c} \mathcal{L}\left(\mathrm{d}_{i}\right)$ 으 $\mathcal{L}\left(\mathrm{f}_{i}\right)$ 으 1 
и $\mathcal{L}\left(\mathrm{e}_{i}^{\prime}\right)$ 드 $\mathcal{L}\left(\mathrm{e}_{i}\right)$ 으 $a^{\epsilon}$ (заметим, что в силу определений 9.12 и 9.4 при измельчениях простота $A$-диаграммы $\Delta$ ранга $\beta$ сохраняется). Добавим затем новые ребра $\mathrm{h}_{i}$ и $\mathrm{g}_{i}$ вдоль путей $\mathrm{d}_{i}^{-1} \mathrm{~N}_{i} \mathrm{~d}_{i+1}$ и $\mathrm{f}_{i+1} \mathrm{M}_{i} \mathrm{f}_{i}^{-1}$ соответственно для каждого $i=0,1, \ldots, k$ (см. рис. 24). Пусть $\mathrm{x} \rightleftharpoons \mathrm{h}_{0} \mathrm{~h}_{1} \ldots \mathrm{h}_{k}$ и у $\rightleftharpoons \mathrm{g}_{k} \mathrm{~g}_{k-1} \ldots \mathrm{g}_{0}$. Так как х и $\mathrm{y}-$ простые непустые пути и $\mathcal{L}(\mathrm{x})$ 으 $\mathcal{L}(\mathrm{y})$ 으 1 , то в силу определения 9.10 пути х и у являются связками ранга 0. В силу определения 9.12 х и у-вырожденные связки ранга $0 . \mathrm{B}$ соответствии с определением 9.11 рассмотрим два случая.

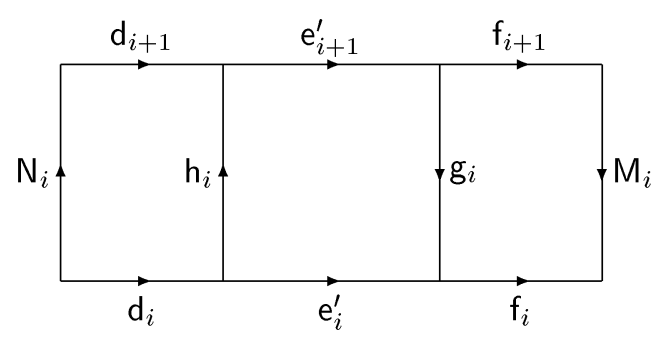

Рис. 24

Случай 1: Один из четырех путей, $\mathrm{x}^{ \pm 1}$ или $\mathrm{y}^{ \pm 1}$, гомотопен пути $\mathrm{P}$, входящему в качестве подпути в некоторый участок границы $\mathrm{S}^{\prime} \in \mathcal{X}_{0}(\Delta)$ (при этом, очевидно, $\mathrm{S}^{\prime}$ есть образ участка границы $\mathrm{S}$ при измельчении диаграммы $\left.\Delta\right)$. Пусть, например, путь $\mathrm{x}^{-1}$ гомотопен такому пути $\mathrm{P}$ (см. рис. 25). Тогда простой замкнутый путь Рх ограничивает дисковую поддиаграмму $\Theta$ диаграммы $\Delta$. По лемме 4.2 имеем $\mathcal{L}(\mathrm{P})=1$ в свободной группе $\mathbf{B}_{0}$, и так как в силу определений 9.1 и 5.7 слово $\mathcal{L}\left(\mathrm{S}^{\prime}\right)$ несократимо, то $\mathcal{L}(\mathrm{P})$ 으 1 . Но тогда в несократимое слово $\mathcal{L}\left(\mathrm{S}^{\prime}\right)$ входит слово $\mathcal{L}\left(\mathrm{e}^{\prime-1}{ }_{k+1} \mathrm{Pe}_{0}^{\prime}\right)$ 으 $a^{-\epsilon} a^{\epsilon}$. Получили противоречие. Аналогично доказывается невозможность всех остальных подслучаев.

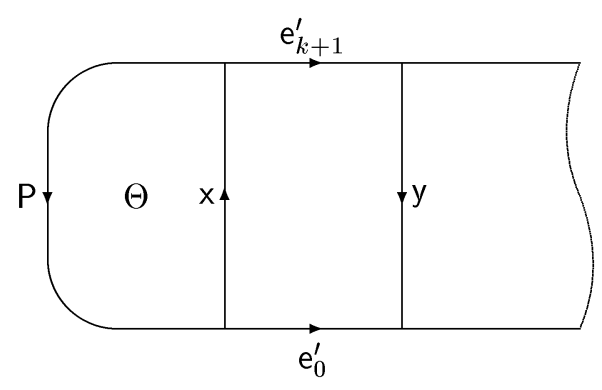

Рис. 25

Случай 2: При некоторых $\epsilon, \delta= \pm 1$ путь х ${ }^{\epsilon}$ гомотопен пути $\mathrm{P}_{1} \mathrm{u}_{1} \mathrm{Q}_{1}$, а путь $\mathrm{y}^{\delta}$ гомотопен пути $\mathrm{P}_{2} \mathrm{u}_{2} \mathrm{Q}_{2}$, где $\mathrm{P}_{i}$ - конец пути из $\mathcal{X}_{0}(\Delta), \mathrm{u}_{i} \in \mathrm{y}_{0}(\Delta), \mathrm{Q}_{i}-$ начало пути из $X_{0}(\Delta)$ и $\mathcal{L}\left(\mathrm{P}_{i}\right)$ 으 $\mathcal{L}\left(\mathrm{Q}_{i}\right)$ 드 1 . Так как $\mathcal{L}\left(\mathrm{P}_{1} \mathrm{u}_{1} \mathrm{Q}_{1}\right)$ 드 1 и $\mathcal{L}\left(\mathrm{e}_{0}^{\prime}\right)$ 드 $\mathcal{L}\left(\mathrm{e}^{\prime-1} k+1\right)$ 卉 1 , то ребра е $\mathrm{e}_{0}^{\prime}$ и $\mathrm{e}_{k+1}^{\prime-1}$ не входят в $\mathrm{P}_{1} \mathrm{u}_{1} \mathrm{Q}_{1}$, т.е. имеем $\epsilon=-1$ и путь $\mathrm{e}_{k+1}^{\prime-1} \mathrm{P}_{1} \mathrm{u}_{1} \mathrm{Q}_{1} \mathrm{e}_{0}^{\prime}$ входит в граничный цикл диаграммы $\Delta$. По той же причине имеем $\delta=-1$ и путь $\mathrm{e}_{0}^{\prime} \mathrm{P}_{2} \mathrm{u}_{2} \mathrm{Q}_{2} \mathrm{e}_{k+1}^{\prime-1}$ также входит в граничньй цикл диаграммы $\Delta$. Тогда некоторьй 
граничный цикл $\Delta$ имеет вид $\mathrm{P}_{1} \mathrm{u}_{1} \mathrm{Q}_{1} \mathrm{e}_{0}^{\prime} \mathrm{P}_{2} \mathrm{u}_{2} \mathrm{Q}_{2} \mathrm{e}_{k+1}^{\prime-1}$, где $\mathrm{Q}_{1} \mathrm{e}_{0}^{\prime} \mathrm{P}_{2}, \mathrm{Q}_{2} \mathrm{e}_{k+1}^{\prime-1} \mathrm{P}_{1} \in$ $X_{0}(\Delta)$ (см. рис. 26). Так как пути $\mathrm{P}_{1} \mathrm{u}_{1} \mathrm{Q}_{1} \mathrm{x}, \mathrm{P}_{2} \mathrm{u}_{2} \mathrm{Q}_{2} \mathrm{y}$ и $\mathrm{e}_{0}^{\prime} \mathrm{y}^{-1} \mathrm{e}_{k+1}^{\prime-1} \mathrm{x}^{-1}$ стягиваемы, то $\Delta$ - дисковая диаграмма. Следовательно, $\left(\# y_{0}(\Delta), \chi(\Delta)\right)=(2,1)$.

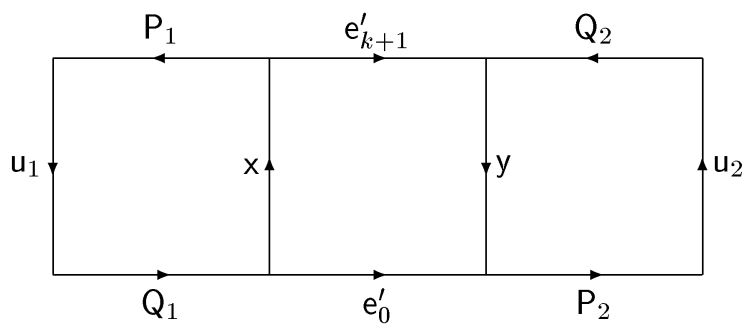

Рис. 26

10.3. В $\S 8$ был введен в рассмотрение вес $\mathcal{W}_{\beta}(X)$ слова $X$ в ранге $\beta$. Мы будем использовать эту функцию для оценки длин меток главных участков ранга $\beta$ $A$-диаграмм ранга $\beta$. Если $X$ есть метка циклического главного участка границы диаграммы $\Delta$, то слово $X$ определено с точностью до циклического сдвига. Для этого случая мы введем следуюшую модификацию функции $\mathcal{W}_{\beta}(X)$. Положим по определению

$$
\mathcal{W}_{\beta}^{\circ}(X) \rightleftharpoons \min \left\{\mathcal{W}_{\beta}(Y) \mid Y \text { есть циклический сдвиг слова } X\right\} .
$$

Если $S$ - участок гранищы диаграммы $\Delta$, то через $\overline{\mathcal{W}}_{\beta}(\mathcal{L}(\mathrm{S}))$ условимся обозначать значение функции $\mathcal{W}_{\beta}^{\circ}(\mathcal{L}(\mathrm{S}))$, если $\mathrm{S}$ - циклический участок границы, и $\mathcal{W}_{\beta}(\mathcal{L}(\mathrm{S}))$ в противном случае.

Следуюшее утверждение для удобства ссылок формулируется для ранга $\alpha-1$ (его формулировку для ранга $\alpha$ можно было бы поместить после предложения 10.11).

10.4. ПРЕДЛОЖЕНИЕ. Пусть $\Delta-A$-диаграмма ранга $\alpha-1, \quad \# y_{\alpha-1}(\Delta)-$ $2 \chi(\Delta) \geqslant 1$ и $\mathcal{S}$ - карта примыканий ранга $\alpha-1$ на $\Delta$. Пусть $\left\{\mathrm{S}_{1}, \mathrm{~S}_{2}, \ldots, \mathrm{S}_{r}\right\}-$ множество всех переходных участков диаграммы $\Delta$ относительно $\mathcal{S}$. Тогда

$$
\sum_{i=1}^{r} \overline{\mathcal{W}}_{\alpha-1}\left(\mathcal{L}\left(\mathrm{S}_{i}\right)\right) \leqslant \eta\left(\# y_{\alpha-1}(\Delta)-2 \chi(\Delta)\right)
$$

ДоКАЗАТЕЛЬСТВО. Напомним, что по определению

$$
\left\{\mathrm{S}_{i}\right\}_{i=1}^{r}=\bigcup_{\Theta \in \mathcal{T}(\Delta, \delta)} x_{\alpha-1}(\Theta) .
$$

В силу условия (ii) определения 9.18 каждая поддиаграмма $\Theta \in \mathcal{T}(\Delta, S)$ есть простая $A$-диаграмма ранга $\alpha-1$, а в силу леммы 9.20 имеем $\# y_{\alpha-1}(\Theta)-2 \chi(\Theta) \geqslant 1$. Если $\alpha=1$, то по лемме 10.2 все слова $\mathcal{L}\left(\mathrm{S}_{i}\right)$ пусты, что сразу влечет искомое неравенство (10.4). Если же $\alpha>1$, то неравенство (10.4) вытекает из индуктивного предположения 10.11 и леммы 9.16 . 
10.5. Лемма. Пусть $\Delta-B$-диаграмма ранга $\alpha,\left(\# y_{\alpha}(\Delta), \chi(\Delta)\right) \neq(0,1) u$ $\mathcal{S}$ - карта примыканий ранга $\alpha-1$ на $A$-диаграмме $\Delta^{\alpha-1}$ ранга $\alpha-1$. Предположим, что выполнены следующие условия (i) $и$ (ii):

(i) если $\mathrm{A} \in \mathbf{R}_{\alpha}(\Delta), \mathrm{S} \in z_{\alpha}(\Delta)$ или $\mathrm{S}=\delta \mathrm{B}$ для некоторой области $\mathrm{B} \in \mathbf{R}_{\alpha}(\Delta), \quad \Pi \in \mathcal{S}-$ поддиаграмма примыкания $\delta \mathrm{A} \kappa \mathrm{S}, \quad \mathrm{P}$ - ее участок примыкания, входящий в $\delta \mathrm{A}, u \mathcal{L}(\delta \mathrm{A}) \approx A^{n}$, где $A \in \mathcal{E}_{\alpha}$, mо $|\mathcal{L}(\mathrm{P})|<p|A|$;

(ii) для каждой области $\mathrm{A} \in \mathbf{R}_{\alpha}(\Delta)$ имеется не более одной поддиаграммы примыкания $\Pi \in \mathcal{S}$ граничного иикла $\delta \mathrm{A} \kappa$ главным участкам ранга $\alpha$ диаграмми $\Delta$.

Тогда если $\left(\# y_{\alpha}(\Delta), \chi(\Delta)\right) \neq(0,1)$, то имеет место неравенство

$$
\# \mathbf{R}_{\alpha}(\Delta) \leqslant \max \left\{0,0,35\left(\# y_{\alpha}(\Delta)-2 \chi(\Delta)\right)\right\} .
$$

ДокАЗАТЕЛЬСТво. Припишем каждому главному участку $\mathrm{S}$ ранга $\alpha-1$ диаграммы $\Delta^{\alpha-1}$ тип 1 , если $\mathrm{S} \in \mathcal{X}_{\alpha}(\Delta)$, тип 2 , если $\mathrm{S} \in \mathcal{Z}_{\alpha}(\Delta)$, и тип 3 , если $\mathrm{S}=\delta \mathrm{A}$ для некоторой области $\mathrm{A} \in \mathcal{Z}_{\alpha}(\Delta)$. Если $\mathrm{P}$ входит в $\mathrm{S}$ в качестве подпути, то пути $\mathrm{P}$ будем приписьвать тот же тип 1,2 или 3 , что и S. Введем обозначения:

$\mathcal{S}_{i j} \rightleftharpoons\{\Pi \in \mathcal{S} \mid$ участки примыкания П имеют типы $i$ и $j\}$,

$N \rightleftharpoons$ множество всех участков примыкания поддиаграмм примыкания $\Pi \in \mathcal{S}$,

$N_{i} \rightleftharpoons\{\mathrm{S} \in N \mid$ С имеет тип $i\}$,

$N_{i \mid j} \rightleftharpoons\left\{\mathrm{S} \in N_{i} \mid \mathrm{S}\right.$ входит в $\left.\delta \Pi, \Pi \in \mathcal{S}_{i j}\right\}$,

$N(\mathrm{~A}) \rightleftharpoons\{\mathrm{S} \in N \mid \mathrm{S}$ входит в $\delta \mathrm{A}\}\left(\mathrm{A} \in \mathbf{R}_{\alpha}(\Delta)\right)$,

$N_{i}(\mathrm{~A}) \rightleftharpoons N(\mathrm{~A}) \cap N_{i}$,

$P \rightleftharpoons \bigcup_{\Theta \in \mathcal{T}\left(\Delta^{\alpha-1,8}\right)} x_{\alpha-1}(\Theta)$ - множество всех переходных участков диаграммы $\Delta^{\alpha-1}$ относительно \&,

$P_{i} \rightleftharpoons\{\mathrm{S} \in P \mid \mathrm{S}$ имеет тип $i\}$,

$P(\mathrm{~A}) \rightleftharpoons\{\mathrm{S} \in P \mid \mathrm{S}$ входит в $\delta \mathrm{A}\}\left(\mathrm{A} \in \mathbf{R}_{\alpha}(\Delta)\right)$.

По условию имеем $\# y_{\alpha}(\Delta)-2 \chi(\Delta) \geqslant-1$. Можно считать, что $\# \mathbf{R}_{\alpha}(\Delta) \geqslant 1$. Тогда из (9.1) и (9.3) следует

$$
\# y_{\alpha-1}\left(\Delta^{\alpha-1}\right)-2 \chi\left(\Delta^{\alpha-1}\right) \geqslant 1 \text {. }
$$

Применив предложение 10.4 и снова используя соотношения $(9.1)$ и $(9.3)$, получаем

$$
\begin{aligned}
\sum_{\mathrm{S} \in P} \overline{\mathcal{W}}_{\alpha-1}(\mathcal{L}(\mathrm{S})) & \leqslant \eta\left(\# y_{\alpha-1}\left(\Delta^{\alpha-1}\right)-2 \chi\left(\Delta^{\alpha-1}\right)\right) \\
& \leqslant 2 \eta \# y_{\alpha}(\Delta)-2 \eta \chi(\Delta)+2 \eta \# \mathbf{R}_{\alpha}(\Delta)
\end{aligned}
$$

Пусть $\mathbf{R}_{\alpha}(\Delta)=\left\{\mathrm{A}_{1}, \mathrm{~A}_{2}, \ldots, \mathrm{A}_{t}\right\}$ и $\mathcal{L}\left(\delta \mathrm{A}_{i}\right) \approx A_{i}^{n}$, где $A_{i} \in \mathcal{E}_{\alpha}$. В силу леммы 10.1 , (i) имеем $|\mathcal{L}(\mathrm{S})|<(n-q)\left|A_{i}\right|$, если $\mathrm{S} \in N_{1}\left(\mathrm{~A}_{i}\right)$, и в силу условия (i) $|\mathcal{L}(\mathrm{S})|<p\left|A_{i}\right|$, если $\mathrm{S} \in N_{2}\left(\mathrm{~A}_{i}\right) \cup N_{3}\left(\mathrm{~A}_{i}\right)$. Следовательно,

$$
\frac{1}{\left|A_{i}\right|} \sum_{\mathrm{S} \in N\left(\mathrm{~A}_{i}\right)}|\mathcal{L}(\mathrm{S})| \leqslant(n-q) \# N_{1}\left(\mathrm{~A}_{i}\right)+p\left(\# N_{2}\left(\mathrm{~A}_{i}\right)+\# N_{3}\left(\mathrm{~A}_{i}\right)\right) .
$$


Далее, из предложения 8.22, (ii) следует

$$
\frac{1}{\left|A_{i}\right|} \sum_{\mathrm{S} \in P\left(\mathrm{~A}_{i}\right)}|\mathcal{L}(\mathrm{S})| \leqslant 13 \sum_{\mathrm{S} \in P\left(\mathrm{~A}_{i}\right)} \overline{\mathcal{W}}_{\alpha-1}(\mathcal{L}(\mathrm{S}))+2 \# P\left(\mathrm{~A}_{i}\right)
$$

Складывая полученные неравенства и учитывая, что

$$
\left|\mathcal{L}\left(\delta \mathrm{A}_{i}\right)\right|=n\left|A_{i}\right|=\sum_{\mathrm{S} \in N\left(\mathrm{~A}_{i}\right)}|\mathcal{L}(\mathrm{S})|+\sum_{\mathrm{S} \in P\left(\mathrm{~A}_{i}\right)}|\mathcal{L}(\mathrm{S})|
$$

и

$$
\# P\left(\mathrm{~A}_{i}\right) \leqslant \# N\left(\mathrm{~A}_{i}\right)+1=\# N_{1}\left(\mathrm{~A}_{i}\right)+\# N_{2}\left(\mathrm{~A}_{i}\right)+\# N_{3}\left(\mathrm{~A}_{i}\right)+1,
$$

получаем

$n \leqslant(n-q+2) \# N_{1}\left(\mathrm{~A}_{i}\right)+(p+2)\left(\# N_{2}\left(\mathrm{~A}_{i}\right)+\# N_{3}\left(\mathrm{~A}_{i}\right)\right)+13 \sum_{\mathrm{S} \in P\left(\mathrm{~A}_{i}\right)} \overline{\mathcal{W}}_{\alpha-1}(\mathcal{L}(\mathrm{S}))+2$,

откуда, просуммировав по всем областям $\mathrm{A}_{i}$, выводим, что

$$
(n-2) \# \mathbf{R}_{\alpha}(\Delta) \leqslant(n-q+2) \# N_{3 \mid 1}+(p+2)\left(\# N_{3 \mid 2}+\# N_{3 \mid 3}\right)+13 \sum_{\mathrm{S} \in P_{3}} \overline{\mathcal{W}}_{\alpha-1}(\mathcal{L}(\mathrm{S})) .
$$

Так как $\# N_{3 \mid 1} \leqslant \# \mathcal{S}_{13}, \# N_{3 \mid 2} \leqslant \# \mathcal{S}_{32}, \# N_{3 \mid 3} \leqslant 2 \# \mathcal{S}_{33}$, то отсюда следует

$$
(n-2) \# \mathbf{R}_{\alpha}(\Delta) \leqslant(p+2) \# \mathcal{S}+(n-q-p) \# \mathcal{S}_{13}+(p+2) \# \mathcal{S}_{33}+13 \sum_{\mathrm{S} \in P_{3}} \overline{\mathcal{W}}_{\alpha-1}(\mathcal{L}(\mathrm{S})) .
$$

По лемме 9.21

$$
\# \mathcal{S} \leqslant \# y_{\alpha-1}\left(\Delta^{\alpha-1}\right)-3 \chi\left(\Delta^{\alpha-1}\right) \leqslant 3 \# \mathbf{R}_{\alpha}(\Delta)+2 \# y_{\alpha}(\Delta)-3 \chi(\Delta) .
$$

В силу условия (ii) имеем

$$
\# \mathcal{S}_{13} \leqslant \# \mathbf{R}_{\alpha}(\Delta)
$$

Докажем неравенство

$$
\# \delta_{33} \leqslant-3 \chi\left(\Delta^{\alpha-1}\right) .
$$

Так как из предположения $\# \mathbf{R}_{\alpha}(\Delta) \geqslant 1$ следует $\chi\left(\Delta^{\alpha-1}\right) \leqslant 0$, то достаточно рассмотреть случай $\# \mathcal{S}_{33} \geqslant 1$. По лемме 9.16 при $\Delta:=\Delta^{\alpha-1}$ и $\mathcal{S}:=\mathcal{S}_{33}$

$$
\sum_{\Theta \in \mathcal{T}\left(\Delta^{\alpha-1}, \delta_{33}\right)}\left(\# y_{\alpha-1}(\Theta)-2 \chi(\Theta)\right)=\# y_{\alpha-1}\left(\Delta^{\alpha-1}\right)-2 \chi\left(\Delta^{\alpha-1}\right) .
$$

Обозначив $y_{\alpha-1}^{\prime}(\Theta) \rightleftharpoons y_{\alpha-1}(\Theta) \backslash y_{\alpha-1}\left(\Delta^{\alpha-1}\right)$, получим

$$
\sum_{\Theta \in \mathcal{T}\left(\Delta^{\alpha-1}, \S_{33}\right)}\left(\# y_{\alpha-1}^{\prime}(\Theta)-2 \chi(\Theta)\right)=-2 \chi\left(\Delta^{\alpha-1}\right) .
$$


Очевидно, при $\# \mathcal{S}_{33} \geqslant 1$ для каждой поддиаграммы $\Theta \in \mathcal{T}\left(\Delta^{\alpha-1}, \mathcal{S}_{33}\right)$ имеем $\# y_{\alpha-1}^{\prime}(\Theta) \geqslant 1$. Допустим, для некоторой поддиаграммы $\Theta \in \mathcal{T}\left(\Delta^{\alpha-1}, \mathcal{S}_{33}\right)$ выполнено $\# y_{\alpha-1}^{\prime}(\Theta)-2 \chi(\Theta) \leqslant 0$. Тогда $\chi(\Theta)=1$, т.е. $\Theta$ имеет ровно один граничный цикл. Так как каждый граничный цикл каждой поддиаграммы из $\mathcal{T}\left(\Delta^{\alpha-1}, \mathcal{S}_{33}\right)$ либо является граничным циклом диаграммы $\Delta$, либо не пересекается с границей $\Delta$, то в этом случае $\# y_{\alpha-1}^{\prime}(\Theta)=\# y_{\alpha-1}(\Theta)$, и мы приходим к противоречию с леммой 9.20. Следовательно, для любой подлиаграммы $\Theta \in \mathcal{T}\left(\Delta^{\alpha-1}, \mathcal{S}_{33}\right)$ выполнено $\# y_{\alpha-1}^{\prime}(\Theta)-2 \chi(\Theta) \geqslant 1$. Так как $\chi(\Theta) \leqslant 1$, то отсюда следует $1 / 2 \# y_{\alpha-1}^{\prime}(\Theta) \leqslant 3 / 2\left(\# y_{\alpha-1}^{\prime}(\Theta)-2 \chi(\Theta)\right)$, откуда, просуммировав по всем $\Theta$, получаем

$$
\# S_{33}=\frac{1}{2} \sum_{\Theta \in \mathcal{T}\left(\Delta^{\alpha-1}, S_{33}\right)} \# y_{\alpha-1}^{\prime}(\Theta) \leqslant \frac{3}{2}\left(-2 \chi\left(\Delta^{\alpha-1}\right)\right)=-3 \chi\left(\Delta^{\alpha-1}\right) .
$$

Тем самым доказано неравенство (10.8).

Подставляя полученные оценки для $\# \mathcal{S}, \# \mathcal{S}_{13}$ и $\# \mathcal{S}_{33}$ и оценку $(10.5)$ в $(10.7)$, с учетом неравенства $26 \eta<11$ получаем

$$
\begin{aligned}
& (n-2) \# \mathbf{R}_{\alpha}(\Delta) \\
& \leqslant(n-q+5 p+12) \# \mathbf{R}_{\alpha}(\Delta)+2(p+2) \# y_{\alpha}(\Delta)-6(p+2) \chi(\Delta)+13 \sum_{\mathrm{S} \in P_{3}} \overline{\mathcal{W}}_{\alpha-1}(\mathcal{L}(\mathrm{S})) \\
& \quad \leqslant(n-q+5 p+23) \# \mathbf{R}_{\alpha}(\Delta)+(2 p+15) \# y_{\alpha}(\Delta)-(6 p+23) \chi(\Delta),
\end{aligned}
$$

откуда, с учетом значений числовых параметров $p$ и $q$,

$$
\# \mathbf{R}_{\alpha}(\Delta) \leqslant \frac{6 p+23}{2(q-5 p-25)}\left(\# y_{\alpha}(\Delta)-2 \chi(\Delta)\right) \leqslant 0,35\left(\# y_{\alpha}(\Delta)-2 \chi(\Delta)\right) .
$$

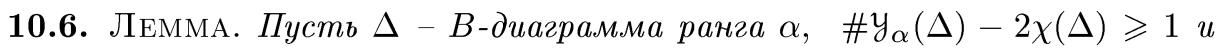
$\mathcal{S}$ - карта примыканий ранга $\alpha-1$ на $\Delta^{\alpha-1}$. Предположим, что выполнены условия (i), (ii) леммы 10.5 и следующее условие:

(i) $\mathcal{S}$ не содержит поддиаграмм примыкания главных участков ранга $\alpha$ диаграммы $\Delta$ кее участкам граничы $\mathrm{S} \in \mathcal{X}_{\alpha}(\Delta) \cup \mathcal{Z}_{\alpha}(\Delta)$.

Тогда имеет место неравенство

$$
\sum_{\mathrm{S} \in \mathcal{X}_{\alpha}(\Delta)} \overline{\mathcal{W}}_{\alpha}(\mathcal{L}(\mathrm{S})) \leqslant \eta\left(\# y_{\alpha}(\Delta)-2 \chi(\Delta)\right)
$$

ДокАЗАТЕЛЬСТво. Будем использовать обозначения, введенные в начале доказательства леммы 10.5. Так как каждый главньй участок ранга $\alpha$ диаграммы $\Delta$ либо лежит в $P_{1}$, либо является произведением чередуюшихся путей из $N_{1}$ и $P_{1}$, то по лемме 8.20 , (ii), (iii) имеем

$$
\sum_{\mathrm{S} \in \mathcal{X}_{\alpha}(\Delta)} \overline{\mathcal{W}}_{\alpha}(\mathcal{L}(\mathrm{S})) \leqslant \sum_{\mathrm{S} \in N_{1}} \mathcal{W}_{\alpha}(\mathcal{L}(\mathrm{S}))+\zeta \sum_{\mathrm{S} \in P_{1}} \overline{\mathcal{W}}_{\alpha-1}(\mathcal{L}(\mathrm{S}))
$$


Пусть $\Pi \in \mathcal{S}$ и $\delta \Pi \approx \mathrm{PuQv}$, где $\mathrm{P}, \mathrm{Q}$ - участки примыкания и $\mathrm{P}$ имеет тип 1 . Согласно условию (i) путь $\mathrm{Q}$ имеет тип 3 ; в частности, имеем $N_{1}=N_{1 \mid 3}$. Далее, так как $\mathcal{L}(\mathrm{Q})$ есть подслово некоторого слова $A^{n}$ при $A \in \mathcal{E}_{\alpha}$, то с учетом лемм 9.14 и $8.20,(\mathrm{i})$ имеем $\mathcal{W}_{\alpha}(\mathcal{L}(\mathrm{P})) \leqslant 1$. Заметим также, что из условия 10.5 , (ii) следует $\# N_{1 \mid 3} \leqslant \# \mathbf{R}_{\alpha}(\Delta)$. Следовательно,

$$
\sum_{\mathrm{S} \in N_{1}} \mathcal{W}_{\alpha}(\mathcal{L}(\mathrm{S})) \leqslant \# \mathbf{R}_{\alpha}(\Delta)
$$

Для оценки второго слагаемого $\zeta \sum_{\mathrm{S} \in P_{1}} \overline{\mathcal{W}}_{\alpha-1}(\mathcal{L}(\mathrm{S}))$ воспользуемся предложением 10.4 , посылка которого верна в силу неравенства \# $y_{\alpha}(\Delta)-2 \chi(\Delta) \geqslant 1$ и соотношений (9.1) и (9.3); имеем

$$
\begin{aligned}
\sum_{\mathrm{S} \in P_{1}} \overline{\mathcal{W}}_{\alpha-1}(\mathcal{L}(\mathrm{S})) & \leqslant \eta\left(\# y_{\alpha-1}\left(\Delta^{\alpha-1}\right)-2 \chi\left(\Delta^{\alpha-1}\right)\right) \\
& \leqslant 2 \eta\left(\# y_{\alpha}(\Delta)-2 \chi(\Delta)\right)+2 \eta \chi(\Delta)+2 \eta \# \mathbf{R}_{\alpha}(\Delta)
\end{aligned}
$$

В результате получаем

$$
\sum_{S \in X_{\alpha}(\Delta)} \overline{\mathcal{W}}_{\alpha}(\mathcal{L}(\mathrm{S})) \leqslant(1+2 \zeta \eta) \# \mathbf{R}_{\alpha}(\Delta)+2 \zeta \eta\left(\# y_{\alpha}(\Delta)-2 \chi(\Delta)\right)+2 \zeta \eta \chi(\Delta) .
$$

Если $\# \mathbf{R}_{\alpha}(\Delta)=0$, то искомое неравенство (10.9) легко следует из (10.11) с учетом неравенства $\chi(\Delta) \leqslant 1$. Пусть $\# \mathbf{R}_{\alpha}(\Delta) \geqslant 1$. Тогда из леммы 10.5 следует $\# y_{\alpha}(\Delta)-2 \chi(\Delta) \geqslant 3$. Подставляя оценку из леммы 10.5 в (10.11), получаем

$$
\sum_{\mathrm{S} \in \mathcal{X}_{\alpha}(\Delta)} \overline{\mathcal{W}}_{\alpha}(\mathcal{L}(\mathrm{S})) \leqslant(0,35(1+2 \zeta \eta)+2 \zeta \eta)\left(\# y_{\alpha}(\Delta)-2 \chi(\Delta)\right)+2 \zeta \eta \chi(\Delta) .
$$

Подставляя значения параметров $\zeta$ и $\eta$, легко убедиться, что при $\# y_{\alpha}(\Delta)-$ $2 \chi(\Delta) \geqslant 3$ значение выражения в правой части полученного неравенства меньше $\eta\left(\# y_{\alpha}(\Delta)-2 \chi(\Delta)\right)$.

10.7. Лемма. Пусть $\Delta$ - дисковая $B$-диаграмма ранга $\alpha$ и $\delta \Delta \approx \mathrm{Ru}$, где $x_{\alpha}(\Delta)=\{\mathrm{R}\}$ и $y_{\alpha}(\Delta)=\{\mathrm{u}\}$. Пусть $\mathcal{S}-$ карта примыканий ранга $\alpha-1$ на $\Delta^{\alpha-1}$. Предположим, $\Delta u \mathcal{S}$ удовлетворяют условию 10.5, (i). Тогда $\Delta$ не содерэит областей ранга $\alpha$.

ДокАЗАТЕльство. Допустим, лемма неверна. Выберем контрпример, состоящий из диаграммы $\Delta$ и карты примыканий $\mathcal{S}$ ранга $\alpha-1$ на $\Delta^{\alpha-1}$ так, чтобы значение $\# \mathbf{R}_{\alpha}(\Delta)$ было минимально возможным. Если $\Delta$ и $\mathcal{S}$ удовлетворяют условию 10.5 , (ii), то мы получаем противоречие с леммой 10.5. Нам остается вывести противоречие в случае, когда не выполнено условие 10.5, (ii). Допустим, условие 10.5 , (ii) не выполнено, т.е. для некоторой области $\mathrm{A} \in \mathbf{R}_{\alpha}(\Delta)$ граничный цикл $\delta$ A имеет две поддиаграммы примыкания $\Pi_{1}, \Pi_{2} \in \mathcal{S}$ к участку границы $\mathrm{R}$. Пусть 
$\delta \Pi_{i} \approx \mathrm{P}_{i} \mathrm{v}_{i} \mathrm{Q}_{i} \mathrm{w}_{i}(i=1,2)$, где $\mathrm{P}_{i}, \mathrm{Q}_{i}$ - участки примыкания, $\delta \mathrm{A} \approx \mathrm{P}_{1} \mathrm{~S}_{1} \mathrm{P}_{2} \mathrm{~S}_{2}$ и $\mathrm{R}=\mathrm{R}_{0} \mathrm{Q}_{1} \mathrm{R}_{1} \mathrm{Q}_{2} \mathrm{R}_{2}$ (см. рис. 27). Пусть $\Delta^{\prime}$ - дисковая поддиаграмма диаграммы $\Delta$ с граничным циклом $\delta \Delta^{\prime} \approx \mathrm{R}_{1} \mathrm{v}_{2}^{-1} \mathrm{~S}_{2} \mathrm{w}_{1}^{-1}$. Определим на $\Delta^{\prime}$ разбиение гранищы типа В, полагая

$$
x_{\alpha}\left(\Delta^{\prime}\right) \rightleftharpoons\left\{R_{1}\right\}, \quad y_{\alpha-1}\left(\Delta^{\prime}\right) \rightleftharpoons\left\{v_{2}^{-1}, w_{1}^{-1}\right\} \quad \text { и } \quad z_{\alpha}\left(\Delta^{\prime}\right) \rightleftharpoons\left\{S_{2}\right\}
$$

Так как, очевидно, $\Delta^{\prime \alpha-1} \in \mathcal{T}\left(\Delta^{\alpha-1},\left\{\Pi_{1}, \Pi_{2}\right\}\right)$, то по лемме 9.22 множество $\mathcal{S}_{1}$ подлиаграмм примыкания П $\in \mathcal{S}$, содержашихся в $\Delta^{\prime \alpha-1}$, образует карту примыканий ранга $\alpha-1$ на $\Delta^{\prime \alpha-1}$. Из выполнения условия 10.5 , (i) для $\Delta$ и $\mathcal{S}$ вытекает выполнение этого условия для $\Delta^{\prime}$ и $\mathcal{S}_{1}$. Но так как $\# \mathbf{R}_{\alpha}\left(\Delta^{\prime}\right)<\# \mathbf{R}_{\alpha}(\Delta)$, мы получаем противоречие в выбором $\Delta$ и $\mathcal{S}$.

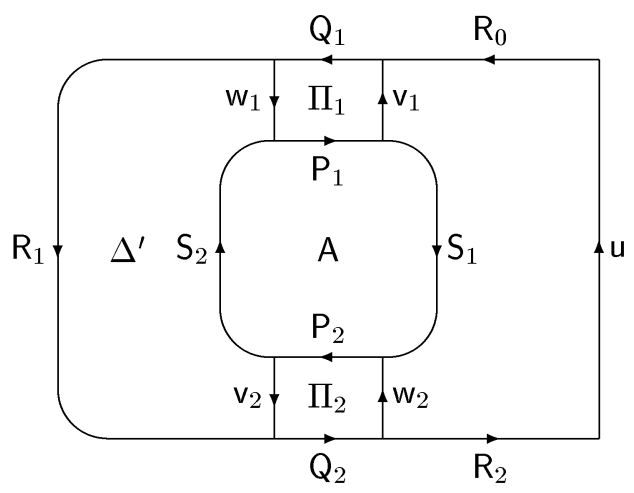

Рис. 27

10.8. ЛЕммА. Пусть $\Delta-B$-диаграмма ранга $\alpha$ и $\mathcal{S}$ - карта примыкания ранга $\alpha-1$ на $\Delta^{\alpha-1}$. Предположим, $\Delta$ и $\mathcal{S}$ удовлетворяют условию 10.5 , (i) (в частности, в силу леммы 10.1, (ii) әто верно в случае, если $\Delta$ есть приведенная $B$-диаграмма ранга $\alpha$ ). Тогда верны следуюшие утверждения.

(i) Пусть $\mathrm{A} \in \mathbf{R}_{\alpha}(\Delta) u \Pi_{1}, \Pi_{2} \in \mathcal{S}-$ две поддиаграммы примыкания граничного иикла области $\mathrm{A} к$ главному участку $\mathrm{S}$ ранга $\alpha$ диаграммы $\Delta$. Пусть $\delta \Pi_{i} \approx \mathrm{P}_{i} \mathrm{v}_{i} \mathrm{Q}_{i} \mathrm{w}_{i} \quad(i=1,2)$, где $\mathrm{P}_{i}, \mathrm{Q}_{i}$ - участки примыкания, $\mathrm{P}_{1} \mathrm{XP}_{2}$ входит в $\delta \mathrm{A} u \mathrm{Q}_{2} \mathrm{YQ}_{1}$ входит в $\mathrm{S}$ (см. рис. 28). Тогда поддиаграмма $\Delta^{\prime}$ диаграммы $\Delta$, ограниченная замкнутым путем $\mathrm{Xw}_{2}^{-1} \mathrm{Yv}_{1}^{-1}$, не является односвязной.

(ii) Пусть $\mathrm{S} \in \mathcal{X}_{\alpha}(\Delta)$ u $\Pi \in \mathcal{S}-$ поддиаграмма примыкания $\mathrm{S} \kappa \mathrm{S}$. Пусть $\delta \Pi \approx \mathrm{PvQw}$, где $\mathrm{P}, \mathrm{Q}$ - участки примыкания и $\mathrm{PYQ}$ входит в $\mathrm{S}$ (см. рис. 29). Тогда поддиаграмма $\Delta^{\prime}$ диаграммы $\Delta$, ограниченная замкнутым путем $\mathrm{Yv}^{-1}$, не является односвязной.

ДокАЗАТЕЛЬСТво. (i) Допустим, $\Delta^{\prime}$ - односвязная поддиаграмма. Определим на $\Delta^{\prime}$ разбиение границы типа $\mathrm{B}$, полагая $X_{\alpha}\left(\Delta^{\prime}\right) \rightleftharpoons\{\mathrm{Y}\}, y_{\alpha-1}\left(\Delta^{\prime}\right) \rightleftharpoons\left\{\mathrm{v}_{2}^{-1}, \mathrm{w}_{1}^{-1}\right\}$ и $Z_{\alpha}\left(\Delta^{\prime}\right) \rightleftharpoons\{\mathrm{X}\}$. Рассмотрев в силу леммы 9.22 карту примыканий ранга $\alpha-1$ на 


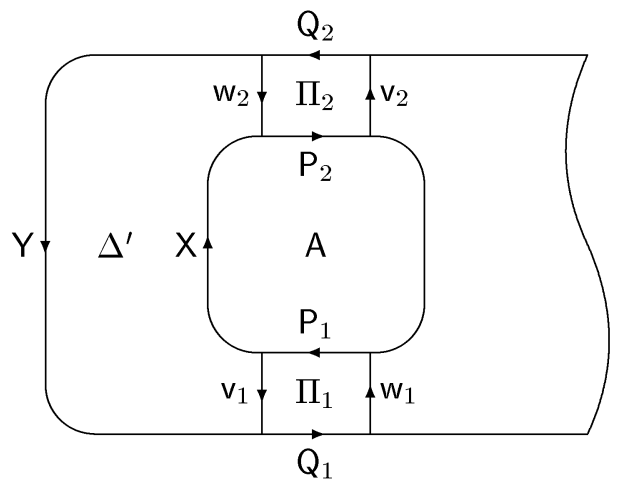

Рис. 28

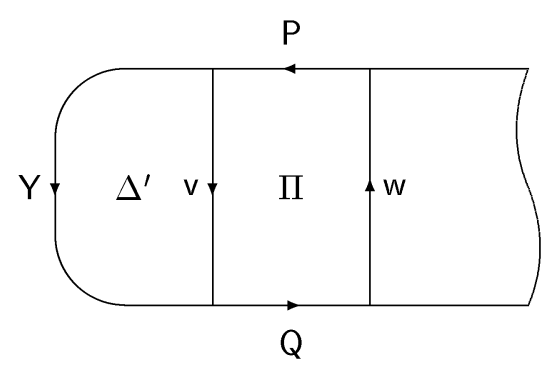

Рис. 29

$\Delta^{\prime \alpha-1}$, образованную поддиаграммами примыкания $\Pi \in \mathcal{S}$, содержашимися в $\Delta^{\prime}$, по лемме 10.7 получаем, что $\Delta^{\prime}$ не содержит областей ранга $\alpha$. Отсюда следует $\Delta^{\prime} \in \mathcal{T}\left(\Delta^{\alpha-1},\left\{\Pi_{1}, \Pi_{2}\right\}\right)$, и так как $\left(y_{\alpha-1}\left(\Delta^{\prime}\right), \chi\left(\Delta^{\prime}\right)\right)=(2,1)$, мы приходим к противоречию с леммой 9.20 .

Для доказательства (ii) проходят те же рассуждения, нужно лишь определить на диаграмме $\Delta^{\prime}$ разбиение гранищы ранга $\alpha$ типа $\mathrm{B}$, полагая $X_{\alpha}\left(\Delta^{\prime}\right) \rightleftharpoons\{\mathrm{Y}\}$, $y_{\alpha-1}\left(\Delta^{\prime}\right) \rightleftharpoons\left\{v^{-1}\right\}$ и $z_{\alpha}\left(\Delta^{\prime}\right) \rightleftharpoons \varnothing$.

10.9. ПРЕДЛОЖЕНИЕ. Пусть $\Delta$ - приведенная $B$-диаграмма ранга $\alpha$ и $\mathcal{S}$ карта примыканий ранга $\alpha-1$ на диаграмме $\Delta^{\alpha-1}$. Пусть $\mathrm{A} \in \mathbf{R}_{\alpha}(\Delta), u$ nусть $t(\mathrm{~S})$ обозначает число поддиаграмм примыкания $\Pi \in \mathcal{S}$ граничного иикла области А к главному участку S ранга $\alpha$ диаграммы $\Delta$. Тогда верны следующие утверәсдения.

(i) Eсли $\Delta-$ дисковая диаграмма, mо $t(\mathrm{~S}) \leqslant 1$ для любого $\mathrm{S} \in \mathcal{X}_{\alpha}(\Delta)$.

(ii) Если $\Delta$ - кольцевая диаграмма, то $t(\mathrm{~S}) \leqslant 1$, если $\mathrm{S}$ - ииклический участок граничь, и $t(\mathrm{~S}) \leqslant 2$, если $\mathrm{S}$ - нециклический участок граничь диаграммы $\Delta$. При әтом в каждый из двух граничных ииклов диаграммы $\Delta$ входит не более одного участка гранищы $\mathrm{S} \in X_{\alpha}(\Delta)$ с $t(\mathrm{~S})=2$.

(iii) Eсли $\Delta$ имеет три граничных иикла и все они принадлежат $X_{\alpha}(\Delta)$, $\operatorname{mot}(\mathrm{S}) \leqslant 2 u \sum_{\mathrm{S} \in X_{\alpha}(\Delta)} t(\mathrm{~S}) \leqslant 4$. 
ДоКАЗАТЕЛЬСТВо аналогично доказательству леммы 9.23 с использованием леммы 10.8, (i) вместо леммы 9.20.

10.10 Лемма. Пусть $\Delta, \Delta^{\prime}-A$-диаграммы ранга $\alpha$ и $\Delta^{\prime}=\Delta \cup \Theta$, әде имеет место один из двух случаев (i) или (ii):

(i) $\delta \Theta=\mathrm{P}^{-1} \mathrm{Q}, \quad \Delta \cap \Theta=\operatorname{supp} \mathrm{P}, \quad \mathrm{vPw} \in y_{\alpha}(\Delta) u y_{\alpha}\left(\Delta^{\prime}\right)=\left(y_{\alpha}(\Delta) \backslash\{\mathrm{vPw}\}\right) \cup$ $\{\mathrm{vQw}\}$ (рис. 30 слева);

(ii) $\delta \Theta=\mathrm{u}^{-1} \mathrm{Su}^{\prime} \mathrm{T}, \quad \Delta \cap \Theta=\operatorname{suppu}, \quad \mathrm{u} \in y_{\alpha}(\Delta), \quad \mathcal{L}(\mathrm{S})$ 드 $\mathcal{L}(\mathrm{T})$ 드 $1 u$ $y_{\alpha}\left(\Delta^{\prime}\right)=\left(y_{\alpha}(\Delta) \backslash\{\mathrm{u}\}\right) \cup\left\{\mathrm{u}^{\prime}\right\}$ (рис. 30 справа).

Тогда $\Delta$ - простая $A$-диаграмма ранга $\alpha$ в том и только том случае, если $\Delta^{\prime}$ - простая $A$-диаграмма ранга $\alpha$.

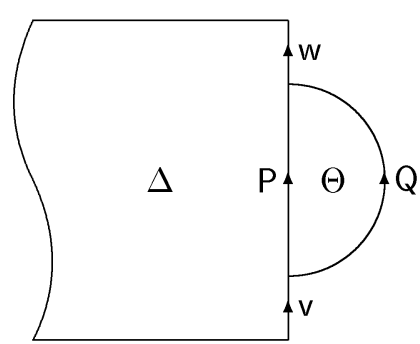

(i)

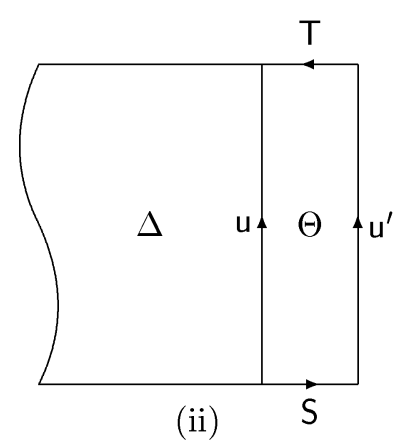

(ii)

Рис. 30

ДоКАЗАТЕЛЬСтво. Предположим, $\Delta$ не является простой $A$-диаграммой ранга $\alpha$. Тогда сушествует последовательность внутренних преобразований ранга $\alpha$, переводящая $\Delta$ в некоторую $A$-диаграмму $\Delta^{*}$ ранга $\alpha$, обладающую невырожденной связкой х ранга $\alpha$. В силу замечания 9.5 можно считать, что $\Delta^{*}$ есть поддиаграмма диаграммы $\Delta^{\prime *}$, полученной из $\Delta^{\prime}$ соответствующей последовательностью внутренних преобразований ранга $\alpha$. Тогда, как легко видеть из определений 9.10 и 9.11, x - невырожденная связка ранга $\alpha$ на $\Delta^{\prime *}$, и, следовательно, $\Delta^{\prime}$ не является простой $A$-диаграммой ранга $\alpha$.

Пусть $\Delta^{\prime}$ не является простой $A$-диаграммой ранга $\alpha$. Мы должны показать, что в этом случае и $\Delta$ не является простой. Заметим, что в силу определения 9.12 и замечания 9.5 свойство $A$-диаграммы ранга $\alpha$ быть простой или не простой сохраняется при выполнении операций измельчения. Поэтому, произведя утолшение пути $\mathrm{P}$, или, соответственно, и (см. 4.9), можно считать, что $\mathrm{P}$ (или и) входит в граничный цикл некоторой дисковой поддиаграммы $\Sigma$ диаграммы $\Delta$. Пусть $\Delta^{\prime \prime}$ - $A$-диаграмма ранга $\alpha$, полученная приклеиванием к $\Delta^{\prime}$ зеркальной копии $\Theta^{\prime}$ диаграммы $\Theta$, как изображено на рис. 31, где, соответственно, $\mathcal{L}\left(\mathrm{P}^{\prime}\right)$ 드 $\mathcal{L}(\mathrm{P})$, $y_{\alpha}\left(\Delta^{\prime \prime}\right)=\left(y_{\alpha}(\Delta) \backslash\{\mathrm{vPw}\}\right) \cup\left\{\mathrm{vP}^{\prime} \mathrm{w}\right\}$ в (i) и $\mathcal{L}\left(\mathrm{u}^{\prime \prime}\right)$ 드 $\mathcal{L}(\mathrm{u}), \mathcal{L}\left(\mathrm{S}^{\prime}\right)$ 드 $\mathcal{L}\left(\mathrm{T}^{\prime}\right)$ 으 1 , $y_{\alpha}\left(\Delta^{\prime \prime}\right)=\left(y_{\alpha}(\Delta) \backslash\{\mathrm{u}\}\right) \cup\left\{\mathrm{u}^{\prime \prime}\right\}$ в (ii). По доказанному при $\Delta:=\Delta^{\prime}$ и $\Delta^{\prime}:=\Delta^{\prime \prime}$ диаграмма $\Delta^{\prime \prime}$ не является простой. Но, с точностью до изотопной деформации, $\Delta^{\prime \prime}$ получается из $\Delta$ заменой дисковой поддиаграммы $\Sigma$ на $\Sigma \cup \Theta \cup \Theta^{\prime}$. Тогда из определения 9.12 следует, что $\Delta$ - не простая $A$-диаграмма ранга $\alpha$. 


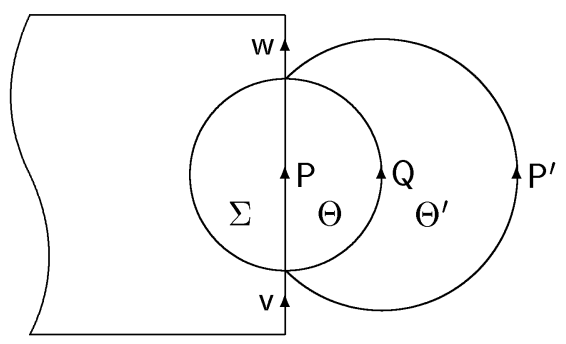

(i)

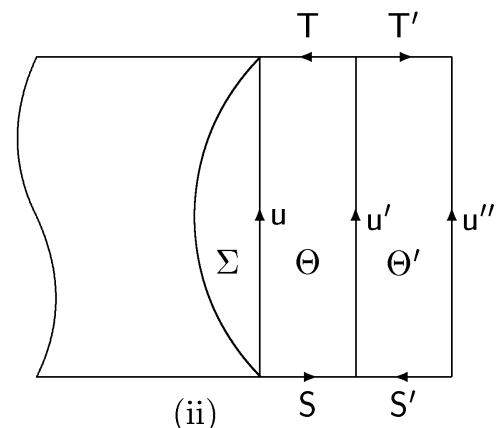

(ii)

Рис. 31

10.11. ПРЕДЛОЖЕНИЕ. Если $\Delta$ - простая А-диаграмма ранга $\alpha u$ $\# y_{\alpha}(\Delta)-2 \chi(\Delta) \geqslant 1, m o$

$$
\sum_{\mathrm{S} \in \mathcal{X}_{\alpha}(\Delta)} \overline{\mathcal{W}}_{\alpha}(\mathcal{L}(\mathrm{S})) \leqslant \eta\left(\# y_{\alpha}(\Delta)-2 \chi(\Delta)\right)
$$

ДоКАЗАТЕЛЬСтво. Без ограничения обшности можно считать, что для некоторого $\mathrm{S} \in X_{\alpha}(\Delta)$ слово $\mathcal{L}(\mathrm{S})$ непусто. Заметим, что в силу определения 9.12 простота диаграммы $\Delta$ сохраняется при внутренних преобразованиях ранга $\alpha$. В частности, произведя измельчение диаграммы $\Delta$, можно считать, что на $\Delta$ задано некоторое разбиение границы ранга $\alpha$ типа В. В силу леммы 10.10 , (i) простота $\Delta$ сохраняется при выполнении операции добавления области ранга $\alpha$. Будем считать, что на множестве всех простых (как $A$-диаграмма ранга $\alpha$ ) $B$-диаграмм ранга $\alpha$, обладающих данным набором параметров $\sum_{\mathrm{S} \in \mathcal{X}_{\alpha}(\Delta)} \overline{\mathcal{W}}_{\alpha}(\mathcal{L}(\mathrm{S})), \# y_{\alpha}(\Delta)$ и $\chi(\Delta)$, диаграмма $\Delta$ удовлетворяет следуюшим условиям:

(i) значение $\# \mathbf{R}_{\alpha}(\Delta)$ минимально возможное;

(ii) для данного значения $\# \mathbf{R}_{\alpha}(\Delta)$ значение $\# z_{\alpha}(\Delta)$ минимально возможное;

(iii) для данных значений $\# \mathbf{R}_{\alpha}(\Delta)$ и $\# Z_{\alpha}(\Delta)$ значение $\sum_{S \in X_{\alpha-1}\left(\Delta^{\alpha-1}\right)}|\mathcal{L}(\mathrm{S})|$ минимально возможное.

Тогда в силу предложения $9.9 \Delta$ - приведенная $B$-диаграмма ранга $\alpha$. Используя, кроме того, предложение 9.19 , можно считать, что на $\Delta^{\alpha-1}$ задана некоторая карта примыканий $\mathcal{S}$ ранга $\alpha-1$. В силу леммы 10.1 , (ii) $\Delta$ и $\mathcal{S}$ удовлетворяют условию 10.5, (i). Следовательно, в силу леммы 10.6 в этом случае нам достаточно проверить выполнение условий 10.5, (ii) и 10.6, (i). Допустим, хотя бы одно из этих условий не выполнено. Рассмотрим два случая.

Случай 1: Не выполнено условие 10.5 , (ii), т.е. для некоторой области $\mathrm{A} \in$ $\mathbf{R}_{\alpha}(\Delta)$ имеются две поддиаграммы примыкания $\Pi_{1}, \Pi_{2} \in \mathcal{S}$ граничного цикла $\delta \mathrm{A}$ к некоторым главным участкам ранга $\alpha$ диаграммы $\Delta$. Пусть $\delta \Pi_{i} \approx \mathrm{P}_{i} \mathrm{v}_{i} \mathrm{Q}_{i} \mathrm{w}_{i}$ $(i=1,2)$, где $\mathrm{P}_{i}$ и $\mathrm{Q}_{i}-$ участки примыкания, $\delta \mathrm{A} \approx \mathrm{P}_{1} \mathrm{~S}_{1} \mathrm{P}_{2} \mathrm{~S}_{2}$ и $\mathrm{Q}_{i}$ входит в $\mathrm{R}_{i} \in X_{\alpha}(\Delta)$ (см. рис. 32). Согласно определению 9.10 пути $\mathrm{x}_{1} \rightleftharpoons \mathrm{w}_{1} \mathrm{~S}_{2}^{-1} \mathrm{v}_{2}$ и $\mathrm{x}_{2} \rightleftharpoons \mathrm{w}_{2} \mathrm{~S}_{1}^{-1} \mathrm{v}_{1}$ являются связками ранга $\alpha$ на $\Delta$. Так как $\Delta$-простая $A$-диаграмма ранга $\alpha$, то $\mathrm{x}_{1}$ - вырожденная связка ранга $\alpha$ на $\Delta$. Из леммы 10.8 , (i) следует, 


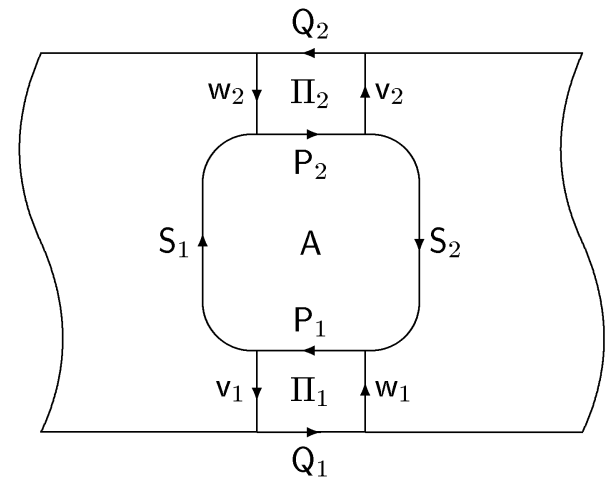

Рис. 32

что случай, когда путь $\mathrm{x}_{1}$ или $\mathrm{x}_{1}^{-1}$ гомотопен некоторому подпути участка границы $\mathrm{R}_{1}=\mathrm{R}_{2}$, невозможен. Рассмотрим остальные случаи из определения 9.11.

Случай 1A: Путь $x_{1}$ гомотопен пути $\mathrm{MuN}$, где $\mathrm{M}$ - конец пути $\mathrm{R}_{1}, \mathrm{~N}$ - начало пути $\mathrm{R}_{2}, \mathrm{u} \in \mathcal{Y}_{\alpha}(\Delta)$ и $\mathcal{L}(\mathrm{M})$ 으 $\mathcal{L}(\mathrm{N})$ 으 1 . Пусть $\Delta^{\prime} \rightleftharpoons \Delta-\Delta_{1}$, где $\Delta_{1}-$ дисковая поддиаграмма диаграммы $\Delta$ с граничным циклом $\delta \Delta_{1} \approx \mathrm{MuNv}_{2}^{-1} \mathrm{P}_{2}^{-1} \mathrm{~S}_{1}^{-1} \mathrm{P}_{1}^{-1} \mathrm{w}_{1}^{-1}$ (см. рис. 33). Пусть $R_{1}=R_{1}^{\prime} M, R_{2}=N R_{2}^{\prime}$ при $R_{1} \neq R_{2}, R_{1}=N R_{1}^{\prime} M$ при $\mathrm{R}_{1}=\mathrm{R}_{2}$, и пусть $\mathrm{u}=\mathrm{u}_{1} \mathrm{Tu}_{2}$, где $\mathrm{u}_{1}, \mathrm{u}_{2} \in \mathrm{y}_{\alpha-1}(\Delta)$ и $\mathrm{T} \in z_{\alpha}(\Delta)$, в случае, если и $\notin y_{\alpha}(\Delta)$. Определим на $\Delta^{\prime}$ разбиение границы ранга $\alpha$ типа $\mathrm{B}$, полагая $X_{\alpha}\left(\Delta^{\prime}\right) \rightleftharpoons\left(X_{\alpha}(\Delta) \backslash\left\{\mathrm{R}_{1}, \mathrm{R}_{2}\right\}\right) \cup\left\{\mathrm{R}_{1}^{\prime}, \mathrm{R}_{2}^{\prime}\right\}$ или $X_{\alpha}\left(\Delta^{\prime}\right) \rightleftharpoons\left(X_{\alpha}(\Delta) \backslash\left\{\mathrm{R}_{1}\right\}\right) \cup\left\{\mathrm{R}_{1}^{\prime}\right\}$ соответственно в случаях $\mathrm{R}_{1} \neq \mathrm{R}_{2}$ и $\mathrm{R}_{1}=\mathrm{R}_{2}$, и $y_{\alpha-1}\left(\Delta^{\prime}\right) \rightleftharpoons\left(y_{\alpha-1}(\Delta) \backslash\{\mathrm{u}\}\right) \cup$ $\left\{\mathrm{w}_{1}, \mathrm{v}_{2}\right\}, z_{\alpha}\left(\Delta^{\prime}\right) \rightleftharpoons z_{\alpha}(\Delta) \cup\left\{\mathrm{P}_{1} \mathrm{~S}_{1} \mathrm{P}_{2}\right\}$ или $\mathrm{y}_{\alpha-1}\left(\Delta^{\prime}\right) \rightleftharpoons\left(\mathrm{y}_{\alpha-1}(\Delta) \backslash\left\{\mathrm{u}_{1}, \mathrm{u}_{2}\right\}\right) \cup$ $\left\{\mathrm{w}_{1}, \mathrm{v}_{2}\right\}, Z_{\alpha}\left(\Delta^{\prime}\right) \rightleftharpoons\left(Z_{\alpha}(\Delta) \backslash\{\mathrm{T}\}\right) \cup\left\{\mathrm{P}_{1} \mathrm{~S}_{1} \mathrm{P}_{2}\right\}$ соответственно в случаях, когда $\mathrm{u} \in y_{\alpha}(\Delta)$ и и $\notin y_{\alpha}(\Delta)$. По лемме 10.10 , (ii) $\Delta^{\prime}$ - простая $A$-диаграмма ранга $\alpha$. Имеем $\# y_{\alpha}\left(\Delta^{\prime}\right)=\# y_{\alpha}(\Delta), \quad \chi\left(\Delta^{\prime}\right)=\chi(\Delta)$, и так как слова $\mathcal{L}(\mathrm{M})$ и $\mathcal{L}(\mathrm{N})$ пусты, то $\sum_{\mathrm{S} \in \mathcal{X}_{\alpha}\left(\Delta^{\prime}\right)} \overline{\mathcal{W}}_{\alpha}(\mathcal{L}(\mathrm{S}))=\sum_{\mathrm{S} \in \mathcal{X}_{\alpha}(\Delta)} \overline{\mathcal{W}}_{\alpha}(\mathcal{L}(\mathrm{S}))$. Но поскольку $\mathbf{R}_{\alpha}\left(\Delta^{\prime}\right) \leqslant \# \mathbf{R}_{\alpha}(\Delta)-1$, мы получаем противоречие с условием (i).

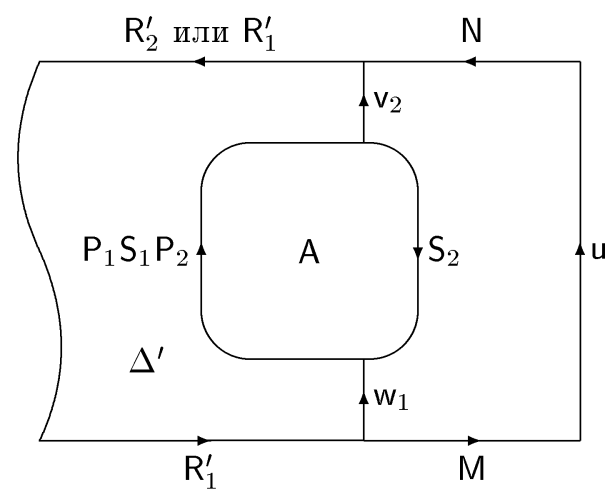

Рис. 33 
Случай 1В: Путь $\mathrm{x}_{1}^{-1}$ гомотопен пути $\mathrm{MuN}$, где $\mathrm{M}$ - конец пути $\mathrm{R}_{2}, \mathrm{~N}$ - начало пути $\mathrm{R}_{1}$, и $\in \mathrm{y}_{\alpha}(\Delta)$ и $\mathcal{L}(\mathrm{M})$ 으 $\mathcal{L}(\mathrm{N})$ 으 1 . Тогда для некоторых путей $\mathrm{M}_{1}$ и $\mathrm{N}_{1}$ имеем $\mathrm{M}=\mathrm{Q}_{2} \mathrm{M}_{1}, \mathrm{~N}=\mathrm{N}_{1} \mathrm{Q}_{1}$ и $\mathcal{L}\left(\mathrm{M}_{1}\right)$ 으 $\mathcal{L}\left(\mathrm{N}_{1}\right)$ 으 1 . Так как при этом путь $\mathrm{x}_{2}$ гомотопен пути $\mathrm{M}_{1} \mathrm{uN} \mathrm{N}_{1}$, то этот случай симметричен случаю $1 \mathrm{~A}$ с заменой $\mathrm{x}_{1}$ на $\mathrm{x}_{2}$.

Случай 2 : Не выполнено условие $10.6,(\mathrm{i})$, т.е. $\mathcal{S}$ содержит поддиаграмму примыкания $\Pi_{0}$ некоторого участка границы $\mathrm{R} \in X_{\alpha}(\Delta)$ к участку гранищы $\mathrm{S} \in X_{\alpha}(\Delta) \cup$ $z_{\alpha}(\Delta)$. Пусть $\delta \Pi_{0} \approx \mathrm{PvQw}$, где $\mathrm{P}, \mathrm{Q}$ - участки примыкания, $\mathrm{P}$ входит в $\mathrm{R}$ и $\mathrm{Q}$ входит в $\mathrm{S}$. Так как из неравенства $\# y_{\alpha}(\Delta)-2 \chi(\Delta) \geqslant 1$ следует $\Pi_{0} \neq \Delta$, то один из путей $v$ или $w$ является невырожденной связкой ранга $\alpha-1$ на $\Delta^{\alpha-1}$. Будем считать, что $v$ - невырожденная связка ранга $\alpha-1$ на $\Delta^{\alpha-1}$ (случай для w рассматривается аналогично). Рассмотрим два подслучая.

Случай $2 \mathrm{~A}: \mathrm{S} \in \mathcal{X}_{\alpha}(\Delta)$. Из определения 9.10 и леммы 5.12 следует, что v - связка ранга $\alpha$ на $\Delta$. Так как $\Delta$ - простая $A$-диаграмма ранга $\alpha$, то $v$ - вырожденная связка ранга $\alpha$ на $\Delta$. Как легко следует из леммы 10.8 , (ii), пути v и $v^{-1}$ не могут быть гомотопны никакому подпути главного участка ранга $\alpha$ диаграммы $\Delta$. Тогда при некотором $\epsilon= \pm 1$ путь $v^{\epsilon}$ гомотопен пути вида MuN, где $\mathrm{M}$ - конец пути $\mathrm{R}, \mathrm{N}$ - начало пути $\mathrm{S}, \mathrm{u} \in \mathfrak{y}_{\alpha}(\Delta)$ и $\mathcal{L}(\mathrm{M})$ 으 $\mathcal{L}(\mathrm{N})$ 으 1 . Пусть сначала $\epsilon=1$ (см. рис. 34 слева). Пусть $\Delta^{\prime} \rightleftharpoons \Delta-\Delta_{1}$, где $\Delta_{1}$ - дисковая поддиаграмма диаграммы $\Delta$ с граничным циклом $\delta \Delta_{1} \approx \mathrm{MuNv}^{-1}$. Пусть, как и в случае $1 \mathrm{~A}, \mathrm{R}=\mathrm{R}^{\prime} \mathrm{M}, \mathrm{S}=\mathrm{NS}^{\prime}$ при $\mathrm{R} \neq \mathrm{S}$, $\mathrm{R}=\mathrm{NR}^{\prime} \mathrm{M}$ при $\mathrm{R}=\mathrm{S}$, и, в случае $\mathrm{u} \notin \mathrm{y}_{\alpha-1}(\Delta), \mathrm{u}=\mathrm{u}_{1} \mathrm{Tu}_{2}$, где $\mathrm{u}_{1}, \mathrm{u}_{2} \in \mathrm{y}_{\alpha-1}(\Delta)$ и $\mathrm{T} \in z_{\alpha}(\Delta)$. Определим на $\Delta^{\prime}$ разбиение границы ранга $\alpha$ типа $\mathrm{B}$, полагая $X_{\alpha}\left(\Delta^{\prime}\right) \rightleftharpoons\left(X_{\alpha}(\Delta) \backslash\{\mathrm{R}, \mathrm{S}\}\right) \cup\left\{\mathrm{R}^{\prime}, \mathrm{S}^{\prime}\right\}$ при $\mathrm{R} \neq \mathrm{S}, X_{\alpha}\left(\Delta^{\prime}\right) \rightleftharpoons\left(X_{\alpha}(\Delta) \backslash\left\{\mathrm{R}_{1}\right\}\right) \cup\left\{\mathrm{R}_{1}^{\prime}\right\}$ при $\mathrm{R}=\mathrm{S}, y_{\alpha-1}\left(\Delta^{\prime}\right) \rightleftharpoons\left(y_{\alpha-1}(\Delta) \backslash\{\mathrm{u}\}\right) \cup\{\mathrm{v}\}, z_{\alpha}\left(\Delta^{\prime}\right) \rightleftharpoons z_{\alpha}(\Delta)$, если $\mathrm{u} \in y_{\alpha}(\Delta)$, и $y_{\alpha-1}\left(\Delta^{\prime}\right) \rightleftharpoons\left(y_{\alpha-1}(\Delta) \backslash\left\{\mathrm{u}_{1}, \mathrm{u}_{2}\right\}\right) \cup\{\mathrm{v}\}, z_{\alpha}\left(\Delta^{\prime}\right) \rightleftharpoons z_{\alpha}(\Delta) \backslash\{\mathrm{T}\}$, если u $\notin y_{\alpha}(\Delta)$. Как и случае $1 \mathrm{~A}$, получаем, что $\Delta^{\prime}$ - простая $A$-диаграмма ранга $\alpha$. Кроме того, имеем \# $y_{\alpha}\left(\Delta^{\prime}\right)=\# y_{\alpha}(\Delta), \chi\left(\Delta^{\prime}\right)=\chi(\Delta)$ и, так как слова $\mathcal{L}(\mathrm{M})$ и $\mathcal{L}(\mathrm{N})$ пусты, то $\sum_{\mathrm{s} \in \mathcal{X}_{\alpha}\left(\Delta^{\prime}\right)} \overline{\mathcal{W}}_{\alpha}(\mathcal{L}(\mathrm{S}))=\sum_{\mathrm{S} \in \mathcal{X}_{\alpha}(\Delta)} \overline{\mathcal{W}}_{\alpha}(\mathcal{L}(\mathrm{S}))$. По лемме 9.20 при $\Delta:=\Delta^{\alpha-1}$ и $\mathcal{S}_{1}:=\left\{\Pi_{0}\right\}$ невозможен случай, когда $\mathbf{R}_{\alpha}\left(\Delta_{1}\right)=\varnothing$ и $\mathrm{u} \in \mathfrak{y}_{\alpha-1}(\Delta)$. Пусть $\mathbf{R}_{\alpha}\left(\Delta_{1}\right) \neq \varnothing$ или $\mathbf{u} \notin y_{\alpha-1}(\Delta)$. Тогда $\# \mathbf{R}_{\alpha}\left(\Delta^{\prime}\right)<\# \mathbf{R}_{\alpha}(\Delta)$ или $\# y_{\alpha}\left(\Delta^{\prime}\right)<\# y_{\alpha}(\Delta)$ и, беря $\Delta^{\prime}$ в качестве $\Delta$, получаем противоречие с одним из условий (i) или (ii) выбора диаграммы $\Delta$.
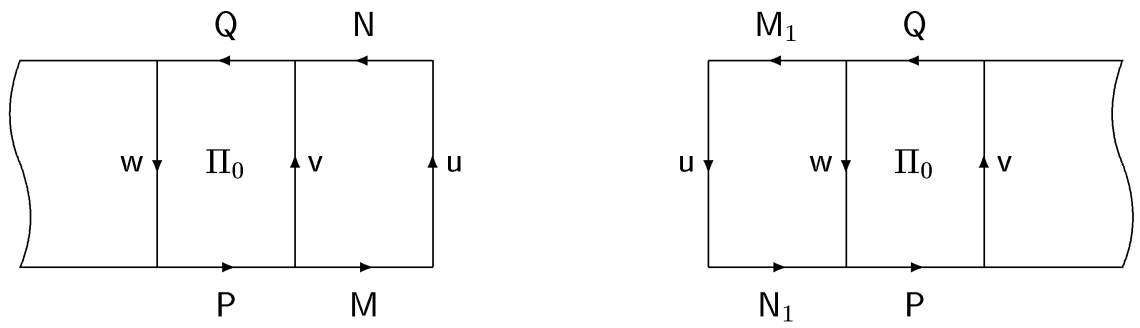

Рис. 34

Пусть теперь $\epsilon=-1$. Тогда $\mathrm{w} \notin \mathrm{y}_{\alpha}(\Delta)$, так как иначе получаем $\mathrm{M}=\mathrm{Q}, \mathrm{u}=\mathrm{w}$ и $\mathrm{N}=\mathrm{P}$, и, значит, $\mathrm{v}-$ вырожденная связка ранга $\alpha-1$ на $\Delta^{\alpha-1}$. Следовательно, 
$\mathrm{w}$ - невырожденная связка ранга $\alpha-1$ на $\Delta^{\alpha-1}$. В этом случае имеем $\mathrm{M}=\mathrm{QM}_{1}$ и $\mathrm{N}=\mathrm{N}_{1} \mathrm{P}$, где $\mathcal{L}\left(\mathrm{M}_{1}\right)$ 으 $\mathcal{L}\left(\mathrm{N}_{1}\right)$ 으 1 , пути ш и $\mathrm{M}_{1} \mathrm{uN}_{1}$ гомотопны (см. рис. 34 справа), и проходят симметричные рассуждения. Рассмотрение случая $2 \mathrm{~A}$ завершено.

Cлучай 2B: $\mathrm{Q}$ входит в некоторый участок границы $\mathrm{T} \in Z_{\alpha}(\Delta)$. Пусть пути $\mathrm{R}_{1} \mathrm{u}_{1} \mathrm{Tu}_{2}$ и $\mathrm{u}_{1} \mathrm{Tu}_{2} \mathrm{R}_{2}$ входят в граничный цикл диаграммы $\Delta$, где $\mathrm{u}_{1}, \mathrm{u}_{2} \in \mathrm{y}_{\alpha-1}(\Delta)$, $\mathrm{R}_{1}, \mathrm{R}_{2} \in X_{\alpha}(\Delta)(i=1,2)$ (возможно, $\left.\mathrm{R}_{1}=\mathrm{R}_{2}\right)$, и пусть $\mathrm{T}=\mathrm{T}_{1} \mathrm{QT}_{2}$ ( $\mathrm{T}_{1}$ - пустой путь в случае $\left.\mathrm{v} \in \mathrm{y}_{\alpha-1}(\Delta)\right)$. Добавив к $\Delta$ две новые вершины, лежашие соответственно на $R_{1}$ и $R_{2}$, можно считать, что ребро е 1 есть конец пути $R_{1}$ и ребро е 2 есть начало пути $\mathrm{R}_{2}$, где $\mathcal{L}\left(\mathrm{e}_{1}\right)$ 으 $\mathcal{L}\left(\mathrm{e}_{2}\right)$ 으 1 . Произведя далее измельчение диаграммы $\Delta$, как описано в п. 4.9 , можно считать, что вершины $\iota\left(\mathrm{e}_{1}\right)$ и $\tau(\mathrm{w})$ соединены простым путем $\mathrm{x}_{1} \mathrm{c} \mathcal{L}\left(\mathrm{x}_{1}\right)$ 으 $\mathcal{L}\left(\mathrm{u}_{1} \mathrm{~T}_{1} \mathrm{Qw}\right)$, а вершины $\tau\left(\mathrm{e}_{2}\right)$ и $\tau(\mathrm{w})$ соединены простым путем $\mathrm{x}_{2} \mathrm{c} \mathcal{L}\left(\mathrm{x}_{2}\right)$ 으 $\mathcal{L}\left(\mathrm{u}_{2}^{-1} \mathrm{~T}_{2}^{-1} \mathrm{w}\right)$, причем $\mathrm{x}_{1}$ и $\mathrm{x}_{2}$ не имееют отличных от $\iota\left(\mathrm{x}_{i}\right)$ и $\tau\left(\mathrm{x}_{i}\right)$ вершин в пересечении с гранищей диаграммы $\Delta$ (см. рис. 35). Согласно определению 9.10 пути х 1 и х 2 являются связками ранга $\alpha$ на $\Delta$, а в силу простоты $\Delta$ - вырожденными связками ранга $\alpha$ на $\Delta$. Заметим, что пути $\mathrm{x}_{1}$ и $\mathrm{x}_{2}^{-1}$ не могут быть гомотопны путям, входяшим в качестве подпутей в главные участки ранга $\alpha$ диаграммы $\Delta$. Рассмотрим остальные случаи из определения 9.11.

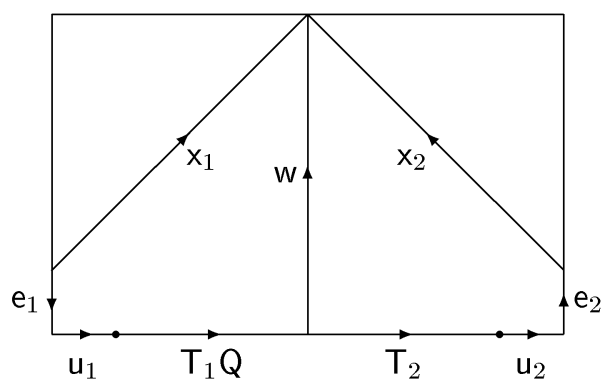

Рис. 35

Cлучай 2B1: $\mathrm{R}=\mathrm{R}_{2}$ и путь $\mathrm{x}_{1}$ гомотопен пути вида е $\mathrm{e}_{1} \mathrm{ue}_{2} \mathrm{M}$, где $\mathrm{e}_{2} \mathrm{M}$ - начало $\mathrm{R}$ и $\mathcal{L}(\mathrm{M})$ 으 1 . Пусть $\Delta^{\prime} \rightleftharpoons \Delta-\Delta_{1}$, где $\Delta_{1}$ - дисковая поддиаграмма диаграммы $\Delta$ с граничным циклом $\delta \Delta_{1} \approx \mathrm{e}_{2} \mathrm{Mw}^{-1} \mathrm{~T}_{2} \mathrm{u}_{2}$. Определим на $\Delta^{\prime}$ разбиение границы ранга $\alpha$ типа В, полагая

$$
\begin{aligned}
x_{\alpha}\left(\Delta^{\prime}\right) & \rightleftharpoons\left(X_{\alpha}(\Delta) \backslash\{\mathrm{R}\}\right) \cup\left\{\mathrm{R}^{\prime}\right\}, \quad \text { где } \mathrm{R}=\mathrm{e}_{2} \mathrm{MR}^{\prime}, \\
y_{\alpha-1}\left(\Delta^{\prime}\right) & \rightleftharpoons\left(\mathcal{Y}_{\alpha-1}(\Delta) \backslash\left\{\mathrm{u}_{2}\right\}\right) \cup\{\mathrm{w}\}, \\
z_{\alpha}\left(\Delta^{\prime}\right) & \rightleftharpoons\left(\mathcal{Z}_{\alpha}(\Delta) \backslash\{\mathrm{T}\}\right) \cup\left\{\mathrm{T}_{1} \mathrm{Q}\right\} .
\end{aligned}
$$

Как и выше, получаем, что $\Delta^{\prime}$ - простая $A$-диаграмма ранга $\alpha$. Если $\mathbf{R}_{\alpha}\left(\Delta_{1}\right) \neq \varnothing$, то $\# \mathbf{R}_{\alpha}\left(\Delta^{\prime}\right)<\# \mathbf{R}_{\alpha}(\Delta)$ вопреки условию (i) выбора $\Delta$. Если же $\mathbf{R}_{\alpha}\left(\Delta_{1}\right)=\varnothing$, то в силу невырожденности связки ш ранга $\alpha-1$ на $\Delta^{\alpha-1}$ имеем $\mathcal{L}\left(\mathrm{T}_{2}\right) \not{f} 1$, и поэтому $\sum_{\mathrm{S} \in X_{\alpha-1}\left(\Delta^{\prime \alpha-1}\right)}|\mathcal{L}(\mathrm{S})|<\sum_{\mathrm{S} \in X_{\alpha-1}\left(\Delta^{\alpha-1}\right)}|\mathcal{L}(\mathrm{S})|$. Так как при этом \# $\mathbf{R}_{\alpha}\left(\Delta^{\prime}\right)=$ $\# \mathbf{R}_{\alpha}(\Delta)$ и \#站 $\left(\Delta^{\prime}\right)=\# z_{\alpha}(\Delta)$, получаем противоречие с условием (iii). 
Случай 2B2: $\mathrm{R}=\mathrm{R}_{1}$ и путь $x_{2}^{-1}$ гомотопен пути вида $\mathrm{Ne}_{1}$ uе $_{2}$, где $\mathrm{Ne}_{1}-$ конец пути $\mathrm{R}$ и $\mathcal{L}(\mathrm{N})$ 으 1 . В этом случае, взяв в качестве $\Delta_{1}$ дисковую поддиаграмму диаграммы $\Delta$ с граничным циклом $\delta \Delta_{1} \approx \mathrm{Ne}_{1} \mathrm{uT}_{1} \mathrm{Qw}$, приходим к противоречию аналогично случаю $2 \mathrm{~B} 1$.

Случай 2B3: $\mathrm{R}=\mathrm{R}_{1}$ и путь $\mathrm{x}_{1}^{-1}$ гомотопен некоторому подпути $\mathrm{M}$ пути $\mathrm{R}$. Так как по условию имеет место хотя бы одно из неравенств $\chi(\Delta) \leqslant 0$ или \# $y_{\alpha}(\Delta) \geqslant 3$, то в этом случае путь $\mathrm{x}_{2}$ не гомотопен никакому подпути главного участка ранга $\alpha$ диаграммы $\Delta$ и никакому пути вида $\mathrm{M}^{\prime} \mathrm{u}^{\prime} \mathrm{N}^{\prime}$, где $\mathrm{M}^{\prime}$ и $\mathrm{N}^{\prime}$ - соответственно конец и начало путей из $X_{\alpha}(\Delta)$ и $\mathrm{u}^{\prime} \in \mathcal{Y}_{\alpha}(\Delta)$. Отсюда следует, что имеет место уже рассмотренный случай $2 \mathrm{~B} 2$.

Случай 2B4: $\mathrm{R}=\mathrm{R}_{2}$ и путь $\mathrm{x}_{2}$ гомотопен некоторому подпути Мпути $\mathrm{R}$. Подобно случаю $2 \mathrm{~B} 3$, этот случай сводится к случаю $2 \mathrm{~B} 1$.

Случай 2В5: Ни один из случаев 2B1-2B4 ни имеет места. Тогда для $\mathrm{x}_{1}$ и $\mathrm{x}_{2}$ остаются единственные возможности из определения 9.11: пути $\mathrm{x}_{1}^{-1}$ их $\mathrm{x}_{2}$ гомотопны путям $\mathrm{M}_{1} \mathrm{y}_{1} \mathrm{~N}_{1}$ и $\mathrm{M}_{2} \mathrm{y}_{2} \mathrm{~N}_{2}$ соответственно, где $\mathrm{R}=\mathrm{N}_{2} \mathrm{M}_{1}, \mathrm{R}_{1}=\mathrm{N}_{1} \mathrm{e}_{1}, \mathrm{R}_{2}=\mathrm{e}_{2} \mathrm{M}_{2}$, $\mathrm{y}_{1}, \mathrm{y}_{2} \in y_{\alpha}(\Delta)$ и $\mathcal{L}\left(\mathrm{M}_{i}\right)$ 드 $\mathcal{L}\left(\mathrm{N}_{i}\right)$ 드 $1(i=1,2)$ (см. рис. 36) Так как в этом случае $\Delta$ - дисковая диаграмма, то $X_{\alpha}(\Delta)=\left\{\mathrm{R}, \mathrm{R}_{1}, \mathrm{R}_{2}\right\}$, и мы получаем противоречие со сделанным в начале доказательства предположением о непустоте слова $\mathcal{L}(\mathrm{S})$ хотя бы одного $\mathrm{S} \in \mathcal{X}_{\alpha}(\Delta)$. Предложение 10.11 доказано.

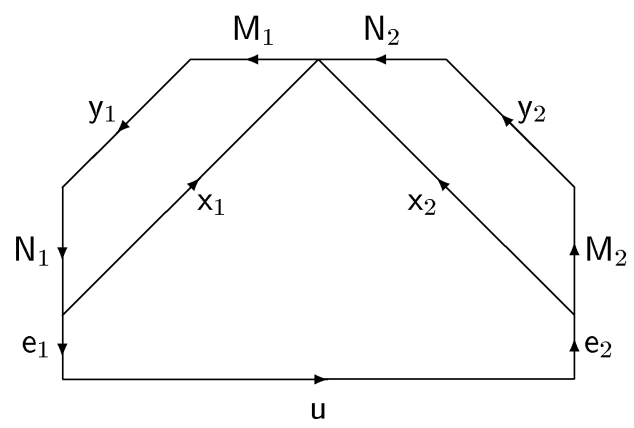

Рис. 36

10.12. ПРЕДЛОЖЕНИЕ. Пусть $\Delta-B$-диаграмма ранга $\alpha,\left(\# y_{\alpha}(\Delta), \chi(\Delta)\right) \neq$ $(0,1)$ u $\# y_{\alpha}(\Delta)-2 \chi(\Delta) \leqslant 2$. Пусть $\mathcal{S}$ - карта примыканий ранга $\alpha-1$ на $\Delta^{\alpha-1}$ и выполнено условие 10.5, (i) (в частности, в силу леммы 10.1, (ii) это верно в случае, если $\Delta$ есть приведенная $B$-диаграмма ранга $\alpha$ ). Тогда граничный чикл каждой области $\mathrm{A} \in \mathbf{R}_{\alpha}(\Delta)$ имеет не менее двух поддиаграмм примыкания $\Pi \in \mathcal{S} \kappa$ главным участкам ранга $\alpha$ диаграммы $\Delta$.

ДокаЗАтельство (индукция по \# $\mathbf{R}_{\alpha}(\Delta)$ ). Допустим, лемма неверна. Выберем контрпример $(\Delta, \mathcal{S})$ с минимальным значением $\# \mathbf{R}_{\alpha}(\Delta)$. Пусть $\mathrm{A}_{0} \in \mathbf{R}_{\alpha}(\Delta)$ область, граничньй цикл которой имеет не более одной поддиаграммы примыкания $\Pi \in \mathcal{S}$ к главным участкам ранга $\alpha$ диаграммы $\Delta$. В силу леммы 10.5 для получения противоречия достаточно доказать выполнение условия 10.5, (ii). 
Допустим, условие 10.5 , (ii) не выполнено, т.е. найдется область $\mathrm{A} \in \mathbf{R}_{\alpha}(\Delta)$ и поддиаграммы примыкания $\Pi_{1}, \Pi_{2} \in \mathcal{S}$ граничного цикла $\delta$ А соответственно к главным участкам $\mathrm{R}_{1}$ и $\mathrm{R}_{2}$ ранга $\alpha$ диаграммы $\Delta$. Пусть $\delta \Pi_{i} \approx \mathrm{P}_{i} \mathrm{v}_{i} \mathrm{Q}_{i} w_{i}(i=1,2)$, где $\mathrm{P}_{i}, \mathrm{Q}_{i}$ - участки примыкания, $\mathrm{P}_{i}$ входит в $\delta \mathrm{A}_{\text {и }} \mathrm{Q}_{i}$ входит в $\mathrm{R}_{i}$. Пусть $\delta \mathrm{A} \approx \mathrm{P}_{1} \mathrm{~S}_{1} \mathrm{P}_{2} \mathrm{~S}_{2}$ (см. рис. 32 ). Пусть (не обязательно связная) диаграмма $\Delta^{\prime}$ получена удалением области А и поддиаграмм $\Pi_{1}, \Pi_{2}$ из $\Delta$, и пусть $\Delta_{1}-$ связная компонента диаграммы $\Delta^{\prime}$, содержащая область $\mathrm{A}_{0}$. Определим на $\Delta_{1}$ разбиение границы ранга $\alpha$ типа В, полагая

$$
\begin{aligned}
y_{\alpha-1}\left(\Delta_{1}\right) \rightleftharpoons & \left\{\mathrm{x} \in \mathrm{y}_{\alpha-1}(\Delta) \cup\left\{\mathrm{v}_{1}^{-1}, \mathrm{v}_{2}^{-1}, \mathrm{w}_{1}^{-1}, \mathrm{w}_{2}^{-1}\right\} \mid\right. \\
& \text { х входит в некоторый граничный цикл } \left.\Delta_{1}\right\}
\end{aligned}
$$

и

$$
z_{\alpha}\left(\Delta_{1}\right) \rightleftharpoons\left\{\mathrm{R} \in z_{\alpha}(\Delta) \cup\left\{\mathrm{S}_{1}, \mathrm{~S}_{2}\right\} \mid \mathrm{R} \text { входит в некоторый граничный цикл } \Delta_{1}\right\} .
$$

Очевидно, если $\mathrm{Q}_{1}$ и $\mathrm{Q}_{2}$ входят в разные граничные циклы диаграммы $\Delta$, то $\Delta^{\prime}=\Delta_{1}$, а если $\mathrm{Q}_{1}$ и $\mathrm{Q}_{2}$ входят в один граничный цикл диаграммы $\Delta$, то $\Delta^{\prime}$ состоит из двух связных компонент $\Delta_{1}$ и $\Delta_{2}$. Из формулы (4.1) легко видеть, что в первом случае $\chi\left(\Delta_{1}\right)=\chi(\Delta)+1$, а во втором случае $\chi\left(\Delta_{1}\right)+\chi\left(\Delta_{2}\right)=\chi(\Delta)+1$, в частности, $\chi\left(\Delta_{1}\right) \geqslant \chi(\Delta)$. Далее, имеем $\# y_{\alpha}\left(\Delta_{1}\right)=\# y_{\alpha}(\Delta)+2$ в случае $\Delta^{\prime}=\Delta_{1}$ и $\# y_{\alpha}\left(\Delta_{1}\right)+\# y_{\alpha}\left(\Delta_{2}\right)=\# y_{\alpha}(\Delta)+2$ в случае $\Delta^{\prime}=\Delta_{1}$, где $y_{\alpha}\left(\Delta_{2}\right)$ - множество участков границы и $\in y_{\alpha}(\Delta)$, входящих в границу $\Delta_{2}$. Во втором случае, если $\chi\left(\Delta_{2}\right)=1$, то в силу леммы 10.8 , (i) \#秋 $\left(\Delta_{2}\right)>1$, и тогда $\# y_{\alpha}\left(\Delta_{1}\right) \leqslant \# y_{\alpha}(\Delta)$. Таким образом, во всех случаях имеем либо $\chi\left(\Delta_{1}\right) \geqslant$ $\chi(\Delta)+1$ и \# $y_{\alpha}\left(\Delta_{1}\right) \leqslant \# y_{\alpha}(\Delta)+2$, либо $\chi\left(\Delta_{1}\right)=\chi(\Delta)$ и \# $y_{\alpha}\left(\Delta_{1}\right) \leqslant \# y_{\alpha}(\Delta)$. Отсюда следует \# $y_{\alpha}\left(\Delta_{1}\right)-2 \chi\left(\Delta_{1}\right) \leqslant \# y_{\alpha}(\Delta)-2 \chi(\Delta) \leqslant 2$. Так как $y_{\alpha}\left(\Delta_{1}\right) \neq \varnothing$, то $\left(\# y_{\alpha}\left(\Delta_{1}\right), \chi\left(\Delta_{1}\right)\right) \neq(0,1)$, и так как, очевидно, $\Delta_{1}^{\alpha-1} \in \mathcal{T}\left(\Delta^{\alpha-1},\left\{\Pi_{1}, \Pi_{2}\right\}\right)$, то по лемме 9.22 множество поддиаграмм примыкания $\Pi \in \mathcal{S}$, содержашихся в $\Delta_{1}^{\alpha-1}$, образует карту примыканий ранга $\alpha-1$ на $\Delta_{1}^{\alpha-1}$. Так как $\# \mathbf{R}_{\alpha}\left(\Delta_{1}\right)<\# \mathbf{R}_{\alpha}(\Delta)$, то, рассматривая $\Delta_{1}$ и $\mathcal{S}_{1}$ в качестве $\Delta$ и $\mathcal{S}$, мы приходим к противоречию с выбором $\Delta$ и $\delta$.

10.13. ПРЕДЛОЖЕНИЕ. Пусть $\Delta$ - приведенная В-диаграм.ма ранга $\alpha u$ $\mathcal{S}$ - карта примыканий ранга $\alpha-1$ на А-диаграмме $\Delta^{\alpha-1}$ ранга $\alpha-1$. Пусть $\mathrm{A} \in \mathbf{R}_{\alpha}(\Delta), \quad \mathcal{L}(\mathrm{A}) \approx A^{n}$, әде $A \in \mathcal{E}_{\alpha}$, и пусть $\left\{\mathrm{R}_{1}, \mathrm{R}_{2}, \ldots, \mathrm{R}_{t}\right\}$ - множество всех участков примыкания поддиаграмм примыкания $\Pi \in \mathcal{S}$ граничного цикла области А $к$ главным участкам ранга $\alpha$ диаграммы $\Delta$. Тогда верны следующие утверждения.

(i) Если $\Delta-$ дисковая диаграмма и $2 \leqslant \# y_{\alpha}(\Delta) \leqslant 4$, mо

$$
\frac{1}{|A|} \sum_{i=1}^{t}\left|\mathcal{L}\left(\mathrm{R}_{i}\right)\right| \geqslant n-(p+16) \# y_{\alpha}(\Delta) .
$$




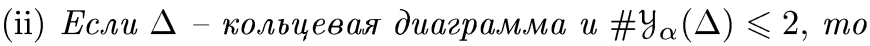

$$
\frac{1}{|A|} \sum_{i=1}^{t}\left|\mathcal{L}\left(\mathrm{R}_{i}\right)\right| \geqslant n-2 p-32-(2 p+16) \# y_{\alpha}(\Delta)
$$

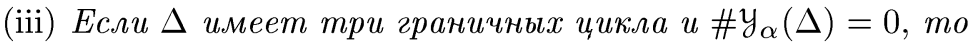

$$
\frac{1}{|A|} \sum_{i=1}^{t}\left|\mathcal{L}\left(\mathrm{R}_{i}\right)\right| \geqslant n-6 p-68
$$

ДокАЗАТЕльСтво. Ослабим посылку предложения, считая вместо условия о том, что диаграмма $\Delta$ приведена, выполненным условие 10.5 , (i) (это можно сделать в силу леммы 10.1, (ii)). Утверждения (i)-(iii) будем доказывать одновременно, используя индукцию по \# $\mathbf{R}_{\alpha}(\Delta)$.

Случай 1: $\# \mathbf{R}_{\alpha}(\Delta)=\{\mathrm{A}\}$ (база индукции). Будем использовать обозначения $N(\mathrm{~A}), N_{i}(\mathrm{~A})$ и $P(\mathrm{~A})$ из доказательства леммы 10.5. В этих обозначениях, в частности, $\left\{\mathrm{R}_{1}, \mathrm{R}_{2}, \ldots \mathrm{R}_{t}\right\}=N_{1}(\mathrm{~A})$. Имеем

$$
\frac{1}{|A|} \sum_{\mathrm{S} \in N_{1}(\mathrm{~A})}|\mathcal{L}(\mathrm{S})|=n-\frac{1}{|A|} \sum_{\mathrm{S} \in N_{2}(\mathrm{~A}) \cup N_{3}(\mathrm{~A})}|\mathcal{L}(\mathrm{S})|-\frac{1}{|A|} \sum_{\mathrm{S} \in P(\mathrm{~A})}|\mathcal{L}(\mathrm{S})| .
$$

Используя предложения 10.4, 8.22, (ii), лемму 8.20, (ii) и соотношения (9.3), (9.1), получаем

$$
\begin{aligned}
\frac{1}{|A|} \sum_{\mathrm{S} \in P(\mathrm{~A})}|\mathcal{L}(\mathrm{S})| & \leqslant 6\left(\# y_{\alpha-1}\left(\Delta_{\alpha-1}\right)-2 \chi\left(\Delta_{\alpha-1}\right)\right)+2 \# P(\mathrm{~A}) \\
& \leqslant 6\left(2 \# y_{\alpha}(\Delta)-2 \chi(\Delta)+2\right)+2 \# P(\mathrm{~A})
\end{aligned}
$$

а в силу условия $10.5,(\mathrm{i})$ имеем

$$
\frac{1}{|A|} \sum_{\mathrm{S} \in N_{2}(\mathrm{~A}) \cup N_{3}(\mathrm{~A})}|\mathcal{L}(\mathrm{S})| \leqslant p\left(\# N_{2}(\mathrm{~A})+\# N_{3}(\mathrm{~A})\right)
$$

Далее, имеем \#P(A) $\leqslant \max \{1, \# N(\mathrm{~A})\}$, а из леммы $9.23,($ iii) $-(\mathrm{v})$, учитывая неравенство (9.3), легко выводим, что

$$
\# N(\mathrm{~A}) \leqslant \begin{cases}2 \# y_{\alpha}(\Delta), & \text { если } \chi(\Delta)=1, \\ 2 \# y_{\alpha}(\Delta)+4, & \text { если } \chi(\Delta)=0, \\ 10, & \text { если } \chi(\Delta)=-1 \text { и } \# y_{\alpha}(\Delta)=0,\end{cases}
$$

и, с учетом неравенства \#z, $\alpha(\Delta) \leqslant \# y_{\alpha}(\Delta)$,

$$
\# N_{2}(\mathrm{~A})+\# N_{3}(\mathrm{~A}) \leqslant \begin{cases}\# y_{\alpha}(\Delta), & \text { если } \chi(\Delta)=1, \\ 2 \# y_{\alpha}(\Delta)+2, & \text { если } \chi(\Delta)=0, \\ 6, & \text { если } \chi(\Delta)=-1 \text { и \#у } y_{\alpha}(\Delta)=0 .\end{cases}
$$


Подставляя все полученные оценки в (10.14), легко убедиться в истинности утверждений (i)-(iii).

Случай 2: $\# \mathbf{R}_{\alpha}(\Delta)>1$ (индуктивный шаг). Пусть $\mathrm{B} \in \mathbf{R}_{\alpha}(\Delta) \backslash\{\mathrm{A}\}$. По лемме 10.12 имеются две поддиаграммы примыкания $\Pi_{1}, \Pi_{2} \in \mathcal{S}$ граничного цикла $\delta \mathrm{B}$ к главным участкам ранга $\alpha$ диаграммы $\Delta$. Пусть $\delta \Pi_{i} \approx \mathrm{P}_{i} \mathrm{v}_{i} \mathrm{Q}_{i} \mathrm{w}_{i}(i=1,2)$, где $\mathrm{P}_{i}, \mathrm{Q}_{i}$ - участки примыкания и $\delta \mathrm{B} \approx \mathrm{P}_{1} \mathrm{~S}_{1} \mathrm{P}_{2} \mathrm{~S}_{2}$. Пусть поддиаграмма $\Delta^{\prime}$ диаграммы $\Delta$ получена удалением области В и поддиаграмм $\Pi_{1}, \Pi_{2}$ из $\Delta$, и пусть $\Delta_{1}$ - связная компонента диаграммы $\Delta^{\prime}$, содержащая область А. Определим разбиение гранищы ранга $\alpha$ типа В на $\Delta_{1}$ равенствами $(10.12),(10.13)$, и в силу леммы 9.22 рассмотрим карту примыканий $\mathcal{S}_{1}$ ранга $\alpha-1$ на $\Delta_{1}^{\alpha-1}$, состоящую из поддиаграмм примыкания $\Pi \in \mathcal{S}$, содержашихся в $\Delta_{1}^{\alpha-1}$. Как в доказательстве предложения 10.12, имеем либо

$$
\chi\left(\Delta_{1}\right) \geqslant \chi(\Delta)+1 \text { и } \# y_{\alpha}\left(\Delta_{1}\right) \leqslant \# y_{\alpha}(\Delta)+2,
$$

либо

$$
\chi\left(\Delta_{1}\right)=\chi(\Delta) \text { и } \# y_{\alpha}\left(\Delta_{1}\right) \leqslant \# y_{\alpha}(\Delta) .
$$

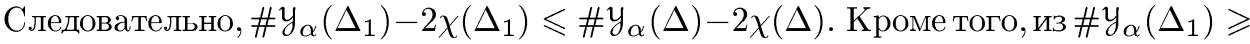
1 и леммы 10.8 , (i) при $\Delta^{\prime}:=\Delta_{1}$ и $\mathcal{S}:=\mathcal{S}_{1}$ вытекает \# $\mathcal{Y}_{\alpha}\left(\Delta_{1}\right)-2 \chi\left(\Delta_{1}\right) \geqslant 0$. Отсюда видно, что истинность посылки одного из утверждений (i)-(iii) влечет истинность посылки одного из утверждений (i)-(iii) при $\Delta:=\Delta_{1}$. Заметим, что при замене $\Delta$ на $\Delta_{1}$ множество $N_{1}(\mathrm{~A})$ не меняется. Тогда из вида правых частей неравенств в (i)-(iii) сразу получаем, что в случае (10.16) соответствуюшее неравенство (i)-(iii) для диаграммы $\Delta$ вытекает из индуктивного предположения для $\Delta:=\Delta_{1}$. В случае (10.15) нужно еше заметить, что правые части неравенств в (i),(ii) при подстановке $\# y_{\alpha}(\Delta):=2$ больше правой части неравенства в (iii), а правая часть неравенства в (i) при подстановке \# $y_{\alpha}(\Delta):=\# y_{\alpha}(\Delta)+2$ становится равной правой части неравенства в (ii).

\section{$\S 11$. Анализ соотношений в группе $\mathbf{B}_{\alpha}$ с помошью диаграмм}

В этом параграфе мы используем полученные в 110 результаты о диаграммах ранга $\alpha$ для анализа соотношений равенства и сопряженности в группе $\mathbf{B}_{\alpha}$.

11.1. ОПРЕДЕЛЕНИЕ. Пусть $u \in \mathcal{H}_{\alpha}$ и для слова $u$ фиксировано разложение в виде $u$ 드 $u_{1} Q u_{2}$, где $u_{1}, u_{2} \in \mathcal{H}_{\alpha-1}$ и $Q$ - подслово слова $B^{n}$ при $B \in \mathcal{E}_{\alpha}$. Предположим, $|Q| \geqslant 2|B|$, и пусть слово $\widehat{Q}$ определено равенством $Q \widehat{Q}^{-1} \approx B^{n}$. Слово $u_{1} \widehat{Q} u_{2} \in \mathcal{H}_{\alpha}$ будем называть дополнительны.м $\kappa u$.

Если слово $u \in \mathcal{H}_{\alpha}$ может быть представлено в виде $u$ 으 $u_{1} Q u_{2}$, где $u_{1}, u_{2} \in$ $\mathcal{H}_{\alpha-1}, Q \in \Pi$ ер $(B), B \in \mathcal{E}_{\alpha}$ и $|Q|<2|B|$, то $u$ назовем ограниченным в ранге $\alpha$. В частности, любое слово $u \in \mathcal{H}_{\alpha-1}$ является ограниченным в ранге $\alpha$.

Из определения непосредственно вытекает, что если $u^{\prime}$ - слово, дополнительное к слову $u \in \mathcal{H}_{\alpha}$, то $u \stackrel{\alpha}{=} u^{\prime}$. 
11.2. ПРЕДЛОЖЕНИЕ. Пусть $X \stackrel{\alpha}{=} u Y v$, əде $X, Y \in \mathcal{R}_{\alpha} u u, v \in \mathcal{H}_{\alpha}$. Пусть для $u, v$ фиксированы некоторые разложсения в виде $u$ 드 $u_{1} Q u_{2} u v$ 으 $v_{1} R v_{2}$, где $u_{i}, v_{i} \in \mathcal{H}_{\alpha-1}(i=1,2)$ и $Q, R$ - подслова слов $B^{n}, C^{n}$ соответственно при $B, C \in \mathcal{E}_{\alpha}$. Тогда верно хотя бы одно из двух следующих утверждений.

(i) $X \stackrel{\alpha=1}{=} \hat{u} Y \hat{v}$, где каждое из слов $\hat{u}$ и $\hat{v}$ либо совпадает с соответствующим словом и или $v$, либо является дополнительным $к$ нему.

(ii) Для некоторого $r \geqslant 1$ существуют слова $K_{i}, L_{i}(i=0,1, \ldots, r), G_{i}$, $H_{i}, S_{i}, U_{i}, T_{i}, V_{i}$, әлементарные периоды $D_{i} \in \mathcal{E}_{\alpha}$ и слова $x_{i}, y_{i}, z_{i}, w_{i} \in \mathcal{H}_{\alpha-1}$ $(i=1,2, \ldots, r)$ такие, что выполнены следуюшие соотношения, где каждое из слов $\hat{u}$ и $\hat{v}$ либо совпадает с соответствующим словом и или $v$, либо является дополнительным $к$ нему:

$$
\begin{gathered}
X \text { 으 } K_{0} G_{1} K_{1} \ldots G_{r} K_{r}, \quad Y \text { 으 } L_{0} H_{1} L_{1} \ldots H_{r} L_{r}, \\
S_{i}^{-1} U_{i} T_{i} V_{i} \approx D_{i}^{n} \quad(i=1,2, \ldots, r), \\
G_{i} \stackrel{\alpha=1}{=} x_{i} S_{i} y_{i}, \quad H_{i} \stackrel{\alpha=1}{=} z_{i} T_{i} w_{i} \quad(i=1,2, \ldots, r), \\
\left\{\begin{array}{l}
K_{0} \stackrel{\alpha=1}{=} \hat{u} L_{0} z_{1} U_{1}^{-1} x_{1}^{-1}, \\
K_{i} \stackrel{\alpha=1}{=} y_{i}^{-1} V_{i}^{-1} w_{i} L_{i} z_{i+1} U_{i+1}^{-1} x_{i+1}^{-1} \quad(i=1,2, \ldots, r-1), \\
K_{r} \stackrel{\alpha=1}{=} y_{r}^{-1} V_{r}^{-1} w_{r} L_{r} \hat{v}, \\
\left|S_{i}\right|,\left|T_{i}\right|>(q-2 p-32)\left|D_{i}\right| \quad(i=1,2, \ldots, r) .
\end{array}\right.
\end{gathered}
$$

Если слова $u, v$ ограничены в ранге $\alpha$, то в (i) $и$ (ii) можно считать $и$ 으 $\hat{u}$ $u v$ 으 $\hat{v}$.

ДокАЗАТЕЛЬСТво. Рассмотрим дисковую $B$-диаграмму $\Delta$ ранга $\alpha$ с граничным циклом $\delta \Delta \approx \mathrm{X}^{-1} \mathrm{u}_{1} \mathrm{Qu}_{2} \mathrm{Yv}_{1} \mathrm{Rv}_{2}$, где метки путей $\mathrm{X}, \mathrm{Y}, \mathrm{Q}, \mathrm{R}, \mathrm{u}_{i}, \mathrm{v}_{i}$ равны соответственно словам $X, Y, Q, R, u_{i}, v_{i}$,

$$
X_{\alpha}(\Delta)=\left\{X^{-1}, Y\right\}, \quad y_{\alpha-1}(\Delta)=\left\{u_{1}, u_{2}, v_{1}, v_{2}\right\} \quad \text { и } Z_{\alpha}(\Delta)=\{\mathrm{Q}, \mathrm{R}\}
$$

Заменим $\Delta$ на приведенную $B$-диаграмму ранга $\alpha$ согласно предложению 9.9. В силу замечания 9.7 при такой замене вместо $\mathcal{L}\left(\mathrm{u}_{1} \mathrm{Qu}_{2}\right)$ 으 $u$ и $\mathcal{L}\left(\mathrm{v}_{1} \mathrm{Rv}_{2}\right)$ 의 $v$ будем иметь $\mathcal{L}\left(\mathrm{u}_{1} \mathrm{Qu}_{2}\right)$ 으 $\hat{u}$ и $\mathcal{L}\left(\mathrm{v}_{1} \mathrm{Rv}_{2}\right)$ 으 $\hat{v}$, где каждое из слов $\hat{u}$ или $\hat{v}$ либо совпадает соответственно с $u$ или $v$, либо является дополнительным словом. По предложению 9.19 можно считать, что на $\Delta^{\alpha-1}$ задана некоторая карта примыканий $\mathcal{S}$ ранга $\alpha-1$. Если $\Delta$ не содержит областей ранга $\alpha$, то имеет место утверждение (i). Пусть $\mathbf{R}_{\alpha}(\Delta)=\left\{\mathrm{A}_{1}, \mathrm{~A}_{2} \ldots, \mathrm{A}_{r}\right\} \neq \varnothing$. В силу предложений 10.12 и 10.9 , (i) граничньй цикл $\delta \mathrm{A}_{i}$ каждой области $\mathrm{A}_{i}$ имеет ровно одну поддиаграмму примыкания ранга $\alpha-1$ к каждому из участков границы $\mathrm{X}_{\text {и }} \mathrm{Y}^{-1}$. Пусть $\Pi_{i}$ и $\Pi_{i}^{\prime}-$ соответствующие поддиаграммы примыкания, $\delta \Pi_{i} \approx \mathrm{G}_{i} \mathrm{y}_{i}^{-1} \mathrm{~S}_{i}^{-1} \mathrm{x}_{i}^{-1}, \delta \Pi_{i}^{\prime} \approx \mathrm{H}_{i}^{-1} \mathrm{z}_{i} \mathrm{~T}_{i} \mathrm{w}_{i}$, где $\mathrm{G}_{i}$, $\mathrm{S}_{i}^{-1}, \mathrm{H}_{i}^{-1}, \mathrm{~T}_{i}$ - участки примыкания, $\mathcal{L}\left(\mathrm{x}_{i}\right), \mathcal{L}\left(\mathrm{y}_{i}\right), \mathcal{L}\left(\mathrm{z}_{i}\right), \mathcal{L}\left(\mathrm{w}_{i}\right) \in \mathcal{H}_{\alpha-1}, \mathrm{G}_{i}$ входит в $\mathrm{X}, \mathrm{H}_{i}^{-1}$ входит в $\mathrm{Y}, \mathrm{S}_{i}^{-1}$ и $\mathrm{T}_{i}$ входят в $\delta \mathrm{A}_{i}$. Пусть $\delta \mathrm{A}_{i} \approx \mathrm{S}_{i}^{-1} \mathrm{U}_{i} \mathrm{~T}_{i} \mathrm{~V}_{i}$ и $\mathcal{L}\left(\delta \mathrm{A}_{i}\right) \approx D_{i}^{n}$, где $D_{i} \in \mathcal{E}_{\alpha}$. Перенумеровав области $\mathrm{A}_{i}$, можно считать, что пути $\mathrm{X}$ и $\mathrm{Y}$ имеют 
вид $\mathrm{X}=\mathrm{K}_{0} \mathrm{G}_{1} \mathrm{~K}_{1} \ldots \mathrm{G}_{r} \mathrm{~K}_{r}$ и $\mathrm{Y}=\mathrm{L}_{0} \mathrm{H}_{1} \mathrm{~L}_{1} \ldots \mathrm{H}_{r} \mathrm{~L}_{r}$ (см. рис. 37 ). Обозначим через $K_{i}, G_{i}, L_{i}, H_{i}, S_{i}, U_{i}, T_{i}, V_{i}, x_{i}, y_{i}, z_{i}, w_{i}$ метки соответствуюших путей $\mathrm{K}_{i}, \mathrm{G}_{i}$, $\mathrm{L}_{i}, \mathrm{H}_{i}, \mathrm{~S}_{i}, \mathrm{U}_{i}, \mathrm{~T}_{i}, \mathrm{~V}_{i}, \mathrm{x}_{i}, \mathrm{y}_{i}, \mathrm{z}_{i}, \mathrm{w}_{i}$ на диаграмме $\Delta$. Тогда из расположения этих путей на $\Delta$ и леммы 4.2 вытекают соотношения (11.1)-(11.4). Далее, по предложению 10.13 имеем $\left|S_{i}\right|+\left|T_{i}\right| \geqslant(n-2 p-32)\left|D_{i}\right|$, а в силу приведенности слов $X$ и $Y$ в ранге $\alpha$ имеем $\left|S_{i}\right|,\left|T_{i}\right|<(n-q)\left|D_{i}\right|$. Отсюда вытекают неравенства (11.5).

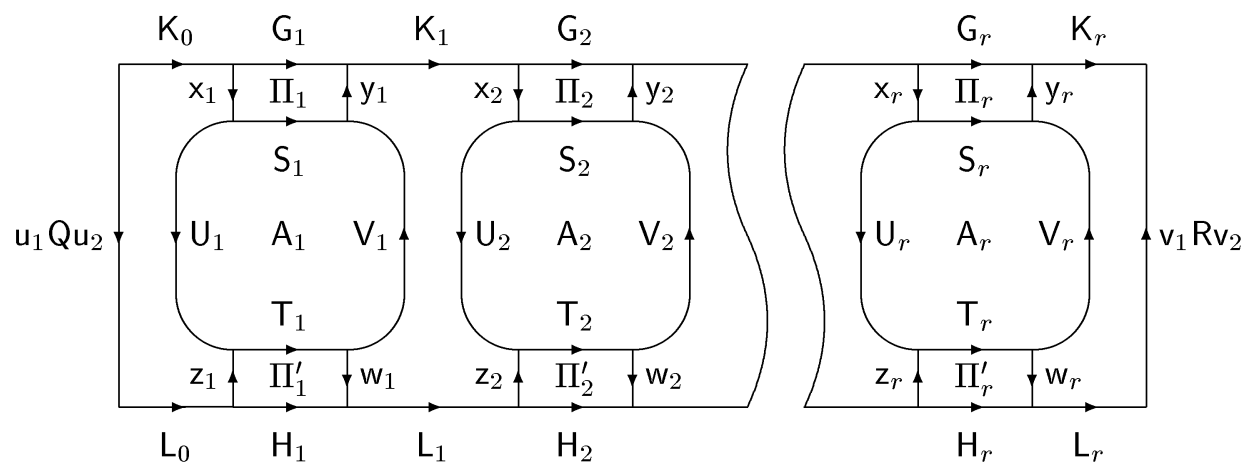

Рис. 37

Наконец, если слова $u, v$ ограничены в ранге $\alpha$, то согласно определению 11.1 в разложениях $u$ 으 $u_{1} Q u_{2}$ и $v$ 으 $v_{1} R v_{2}$ можно считать $|Q|<2|B|$ и $|R|<2|C|$. Тогда в силу замечания 9.7 выполнены равенства $u$ 으 $\hat{u}$ и $v$ 으 $\hat{v}$.

Прежде чем перейти к выводу следствий из предложения 11.2, сделаем одно наблюдение. Пусть дано соотношение $X \stackrel{\alpha}{=} u Y v$, где $X, Y \in \mathcal{R}_{\alpha}$ и $u, v \in \mathcal{H}_{\alpha}$, и пусть для слов $u$ и $v$ фиксированы некоторые разложения $u$ 으 $u_{1} Q u_{2}$ и $v$ 드 $v_{1} R v_{2}$, где $u_{i}, v_{i} \in \mathcal{H}_{\alpha-1}(i=1,2)$ и $Q, R$ - подслова слов $B^{n}, C^{n}$ соответственно при $B, C \in \mathcal{E}_{\alpha}$. Предположим, имеет место случай (ii) из формулировки предложения 11.2. Тогда из соотношений $(11.3),(11.4)$, равенства $q_{4}=q-2 p-68$ и следствия 8.24 вытекает, что в каждое из подслов $G_{i}, H_{i}$ слов $X$ и $Y$ входит элементарная $q_{4}$-степень ранга $\alpha$. Отсюда следует, что если хотя бы одно из слов $X$ или $Y$ не содержит элементарных $q_{4}$-степеней ранга $\alpha$, то имеет место случай (i) из предложения 11.2 , т.е. $X \stackrel{\alpha=1}{=} \hat{u} Y \hat{v}$, где каждое из слов $\hat{u}$ и $\hat{v}$ либо совпадает с соответствуюшим словом $u$ или $v$, либо является дополнительным к нему. В частности, имеет место

11.3. СлЕДСТвИЕ. Если слова $X, Y \in \mathcal{R}_{\alpha}, \quad u, v \in \mathcal{H}_{\alpha-1}$ удовлетворяют соотношению $X \stackrel{\alpha}{=} u Y v$ и хотя бы одно из слов $X$ или $Y$ не содержит әлементарных $q_{4}$-степеней ранга $\alpha$, то $X \stackrel{\alpha=1}{=} u Y v$.

Более того, утверждение имеет место, если вместо $u, v \in \mathcal{H}_{\alpha-1}$ предполагать, что $u, v \in \mathcal{H}_{\alpha}$ и слова $u$, v ограничены в ранге $\alpha$.

11.4. СЛЕДСТВИЕ $(\alpha \geqslant 0)$. Если $X \stackrel{\alpha}{=} 1 u X \in \mathcal{R}_{\alpha}$, mo $X$ - nустое слово. 
ДОКАЗАТЕЛЬСТвО. Если $\alpha=0$, то это следует из определения 5.1. При $\alpha \geqslant 1$ нужно применить следствие 11.3 при $u:=Y:=v:=1$ и воспользоваться индуктивным предположением.

Из предыдушего утверждения непосредственно вытекает

11.5. СлЕДСТВИЕ $(\alpha \geqslant 0)$. Любой путь $\mathrm{X}$ на графе $\Gamma_{\alpha}$ с $\mathcal{L}(\mathrm{X}) \in \mathcal{R}_{\alpha}$ является простылм.

11.6. СлЕДСТВИЕ. Пусть $\gamma \leqslant \beta \leqslant \alpha-1$ u $\mathrm{X}, \mathrm{Y}-$ - блзкие в ранге $\gamma$ пути на графе $\Gamma_{\alpha}$. Если для каждого $\delta=\beta+1, \beta+2, \ldots, \alpha$ хотя бы одно из множеств

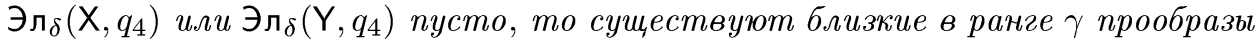
путей $\mathrm{X} u \mathrm{Y}$ на графе $\Gamma_{\beta}$.

ДОКАЗАТЕЛЬСтво. Соединим вершины $\iota(\mathrm{X})$ и $\iota(\mathrm{Y})$ путем $\mathrm{u}$, а вершины $\tau(\mathrm{X})$ и $\tau(\mathrm{Y})$ - путем v, где $\mathcal{L}(\mathrm{u}), \mathcal{L}(\mathrm{v}) \in \mathcal{H}_{\gamma}$. Выберем произвольньй прообраз $\overline{\mathrm{X}} \overline{\mathrm{v}} \overline{\mathrm{Y}}^{-1} \overline{\mathrm{v}}^{-1}$ на графе $\Gamma_{\beta}$ пути $X v Y^{-1} v^{-1}$. Применяя следствие 11.3, получаем $\mathcal{L}\left(\bar{X} \bar{v} \bar{Y}^{-1} \bar{v}^{-1}\right) \stackrel{\beta}{=}$ 1. Тогда $\bar{X} \bar{v} \bar{Y}^{-1} \bar{v}^{-1}$ - замкнутый путь и, следовательно, $\bar{X}$ и $\bar{Y}-$ близкие в ранге $\gamma$ пути.

Теперь докажем одно вспомогательное утверждение для соотношений в группе $\mathbf{B}_{\alpha-1}$.

11.7. Лемма. Пусть $r \geqslant 2, \mathrm{X} u \mathrm{~W} \rightleftharpoons \mathrm{u}_{0} \mathrm{Z}_{1} \mathrm{u}_{1} \ldots \mathrm{Z}_{r} \mathrm{u}_{r}-$ nути на графе $\Gamma_{\alpha-1}$ с общими начальной и конечной вершинами, әде $\mathcal{L}(\mathrm{X}), \mathcal{L}\left(\mathrm{Z}_{1}\right), \ldots, \mathcal{L}\left(\mathrm{Z}_{r}\right) \in \mathcal{R}_{\alpha-1}$ $u \mathcal{L}\left(\mathrm{u}_{0}\right), \mathcal{L}\left(\mathrm{u}_{1}\right), \ldots, \mathcal{L}\left(\mathrm{u}_{r}\right) \in \mathcal{H}_{\alpha-1}$. Тогда

(i) любой подпуть $\mathrm{Y}$ пути $\mathrm{X}$ можно разложить в произведение $\mathrm{Y}=$ $\mathrm{w}_{0} \mathrm{~S}_{1} \mathrm{w}_{1} \ldots \mathrm{S}_{t} \mathrm{w}_{t}$ (возможно, $\left.t=0\right)$, где $\sum_{i=0}^{t} \mathcal{W}_{\alpha-1}\left(\mathcal{L}\left(\mathrm{w}_{i}\right)\right) \leqslant(r-1) \eta$ и для некоторых $1 \leqslant k_{1}<k_{2}<\cdots<k_{t} \leqslant r$ каждый путь $\mathrm{S}_{i}$ входит в подпуть пути $\mathrm{X}$, близкий в ранге $\alpha-1 \kappa$ некоторому подпути $\mathrm{R}_{i}$ пути $\mathrm{Z}_{k_{i}}$;

(ii) если $\mathrm{E} \in \Pi$ Пер $\alpha(A)$ - подпуть пути $\mathrm{X} u \ell_{\alpha, A}(\mathrm{E}) \geqslant 45 r$, то найдутся подпути $\mathrm{F}_{1}, \mathrm{~F}_{2}, \ldots, \mathrm{F}_{t} \in \Pi$ Пер $\alpha(A)$ попарно различных путей $\mathrm{Z}_{i}$ такие, что ПСогл $\Omega_{\alpha}\left(\mathrm{E}, \mathrm{F}_{i}\right)$ при всех $і$ и $\sum_{i=1}^{t} \ell_{\alpha, A}\left(\mathrm{~F}_{i}\right) \geqslant \ell_{\alpha, A}(\mathrm{E})-45 r+5$. При этом пути $\mathrm{F}_{i}$ можно выбрать таким образом, чтобы каждый $\mathrm{F}_{i}$ и некоторый подпуть $\overline{\mathrm{F}}_{i} \in \Pi$ Пер $\alpha(A)$ пути Е бълли строго близки в ранге $\rho(A)$.

ДоКАЗАТЕЛЬСТво. (i) Ввиду леммы 8.20, (iv) достаточно рассмотреть случай, когда $\mathrm{Y}=\mathrm{X}$. Рассмотрим $A$-диаграмму $\Delta$ ранга $\alpha-1$ с граничным циклом $\delta \Delta=$ $\overline{\mathrm{X}} \overline{\mathrm{u}}_{r}^{-1} \overline{\mathrm{Z}}_{r}^{-1} \overline{\mathrm{u}}_{r-1}^{-1} \ldots \overline{\mathrm{Z}}_{1}^{-1} \overline{\mathrm{u}}_{0}^{-1}$, где

$$
\begin{gathered}
\mathcal{L}(\overline{\mathrm{X}}) \text { 으 } \mathcal{L}(\mathrm{X}), \quad \mathcal{L}\left(\overline{\mathrm{u}}_{i}\right) \text { 으 } \mathcal{L}\left(\mathrm{u}_{i}\right) \quad(i=0,1, \ldots, r), \quad \mathcal{L}\left(\overline{\mathrm{Z}}_{i}\right) \text { 으 } \mathcal{L}\left(\mathrm{Z}_{i}\right) \quad(i=1,2, \ldots, r), \\
x_{\alpha-1}(\Delta)=\left\{\overline{\mathrm{X}}, \overline{\mathrm{Z}}_{r}^{-1}, \overline{\mathrm{Z}}_{r-1}^{-1}, \ldots, \overline{\mathrm{Z}}_{1}^{-1}\right\} \quad \text { и } \quad \mathrm{y}_{\alpha-1}(\Delta)=\left\{\overline{\mathrm{u}}_{r}^{-1}, \overline{\mathrm{u}}_{r-1}^{-1}, \ldots, \overline{\mathrm{u}}_{0}^{-1}\right\} .
\end{gathered}
$$

По предложению 9.19 можно считать, что на $\Delta$ задана некоторая карта примыканий $\mathcal{S}$ ранга $\alpha-1$. Пусть $\overline{\mathrm{X}}=\overline{\mathrm{w}}_{0} \overline{\mathrm{S}}_{1} \overline{\mathrm{w}}_{1} \ldots \overline{\mathrm{S}}_{t} \overline{\mathrm{w}}_{t}$, где $\overline{\mathrm{S}}_{i}-$ участки примыкания 
поддиаграмм примыкания $\Pi \in \mathcal{S}$ и $\overline{\mathbf{w}}_{i}-$ переходные участки диаграммы $\Delta$ относительно $\mathcal{S}$ либо, возможно, пустые пути при $i=0$ или $i=t$. По предложению 10.4

$$
\sum_{i=0}^{t} \mathcal{W}_{\alpha-1}\left(\mathcal{L}\left(\overline{\mathbf{w}}_{i}\right)\right) \leqslant(r-1) \eta
$$

Пусть $\overline{\mathrm{S}}_{i}$ входит в граничный цикл поддиаграммы примыкания $\Pi_{i} \in \mathcal{S}$, и пусть $\overline{\mathrm{R}}_{i}^{-1}$ - другой участок примыкания поддиаграммы $\Pi_{i}$. По лемме 9.23 , (i) каждый путь $\overline{\mathrm{R}}_{i}^{-1}$ входит в некоторый путь $\overline{\mathrm{Z}}_{k_{i}}^{-1}$ и все $k_{i}$ попарно различны, а из расположения поддиаграмм $\Pi_{i}$ вытекает $k_{1}<k_{2}<\cdots<k_{t}$ (см. рис. 38). Рассмотрим отображение $\omega$ множества путей на диаграмме $\Delta$ во множество путей на графе $\Gamma_{\alpha-1}$ такое, что $\omega(\iota(\overline{\mathrm{X}}))=\iota(\mathrm{X})\left(\right.$ см. 4.12). Очевидно, $\omega(\overline{\mathrm{X}})=\mathrm{X}$ и $\omega\left(\bar{Z}_{i}\right)=\mathrm{Z}_{i}$ при всех $i$. Тогда в качестве искомых путей $\mathrm{S}_{i}, \mathrm{w}_{i}$ и $\mathrm{R}_{i}$ можно взять соответственно $\omega\left(\overline{\mathrm{S}}_{i}\right)$, $\omega\left(\overline{\mathrm{w}}_{i}\right)$ и $\omega\left(\overline{\mathrm{R}}_{i}\right)$.
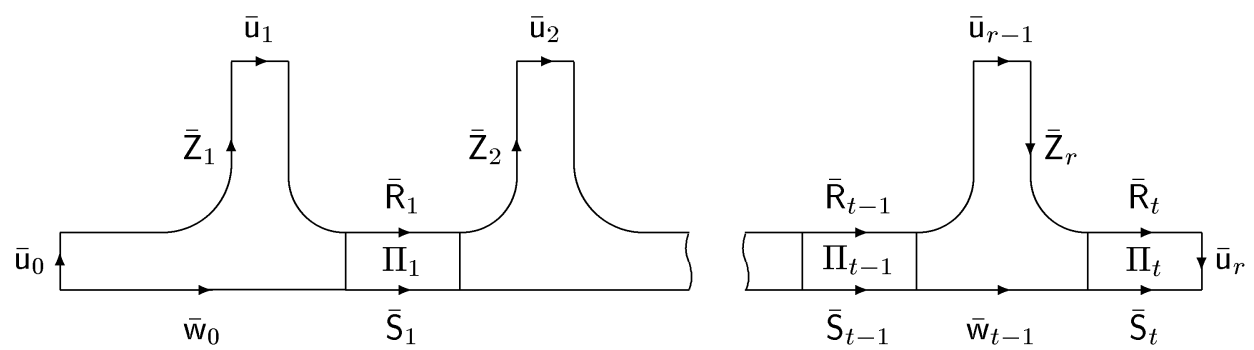

Рис. 38

(ii) Согласно (i) имеем $\mathrm{E}=\mathrm{w}_{0} \mathrm{~S}_{1} \mathrm{w}_{1} \ldots \mathrm{S}_{s} \mathrm{w}_{s}$, где $\sum_{i=0}^{s} \mathcal{W}_{\alpha-1}\left(\mathcal{L}\left(\mathrm{w}_{i}\right)\right) \leqslant(r-1) \eta$ и каждый $\mathrm{S}_{i}$ входит в подпуть пути $\mathrm{X}$, близкий к подпути пути $\mathrm{Z}_{k_{i}}$, причем все $k_{i}$ попарно различны. По предложению 8.22 , (i)

$$
\sum_{i=0}^{s} \ell_{\alpha, A}\left(\mathcal{L}\left(\mathrm{w}_{i}\right)\right) \leqslant 13(r-1) \eta+s+1<6(r-1)+r+1=7 r-5,
$$

откуда в силу предложения 8.14 , (ii)

$$
\sum_{i=1}^{s} \ell_{\alpha, A}\left(\mathcal{L}\left(\mathrm{S}_{i}\right)\right) \geqslant \ell_{\alpha, A}(\mathrm{E})-11 r+5
$$

Предположим, $\ell_{\alpha, A}\left(\mathcal{L}\left(\mathrm{S}_{i}\right)\right) \geqslant 35$ для некоторого $i$. Пусть $\mathrm{E}_{i} \in$ ППер $\alpha(A, 35)-$ подпуть пути $\mathrm{S}_{i}$, где $\ell_{\alpha, A}\left(\mathrm{E}_{i}\right)=\ell_{\alpha, A}\left(\mathcal{L}\left(\mathrm{S}_{i}\right)\right)$. В силу леммы 8.25 найдется подпуть $\mathrm{F}_{i} \in$ ППер $\alpha(A)$ пути $\mathrm{Z}_{k_{i}}$ такой, что $\ell_{\alpha, A}\left(\mathrm{~F}_{i}\right) \geqslant \ell_{\alpha, A}\left(\mathcal{L}\left(\mathrm{S}_{i}\right)\right)-34$ и $\mathrm{F}_{i}$ и некоторьй подпуть $\overline{\mathrm{F}}_{i} \in$ ППер $\alpha(A)$ пути $\mathrm{E}_{i}$ строго близки в ранге $\rho(A)$, причем $\Pi$ Сог $\Omega_{\alpha}\left(\mathrm{E}_{i}, \overline{\mathrm{F}}_{i}\right)$. Так как в силу предложения $8.9 \Pi_{\text {Cог } \Omega_{\alpha}}\left(\mathrm{E}_{1} \mathrm{E}_{i}\right)$, то, используя лемму 8.3 при $\mathrm{E}:=\mathrm{E}_{i}$, получаем $\Pi$ Согл $\Omega_{\alpha}\left(\mathrm{E}, \overline{\mathrm{F}}_{i}\right)$, откуда в силу леммы 8.12 выводим ПСогл $\Omega_{\alpha}\left(\mathrm{E}, \mathrm{F}_{i}\right)$ (мы не можем здесь воспользоваться следствием 8.6, (ii), так 
как, вообше говоря, неверно, что $\left.\ell_{\alpha, A}\left(\overline{\mathrm{F}}_{i}\right), \ell_{\alpha, A}\left(\mathrm{~F}_{i}\right) \geqslant 8\right)$. Наконец, остается заметить, что

$$
\sum_{i} \ell_{\alpha, A}\left(\mathrm{~F}_{i}\right) \geqslant \sum_{i} \ell_{\alpha, A}\left(\mathcal{L}\left(\mathrm{S}_{i}\right)\right)-34 \#\left\{\mathrm{~F}_{i}\right\} \geqslant \ell_{\alpha, A}(\mathrm{E})-45 r+5
$$

Теперь мы используем лемму 11.7 для доказательства еще одного следствия из предложения 11.2.

11.8. ПРЕДЛОЖЕНИЕ. Пусть пути $\mathrm{X} u \mathrm{Y}$ на графе $\Gamma_{\alpha}$ близки в ранге $\alpha u$ $\mathcal{L}(\mathrm{X}), \mathcal{L}(\mathrm{Y}) \in \mathcal{R}_{\alpha}$. Пусть $\mathrm{X}=\mathrm{X}_{1} \mathrm{EX}_{2}$, әде $\mathcal{W}_{\alpha}(\mathcal{L}(\mathrm{E}))>2+2 \zeta \eta u$ Эл $_{\alpha}\left(\mathrm{E}, q_{4}\right)=\varnothing$. Тогда $\mathrm{E}=\mathrm{U}_{1} \mathrm{E}^{\prime} \mathrm{U}_{2} u \mathrm{Y}=\mathrm{V}_{1} \mathrm{Y}^{\prime} \mathrm{V}_{2}$, где пути $\mathrm{E}^{\prime}$ и $\mathrm{Y}^{\prime}$ близки в ранге $\alpha-1 u$ $\mathcal{W}_{\alpha}\left(\mathcal{L}\left(\mathrm{U}_{i}\right)\right) \leqslant 1+2 \zeta \eta \quad(i=1,2)$.

ДокаЗАТЕЛЬСТво. Соединим вершины $\iota(\mathrm{X})$ и $\iota(\mathrm{Y})$ путем $\mathrm{u}$, а вершины $\tau(\mathrm{Y})$ и $\tau(\mathrm{X})$ - путем v, где $u \rightleftharpoons \mathcal{L}(\mathrm{u}) \in \mathcal{H}_{\alpha}$ и $v \rightleftharpoons \mathcal{L}(\mathrm{v}) \in \mathcal{H}_{\alpha}$. Имеем $\mathcal{L}(\mathrm{X}) \stackrel{\alpha}{=} u \mathcal{L}(\mathrm{Y}) v$ и, следовательно, в соответствии с предложением 11.2 при $X \rightleftharpoons \mathcal{L}(\mathrm{X})$ и $Y \rightleftharpoons \mathcal{L}(\mathrm{Y})$ можно рассмотреть два случая.

Случай 1: Имеет место случай (i) из предложения 11.2 , т.е. $\mathcal{L}(\mathrm{X}) \stackrel{\alpha=1}{=} \hat{u} \mathcal{L}(\mathrm{Y}) \hat{v}$, где $\hat{u}, \hat{v}$ - либо сами слова $u, v$, либо некоторые дополнительные к ним слова. Так как $u \stackrel{\alpha}{=} \hat{u}$ и $v \stackrel{\alpha}{=} \hat{v}$, то, заменяя пути и и $\mathrm{v}$ на пути с метками $\hat{u}$ и $\hat{v}$ соответственно и переходя к новым обозначениям, можно считать $\mathcal{L}(\mathrm{X}) \stackrel{\alpha=1}{=} u \mathcal{L}(\mathrm{Y}) v$. Рассмотрим прообразы $\bar{X}, \bar{Y}, \bar{u}$ и $\bar{v}$ на графе $\Gamma_{\alpha-1}$ путей $X, Y, u$ и v, образующие замкнутый путь $\bar{X}^{-1} \bar{u} \bar{Y} \bar{v}$. Пусть $\bar{E}$ - подпуть пути $\bar{X}$, для которого $\pi_{\alpha-1, \alpha}(\bar{E})=E$. Представим $\overline{\mathrm{u}}$ и $\overline{\mathrm{v}}$ в виде $\overline{\mathrm{u}}=\mathrm{u}_{1} \mathrm{Qu}_{2}$ и $\overline{\mathrm{v}}=\mathrm{v}_{1} \mathrm{Rv}_{2}$, где $\mathcal{L}\left(\mathrm{u}_{i}\right), \mathcal{L}\left(\mathrm{v}_{i}\right) \in \mathcal{H}_{\alpha-1} \quad(i=1,2)$ и $\mathcal{L}(\mathrm{Q}), \mathcal{L}(\mathrm{R})$ - подслова некоторых слов $B^{n}$ и $C^{n}$ при $B, C \in \mathcal{E}_{\alpha}$. Заметим, что если произвольный путь $S$ и некоторый подпуть пути $Q$ или пути $R$ близки в ранге $\alpha-1$, то в силу леммы 8.20 , (i) $\mathcal{W}_{\alpha}(\mathcal{L}(\mathrm{S})) \leqslant 1$. По лемме 11.7 , (i) при $\mathrm{W}:=\mathrm{u}_{1} \mathrm{Qu}_{2} \overline{\mathrm{Y}} \mathrm{v}_{1} \mathrm{Rv}_{2}, \mathrm{X}:=\overline{\mathrm{X}}$ и $\mathrm{Y}:=\overline{\mathrm{E}}$ путь $\overline{\mathrm{E}}$ можно разложить в произведение $\overline{\mathrm{E}}=\mathrm{w}_{0} \mathrm{E}_{1} \mathrm{w}_{1} \mathrm{E}_{2} \mathrm{w}_{2} \mathrm{E}_{3} \mathrm{w}_{3}$, где $\sum_{i=0}^{3} \mathcal{W}_{\alpha-1}\left(\mathcal{L}\left(\mathrm{w}_{i}\right)\right) \leqslant 2 \eta$ и каждый путь $\mathrm{E}_{i}$ либо пуст, либо близок в ранге $\alpha-1$ к некоторому подпути соответствующего пути $\mathrm{Q}, \overline{\mathrm{Y}}$ или R. В силу леммы 8.20, (ii), (iii) имеем $\mathcal{W}_{\alpha}\left(\mathcal{L}\left(\mathrm{w}_{0} \mathrm{E}_{1} \mathrm{w}_{1}\right)\right) \leqslant 1+2 \zeta \eta$, $\mathcal{W}_{\alpha}\left(\mathcal{L}\left(\mathrm{w}_{2} \mathrm{E}_{3} \mathrm{w}_{3}\right)\right) \leqslant 1+2 \zeta \eta$ и $\mathcal{W}_{\alpha}\left(\mathcal{L}\left(\mathrm{w}_{0} \mathrm{E}_{1} \mathrm{w}_{1}\right)\right)+\mathcal{W}_{\alpha}\left(\mathcal{L}\left(\mathrm{w}_{2} \mathrm{E}_{3} \mathrm{w}_{3}\right)\right) \leqslant 2+2 \zeta \eta$. Так как по условию $\mathcal{W}_{\alpha}(\mathcal{L}(\overline{\mathrm{E}}))>2+2 \zeta \eta$, то отсюда следует, что $\mathrm{E}_{2}-$ непустой путь, и, следовательно, $\mathrm{E}_{2}$ близок в ранге $\alpha-1$ к некоторому подпути $\overline{\mathrm{Y}}^{\prime}$ пути $\overline{\mathrm{Y}}$. Тогда в качестве искомых путей $\mathrm{U}_{1}, \mathrm{E}^{\prime}, \mathrm{U}_{2}$ и $\mathrm{Y}^{\prime}$ можно взять $\pi_{\alpha-1, \alpha}\left(\mathrm{w}_{0} \mathrm{E}_{1} \mathrm{w}_{1}\right), \pi_{\alpha-1, \alpha}\left(\mathrm{E}_{2}\right)$, $\pi_{\alpha-1, \alpha}\left(\mathrm{w}_{2} \mathrm{E}_{3} \mathrm{w}_{3}\right)$ и $\pi_{\alpha-1, \alpha}\left(\overline{\mathrm{Y}}^{\prime}\right)$ соответственно.

Случай 2: Имеет место случай (ii) из предложения 11.2 , т.е. найдутся слова $D_{i} \in \mathcal{E}_{\alpha}, x_{i}, y_{i}, z_{i}, w_{i} \in \mathcal{H}_{\alpha-1}, G_{i}, H_{i}, S_{i}, U_{i}, T_{i}, V_{i}(i=1,2, \ldots, r), K_{i}, L_{i}$ $(i=0,1, \ldots, r)$ такие, что выполнены соотношения (11.1)-(11.5). Как и в случае 1 , в (11.4) можно считать $u$ 으 $\hat{u}$ и $v$ 드 $\hat{v}$. Пусть $\mathrm{X}=\mathrm{K}_{0} \mathrm{G}_{1} \mathrm{~K}_{1} \ldots \mathrm{G}_{r-1} \mathrm{~K}_{r}$ и $\mathrm{Y}=$ $\mathrm{L}_{0} \mathrm{H}_{1} \mathrm{~L}_{1} \ldots \mathrm{H}_{r-1} \mathrm{~L}_{r}$, где метки путей $\mathrm{K}_{i}, \mathrm{G}_{i}, \mathrm{~L}_{i}$ и $\mathrm{H}_{i}$ равны словам $K_{i}, G_{i}, L_{i}$ и $H_{i}$ соответственно. В силу соотношений (11.1)-(11.5) рассмотрим пути на графе $\Gamma_{\alpha}$, расположенные, как на рис. 39 , и обозначенные теми же буквами, что и их метки. $\mathrm{B}$ силу сделанного выше замечания каждый путь $\mathrm{G}_{i}$ содержит некоторый подпуть 
$\widetilde{\mathrm{G}}_{i} \in$ Эл $_{\alpha}\left(\Gamma_{\alpha}, q_{4}\right)$. Отсюда следует, что подпуть $\mathrm{E}$ пути $\mathrm{X}$ содержится в одном из подпутей $\mathrm{Z}_{0} \rightleftharpoons \mathrm{K}_{0} \mathrm{G}_{1}, \mathrm{Z}_{1} \rightleftharpoons \mathrm{G}_{1} \mathrm{~K}_{1} \mathrm{G}_{2}, \mathrm{Z}_{2} \rightleftharpoons \mathrm{G}_{2} \mathrm{~K}_{2} \mathrm{G}_{3}, \ldots, \mathrm{Z}_{r} \rightleftharpoons \mathrm{G}_{r} \mathrm{~K}_{r}$. Пусть $\mathrm{E}$ содержится в $Z_{k}$. Положим

$$
\mathrm{u}^{*} \rightleftharpoons\left\{\begin{array} { l l } 
{ \mathrm { u } , } & { \text { если } k = 0 , } \\
{ \mathrm { x } _ { k } \mathrm { S } _ { k } \mathrm { V } _ { k } ^ { - 1 } \mathrm { w } _ { k } , } & { \text { если } k > 0 , }
\end{array} \quad \mathrm { v } ^ { * } \rightleftharpoons \left\{\begin{array}{ll}
\mathrm{v}, & \text { если } k=r, \\
\mathrm{z}_{k+1} \mathrm{U}_{k+1}^{-1} \mathrm{~S}_{k+1} \mathrm{y}_{k+1}, & \text { если } k<r .
\end{array}\right.\right.
$$

Так как $\mathcal{L}\left(\mathrm{u}^{*}\right), \mathcal{L}\left(\mathrm{v}^{*}\right) \in \mathcal{H}_{\alpha}$ и из $(11.2)-(11.4)$ следует $\mathcal{L}\left(\mathrm{Z}_{k}\right) \stackrel{\alpha=1}{=} \mathcal{L}\left(\mathrm{u}^{*}\right) L_{k} \mathcal{L}\left(\mathrm{v}^{*}\right)$, то можно воспользоваться рассуждениями из случая 1 при $\mathrm{X}:=\mathrm{Z}_{k}$ и $\mathrm{Y}:=\mathrm{L}_{k}$.
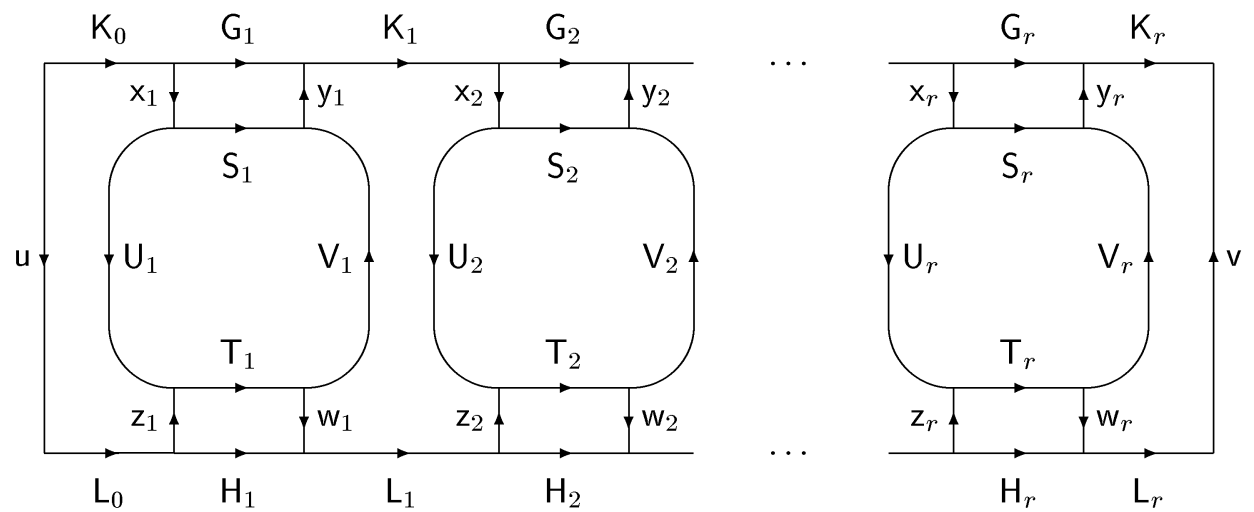

Рис. 39

Из предыдущего утверждения по индукции с использованием следствия 11.3, леммы 8.20 , (ii), (iii) и неравенств $\frac{1+\zeta \eta}{1-\zeta}<1+\zeta, \frac{1+2 \zeta \eta}{1-\zeta}<1+2 \zeta$ легко выводится

11.9. СлЕДСТВИЕ. Пусть пути $\mathrm{X} u \mathrm{Y}$ на графе $\Gamma_{\alpha}$ близки в ранге $\alpha, \mathcal{L}(\mathrm{X})$, $\mathcal{L}(\mathrm{Y}) \in \mathcal{R}_{\alpha}$, u nусть $\mathrm{X}=\mathrm{X}_{1} \mathrm{EX}_{2}$, zде $\mathcal{W}_{\alpha}(\mathcal{L}(\mathrm{E}))>2+2 \zeta$ u эл $_{\gamma}\left(\mathrm{E}, q_{4}\right)=\varnothing n p u$ $\gamma=\beta+1, \beta+2, \ldots, \alpha$. Тогда $\mathrm{E}=\mathrm{U}_{1} \mathrm{E}^{\prime} \mathrm{U}_{2} u \mathrm{Y}=\mathrm{V}_{1} \mathrm{Y}^{\prime} \mathrm{V}_{2}$, әде пути $\mathrm{E}^{\prime}$ и $\mathrm{Y}^{\prime}$ близки в ранге $\beta$ и $\mathcal{W}_{\alpha}\left(\mathcal{L}\left(\mathrm{U}_{i}\right)\right)<1+2 \zeta \quad(i=1,2)$.

11.10. ЛЕмма. Пусть $\mathrm{X} u \mathrm{uYv}-$ nути на графе $\Gamma_{\alpha-1}$ с общей начальной и конечной вершинами, где $\mathcal{L}(\mathrm{X}), \mathcal{L}(\mathrm{Y}) \in \mathcal{R}_{\alpha-1}$ и $\mathcal{L}(\mathrm{u}), \mathcal{L}(\mathrm{v}) \in \mathcal{H}_{\alpha}$. Пусть $\mathrm{u}=\mathrm{u}_{1} \mathrm{Qu}_{2}, \quad \mathrm{v}=\mathrm{v}_{1} \mathrm{Rv}_{2}$, әде $\mathcal{L}\left(\mathrm{u}_{i}\right), \mathcal{L}\left(\mathrm{v}_{i}\right) \in \mathcal{H}_{\alpha-1} u \mathcal{L}(\mathrm{Q}), \mathcal{L}(\mathrm{R})-$ подслова некоторых слов $B^{n}, C^{n}$ соответственно при $B, C \in \mathcal{E}_{\alpha}$. Пусть $\mathrm{E} \in \Pi$ Пер $\alpha(A, h+8)$ - подпуть пути $\mathrm{X}, \mathrm{P} \in \Pi$ Пер $\left(\Gamma_{\alpha-1}, A, 2\right)$ - периодическая основа для $\mathrm{E}$ и выполнены следуюшие условия:

(i) либо $|\mathrm{Q}|<2|B|$, либо $|\mathrm{Q}| \geqslant 2|B|$ u $\neg \operatorname{Cогл~}(\mathrm{P}, \mathrm{Q})$;

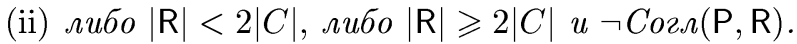

Тогда существует подпуть $\mathrm{F} \in \Pi_{\text {пер }}(A, 8)$ пути $\mathrm{Y}$ такой, что $\Pi C o \Omega_{\alpha}(\mathrm{E}, \mathrm{F}) u \ell_{\alpha, A}(\mathrm{~F}) \geqslant \ell_{\alpha, A}(\mathrm{E})-h$. 
ДоКАЗАТЕЛЬСТВо. По лемме 11.7 , (ii) при $\mathrm{W}:=\mathrm{u}_{1} \mathrm{Qu}_{2} \mathrm{Yv}_{1} \mathrm{Rv}_{2}$ найдутся подпути $\mathrm{F}_{1}, \mathrm{~F}_{2}, \ldots, \mathrm{F}_{t} \in$ ППер $\alpha(A)$ попарно различных путей $\mathrm{Q}, \mathrm{Y}$ и $\mathrm{R}$ такие, что ПСогл $\alpha\left(\mathrm{E}_{1} \mathrm{~F}_{i}\right)$ при всех $i$ и $\sum_{i} \ell_{\alpha, A}\left(\mathrm{~F}_{i}\right) \geqslant \ell_{\alpha, A}(\mathrm{E})-130$. Допустим, $\mathrm{F}_{i}-$ подпуть пути $\mathrm{Q}$ и $\ell_{\alpha, A}\left(\mathrm{~F}_{i}\right) \geqslant p$. Пусть $\mathrm{P}_{i} \in$ Пер $\left(\Gamma_{\alpha-1}, A, 2\right)$ - периодическая основа для $\mathrm{F}_{i}$. Так как в силу леммы 8.2 , (ii) пути $\mathrm{F}_{i}$ и $\mathrm{P}_{i}$ близки в ранге $\alpha-1$, то в силу предложения 8.44, (i) получаем $|\mathrm{Q}|>2|B|$, а в силу предложения 8.36 получаем $C$ олл $\left(\mathrm{P}_{i}, \mathrm{Q}\right)$. Далее, так как в силу предложений 8.9 и 8.5 имеем $\operatorname{Cozл}\left(\mathrm{P}_{i}, \mathrm{P}\right)$, то отсюда следует $\operatorname{Cozл(P,Q),~что~противоречит~(i).~Аналогично,~из~(ii)~следует,~что~если~} \mathrm{F}_{i}$ - подпуть пути $\mathrm{R}$, то $\ell_{\alpha, A}\left(\mathrm{~F}_{i}\right) \leqslant p-1$. Тогда некоторьй $\mathrm{F} \rightleftharpoons \mathrm{F}_{j}-$ подпуть пути $\mathrm{Y}_{\text {и }}$ $\ell_{\alpha, A}(\mathrm{~F}) \geqslant \ell_{\alpha, A}(\mathrm{E})-2 p-128=\ell_{\alpha, A}(\mathrm{E})-h$.

11.11. ОПРЕДЕЛЕНИЕ. Пусть $\mathrm{E} \in \ni_{\alpha}\left(\Gamma_{\alpha}\right)$ и u-путь на графе $\Gamma_{\alpha} \mathrm{c} \mathcal{L}(\mathrm{u}) \in \mathcal{H}_{\alpha}$. Будем говорить, что $\mathrm{E}$ устойчив относительно и, если и можно представить в виде $\mathbf{u}=\mathrm{u}_{1} \mathrm{Qu}_{2}, \mathcal{L}\left(\mathrm{u}_{1}\right), \mathcal{L}\left(\mathrm{u}_{2}\right) \in \mathcal{H}_{\alpha-1}, \mathcal{L}(\mathrm{Q})$ есть подслово слова $B^{n}, B \in \mathcal{E}_{\alpha}$, так что либо $|\mathrm{Q}|<2|B|$, либо $|\mathrm{Q}| \geqslant 2|B|$ и для любых прообразов $\overline{\mathrm{Q}}$ и $\overline{\mathrm{E}}$ на графе $\Gamma_{\alpha-1}$ путей $\mathrm{Q}$ и $\mathrm{E}$ вьполнено $\neg \operatorname{Coлл}(\mathrm{P}, \overline{\mathrm{Q}})$ и $\neg \operatorname{Cozл}\left(\mathrm{P}, \overline{\mathrm{Q}}^{-1}\right)$, где $\mathrm{P}$ - периодическая основа для $\overline{\mathrm{E}}$.

11.12. Лемма. Пусть и - путь на графе $\Gamma_{\alpha} c \mathcal{L}(\mathrm{u}) \in \mathcal{H}_{\alpha}$. Тогда верны следующие утверждения.

(i) $Е с л и \mathrm{E}, \mathrm{F} \in \ni_{\alpha}\left(\Gamma_{\alpha}\right)$ и $\neg$ Эогл $\alpha(\mathrm{E}, \mathrm{F})$, то по крайней мере один из путей $\mathrm{E}$ или $\mathrm{F}$ устойчив относительно и.

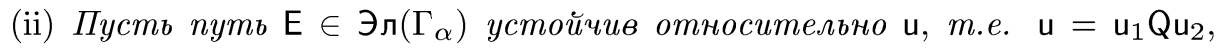
где $\mathcal{L}\left(\mathrm{u}_{1}\right), \mathcal{L}\left(\mathrm{u}_{2}\right) \in \mathcal{H}_{\alpha-1}, \quad \mathcal{L}(\mathrm{Q})$ есть подслово слова $B^{n}, \quad B \in \mathcal{E}_{\alpha}, u \mathrm{Q} u \mathrm{E}$ удовлетворяют условию из определения 11.11. Предположим, $|\mathrm{Q}| \geqslant 2|B| u$ $\mathrm{u}^{\prime}$ - слово, дополнительное $\kappa \mathcal{L}(\mathrm{u})$ в смысле определения 11.1 при $u_{i}:=\mathcal{L}\left(\mathrm{u}_{i}\right)$

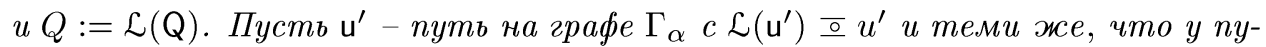
ти и, начальной и конечной вершинами. Тогда $\mathrm{E}$ устойчив относительно $\mathrm{u}^{\prime}$.

ДокАЗАтЕльство. (i) Предположим, например, что Е не устойчив относительно и. Пусть и $=\mathrm{u}_{1} \mathrm{Qu} \mathrm{u}_{2}$, где $\mathcal{L}\left(\mathrm{u}_{1}\right), \mathcal{L}\left(\mathrm{u}_{2}\right) \in \mathcal{H}_{\alpha-1}$ и $\mathcal{L}(\mathrm{Q})$ есть подслово слова $B^{n}$ при $B \in \mathcal{E}_{\alpha}$. Согласно определению 11.11 имеем $|\mathrm{Q}| \geqslant 2|B|$ и найдутся прообразы $\overline{\mathrm{E}}$ и $\overline{\mathrm{Q}}$ на графе $\Gamma_{\alpha-1}$ путей $\mathrm{E}$ и $\mathrm{Q}$ такие, что $\operatorname{Cozл}(\mathrm{P}, \overline{\mathrm{Q}})$ или $\operatorname{Cozл}\left(\mathrm{P}, \overline{\mathrm{Q}}^{-1}\right)$, где $\mathrm{P}$ - периодическая основа для $\overline{\mathrm{E}}$. Пусть $\overline{\mathrm{F}}$ - произвольный прообраз на графе $\Gamma_{\alpha-1}$ пути $F, R$ - периодическая основа для $F$ и $\bar{Q}^{\prime}$ - любой другой прообраз на графе $\Gamma_{\alpha-1}$ пути $\mathbf{Q}$. Пусть $\overline{\mathbf{Q}}^{\prime}=\mathbf{g} \overline{\mathbf{Q}}$, где $\mathbf{g} \in \operatorname{ker} \pi_{\alpha-1, \alpha}$. Так как $\pi_{\alpha-1, \alpha}(\mathbf{g} \overline{\mathrm{E}})=\mathrm{E}$ и $\mathbf{g P}$ - периодическая основа для $\mathbf{g E}$, то из $\neg$ ЭСогл $\alpha(\mathrm{E}, \mathrm{F})$ следует $\neg \operatorname{Coгл~}(\mathrm{gP}, \mathrm{R})$ и $\neg$ Согл $\left(\mathrm{gP}, \mathrm{R}^{-1}\right)$. Следовательно, $\neg$ Согл $(\mathrm{R}, \mathrm{g} \overline{\mathrm{Q}})$ и $\neg \operatorname{Coгл}\left(\mathrm{R}, \mathrm{g} \overline{\mathrm{Q}}^{-1}\right)$. Тогда согласно определению $11.11 \mathrm{~F}$ устойчив относительно $\mathrm{u}$.

(ii) Согласно определению 11.1 имеем $u^{\prime}$ б $u_{1} Q^{\prime} u_{2}$, где $u_{i} \rightleftharpoons \mathcal{L}\left(\mathrm{u}_{i}\right)$ и $\mathcal{L}(\mathrm{Q}) Q^{\prime} \approx$ $B^{n}$. Пусть $\mathrm{u}^{\prime} \rightleftharpoons \mathrm{u}_{1} \mathrm{Q}^{\prime} \mathrm{u}_{2}^{\prime}$, где $\mathcal{L}\left(\mathrm{Q}^{\prime}\right)$ 으 $Q^{\prime}$ и $\mathcal{L}\left(\mathrm{u}_{2}^{\prime}\right)$ 으 $u_{2}$. Если $\left|Q^{\prime}\right|<2|B|$, то доказывать нечего. Пусть $\left|Q^{\prime}\right| \geqslant 2|B|$. Пусть $\overline{\mathrm{Q}}^{\prime}$ и $\overline{\mathrm{E}}$ - произвольные прообразы на графе $\Gamma_{\alpha-1}$ путей $\mathrm{Q}^{\prime}$ и $\mathrm{E}$. Так как $\iota\left(\mathrm{Q}^{\prime}\right)=\iota(\mathrm{Q})$, то для некоторого прообраза $\overline{\mathrm{Q}}$ на графе $\Gamma_{\alpha-1}$ пути $\mathrm{Q}$ имеем $\iota\left(\overline{\mathrm{Q}}^{\prime}\right)=\iota(\overline{\mathrm{Q}})$. Согласно определению 11.11

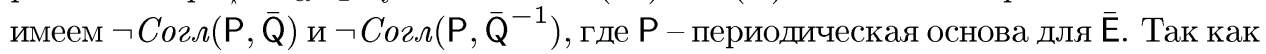
$\mathcal{L}\left(\overline{\mathrm{Q}} \overline{\mathrm{Q}}^{\prime-1}\right) \in$ Пер $\left(\Gamma_{\alpha-1}, B, 2\right)$, то $\overline{\mathrm{Q}} \overline{\mathrm{Q}}^{\prime-1}$ - общее периодическое продолжение $\overline{\mathrm{Q}}$ 
и $\overline{\mathrm{Q}}^{\prime-1}$. Следовательно, $\neg$ Cогл $\left(\mathrm{P}, \overline{\mathrm{Q}}^{\prime}\right)$ и $\neg \operatorname{Co\imath л}\left(\mathrm{P}, \overline{\mathrm{Q}}^{\prime}-1\right)$. Тогда согласно определению $11.11 \mathrm{E}$ устойчив относительно и.

11.13. ОПРЕДЕЛЕНИЕ. Пусть $\mathrm{E} \in \ni_{\alpha}\left(\Gamma_{\alpha}\right)$ и $\mathrm{E}=\pi_{\alpha-1, \alpha}(\overline{\mathrm{E}})$, где $\overline{\mathrm{E}} \in$ ППер $\alpha(A, 8)\left(A \in \mathcal{E}_{\alpha}\right)$. Пусть $\mathrm{P} \in$ Пер $\left(\Gamma_{\alpha-1}, A, 2\right)$ - периодическая основа для $\overline{\mathrm{E}}$. Тогда путь $\pi_{\alpha-1, \alpha}(\mathrm{P})$ также будем называть периодической основой для $\mathrm{E}$.

11.14. Лемма. Пусть $\mathrm{E}, \mathrm{F} \in \jmath_{\alpha}\left(\Gamma_{\alpha}\right)$ u $\mathrm{P}, \mathrm{Q}$ - периодические основы для $\mathrm{E}$ и $\mathrm{F}$ соответственно. Если $\mathrm{P} u \mathrm{Q}$ или $\mathrm{P} u \mathrm{Q}^{-1}$ имеют общее периодическое продолжение, то ЭСогл $(\mathrm{E}, \mathrm{F})$.

ДокаЗАтЕльСтво. Пусть $\overline{\mathrm{E}}, \overline{\mathrm{F}}, \overline{\mathrm{P}}, \overline{\mathrm{Q}}$ - прообразы на графе $\Gamma_{\alpha-1}$ путей $\mathrm{E}, \mathrm{F}, \mathrm{P}$ и $Q$ соответственно такие, что $\bar{P}$ есть периодическая основа для $\bar{E}$ и $\bar{Q}$ есть периодическая основа для $\overline{\mathrm{F}}$. Из посылки следует, что для некоторого $\mathbf{g} \in \operatorname{ker} \pi_{\alpha-1, \alpha}$ пути $\overline{\mathrm{P}}$ и $\mathbf{g} \overline{\mathrm{Q}}^{ \pm 1}$ имеют общее периодическое продолжение. Тогда в силу леммы 3.4, (iv) имеем $C$ огл $(\overline{\mathrm{P}}, \mathbf{g} \overline{\mathrm{Q}})$ или $\operatorname{Cогл}\left(\overline{\mathrm{P}}, \mathbf{g} \overline{\mathrm{Q}}^{-1}\right)$. Так как $\pi_{\alpha-1, \alpha}(\mathrm{gF})=\mathrm{F}$ и $\mathbf{g} \overline{\mathrm{Q}}-$ периодическая основа для $\mathbf{g} \overline{\mathrm{F}}$, то согласно определению 5.18 имеем $П \mathrm{Coz}_{\alpha}(\overline{\mathrm{E}}, \overline{\mathrm{F}})$ или ПСогл $\alpha\left(\overline{\mathrm{E}}, \overline{\mathrm{F}}^{-1}\right)$, а согласно определению $5.20-$ ЭСогл $\alpha_{\alpha}(\mathrm{E}, \mathrm{F})$.

11.15. ЛЕмма. Пусть $\mathrm{E} u$ uPv - пути на графе $\Gamma_{\alpha}$ с общими начальной $и$ конечной вершинами, где $\mathcal{L}(\mathrm{E}) \in \mathcal{R}_{\alpha-1}, \mathcal{L}(\mathrm{u}), \mathcal{L}(\mathrm{v}) \in \mathcal{H}_{\alpha-1} u \mathrm{P} \in \Pi_{\mathrm{ep}}\left(\Gamma_{\alpha}, A, r\right)$ при $A \in \mathcal{E}_{\alpha}$. Пусть $\mathcal{L}\left(\mathrm{E}^{-1} \mathrm{uPv}\right) \stackrel{\alpha=1}{=} 1$. Тогда если $r \geqslant 44$, то существуют nодпути $\mathrm{E}^{*} u \mathrm{P}^{*}$ путей $\mathrm{E}$ u $\mathrm{P}$ такие, что $\mathrm{E}^{*} \in \ni_{\alpha}\left(\Gamma_{\alpha}, r-36\right) u \mathrm{P}^{*}-$ nериодическая основа для $\mathrm{E}^{*}$.

ДоказАТЕЛЬСТво. Нужно перейти к прообразу $\bar{E}^{-1} \bar{u} \bar{P} \bar{v}$ на графе $\Gamma_{\alpha-1}$ замкнутого пути $\mathrm{E}^{-1} \mathrm{uPv}$ и воспользоваться предложением 8.23.

11.16. Лемма. Пусть $\mathrm{E}, \mathrm{F} \in \ni\left(\Gamma_{\alpha}\right), \neg Э$ Согл $\Omega_{\alpha}(\mathrm{E}, \mathrm{F})$ u $\mathrm{P}-$ периодическая основа для $\mathrm{E}$. Пусть $\mathrm{u}=\mathrm{u}_{1} \mathrm{Qu}_{2}-$ путь на графе $\Gamma_{\alpha}$, әде $\mathcal{L}\left(\mathbf{u}_{i}\right) \in \mathcal{H}_{\alpha-1}(i=1,2)$ и $\mathcal{L}(\mathrm{Q})$ - подслово слова $B^{n}$ при $B \in \mathcal{E}_{\alpha}$. Тогда если $\mathrm{Q}$ или $\mathrm{Q}^{-1}$ содержится в некотором периодическом продолжении $\mathrm{P}$, то $\mathrm{F}$ устойчив относительно $\mathrm{u}$.

ДокАЗАТЕЛЬСтво. Если $|\mathrm{Q}|<2|B|$, то доказывать нечего. Пусть $|\mathrm{Q}| \geqslant 2|B|$. Пусть $\bar{Q}$ и $\overline{\mathrm{F}}-$ произвольные прообразы на графе $\Gamma_{\alpha-1}$ путей $\mathrm{Q}$ и $\mathrm{F}$, и пусть $\mathrm{R}-$ периодическая основа для $\bar{F}$. Как в доказательстве леммы 11.14 , найдем прообразы $\overline{\mathrm{E}}$ и $\overline{\mathrm{P}}$ на $\Gamma_{\alpha-1}$ путей $\mathrm{E}$ и $\mathrm{P}$ такие, что $\overline{\mathrm{P}}$ - периодическая основа для $\overline{\mathrm{E}}$ и $\overline{\mathrm{P}}$ и $\overline{\mathrm{Q}}^{ \pm 1}$ имеют общее периодическое продолжение. Тогда имеем $\left.\operatorname{Coгл(~} \overline{\mathrm{P}}, \overline{\mathrm{Q}}\right)$ или $\operatorname{Coгл}\left(\overline{\mathrm{P}}, \overline{\mathrm{Q}}^{-1}\right)$, а из $\neg$ ЭСол $(\mathrm{E}, \mathrm{F})$ следует $\neg \operatorname{Cолл~}(\overline{\mathrm{P}}, \mathrm{R})$ и $\neg \operatorname{Coлл}\left(\overline{\mathrm{P}}, \mathrm{R}^{-1}\right)$. Следова-

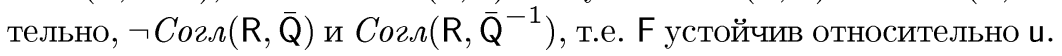

11.17. ПРЕДЛОЖЕНИЕ. Пусть $\mathrm{X} u \mathrm{u} \mathrm{Yv}-$ nути на графе $\Gamma_{\alpha}$ с общей начальной и конечной вершинами, где $\mathcal{L}(\mathrm{X}), \mathcal{L}(\mathrm{Y}) \in \mathcal{R}_{\alpha}$ и $\mathcal{L}(\mathrm{u}), \mathcal{L}(\mathrm{v}) \in \mathcal{H}_{\alpha}$. Пусть $\mathrm{E} \in \exists л_{\alpha}\left(\Gamma_{\alpha}, h+8\right)$ - подпуть пути $\mathrm{X} u \mathrm{E}$ устойчив относительно и $u$ v. Тогда для некоторого подпути $\mathrm{F} \in \ni_{\alpha}\left(\Gamma_{\alpha}\right)$ пути $\mathrm{Y}$ выполнено ЭСогл $(\mathrm{E}, \mathrm{F})$ и $\ell_{\alpha}(\mathrm{F}) \geqslant \min \left\{q_{4}, \ell_{\alpha}(\mathrm{E})-h\right\}$. 
ДоказАТЕЛЬСТво. Обозначим $X \rightleftharpoons \mathcal{L}(\mathrm{X}), u \rightleftharpoons \mathcal{L}(\mathrm{u}), Y \rightleftharpoons \mathcal{L}(\mathrm{Y})$ и $v \rightleftharpoons \mathcal{L}(\mathrm{v})$. Так как $X \stackrel{\alpha}{=} u Y v$, то в силу предложения 11.2 можно рассмотреть два случая.

Случай 1: Имеет место случай 11.2 , (i), т.е. $X \stackrel{\alpha=1}{=} \hat{u} Y \hat{v}$, где каждое из слов $\hat{u}$ или $\hat{v}$ либо совпадает с соответствующим словом $u$ или $v$, либо является дополнительным к нему. Заменяя в силу леммы 11.12 , (ii) пути u и v на пути с теми же начальными и конечными вершинами и с метками соответственно $\hat{u}$ и $\hat{v}$, можно считать, что $\mathcal{L}(\mathrm{u})=\hat{u}$ и $\mathcal{L}(\mathrm{v})=\hat{v}$. Рассмотрим прообразы $\overline{\mathrm{X}}, \overline{\mathrm{u}}, \overline{\mathrm{Y}}, \overline{\mathrm{v}}$ на графе $\Gamma_{\alpha-1}$ путей $\mathrm{X}, \mathrm{u}, \mathrm{Y}, \mathrm{v}$, образуюшие замкнутый путь $\overline{\mathrm{X}}^{-1} \overline{\mathrm{u}} \overline{\mathrm{Y}} \overline{\mathrm{v}}$. Пусть $\overline{\mathrm{E}} \in \Pi_{\text {Пер }}(A, h+8)-$ прообраз пути $E$, содержащийся в $\bar{X}$. Из определения 11.11 следует, что выполнены условия (i) и (ii) посылки леммы 11.10. Тогда по лемме 11.10 найдется подпуть $\overline{\mathrm{F}} \in$ ППер $\alpha(A, 8)$ пути $\overline{\mathrm{Y}}$, для которого ПСогл $\Omega_{\alpha}(\overline{\mathrm{E}}, \overline{\mathrm{F}})$ и $\ell_{\alpha, A}(\overline{\mathrm{F}}) \geqslant \ell_{\alpha, A}(\mathrm{E})-h$. Следовательно, можно положить $\mathrm{F} \rightleftharpoons \pi_{\alpha-1, \alpha}(\overline{\mathrm{F}})$.

Случай 2: Имеет место случай 11.2 , (ii), т.е. найдутся слова $D_{i} \in \mathcal{E}_{\alpha}$, $x_{i}, y_{i}, z_{i}, w_{i} \in \mathcal{H}_{\alpha-1}, G_{i}, H_{i}, S_{i}, U_{i}, T_{i}, V_{i}$ такие, что выполнены соотношения (11.1)-(11.5). В соответствии с равенствами (11.1)-(11.4) рассмотрим пути $\mathrm{x}_{i}, \mathrm{y}_{i}, \mathrm{z}_{i}, \mathrm{w}_{i}, \mathrm{G}_{i}, \mathrm{H}_{i}, \mathrm{~S}_{i}, \mathrm{U}_{i}, \mathrm{~T}_{i}, \mathrm{~V}_{i}$ на графе $\Gamma_{\alpha}$, обозначенные теми же буквами, что и их метки, и расположенные как на рис. 39 , где $\mathrm{X}=\mathrm{K}_{0} \mathrm{G}_{1} \mathrm{~K}_{1} \ldots \mathrm{G}_{r-1} \mathrm{~K}_{r}$ и $\mathrm{Y}=\mathrm{L}_{0} \mathrm{H}_{1} \mathrm{~L}_{1} \ldots \mathrm{H}_{r-1} \mathrm{~L}_{r}$. В силу (11.5) и леммы 11.15 каждый путь $\mathrm{G}_{i}$ содержит подпуть $\overline{\mathrm{G}}_{i}^{*} \in \ni_{\alpha}\left(\Gamma_{\alpha}, q_{4}\right)$ с периодической основой, содержащейся в $\mathrm{S}_{i}$, и каждый путь $\mathrm{H}_{i}$ содержит подпуть $\overline{\mathrm{H}}_{i}^{*} \in$ Эл $_{\alpha}\left(\Gamma_{\alpha}, q_{4}\right)$ с периодической основой, содержащейся в $\mathrm{T}_{i}$. В силу леммы 11.14 имеем $Э С о г \Omega_{\alpha}\left(\mathrm{G}_{i}^{*}, \mathrm{H}_{i}^{*}\right)$ при всех $i$. Если для некоторого $i$ вьполнено $Э$ Согл $\alpha\left(\mathrm{G}_{i}^{*}, \mathrm{E}\right)$, то в качестве $\mathrm{F}$ можно взять $\mathrm{H}_{i}^{*}$. Пусть $\neg$ ЭСогл $\Omega_{\alpha}\left(\mathrm{G}_{i}^{*}, \mathrm{E}\right)$ ни для какого $i$. Тогда в силу предложения $8.40 \mathrm{E}$ содержится в одном из подпутей $\mathrm{K}_{0} \mathrm{G}_{1}, \mathrm{G}_{1} \mathrm{~K}_{1} \mathrm{G}_{2}, \mathrm{G}_{2} \mathrm{~K}_{2} \mathrm{G}_{3}, \ldots, \mathrm{G}_{r} \mathrm{~K}_{r}$ пути $\mathrm{X}$. Пусть, например, $\mathrm{E}$ содержится в $\mathrm{G}_{i} \mathrm{~K}_{i} \mathrm{G}_{i+1}$ для некоторого $i$. По лемме 11.16 путь $\mathrm{E}$ устойчив относительно х $\mathrm{S}_{i} \mathrm{~V}_{i}^{-1} \mathrm{w}_{i}$ и $\mathrm{z}_{i+1} \mathrm{U}_{i+1}^{-1} \mathrm{~S}_{i+1} \mathrm{y}_{i+1}$. Так как $\mathrm{x}_{i} \mathrm{~S}_{i} \mathrm{~V}_{i}^{-1} \mathrm{w}_{i} \mathrm{~L}_{i} \mathrm{z}_{i+1} \mathrm{U}_{i+1}^{-1} \mathrm{~S}_{i+1} \mathrm{y}_{i+1}-$ путь с теми же начальной и конечной вершинами, что и $\mathrm{G}_{i} \mathrm{~K}_{i} \mathrm{G}_{i+1}$ (см. рис. 39 ), и из $(11.3),(11.4)$ следует $G_{i} K_{i} G_{i+1} \stackrel{\alpha=1}{=} x_{i} S_{i} V_{i}^{-1} w_{i} L_{i} z_{i+1} U_{i+1}^{-1} S_{i+1} y_{i+1}$, то тем самым утверждение сводится к случаю 1 при $\mathrm{X}:=\mathrm{G}_{i} \mathrm{~K}_{i} \mathrm{G}_{i+1}, \mathrm{u}:=\mathrm{x}_{i} \mathrm{~S}_{i} \mathrm{~V}_{i}^{-1} \mathrm{w}_{i}$, $\mathrm{Y}:=\mathrm{L}_{i}$ и $\vee:=\mathrm{z}_{i+1} \mathrm{U}_{i+1}^{-1} \mathrm{~S}_{i+1} \mathrm{y}_{i+1}$. Аналогично рассматриваются случаи, когда $\mathrm{E}$ содержится в $\mathrm{K}_{0} \mathrm{G}_{1}$ или в $\mathrm{G}_{r} \mathrm{~K}_{r}$, при этом в первом случае роль пути $\mathrm{x}_{i} \mathrm{~S}_{i} \mathrm{~V}_{i}^{-1} \mathrm{w}_{i}$ играет $\mathrm{u}$, а во втором роль пути $\mathrm{z}_{i+1} \mathrm{U}_{i+1}^{-1} \mathrm{~S}_{i+1} \mathrm{y}_{i+1}$ играет v.

11.18. СлЕДСТВИЕ. Пусть $\mathrm{X} u \mathrm{Y}$ - близкие в ранге $\alpha-1$ пути на графе $\Gamma_{\alpha}$ c $\mathcal{L}(\mathrm{X}), \mathcal{L}(\mathrm{Y}) \in \mathcal{R}_{\alpha}$. Тогда для любого подпути $\mathrm{E} \in \ni_{\alpha}\left(\Gamma_{\alpha}, h+8\right)$ пути $\mathrm{X}$ существует подпуть $\mathrm{F} \in$ Эл $_{\alpha}\left(\Gamma_{\alpha}\right)$ пути $\mathrm{Y}$ такой, что $Э$ Согл $\Omega_{\alpha}(\mathrm{E}, \mathrm{F})$ u $\ell_{\alpha}(\mathrm{F}) \geqslant$ $\min \left\{q_{4}, \ell_{\alpha}(\mathrm{E})-h\right\}$.

ДокАЗАТЕЛЬСтво. Достаточно заметить, что если $\mathbf{w}-$ путь на $\Gamma_{\alpha} \mathrm{c} \mathcal{L}(\mathrm{w}) \in$ $\mathcal{H}_{\alpha-1}$, то Е устойчив относительно $\mathrm{w}$, так как $\mathrm{w}=\mathrm{w}_{1} \mathrm{Q} \mathrm{w}_{2}$, где $\mathrm{w}_{1} \rightleftharpoons \mathrm{w}$ и $\mathrm{Q}, \mathrm{w}_{2}-$ пустые пути.

11.19. Лемма. Пусть $\mathrm{E}, \mathrm{F} \in \ni\left(\Gamma_{\alpha}\right)$ и $Э$ Согл $_{\alpha}(\mathrm{E}, \mathrm{F})$. Тогда каждая из вериин $\iota(\mathrm{E})$ или $\tau(\mathrm{E})$ может быть соединена $с$ каждой из вериин $\iota(\mathrm{F})$ или $\tau(\mathrm{F})$ путем и $с \mathcal{L}(\mathrm{u}) \in \mathcal{H}_{\alpha}$ таким, что относительно и устойчив любой путь $\mathrm{G} \in \mathrm{\ni}_{\alpha}\left(\Gamma_{\alpha}\right)$, для которого $\neg \mathcal{Э С о г л ~} \alpha_{\alpha}(\mathrm{G}, \mathrm{E})$. 
ДокАЗАТЕЛЬСТво. Пусть $\overline{\mathrm{E}}, \overline{\mathrm{F}}$ - прообразы на графе $\Gamma_{\alpha-1}$ путей $\mathrm{E}$ и $\mathrm{F}$ такие, что ПСогл $\Omega_{\alpha}(\overline{\mathrm{E}}, \overline{\mathrm{F}})$ или ПСол $\Omega_{\alpha}\left(\overline{\mathrm{E}}, \overline{\mathrm{F}}^{-1}\right)$. Пусть $\overline{\mathrm{P}} \in \Pi_{\mathrm{ep}}\left(\Gamma_{\alpha-1}, A, 2\right)\left(A \in \mathcal{E}_{\alpha}\right)-$ периодическая основа для $\overline{\mathrm{E}}$. Используя предложение 7.7 и индуктивное предположение 11.23 (см. доказательство предложения 8.45), легко видеть, что каждая из двух вершин $\iota(\overline{\mathrm{E}})$ или $\tau(\overline{\mathrm{E}})$ может быть соединена с каждой из двух вершин $\iota(\overline{\mathrm{F}})$ или $\tau(\overline{\mathrm{F}})$ путем $\overline{\mathrm{w}}$ вида $\overline{\mathrm{w}}=\overline{\mathrm{u}}_{1} \overline{\mathrm{Qu}}_{2}$, где $\mathcal{L}\left(\overline{\mathrm{u}}_{1}\right), \mathcal{L}\left(\overline{\mathrm{u}}_{2}\right) \in \mathcal{H}_{\alpha-1}$ и $\overline{\mathrm{Q}}$ или $\overline{\mathrm{Q}}^{-1}$ содержится в некотором периодическом продолжении $\overline{\mathrm{P}}$. Пусть $\mathrm{u}_{i} \rightleftharpoons \pi_{\alpha-1, \alpha}\left(\overline{\mathrm{u}}_{i}\right)$, $\mathrm{Q} \rightleftharpoons \pi_{\alpha-1, \alpha}(\overline{\mathrm{Q}})$ и $\mathrm{w} \rightleftharpoons \pi_{\alpha-1, \alpha}(\overline{\mathrm{w}})=\mathrm{u}_{1} \mathrm{Qu}_{2}$. Очевидно, $\mathrm{Q}$ или $\mathrm{Q}^{-1}$ содержится в некотором периодическом продолжении $\mathrm{P} \rightleftharpoons \pi_{\alpha-1, \alpha}(\overline{\mathrm{P}})$. Так как $A^{n} \stackrel{\alpha}{=} 1$, то $\tau\left(\mathrm{Q}^{\prime}\right)=\tau(\mathrm{Q})$ для некоторого начала $\mathrm{Q}^{\prime}$ пути $\mathrm{Q}$ длины $\left|\mathrm{Q}^{\prime}\right|<n|A|$. Тогда $\mathcal{L}\left(\mathrm{Q}^{\prime}\right)-$ подслово слова $A^{\prime n}$, где $A^{\prime}$ - некоторый циклический сдвиг слова $A$, и в силу леммы 11.16 путь $\mathrm{u} \rightleftharpoons \mathrm{u}_{1} \mathrm{Q}^{\prime} \mathrm{u}_{2}$ является искомым.

11.20. СЛЕДСТВИЕ. Пусть $\mathrm{X} u \mathrm{Y}$ - близкие в ранге $\alpha$ пути на графе $\Gamma_{\alpha}$ $c \mathcal{L}(\mathrm{X}), \mathcal{L}(\mathrm{Y}) \in \mathcal{R}_{\alpha}$. Пусть $\mathrm{E}_{i} \in \ni л_{\alpha}(\mathrm{X}, h+8) \quad(i=1,2,3), \neg$ ЭСогл $\alpha_{\alpha}\left(\mathrm{E}_{i}, \mathrm{E}_{j}\right)$ при $i \neq j u \mathrm{E}_{1}<\mathrm{x} \mathrm{E}_{2}<\mathrm{x} \mathrm{E}_{3}$. Тогда существует $\mathrm{F} \in \ni_{\alpha}(\mathrm{Y})$ такой, что $\exists \operatorname{Coz} \Omega_{\alpha}\left(\mathrm{E}_{2}, \mathrm{~F}\right) u \ell_{\alpha}(\mathrm{F}) \geqslant \min \left\{q_{4}, \ell_{\alpha}\left(\mathrm{E}_{2}\right)-h\right\}$.

ДокАЗАТЕЛЬСТво. Соединим вершины $\iota(\mathrm{X})$ и $\iota(\mathrm{Y})$ путем $\mathrm{u}$, а вершины $\tau(\mathrm{Y})$ и $\tau(\mathrm{X})$ путем $\mathrm{v}$, где $\mathcal{L}(\mathrm{u}), \mathcal{L}(\mathrm{v}) \in \mathcal{H}_{\alpha}$. По лемме $11.12,(\mathrm{i})$ хотя бы один из $\mathrm{E}_{i}$ устойчив как относительно u, так и относительно v. Если это $\mathrm{E}_{2}$, то можно непосредственно воспользоваться предложением 11.17. Из двух оставшихся симметричных случаев рассмотрим случай, когда $\mathrm{E}_{1}$ устойчив относительно и и v. По предложению 11.17 найдется $\mathrm{F}_{1} \in Э \boldsymbol{л}_{\alpha}(\mathrm{Y})$ такой, что ЭСог, $\boldsymbol{\Omega}_{\alpha}\left(\mathrm{E}_{1}, \mathrm{~F}_{1}\right)$. Тогда по лемме 11.19 начальные вершины путей $\mathrm{E}_{1}$ и $\mathrm{F}_{1}$ соединены путем u' с $\mathcal{L}\left(\mathrm{u}^{\prime}\right) \in \mathcal{H}_{\alpha}$, относительно которого устойчивы $E_{2}$ и $E_{3}$. Если теперь $E_{2}$ устойчив относительно $v$, то снова можно воспользоваться предложением 11.17 , рассмотрев вместо путей $\mathrm{X}$ и $\mathrm{Y}$ их конщы, начинающиеся с $E_{1}$ и с $F_{1}$ соответственно. Если $E_{2}$ не устойчив относительно v, то по лемме 11.12 , (i) $\mathrm{E}_{3}$ устойчив относительно $\mathrm{v}$, и, повторив рассуждения, найдем сначала $F_{3} \in$ Эл $_{\alpha}(\mathrm{Y})$ такой, что $\mathrm{F}_{1}<_{\mathrm{Y}} \mathrm{F}_{3}$, а затем путь $\mathrm{v}^{\prime}$ с $\mathcal{L}\left(\mathrm{v}^{\prime}\right) \in \mathcal{H}_{\alpha}$, соединяюший вершины $\tau\left(\mathrm{F}_{3}\right)$ и $\tau\left(\mathrm{E}_{3}\right)$, такой, что $\mathrm{E}_{2}$ устойчив относительно $\mathrm{v}^{\prime}$. Теперь можно применить предложение 11.17 при $X:=E_{1} \cup_{X} E_{3}$ и $Y:=F_{1} \cup_{Y} F_{3}$.

11.21. СлЕДСТВИЕ. Пусть $1 \leqslant \beta \leqslant \alpha, \mathbf{X} u \mathrm{Y}-$ близкие в ранге $\beta-1$ пути на графе $\Gamma_{\alpha} u \mathcal{L}(\mathrm{X}), \mathcal{L}(\mathrm{Y}) \in \mathcal{R}_{\alpha}$. Пусть Эл $\gamma\left(\mathrm{X}, q_{4}\right)=\varnothing$ или Эл $\gamma\left(\mathrm{Y}, q_{4}\right)=\varnothing д л я$ каждого $\gamma=\beta+1, \beta+2, \ldots, \alpha$. Тогда для любого $\mathrm{M} \in \ni(\mathrm{X}, t)$ nри $t \geqslant h+8$ существует $\mathrm{M}^{\prime} \in \ni_{\beta}\left(\mathrm{Y}, t^{\prime}\right)$, әде $t^{\prime}=\min \left\{q_{4}, t-h\right\}$, такой, что $Э$ Согл $_{\beta}\left(\mathrm{M}, \mathrm{M}^{\prime}\right)$.

ДоКАЗАТЕЛЬСТво. Если $\beta=\alpha$, то утверждения следствий 11.21 и 11.18 совпадают. Пусть $\beta<\alpha$. В силу следствия 11.6 найдутся близкие в ранге $\beta-1$ прообразы $\overline{\mathrm{X}}$ и $\overline{\mathrm{Y}}$ на графе $\Gamma_{\beta}$ путей $\mathrm{X}$ и $\mathrm{Y}$. Из определения 5.20 легко видеть, что для любых $\mathrm{K}, \mathrm{L} \in \ni{ }_{\beta}\left(\Gamma_{\beta}\right)$ из Согл $\beta_{\beta}(\mathrm{K}, \mathrm{L})$ следует $Э \operatorname{Cогл}_{\beta}\left(\pi_{\beta, \alpha}(\mathrm{K}), \pi_{\beta, \alpha}(\mathrm{L})\right)$. Тогда, очевидно, можно воспользоваться индуктивным предположением 11.18 при $\alpha:=\beta$.

11.22. СлЕДСТвИЕ $(\alpha \geqslant 0)$. Пусть $0 \leqslant \beta \leqslant \alpha, \quad \mathrm{X} u \mathrm{Y}$ - близкие в ранге $\beta$ пути на графе $\Gamma_{\alpha}$ и $\mathcal{L}(\mathrm{X}), \mathcal{L}(\mathrm{Y}) \in \mathcal{R}_{\alpha}$. Пусть Эл $\gamma\left(\mathrm{X}, q_{4}\right)=\varnothing$ или Эл $\gamma\left(\mathrm{Y}, q_{4}\right)=\varnothing$ для каждого $\gamma=\beta+1, \beta+2, \ldots, \alpha$. Пусть $\mathrm{K}, \mathrm{L} \in \ni(\mathrm{X}, h+8), \mathrm{M} \in$ Эл $_{\beta}(\mathrm{X}, t)$ 
для некоторого $t \geqslant h+8, \quad \mathrm{~K}<\mathrm{x} \mathrm{M}<\mathrm{x} \mathrm{L} u \mathrm{~K}, \mathrm{~L}, \mathrm{M}$ попарно не согласованвь. Тогда существует $\mathrm{M}^{\prime} \in \ni_{\beta}\left(\mathrm{Y}, t^{\prime}\right)$, где $t^{\prime}=\min \left\{q_{4}, t-h\right\}$, такой, что $\vartheta \operatorname{Coz}_{\beta}\left(\mathrm{M}, \mathrm{M}^{\prime}\right)$.

ДокАЗАТЕЛЬСтво. Пусть $\bar{X}$ и $\bar{Y}$ - близкие в ранге $\beta$ прообразы на графе $\Gamma_{\beta}$ путей $\mathrm{X}$ и $\mathrm{Y}$, существующие в силу следствия 11.6. Если $\beta=0$, то в силу определения 7.4 пути $\bar{X}$ и $\bar{Y}$ имеют обшие начальную и конечную вершины. В этом случае $\mathcal{L}(\overline{\mathrm{X}}) \stackrel{0}{=} \mathcal{L}(\overline{\mathrm{Y}})$ в свободной группе $\mathbf{B}_{0}$, и так как $\mathcal{L}(\overline{\mathrm{X}}), \mathcal{L}(\overline{\mathrm{Y}})$ - несократимые слова, то $\mathcal{L}(\bar{X})$ 으 $\mathcal{L}(\bar{Y})$, т.е. $X=Y$. Тогда утверждение становится тривиальныт. Если $\beta \geqslant 1$, то утверждение вытекает из следствия 11.20.

11.23. СлеДСТвИЕ $(\alpha \geqslant 0)$. Пусть $0 \leqslant \beta \leqslant \alpha, \quad \mathrm{X} u \mathrm{Y}-$ nути на графе $\Gamma_{\alpha}$

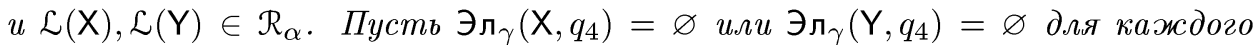
$\gamma=\beta+1, \beta+2, \ldots, \alpha$. Пусть $\mathrm{K}, \mathrm{L} \in$ Эл $_{\beta}(\mathrm{X}, h+8), \quad \mathrm{M} \in \ni(\mathrm{X}, t)$ для некоmорого $t \geqslant h+8, \quad \mathrm{~K}^{\prime}, \mathrm{L}^{\prime} \in$ Эл $\beta_{\beta}(\mathrm{Y}, p+34), \mathrm{K}<\mathrm{x} \mathrm{M}<\mathrm{x} \mathrm{L}, \mathrm{K}^{\prime}<\mathrm{Y} \mathrm{L}^{\prime}, \mathrm{K}, \mathrm{L}, \mathrm{M}$ попарно не согласованы, ЭСогл ${ }_{\beta}\left(\mathrm{K}_{1} \mathrm{~K}^{\prime}\right)$ и $Э С о г \Omega_{\beta}\left(\mathrm{L}, \mathrm{L}^{\prime}\right)$. Тогда существует $\mathrm{M}^{\prime} \in Э{ }_{\beta}\left(\mathrm{Y}, t^{\prime}\right)$, где $t^{\prime}=\min \left\{q_{4}, t-h\right\}$, такой, что ЭСогл $\beta\left(\mathrm{M}^{\prime} \mathrm{M}^{\prime}\right)$. Если при этом $t \geqslant h+p+34$, mо $\mathrm{K}^{\prime}<_{\mathrm{Y}} \mathrm{M}^{\prime}<_{\mathrm{Y}} \mathrm{L}^{\prime}$.

ДокАЗАТЕЛЬСТво. Так как в силу предложения 8.45 пути $K \cup_{X} L_{\text {и }} K^{\prime} \cup_{Y} L^{\prime}$ близки в ранге $\beta$, то первое утверждение вытекает из следствия 11.22. Предположим, $t \geqslant h+p+34$. Тогда $t^{\prime} \geqslant p+34$, и так как $\neg$ Эогл $\Omega_{\beta}\left(\mathrm{K}^{\prime}, \mathrm{M}^{\prime}\right)$ и $\neg$ Эогл $\Omega_{\beta}\left(\mathrm{L}^{\prime}, \mathrm{M}^{\prime}\right)$, то по предложению 8.40 путь $\mathrm{M}^{\prime}$ не содержится в $\mathrm{K}^{\prime}$ или в $\mathrm{L}^{\prime}$ и ни один из путей $\mathrm{K}^{\prime}, \mathrm{L}^{\prime}$ не содержится в $\mathrm{M}^{\prime}$. Отсюда следует, что $\mathrm{K}^{\prime}<_{\mathrm{Y}} \mathrm{M}^{\prime}<_{\mathrm{Y}} \mathrm{L}^{\prime}$.

11.24. ПРЕДЛОЖЕНИЕ. Пусть $1 \leqslant \beta \leqslant \alpha, \mathrm{X}$ - путь на графе $\Gamma_{\beta-1} c \mathcal{L}(\mathrm{X}) \in$

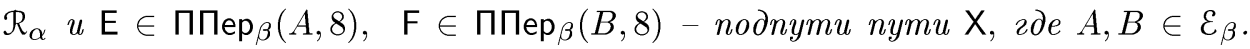
Тогда

$$
{ } \operatorname{Coгл}_{\beta}\left(\pi_{\beta-1, \alpha}(\mathrm{E}), \pi_{\beta-1, \alpha}(\mathrm{F})\right) \Leftrightarrow \Pi \operatorname{Coгл}_{\beta}(\mathrm{E}, \mathrm{F}) \text { или } \Pi \operatorname{Coгл}_{\beta}\left(\mathrm{E}, \mathrm{F}^{-1}\right) .
$$

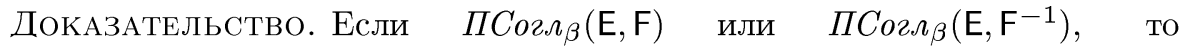
$\vartheta \operatorname{Coгл}_{\beta}\left(\pi_{\beta-1, \alpha}(\mathrm{E}), \pi_{\beta-1, \alpha}(\mathrm{F})\right)$ согласно определению 5.20. Докажем, что из $\vartheta{\operatorname{Coл} \Omega_{\beta}}_{\beta}\left(\pi_{\beta-1, \alpha}(\mathrm{E}), \pi_{\beta-1, \alpha}(\mathrm{F})\right)$ следует ПСогл $\beta\left(\mathrm{E}, \mathrm{F}^{\epsilon}\right)$ при некотором $\epsilon= \pm 1$. Согласно определению 5.20 найдутся пути $\widetilde{E}$ и $\widetilde{F}$ на графе $\Gamma_{\beta-1}$ такие, что $\pi_{\beta-1, \alpha}(\widetilde{\mathrm{E}})=\mathrm{E}, \pi_{\beta-1, \alpha}(\widetilde{\mathrm{F}})=\mathrm{F}$ и ПСол $\Omega_{\beta}\left(\widetilde{\mathrm{E}}, \widetilde{\mathrm{F}}^{\epsilon}\right)$. Имеем $\widetilde{\mathrm{E}}=\mathbf{g E}$ и $\widetilde{\mathrm{F}}=\mathbf{h F}$ для некоторых $\mathbf{g}, \mathbf{h} \in \operatorname{ker} \pi_{\beta-1, \alpha}$ и, заменяя $\widetilde{\mathrm{E}}_{\text {и }} \widetilde{\mathrm{F}}_{\text {на }} \mathbf{h}^{-1} \widetilde{\mathrm{E}}_{\text {и }} \mathbf{h}^{-1} \widetilde{\mathrm{F}}$, можем считать, что $\mathbf{h}=1$, т.е. $\widetilde{\mathrm{F}}=\mathrm{F}$. Достаточно доказать, что $\Pi \operatorname{Coл}_{\beta}(\widetilde{\mathrm{E}}, \mathrm{E})$. Пусть $\mathrm{P} \in \Pi_{\mathrm{ep}}\left(\Gamma_{\beta-1}, A, 2\right)-$ периодическая основа для Е. Тогда $\mathbf{g P}$ - периодическая основа для Ё. Без ограничения обшности можно считать, что слово $\mathcal{L}(\mathrm{P})$ начинается с $A$. В силу леммы 8.2, (ii) вершины $\iota(\mathrm{E})$ и $\iota(\mathrm{F})$ соединены некоторым путем и с $\mathcal{L}(\mathrm{u}) \in \mathcal{H}_{\beta-1}$. Так как $\Pi$ Согл $\beta\left(\widetilde{\mathrm{E}}, \mathrm{F}^{\epsilon}\right)$, то, используя предложение 7.7 и индуктивное предположение 11.23 , легко видеть, что вершины $\iota(\mathrm{gP})$ и $\iota(\mathrm{F})$ соединены путем вида $\mathrm{Rv}$, где $\mathcal{L}(\mathrm{v}) \in \mathcal{H}_{\beta-1}$ и $\mathcal{L}(\mathrm{R})$ - начало слова вида $A^{ \pm t}$. Пусть $\mathrm{Y}$ - подпуть пути $\mathrm{X}$ или $\mathrm{X}^{-1}$, соединяюший вершины $\iota(\mathrm{F})$ и $\iota(\mathrm{E})$. Мы имеем, таким образом, путь RvYu, соединяюший вершины $\iota(\mathrm{gP})$ и $\iota(\mathrm{P})$ (см. рис. 40$)$. Так как вершины $\iota(\mathrm{gP})$ и $\iota(\mathrm{P})$ отображаются в одну и ту же вершину графа $\Gamma_{\alpha}$, то $\mathcal{L}(\mathrm{RvYu}) \stackrel{\alpha}{=} 1$. Пусть $\mathcal{L}(\mathrm{R}) \stackrel{0}{=} A^{k n} S$, где 
$S$-начало слова $A^{n}$. Так как $A^{k n} \stackrel{\alpha}{=} 1$, то $(\mathcal{L}(\mathrm{Y}))^{-1} \stackrel{\alpha}{=} \mathcal{L}(\mathrm{u}) S \mathcal{L}(\mathrm{v})$. Тогда по предложению 11.2 при $X:=(\mathcal{L}(\mathrm{Y}))^{-1}, u:=\mathcal{L}(\mathrm{u}) S \mathcal{L}(\mathrm{v}), Y:=v:=1$ и $\alpha:=\alpha, \alpha-1, \ldots, \beta$, учитывая, что в силу предложения 8.18 пустое слово не может содержать элементарных $q_{4}$-степеней ранга $\geqslant 1$, получаем $(\mathcal{L}(\mathrm{Y}))^{-1} \stackrel{\beta=1}{=} \mathcal{L}(\mathrm{u}) \widehat{S} \mathcal{L}(\mathrm{v})$, где $\widehat{S}$ 으 $S$ или, в случае $S \geqslant 2|A|$, возможно, $S \widehat{S}^{-1}$ 으 $A^{n}$. Поменяв $k$, полученное равенство можно переписать в виде $(\mathcal{L}(\mathrm{Y}))^{-1} \stackrel{\beta=1}{=} \mathcal{L}(\mathrm{u}) A^{k n} \mathcal{L}(\mathrm{Rv})$, т.е. $\mathcal{L}(\mathrm{Rv} Y \mathrm{u}) \stackrel{\beta=1}{=} A^{-k n}$. Отсюда следует, что вершины $\iota(\mathrm{gP})$ и $\iota(\mathrm{P})$ соединены путем $\mathrm{T}$ с $\mathcal{L}(\mathrm{T})$ 으 $A^{-k n}$. Так как слово $\mathcal{L}(\mathrm{gP})$ 은 $\mathcal{L}(\mathrm{P})$ начинается с $A$, то в этом случае $\mathrm{gP}$ и $\mathrm{P}$ имеют общее периодическое продолжение. Тогда имеем Согл( $\mathbf{g P}, \mathrm{P})$, и, следовательно, ПСогл $\boldsymbol{}_{\beta}(\widetilde{\mathrm{E}}, \mathrm{E})$, что и требовалось.

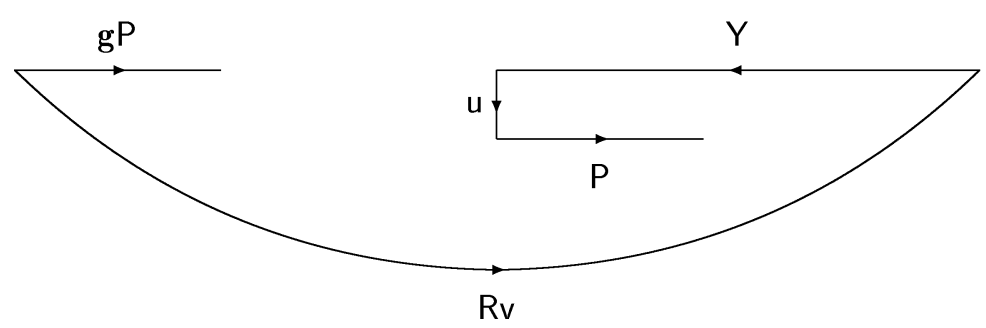

Рис. 40

11.25. ПРЕДЛОЖЕНИЕ $(\alpha \geqslant 0)$. Пусть $\beta \leqslant \alpha, \quad \mathrm{X}-$ nуть на графе $\Gamma_{\alpha} c$

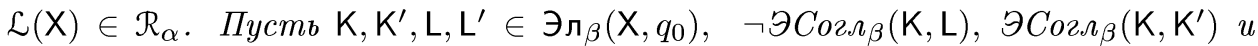
ЭСогл $\beta_{\beta}\left(\mathrm{L}, \mathrm{L}^{\prime}\right)$. Тогда $\mathrm{K}<\mathrm{x} \mathrm{L} \Leftrightarrow \mathrm{K}^{\prime}<\mathrm{x} \mathrm{L}^{\prime}$.

ДоКАЗАТЕЛЬСтво. При $\beta=0$ утверждение тривиально. При $\beta \geqslant 1$ нужно перейти к прообразу на графе $\Gamma_{\beta-1}$ пути $\mathrm{X}$ и воспользоваться предложениями 11.24 , 8.46 и 8.39 .

11.26. ЛЕмма $(\alpha \geqslant 0)$. Пусть $\mathrm{X}, \mathrm{Y}-$ nути на графе $\Gamma_{\alpha} c \mathcal{L}(\mathrm{X}), \mathcal{L}(\mathrm{Y}) \in \mathcal{R}_{\alpha}$.

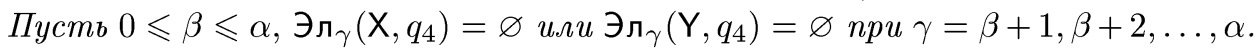

Пусть , далее, $\mathrm{K}_{i} \in$ Эл $_{\beta}\left(\mathrm{X}, q_{0}+h\right), \mathrm{K}_{i}^{\prime} \in \ni_{\beta}\left(\mathrm{Y}, q_{0}+h\right) \quad(i=1,2,3)$, $\vartheta$ Согл $\beta\left(\mathrm{K}_{i}, \mathrm{~K}_{i}^{\prime}\right)$ nри всех $i, \neg Э$ Cогл $\beta_{\beta}\left(\mathrm{K}_{i}, \mathrm{~K}_{j}\right)$ nрu $i \neq j$ u $\mathrm{K}_{1}<\mathrm{x} \mathrm{K}_{2}<\mathrm{x} \mathrm{K}_{3}$. Тогда если для некоторых $1 \leqslant i<j \leqslant 3$ выполнено $\mathrm{K}_{i}^{\prime}<_{\mathrm{Y}} \mathrm{K}_{j}^{\prime}$, mо $\mathrm{K}_{1}^{\prime}<_{\mathrm{Y}} \mathrm{K}_{2}^{\prime}<_{\mathrm{Y}} \mathrm{K}_{3}^{\prime}$.

ДокаЗАТЕЛЬСтво. Допустим, $\mathrm{K}_{i}^{\prime}<_{\mathrm{Y}} \mathrm{K}_{j}^{\prime}$ для некоторых $1 \leqslant i<j \leqslant 3$, но неверно, что $\mathrm{K}_{1}^{\prime}<_{\mathrm{Y}} \mathrm{K}_{2}^{\prime}<_{\mathrm{Y}} \mathrm{K}_{3}^{\prime}$. В силу предложения 8.40 имеет место один из четырех случаев: $\mathrm{K}_{1}^{\prime}<_{\mathrm{Y}} \mathrm{K}_{3}^{\prime}<_{\mathrm{Y}} \mathrm{K}_{2}^{\prime}, \mathrm{K}_{2}^{\prime}<_{\mathrm{Y}} \mathrm{K}_{1}^{\prime}<_{\mathrm{Y}} \mathrm{K}_{3}^{\prime}, \mathrm{K}_{2}^{\prime}<_{\mathrm{Y}} \mathrm{K}_{3}^{\prime}<_{\mathrm{Y}} \mathrm{K}_{1}^{\prime}$ или $\mathrm{K}_{3}^{\prime}<_{\mathrm{Y}} \mathrm{K}_{1}^{\prime}<_{\mathrm{Y}} \mathrm{K}_{2}^{\prime}$. Если $\mathrm{K}_{1}^{\prime}<_{\mathrm{Y}} \mathrm{K}_{3}^{\prime}<_{\mathrm{Y}} \mathrm{K}_{2}^{\prime}$, то в силу следствия 11.23 при $\mathrm{X}:=\mathrm{Y}, \mathrm{Y}:=\mathrm{X}, \mathrm{K}:=\mathrm{K}_{1}^{\prime}, \mathrm{L}:=\mathrm{K}_{2}^{\prime}$ и $\mathrm{M}:=\mathrm{K}_{3}^{\prime}$ найдется $\mathrm{L} \in$ Эл $_{\beta}\left(\mathrm{X}, q_{0}\right)$ такой, что ЭСогл $\beta\left(\mathrm{K}_{3}^{\prime}, \mathrm{L}\right)$ и $\mathrm{K}_{1}<\mathrm{x} \mathrm{L}<\mathrm{x} \mathrm{K}_{2}$, и мы приходим к противоречию с предложением 11.25. Аналогично рассматривается случай $\mathrm{K}_{2}^{\prime}<_{\mathrm{Y}} \mathrm{K}_{1}^{\prime}<_{\mathrm{Y}} \mathrm{K}_{3}^{\prime}$, с применением следствия 11.23 при $\mathrm{M}:=\mathrm{K}_{1}^{\prime}$. Из двух оставшихся случаев в силу полной аналогии ограничимся рассмотрением случая, когда $\mathrm{K}_{2}^{\prime}<_{\mathrm{Y}} \mathrm{K}_{3}^{\prime}<_{\mathrm{Y}} \mathrm{K}_{1}^{\prime}$. Для вывода противоречия рассмотрим два подслучая. 
Случай 1: $\beta=0$. Согласно определению $5.14 \mathrm{~K}_{i}=\mathrm{K}_{i}^{\prime}(i=1,2,3)$ и $\mathcal{L}\left(\mathrm{K}_{i}\right)-$ однобуквенные слова. Пусть $\mathrm{X}=\mathrm{R}_{0} \mathrm{~K}_{1} \mathrm{R}_{1} \mathrm{~K}_{2} \mathrm{R}_{2} \mathrm{~K}_{3} \mathrm{R}_{3}$ и $\mathrm{Y}=\mathrm{S}_{0} \mathrm{~K}_{2} \mathrm{~S}_{1} \mathrm{~K}_{3} \mathrm{~S}_{2} \mathrm{~K}_{1} \mathrm{~S}_{3}$. Тогда $\mathrm{W} \rightleftharpoons \mathrm{K}_{1} \mathrm{R}_{1} \mathrm{~K}_{2} \mathrm{R}_{2} \mathrm{~K}_{3} \mathrm{~S}_{2}$ - замкнутый путь на $\Gamma_{\alpha}$, и в силу следствия 11.6 при $\mathrm{X}:=\mathrm{K}_{1} \mathrm{R}_{1} \mathrm{~K}_{2} \mathrm{R}_{2} \mathrm{~K}_{3}$ и $\mathrm{Y}:=\mathrm{S}_{2}^{-1}$ сушествует замкнутый путь $\overline{\mathrm{W}}$ на $\Gamma_{0}$, являюшийся прообразом $\mathrm{W}$. Так как $\mathcal{L}\left(\mathrm{K}_{1} \mathrm{R}_{1} \mathrm{~K}_{2} \mathrm{R}_{2} \mathrm{~K}_{3}\right)$ и $\mathcal{L}\left(\mathrm{K}_{3} \mathrm{~S}_{2}\right)$ - подслова несократимых слов $\mathcal{L}(\mathrm{X})$ и $\mathcal{L}(\mathrm{Y})$ соответственно, то слово $\mathcal{L}(\overline{\mathrm{W}})$ несократимо. Но это невозможно, так как $\mathcal{L}(\overline{\mathrm{W}}) \stackrel{0}{=} 1$ в свободной группе $\mathbf{B}_{0}$.

Случай 2: $\beta>0$. Применяя следствие 11.23 при $\left(\mathrm{X}, \mathrm{Y}, \mathrm{K}, \mathrm{L}, \mathrm{M}, \mathrm{K}^{\prime}, \mathrm{L}^{\prime}\right):=$ $\left(\mathrm{X}, \mathrm{Y}^{-1}, \mathrm{~K}_{1}, \mathrm{~K}_{3}, \mathrm{~K}_{2}, \mathrm{~K}_{1}^{\prime-1}, \mathrm{~K}_{3}^{\prime-1}\right)$ (с учетом леммы 8.42), находим $\mathrm{L} \in$ Эл $_{\beta}\left(\mathrm{Y}, q_{0}\right)$ такой, что $Э \mathrm{Coz}_{\beta}\left(\mathrm{K}_{2}, \mathrm{~L}\right)$ и $\mathrm{K}_{3}^{\prime}<_{Y} \mathrm{~L}<_{Y} \mathrm{~K}_{1}^{\prime}$. Вновь противоречие с предложением 11.25 .

11.27. ПРЕДЛОЖЕНИЕ $(\alpha \geqslant 0)$. Пусть $\mathrm{X}, \mathrm{Y}-$ nути на графе $\Gamma_{\alpha} c$ $\mathcal{L}(\mathrm{X}), \mathcal{L}(\mathrm{Y}) \in \mathcal{R}_{\alpha} . \quad$ Пусть $0 \leqslant \beta \leqslant \alpha, \quad$ Эл $_{\gamma}\left(\mathrm{X}, q_{4}\right)=\varnothing$ или Эл $\gamma\left(\mathrm{Y}, q_{4}\right)=\varnothing$ при $\gamma=\beta+1, \beta+2, \ldots, \alpha$. Предположим, выполнено хотя бы одно из двух следующих условий (i) или (ii):

(i) для некоторых $\mathrm{K}_{1}, \mathrm{~K}_{2} \in \ni_{\beta}\left(\mathrm{X}, q_{0}+h\right), \mathrm{K}_{1}^{\prime}, \mathrm{K}_{2}^{\prime} \in \ni_{\beta}\left(\mathrm{Y}, q_{0}+h\right)$ выполнено $\neg \ni \operatorname{Coгл}_{\beta}\left(\mathrm{K}_{1}, \mathrm{~K}_{2}\right), \quad \exists \operatorname{Coz}_{\beta}\left(\mathrm{K}_{i}, \mathrm{~K}_{i}^{\prime}\right) \quad(i=1,2), \mathrm{K}_{1}<\mathrm{x} \mathrm{K}_{2} u \mathrm{~K}_{1}^{\prime}<\mathrm{Y} \mathrm{K}_{2}^{\prime}$;

(ii) пути $\mathrm{X} u \mathrm{Y}$ близки в ранге $\beta$ и $\mathrm{X}$ содержит по крайней мере 4 подпути $\mathrm{K}_{i} \in$ Эл $_{\beta}\left(\mathrm{X}, q_{0}+2 h\right) \quad(1 \leqslant i \leqslant 4)$ maкux, что $\neg Э \operatorname{Cогл}_{\beta}\left(\mathrm{K}_{i}, \mathrm{~K}_{j}\right)$ nри $i \neq j$.

Тогда если $\mathrm{M}, \mathrm{N} \in \ni \boldsymbol{л}_{\beta}\left(\mathrm{X}, q_{0}+h\right), \mathrm{M}^{\prime}, \mathrm{N}^{\prime} \in \mathrm{\jmath}_{\beta}\left(\mathrm{Y}, q_{0}+h\right), \neg \operatorname{ЭСогл}_{\beta}(\mathrm{M}, \mathrm{N})$, $\exists \operatorname{Coz}_{\beta}\left(\mathrm{M}, \mathrm{M}^{\prime}\right) u \vartheta \operatorname{Coz}_{\beta}\left(\mathrm{N}, \mathrm{N}^{\prime}\right)$, mo $\mathrm{M}<\mathrm{x} \mathrm{N} \Leftrightarrow \mathrm{M}^{\prime}<\mathrm{Y} \mathrm{N}^{\prime}$.

ДокАЗАТЕЛЬСТво. Пусть сначалавыполнено условие (i). Если $Э С о љ \Omega_{\beta}\left(\mathrm{M}, \mathrm{K}_{i}\right)$ или $Э \operatorname{Coл}_{\beta}\left(\mathrm{N}, \mathrm{K}_{i}\right)$ для некоторого $i=1,2$, то утверждение вытекает из предложения 11.25 и леммы 11.26 (предложением 11.25 нужно воспользоваться в случае, когда $Э \operatorname{Cогл~}_{\beta}\left(\mathrm{M}, \mathrm{K}_{i}\right)$ и $Э \operatorname{Cогл~}_{\beta}\left(\mathrm{N}, \mathrm{K}_{j}\right)$, где $\left.\{i, j\}=\{1,2\}\right)$. В случае $\neg$ ЭСогл $\beta\left(\mathrm{M}, \mathrm{K}_{i}\right)$

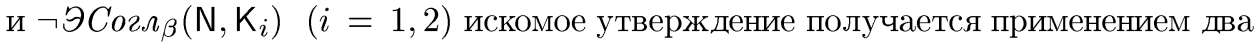
раза леммы 11.26 при $\left\{\mathrm{K}_{1}, \mathrm{~K}_{2}, \mathrm{~K}_{3}\right\}:=\left\{\mathrm{K}_{1}, \mathrm{~K}_{2}, \mathrm{M}\right\},\left\{\mathrm{K}_{1}, \mathrm{~K}_{2}, \mathrm{~K}_{3}\right\}:=\left\{\mathrm{K}_{1}, \mathrm{~K}_{2}, \mathrm{~N}\right\}$ и, в случае, если $\mathrm{K}_{1}<\mathrm{x} M{ }_{\mathrm{x}} \mathrm{K}_{2}$ и $\mathrm{K}_{1}<\mathrm{x} \mathrm{N}<\mathrm{x} \mathrm{K}_{2}$, дополнительным применением леммы 11.26 при $\left\{\mathrm{K}_{1}, \mathrm{~K}_{2}, \mathrm{~K}_{3}\right\}:=\left\{\mathrm{K}_{1}, \mathrm{M}, \mathrm{N}\right\}$.

Пусть вьполнено условие (ii). В силу предложения 8.40 можно считать $\mathrm{K}_{1}<\mathrm{x} \mathrm{K}_{2}<\mathrm{x} \mathrm{K}_{3}<\mathrm{x} \mathrm{K}_{4}$. С помощью следствия 11.22 найдем сначала $\mathrm{K}_{2}^{\prime} \in$ Эл $_{\beta}(\mathrm{Y}$, $\left.q_{0}+h\right)$ такой, что ЭСогл $\beta\left(\mathrm{K}_{2}, \mathrm{~K}_{2}^{\prime}\right)$. Далее, рассматривая вместо $\mathrm{X}$ конец $\mathrm{X}$, начинаюшийся с $\mathrm{K}_{2}$, а вместо $\mathrm{Y}$ - конец $\mathrm{Y}$, начинаюшийся с $\mathrm{K}_{2}^{\prime}$, в силу предложения 8.45 и следствия 11.22 найдем $\mathrm{K}_{3}^{\prime} \in$ Эл $_{\beta}\left(\mathrm{Y}, q_{0}+h\right)$ такой, что $Э$ Согл $\beta\left(\mathrm{K}_{3}, \mathrm{~K}_{3}^{\prime}\right)$. Тогда в силу предложения 8.40 получим $\mathrm{K}_{2}^{\prime}<_{\mathrm{Y}} \mathrm{K}_{3}^{\prime}$. Остается воспользоваться уже рассмотренным случаем (i).

Перейдем к анализу соотношений вида $X_{1} \stackrel{\alpha}{=} u_{1} X_{2} u_{2} X_{3} u_{3}$, где $X_{1}, X_{2}, X_{3} \in \mathcal{R}_{\alpha}$ и $u_{1}, u_{2}, u_{3} \in \mathcal{H}_{\alpha}$. Наша цель сейчас - получить аналог предложения 11.17 для соотношений указанного вида (предложение 11.30). Схема рассуждений в этом случае такая же, как и для соотношений вида $X \stackrel{\alpha}{=} u Y v$ с тем лиш отличием, что вместо аналога предложения 11.2 (которое используется также и для других целей) мы докажем более слабое утверждение. 
11.28. Лемма. Пусть $X_{1} \stackrel{\alpha}{=} u_{1} X_{2} u_{2} X_{3} u_{3}$, әде $X_{i} \in \mathcal{R}_{\alpha}, \quad u_{i} \in \mathcal{H}_{\alpha} \quad(i=$ $1,2,3)$. Пусть для каждого слова $u_{i}$ фиксировано некоторое разложение $u_{i}$ 으 $v_{i} Q_{i} w_{i}$, где $v_{i}, w_{i} \in \mathcal{H}_{\alpha-1}$ и $Q_{i}-$ подслово слова $B_{i}^{n}$ при $B_{i} \in \mathcal{E}_{\alpha}$. Тогда верно по крайней мере одно из двух следующих утверждений (i) или (ii).

(i) $X_{1} \stackrel{\alpha=1}{=} \hat{u}_{1} X_{2} \hat{u}_{2} X_{3} \hat{u}_{3}$, где либо $\hat{u}_{i}$ 드 $u_{i}$, либо $\hat{u}_{i}$ есть слово, дополнительное $\kappa u_{i}$.

(ii) Пусть $\mathrm{X}_{1} u \mathrm{u}_{1} \mathrm{X}_{2} \mathrm{u}_{2} \mathrm{X}_{3} \mathrm{u}_{3}$ - пути на графе $\Gamma_{\alpha}$ с общими начальной $и$ конечной веричнами, где $\mathcal{L}\left(\mathrm{X}_{i}\right)$ 으 $X_{i} u \mathcal{L}\left(\mathrm{u}_{i}\right)$ 으 $u_{i}$. Тогда либо каждый nуть $\mathrm{X}_{i}$ содержит подпуть $\mathrm{E}_{i} \in \ni_{\alpha}\left(\Gamma_{\alpha}\right)$, где $Э$ Согл $\Omega_{\alpha}\left(\mathrm{E}_{1}, \mathrm{E}_{j}\right) \quad(j=2,3) u$ для любых $i \neq j$ выполнено $\ell_{\alpha}\left(\mathrm{E}_{i}\right)+\ell_{\alpha}\left(\mathrm{E}_{j}\right) \geqslant q-3 p-166$, либо некоторые два $\mathrm{X}_{i}$ и $\mathrm{X}_{j}$ содержат подпути $\mathrm{E}_{1}, \mathrm{E}_{2} \in{ } л_{\alpha}\left(\Gamma_{\alpha}\right)$ соответственно такие, umo $\ni$ Согл $\alpha\left(\mathrm{E}_{1}, \mathrm{E}_{2}\right)$ u $\ell_{\alpha}\left(\mathrm{E}_{i}\right) \geqslant q-3 p-129 \quad(i=1,2)$.

ДокАЗАтЕльство. Рассмотрим дисковую $B$-диаграмму $\Delta$ ранга $\alpha$ с граничным циклом $\delta \Delta \approx \widetilde{\mathrm{X}}_{1} \widetilde{\mathrm{w}}_{3}^{-1} \widetilde{\mathrm{Q}}_{3}^{-1} \tilde{\mathrm{v}}_{3}^{-1} \widetilde{\mathrm{X}}_{3}^{-1} \widetilde{\mathrm{w}}_{2}^{-1} \widetilde{\mathrm{Q}}_{2}^{-1} \tilde{\mathrm{v}}_{2}^{-1} \widetilde{\mathrm{X}}_{2}^{-1} \widetilde{\mathrm{w}}_{1}^{-1} \widetilde{\mathrm{Q}}_{1}^{-1} \tilde{\mathrm{v}}_{1}^{-1}$, где метки путей $\widetilde{\mathrm{X}}_{i}, \tilde{\mathrm{v}}_{i}, \widetilde{\mathrm{Q}}_{i}, \widetilde{\mathrm{w}}_{i}$ равны словам $X_{i}, v_{i}, Q_{i}, w_{i}$ соответственно,

$$
\begin{gathered}
x_{\alpha}(\Delta)=\left\{\widetilde{\mathrm{X}}_{1}, \widetilde{\mathrm{X}}_{3}^{-1}, \widetilde{\mathrm{X}}_{2}^{-1}\right\}, \quad y_{\alpha-1}(\Delta)=\left\{\widetilde{\mathrm{w}}_{3}^{-1}, \widetilde{\mathrm{v}}_{3}^{-1}, \widetilde{\mathrm{w}}_{2}^{-1}, \tilde{\mathrm{v}}_{2}^{-1}, \widetilde{\mathrm{w}}_{1}^{-1}, \tilde{\mathrm{v}}_{1}^{-1}\right\}, \\
z_{\alpha}(\Delta)=\left\{\widetilde{\mathrm{Q}}_{3}^{-1}, \widetilde{\mathrm{Q}}_{2}^{-1}, \widetilde{\mathrm{Q}}_{1}^{-1}\right\} .
\end{gathered}
$$

Так же, как и в доказательстве предложения 11.2 , заменяя слова $u_{i}$ на $\hat{u}_{i}$ и используя предложения 9.9 и 9.19 , можно считать, что $\Delta$ есть приведенная $B$-диаграмма ранга $\alpha$ и на $\Delta^{\alpha-1}$ задана некоторая карта примыканий $\mathcal{S}$ ранга $\alpha-1$. Если $\Delta$ не содержит областей ранга $\alpha$, то мы приходим к случаю (i). Пусть $\mathrm{A} \in \mathbf{R}_{\alpha}(\Delta)$ и $\mathcal{L}(\delta \mathrm{A}) \approx A^{n}$, где $A \in \mathcal{E}_{\alpha}$. В силу предложения 10.9 , (i) граничный цикл $\delta \mathrm{A}$ области А имеет не более одной подлиаграмм примыкания $\Pi \in \mathcal{S}$ к каждому участку границы $\widetilde{\mathrm{X}}_{1}, \widetilde{\mathrm{X}}_{3}^{-1}$ или $\widetilde{\mathrm{X}}_{2}^{-1}$ диаграммы $\Delta^{\alpha-1}$. Пусть $\Pi_{i}(1 \leqslant i \leqslant r, r \leqslant 3)$ - все такие поддиаграммы. Пусть $\delta \Pi_{i} \approx \mathrm{G}_{i} \mathrm{y}_{i}^{-1} \mathrm{~S}_{i} \mathrm{x}_{i}^{-1}$, где $\mathrm{G}_{i}, \mathrm{~S}_{i}$ - участки примыкания, $\mathrm{G}_{i}$ входит в $\mathrm{X}_{1}, \mathrm{X}_{2}^{-1}$ или $\mathrm{X}_{3}^{-1}, \mathrm{~S}_{i}$ входит в $\delta \mathrm{A}$ и $\mathcal{L}\left(\mathrm{x}_{i}\right), \mathcal{L}\left(\mathrm{y}_{i}\right) \in \mathcal{H}_{\alpha-1}$. По предложению 10.13 , (i) имеем

$$
\sum_{i=1}^{r}\left|\mathcal{L}\left(\mathrm{S}_{i}\right)\right| \geqslant(n-3 p-48)|A|,
$$

а в силу приведенности слов $X_{i}$ в ранге $\alpha$ для каждого $i$

$$
\left|\mathcal{L}\left(\mathrm{S}_{i}\right)\right|<(n-q)|A| .
$$

Отбросим те поддиаграммы примыкания $\Pi_{i}$, для которых $\left|\mathcal{L}\left(\mathrm{S}_{i}\right)\right|<44|A|$. Из (11.7) и (11.8) следует, что таких поддиаграмм примыкания $\Pi_{i}$ не более одной. Следовательно, вместо (11.7) будем иметь

$$
\sum_{i=1}^{r}\left|\mathcal{L}\left(\mathrm{S}_{i}\right)\right|>(n-3 p-92)|A| .
$$


Из (11.8) и (11.9) следует $r \geqslant 2$ для нового значения $r$. Рассмотрим теперь пути $\mathrm{X}_{1}$ и $\mathrm{u}_{1} \mathrm{X}_{2} \mathrm{u}_{2} \mathrm{X}_{3} \mathrm{u}_{3}$ на графе $\Gamma_{\alpha}$, как указано в (ii). Рассмотрим отображение $\omega$ множества путей на диаграмме $\Delta$ во множество путей на графе $\Gamma_{\alpha}$, как указано в 4.12 , такое, что $\omega\left(\iota\left(\widetilde{\mathrm{X}}_{1}\right)\right)=\iota\left(\mathrm{X}_{1}\right)$. Очевидно, $\omega\left(\widetilde{\mathrm{X}}_{i}\right)=\mathrm{X}_{i} \quad(i=1,2,3)$. По лемме 11.15 каждый путь $\omega\left(\mathrm{G}_{i}\right)$ содержит подпуть $\mathrm{G}_{i}^{*} \in$ Эл $_{\alpha}\left(\Gamma_{\alpha}\right)$ с периодической основой, содержашейся в $\omega\left(\mathrm{S}_{i}\right)$, причем $\ell_{\alpha}\left(\mathrm{G}_{i}^{*}\right) \geqslant\left|\mathcal{L}\left(\mathrm{S}_{i}\right)\right| /|A|-37$. Так как все $\omega\left(\mathrm{S}_{i}\right)$ имеют общее периодическое продолжение, то по лемме 11.14 имеем $Э \operatorname{Coz}_{\alpha}\left(\mathrm{G}_{i}^{*}, \mathrm{G}_{j}^{*}\right)$ при всех $i, j$. Из (11.8) и (11.9) следует, что если $r=2$, то $\ell_{\alpha}\left(\mathrm{G}_{i}^{*}\right) \geqslant q-3 p-129$ для каждого $i=1,2$, а если $r=3$, то $\ell_{\alpha}\left(\mathrm{G}_{i}^{*}\right)+\ell_{\alpha}\left(\mathrm{G}_{j}^{*}\right) \geqslant q-3 p-166$ для любых $i \neq j$. Поэтому в качестве $\mathrm{E}_{i}$ можно взять $\mathrm{G}_{i}^{*}$, если $\mathrm{G}_{i}^{*}$ содержится в $\mathrm{X}_{1}$, и $\mathrm{G}_{i}^{*-1}$, если $\mathrm{G}_{i}^{*}$ содержится в $\mathrm{X}_{2}^{-1}$ или в $\mathrm{X}_{3}^{-1}$.

11.29. Лемма. Пусть $\mathrm{X}_{1} u \mathrm{u}_{1} \mathrm{X}_{2} \mathrm{u}_{2} \mathrm{X}_{3} \mathrm{u}_{3}-$ пути на графе $\Gamma_{\alpha-1}$ с общей начальной и конечной вериинами, где $\mathcal{L}\left(\mathrm{X}_{i}\right) \in \mathcal{R}_{\alpha-1}$ и $\mathcal{L}\left(\mathrm{u}_{i}\right) \in \mathcal{H}_{\alpha}$, и пусть $\mathrm{E} \in \Pi$ Пер $\alpha(A, 3 p+232)\left(A \in \mathcal{E}_{\alpha}\right)-$ nодпуть пути $\mathrm{X}_{1}$. Пусть для каждого $i$ $\mathrm{u}_{i}=\mathrm{v}_{i} \mathrm{Q}_{i} \mathrm{w}_{i}$, әде $\mathcal{L}\left(\mathrm{v}_{i}\right), \mathcal{L}\left(\mathrm{w}_{i}\right) \in \mathcal{H}_{\alpha-1}, \mathcal{L}\left(\mathrm{Q}_{i}\right)-$ подслово слова $B_{i}^{n}$ при $B_{i} \in \mathcal{E}_{\alpha}$ и либо $\left|\mathrm{Q}_{i}\right|<2\left|B_{i}\right|$, либо $\left|\mathrm{Q}_{i}\right| \geqslant 2\left|B_{i}\right|$ и $\neg$ Согл $\left(\mathrm{P}, \mathrm{Q}_{i}\right)$, где $\mathrm{P}-$ периодическая основа для Е. Тогда верно хотя бь одно из утверждений (i) или (ii):

(i) существует подпуть $\mathrm{F} \in \Pi_{\text {- }}(A, 8)$ пути $\mathrm{X}_{2}$ или $\mathrm{X}_{3}$ такой, что $\Pi C o 2 \Omega_{\alpha}(\mathrm{E}, \mathrm{F}) u \ell_{\alpha, A}(\mathrm{~F}) \geqslant \ell_{\alpha, A}(\mathrm{E})-3 p-224$;

(ii) существуют подпути $\mathrm{F}, \mathrm{G} \in \Pi_{\text {Пер }}(A, 8)$ путей $\mathrm{X}_{2}$ и $\mathrm{X}_{3}$ соответственно такие, что ПСог $\Omega_{\alpha}(\mathrm{E}, \mathrm{F})$, ПСог $\Omega_{\alpha}(\mathrm{E}, \mathrm{G})$ и $\ell_{\alpha, A}(\mathrm{~F})+\ell_{\alpha, A}(\mathrm{G}) \geqslant \ell_{\alpha, A}(\mathrm{E})-$ $3 p-217$.

ДоКАЗАТЕЛЬСТво. По существу, нужно повторить рассуждения из доказательства леммы 11.10. По лемме 11.7, (ii) при $\mathrm{X}:=\mathrm{X}_{1}$ и $\mathrm{W}:=\mathrm{v}_{1} \mathrm{Q}_{1} \mathrm{w}_{1} \mathrm{X}_{2} \mathrm{v}_{2} \mathrm{Q}_{2} \mathrm{w}_{2} \times$ $\mathrm{X}_{3} \mathrm{v}_{3} \mathrm{Q}_{3} \mathrm{w}_{3}$ найдутся подпути $\mathrm{F}_{1}, \mathrm{~F}_{2}, \ldots, \mathrm{F}_{t} \in \Pi_{\text {Пер }}(A)$ попарно различных путей $\mathrm{Q}_{1}, \mathrm{X}_{2}, \mathrm{Q}_{2}, \mathrm{X}_{3}$ и $\mathrm{Q}_{3}$ такие, что ПСогл $\alpha\left(\mathrm{E}, \mathrm{F}_{i}\right)$ при всех $i$ и $\sum_{i=1}^{t} \ell_{\alpha, A}\left(\mathrm{~F}_{i}\right) \geqslant$ $\ell_{\alpha, A}(\mathrm{E})-220$. Если $\mathrm{F}_{i}$ - подпуть некоторого $\mathrm{Q}_{j}$, то из посылки леммы аналогично тому, как в доказательстве леммы 11.10 , вытекает $\ell_{\alpha, A}\left(\mathrm{~F}_{i}\right) \leqslant p-1$. Следовательно, отбросив $\mathrm{F}_{i}$, содержашиеся во всех $\mathrm{Q}_{j}$, а также в случае $\ell_{\alpha, A}\left(\mathrm{~F}_{i}\right) \leqslant 7$ и содержашиеся в $\mathrm{X}_{2}$ или в $\mathrm{X}_{3}$, придем к одному из утверждений (i) или (ii).

11.30. ПРЕДЛОЖЕНИЕ. Пусть $\mathrm{X}_{1} u \mathrm{u}_{1} \mathrm{X}_{2} \mathrm{u}_{2} \mathrm{X}_{3} \mathrm{u}_{3}$ - nути на графе $\Gamma_{\alpha}$ с общей начальной и конечной вершинами, где $\mathcal{L}\left(\mathrm{X}_{i}\right) \in \mathcal{R}_{\alpha}$ u $\mathcal{L}\left(\mathrm{u}_{i}\right) \in \mathcal{H}_{\alpha}$. Пусть $\mathrm{E} \in \ni_{\alpha}\left(\mathrm{X}_{1}, 3 p+232\right)$ и $\mathrm{E}$ устойчив относительно каждого $\mathrm{u}_{i}$. Тогда верно хотя бъ одно из следующих утверждений:

(i) существует подпуть $\mathrm{F} \in$ Эл $\alpha_{\alpha}\left(\Gamma_{\alpha}\right)$ пути $\mathrm{X}_{2}$ или $\mathrm{X}_{3}$ такой, что $\ni C о \Omega_{\alpha}(\mathrm{E}, \mathrm{F}) u \ell_{\alpha}(\mathrm{F}) \geqslant \min \left\{q-3 p-129, \ell_{\alpha}(\mathrm{E})-3 p-224\right\}$;

(ii) существуют подпути $\mathrm{F}, \mathrm{G} \in \ni_{\alpha}\left(\Gamma_{\alpha}\right)$ путей $\mathrm{X}_{2}$ и $\mathrm{X}_{3}$ соответственно такие, что $Э$ Согл $\Omega_{\alpha}(\mathrm{E}, \mathrm{F}), \quad Э С о г \Omega_{\alpha}(\mathrm{E}, \mathrm{G})$ и $\ell_{\alpha}(\mathrm{F})+\ell_{\alpha}(\mathrm{G}) \geqslant \min \{q-3 p-$ $\left.166, \ell_{\alpha}(\mathrm{E})-3 p-217\right\}$.

ДоКАЗАТЕЛЬСТво. БУдем использовать индукцию по $\left|\mathrm{X}_{1}\right|+\left|\mathrm{X}_{2}\right|+\left|\mathrm{X}_{3}\right|$. Пусть $\mathrm{u}_{i}=\mathrm{v}_{i} \mathrm{Q}_{i} \mathrm{w}_{i} \quad(i=1,2,3)$, где $\mathcal{L}\left(\mathrm{v}_{i}\right), \mathcal{L}\left(\mathrm{w}_{i}\right) \in \mathcal{H}_{\alpha-1}, \mathcal{L}\left(\mathrm{Q}_{i}\right)$ - подслово слова $B_{i}^{n}$ при $B_{i} \in \mathcal{E}_{\alpha}$ и $\mathrm{Q}_{i}$ и $\mathrm{E}$ удовлетворяют условию из определения 11.11. Применим 
лемму 11.28 , где роль слов $X_{i}, u_{i}, v_{i}, Q_{i}$, и $w_{i}$ играют метки соответствуюших путей на графе $\Gamma_{\alpha}$. Если имеет место случай $11.28,(\mathrm{i})$, то утверждение сводится к лемме 11.29 точно так же, как в доказательстве предложения 11.17. Пусть имеет место случай 11.28 , (ii), т.е. либо каждый путь $\mathrm{X}_{i}$ содержит подпуть $\mathrm{E}_{i} \in \ni_{\alpha}\left(\Gamma_{\alpha}\right)$, где ЭСогл $\alpha\left(\mathrm{E}_{1}, \mathrm{E}_{j}\right)(j=2,3)$ и для любых $i \neq j$ выполнено $\ell_{\alpha}\left(\mathrm{E}_{i}\right)+\ell_{\alpha}\left(\mathrm{E}_{j}\right) \geqslant q-$ $3 p-166$, либо некоторые $\mathrm{X}_{i}$ и $\mathrm{X}_{j}$ содержат подпути $\mathrm{E}_{1}, \mathrm{E}_{2} \in$ Эл $_{\alpha}\left(\Gamma_{\alpha}\right)$ такие, что ЭСогл $\alpha\left(\mathrm{E}_{1}, \mathrm{E}_{2}\right)$ и $\ell_{\alpha}\left(\mathrm{E}_{i}\right) \geqslant q-3 p-129(i=1,2)$. Рассмотрим два случая.

Случай 1: Каждый путь $\mathrm{X}_{i}$ содержит подпуть $\mathrm{E}_{i} \in \ni_{\alpha}\left(\Gamma_{\alpha}\right)$, где ЭСогл $\Omega_{\alpha}\left(\mathrm{E}_{1}, \mathrm{E}_{j}\right)$ $(j=2,3)$ и для любых $i \neq j$ выполнено $\ell_{\alpha}\left(\mathrm{E}_{i}\right)+\ell_{\alpha}\left(\mathrm{E}_{j}\right) \geqslant q-3 p-166$. Если $Э С о \Omega_{\alpha}\left(E, E_{1}\right)$, то верно утверждение (ii) при $F=E_{2}$ и $G=E_{3}$. Пусть $\neg \mathcal{Э С о г л ~}_{\alpha}\left(\mathrm{E}_{1} \mathrm{E}_{1}\right)$. Пусть $\mathrm{X}_{i}=\mathrm{X}_{i}^{\prime} \mathrm{E}_{i} \mathrm{X}_{i}^{\prime \prime} \quad(i=2,3)$. По лемме 11.19 соединим вершины $\iota\left(\mathrm{E}_{2}\right)$ и $\tau\left(\mathrm{E}_{3}\right)$ путем $\mathrm{u}_{2}^{\prime}$ с $\mathcal{L}\left(\mathrm{u}_{2}^{\prime}\right) \in \mathcal{H}_{\alpha}$, относительно которого устойчив путь $\mathrm{E}$. Так как слова $\mathcal{L}\left(\mathrm{E}_{i}\right)$ непусты (это следует, например, из предложения 8.16), то можно воспользоваться индуктивным предположением для $\mathrm{u}_{1} \mathrm{X}_{2}^{\prime} \mathrm{u}_{2}^{\prime} \mathrm{X}_{3}^{\prime \prime} \mathrm{u}_{3}$ вместо $\mathrm{u}_{1} \mathrm{X}_{2} \mathrm{u}_{2} \mathrm{X}_{3} \mathrm{u}_{3}$.

Случай 2: Для некоторых $i \neq j$ пути $\mathrm{X}_{i}$ и $\mathrm{X}_{j}$ содержат подпути $\mathrm{E}_{1}, \mathrm{E}_{2} \in$ Эл $\alpha\left(\Gamma_{\alpha}\right)$ такие, что ЭСогл $\alpha\left(\mathrm{E}_{1}, \mathrm{E}_{2}\right)$ и $\ell_{\alpha}\left(\mathrm{E}_{i}\right) \geqslant q-3 p-129(i=1,2)$. Если $\{i, j\}=\{2,3\}$, то проходят рассуждения из предыдушего случая. Предположим, $\{i, j\}=\{1,2\}$. Можно считать, что $\mathrm{E}_{k}$ содержится в $\mathrm{X}_{k}(k=1,2)$. Если $Э С о \boldsymbol{C}_{\alpha}\left(\mathrm{E}, \mathrm{E}_{1}\right)$, то верно утверждение (i) при $\mathrm{F}:=\mathrm{E}_{2}$. Пусть $\neg Э \operatorname{Coгл}_{\alpha}\left(\mathrm{E}, \mathrm{E}_{1}\right)$. Пусть $\mathrm{X}_{i}=\mathrm{X}_{i}^{\prime} \mathrm{E}_{i} \mathrm{X}_{i}^{\prime \prime} \quad(i=1,2)$. По лемме 11.19 соединим вершины $\iota\left(\mathrm{E}_{1}\right)$ и $\tau\left(\mathrm{E}_{2}\right)$ путем $\mathrm{u}_{1}^{\prime}$, а вершины $\tau\left(\mathrm{E}_{1}\right)$ и $\tau\left(\mathrm{E}_{2}\right)$ путем $\mathrm{u}_{1}^{\prime \prime}$, где $\mathcal{L}\left(\mathrm{u}_{1}^{\prime}\right), \mathcal{L}\left(\mathrm{u}_{1}^{\prime \prime}\right) \in \mathcal{H}_{\alpha}$ и $\mathrm{E}$ устойчив относительно $\mathbf{u}_{1}^{\prime}$ и $\mathbf{u}_{1}^{\prime \prime}$ (см. рис. 41). По предложению 8.40 путь $\mathrm{E}$ содержится либо в $\mathrm{X}_{1}^{\prime} \mathrm{E}_{1}$, либо в $\mathrm{E}_{1} \mathrm{X}_{1}^{\prime \prime}$. В первом случае по предложению 11.17 при $\mathrm{X}:=\mathrm{X}_{1}^{\prime} \mathrm{E}_{1}$ и u $\mathrm{Yv}:=\mathrm{u}_{1} \mathrm{X}_{2}^{\prime} \mathrm{E}_{2} \mathrm{u}_{1}^{\prime \prime-1}$ найдется подпуть $\mathrm{F} \in$ Эл $_{\alpha}\left(\Gamma_{\alpha}\right)$ пути $\mathrm{X}_{2}$ такой, что $Э \operatorname{Coz}_{\alpha}(\mathrm{E}, \mathrm{F})$ и $\ell_{\alpha}(\mathrm{F}) \geqslant \min \left\{q_{4}, \ell_{\alpha}(\mathrm{E})-h\right\}$, т.е. мы снова приходим к утверждению (i). Во втором случае можно воспользоваться индуктивным предположением при $\mathrm{X}_{1}:=\mathrm{E}_{1} \mathrm{X}_{1}^{\prime \prime}$ и $\mathrm{u}_{1} \mathrm{X}_{2} \mathrm{u}_{2} \mathrm{X}_{3} \mathrm{u}_{3}:=\mathrm{u}_{1}^{\prime} \mathrm{X}_{2}^{\prime \prime} \mathrm{u}_{2} \mathrm{X}_{3} \mathrm{u}_{3}$. Аналогично рассматривается случай, когда $\{i, j\}=\{1,3\}$.

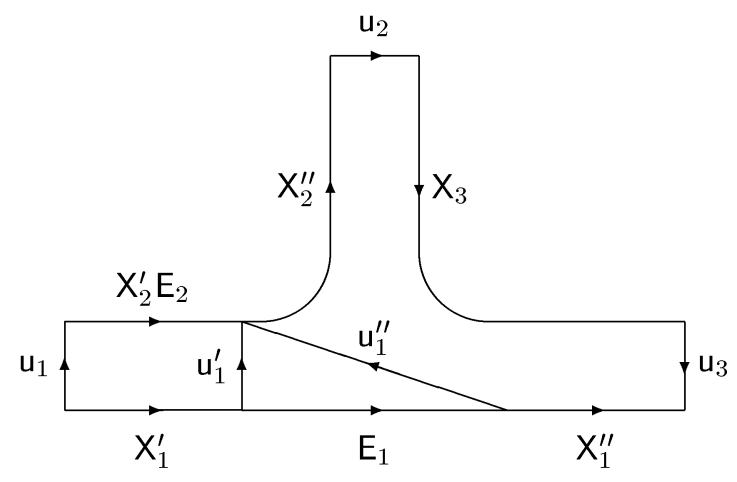

Рис. 41

В оставшейся части параграфа изучаются соотношения сопряженности в груп- 
пе $\mathbf{B}_{\alpha}$.

11.31. Лемма. Пусть слова $X$ и $Y$ ииклически приведены в ранге $\alpha$, әлемент $[X]_{\alpha}$ имеет бесконечный порядок и $T^{-1} X T \stackrel{\alpha}{=} Y$. Тогда верно по крайней мере одно из утверждений (i) или (ii):

(i) для некоторого $T^{\prime}$ выполнено $T \stackrel{\alpha}{=} T^{\prime}$ и $T^{\prime-1} X T^{\prime} \stackrel{\alpha=1}{=} Y$;

(ii) в некоторый ииклический сдвиг каждого из слов $X$ и $Y$ входят әлементарнье $q_{4}$-степени ранга $\alpha$ и $T \stackrel{\alpha}{=} X^{t} X_{1} u Y_{1}^{-1}$ для некоторых $u \in \mathcal{H}_{\alpha}$, начала $X_{1}$ слова $X$, начала $Y_{1}$ слова $Y$ и $t \in \mathbb{Z}$.

ДокАЗАТЕЛЬСТво. Пусть $\widetilde{\Delta}$ - дисковая диаграмма ранга $\alpha$ с граничным циклом $\delta \Delta \approx \widetilde{\mathrm{T}}^{-1} \widetilde{\mathrm{X}} \widetilde{\mathrm{T}}^{\prime} \widetilde{\mathrm{Y}}^{-1}$, где $\mathcal{L}(\widetilde{\mathrm{T}})$ 으 $\mathcal{L}\left(\widetilde{\mathrm{T}}^{\prime}\right)$ 으 $T, \mathcal{L}(\widetilde{\mathrm{X}})$ 으 $X$ и $\mathcal{L}(\widetilde{\mathrm{Y}})$ 으 $Y$. Склеивая участки границы диаграммы $\widetilde{\Delta}$ вдоль путей $\widetilde{\top}$ и $\widetilde{\mathrm{T}}^{\prime}$, как описано в п. 4.6, получим кольцевую диаграмму $\Delta$ с граничными циклами $\mathrm{X}$ и $\mathrm{Y}^{-1}$, где $\mathcal{L}(\mathrm{X})$ 으 $X$, $\mathcal{L}(\mathrm{Y})$ 으 $Y$, и вершины $\iota(\mathrm{X})$ и $\iota(\mathrm{Y})$ соединены путем $\mathrm{T}$ с $\mathcal{L}(\mathrm{T})$ 으 $T$. Так как слова $X$ и $Y^{-1}$ циклически приведены в ранге $\alpha$, то $\Delta$ можно рассматривать как $B$-диаграмму ранга $\alpha$ с $X_{\alpha}(\Delta)=\{\mathrm{X}, \mathrm{Y}\}$ и $y_{\alpha-1}(\Delta)=Z_{\alpha}(\Delta)=\varnothing$. В силу предложений 9.9 и 9.19 существует последовательность внутренних преобразований ранга $\alpha$

$$
\Delta=\Delta_{0} \rightarrow \Delta_{1} \rightarrow \cdots \rightarrow \Delta_{r}=\Delta^{\prime},
$$

переводящая $\Delta$ в приведенную $B$-диаграмму $\Delta^{\prime}$ ранга $\alpha$ такую, что на $\Delta^{\alpha-1}$ сушествует некоторая карта примыканий $\mathcal{S}$. Докажем, что в последовательности (11.10) можно обойтись без преобразований вида 9.4 , (iii), т.е. только измельчениями и заменами дисковых поддиаграмм. Предположим, диаграмма $\Delta_{i+1}$ получена из $\Delta_{i}$ преобразованием 9.4 , (iii), т.е. $\Delta_{i+1}$ есть результат замены кольцевой поддиаграммы $\Theta$ диаграммы $\Delta_{i}$ на некоторую кольцевую диаграмму $\Theta^{\prime}$, где для каждого из двух граничных циклов $\mathrm{C}_{1}$ и $\mathrm{C}_{2}$ диаграммы $\Theta$ элемент $\left[\mathcal{L}\left(\mathrm{C}_{i}\right)\right]_{\alpha}$ имеет конечньй порядок. Диаграмма $\Delta_{i}-\operatorname{int} \Theta$ состоит из двух связных компонент $\Sigma_{1}, \Sigma_{2}$ и имеет четыре граничных цикла $\mathrm{X}, \mathrm{Y}^{-1}, \mathrm{C}_{1}^{-1}$ и $\mathrm{C}_{2}^{-1}$, причем $\mathrm{C}_{1}^{-1}$ и $\mathrm{C}_{2}^{-1}$ - граничные циклы разных поддиаграмм $\Sigma_{1}$ и $\Sigma_{2}$. Если бы каждая из поддиаграмм $\Sigma_{j}$ имела по два граничных цикла, то по предложению 4.3 мы получили бы, что $[X]_{\alpha}-$ элемент конечного порядка вопреки условию. Следовательно, одна из поддиаграмм $\Sigma_{j}$, скажем $\Sigma_{1}$, является дисковой диаграммой, и тогда $\Delta_{i+1}$ есть результат замены дисковой поддиаграммы $\Theta \cup \Sigma_{1}$ диаграммы $\Delta_{i}$ на диаграмму $\Theta^{\prime} \cup \Sigma^{\prime}$, где $\Sigma^{\prime}$ - копия диаграммы $\Sigma_{1}$, приклеенная к $\Theta^{\prime}$.

Итак, для каждого $i$ диаграмма $\Delta_{i+1}$ есть либо результат измельчения диаграммы $\Delta_{i}$, либо результат замены дисковой поддиаграммы диаграммы $\Delta_{i}$. Тогда по лемме 4.10 вершины $\iota(\mathrm{X})$ и $\iota(\mathrm{Y})$ соединены путем $\mathrm{T}^{\prime}$ на $\Delta^{\prime}$ с $\mathcal{L}\left(\mathrm{T}^{\prime}\right)$ 으 $T^{\prime} \stackrel{\underline{\alpha}}{=} T$. Если $\Delta^{\prime}$ не имеет областей ранга $\alpha$, то по предложению 4.3 выполнено равенство $T^{\prime-1} X T^{\prime} \stackrel{\alpha=1}{=} Y$, и мы приходим к случаю (i). Пусть $\Delta^{\prime}$ имеет некоторую область А ранга $\alpha$. Изменив обозначения, будем считать $\Delta=\Delta^{\prime}$ и $\mathrm{T}=\mathrm{T}^{\prime}$ (соответственно $T$ 으 $\left.T^{\prime}\right)$. В силу предложений 10.12 и 10.9 граничньй цикл области А имеет ровно одну поддиаграмму примыкания $\Pi_{1} \in \mathcal{S}$ к $\mathrm{X}$ и ровно одну поддиаграмму примыкания $\Pi_{2} \in \mathcal{S}$ к $\mathrm{Y}^{-1}$. Пусть $\mathrm{S}_{1}$ и $\mathrm{S}_{2}$ - соответствуюшие участки 
примыкания, входяшие в $\delta \mathrm{A}$, и пусть $\mathcal{L}(\delta \mathrm{A}) \approx A^{n}$, где $A \in \mathcal{E}_{\alpha}$. В силу предложения 10.13, (ii) $\left|\mathcal{L}\left(\mathrm{S}_{1}\right)\right|+\left|\mathcal{L}\left(\mathrm{S}_{2}\right)\right| \geqslant(n-2 p-32)|A|$. Так как слова $\mathcal{L}\left(\mathrm{S}_{1}\right)$ и $\mathcal{L}\left(\mathrm{S}_{2}\right)$ близки в ранге $\alpha-1$ к некоторым подсловам циклических сдвигов слов $X^{-1}$ и $Y$ соответственно, то в силу циклической приведенности слов $X^{-1}$ и $Y$ имеем $\left|\mathcal{L}\left(\mathrm{S}_{i}\right)\right|<(n-q)|A|$. Отсюда следует $\left|\mathcal{L}\left(\mathrm{S}_{i}\right)\right| \geqslant(q-2 p-32)|A|(i=1,2)$. Тогда в силу следствия 8.24 в циклический сдвиг каждого слова $X^{-1}$ и $Y$ (а значит, и $\left.X\right)$ входит элементарная $q_{4}$-степень ранга $\alpha$. Тем самым доказана первая часть утверждения (ii). Для доказательства второй части рассмотрим путь и на диаграмме $\Delta$ с $u \rightleftharpoons \mathcal{L}(\mathrm{u}) \in \mathcal{H}_{\alpha}$, изображенный более толстой линией на рис. 42 . Пусть $\mathrm{X}_{1}$ - начало пути $\mathrm{X}_{\text {и }} \mathrm{Y}_{1}$ - начало пути $\mathrm{Y}$ с конечными вершинами $\iota(\mathrm{u})$ и $\tau(\mathrm{u})$ соответственно. Положим $X_{1} \rightleftharpoons \mathcal{L}\left(\mathrm{X}_{1}\right)$ и $Y_{1} \rightleftharpoons \mathcal{L}\left(\mathrm{Y}_{1}\right)$. Так как циклическая группа $\pi_{1}(\Delta, \iota(\mathrm{X}))$ порождается классом $[\mathrm{X}]_{\Delta}$, то замкнутый путь $\mathrm{TY}_{1} \mathrm{u}^{-1} \mathrm{X}_{1}^{-1}$ гомотопен пути $\mathrm{X}^{t}$ для некоторого $t \in \mathbb{Z}$. Тогда по лемме 4.2 имеем $T Y_{1} u^{-1} X_{1}^{-1} \stackrel{\alpha}{=} X^{t}$, т.е. $T \stackrel{\alpha}{=} X^{t} X_{1} u Y_{1}^{-1}$.

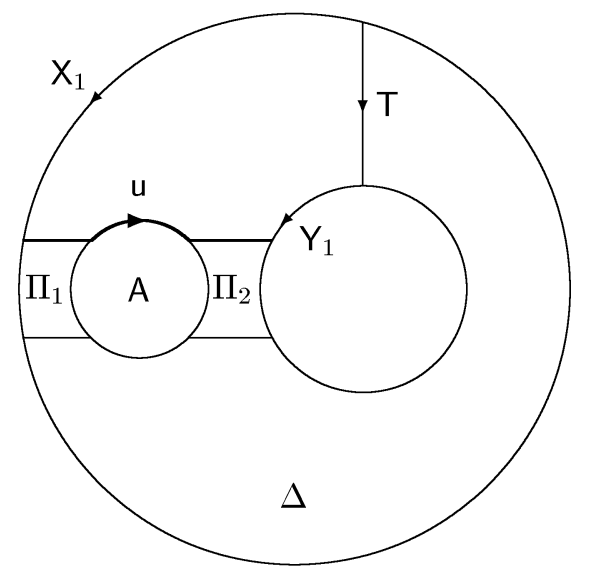

Рис. 42

11.32. СлЕДСТВИЕ $(\alpha \geqslant 0)$. Пусть слова $X$ и $Y$ ииклически приведены $в$ ранге $\alpha$, әлемент $[X]_{\alpha}$ имеет бесконечный порядок и $T^{-1} X T \stackrel{\alpha}{=} Y$. Пусть $\beta \leqslant \alpha$ и никакой ииклический сдвиг слова $X$ не содержит әлементарных $q_{4}$-степеней ранга $\gamma$ при $\beta+1 \leqslant \gamma \leqslant \alpha$. Тогда $T \stackrel{\alpha}{=} X^{t} X_{1} u Y_{1}^{-1}$ для некоторых $u \in \mathcal{H}_{\beta}$, начала $X_{1}$ слова $X$, начала $Y_{1}$ слова $Y$ u $t \in \mathbb{Z}$.

ДоКАЗАТЕЛЬСТВО. При $\alpha=0$ из циклической несократимости слов $X$ и $Y$ и равенства $T^{-1} X T \stackrel{0}{=} Y$ легко следует, что $X$ 으 $X_{1} X_{2}, Y$ 으 $X_{2} X_{1}$ и $T \stackrel{0}{=} X^{t} X_{1}$ для некоторых $X_{1}, X_{2}$ и $t$, т.е. в качестве $u$ и $Y_{1}$ можно взять пустые слова. При $\alpha \geqslant 1$ нужно применить лемму 11.31 и в случае $\beta<\alpha$ воспользоваться индуктивньгм предположением для $\alpha:=\alpha-1$.

11.33. ПРЕДЛОЖЕНИЕ. Если чиклически приведенные в ранге $\alpha$ слова $X$ и $Y$ сопряжены в группе $\mathbf{B}_{\alpha}, \beta \leqslant \alpha-1$ и никакой ииклический сдвиг слова $X$ 
не содержит әлементарных $q_{4}$-степеней ранга $\gamma$ при $\beta+1 \leqslant \gamma \leqslant \alpha$, mо $X$ и $Y$ сопряжены в $\mathbf{B}_{\beta}$.

ДокАЗАТЕЛЬство. Достаточно рассмотреть случай $\beta=\alpha-1$. Пусть $\Delta-$ кольцевая диаграмма ранга $\alpha$ с граничными циклами $\mathrm{X}$ и $\mathrm{Y}^{-1}$, где $\mathcal{L}(\mathrm{X})$ 으 $X$ и $\mathcal{L}(\mathrm{Y})$ 으 $Y$. Рассмотрим $\Delta$ как $B$-диаграмму ранга $\alpha$, где $\mathcal{X}_{\alpha}(\Delta)=\left\{\mathrm{X}, \mathrm{Y}^{-1}\right\}$ и $y_{\alpha-1}(\Delta)=z_{\alpha}(\Delta)=\varnothing$. В силу предложений 9.9 и 9.19 можно считать, что $\Delta-$ приведенная $B$-диаграмма ранга $\alpha$ и $\mathcal{S}$ - некоторая карта примыканий ранга $\alpha-1$ на $\Delta^{\alpha-1}$. Повторяя рассуждения из доказательства леммы 11.31 , получаем, что либо $\Delta$ есть диаграмма ранга $\alpha-1$, и тогда $X$ и $Y$ сопряжены в $\mathbf{B}_{\alpha-1}$, либо некоторый циклический сдвиг слова $X$ содержит элементарую $q_{4}$-степень ранга $\alpha$.

11.34. Лемма. Пусть слова $X u Y v$ и $Z$ сопряжены в группе $\mathbf{B}_{\alpha}$, әде $X, Y \in$ $\mathcal{R}_{\alpha}, u, v \in \mathcal{H}_{\alpha}$ и слово $Z$ чиклически приведено в ранге $\alpha$. Пусть для слов $и, v$ фиксированы представления в виде $u$ 으 $u_{1} Q u_{2}, v$ 으 $v_{1} R v_{2}$, zде $u_{i}, v_{i} \in \mathcal{H}_{\alpha-1}$ $(i=1,2)$ и $Q, R-$ подслова слов $B^{n}, C^{n}$ соответственно при $B, C \in \mathcal{E}_{\alpha}$.

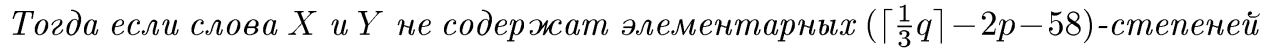

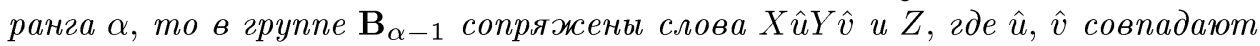
соответственно с и, $v$ или являются дополнительными к ним словами.

ДокаЗАтельство. Рассмотрим кольцевую $B$-диаграмму $\Delta$ ранга $\alpha$ с граничными циклами $\mathrm{Xu}_{1} \mathrm{Qu}_{2} \mathrm{Yv}_{1} \mathrm{Ru}_{2}$ и $\mathrm{Z}^{-1}$, где метки путей $\mathrm{X}, \mathrm{u}_{i}, \mathrm{Q}, \mathrm{Y}, \mathrm{v}_{i}, \mathrm{R}$ и $\mathrm{Z}$ равны соответственно словам $X, u_{i}, Q, Y, v_{i}, R$ и $Z$,

$$
x_{\alpha}(\Delta)=\left\{\mathrm{X}, \mathrm{Y}, \mathrm{Z}^{-1}\right\}, \quad \mathrm{y}_{\alpha-1}(\Delta)=\left\{\mathrm{u}_{1}, \mathrm{u}_{2}, \mathrm{v}_{1}, \mathrm{v}_{2}\right\} \quad \text { и } \quad Z_{\alpha}(\Delta)=\left\{\mathrm{Q}^{-1}, \mathrm{R}^{-1}\right\}
$$

Так же, как и в доказательстве предложения 11.2 , заменяя слова $u_{i}$ на $\hat{u}_{i}$ и используя предложение 9.9 , можно считать, что $\Delta$ есть приведенная $B$-диаграмма ранга $\alpha$, а в силу предложения 9.19 можно считать, что на $\Delta^{\alpha-1}$ задана некоторая карта примыканий $\mathcal{S}$ ранга $\alpha-1$. Достаточно доказать, что $\Delta$ не имеет областей ранга $\alpha$. Допустим, $\mathrm{A} \in \mathbf{R}_{\alpha}(\Delta)$. Пусть $\Pi_{1}, \Pi_{2}, \ldots, \Pi_{r} \in \mathcal{S}$ - поддиаграммы примыкания граничного цикла области А к главным участкам ранга $\alpha$ диаграммы $\Delta$, и пусть $\mathrm{S}_{i}$ - участок примыкания поддиаграммы примыкания $\Pi_{i}$, входящий в $\delta \mathrm{A}$. Пусть $\mathcal{L}(\mathrm{A}) \approx A^{n}$, где $A \in \mathcal{E}_{\alpha}$. По предложению 10.13 , (ii)

$$
\sum_{i=1}^{r}\left|\mathcal{L}\left(\mathrm{S}_{i}\right)\right| \geqslant(n-6 p-64)|A| .
$$

В силу предложения 10.9, (ii) имеется не более одной поддиаграммы примыкания $\Pi_{i}$ граничного цикла $\mathrm{A}_{\mathrm{k}} \mathrm{Z}^{-1}$. Так как слово $Z$ циклически приведено в ранге $\alpha$, то для такой поддиаграммы примыкания $\Pi_{i}$ выполнено $\left|\mathcal{L}\left(\mathrm{S}_{i}\right)\right|<(n-q)|A|$. Следовательно, выбросив такую подлиаграмму $\Pi_{i}$, если она сушествует, вместо (11.11) получим

$$
\sum_{i=1}^{r}\left|\mathcal{L}\left(\mathrm{S}_{i}\right)\right| \geqslant(q-6 p-64)|A|
$$


По предложению 10.9 , (ii) для нового значения $r$ имеем $r \leqslant 3$, поэтому для некоторого $i$ выполнено $\mathcal{L}\left(\mathrm{S}_{i}\right) \geqslant\left(\left\lceil\frac{1}{3} q\right\rceil-2 p-22\right)|A|$. Тогда в силу следствия 8.24 одно из слов $X$ или $Y$ содержит элементарную ( $\left.\left.\frac{1}{3} q\right\rceil-2 p-58\right)$-степень ранга $\alpha$ вопреки условию. Следовательно, $\Delta$ не имеет областей ранга $\alpha$.

\section{§ 12. Представление элементов группы $\mathrm{B}_{\alpha}$ приведенными словами}

Часть рассуждений этого параграфа будет проводиться на языке слов и вхождений. Нам будет удобно использовать следующие два понятия, которые являются аналогами соответствуюших понятий из [1].

12.1. ОПРЕДЕЛЕНИЕ. Пусть $\mathrm{U}=K * E * L, \mathrm{~V}=M * F * N$ - два вхождения в слово $X$ элементарных слов $E$ и $F$ ранга $\alpha$. Будем говорить, что $U$ и $V$ согласованы, если для некоторого (или, равносильно, для любого) пути $\mathrm{X}$ на графе $\Gamma_{\alpha-1}$ с $\mathcal{L}(\mathrm{X})$ 으 $X$ выполнено ПСогл $\Omega_{\alpha}\left(\phi_{\mathbf{X}}(\mathrm{U}), \phi_{\mathbf{X}}(\mathrm{V})\right)$ (обозначение $\phi_{\mathrm{X}}(\mathrm{U})$ было введено в $\S 3)$. Отношение согласованности вхождений $U$ и $\mathrm{V}$ будем обозначать той же записью $\vartheta_{\text {Coгл }}(\mathrm{U}, \mathrm{V})$, что и для путей на графе $\Gamma_{\beta}$.

12.2. ОПРЕДЕЛЕНИЕ. Пусть $\mathrm{U}, \mathrm{V}$ - два вхождения в слово $X$ элементарных слов ранга $\alpha$, порожденных одним элементарным периодом $A \in \mathcal{E}_{\alpha}$. Будем говорить, что $\mathrm{V}$ есть продолжение $\mathrm{U}$ относительно периода $A$, если $\mathrm{U}$ содержится в V. Продолжение $\mathrm{V}$ вхождения U относительно периода $A$ назовем максимальным, если не сушествует отличных от $\mathrm{V}$ продолжений вхождения $\mathrm{U}$ относительно $A$, содержаших $\mathrm{V}$.

12.3. Лемма. (i) Отношение ЭСогл $(\mathrm{U}, \mathrm{V})$ на множестве вхождений әлементарных слов ранга $\alpha$ в данное слово $X$ яв.ляется отношением әквивалентности.

(ii) $E$ сли $P * E * Q$ содержится в $R * F * S$ и $\ell_{\alpha}(E) \geqslant p+34$, то $P * E * Q$ u $R * F * S$ согласованы.

(iii) Для любой әлементарной $(2 p+80)$-степени $E$ ранга $\alpha$ существуют әлементарное слово $\bar{E}$ ранга $\alpha$ и слова $U$ u $V$ такие, что $E$ 으 $U \bar{E} V, \ell_{\alpha}(\bar{E}) \geqslant$ $\ell_{\alpha}(E)-2 p-72$ и для любых двух несогласованных вхождений $P * E * Q$ u $R * F * S$ в слово $X \in \mathcal{R}_{\alpha-1}$ вхождения $P U * \bar{E} * V Q$ и $R * F * S$ не пересекаются.

(iv) Каждое вхождение в слово $X \in \mathcal{R}_{\alpha-1}$ әлементарного слова ранга $\alpha$, порожденного данным әлементарным периодом А ранга $\alpha$, обладает единственным максимальным продолжением относительно $A$.

(v) Если вхождения әлементарных слов $F$ и $F^{\prime}$ ранга $\alpha$ в слово $X \in \mathcal{R}_{\alpha-1}-$ продолжения некоторого вхождения әлементарного слова ранга $\alpha$ в $X$ относительно периода $A$ и $F$ максимально, то $\ell_{\alpha, A}(F) \geqslant \ell_{\alpha, A}\left(F^{\prime}\right)$.

ДокаЗАТЕльство. Утверждение (i) вытекает из предложения 8.6, (ii). Утверждение (ii) вытекает из предложения 8.38, (ii), a (iii) - из предложений 8.38, (ii) и 8.14, (i). Наконец, (iv) и (v) легко следуют из предложений 8.9 и 8.11. 
12.4. ОПРЕДЕЛЕНИЕ. Пусть $X \in \mathcal{R}_{\alpha-1}$ и $X$ 으 $K_{0} E_{1} K_{1} \ldots E_{r} K_{r}$, где $E_{1}$, $E_{2}, \ldots, E_{r}$ - элементарные слова ранга $\alpha$. Пусть для каждого слова $E_{i}$ фиксирован некоторый элементарный период $A_{i} \in \mathcal{E}_{\alpha}$, где $E_{i} \in \Pi \Pi е p_{\alpha}\left(A_{i}, 8\right)$. Слово $Y \in \mathcal{R}_{\alpha-1}$ будем называть результатом поворота в слове $X$ набора вхождений

$$
K_{0} E_{1} K_{1} \ldots K_{i-1} * E_{i} * K_{i} \ldots E_{r} K_{r} \quad(i=1,2, \ldots, r),
$$

если существуют слова $P_{i} \in \Pi е p\left(A_{i}, 2\right), u_{i}, v_{i} \in \mathcal{H}_{\alpha-1}$ и $Q_{i}(i=1,2, \ldots, r)$, удовлетворяюшие следуюшим условиям:

(i) для каждого $i=1,2, \ldots, r$ сушествуют пути $\mathrm{E}_{i}$ и $\mathrm{u}_{i} \mathrm{P}_{i} \mathrm{v}_{i}$ на графе $\Gamma_{\alpha-1} \mathrm{c}$ обшими начальной и конечной вершинами такие, что метки путей $\mathrm{E}_{i}, \mathrm{u}_{i}, \mathrm{P}_{i}$ и $\mathrm{v}_{i}$ равны словам $E_{i}, u_{i}, P_{i}$ и $v_{i}$ соответственно и $\mathrm{P}_{i}-$ периодическая основа для $\mathrm{E}_{i} ;$ в частности, $E_{i} \stackrel{\alpha=1}{=} u_{i} P_{i} v_{i}$;

(ii) для каждого $i=1,2, \ldots, r$ вьполнено $A_{i}^{n} \approx P_{i} Q_{i}$;

(iii) $Y \stackrel{\alpha=1}{=} K_{0} u_{1} Q_{1}^{-1} v_{1} K_{1} \ldots u_{r} Q_{r}^{-1} v_{r} K_{r}$.

Из определения вытекает

12.5. Лемма. Если слово $Y$ есть результат поворота в $X$ некоторого набора вхождений (12.1), то $X \stackrel{\alpha}{=} Y$.

12.6. Лемма. Пусть $X \in \mathcal{R}_{\alpha-1}, \quad X$ 으 $K_{0} E_{1} K_{1} \ldots E_{r} K_{r}$, әде $E_{i}$ - әлементарное слово ранга $\alpha$, порожденное әлементарным периодом $A_{i} \in \mathcal{E}_{\alpha}$. Пусть $\ell_{\alpha, A_{i}}\left(E_{i}\right) \leqslant n-2$ для всех $i$. Тогда существует слово $Y$, являюшееся результатом поворота в $X$ набора вхождений (12.1).

ДоказательСтво. Сушествование слов $P_{i} \in \Pi е p\left(A_{i}, 2\right), u_{i}, v_{i} \in \mathcal{H}_{\alpha-1}$, удовлетворяющих условию (i) определения 12.4 , вытекает из определений $5.19,5.16$ и леммы 8.2, (ii). Так как $\ell_{\alpha, A_{i}}\left(E_{i}\right) \leqslant n-2$, то из 12.4 , (i), определения 5.17 и леммы 7.6 следует $\left|P_{i}\right| \leqslant n\left|A_{i}\right|$. Поэтому для некоторых $Q_{i}$ выполнено условие 12.4 , (ii). Наконец, по индуктивному предположению 12.13 существует слово $Y \in \mathcal{R}_{\alpha-1}$ такое, что имеет место равенство 12.4 , (iii).

12.7. Лемма. Пусть $X \in \mathcal{R}_{\alpha-1}, \quad X$ 으 $K_{0} E_{1} K_{1} \ldots E_{r} K_{r}$, əде $E_{i} \in$ ППер ${ }_{\alpha}\left(A_{i}, 44\right) \quad\left(A_{i} \in \mathcal{E}_{\alpha}\right)$, и пусть слово $Y$ есть результат поворота в $X$ набора вхождений

$$
\mathrm{U}_{i} \rightleftharpoons K_{0} E_{1} K_{1} \ldots K_{i-1} * E_{i} * K_{i} \ldots E_{r} K_{r} \quad(i=1,2, \ldots, r)
$$

т.е. выполнены условия (i)-(iii) определения 12.4 для некоторых $P_{i} \in$ $\Pi е p\left(A_{i}, 2\right), \quad u_{i}, v_{i} \in \mathcal{H}_{\alpha-1} u Q_{i} \quad(i=1,2, \ldots, r)$.

Пусть вхождение слова $\widetilde{E}_{i}$ в слово $X$ есть максимальное продолжение вхождения $\mathrm{U}_{i}$ относительно $A_{i}$ и для каждого $i=1,2, \ldots, r$ выполнено

$$
\ell_{\alpha, A_{i}}\left(\widetilde{E}_{i}\right) \leqslant n-2 p-111
$$


ПРЕДСТАВЛЕНИЕ ЭЛЕМЕНТОВ ГРУППЫ $\mathbf{B}_{\alpha}$ ПРИВЕДЕННЫМИ СЛОВАМИ 125

Тогда существуют әлементарные слова $F_{1}, F_{2}, \ldots, F_{r}$ ранга $\alpha$ и слова $w_{i}, z_{i} \in \mathcal{H}_{\alpha-1}, \quad S_{i} \in \Pi e p\left(A_{i}^{-1}, 2\right), \quad U_{i}, V_{i} \quad(i=1,2, \ldots, r)$ u $L_{i} \quad(i=0,1, \ldots, r)$ такие, что $F_{i} \in \Pi \Pi е p_{\alpha}\left(A_{i}^{-1}, 8\right)$ и верны следующие утверждения:

(i) $Y$ 드 $L_{0} F_{1} L_{1} \ldots F_{r} L_{r}$;

(ii) для каждого $i=1,2, \ldots, r$ существуют пути $\mathrm{F}_{i} u \mathrm{w}_{i} \mathrm{~S}_{i} \mathrm{z}_{i}$ на графе $\Gamma_{\alpha-1}$ с общими начальной и конечной вершинами такие, что метки путей $\mathrm{F}_{i}, \mathrm{w}_{i}$, $\mathrm{S}_{i}$ и $\mathrm{z}_{i}$ равны словам $F_{i}, v_{i}, S_{i}$ и $z_{i}$ соответственно и $\mathrm{S}_{i}$ - периодическая основа для $\mathrm{F}_{i}$;

(ii) $Q_{i}$ 으 $U_{i} S_{i}^{-1} V_{i} \quad(i=1,2, \ldots, r)$

(iv) имеют место соотношения

$$
\begin{aligned}
& L_{0} \stackrel{\alpha=1}{=} K_{0} u_{1} V_{1}^{-1} w_{1}^{-1}, \\
& L_{i} \stackrel{\alpha=1}{=} z_{i}^{-1} U_{i}^{-1} v_{i} K_{i} u_{i+1} V_{i+1}^{-1} w_{i+1}^{-1} \quad(1 \leqslant i \leqslant r-1), \\
& L_{r} \stackrel{\alpha=1}{=} z_{r}^{-1} U_{r}^{-1} v_{r} K_{r}
\end{aligned}
$$

(v) $\ell_{\alpha, A_{i}}\left(\widetilde{E}_{i}\right)+\ell_{\alpha, A_{i}^{-1}}\left(F_{i}\right) \geqslant n-2 p-103 \quad(i=1,2, \ldots, r)$.

ДоКАЗАТЕЛЬСТВО. В соответствии с условием 12.4 , (iii) рассмотрим дисковую $A$-диаграмму $\Delta$ ранга $\alpha-1$ с граничным циклом

$$
\delta \Delta \approx \mathrm{K}_{0} \mathrm{u}_{1} \mathrm{Q}_{1}^{-1} \mathrm{v}_{1} \mathrm{~K}_{1} \ldots \mathrm{u}_{r} \mathrm{Q}_{r}^{-1} \mathrm{v}_{r} \mathrm{~K}_{r} \times \mathrm{Y}^{-1} \mathrm{y}
$$

где метки путей $\mathrm{K}_{i}, \mathrm{u}_{i}, \mathrm{Q}_{i}, \mathrm{v}_{i}$ и $\mathrm{Y}$ равны словам $K_{i}, u_{i}, Q_{i}, v_{i}$ и $Y$ соответственно, $\mathcal{L}(\mathrm{x})$ 드 $\mathcal{L}(\mathrm{y})$ 으 1,

$$
\begin{aligned}
& x_{\alpha-1}(\Delta)=\left\{\mathrm{K}_{0}, \mathrm{~K}_{1}, \ldots, \mathrm{K}_{r}, \mathrm{Q}_{1}^{-1}, \mathrm{Q}_{2}^{-1}, \ldots \mathrm{Q}_{r}^{-1}, \mathrm{Y}^{-1}\right\} \\
& y_{\alpha-1}(\Delta)=\left\{\mathrm{u}_{1}, \mathrm{u}_{2}, \ldots, \mathrm{u}_{r}, \mathrm{v}_{1}, \mathrm{v}_{2}, \ldots, \mathrm{v}_{r}, \mathrm{x}, \mathrm{y}\right\} .
\end{aligned}
$$

Можно считать, что на $\Delta$ задана некоторая карта примыканий $\mathcal{S}$ ранга $\alpha-1$.

Наша цель - доказать, что каждый из участков границы $\mathrm{Q}_{i}^{-1}$ диаграммы $\Delta$ имеет поддиаграмму примыкания к участку границы $\mathrm{Y}^{-1}$ с достаточно длинным участком примыкания, откуда несложно будет следовать искомое утверждение. Доказательство разбивается на ряд шагов, на каждом из которых мы изучим поддиаграммы примыкания $\Pi \in \mathcal{S}$ определенного сорта.

Прежде всего заметим, что по лемме 9.23, (i)

не существует поддиаграмм примыкания $\Pi \in \mathcal{S}$ участка гранищы $\mathrm{Q}_{i}^{-1}$ к самому себе и для любого другого участка граничь $\mathrm{Z} \in \mathcal{X}_{\alpha-1}(\Delta)$

диаграммы $\Delta$ существует не более одной поддиаграмми примыкания $\mathrm{Q}_{i}^{-1} \kappa \mathrm{Z}$. 


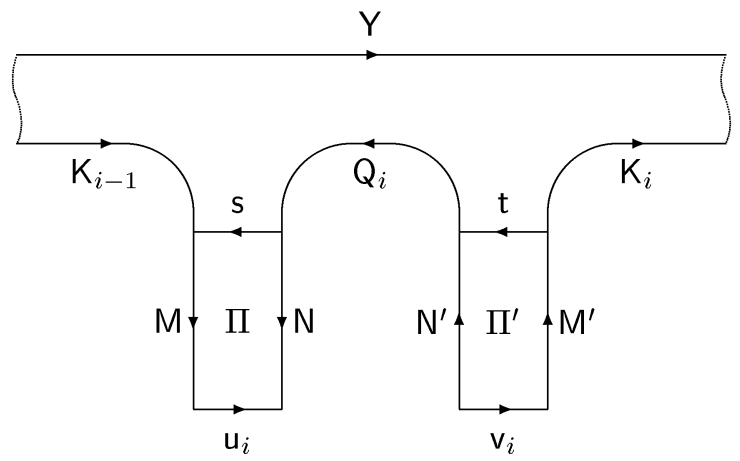

Рис. 43

Изучим поддиаграммы примыкания участка границы $Q_{i}^{-1}$ к соседним участкам границы $\mathrm{K}_{i-1}$ и $\mathrm{K}_{i}$. Предположим, имеются две поддиаграммы примыкания П, $\Pi^{\prime} \in \mathcal{S}$ участка границы $\mathrm{Q}_{i}^{-1}$ к $\mathrm{K}_{i-1}$ и к $\mathrm{K}_{i}$ соответственно. Пусть $\delta \Pi \approx \mathrm{Mu}_{i} \mathrm{~N}^{-1} \mathrm{~s}$ и $\delta \Pi^{\prime} \approx \mathrm{N}^{\prime-1} \mathrm{v}_{i} \mathrm{M}^{\prime} \mathrm{t}$, где $\mathrm{M}, \mathrm{N}^{-1}, \mathrm{~N}^{\prime-1}$ и $\mathrm{M}^{\prime}-$ участки примыкания и $\mathcal{L}(\mathrm{s}), \mathcal{L}(\mathrm{t}) \in \mathcal{H}_{\alpha-1}$ (см. рис. 43 ; тот факт, что пути и $i$ и $\mathrm{v}_{i}$ входят в граничные циклы поддиаграмм П и П' ${ }^{\prime}$ вытекает из леммы 9.20). Допустим,

$$
\left|P_{i}\right|+|\mathcal{L}(\mathrm{N})|+\left|\mathcal{L}\left(\mathrm{N}^{\prime}\right)\right| \geqslant\left(\ell_{\alpha, A_{i}}\left(\widetilde{E}_{i}\right)+37\right)\left|A_{i}\right| .
$$

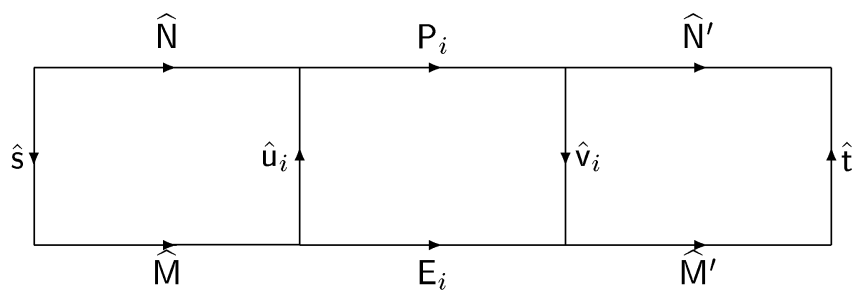

Рис. 44

В силу условия 12.4 , (i) сушествуют пути $\mathrm{E}_{i}$ и $\hat{\mathrm{u}}_{i} \mathrm{P}_{i} \hat{\mathrm{v}}_{i}$ на графе $\Gamma_{\alpha-1}$ с общими начальной и конечной вершинами, где метки путей $\mathrm{E}_{i}, \hat{u}_{i}, \mathrm{P}_{i}, \hat{\mathrm{v}}_{i}$ равны словам $E_{i}, u_{i}, P_{i}, v_{i}$ соответственно и $\mathrm{P}_{i}$ - периодическая основа для $\mathrm{E}_{i}$. Рассмотрим пути $\widehat{\mathrm{M}}, \widehat{\mathrm{S}}, \widehat{\mathrm{N}}, \widehat{\mathrm{M}}^{\prime}, \hat{\mathrm{t}}, \widehat{\mathrm{N}}^{\prime}$ на графе $\Gamma_{\alpha-1}$ с метками $\mathcal{L}(\mathrm{M}), \mathcal{L}(\mathrm{S}), \mathcal{L}(\mathrm{N})$, $\mathcal{L}\left(\mathrm{M}^{\prime}\right), \mathcal{L}(\mathrm{t}), \mathcal{L}\left(\mathrm{N}^{\prime}\right)$ соответственно, расположенные, как на рис. 44. Заметим, что $\mathcal{L}\left(\widehat{\mathrm{M}}_{i} \widehat{\mathrm{M}}^{\prime}\right)$ 으 $\mathcal{L}(\mathrm{M}) E_{i} \mathcal{L}\left(\mathrm{M}^{\prime}\right)$ - подслово слова $X \in \mathcal{R}_{\alpha-1}$, и в силу 12.4 , (ii) имеем $\widehat{\mathrm{N}} \mathrm{P}_{i} \widehat{\mathrm{N}}^{\prime} \in$ Пер $\left(\Gamma_{\alpha-1}, A_{i}\right)$. Так как пути $\widehat{\mathrm{M}} \mathrm{E}_{i} \widehat{\mathrm{M}}^{\prime}$ и $\widehat{\mathrm{N}} \mathrm{P}_{i} \widehat{\mathrm{N}}^{\prime}$ близки в ранге $\alpha-1$, то в силу (12.4) и предложения 8.23 путь $\widehat{\mathrm{M}} \mathrm{E}_{i} \widehat{\mathrm{M}}^{\prime}$ содержит подпуть $\widetilde{\mathrm{E}} \in \Pi_{\text {Пер }}\left(A_{i}, 8\right)$ такой, что $\ell_{\alpha, A_{i}}(\widetilde{\mathrm{E}}) \geqslant \ell_{\alpha, A_{i}}\left(\widetilde{E}_{i}\right)+1$. Но это противоречит лемме 12.3 , (v). Следовательно, неравенство (12.4) неверно. Аналогично доказывается, что если имеется одна поддиаграмма примыкания $\Pi \in \mathcal{S}$ участка границы $\mathrm{Q}_{i}^{-1}$ к $\mathrm{K}_{i-1}$ или к $\mathrm{K}_{i}$ и $\mathrm{N}-$ ее участок примыкания, содержащийся в $\mathrm{Q}_{i}^{-1}$, то $\left|P_{i}\right|+|\mathcal{L}(\mathrm{N})|<\left(\ell_{\alpha . A_{i}}\left(\widetilde{E}_{i}\right)+\right.$ 
ПРЕДСТАВЛЕНИЕ ЭЛЕМЕНТОВ ГРУППЫ $\mathbf{B}_{\alpha}$ ПРИВЕДЕННЫМИ СЛОВАМИ 127

37) $\left|A_{i}\right|$. Так как $\left|P_{i}\right|+\left|Q_{i}\right|=n\left|A_{i}\right|$ в силу 12.4 , (ii), то доказанное нами можно сформулировать в виде следуюшего утверждения:

суммарная длина меток содержащихся в $\mathrm{Q}_{i}^{-1}$ участков примыкания поддиаграмм примыкания $\Pi \in \mathcal{S}$ участка границы $\mathrm{Q}_{i}^{-1} \kappa$ участкам границь $\mathrm{K}_{i-1}$ и $\mathrm{K}_{i}$ меньше $\left|Q_{i}\right|-\left(n-\ell_{\alpha, A_{i}}\left(\widetilde{E}_{i}\right)-37\right)\left|A_{i}\right|$, в частности, с учетом (12.2), меньше $\left|Q_{i}\right|-(2 p+74)\left|A_{i}\right|$.

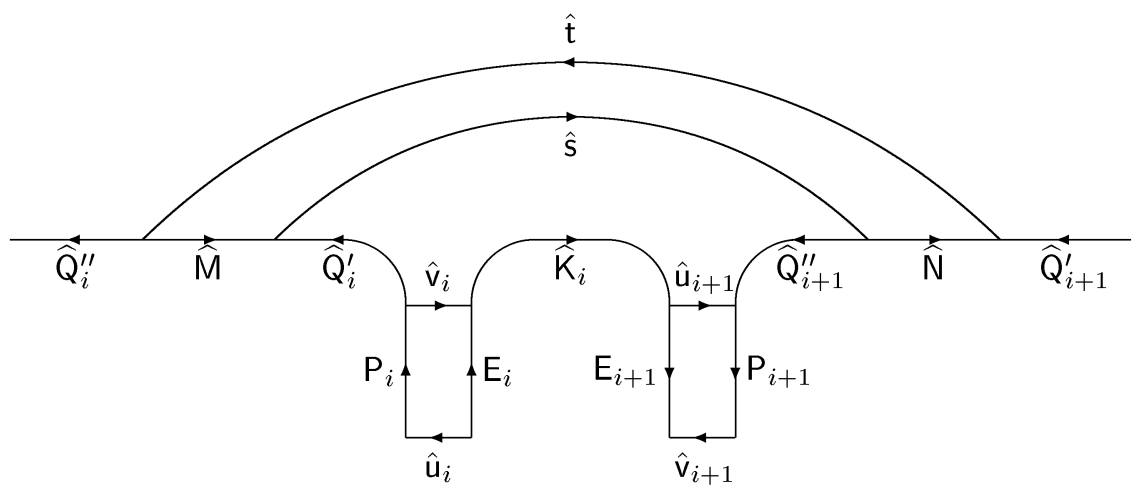

Рис. 45

Теперь предположим, что $\Pi \in \mathcal{S}$ - поддиаграмма примыкания $\mathrm{Q}_{i}^{-1}$ к $\mathrm{Q}_{i+1}^{-1}$. Пусть $\delta \Pi \approx \mathrm{MsNt}, \mathrm{Q}_{i}=\mathrm{Q}_{i}^{\prime} \mathrm{M}^{-1} \mathrm{Q}_{i}^{\prime \prime}, \mathrm{Q}_{i+1}=\mathrm{Q}_{i+1}^{\prime} \mathrm{N}^{-1} \mathrm{Q}_{i+1}^{\prime \prime}$, где $\mathrm{M}, \mathrm{N}$ - участки примыкания. Допустим, $|\mathcal{L}(\mathrm{M})| \geqslant p\left|A_{i}\right|$ или $|\mathcal{L}(\mathrm{N})| \geqslant p\left|A_{i+1}\right|$. Рассмотрим пути $\widehat{Q}_{i}^{\prime}, \widehat{M}, \widehat{Q}_{i}^{\prime \prime}, \hat{v}_{i}, \widehat{\mathrm{K}}_{i}, \hat{\mathrm{u}}_{i+1}, \widehat{\mathrm{Q}}_{i+1}^{\prime}, \widehat{\mathrm{N}}, \widehat{\mathrm{Q}}_{i+1}^{\prime \prime}$ на графе $\Gamma_{\alpha-1}$ с теми же метками, что и соответствуюшие пути на диаграмме $\Delta$, и с учетом условия 12.4, (i) добавим к ним пути $\mathrm{E}_{i}, \hat{\mathrm{u}}_{i}, \mathrm{P}_{i}, \mathrm{E}_{i+1}, \mathrm{P}_{i+1}, \hat{\mathrm{v}}_{i+1}$, как изображено на рис. 45 . Так как пути $\widehat{\mathrm{M}}$ и $\widehat{\mathrm{N}}^{-1}$ близки в ранге $\alpha-1$, то по предложению 8.36 получаем $C$ огл $\left(\widehat{\mathrm{Q}}_{i}, \widehat{\mathrm{Q}}_{i+1}^{-1}\right)$. В силу условия 12.4, (i) $\mathrm{P}_{i}$ и $\mathrm{P}_{i+1}$ - периодические основы для $\mathrm{E}_{i}$ и $\mathrm{E}_{i+1}$ соответственно. Так как $\mathrm{P}_{i} \widehat{\mathrm{Q}}_{i} \in \Pi$ ер $\left(\Gamma_{\alpha-1}, A_{i}, n\right)$ и $\mathrm{P}_{i+1} \widehat{\mathrm{Q}}_{i+1} \in \Pi$ Пер $\left(\Gamma_{\alpha-1}, A_{i+1}, n\right)$ в силу 12.4 , (ii), то имеем, следовательно, Согл $\left(\mathrm{P}_{i}, \mathrm{P}_{i+1}^{-1}\right)$, т.е. ПСогл $\left.\boldsymbol{\mathrm { E }}_{i}, \mathrm{E}_{i+1}^{-1}\right)$. Но это невозможно по предложению 8.46 при $\mathrm{X}:=\mathrm{E}_{i} \widehat{\mathrm{K}}_{i} \mathrm{E}_{i+1}$. Мы доказали следующее утверждение:

суммарная длина меток содержащихся в $\mathrm{Q}_{i}^{-1}$ участков примыкания поддиаграмм примыкания участка границы $\mathrm{Q}_{i}^{-1} \kappa$ участкам границы $\mathrm{Q}_{i-1}^{-1}$ и $\mathrm{Q}_{i+1}^{-1}$ меньше $2 p\left|A_{i}\right|$.

Теперь докажем, что

Не существует поддиаграмм примыкания $\Pi \in \mathcal{S}$ участка границы $\mathrm{Q}_{i}^{-1}$ к участкам гранищы $\mathrm{K}_{j}$ при $j \neq i-1, i$ u $\mathrm{Q}_{j}^{-1}$ при $j \neq i-1, i+1$. 


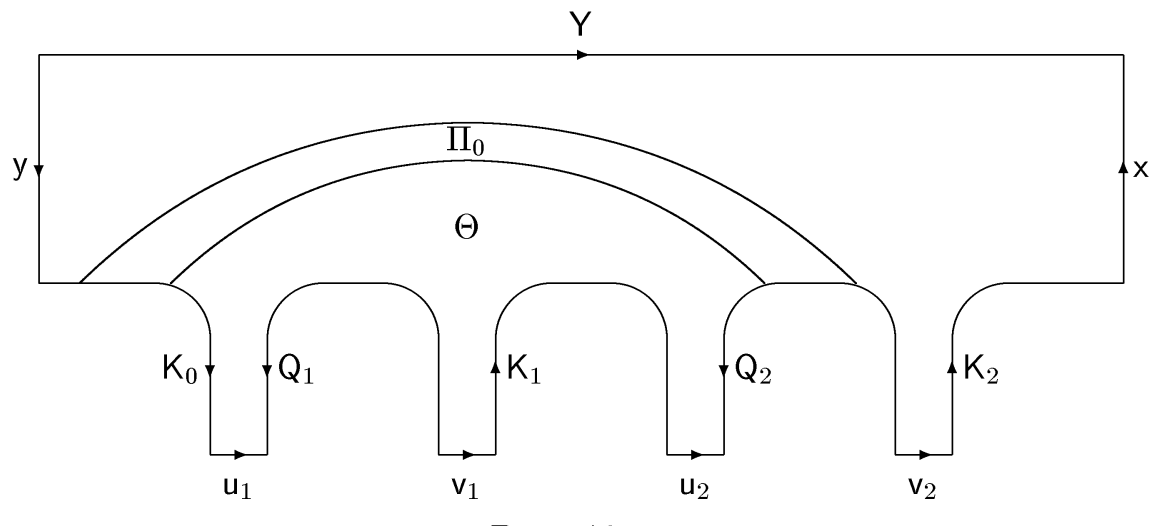

Рис. 46

Действительно, допустим, при некоторых $i_{0}, j_{0}$ такая поддиаграмма $\Pi_{0}$ сушествует. Пусть $\Theta$ - связная компонента диаграммы $\Delta-\Pi_{0}$, в граничный цикл которой не входит $\mathrm{Y}^{-1}$ (см. рис. 46). Если диаграмма $\Theta$, в свою очередь, содержит поддиаграмму $\Pi_{1} \in \mathcal{S}$, нарушающую условие из $(12.7)$, то вместо $\Theta$ можно рассмотреть соответствуюшую связную компоненту диаграммы $\Delta-\Pi_{1}$, которая, очевидно, является собственной поддиаграммой $\Theta$. Следовательно, можно считать, что $\Theta$ уже не содержит поддиаграмм П, нарушаюших (12.7), т.е. каждая поддиаграмма $\Pi \in \mathcal{S}$, содержашаяся в $\Theta$, является поддиаграммой одного из видов, учтенных в (12.5) или (12.6). Обозначим

$$
\mathrm{Z}_{1} \rightleftharpoons \mathrm{K}_{0}, \quad \mathrm{Z}_{2} \rightleftharpoons \mathrm{Q}_{1}^{-1}, \quad \mathrm{Z}_{3} \rightleftharpoons \mathrm{K}_{1}, \quad \ldots, \mathrm{Z}_{2 r} \rightleftharpoons \mathrm{Q}_{r}^{-1}, \quad \mathrm{Z}_{2 r+1} \rightleftharpoons \mathrm{K}_{r} .
$$

Тогда $\Pi_{0}$ - поддиаграмма примыкания некоторых $Z_{i_{1}}$ и $Z_{j_{1}}$, где $j_{1}-i_{1}>1$. Легко видеть, что $X_{\alpha-1}(\Theta)=\left\{Z_{i_{1}}^{\prime}, Z_{i_{1}+1}, Z_{i_{1}+2}, \ldots, Z_{j_{1}-1}, Z_{j_{1}}^{\prime}\right\}$, где $Z_{i_{1}}^{\prime}$ и $Z_{j_{1}}^{\prime}$ - некоторые конец и начало путей $Z_{i_{1}}$ и $Z_{j_{1}}$ соответственно. Отсюда следует $\# y_{\alpha-1}(\Theta)=$ $\# X_{\alpha-1}(\Theta)=j_{1}-i_{1}+1$ и, в частности, $\# y_{\alpha-1}(\Theta)-2 \chi(\Theta)=j_{1}-i_{1}-1 \geqslant 1$. По лемме 9.22 множество $\mathcal{S}_{1}$ поддиаграмм примыкания $\Pi \in \mathcal{S}$, содержашихся в $\Theta$, образует карту примыканий ранга $\alpha-1$ на $\Theta$. Обозначим через $U$ множество всех переходных участков диаграммы $\Theta$ относительно $S_{1}$. По предложению 10.4

$$
\sum_{\mathrm{S} \in U} \mathcal{W}_{\alpha-1}(\mathcal{L}(\mathrm{S})) \leqslant \eta\left(j_{1}-i_{1}-1\right)
$$

С другой стороны, пусть $U_{i}\left(i=i_{1}+1, i_{1}+2, \ldots, j_{1}-1\right)$ обозначает множество переходных участков диаграммы $\Theta$ относительно $\mathcal{S}_{1}$, содержащихся в $Z_{i}$. Предположим, $Z_{i}$ есть некоторый $Q_{j}^{-1}$. Тогда из (12.5) и (12.6) следует

$$
\sum_{\mathrm{S} \in U_{i}}|\mathcal{L}(\mathrm{S})|>74\left|A_{i}\right| \text {. }
$$

Так как в силу $(12.3)$ и выбора $\Theta$ в $Q_{j}^{-1}$ входит не более четырех участков примыкания поддиаграмм примыкания из $\mathcal{S}$, то $\# U_{i} \leqslant 5$. Тогда, используя предложение 8.22 , (ii), получаем

$$
\sum_{\mathrm{S} \in U_{i}} \mathcal{W}_{\alpha-1}(\mathcal{L}(\mathrm{S}))>\frac{64}{13}>4 \eta
$$


Заметим теперь, что по крайней мере $\left\lceil 1 / 3\left(j_{1}-i_{1}-1\right)\right\rceil$ путей $\mathrm{Q}_{j}^{-1}$ находятся среди $\mathrm{Z}_{i}$ (в наихудшем случае $j_{1}-i_{1}-1=3$ и среди $\mathrm{Z}_{i}$ ровно один $\mathrm{Q}_{j}^{-1}$ ). Учитывая это, из предыдушего неравенства выводим

$$
\sum_{\mathrm{S} \in U} \mathcal{W}_{\alpha-1}(\mathcal{L}(\mathrm{S}))>\eta\left(j_{1}-i_{1}-1\right)
$$

Мы получили противоречие с неравенством (12.9), что и доказывает (12.7).

Теперь докажем, что для каждого $i=1,2, \ldots, r$ имеется поддиаграмма примыкания $\Pi_{i} \in \mathcal{S}$ участков гранищы $Q_{i}^{-1}$ и $Y^{-1}$. Допустим, для некоторого $i_{0}$ это не так. Пусть $\Pi_{1}, \Pi_{2}, \ldots, \Pi_{t} \in \mathcal{S}$ - все поддиаграммы примыкания участков границы $Q_{i}^{-1}$ и $\mathrm{Y}^{-1}$. Из допушения следует, что путь $\mathrm{Q}_{i_{0}}^{-1}$ входит целиком в граничньй цикл некоторой связной компоненты $\Theta$ диаграммы $\Delta-\bigcup_{i=1}^{t} \Pi_{i}$. Пусть $i_{1} \leqslant i_{0}$ и $i_{2} \geqslant i_{0}-$ соответственно наименьший и наибольший индексы, для которых пути $\mathrm{Q}_{i}^{-1}$ входят в $\delta \Theta$ при $i_{1} \leqslant i \leqslant i_{2}$. Легко видеть, что кроме путей $\mathrm{Q}_{i}^{-1}$ при $i_{1} \leqslant i \leqslant i_{2}$, множество $X_{\alpha-1}(\Theta)$ состоит из путей $\mathrm{K}_{i_{1}-1}, \mathrm{~K}_{i_{1}}, \ldots, \mathrm{K}_{i_{2}}$, некоторого подпути пути $\mathrm{Y}^{-1}$, некоторого конца пути $\mathrm{Q}_{i_{1}-1}^{-1}$, если $i_{1}>1$, и некоторого начала пути $\mathrm{Q}_{i_{2}+1}^{-1}$, если $i_{2}<r$. Следовательно, \# $y_{\alpha-1}(\Theta)=\# X_{\alpha-1}(\Theta) \leqslant 2\left(i_{2}-i_{1}\right)+6$, и поэтому $\# y_{\alpha-1}(\Theta)-2 \chi(\Theta) \leqslant 2\left(i_{2}-i_{1}\right)+4 \leqslant 4\left(i_{2}-i_{1}+1\right)$. Тогда, используя $(12.5)-(12.7)$ и предложения $10.4,8.22$, (ii), приходим к противоречию аналогично тому, как в доказательстве утверждения (12.7).

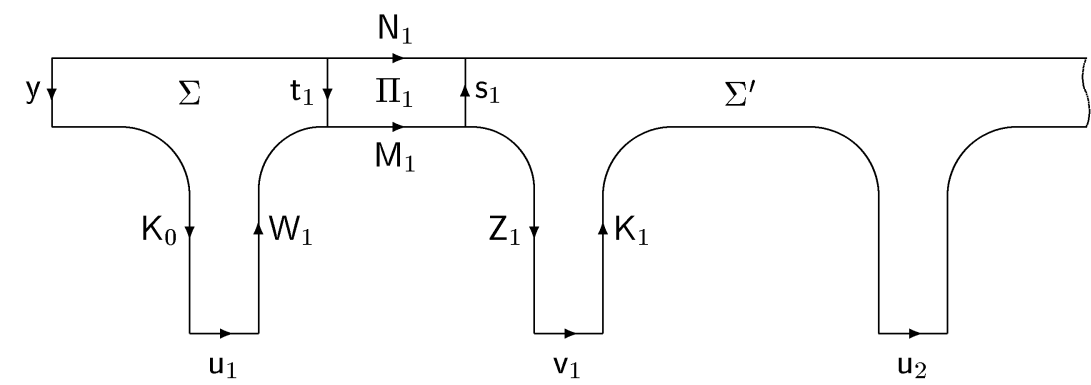

Рис. 47

Итак, каждый участок границы $\mathrm{Q}_{i}^{-1}$ имеет поддиаграмму примыкания $\Pi_{i} \in \mathcal{S}$ к $\mathrm{Y}^{-1}$. Пусть $\delta \Pi_{i} \approx \mathrm{M}_{i} \mathrm{~s}_{i} \mathrm{~N}_{i}^{-1} \mathrm{t}_{i}$, где $\mathrm{M}_{i}, \mathrm{~N}_{i}^{-1}$ - участки примыкания, $\mathcal{L}\left(\mathrm{s}_{i}\right), \mathcal{L}\left(\mathrm{t}_{i}\right) \in$ $\mathcal{H}_{\alpha-1}, \mathrm{Q}_{i}^{-1}=\mathrm{W}_{i} \mathrm{M}_{i} \mathrm{Z}_{i}$ и $\mathrm{N}_{i}$ входит в $\mathrm{Y}$. Оценим снизу длину слова $\mathcal{L}\left(\mathrm{M}_{i}\right)$. Заметим, что пути $\mathrm{W}_{i}$ и $\mathrm{Z}_{i}$ входят в граничные циклы некоторых дисковых поддиаграмм $\Sigma$ и $\Sigma^{\prime}$ диаграммы $\Delta$, являюшихся связными компонентами диаграммы $\Delta-\bigcup_{i=1}^{r} \Pi_{i}$ (см. рис. 47 , где изображен случай $i=1$ ). Ясно, что $\# y_{\alpha-1}(\Sigma), \# y_{\alpha-1}\left(\Sigma^{\prime}\right) \leqslant 4$. Пусть $\mathcal{S}_{1}$ и $\mathcal{S}_{2}$ - множество содержащихся соответственно в $\Sigma$ и в $\Sigma^{\prime}$ поддиаграмм из $\mathcal{S}$. Так как каждьй переходный участок диаграммы $\Delta$ относительно $\mathcal{S}$, содержашийся в $Q_{i}^{-1}$, является либо переходным участком диаграммы $\Sigma$

Серия математическая, №3 
относительно $\mathcal{S}_{1}$, либо переходньм участком диаграммы $\Sigma^{\prime}$ относительно $\mathcal{S}_{2}$, то, применяя предложение 10.4 два раза для $(\Delta, \mathcal{S}):=\left(\Sigma, \mathcal{S}_{1}\right),\left(\Sigma^{\prime}, \mathcal{S}_{2}\right)$, получаем $\sum_{\mathrm{S} \in V_{i}} \mathcal{W}_{\alpha-1}(\mathcal{L}(\mathrm{S})) \leqslant 4 \eta$, где $V_{i}$ - множество переходных участков диаграммы $\Delta$ относительно $\mathcal{S}$, содержащихся в $\mathrm{Q}_{i}^{-1}$. Так как $\# V_{i} \leqslant 4$ по лемме 9.23, (i) при $\Delta:=\Sigma, \Sigma^{\prime}$, то по предложению 8.22 , (ii) получаем

$$
\sum_{\mathrm{S} \in V_{i}}|\mathcal{L}(\mathrm{S})| \leqslant(52 \eta+8)\left|A_{i}\right|<30\left|A_{i}\right|
$$

Используя, далее,(12.3), (12.5)-(12.7), получаем наконец

$$
\left|\mathcal{L}\left(\mathrm{M}_{i}\right)\right|>\left(n-\ell_{\alpha, A_{i}}\left(\widetilde{E}_{i}\right)-2 p-67\right)\left|A_{i}\right| .
$$

В частности, ввиду (12.2) имеем $\left|\mathcal{L}\left(\mathrm{M}_{i}\right)\right| \geqslant 44\left|A_{i}\right|$.

Теперь уже несложно вывести все требуемые утверждения. Рассмотрим отображение $\omega$ множества путей на диаграмме $\Delta$ во множество путей на графе $\Gamma_{\alpha-1}$ (см. 4.12). Так как пути $\omega\left(\mathrm{M}_{i}\right)$ и $\omega\left(\mathrm{N}_{i}\right)$ близки в ранге $\alpha-1$, то по предложению 8.23 пути $\omega\left(\mathrm{M}_{i}\right)$ и $\omega\left(\mathrm{N}_{i}\right)$ содержат подпути $\mathrm{S}_{i}$ и $\mathrm{F}_{i}$ соответственно, где $\mathrm{F}_{i} \in \Pi$ Пер $\alpha\left(A_{i}^{-1}, 8\right), \mathrm{S}_{i}$ - периодическая основа для $\mathrm{F}_{i}$ и

$$
\ell_{\alpha, A^{-1}}\left(\mathrm{~F}_{i}\right) \geqslant n-\ell_{\alpha, A}\left(\widetilde{E}_{i}\right)-2 p-103 .
$$

Пусть $\omega(\mathrm{Y})=\mathrm{L}_{0} \mathrm{~F}_{1} \mathrm{~L}_{1} \ldots \mathrm{F}_{r} \mathrm{~L}_{r}$ и $\omega\left(\mathrm{Q}_{i}\right)=\mathrm{U}_{i} \mathrm{~S}_{i}^{-1} \mathrm{~V}_{i}(i=1,2, \ldots, r)$. В силу леммы 8.2, (ii) соединим вершины $\iota\left(\mathrm{F}_{i}\right)$ и $\iota\left(\mathrm{S}_{i}\right)$ путем $\mathrm{w}_{i}$, а вершины $\tau\left(\mathrm{S}_{i}\right)$ и $\tau\left(\mathrm{F}_{i}\right)$ путем $\mathrm{z}_{i}$, где $\mathcal{L}\left(\mathrm{w}_{i}\right), \mathcal{L}\left(\mathrm{z}_{i}\right) \in \mathcal{H}_{\alpha-1}$. Мы имеем, таким образом, набор путей на графе $\Gamma_{\alpha-1}$, расположенных, как на рис. 48 (заметим, что $\omega(\mathrm{x})$ и $\omega(\mathrm{y})$ - пустые пути). Рассматривая в качестве слов $F_{i}, L_{i}, w_{i}, Z_{i}, S_{i}, U_{i}, V_{i}$ метки соответствующих путей на рис. 48 , мы сразу получаем вьполнение условий (i)-(iii), а из рис. 48 видно, что имеют место соотношения (iv). Наконец, искомые неравенства (v) выполнены ввиду (12.10). Доказательство леммы 12.7 завершено.

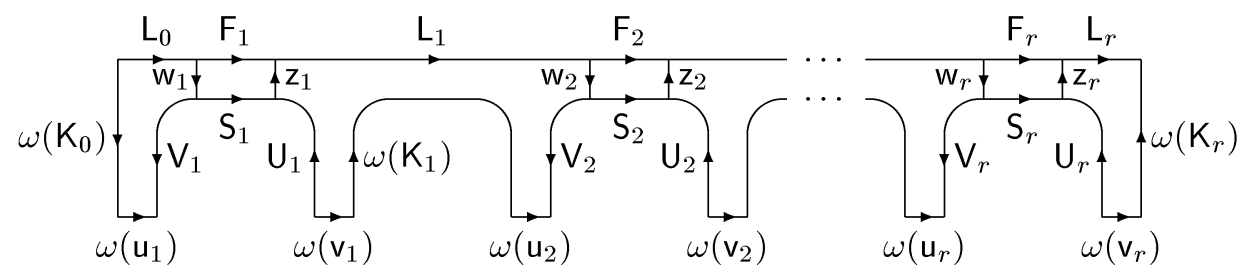

Рис. 48

12.8. ОПРЕДЕлЕнИЕ. Если выполнены посылка и заключение леммы 12.7 , то набор вхождений $\mathrm{V}_{i} \rightleftharpoons L_{0} F_{1} \ldots L_{i-1} * F_{i} * L_{i} \ldots F_{r} L_{r} \quad(i=1,2, \ldots, r)$ элементарных слов $F_{i}$ ранга $\alpha$ в слово $Y$ будем называть образами вхождений $\mathrm{U}_{i}$ в повоpome $X \rightarrow Y$. 
12.9. Лемма. Пусть слово $Y$ есть результат поворота в слове $X$ некоторого набора вхождений $\mathrm{U}_{i}=K_{0} E_{1} K_{1} \ldots K_{i-1} * E_{i} * K_{i} \ldots E_{r} K_{r}(i=1,2, \ldots, r)$ и $\mathrm{V}_{i}=L_{0} F_{1} \ldots L_{i-1} * F_{i} * L_{i} \ldots F_{r} L_{r}-$ образы вхождений $\mathrm{U}_{i}$ в повороте $X \rightarrow Y$. Предположим, ито $\ell_{\alpha}\left(E_{i}\right) \geqslant q_{0} u \ell_{\alpha}\left(F_{i}\right) \geqslant q_{0}$ при всех $i$. Пусть $\mathrm{W}=M * G * N-$ вхождение әлементарной $(h+8)$-степени $G$ ранга $\alpha$ в слово $Y$ такое, что $\neg Э С о г \Omega_{\alpha}\left(\mathrm{W}, \mathrm{V}_{i}\right)$ при всех $i=1,2, \ldots, r$. Тогда найдется вхождение $\mathrm{W}^{\prime}=R * H * T$ әлементарного слова $H$ ранга $\alpha$ в слово $X$ такое, что $\ell_{\alpha}(H) \geqslant \ell_{\alpha}(G)-h$ u $Э$ ЭСог, $\Omega_{\alpha}\left(\mathrm{W}^{\prime}, \mathrm{U}_{i}\right)$ при $i=1,2, \ldots, r$.

ДОКАЗАТЕЛЬСТвО. Из $\neg$ ЭСољ $\Omega_{\alpha}\left(\mathrm{W}, \mathrm{V}_{i}\right)(i=1,2, \ldots, r)$ в силу леммы $12.3,(\mathrm{ii})$ следует, что $G$ содержится в одном из подслов $L_{0} F_{1}, F_{1} L_{1} F_{2}, \ldots, F_{r-1} L_{r-1} F_{r}$, $F_{r} L_{r}$ слова $Y$. Рассмотрим случай, когда $G$ содержится в некотором подслове $F_{k} L_{k} F_{k+1}$. В соответствии с 12.4 , (i), 12.7 , (ii) и 12.7 , (iv) рассмотрим пути на графе $\Gamma_{\alpha-1}$, расположенные, как на рис. 49 , и обозначенные теми же буквами, что и их метки. Пусть $\mathrm{X} \rightleftharpoons \mathrm{K}_{0} \mathrm{E}_{1} \mathrm{~K}_{1} \ldots \mathrm{E}_{r} \mathrm{~K}_{r}, \mathrm{Y} \rightleftharpoons \mathrm{L}_{0} \mathrm{~F}_{1} \mathrm{E}_{1} \ldots \mathrm{F}_{r} \mathrm{~L}_{r}$ и $\mathrm{G} \rightleftharpoons \phi_{\mathrm{Y}}(\mathrm{W})$ - подпуть пути $\mathrm{F}_{k} \mathrm{~L}_{k} \mathrm{~F}_{k+1}$ с $\mathcal{L}(\mathrm{G})$ 으 $G$. Так как по условию $\neg$ ЭСогл $\alpha\left(\mathrm{W}, \mathrm{V}_{k}\right)$ и $\neg \operatorname{SCozл}_{\alpha}\left(\mathrm{W}, \mathrm{V}_{k+1}\right)$, то в силу определения 12.1 имеем $\neg \Pi \operatorname{Coг}_{\alpha}\left(\mathrm{G}, \mathrm{F}_{k}\right)$ и $\neg \Pi \operatorname{Coz}_{\alpha}\left(\mathrm{G}, \mathrm{F}_{k+1}\right)$. Так как $\mathrm{S}_{k}, \mathrm{~S}_{k+1}$ - периодические основы для $\mathrm{F}_{k}, \mathrm{~F}_{k+1}$ в силу 12.7 , (ii), а в силу 12.4 , (ii), 12.7 , (iii) пути $\mathrm{S}_{k} \mathrm{U}_{k}^{-1} \mathrm{P}_{k}^{-1}$ и $\mathrm{P}_{k+1}^{-1} \mathrm{~V}_{k+1}^{-1} \mathrm{~S}_{k+1}-$ периодические продолжения для $\mathrm{S}_{k}$ и $\mathrm{S}_{k+1}$, то в силу определения 11.11 путь $\mathrm{G}$ устойчив относительно $\mathrm{w}_{k} \mathrm{~S}_{k} \mathrm{U}_{k}^{-1} \mathrm{v}_{k}$ и $\mathrm{u}_{k+1} \mathrm{~V}_{k+1}^{-1} \mathrm{~S}_{k+1} \mathrm{z}_{k+1}$. Тогда по лемме 11.10 найдется подпуть $\mathrm{H} \in$ ППер $\alpha(B, 8)$ пути $\mathrm{K}_{k}\left(B \in \mathcal{E}_{\alpha}\right)$ такой, что $\Pi$ Погл $\Omega_{\alpha}(\mathrm{G}, \mathrm{H})$ и $\ell_{\alpha}(\mathrm{H}) \geqslant \ell_{\alpha}(\mathrm{G})-h$. Проверим, что $\neg \Pi$ Согл $\Omega_{\alpha}\left(\mathrm{E}_{i}, \mathrm{H}\right)$ при всех $i$. Так как $\mathrm{P}_{k}$ и $\mathrm{P}_{k+1}$ - периодические основы для $\mathrm{E}_{k}$ и $\mathrm{E}_{k+1}$ в силу 12.4, (i), то согласно определению 5.18 имеем ПСогл $\Omega_{\alpha}\left(\mathrm{E}_{k}, \mathrm{~F}_{k}^{-1}\right)$ и ПСогл $\Omega_{\alpha}\left(\mathrm{E}_{k+1}, \mathrm{~F}_{k+1}^{-1}\right)$. Тогда если бы вьполнялось ПСогл $\Omega_{\alpha}\left(\mathrm{E}_{i}, \mathrm{H}\right)$ при $i=k$ или $i=k+1$, то мы получили бы $\Pi \operatorname{Coz}_{\alpha}\left(\mathrm{G}_{1} \mathrm{~F}_{i}^{-1}\right)$ вопреки предложению 8.46. Следовательно, $\neg \Pi \operatorname{Coг}_{\alpha}\left(\mathrm{E}_{i}, \mathrm{H}\right)$ при $i=k, k+1$.

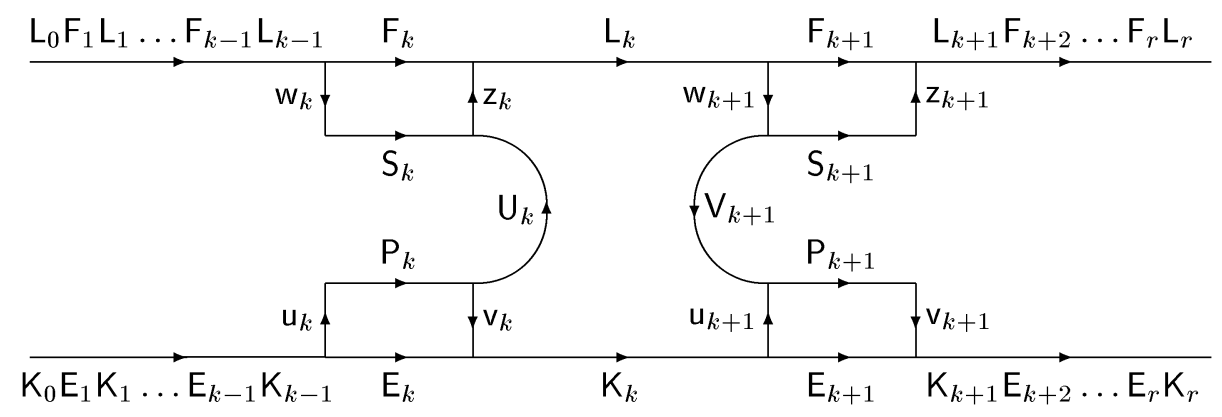

Рис. 49

Допустим, ПСогл $\Omega_{\alpha}\left(\mathrm{E}_{i}, \mathrm{H}\right)$ при некотором $i<k$. Тогда по предложению 8.35 имеем $\widetilde{\mathrm{E}}_{i} \in \Pi$ Пер $\alpha(B, p+34)$ для некоторого подпути $\widetilde{\mathrm{E}}_{i}$ пути $\mathrm{E}_{i}$, откуда в силу предложений 8.11 и 8.38 , (ii) получаем $\widetilde{\mathrm{E}}_{i} \cup \mathrm{x} \mathrm{H} \in \Pi$ Пер $\alpha(B, p+34), \Pi$ Согл $\Omega_{\alpha}\left(\mathrm{E}_{k}, \widetilde{\mathrm{E}}_{i} \cup_{\mathrm{X}} \mathrm{H}\right)$, 
и, следовательно, ПСогл $\Omega_{\alpha}\left(\mathrm{E}_{k}, \mathrm{H}\right)$ вопреки доказанному. Аналогично доказывается $\neg \Pi$ Согл $\Omega_{\alpha}\left(\mathrm{E}_{i}, \mathrm{H}\right)$ при $i>k+1$. Таким образом, $\neg \Pi$ Сог $\Omega_{\alpha}\left(\mathrm{E}_{i}, \mathrm{H}\right)$ при всех $i$, и в качестве искомого вхождения $W^{\prime}$ в слово $X$ можно взять соответствующее вхождение слова $\mathcal{L}(\mathrm{H})$ в $X$.

Мы рассмотрели случай, когда $G$ содержится в некотором подслове $F_{k} L_{k} F_{k+1}$ слова $Y$. Аналогично рассматриваются случаи, когда $G$ содержится в $L_{0} F_{1}$ или в $F_{r} L_{r}$, с тем лишь отличием, что роль пути $\mathrm{w}_{k} \mathrm{~S}_{k} \mathrm{U}_{k}^{-1} \mathrm{v}_{k}$ или, соответственно, $\mathrm{u}_{k+1} \mathrm{~V}_{k+1}^{-1} \mathrm{~S}_{k+1} \mathrm{z}_{k+1}$ при использовании леммы 11.10 будет играть пустой путь.

12.10. Лемма. Пусть слово $Y$ есть результат поворота в слове $X$ некоторого набора вхождений $\mathrm{U}_{i}=K_{0} E_{1} K_{1} \ldots K_{i-1} * E_{i} * K_{i} \ldots E_{r} K_{r} \quad(i=$ $1,2, \ldots, r)$, где $E_{i}$ - әлементарное слово ранга $\alpha$, порожденное периодом $A_{i} \in \mathcal{E}_{\alpha}$. Пусть $\mathrm{V}_{i}=L_{0} F_{1} \ldots L_{i-1} * F_{i} * L_{i} \ldots F_{r} L_{r} \quad(i=1,2, \ldots, r)-$ образы вхождений $\mathrm{U}_{i}$ в повороте $X \rightarrow Y$. Предположим, $\ell_{\alpha}\left(E_{i}\right) \geqslant p+34$, $\ell_{\alpha}\left(F_{i}\right) \geqslant p+34$ nри всех $i$ u $\neg$ ЭСогл $\alpha\left(\mathrm{U}_{i}, \mathrm{U}_{i+1}\right) \quad(1 \leqslant i \leqslant r-1)$. Пусть вхождение слова $\widetilde{E}_{i}$ в слово $X$ и вхождение слова $\widetilde{F}_{i}$ в слово $Y$ - максимальнье продолэсения вхождений $\mathrm{U}_{i} u \mathrm{~V}_{i}$ относительно периодов $A_{i} u A_{i}^{-1}$ соответственно. Тогда

$$
\ell_{\alpha, A_{i}}\left(\widetilde{E}_{i}\right)+\ell_{\alpha, A_{i}^{-1}}\left(\widetilde{F}_{i}\right)<n+2 p+120 .
$$

ДокАЗАТЕльство. Пусть для некоторых слов $u_{i}, v_{i}, w_{i}, z_{i} \in \mathcal{H}_{\alpha-1}, P_{i} \in$ $\Pi е p\left(A_{i}, 2\right), S_{i} \in \Pi e p\left(A_{i}^{-1}, 2\right), U_{i}, V_{i}(i=1,2, \ldots, r)$ выполнены условия (i)-(iii) определения 12.4 и верны утверждения 12.7, (i)-(v). Выберем произвольные пути

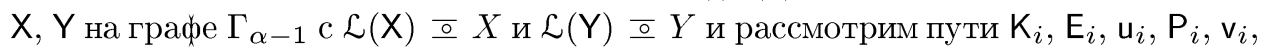
$\mathrm{L}_{i}, \mathrm{~F}_{i}, \mathrm{w}_{i}, \mathrm{~S}_{i}, \mathrm{z}_{i}$, обозначенные теми же буквами, что и их метки, расположенные, как на рис. 50, где $\mathrm{X}=\mathrm{K}_{0} \mathrm{E}_{0} \mathrm{~K}_{1} \ldots \mathrm{E}_{r} \mathrm{~K}_{r}$ и $\mathrm{Y}=\mathrm{L}_{0} \mathrm{~F}_{0} \mathrm{~L}_{1} \ldots \mathrm{F}_{r} \mathrm{~L}_{r}$. Согласно 12.4, (i) и 12.7, (ii) $\mathrm{P}_{i}$ и $\mathrm{S}_{i}$ - периодические основы для $\mathrm{E}_{i}$ и $\mathrm{F}_{i}$ соответственно.

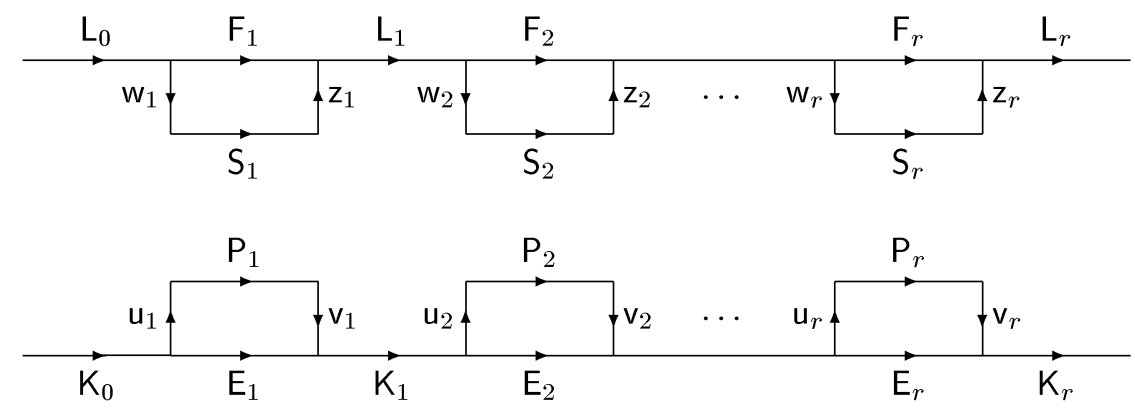

Рис. 50

Зафиксируем индекс $i$, и пусть $\mathrm{U}$ и $\mathrm{V}$ - максимальные продолжения вхождений $\mathrm{U}_{i}$ и $\mathrm{V}_{i}$ соответственно. Обозначим $\widetilde{\mathrm{E}} \rightleftharpoons \phi_{\mathbf{X}}(\widetilde{\mathrm{U}})$ и $\widetilde{\mathrm{F}} \rightleftharpoons \phi_{\mathrm{Y}}(\widetilde{\mathrm{V}})$. Мы должны доказать неравенство $\ell_{\alpha, A_{i}}\left(\widetilde{E}_{i}\right)+\ell_{\alpha, A_{i}^{-1}}\left(\widetilde{F}_{i}\right)<n+2 p+120$. В силу предложений 8.9 и 8.10 , (ii) найдутся периодические основы $\widetilde{P}$ и $\widetilde{S}$ для $\widetilde{E}$ и $\widetilde{F}$ соответственно, 
где $\widetilde{\mathrm{P}}$ есть периодическое продолжение $\mathrm{P}_{i}$ и $\widetilde{\mathrm{S}}$ есть периодическое продолжение $\mathrm{S}_{i}$, причем можно считать $|\widetilde{\mathrm{P}}| \geqslant \ell_{\alpha, A_{i}}(\widetilde{\mathrm{E}})\left|A_{i}\right|$ и $|\widetilde{\mathrm{S}}| \geqslant \ell_{\alpha, A_{i}^{-1}}(\widetilde{\mathrm{F}})\left|A_{i}\right|$. Пусть $\widetilde{\mathrm{P}}=\mathrm{G}^{\prime} \mathrm{P}_{i} \mathrm{G}^{\prime \prime}$ и $\widetilde{\mathrm{S}}=\mathrm{H}^{\prime} \mathrm{S}_{i} \mathrm{H}^{\prime \prime}$. Так как согласно 12.4 , (ii) и 12.7 , (iii) имеем $A_{i}^{n} \approx P_{i} U_{i} S_{i}^{-1} V_{i}$, то для доказательства искомой оценки достаточно установить два неравенства:

$$
\left|\mathrm{G}^{\prime}\right|+\left|\mathrm{H}^{\prime}\right|<\left|V_{i}\right|+(p+60)\left|A_{i}\right| \text { и }\left|\mathrm{G}^{\prime \prime}\right|+\left|\mathrm{H}^{\prime \prime}\right|<\left|U_{i}\right|+(p+60)\left|A_{i}\right| .
$$

Эти два неравенства симметричны. Ограничимся доказательством первого из них. Рассмотрим два случая.

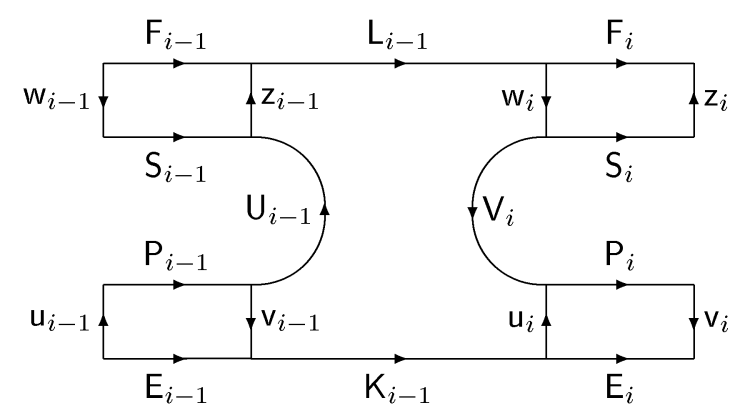

Рис. 51

Случай 1: $i>1$. Согласно 12.7, (iv) имеем $v_{i-1} K_{i-1} u_{i} \stackrel{\alpha=1}{=} U_{i-1} z_{i-1} L_{i-1} w_{i} V_{i}$. Рассмотрим путь $\mathrm{U}_{i-1} \mathrm{z}_{i-1}^{*} \mathrm{~L}_{i-1}^{*} \mathrm{w}_{i}^{*} \mathrm{~V}_{i}$ с теми же, что и у пути $\mathrm{v}_{i-1} \mathrm{~K}_{i-1} \mathrm{u}_{i}$, начальной и конечной вершинами, где метки путей $\mathrm{U}_{i-1}, \mathrm{z}_{i-1}^{*}, \mathrm{~L}_{i-1}^{*}, \mathrm{w}_{i}^{*}$ и $\mathrm{V}_{i}$ равны словам $U_{i-1}, z_{i-1}, L_{i-1}, w_{i}$ и $V_{i}$ соответственно. Так как путь $\mathrm{Y}$ расположен произвольным образом относительно $\mathrm{X}$, то можно считать $\mathrm{z}_{i-1}^{*}=\mathrm{z}_{i-1}, \mathrm{~L}_{i-1}^{*}=\mathrm{L}_{i-1}$ и $\mathrm{w}_{i}^{*}=\mathrm{w}_{i}$ (см. рис. 51). Если $\left|\mathrm{G}^{\prime}\right|+\left|\mathrm{H}^{\prime}\right| \leqslant\left|\mathrm{V}_{i}\right|$, то доказывать нечего. Пусть

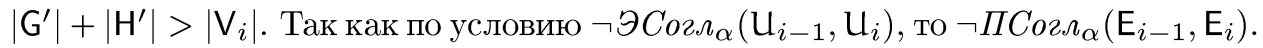
Так как периодические основы $\mathrm{P}_{i-1}$ и $\mathrm{S}_{i-1}^{-1}$ для $\mathrm{E}_{i-1}$ и $\mathrm{F}_{i-1}^{-1}$ имеют общее периодическое продолжение $\mathrm{P}_{i-1} \mathrm{U}_{i} \mathrm{~S}_{i-1}^{-1}$, то имеем ПСогл $\alpha\left(\mathrm{E}_{i-1}, \mathrm{~F}_{i-1}^{-1}\right)$. По той же причине $\Pi$ Согл $\Omega_{\alpha}\left(\mathrm{E}_{i}, \mathrm{~F}_{i}^{-1}\right)$. Следовательно, $\neg \Pi$ Согл $\Omega_{\alpha}\left(\mathrm{F}_{i-1}, \mathrm{~F}_{i}\right)$. Так как в силу предложения 8.9 имеем ПСогл $\alpha\left(\mathrm{E}_{i}, \widetilde{\mathrm{E}}\right)$ и $\Pi$ Согл $\Omega_{\alpha}\left(\mathrm{F}_{i}, \widetilde{\mathrm{F}}\right)$, то в силу предложения 8.38, (ii) $\mathrm{E}_{i-1}$ не может содержаться в $\widetilde{\mathrm{E}}$ и $\mathrm{F}_{i-1}$ не может содержаться в $\widetilde{\mathrm{F}}$, т.е. $\mathrm{E}_{i-1}<\mathrm{x} \widetilde{\mathrm{E}}$ и $\mathrm{F}_{i-1}<_{\mathrm{Y}} \widetilde{\mathrm{F}}$. Пусть $\mathrm{W}$ - начало пути $\mathrm{E}_{i-1} \mathrm{~K}_{i-1}$ с $\tau(\mathrm{W})=\iota(\widetilde{\mathrm{E}})$ и $\mathrm{Z}$ - начало пути $\mathrm{F}_{i-1} \mathrm{~L}_{i-1} \mathrm{c} \tau(\mathrm{Z})=\iota(\widetilde{\mathrm{F}})$. Так как $\mathrm{G}^{\prime} \mathrm{P}_{i} \mathrm{G}^{\prime \prime}-$ периодическая основа для $\widetilde{\mathrm{E}}$, то в силу леммы 8.2 , (ii) вершины $\tau(\mathrm{W})$ и $\iota\left(\mathrm{G}^{\prime}\right)$ можно соединить путем и̃ с $\mathcal{L}(\tilde{\mathrm{u}}) \in \mathcal{H}_{\alpha-1}$. По той же причине вершины $\tau(\mathrm{Z})$ и $\iota\left(\mathrm{H}^{\prime}\right)$ можно соединить путем v с $\mathcal{L}(\tilde{\mathrm{v}}) \in \mathcal{H}_{\alpha-1}$. Из предположения $\left|\mathrm{G}^{\prime}\right|+\left|\mathrm{H}^{\prime}\right|>\left|\mathrm{V}_{i}\right|$ следует, что вершины $\iota\left(\mathrm{G}^{\prime}\right)=\tau(\tilde{\mathrm{u}})=\iota(\widetilde{\mathrm{P}})$ и $\iota\left(\mathrm{H}^{\prime}\right)=\tau(\widetilde{\mathrm{v}})=\iota(\widetilde{\mathrm{S}})$ соединены путем $\mathrm{T} \in$ Пер $\left(\Gamma_{\alpha-1}, A_{i}^{-1}\right)$, содержащимся в некотором периодическом продолжении $\mathrm{S}_{i}^{-1} \mathrm{~V}_{i} \mathrm{P}_{i}$,

$$
|\mathrm{T}|=\left|\mathrm{G}^{\prime}\right|+\left|\mathrm{H}^{\prime}\right|-\left|\mathrm{V}_{i}\right|
$$


Мы имеем, таким образом, замкнутый путь $\mathrm{WũT}^{-1} \mathrm{Z}^{-1} \mathrm{~W}_{i-1} \mathrm{~S}_{i-1} \mathrm{U}_{i-1}^{-1} \mathrm{P}_{i-1}^{-1} \mathrm{u}_{i-1}^{-1}$ (см. рис. 52). Предположим, некоторый подпуть $\mathrm{R}$ пути $\mathrm{T}$ и некоторый подпуть $\mathrm{Q}$ пути $\mathrm{W}^{-1}$ близки в ранге $\alpha-1$. Тогда, применив лемму 11.7 , (ii) при $\mathrm{E}:=\widetilde{\mathrm{E}}$, $\mathrm{P}:=\widetilde{\mathrm{P}}, \mathrm{U}_{1}:=\mathrm{W}$, где в качестве $\mathrm{V}_{1}$ нужно взять начало пути $\widetilde{\mathrm{P}}$ длины $20|A|$, получим $|\mathrm{R}|<20\left|A_{i}\right|$. Аналогично, используя лемму 8.43 , (ii) при $\mathrm{E}:=\widetilde{\mathrm{F}}$ и $\mathrm{P}:=\widetilde{\mathrm{S}}$, получим, что если некоторый подпуть $\mathrm{R}$ пути $\mathrm{T}$ и некоторый подпуть пути $\mathrm{Z}$ близки в ранге $\alpha-1$, то $|\mathrm{R}|<20\left|A_{i}\right|$. Далее, из $\neg \Pi C о \Omega_{\alpha}\left(\mathrm{E}_{i-1}, \mathrm{E}_{i}\right)$ следует $\neg$ Согл $\left(\mathrm{P}_{i-1} \mathrm{U}_{i-1} \mathrm{~S}_{i-1}^{-1}, \mathrm{~S}_{i}^{-1} \mathrm{~V}_{i} \mathrm{P}_{i}\right)$, откуда по предложению 8.36 заключаем, что если подпуть $\mathrm{R}$ пути $\mathrm{T}$ и подпуть пути $\mathrm{P}_{i-1} \mathrm{U}_{i-1} \mathrm{~S}_{i-1}^{-1}$ близки в ранге $\alpha-1$, то $|\mathrm{R}|<p\left|A_{i}\right|$. Тогда, используя лемму 11.7 , (i) при $\mathrm{X}:=\mathrm{Y}:=\mathrm{T}$, $\mathrm{W}:=\tilde{\mathrm{u}}^{-1} \mathrm{~W}^{-1} \mathrm{u}_{i-1} \mathrm{P}_{i-1} \mathrm{U}_{i-1} \mathrm{~S}_{i-1}^{-1} \mathrm{w}_{i-1}^{-1} \mathrm{Z} \tilde{\mathrm{v}}$ и предложение 8.22, (ii), легко выводим $|\mathrm{T}|<(p+60)\left|A_{i}\right|$, что вместе с (12.12) дает искомое неравенство (12.11).

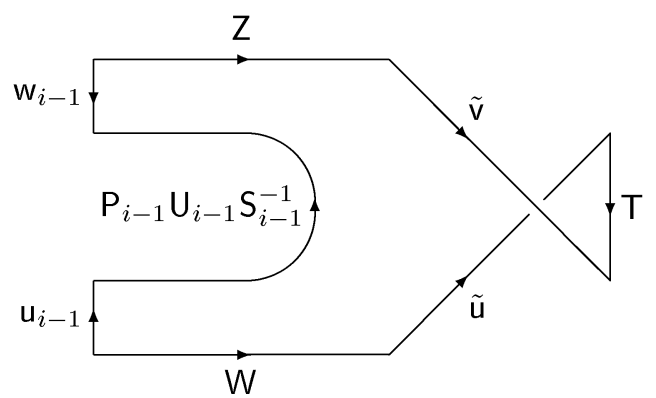

Рис. 52

Случай $2: i=1$. Этот случай рассматривается аналогично случаю 1, с той лишь разнищей, что вместо пути $\mathrm{u}_{i-1} \mathrm{P}_{i-1} \mathrm{U}_{i-1} \mathrm{~S}_{i-1}^{-1} \mathrm{w}_{i-1}^{-1}$ на рис. 52 будет пустой путь.

12.11. Лемма. Для любого слова $X \in \mathcal{R}_{\alpha-1}$, не содержащего әлементарных $\left(n-q_{0}-2 p-102\right)$-степеней ранга $\alpha$, существует слово $Y \in \mathcal{R}_{\alpha-1}$, не содержащее әлементарных $\left(\frac{n}{2}+2 p+142\right)$-степеней ранга $\alpha$, такое, что $X \stackrel{\alpha}{=} Y$.

ДоКАЗАТЕЛЬСТво. Без ограничения обшности можно считать, что $X$ содержит некоторую элементарную $\left(\frac{n}{2}+14\right)$-степень ранга $\alpha$. Рассмотрим множество всех вхождений в слово $X$ элементарных $\left(\frac{n}{2}+14\right)$-степеней ранга $\alpha$ и выберем из него подмножество $\left\{\mathrm{U}_{i}\right\}_{i=1}^{r}$ представителей по отношению $Э С о г \Omega_{\alpha}(\mathrm{U}, \mathrm{V})$, которое в силу леммы 12.3 , (i) является отношением эквивалентности на множестве вхождений в слово $X$ элементарных слов ранга $\alpha$. Пусть $\mathrm{U}_{i}$ есть вхождение слова $E_{i} \in \Pi$ Пер $\alpha\left(A_{i}, \frac{n}{2}+14\right) \quad\left(A_{i} \in \mathcal{E}_{\alpha}\right)$. По лемме 12.3 , (iii) укоротим каждое $\mathrm{U}_{i}$, чтобы получились попарно непересекающиеся вхождения $\mathrm{U}_{i}^{\prime}$ слов $E_{i}^{\prime} \in \Pi$ Пер $\alpha\left(A_{i}, \frac{n}{2}-2 p-68\right)$. Тем самым мы получим набор $\left\{\mathrm{U}_{i}^{\prime}\right\}_{i=1}^{r}$ попарно непересекаюшихся вхождений элементарных слов ранга $\alpha$ в слово $X$, удовлетворяюший следуюшим условиям: 
(i) если вхождение слова $\widetilde{E}_{i}$ в $X$ есть максимальное продолжение вхождения $\mathrm{U}_{i}^{\prime}$ относительно периода $A_{i}$, то $\frac{n}{2}+14 \leqslant \ell_{\alpha, A_{i}}\left(\widetilde{E}_{i}\right) \leqslant n-q_{0}-2 p-103$;

(ii) $\neg \ni$ Согл $_{\alpha}\left(\mathrm{U}_{i}^{\prime}, \mathrm{U}_{j}^{\prime}\right)$ при $i \neq j$ (это верно в силу леммы $\left.12.3,(\mathrm{i}),(\mathrm{ii})\right)$;

(iii) если $\mathrm{U}$ - вхождение в слово $X$ элементарной $\left(\frac{n}{2}+14\right)$-степени ранга $\alpha$, то ЭСогл $\Omega_{\alpha}\left(\mathrm{U}_{i}^{\prime}, \mathrm{U}\right)$ для некоторого $i$.

По лемме 12.6 существует поворот $X \rightarrow Y$ набора вхождений $\left\{\mathrm{U}_{i}^{\prime}\right\}_{i=1}^{r}$. Проверим, что $Y$ - искомое слово. По лемме 12.5 имеем $X \stackrel{\alpha}{=} Y$. Допустим, $W-$ вхождение в слово $Y$ элементарной $\left(\frac{n}{2}+2 p+142\right)$-степени ранга $\alpha$. Пусть $\mathrm{V}_{i}$ $(i=1,2, \ldots, r)$ - образы вхождений $\mathrm{U}_{i}^{\prime}$ в повороте $X \rightarrow Y$. Если $\neg Э C о г \Omega_{\alpha}\left(\mathrm{V}_{i}, W\right)$ при всех $i$, то по лемме 12.9 , посылка $\ell_{\alpha, A_{i}^{-1}}\left(F_{i}\right) \geqslant q_{0}$ которой выполнена в силу (i) и условия $12.7,(\mathrm{v})$, найдется вхождение $W^{\prime}$ элементарной $\left(\frac{n}{2}+2 p+142-h\right)$-степени ранга $\alpha$ в слово $X$ такое, что $\neg$ Эогл $\Omega_{\alpha}\left(\mathrm{U}_{i}^{\prime}, W^{\prime}\right)$ для всех $i$. Но это невозможно в силу (iii), так как $h=2 p+128$. Пусть $Э С о г \Omega_{\alpha}\left(\mathrm{V}_{i}, W\right)$ для некоторого $i$. Используя предложения 8.35 и 8.11, легко видеть, что в этом случае найдется содержашее $\mathrm{V}_{i}$ вхождение $W^{*}$ в слово $Y$ элементарной $\left(\frac{n}{2}+2 p+106\right)$-степени ранга $\alpha$, порожденной периодом $A_{i}^{-1}$. Тогда по лемме $12.3,(\mathrm{v})$ получаем $\ell_{\alpha, A_{i}^{-1}}\left(\widetilde{F}_{i}\right) \geqslant \frac{n}{2}+2 p+106$, где вхождение слова $\widetilde{F}_{i}$ есть максимальное продолжение $\mathrm{V}_{i}$ относительно $A_{i}^{-1}$. Но в этом случае мы получаем противоречие с (i) и леммой 12.10 , посылка которой выполнена в силу (ii). Таким образом, $Y$ не содержит вхождений элементарных $\left(\frac{n}{2}+2 p+142\right)$-степеней ранга $\alpha$.

12.12. Лемма. Если $X \in \mathcal{R}_{\alpha}$, то $X$ не содержит әлементарных $(n-q)$-степеней ранга $\alpha$. Если $X \in \mathcal{R}_{\alpha-1}$ u $X$ не содержит элементарных $(n-q-36)$-степеней ранга $\alpha$, mо $X \in \mathcal{R}_{\alpha}$.

ДокАЗАТЕльство. Первое утверждение вытекает их определений 5.7, 5.16, 5.17 и леммы 8.2, (ii), а второе - из определения 5.7 и следствия 8.27.

12.13. ПРЕДЛОЖЕНИЕ $(\alpha \geqslant 0)$. Для любого слова $X$ существует слово $Y$ maкое, что $X \stackrel{\alpha}{=} Y$ u $Y \in \mathcal{R}_{\alpha}$.

ДокАЗАТЕЛЬСТво. При $\alpha=0$ утверждается сушествование несократимого слова $Y$, равного данному слову $X$ в свободной группе $\mathbf{B}_{0}$. Пусть $\alpha \geqslant 1$. Воспользуемся индукцией по длине слова $X$. Если $|X|=1$, то включение $X \in \mathcal{R}_{\alpha}$ вытекает из леммы 12.12 и предложения 8.18. Пусть $|X|>1$ и $X$ 으 $X_{1} a^{\epsilon}$, где $a^{\epsilon} \in \mathcal{A}^{ \pm 1}$. По индуктивному предположению найдется слово $Y_{1} \in \mathcal{R}_{\alpha}$ такое, что $X_{1} \stackrel{\alpha}{=} Y_{1}$. По индуктивному предположению при $\alpha:=\alpha-1$ найдется слово $Y_{2} \in \mathcal{R}_{\alpha-1}$ такое, что $Y_{2} \stackrel{\alpha=1}{=} Y_{1} a^{\epsilon}$. Так как в силу леммы 12.12 слово $Y_{1}$ не содержит элементарных $(n-q)$-степеней ранга $\alpha$, а в силу леммы 8.16 слово $a^{\epsilon}$ не содержит элементарных 2 -степеней ранга $\alpha$, то, переходя к путям на $\Gamma_{\alpha-1}$ и применяя лемму 11.7, (ii), получаем, что слово $Y_{2}$ не содержит элементарных $(n-q+87)$-степеней ранга $\alpha$. Так как $n-q+87<n-q_{0}-2 p-102$, то по предложению 12.11 найдется слово $Y \in \mathcal{R}_{\alpha-1}$ такое, что $Y_{2} \stackrel{\alpha}{=} Y$ и $Y$ не содержит элементарных $\left(\frac{n}{2}+2 p+142\right)$-степеней ранга $\alpha$. Так как $n-q-36=\frac{n}{2}+2 p+142$, то снова по лемме 12.12 получаем $Y \in \mathcal{R}_{\alpha}$.

Теперь перейдем к доказательству “циклического” аналога предложения 12.13 
(предложение 12.24 ниже). Основная схема рассуждений в этом случае повторяет схему рассуждений из доказательства предложения 12.13, начиная с определения 12.4, за исключением последнего шага: в доказательстве предложения 12.13 мы использовали индукцию по длине слова $X$, строя искомое слово $Y \in \mathcal{R}_{\alpha}$ исходя из уже построенного слова $Y_{1} \in \mathcal{R}_{\alpha}$, где $Y_{1} \stackrel{\alpha}{=} X_{1}$ и слово $X_{1}$ получено из $X$ удалением последней буквы. Этот подход неприменим для построения циклически приведенного слова в ранге $\alpha$, сопряженного данному слову $X$. Вместо этого будет доказана специальная лемма 12.23 .

12.14. ОПРЕДЕЛЕНИЕ. Пусть $X$ - период ранга $\alpha$ и $X \approx E_{1} K_{1} E_{2} K_{2} \ldots E_{r} K_{r}$, где $E_{1}, E_{2}, \ldots, E_{r}$ - элементарные слова ранга $\alpha$. Пусть для каждого слова $E_{i}$ фиксирован некоторый элементарный период $A_{i} \in \mathcal{E}_{\alpha}$, где $E_{i} \in \Pi \Pi е p_{\alpha}\left(A_{i}, 8\right)$. Слово $Y$ будем называть результатом ииклического поворота в слове $X$ набора вхождений

$$
E_{1} K_{1} \ldots K_{i-1} * E_{i} * K_{i} \ldots E_{r} K_{r} \quad(i=1,2, \ldots, r),
$$

если $Y$ - период ранга $\alpha$ и существуют слова $P_{i} \in \Pi е p\left(A_{i}, 2\right), u_{i}, v_{i} \in \mathcal{H}_{\alpha-1}$ и $Q_{i}$ $(i=1,2, \ldots, r)$, удовлетворяющие следующим условиям:

(i) для каждого $i=1,2, \ldots, r$ сушествуют пути $\mathrm{E}_{i}$ и $\mathrm{u}_{i} \mathrm{P}_{i} \mathrm{v}_{i}$ на граф̆е $\Gamma_{\alpha-1}$ с общими начальной и конечной вершинами такие, что метки путей $\mathrm{E}_{i}, \mathrm{u}_{i}, \mathrm{P}_{i}$ и $\mathrm{v}_{i}$ равны словам $E_{i}, u_{i}, P_{i}$ и $v_{i}$ соответственно и $\mathrm{P}_{i}$ - периодическая основа для $\mathrm{E}_{i}$;

(ii) для каждого $i=1,2, \ldots, r$ выполнено $A_{i}^{n} \approx P_{i} Q_{i}$;

(iii) слова $Y$ и $u_{1} Q_{1}^{-1} v_{1} K_{1} u_{2} Q_{2}^{-1} v_{2} K_{2} \ldots u_{r} Q_{r}^{-1} v_{r} K_{r}$ сопряжены в группе $\mathbf{B}_{\alpha-1}$.

Из определения вытекает

12.15. Лемма. Если слово $Y$ есть результат ииклического поворота некоторого набора вхождений в слове $X$, то слова $X$ и $Y$ сопряжсны в групne $\mathbf{B}_{\alpha}$.

Подобно тому, как из индуктивного предположения 12.13 вытекает лемма 12.6, из индуктивного предположения 12.24 вытекает

12.16. Лемма. Пусть $X$ - период ранга $\alpha$ и әлемент $[X]_{\alpha-1}$ әруппьи $\mathbf{B}_{\alpha-1}$ не сопряжен никакому әлементу $\mathbf{g} \in \mathbf{S}_{\alpha-1}^{+}(A)$ при $A \in \bigcup_{\beta \leqslant \alpha-1} \mathcal{E}_{\beta}$. Пусть $X \approx E_{1} K_{1} E_{2} K_{2} \ldots E_{r} K_{r}$, әде $E_{1}, E_{2}, \ldots, E_{r}$ - элементарные слова ранга $\alpha$, и даны элементарные периоды $A_{i} \in \mathcal{E}_{\alpha}$, где $E_{i} \in \Pi$ Пер ${ }_{\alpha}\left(A_{i}, 8\right)$. Предположим, $\ell_{\alpha, A_{i}}\left(E_{i}\right) \leqslant n-2$ для всех $i$. Тогда существует слово $Y$, являющееся результатом поворота в $X$ набора вхождений (12.13).

12.17. Лемма. Пусть $X$ - период ранга $\alpha, X \approx E_{1} K_{1} E_{2} K_{2} \ldots E_{r} K_{r}$, әде $E_{i} \in \Pi$ Пер ${ }_{\alpha}\left(A_{i}, 44\right) \quad\left(A_{i} \in \mathcal{E}_{\alpha}\right)$, и пусть слово $Y$ есть результат ииклического поворота в слове $X$ набора вхождений

$$
\mathrm{U}_{i} \rightleftharpoons E_{1} K_{1} \ldots K_{i-1} * E_{i} * K_{i} \ldots E_{r} K_{r} \quad(i=1,2, \ldots, r)
$$

Пусть вхождение слова $\widetilde{E}_{i}$ в слово $E_{i-1} K_{i-1} E_{i} K_{i} E_{i+1}(i \bmod r)$ естьмаксимальное продолжение вхождения $E_{i-1} K_{i-1} * E_{i} * K_{i} E_{i+1}$ относительно $A_{i}$ $u$ для каждого $i=1,2, \ldots, r$ выполнено $\ell_{\alpha, A_{i}}\left(\widetilde{E}_{i}\right) \leqslant n-2 p-111$. 
ПРЕДСТАВЛЕНИЕ ЭЛЕМЕНТОВ ГРУППЫ В $\alpha$ ПРИВЕДЕННЫМИ СЛОВАМИ 137

Пусть, кроме того, слова $P_{i} \in \Pi$ Пер $\left(A_{i}, 2\right), \quad u_{i}, v_{i} \in \mathcal{H}_{\alpha-1}, \quad Q_{i} \quad(i=$ $1,2, \ldots, r)$ удовлетворяют условиям (i)-(iii) определения 12.14.

Тогда существуют әлементарные слова $F_{1}, F_{2}, \ldots, F_{r}$ ранга а и слова $w_{i}, z_{i} \in \mathcal{H}_{\alpha-1}, \quad S_{i} \in \Pi e p\left(A_{i}^{-1}, 2\right), \quad U_{i}, V_{i}$ u $L_{i} \quad(i=0,1, \ldots, r)$ maкuе, что $F_{i} \in \Pi \Pi е p_{\alpha}\left(A_{i}^{-1}, 8\right)$ и верны следующие утверждения:

(i) $Y \approx F_{1} L_{1} F_{2} L_{2} \ldots F_{r} L_{r}$;

(ii) для каждого $i=1,2, \ldots, r$ существуют пути $\mathrm{F}_{i} u \mathrm{w}_{i} \mathrm{~S}_{i} \mathrm{z}_{i}$ на графе $\Gamma_{\alpha-1}$ с общими начальной и конечной вершинами такие, что метки путей $\mathrm{F}_{i}, w_{i}$, $\mathrm{S}_{i}$ и $\mathrm{z}_{i}$ равны словам $F_{i}, v_{i}, S_{i}$ и $z_{i}$ соответственно и $\mathrm{S}_{i}$ - периодическая основа для $\mathrm{F}_{i}$;

(iii) $Q_{i}$ 으 $U_{i} S_{i}^{-1} V_{i} \quad(i=1,2, \ldots, r)$;

(iv) имеют место соотношения

$$
L_{i} \stackrel{\alpha=1}{=} z_{i}^{-1} U_{i}^{-1} v_{i} K_{i} u_{i+1} V_{i+1}^{-1} w_{i+1}^{-1} \quad(i=1,2, \ldots, r, i \bmod r) ;
$$

(v) $\ell_{\alpha, A_{i}}\left(\widetilde{E}_{i}\right)+\ell_{\alpha, A_{i}^{-1}}\left(F_{i}\right) \geqslant n-2 p-103 \quad(i=1,2, \ldots, r)$.

ДоКАЗАТЕЛЬСТВо получается модификацией доказательства леммы 12.7. Исходя из условия 12.14 , (iii), рассмотрим кольцевую $A$-диаграмму $\Delta$ ранга $\alpha-1$ с граничными циклами $\mathrm{u}_{1} \mathrm{Q}_{1}^{-1} \mathrm{v}_{1} \mathrm{~K}_{1} \mathrm{u}_{2} \mathrm{Q}_{2}^{-1} \mathrm{v}_{2} \mathrm{~K}_{2} \ldots \mathrm{u}_{r} \mathrm{Q}_{r}^{-1} \mathrm{v}_{r} \mathrm{~K}_{r}$ и $\mathrm{Y}^{-1}$, где пути обозначены теми же буквами, что и их метки, $X_{\alpha-1}(\Delta)=\left\{\mathrm{K}_{1}, \mathrm{~K}_{2}, \ldots\right.$ $\left.\ldots, \mathrm{K}_{r}, \mathrm{Q}_{1}^{-1}, \mathrm{Q}_{2}^{-1}, \ldots \mathrm{Q}_{r}^{-1}, \mathrm{Y}^{-1}\right\}$ и $\mathcal{y}_{\alpha-1}(\Delta)=\left\{\mathrm{u}_{1}, \mathrm{u}_{2}, \ldots, \mathrm{u}_{r}, \mathrm{v}_{1}, \mathrm{v}_{2}, \ldots, \mathrm{v}_{r}\right\}$. Пусть $\mathcal{S}$ - карта примыканий ранга $\alpha-1$ на $\Delta$.

Основное отличие от доказательства леммы 12.7 состоит в том, что теперь утверждение (12.3) неверно. Пусть $\Pi \in \mathcal{S}$ - поддиаграмма примыкания участка гранищы $\mathrm{Q}_{i}^{-1}$ к участку гранищы $\mathrm{R} \in X_{\alpha-1}(\Delta)$, где $\mathrm{R}=\mathrm{Q}_{j}^{-1}$ или $\mathrm{R}=\mathrm{K}_{j}^{-1}$ для некоторого $j$. Назовем П правильной, если имеет место один из четырех случаев:

1) $\mathrm{R}=\mathrm{K}_{i}$ и $\mathrm{v}_{i}$ входит в граничный цикл $\Pi$;

2) $\mathrm{R}=\mathrm{K}_{j}, j \equiv i-1(\bmod r), \mathbf{u}_{i}$ входит в граничный цикл $\Pi$;

3) $\mathrm{R}=\mathrm{Q}_{j}^{-1}, j \equiv i+1(\bmod r)$, связная компонента диаграммы $\Delta-\Pi$, в граничньй цикл которой входит $\mathrm{v}_{i}$, является дисковой диаграммой;

4) $\mathrm{R}=\mathrm{Q}_{j}^{-1}, j \equiv i-1(\bmod r)$, связная компонента диаграммы $\Delta-\Pi$, в граничный цикл которой входит $\mathbf{u}_{i}$, является дисковой диаграммой.

Из доказательства утверждений (12.5) и (12.6) легко видеть, что в нашей ситуации эти утверждения верны, если вместо $\mathcal{S}$ рассмотреть множество всех правильных поддиаграмм примыкания $\Pi \in \mathcal{S}$. Утверждение (12.7) модифицируется следуюшим образом:

Ни для каких $i, j$ не существует неправильных поддиаграммм примыкания $\Pi \in \mathcal{S}$ участка границы $\mathrm{Q}_{i}^{-1} \kappa$ участкам границы $\mathrm{K}_{j} u \mathrm{Q}_{j}^{-1}$.

Его доказательство почти дословно повторяет доказательство (12.7). Небольшая модификация связана с тем, что индексы у путей $\mathrm{Q}_{i}^{-1}, \mathrm{~K}_{i}$ следует считать упорядоченными не линейно, а циклически. Формально, вместо (12.8) нужно занумеровать 
пути $\mathrm{Q}_{i}^{-1}$ и $\mathrm{K}_{i}$ с помощью $\mathrm{Z}_{i}$ таким образом, чтобы в граничный цикл поддиаграммы $\Theta$ последовательно входили пути $Z_{i_{1}}^{\prime}, Z_{i_{1}+1}, Z_{i_{1}+2}, \ldots, Z_{j_{1}-1}, Z_{j_{1}}^{\prime}$, где $Z_{i_{1}}^{\prime}$ и $Z_{j_{1}}^{\prime}-$ некоторые конец и начало путей $Z_{i_{1}}$ и $Z_{j_{1}}$ соответственно (при этом если $\Pi_{0}$ - поддиаграмма примыкания $\mathrm{Q}_{i_{0}}^{-1}$ к $\mathrm{Q}_{i_{0}}^{-1}$, то $\mathrm{Z}_{i_{1}}=\mathrm{Z}_{j_{1}}=\mathrm{Q}_{i_{0}}^{-1}$, но индексы $i_{1}$ и $j_{1}$ будут различаться на $r$ ).

Допустим далее, что ни для какого $i$ не существует поддиаграммы примыкания $\mathrm{Q}_{i}^{-1} \mathrm{\kappa} \mathrm{Y}^{-1}$. Пусть $W_{i}$ - множество всех переходных участков диаграммы $\Delta$, содержашихся в $Q_{i}^{-1}$. Тогда из утверждений (12.5), (12.6) в новой формулировке и (12.14) следует $\sum_{\mathrm{S} \in W_{i}}|\mathcal{L}(\mathrm{S})|>74\left|A_{i}\right|$, откуда по предложению 8.22 , (ii) получаем $\sum_{S \in W_{i}}\left|\mathcal{W}_{\alpha-1}(\mathcal{L}(\mathrm{S}))\right|>\frac{64}{13}>2 \eta$. С другой стороны, по предложению 10.4 $\sum_{i} \sum_{\mathrm{S} \in W_{i}}\left|\mathcal{W}_{\alpha-1}(\mathcal{L}(\mathrm{S}))\right| \leqslant 2 \eta$. Противоречие. Таким образом, для некоторого $i$ существует поддиаграмма примыкания $\Pi \in \mathcal{S}$ участков границы $\mathrm{Q}_{i}$ и $\mathrm{Y}^{-1}$. C точностью до нумерации, можно считать $i=1$. Повторяя дословно рассуждения из доказательства леммы 12.7 , получаем, что для каждого $i$ найдется поддиаграмма примыкания $\Pi \in \mathcal{S}$ участков границы $\mathrm{Q}_{i}$ и $\mathrm{Y}^{-1}$. Далее можно повторить заключительный шаг доказательства 12.7, при этом, когда рассматривается отображение $\omega$ множества путей на $\Delta$ во множество путей на графе $\Gamma_{\alpha-1}$, вместо $\Delta$ нужно взять дисковую диаграмму, полученную из $\Delta$ разрезом вдоль некоторого простого пути, соединяюшего две вершины, лежашие на разных граничных циклах диаграммы $\Delta$. Для этой цели можно взять, например, путь $\mathrm{s}_{1}$, входящий в граничный цикл поддиаграммы примыкания П 1 участков границы $Q_{1}^{-1}$ и $Y^{-1}$.

12.18. ОПРЕДЕлЕНИЕ. Если выполнены посылка и заключение предложения 12.17 , то набор вхождений $\mathrm{V}_{i} \rightleftharpoons F_{1} L_{1} \ldots L_{i-1} * F_{i} * L_{i} \ldots F_{r} L_{r}$ будем называть образами вхождений $\mathrm{U}_{i}$ в ииклическом повороте $X \rightarrow Y$.

12.19. Лемма. Пусть слово $Y$ есть результат ииклического поворота в слове $Х$ набора вхождений

$$
\mathrm{U}_{i}=E_{1} K_{1} E_{2} K_{2} \ldots K_{i-1} * E_{i} * K_{i} \ldots E_{r} K_{r} \quad(i=1,2, \ldots, r) .
$$

Пусть

$$
\mathrm{V}_{i}=F_{1} L_{1} F_{2} L_{2} \ldots L_{i-1} * F_{i} * L_{i} \ldots F_{r} L_{r} \quad(i=1,2, \ldots, r)
$$

- образы вхождений $\mathrm{U}_{i}$ в чиклическом повороте $X \rightarrow Y$. Предположим, $\ell_{\alpha}\left(E_{i}\right) \geqslant q_{0}, \quad \ell_{\alpha}\left(F_{i}\right) \geqslant q_{0}$ при всех $i$. Пусть $\mathrm{W}-$ вхождение әлементарной $(h+8)$-степени $G$ ранга $\alpha$ в слово $F_{i} L_{i} F_{i+1}(i \bmod r)$ mакое, что $\neg$ ЭСогл $\Omega_{\alpha}\left(* F_{i} * L_{i} F_{i+1}, W\right)$ u $\neg$ ЭСогл $\alpha\left(F_{i} L_{i} * F_{i+1} *, W\right)$. Тогда найдется вхождение $W^{\prime}$ әлементарного слова $H$ ранга $\alpha$ в слово $E_{i} K_{i} E_{i+1}$ такое, что

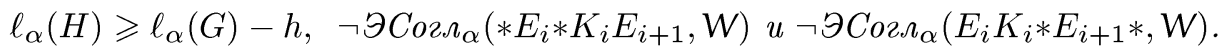

ДоКАЗАТЕЛЬСТво аналогично доказательству леммы 12.9.

12.20. ЛЕмма. Пусть слово $Y$ есть результат ииклического поворота в слове X набора вхождений

$$
\mathrm{U}_{i}=E_{1} K_{1} E_{2} K_{2} \ldots K_{i-1} * E_{i} * K_{i} \ldots E_{r} K_{r} \quad(i=1,2, \ldots, r),
$$


ПРЕДСТАВЛЕНИЕ ЭЛЕМЕНТОВ ГРУППЫ $\mathbf{B}_{\alpha}$ ПРИВЕДЕННЫМИ СЛОВАМИ 139

где $E_{i}$ - әлементарное слово ранга $\alpha$, порохсденное периодом $A_{i} \in \mathcal{E}_{\alpha}, u$ nycmb

$$
\mathrm{V}_{i}=F_{1} L_{1} F_{2} L_{2} \ldots L_{i-1} * F_{i} * L_{i} \ldots F_{r} L_{r} \quad(i=1,2, \ldots, r)
$$

- образы вхождений $\mathrm{U}_{i}$ в ииклическом повороте $X \rightarrow Y$.

Предположим, $\ell_{\alpha}\left(E_{i}\right) \geqslant p+34, \quad \ell_{\alpha}\left(F_{i}\right) \geqslant p+34$ при всех $i u$ $\neg$ ЭСог $\Omega_{\alpha-1}\left(* E_{i} * K_{i} E_{i+1}, E_{i} K_{i} * E_{i+1} *\right)(i=1,2, \ldots, r, i \bmod r)$. Пусть вхождение слова $\widetilde{E}_{i}$ в слово $E_{i-1} K_{i-1} E_{i} K_{i} E_{i+1}$ и вхохдение слова $\widetilde{F}_{i}$ в слово $F_{i-1} L_{i-1} F_{i} L_{i} F_{i+1}$ - максимальные продолжсения вхождений $E_{i-1} K_{i-1} * E_{i} *$ $K_{i} E_{i+1}$ и $F_{i-1} L_{i-1} * F_{i} * L_{i} F_{i+1}$ относительно периодов $A_{i}$ и $A_{i}^{-1}$ coответственно.

Тогда имеет место неравенство

$$
\ell_{\alpha, A_{i}}\left(\widetilde{E}_{i}\right)+\ell_{\alpha, A_{i}^{-1}}\left(\widetilde{F}_{i}\right)<n+2 p+120
$$

ДокАЗАТЕЛЬСТВо аналогично доказательству леммы 12.10.

12.21. Лемма. Пусть $X$ - период ранга $\alpha$ и элемент $[X]_{\alpha}$ не сопряжен никакому әлементу $\mathbf{g} \in \mathbf{S}_{\alpha}^{+}(A)$ при $A \in \mathcal{E}_{\alpha}$. Тогда если в некоторое слово $P \in \Pi$ Пр $(X)$ входит слово $E \in \Pi \Pi е p_{\alpha}(B, t) \quad\left(B \in \mathcal{E}_{\alpha}\right)$, где $t \geqslant 15$, то для некоторого начала $E^{\prime}$ слова $E$ выполнено $E^{\prime} \in \Pi$ пер $\alpha(B, t-9) u\left|E^{\prime}\right| \leqslant|X| . B$ частности, некоторый ииклический сдвиг слова $X$ содержит әлементарную $(t-9)$-степень ранга $\alpha$.

ДокаЗАтельство. Можно считать $|P| \geqslant 2|X|$. Рассмотрим произвольный путь $\mathrm{P}$ на графе $\Gamma_{\alpha-1} \mathrm{c} \mathcal{L}(\mathrm{P})$ 으 $P$. По условию $\mathrm{P}$ содержит подпуть $\mathrm{E} \in \Pi_{\text {Пер }}(B, t)$, где $B \in \mathcal{E}_{\alpha}$. Допустим, ПСог $\Omega_{\alpha}\left(\mathrm{E}, \mathbf{s}_{X, \mathrm{P}} \mathrm{E}\right)$. Пусть $\mathrm{Q} \in$ Пер $\left(\Gamma_{\alpha-1}, A, 2\right)$ - периодическая основа для $\mathrm{E}$. В силу следствия 8.6, (i) и предложения 7.3 имеем $\tilde{\mathbf{g}} \rightleftharpoons(\iota(\mathrm{Q}))^{-1} \mathbf{s}_{X, \mathrm{P}} \iota(\mathrm{Q}) \in \mathbf{N}_{\alpha-1}^{+}\left(B^{\prime}\right)$, где $B^{\prime}$ - некоторый циклический сдвиг слова $B$. Так как элемент $\mathbf{s}_{X, \mathrm{P}}$ сопряжен элементу $[X]_{\alpha-1}$, то отсюда следует сопряженность элементов $[X]_{\alpha-1}$ и $\mathbf{g}$. Но тогда сопряжены и их гомоморфные образы $[X]_{\alpha}$ и $\pi_{\alpha-1, \alpha}(\tilde{\mathbf{g}}) \in \mathbf{S}_{\alpha}^{+}\left(B^{\prime}\right)$ в группе $\mathbf{B}_{\alpha}$, что невозможно по условию. Следовательно, $\neg \Pi \operatorname{Coz}_{\alpha}\left(\mathrm{E}, \mathbf{s}_{X, P} \mathrm{E}\right)$. Тогда в силу предложений 8.9 и 8.14, (i) найдется начало $\mathrm{E}^{\prime}$ пути $\mathrm{E}$ такое, что $\mathrm{E}^{\prime} \in$ ППер $\alpha(A, t-9)$ и $\mathrm{E}^{\prime}$ не имеет общих ребер c $\mathbf{s}_{A, \mathrm{P}} \mathrm{E}$. В этом случае, очевидно, $\left|\mathrm{E}^{\prime}\right| \leqslant|X|$ и в качестве $E^{\prime}$ можно взять $\mathcal{L}\left(\mathrm{E}^{\prime}\right)$.

12.22. ЛеммА. Пусть $X$ - период ранга $\alpha$, элемент $[X]_{\alpha}$ группь $\mathbf{B}_{\alpha}$ не сопряжен никакому әлементу $\mathbf{g} \in \mathbf{S}_{\alpha}^{+}(A)$ при $A \in \bigcup_{\beta \leqslant \alpha} \mathcal{E}_{\beta}$ и никакое слово $P \in \Pi е р(X)$ не содержит әлементарных $\left(n-q_{0}-2 p-102\right)$-степеней ранга $\alpha$. Тогда существует период $Y$ ранга $\alpha$, сопряэсенный в группе $\mathbf{B}_{\alpha}$ периоду $X$, такой, что никакое слово $Q \in$ Пер $(Y)$ не содержит әлементарных $\left(\frac{n}{2}+\right.$ $2 p+142)$-степеней ранга $\alpha$. 
ДокАЗАТЕльство. Схема рассуждений такая же, как и в доказательстве леммы 12.11. Без ограничения обшности можно считать, что при некотором $t>0$ в слово $X^{t}$ входит слово $E \in$ ППер $\alpha\left(A_{1}, \frac{n}{2}+14\right)\left(A_{1} \in \mathcal{E}_{\alpha}\right)$. Подобно доказательству леммы 12.11 , покажем, что существует набор вхождений

$$
\mathrm{U}_{i}=E_{1} K_{1} \ldots K_{i-1} * E_{i} * K_{i} \ldots E_{r} K_{r} \quad(i=1,2, \ldots, r),
$$

в некоторый циклический сдвиг слова $X$ элементарных слов $E_{i}$ ранга $\alpha$, удовлетворяющий следующим условиям:

(i) если вхождение слова $\widetilde{E}_{i}$ в слово $E_{i-1} K_{i-1} E_{i} K_{i} E_{i+1} \quad(i \bmod r)$ есть максимальное продолжение вхождения $E_{i-1} K_{i-1} * E_{i} * K_{i} E_{i+1}$ относительно $A_{i}$, то $\frac{n}{2}+14 \leqslant \ell_{\alpha, A_{i}}\left(\widetilde{E}_{i}\right) \leqslant n-q_{0}-2 p-103 ;$

(ii) $\neg$ ЭCo२ $\Omega_{\alpha}\left(* E_{i} * K_{i} E_{i+1}, E_{i} K_{i} * E_{i+1} *\right)(i=1,2, \ldots, r, i \bmod r)$;

(iii) если $\mathrm{U}-$ вхождение в слово $E_{i} K_{i} E_{i+1} \quad(i \bmod r)$ элементарной $\left(\frac{n}{2}+14\right)$-степени ранга $\alpha$, то либо $Э С о г \Omega_{\alpha}\left(* E_{i} * K_{i} E_{i+1}, \mathrm{U}\right)$, либо ЭСогл $\Omega_{\alpha}\left(E_{i} K_{i} * E_{i+1} *, \mathrm{U}\right)$.

В силу леммы 12.21 имеем $X \approx E_{1} K_{1}$, где $E_{1} \in \Pi \Pi е p_{\alpha}\left(A_{1}, \frac{n}{2}+5\right)-$ начало слова $E$. Проверим, что выполнены условия (i) и (ii) при $r:=1$ и $\mathrm{U}_{1}:=* E_{1} * K_{1}$. Можно считать, что $X^{t}$ ㅇ $U E_{1} K_{1} E_{1} V$, где $E$ есть начало слова $E_{1} K_{1} E_{1} V$. Если бы выполнялось ЭСогл $\Omega_{\alpha}\left(* E_{1} * K_{1} E_{1}, E_{1} K_{1} * E_{1} *\right)$, то, аналогично тому, как в доказательстве леммы 12.21 , переходя к путям на графе $\Gamma_{\alpha-1}$, мы получили бы, что элемент $[X]_{\alpha}$ сопряжен элементу $\mathbf{g} \in \mathbf{S}_{\alpha}^{+}\left(A_{1}^{\prime}\right)$, где $A_{1}^{\prime}-$ циклический сдвиг $A_{1}$. Но это невозможно по условию леммы. Следовательно, $\neg$ ЭСогл $\Omega_{\alpha}\left(* E_{1} * K_{1} E_{1}, E_{1} K_{1} * E_{1} *\right)$, что доказывает (ii). В силу леммы 12.3 , (ii) вхождение $W$ слова $E$ в $X^{t}$, началом которого является вхождение $* E_{1} * K_{1} E_{1}$, не может содержать вхождение $E_{1} K_{1} * E_{1} *$. Следовательно, W содержится в $U * E_{1} K_{1} E_{1} * V$, а это означает, что имеет место нижняя оценка $\frac{n}{2}+14 \leqslant \ell_{\alpha, A_{1}}\left(\widetilde{E}_{1}\right)$ в (i). Так как верхняя оценка $\ell_{\alpha, A_{i}}\left(\widetilde{E}_{i}\right) \leqslant n-q_{0}-2 p-103$ имеет место по условию леммы, то тем самым мы проверили выполнение условий (i) и (ii) при $r=1$.

Если теперь для некоторого $r \geqslant 1$ имеется набор вхождений (12.15), удовлетворяющий условиям (i), (ii), но не условию (iii), то, используя лемму 12.3, (ii), (iii), легко видеть, что набор вхождений (12.15), удовлетворяюший условиям (i) и (ii), сушествует при $r:=r+1$. Отсюда по индукции следует, что существует набор вхождений (12.15), удовлетворяюший всем условиям (i)-(iii).

По лемме 12.16 сушествует слово $Y$, являюшееся результатом циклического поворота набора вхождений (12.15) в слове $X$. Проверим, что $Y$ - искомое слово. По лемме 12.15 слова $X$ и $Y$ сопряжены в $\mathbf{B}_{\alpha}$. Допустим, при некотором $t>0$ слово $Y^{t}$ содержит элементарную $\left(\frac{n}{2}+2 p+142\right)$-степень $G$ ранга $\alpha$. Пусть

$$
\mathrm{V}_{i}=F_{1} L_{1} \ldots L_{i-1} * F_{i} * L_{i} \ldots F_{r} L_{r} \quad(i=1,2, \ldots, r)
$$

- образы вхождений $\mathrm{U}_{i}$ в циклическом повороте $X \rightarrow Y$. Заметим, что в силу (i) и $12.17,(\mathrm{v})$ имеем $\ell_{\alpha, A_{i}^{-1}}\left(F_{i}\right) \geqslant q_{0}$ при всех $i$. Используя этот факт, а также условие (ii) и лемму 12.3 , (ii), легко видеть, что либо какое-нибудь из слов $F_{i} L_{i} F_{i+1}$ 
$(i \bmod r)$ содержит вхождение $\mathrm{W}$ слова $G$ такое, что $\neg$ ЭСогл $\Omega_{\alpha}\left(* F_{i} * L_{i} F_{i+1}, W\right)$ и $\neg$ ЭСогл $\alpha\left(F_{i} L_{i} * F_{i+1} *, W\right)$, либо какое-нибудь из слов $F_{i-1} L_{i-1} F_{i} L_{i} F_{i+1}(i \mathrm{mod}$ $r)$ содержит вхождение $W$ слова $G$ такое, что $Э С о љ \Omega_{\alpha}\left(F_{i-1} L_{i-1} * F_{i} * L_{i} F_{i+1}, W\right)$. Тогда, аналогично доказательству леммы 12.11 , в первом случае мы приходим к противоречию с условием (iii) и предложением 12.19 , а во втором - используя предложения 8.35 и 8.11, приходим к противоречию с леммой 12.20 и условиями (i), (ii).

12.23. Лемма. Если $X$ - период ранга $\alpha, X \in \mathcal{R}_{\alpha}$ и әлемент $[X]_{\alpha}$ группы $\mathbf{B}_{\alpha}$ не сопряжен никакому әлементу $\mathbf{g} \in \mathbf{S}_{\alpha}^{+}(B)$ при $B \in \bigcup_{\beta \leqslant \alpha} \mathcal{E}_{\beta}$, то существует период $Y$ ранга $\alpha$ такой, что слова $X$ и $Y$ сопряжсны в группе $\mathbf{B}_{\alpha}$ и никакое слово $Y^{t}$ не содержит әлементарных $\left(\frac{n}{2}+4 p+321\right)$-степеней ранга $\alpha$.

ДоКАЗАТЕЛЬСТВо. В силу леммы 12.21 вместо условия, что никакое слово $Y^{t}$ не содержит элементарных $\left(\frac{n}{2}+4 p+321\right)$-степеней ранга $\alpha$, достаточно проверить, что никакой циклический сдвиг слова $Y$ не содержит элементарных $\left(\frac{n}{2}+\right.$ $4 p+312)$-степеней ранга $\alpha$.

Выберем слова $X_{1}, H, X_{2}$ и $w \in \mathcal{H}_{\alpha}$ таким образом, чтобы выполнялись равенства

$$
X \text { 으 } X_{1} H X_{2} \text { и } X_{2} X_{1} \stackrel{\alpha}{=} w
$$

и при этом длина слова $H$ была минимально возможной (так как равенства (12.16) выполнены при $X_{1}$ 으 $X_{2}$ 드 $w$ 으 1 и $H$ 드 $X$, то такие слова $X_{1}, H, X_{2}$ и $w$ сушествуют). Пусть $w$ 으 $u P v$, где $u, v \in \mathcal{H}_{\alpha-1}$ и $P$ - подслово слова $A^{n}$ при $A \in \mathcal{E}_{\alpha}$. Заменяя при необходимости $P$ на слово $P^{\prime}$, определяемое равенством $P P^{\prime-1} \approx A^{n}$, можно считать $|P| \leqslant \frac{n}{2}|A|$. По индуктивному предположению 12.24 сушествует период $Y$ ранга $\alpha$ такой, что слова $H w$ и $Y$ сопряжены в группе $\mathbf{B}_{\alpha-1}$. Так как из равенств (12.16) следует $X \stackrel{\alpha}{=} X_{1} H w X_{1}^{-1}$, то $X$ и $Y$ сопряжены в $\mathbf{B}_{\alpha}$. Докажем, что слово $Y$ является искомым, т.е. что никакой циклический сдвиг $Y$ не содержит элементарных $\left(\frac{n}{2}+4 p+312\right)$-степеней ранга $\alpha$.

Допустим, в некоторый циклический сдвиг слова $Y$ входит слово $E \in \Pi$ ПГер $\alpha$ ( $B$, $\left.\frac{n}{2}+4 p+312\right)$, где $B \in \mathcal{E}_{\alpha}$.

Рассмотрим кольцевую $A$-диаграмму $\Delta$ ранга $\alpha-1$ с граничными циклами $\mathrm{HuPv}$ и $\mathrm{Y}^{-1}$, где метки путей $\mathrm{H}, \mathrm{u}, \mathrm{P}, \mathrm{v}$ и $\mathrm{Y}$ равны словам $H, u, P, v$ и $Y$ соответственно,

$$
X_{\alpha-1}(\Delta)=\left\{\mathrm{H}, \mathrm{P}, \mathrm{Y}^{-1}\right\} \text { и } y_{\alpha-1}(\Delta)=\{\mathrm{u}, \mathrm{v}\} .
$$

По предложению 9.19 можно считать, что на $\Delta$ задана карта примыканий $\mathcal{S}$ ранга $\alpha-1$. Заметим, что в силу леммы 9.23 , (iii) не существует поддиаграмм примыкания $\Pi \in \mathcal{S}$ граничного цикла $\mathrm{Y}^{-1}$ к самому себе и существует не более одной поддиаграммы примыкания $\mathrm{Y}^{-1}$ к каждому из участков границы $\mathrm{H}$ и $\mathrm{P}$. Ввиду этого рассмотрим четыре случая.

Случай 1: $\mathcal{S}$ не содержит поддиаграмм примыкания граничного цикла $\mathrm{Y}^{-1} \mathrm{~K}$ участкам границы $\mathrm{H}$ и $\mathrm{P}$. Тогда $\mathrm{Y}^{-1}$ является переходным участком диаграммы $\Delta$ относительно $\mathcal{S}$. По предложению 10.4 (см. также 10.3) имеем $\mathcal{W}_{\alpha-1}^{\circ}\left(\mathcal{L}\left(\mathrm{Y}^{-1}\right)\right) \leqslant$ $2 \eta$. С другой стороны, из наличия $E$ в силу предложения 8.14 , (ii) легко следует, 
что любой циклический сдвиг $Z$ слова $\mathcal{L}\left(\mathrm{Y}^{-1}\right)$ содержит в качестве подслова элементарную $\left(\frac{n}{4}+2 p+155\right)$-степень ранга $\alpha$. Тогда по предложению 8.22 , (i) имеем $\mathcal{W}_{\alpha-1}(Z)>2 \eta$ и, следовательно, $\mathcal{W}_{\alpha-1}^{\circ}\left(\mathcal{L}\left(\mathrm{Y}^{-1}\right)\right)>2 \eta$. Противоречие.

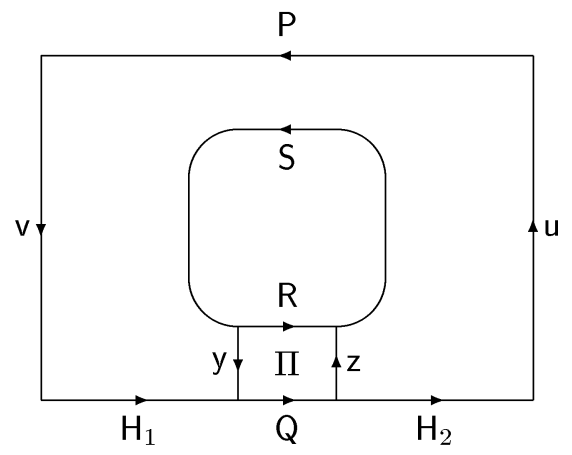

Рис. 53

Случай 2: $\mathcal{S}$ содержит поддиаграмму примыкания П граничного цикла $\mathrm{Y}^{-1} \mathrm{~K} \mathrm{H}$ и не содержит поддиаграммы примыкания $\mathrm{Y}^{-1}$ к $\mathrm{P}$. Пусть $\delta \Pi \approx \mathrm{R}^{-1} \mathrm{yQz}, \mathrm{Y} \approx \mathrm{RS}$ и $\mathrm{H}=\mathrm{H}_{1} \mathrm{QH}_{2}$, где $\mathrm{R}^{-1}$ и $\mathrm{Q}$ - участки примыкания (см. рис. 53). В силу нашего допушения $E$ входит в одно из слов $\mathcal{L}(\mathrm{R}) \mathcal{L}(\mathrm{S}) \mathcal{L}(\mathrm{R})$ или $\mathcal{L}(\mathrm{S}) \mathcal{L}(\mathrm{R}) \mathcal{L}(\mathrm{S})$. В силу предложения 10.4 имеем $\mathcal{W}_{\alpha-1}(\mathcal{L}(\mathrm{S})) \leqslant 2 \eta$. Тогда в силу предложения 8.22 , (i) слово $\mathcal{L}(\mathrm{S})$ не содержит элементарных 12 -степеней ранга $\alpha$. Так как слова $\mathcal{L}(\mathrm{R}) \in \mathcal{R}_{\alpha-1}$ и $\mathcal{L}(\mathrm{Q}) \in \mathcal{R}_{\alpha}$ близки в ранге $\alpha-1$, то из леммы 12.12 и предложения 8.27 следует, что $\mathcal{L}(\mathrm{R})$ не содержит элементарных $(n-q+34)$-степеней ранга $\alpha$. Тогда, используя предложение 8.14 , (ii), легко видеть, что $E$ может содержаться только в $\mathcal{L}(\mathrm{R}) \mathcal{L}(\mathrm{S}) \mathcal{L}(\mathrm{R})$, причем, $\mathcal{L}(\mathrm{R})$ 으 $L U K$, где $E$ 드 $K \mathcal{L}(\mathrm{S}) L$ и $\ell_{\alpha, B}(K), \ell_{\alpha, B}(L)>42$.

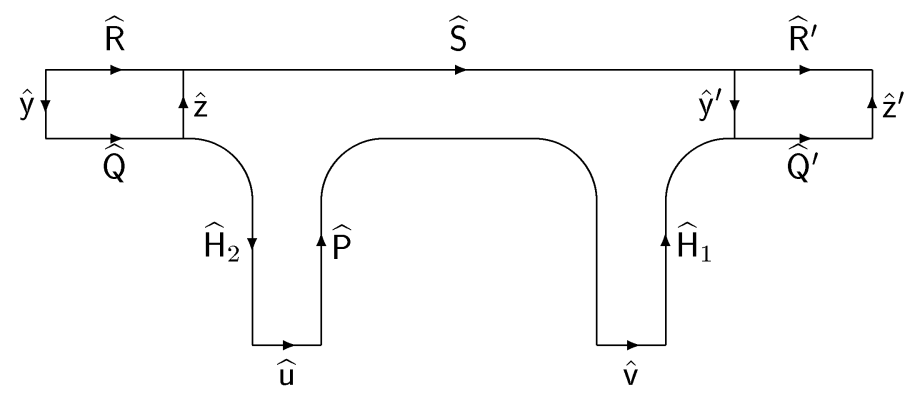

Рис. 54

Рассмотрим пути на графе $\Gamma_{\alpha-1}$, расположенные, как на рис. 54, метки которых равны меткам соответствующих путей на диаграмме $\Delta$. Пусть $\widehat{\mathrm{R}}=\mathrm{LUK}$, где $\mathcal{L}(\mathrm{L})$ 으 $L, \mathcal{L}(\mathrm{U})$ 드 $U$ и $\mathcal{L}(\mathrm{K})$ 으 $K$. Обозначим через $\mathbf{h}$ элемент группы $\mathbf{B}_{\alpha-1}$, для которого $\widehat{R}^{\prime}=\mathbf{h} \widehat{R}$. Очевидно, $\widehat{Q}^{\prime}=\mathbf{h} \widehat{\mathbf{Q}}$. Так как $\ell_{\alpha, B}(\mathrm{~K}), \ell_{\alpha, B}(\mathrm{~L}) \geqslant 42$, то 
для некоторых подпутей $\overline{\mathrm{K}}$ и $\overline{\mathrm{L}}$ путей $\mathrm{K}$ и $\mathrm{L}$ выполнено $\overline{\mathrm{K}}, \overline{\mathrm{L}} \in$ ППер $\alpha(B, 42)$. Используя лемму 8.29, (i), найдем подпути $\mathrm{M}, \mathrm{N} \in$ ППер $\alpha(B, 8)$ пути $\widehat{\mathrm{Q}}$ такие, что $\Pi C о \Omega_{\alpha}(\overline{\mathrm{L}}, \mathrm{M}), \Pi \operatorname{Coг}_{\alpha}(\overline{\mathrm{K}}, \mathrm{N})$ и $\mathrm{M} \ll_{\widehat{Q}} \mathrm{~N}$. Так как $\mathrm{K} \widehat{\mathrm{S}} \mathrm{L}^{\prime} \in \Pi$ Пер $\alpha(B)$, где $\mathrm{L}^{\prime} \rightleftharpoons \mathbf{h L}$, то по предложению 8.9 имеем ПСогл $\alpha(\overline{\mathrm{K}}, \mathbf{h} \overline{\mathrm{L}})$, и, следовательно, ПСогл $\alpha(\mathrm{N}, \mathbf{h M})$. Тогда, используя предложение 8.10 , (i) и лемму 8.2 , (ii), легко видеть, что вершины $\iota(\mathrm{N})$ и $\tau(\mathbf{h M})$ соединены путем $\mathrm{w}^{*}$ с $\mathcal{L}\left(\mathrm{w}^{*}\right)$ 으 $u^{*} P^{*} v^{*}$, где $u^{*}, v^{*} \in \mathcal{H}_{\alpha-1}$ и $P^{*} \in \Pi е p(B) \cup \Pi е p\left(B^{-1}\right)$. Заметим, что $\mathbf{h M}$ содержится в $\widehat{\mathbf{Q}}^{\prime}=\mathbf{h} \widehat{\mathbf{Q}}$. Пусть $\mathrm{T}_{1}-$ начало пути $\widehat{\mathrm{H}}_{1} \widehat{\mathrm{Q}}^{\prime}$, оканчиваюшееся на $\mathbf{h M}$, и $\mathrm{T}_{2}-$ конец пути $\widehat{\mathrm{Q}} \widehat{\mathrm{H}}_{2}$, начинаюшийся c N (см. рис. 55). Так как пути $w^{*}$ и $\mathrm{T}_{2} \hat{\mathrm{u}} \widehat{\mathrm{P}} \hat{\mathrm{v}} \mathrm{T}_{1}$ имеют обшие начальную и конечную вершины, то, обозначив $T_{i} \rightleftharpoons \mathcal{L}\left(\mathbf{T}_{i}\right)(i=1,2)$, получим

$$
u^{*} P^{*} v^{*} \stackrel{\alpha=1}{=} T_{2} u P v T_{1} .
$$

С учетом равенств $w$ 으 $u P v$ и (12.16) отсюда следует

$$
u^{*} P^{*} v^{*} \stackrel{\alpha}{=} T_{2} X_{2} X_{1} T_{1} \text {. }
$$

Так как $\mathrm{M} \ll_{\widehat{Q}} \mathrm{~N}$, то $H$ 으 $T_{1} H^{*} T_{2}$ для некоторого $H^{*}$, и так как $B^{n} \stackrel{\alpha}{=} 1$, то $P^{*} \stackrel{\underline{\alpha}}{=}$ $P^{\prime}$ для некоторого слова $P^{\prime} \in \Pi е p(B) \cup \Pi е p\left(B^{-1}\right)$ с $\left|P^{\prime}\right|<n|B|$. Мы получили, что равенства (12.16) выполнены при $X_{1}:=X_{1} T_{1}, X_{2}:=T_{2} X_{2}, H:=H^{*}$ и $w:=u^{*} P^{\prime} v^{*}$. Так как слова $T_{1}$ и $T_{2}$ содержат элементарные слова $\mathcal{L}(\mathrm{M})$ и $\mathcal{L}(\mathrm{N})$ ранга $\alpha$, то они непусты, следовательно, $\left|H^{*}\right|<|H|$. Получили противоречие с выбором слов $X_{1}, X_{2}, H$ и $w$. Случай 2 рассмотрен.

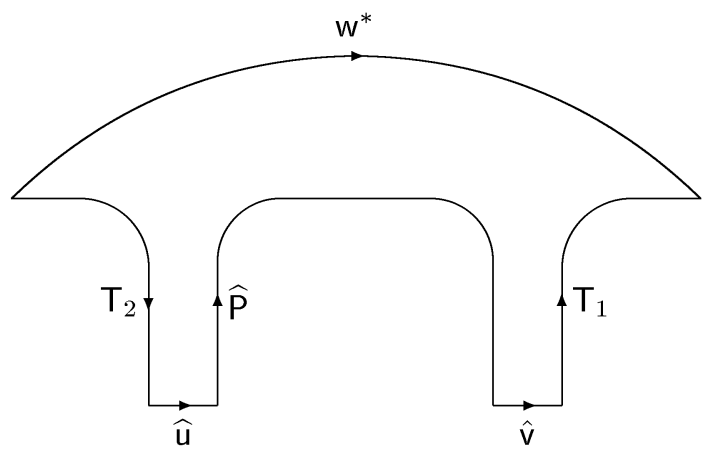

Рис. 55

Случай 3: $\mathcal{S}$ содержит поддиаграмму примыкания П граничного цикла $\mathrm{Y}^{-1} \mathrm{\kappa} \mathrm{P}$ и не содержит подлиаграммы примыкания $\mathrm{Y}^{-1}$ к $\mathrm{H}$. Пусть $\delta \Pi \approx \mathrm{R}^{-1} \mathrm{yQz}, \mathrm{Y} \approx \mathrm{RS}$ и $\mathrm{P}=\mathrm{P}_{1} \mathrm{QP}_{2}$, где $\mathrm{R}^{-1}$ и $\mathrm{Q}$ - участки примыкания (см. рис. 56 ).

Согласно допушению $E$ входит в одно из слов $\mathcal{L}(\mathrm{R}) \mathcal{L}(\mathrm{S}) \mathcal{L}(\mathrm{R})$ или $\mathcal{L}(\mathrm{S}) \mathcal{L}(\mathrm{R}) \mathcal{L}(\mathrm{S})$. $\mathrm{B}$ силу предложений 10.4 и 8.22 , (i) слово $\mathcal{L}(\mathrm{S})$ не содержит элементарных 12 -степеней ранга $\alpha$, а в силу неравенства $|P| \leqslant \frac{n}{2}|A|$ и предложения 8.44, (ii) слово $\mathcal{L}(\mathrm{R})$ не содержит элементарных $\left(\frac{n}{2}+78\right)$-степеней ранга $\alpha$. Так как $\ell_{\alpha, B}(E) \geqslant \frac{n}{2}+3 p+312$, то $E$ может содержаться только в $\mathcal{L}(\mathrm{R}) \mathcal{L}(\mathrm{S}) \mathcal{L}(\mathrm{R})$, причем $\mathcal{L}(\mathrm{R})$ 으 $L U K$, где 


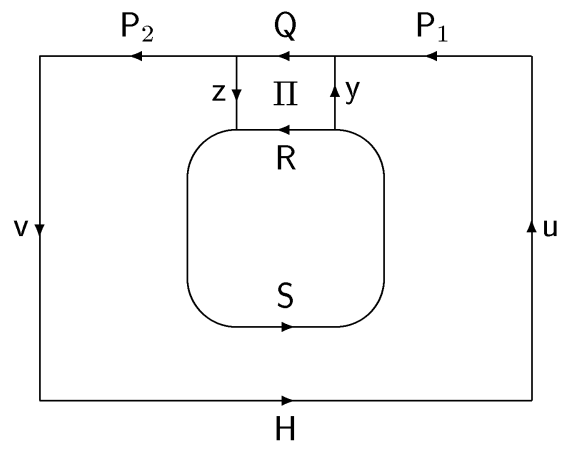

Рис. 56

$E$ 으 $K \mathcal{L}(\mathrm{S}) L$ и $\ell_{\alpha, B}(K), \ell_{\alpha, B}(L)>p+34$. Рассмотрим пути на $\Gamma_{\alpha-1}$, расположенные, как на рис. 57 , метки которых равны меткам соответствуюших путей на диаграмме $\Delta$. Пусть $\widehat{R}=\mathrm{LUK}$, где $\mathcal{L}(\mathrm{L})$ 으 $L, \mathcal{L}(\mathrm{U})$ 으 $U$ и $\mathcal{L}(\mathrm{K})$ 으 $K$. Пусть $\mathbf{h}=\iota(\widehat{\mathrm{R}})[\mathcal{L}(\mathrm{RS})]_{\alpha-1}(\iota(\widehat{\mathrm{R}}))^{-1}$ - элемент группы $\mathbf{B}_{\alpha-1}$, для которого $\widehat{\mathrm{R}}^{\prime}=\mathbf{h} \widehat{\mathrm{R}}$, пусть $\overline{\mathrm{K}}, \overline{\mathrm{L}} \in$ ППер $\alpha(B, p+34)$ - подпути путей $\mathrm{K}$ и $\mathrm{L}$, и пусть $\mathrm{V}, \mathrm{W} \in \Pi_{\mathrm{ep}}\left(\Gamma_{\alpha-1}, B, 2\right)-$ периодические основы для $\overline{\mathrm{K}}$ и $\overline{\mathrm{L}}$ соответственно. В силу предложения 8.44, (ii) имеем $|\widehat{\mathrm{Q}}|,\left|\widehat{\mathrm{Q}}^{\prime}\right|>2|A|$. Применяя предложение 8.38 , (i) два раза при $\mathrm{E}:=\overline{\mathrm{K}}, \mathrm{P}:=\mathrm{V}$, $\mathrm{F}:=\widehat{\mathrm{R}}, \mathrm{Q}:=\widehat{\mathrm{Q}}$ и при $\mathrm{E}:=\overline{\mathrm{L}}, \mathrm{P}:=\mathrm{W}, \mathrm{F}:=\widehat{\mathrm{R}}^{\prime}, \mathrm{Q}:=\widehat{\mathrm{Q}}^{\prime}$, получаем $\operatorname{Cолл~}(\mathrm{V}, \widehat{\mathrm{Q}})$ и

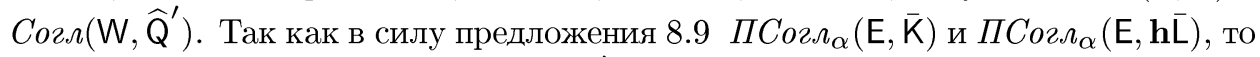

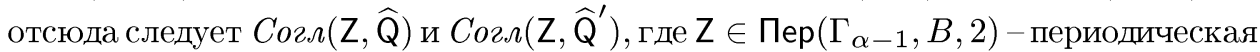
основа для $\mathrm{E} \rightleftharpoons \mathrm{K} \widehat{\mathrm{S}} \mathrm{L}^{\prime}, \mathrm{L}^{\prime} \rightleftharpoons \mathbf{h L}$. Так как $\widehat{\mathrm{Q}}^{\prime}=\mathbf{h} \widehat{\mathbf{Q}}$, то получаем $\operatorname{Cогл}(\mathrm{Z}, \mathbf{h Z})$. Тогда по предложению 7.3 имеем $\mathbf{g} \rightleftharpoons(\iota(\mathrm{Z}))^{-1} \mathbf{h} \iota(\mathrm{Z}) \in \mathbf{N}_{\alpha-1}^{+}\left(B^{\prime}\right)$, где $B^{\prime}-$ циклический сдвиг слова $B$, с которого начинается слово $\mathcal{L}(\mathrm{Z})$. Так как $\mathcal{L}(\mathrm{RS})$ - циклический сдвиг слова $Y$, то элементы $[Y]_{\alpha-1}$ и $\mathbf{h}$ сопряжены. Тогда сопряжены $[Y]_{\alpha-1}$ и $\mathbf{g}$, откуда вытекает сопряженность элементов $[X]_{\alpha}$ и $\pi_{\alpha-1, \alpha}(\mathbf{g}) \in \mathbf{S}_{\alpha}^{+}\left(B^{\prime}\right)$. Но это невозможно по условию леммы. Получили противоречие. Случай 3 рассмотрен.

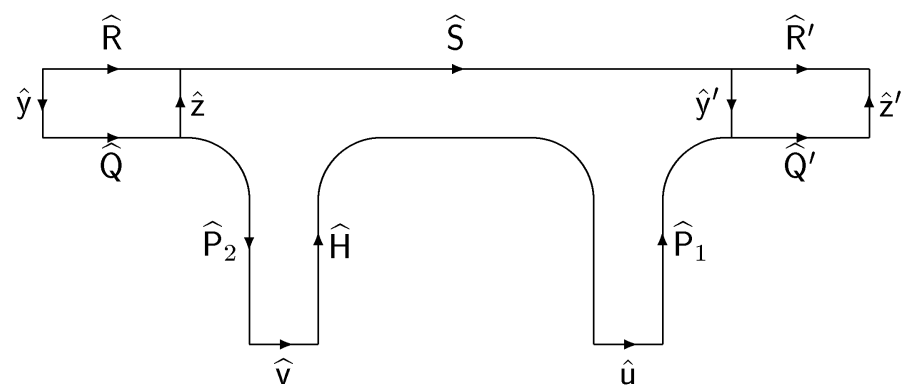

Рис. 57

Случай 4: $\mathcal{S}$ содержит поддиаграммы примыкания $\Pi_{1}$ и $\Pi_{2}$ граничного цикла $\mathrm{Y}^{-1} \mathrm{\kappa} \mathrm{H}$ и $\mathrm{P}$ соответственно. Пусть $\delta \Pi_{i} \approx \mathrm{R}_{i}^{-1} \mathrm{y}_{i} \mathrm{Q}_{i} \mathrm{z}_{i}(i=1,2), \mathrm{Y} \approx \mathrm{R}_{1} \mathrm{~S}_{1} \mathrm{R}_{2} \mathrm{~S}_{2}$, $\mathrm{H}=\mathrm{H}_{1} \mathrm{Q}_{1} \mathrm{H}_{2}$ и $\mathrm{P}=\mathrm{P}_{1} \mathrm{Q}_{2} \mathrm{P}_{2}$, где $\mathrm{R}_{i}^{-1}$ и $\mathrm{Q}_{i}-$ участки примыкания и $\mathrm{S}_{i}^{-1}$-переходные участки диаграммы $\Delta$ относительно $\mathcal{S}$ (см. рис. 58 ). 
ПРЕДСТАВЛЕНИЕ ЭЛЕМЕНТОВ ГРУППЫ $\mathbf{B}_{\alpha}$ ПРИВЕДЕННЫМИ СЛОВАМИ 145

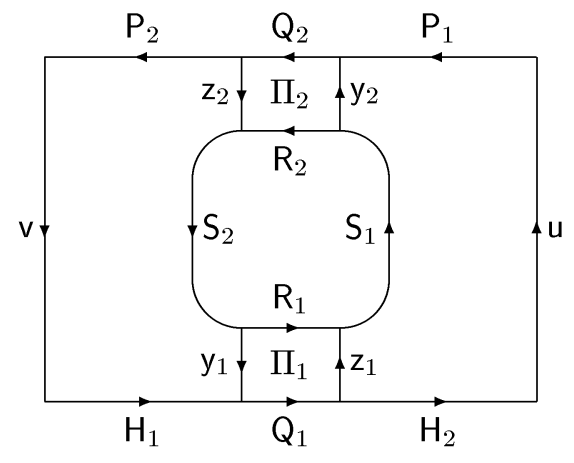

Рис. 58

Обозначим $R_{i} \rightleftharpoons \mathcal{L}\left(\mathrm{R}_{i}\right)$ и $S_{i} \rightleftharpoons \mathcal{L}\left(\mathrm{S}_{i}\right)$. Тогда $E$ содержится в одном из четырех слов $W_{1} \rightleftharpoons R_{1} S_{1} R_{2} S_{2} R_{1}, W_{2} \rightleftharpoons S_{1} R_{2} S_{2} R_{1} S_{1}, W_{3} \rightleftharpoons R_{2} S_{2} R_{1} S_{1} R_{2}$ или $W_{4} \rightleftharpoons S_{2} R_{1} S_{1} R_{2} S_{2}$. В силу предложений 10.4 и 8.22 , (i) каждое слово $S_{i}$ не содержит элементарных 12-степеней ранга $\alpha$ и, как было замечено выше, слово $R_{1}$ не содержит элементарных $(n-q+34)$-степеней ранга $\alpha$, а в слово $R_{2}$ не входят элементарные $\left(\frac{n}{2}+78\right)$-степени ранга $\alpha$. Далее, если бы $E$ содержалось в $W_{1}$ и вхождения $* R_{1} * S_{1} R_{2} S_{2} R_{1}$ и $R_{1} S_{1} R_{2} S_{2} * R_{1} *$ в слово $W_{1}$ содержали бы соответственно подслова $K$ и $L$ слова $E$ с $\ell_{\alpha, B}(K), \ell_{\alpha, B}(L) \geqslant 42$, то мы пришли бы к противоречию, дословно повторив рассуждения из случая 1 . Если бы $E$ содержалось в $W_{3}$ и вхождения $* R_{2} * S_{2} R_{1} S_{1} R_{2}$ и $R_{2} S_{2} R_{1} S_{1} * R_{2} *$ в слово $W_{3}$ содержали бы подслова $K$ и $L$ слова $E$ с $\ell_{\alpha, B}(K), \ell_{\alpha, B}(L) \geqslant p+34$, то мы пришли бы к противоречию, используя рассуждения из случая 2 . Так как

$$
\ell_{\alpha, B}(E) \geqslant \frac{n}{2}+4 p+312=\max \left\{n-q+34, \frac{n}{2}+78\right\}+2(p+34)+2 \cdot 12+2 \cdot 4,
$$

то, используя предложение 8.14 , (ii), легко видеть, что $\mathcal{L}\left(\mathrm{R}_{1}\right)$ 으 $K L$ и $\mathcal{L}\left(\mathrm{R}_{2}\right)$ 으 $M N$, где либо $L S_{1} M$ входит в $E$ и $\ell_{\alpha, B}(L), \ell_{\alpha, B}(M) \geqslant p+34$, либо $N S_{2} K$ входит в $E$ и $\ell_{\alpha, B}(M), \ell_{\alpha, B}(K) \geqslant p+34$. Эти два случая рассматриваются аналогично. Будем считать, что $L S_{1} M$ входит в $E$ и $\ell_{\alpha, B}(L), \ell_{\alpha, B}(M) \geqslant p+34$.

Рассмотрим пути $\widehat{\mathrm{R}}_{i}, \hat{\mathrm{y}}_{i}, \widehat{\mathrm{Q}}_{i}, \hat{\mathrm{z}}_{i}, \widehat{\mathrm{P}}_{i}(i=1,2), \widehat{\mathrm{S}}_{1}, \hat{\mathrm{u}}, \widehat{\mathrm{H}}_{2}$ на $\Gamma_{\alpha-1}$ с метками соответственно $\mathcal{L}\left(\mathrm{R}_{i}\right), \mathcal{L}\left(\mathrm{y}_{i}\right), \mathcal{L}\left(\mathrm{Q}_{i}\right), \mathcal{L}\left(\mathrm{z}_{i}\right), \mathcal{L}\left(\mathrm{P}_{i}\right), \mathcal{L}\left(\mathrm{S}_{1}\right)$, u и $\mathcal{L}\left(\mathrm{H}_{2}\right)$, расположенные, как на рис. 59.

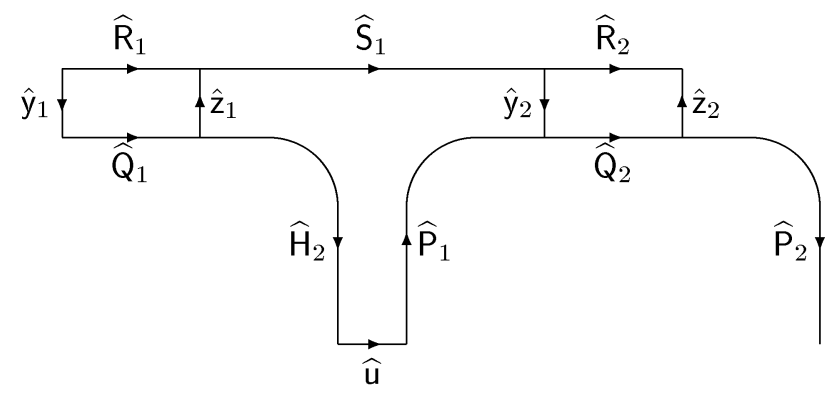

Рис. 59 
Пусть $\widehat{\mathrm{R}}_{1}=\mathrm{KL}, \widehat{\mathrm{R}}_{2}=\mathrm{MN}$, где метки путей $\mathrm{K}, \mathrm{L}, \mathrm{M}$ и $\mathrm{N}$ равны словам $K$, $L, M$ и $N$ соответственно, и пусть $\overline{\mathrm{L}}$ и $\overline{\mathrm{M}}$ - подпути путей $\mathrm{L}$ и $\mathrm{M}$, для которых $\overline{\mathrm{L}}, \overline{\mathrm{M}} \in \Pi_{\text {Пер }}(B, p+34)$. Пусть $\mathrm{V}, \mathrm{W} \in \Pi_{\mathrm{ep}}\left(\Gamma_{\alpha-1}, B, 2\right)$ - периодические основы для $\overline{\mathrm{L}}$ и $\overline{\mathrm{M}}$ соответственно. Так как $\overline{\mathrm{L}}$ и $\overline{\mathrm{M}}$ - подпути пути $\mathrm{L} \widehat{\mathrm{S}}_{1} \mathrm{M} \in \Pi$ Пер ${ }_{\alpha}\left(\Gamma_{\alpha-1}, B\right)$, то в силу предложения 8.9 имеем $\Pi C о г \Omega_{\alpha}(\overline{\mathrm{L}}, \overline{\mathrm{M}})$. По предложению 8.44 , (ii) имеем $\left|\widehat{\mathrm{Q}}_{2}\right| \geqslant 2|A|$, а по предложению 8.38 , (i) при $\mathrm{E}:=\overline{\mathrm{M}}, \mathrm{P}:=\mathrm{W}, \mathrm{F}:=\widehat{\mathrm{R}}_{2}$ и $\mathrm{Q}:=\widehat{\mathrm{Q}}_{2}$ получаем $\operatorname{Cozл}\left(\mathrm{W}, \widehat{\mathrm{Q}}_{2}\right)$. Отсюда следует $\operatorname{Cozл}\left(\mathrm{V}, \widehat{\mathrm{Q}}_{2}\right)$. Тогда, укоротив $\overline{\mathrm{L}}$ в силу предложения 8.35 и используя предложение 8.10 , найдем подпуть $\mathrm{L}^{*} \in \Pi_{\text {Пер }}(A, p)$ пути $\bar{L}$ такой, что $\widehat{P}_{1} \widehat{Q}_{2} \widehat{P}_{2}$ и периодическая основа $V^{*}$ для $L^{*}$ имеют общее периодическое продолжение $\mathrm{U} \in$ Пер $\left(\Gamma_{\alpha-1}, A, 2\right)$. Пусть $\mathrm{P}^{*}$ - подпуть пути $\mathrm{U}$ или $\mathrm{U}^{-1}$, соединяюший вершины $\iota\left(\mathrm{V}^{*}\right)$ и $\tau\left(\widehat{\mathrm{P}}_{2}\right), \mathrm{u}^{*}-$ путь с $\mathcal{L}\left(\mathrm{u}^{*}\right) \in \mathcal{H}_{\alpha-1}$, соединяющий вершины $\iota\left(\mathrm{L}^{*}\right)$ и $\iota\left(\mathrm{W}^{*}\right)$, и $\mathrm{Z}$ - конец пути $\widehat{\mathrm{Q}}_{1} \widehat{\mathrm{H}}_{2}$, начинаюшийся $\mathrm{c} \mathrm{L}^{*}$ (см. рис. 60 ).

Пути Zûि $\widehat{P}_{1} \widehat{Q}_{2} \widehat{P}_{2}$ и u $\mathrm{u}^{*}$ имеют обшие начальную и конечную вершины, следовательно, обозначив $Z \rightleftharpoons \mathcal{L}(\mathrm{Z}), u^{*} \rightleftharpoons \mathcal{L}\left(\mathrm{u}^{*}\right)$ и $P^{*} \rightleftharpoons \mathcal{L}\left(\mathrm{P}^{*}\right)$, получим

$$
Z u P^{\alpha=1} u^{*} P^{*}
$$

С учетом равенств $w$ 드 $u P v$ и (12.16) отсюда следует

$$
Z X_{2} X_{1} \stackrel{\alpha}{=} u^{*} P^{*} v
$$

Так как $Z$ есть конец слова $H$, то $H$ 으 $H^{*} Z$ для некоторого $H^{*}$. Далее, так как $P^{*} \in \Pi е p(A) \cup \Pi е p\left(A^{-1}\right)$, то $P^{*} \stackrel{\alpha}{=} P^{\prime}$ для некоторого слова $P^{\prime} \in \Pi е p(A) \cup$ Пер $\left(A^{-1}\right)$ с $\left|P^{\prime}\right|<n|A|$. Мы получили, что равенства (12.16) выполнены при $X_{1}:=X_{1}, X_{2}:=Z X_{2}, H:=H^{*}$ и $w:=u^{*} P^{\prime} v$. Так как в $Z$ входит элементарное слово $\mathcal{L}(\overline{\mathrm{M}})$ ранга $\alpha$, то $Z$ непусто, следовательно, $\left|H^{*}\right|<|H|$. Получили противоречие с выбором слов $X_{1}, X_{2}, H$ и $w$. Лемма 12.23 доказана.

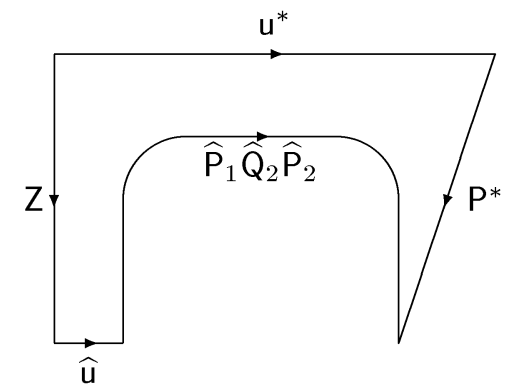

Рис. 60

12.24. ПРЕДЛОЖЕНИЕ $(\alpha \geqslant 0)$. Любой элемент $1 \neq \mathbf{g} \in \mathbf{B}_{\alpha}$, не сопряженный при $\alpha \geqslant 1$ никакому әлементу $\mathbf{h} \in \mathbf{S}_{\alpha}^{+}(A)$, где $A \in \bigcup_{1 \leqslant \beta \leqslant \alpha} \mathcal{E}_{\beta}$, сопряжен некоторому әлементу $[A]_{\alpha}$, где $A$ - период ранга $\alpha+1$. B частности, әто верно для любого әлемента $\mathbf{g}$ бесконечного порядка группы $\mathbf{B}_{\alpha}$. 
ДокАЗАТЕЛЬСтво. Предложение тривиально при $\alpha=0$. При $\alpha>0$ первое утверждение непосредственно вытекает из индуктивного предположения, лемм $12.23,12.22,12.12$ и определения 5.2 с учетом неравенства $\frac{n}{2}+4 p+321<n-$ $q_{0}-2 p-102$, а второе следует из первого в силу предложения 7.14 , (iii).

12.25. СЛЕДСТВИЕ. Любой әлемент конечного порядка группы $\mathbf{B}_{\alpha}$ сопряжсен некоторому әлементу $\mathbf{g} \in \mathbf{S}_{\alpha}^{+}(A)$ при $A \in \bigcup_{1 \leqslant \beta \leqslant \alpha} \mathcal{E}_{\beta}$.

ДокАЗАТЕльСтво. Нужно лишш заметить, что в силу определения 5.2 и предложения 11.4 любой период ранга $\alpha+1$ представляет элемент бесконечного порядка группы $\mathbf{B}_{\alpha}$.

12.26. ПРЕДЛОЖЕНИЕ. Для любого периода А ранга $\alpha+1$ существует минимальный период $B$ ранга $\alpha+1$ такой, что слова $A^{t} u B^{k t}$ сопряжены в zруппе $\mathbf{B}_{\alpha}$ nрu некоторых $k, t>0$.

ДокАЗАтельство. Предположим, $A$ и $B$ - периоды ранга $\alpha+1$ и слова $A^{k}$ и $B^{k t}$ сопряжены в $\mathbf{B}_{\alpha}$ при некоторых $k, t>0$. Если $B$ не является минимальным периодом ранга $\alpha+1$, то в силу определения 5.3 и предложения 12.24 найдется период $C$ ранга $\alpha+1$ такой, что слова $B^{r}$ и $C^{r s}$ сопряжены в $\mathbf{B}_{\alpha}$ при некоторых $r>0$ и $s \geqslant 2$. Так как в этом случае мы имеем сопряженные в $\mathbf{B}_{\alpha}$ слова $A^{t r}$ и $C^{k r t s}$, причем, $t s>t$, то, очевидно, достаточно ограничиться доказательством следуюшего факта:

если $A, B$ - периоды ранга $\alpha+1$ и слова $A^{k} u B^{k t}$ сопряженьи в $\mathbf{B}_{\alpha}$ при $k, t>0$, то $t \leqslant|A|+3$.

Пусть $\beta \leqslant \alpha$ - наибольший ранг такой, что некоторое слово $B^{r}$ содержит элементарную $q_{4}$-степень ранга $\beta$. Применив предложение 11.33 в случае $\beta<\alpha$, получаем, что слова $A^{k}$ и $B^{k t}$ сопряжены в группе $\mathbf{B}_{\beta}$. Увеличив $k$ и заменив слова $A$ и $B$ на их некоторые циклические сдвиги, в силу следствия 11.32 можно считать $A^{k} \stackrel{\beta}{=} u^{-1} B^{k t} u$ для некоторого $u \in \mathcal{H}_{\beta}$. Следовательно, найдутся близкие в ранге $\beta$ пути $\mathrm{P}$ и $\mathrm{Q}$ на графе $\Gamma_{\beta}$, где $\mathcal{L}(\mathrm{P})$ 으 $A^{t}$ и $\mathcal{L}(\mathrm{Q})$ 으 $B^{k t}$. В силу лемм 5.10 и 8.4 путь $\mathrm{Q}$ содержит $k t-1$ подпутей $\mathrm{K}, \mathbf{s}_{B, \mathrm{Q}} \mathrm{K}, \ldots, \mathrm{s}_{B, \mathrm{Q}}^{k t-2} \mathrm{~K}$, где $\mathrm{K} \in \ni \boldsymbol{л}_{\beta}\left(\Gamma_{\beta}, q_{4}-9\right)$. Так как в силу леммы 6.7 имеем $\neg Э \operatorname{Coгл}, \beta_{\beta}\left(\mathbf{s}_{B, \mathrm{Q}}^{i} \mathrm{~K}, \mathrm{~s}_{B, \mathrm{Q}}^{j} \mathrm{~K}\right)$ при $i \neq j$, то в силу следствия 11.23 для каждого $i=1,2, \ldots, k t-3$ найдется подпуть $\mathrm{L}_{i} \in \ni{ }_{\beta}\left(\Gamma_{\beta}, q_{4}-h-9\right)$ пути $\mathrm{P}$ такой, что $Э \operatorname{Coz}_{\beta}\left(\mathbf{s}_{B, \mathrm{Q}}^{i} \mathrm{~K}, \mathrm{~L}_{i}\right)$. Тогда по предложению 8.41 каждый путь $\mathrm{L}_{i}$ содержит некоторый подпуть $\overline{\mathrm{L}}_{i} \in \ni{ }_{\beta}\left(\Gamma_{\beta}, q_{4}-h-2 p-79\right)$, не имеющий обших ребер с $\mathrm{L}_{j}$ при $i \neq j$. Так как слова $\mathcal{L}\left(\overline{\mathrm{L}}_{i}\right)$ непусты, то отсюда следует $k t-3 \leqslant k|A|$, т.е. $t \leqslant|A|+\frac{3}{k} \leqslant|A|+3$, что и требовалось.

12.27. ПРЕДЛОЖЕНИЕ. Если $A$ - период ранга $\alpha+1$, то некоторый ииклический сдвиг слова $A$ содержит әлементарную 109-степень ранга $\alpha$. 
ДокАЗАТЕЛЬСтво. В силу леммы 8.41 достаточно доказать, что некоторое слово $A^{k}$ содержит элементарную 118-степень ранга $\alpha$. По предложению 12.26 для некоторых $B \in \mathcal{M}_{\alpha+1}$ и $k, t>0$ слова $A^{k}$ и $B^{k t}$ сопряжены в $\mathbf{B}_{\alpha}$. Как мы видели в доказательстве предложения 12.26 , заменяя $A$ и $B$ на их циклические сдвиги, можно считать, что $A^{k} \stackrel{\beta}{=} u^{-1} B^{k t} u$ для некоторого $u \in \mathcal{H}_{\beta}$ при $\beta \leqslant \alpha$ и в слово $B^{k t}$ входит элементарная $\left(q_{4}-9\right)$-степень ранга $\beta$. Увеличив $k$, можно считать $k t \geqslant 5$. Если $\beta=\alpha$, то точно так же, как в доказательстве предложения 12.26 , устанавливается, что слово $A^{k}$ содержит элементарную $\left(q_{4}-h-9\right)$-степень ранга $\alpha$. Если $\beta<\alpha$, то слова $A^{k}$ и $B^{k t}$ близки в ранге $\alpha-1$, и тогда можно воспользоваться предложениями 8.34 и 8.27 и леммой 5.13 .

\section{§ 13. Нормальная форма и сопряженность элементов конечного порядка группы $\mathbf{B}_{\alpha}$}

13.1. ОПРЕДЕЛЕНИЕ. Элементарньй период $A$ ранга $\alpha$ назовем нормализованным, если слово $A$ начинается или оканчивается на элементарную $q_{2}$-степень ранга $\rho(A)$. Множество нормализованных элементарных периодов ранга $\alpha$ обозначим через $\bar{\varepsilon}_{\alpha}$.

Из определения вытекает, что если $A \in \bar{\varepsilon}_{\alpha}$, то $A^{-1} \in \overline{\mathcal{E}}_{\alpha}$. Кроме того, в силу определения 5.15 и леммы 7.6 для любого $A \in \mathcal{E}_{\alpha}$ некоторый циклический сдвиг слова $A$ принадлежит $\bar{\varepsilon}_{\alpha}$.

13.2. Лемма. Для любого әлементарного периода $A \in \overline{\mathcal{E}}_{\alpha}$ верны следующие утверждения:

(i) любой әлемент $\mathbf{g} \in \mathbf{N}_{\alpha-1}^{*}(A)$ можно представить словом $w \in \mathcal{H}_{\rho(A)}$;

(ii) любой әлемент $\mathbf{g} \in \mathbf{N}_{\alpha-1}^{+}(A)$ можно представить словом $A^{t} w$, где $w \in \mathcal{H}_{\alpha-1}, \quad t \rightleftharpoons \operatorname{ind}_{A}(\mathbf{g}) u[w]_{\alpha-1} \in \mathbf{N}_{\alpha-1}^{*}(A)$;

(iii) любой әлемент $\mathbf{g} \in \mathbf{N}_{\alpha-1}^{-}(A)$ можно представить словом вида $P w$, әде $P$ - начало слова $A^{t}$ или $A^{-t} u w \in \mathcal{H}_{\alpha-1}$, и словом вида $A^{r} P w A^{-r}$, где $P$ - начало слова $A$ или $A^{-1}$ и $w \in \mathcal{H}_{\alpha-1}$;

(iv) если $\mathbf{G}-$ nодгруппа группь $\mathbf{N}_{\alpha-1}(A) u \mathbf{G} \subseteq \mathbf{N}_{\alpha-1}^{*}(A) \cup \mathbf{N}_{\alpha-1}^{-}(A)$, mo для некоторого $r \in \mathbb{Z}$ каждый әлемент $\mathbf{g} \in \mathbf{G}$ можно представить словом вида $A^{r} P w A^{-r}$, где $P$ - начало слова $A$ или $A^{-1} u w \in \mathcal{H}_{\alpha-1}$.

ДокаЗАтельство. Заменяя, если нужно, $A$ на $A^{-1}$, можно считать, что $A$ 으 $E X$, где $E$ - элементарная $q_{2}$-степень ранга $\rho(A)$.

(i) Пусть $\mathrm{g} \in \mathbf{N}_{\alpha-1}^{*}(A)$. Рассмотрим путь $\mathrm{R}$ на графе $\Gamma_{\alpha-1}$ с $\mathcal{L}(\mathrm{R})$ 드 $A^{2}$, $\iota(\mathrm{R})=1$, и пусть $\mathrm{E}-$ начало пути $\mathrm{R}$ с $\mathcal{L}(\mathrm{E})$ 으 $E$. В силу предложений $7.3,7.8$ и следствия 7.11 имеем $Э С о г \Omega_{\rho(A)}(\mathrm{E}, \mathrm{gE})$. Если $\rho(A)=0$, то согласно определению $5.14 \mathrm{E}=\mathbf{g E}$, т.е. $\mathbf{g}=1$ и в этом случае в качестве $\mathbf{w}$ можно взять пустое слово. В случае $\rho(A)>0$ по индуктивному предположению 8.45 найдется путь w c $w \rightleftharpoons \mathcal{L}(\mathrm{w}) \in \mathcal{H}_{\rho(A)}$, соединяюший вершины $\iota(\mathrm{E})=1$ и $\iota(\mathrm{gE})=\mathrm{g}$. Тогда $w-$ искомое слово, представляющее элемент $\mathbf{g}$.

(ii) По предложению 7.10, (iii), (iv) имеем $[A]_{\alpha-1}^{-t} \mathbf{g} \in \mathbf{N}_{\alpha-1}^{*}(A)$, где $t \rightleftharpoons \operatorname{ind}_{A}(\mathbf{g})$, и тогда утверждение вытекает из (i). 
(iii) Как в доказательстве (i), пусть $\mathrm{R}$ - путь на графе $\Gamma_{\alpha-1}$ с $\mathcal{L}(\mathrm{R})$ 으 $A^{2}$, $\iota(\mathrm{R})=1$, и пусть $\mathrm{E}$ - начало $\mathrm{R}$ c $\mathcal{L}(\mathrm{E})$ 으 $E$. По предложению 7.3 имеем Cогл $\left(\mathrm{R}, \mathrm{gR}^{-1}\right)$. Тогда, используя предложение 7.7 и лемму 8.2, (iii), найдем путь $\mathrm{F} \in \ni_{\rho(A)}\left(\overline{\mathrm{R}}, q_{1}\right)$ такой, что $Э \operatorname{Coz}_{\rho(A)}\left(\mathrm{gE}^{-1}, \mathrm{~F}\right)$, где $\overline{\mathrm{R}}$ - некоторое периодическое продолжение $\mathrm{R}$. Пусть $\mathrm{P}$ - подпуть пути $\overline{\mathrm{R}}$ или $\overline{\mathrm{R}}^{-1}$, соединяюший вершины $1=\iota(\mathrm{R})$ и $\iota(\mathrm{F})$. Очевидно, $P \rightleftharpoons \mathcal{L}(\mathrm{P})$ есть начало слова вида $A^{ \pm t}$. Если $\rho(A)=0$, то из непустоты множества $\mathbf{N}_{\alpha-1}^{-}(A)$ и предложения 11.33 легко вытекало бы, что для некоторого $t \neq 0$ слова $A^{t}$ и $A^{-t}$ сопряжены в свободной группе $\mathbf{B}_{0}$. Следовательно, $\rho(A)>0$. Тогда по индуктивному предположению 8.45 вершины $\iota(\mathrm{F})$ и $\tau(\mathbf{g E})=\mathbf{g}$ соединены некоторым путем w $\mathrm{c} w \rightleftharpoons \mathcal{L}(\mathrm{w}) \in \mathcal{H}_{\rho(A)} \subseteq \mathcal{H}_{\alpha-1}$. Так как $\mathrm{g}=[\mathcal{L}(\mathrm{Pw})]_{\alpha-1}$, то тем самым доказано первое утверждение. Докажем второе. $\mathrm{B}$ силу лемм 7.21 и $3.2,(\mathrm{v})$ для любого $r \in \mathbb{Z}$ имеем ЭСогл $\Omega_{(A)}\left(\mathbf{s}_{A, \mathbf{g R}}^{r} \mathbf{g E}^{-1}, \mathbf{s}_{A, \overline{\mathrm{R}}}^{-r} \mathrm{~F}\right)$. Соединим вершины $\mathbf{s}_{A, \overline{\mathrm{R}}}^{-r} \iota(\mathrm{F})$ и $\mathbf{s}_{A, \mathbf{g R}}^{r} \iota(\mathrm{gE})$ путем $\mathrm{w}_{1}$ с $w_{1} \rightleftharpoons \mathcal{L}\left(\mathrm{w}_{1}\right) \in \mathcal{H}_{\alpha-1}$. Очевидно, имеем путь на $\Gamma_{\alpha-1}$, где над стрелками указаны метки соответствующих подпутей и $A_{1}$ - циклический сдвиг слова $A$, для которого $P^{-1} A P \stackrel{0}{=} A_{1}$ :

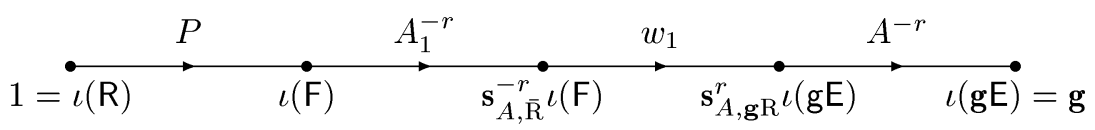

Отсюда следует

$$
\mathbf{g}=\left[P A_{1}^{-r} w_{1} A^{-r}\right]_{\alpha-1}=\left[A^{-r} P w_{1} A^{-r}\right]_{\alpha-1}=\left[A^{r} \cdot A^{-2 r} P \cdot w_{1} \cdot A^{-r}\right]_{\alpha-1} .
$$

Так как $P$ - начало слова вида $A^{ \pm t}$, то для некоторого $r$ имеем $A^{-2 r} P \stackrel{0}{=} P_{1}$, где $P_{1}$ - начало слова $A^{ \pm 1}$. Тогда в качестве искомого слова $A^{r} P w A^{-r}$ можно взять $A^{r} P_{1} w_{1} A^{-r}$.

(iv) Если $\mathbf{G} \subseteq \mathbf{N}_{\alpha-1}^{*}(A)$, то, взяв $r=0$, можно воспользоваться уже доказанным утверждением (i). Пусть $\mathbf{G} \cap \mathbf{N}_{\alpha-1}^{-}(A) \neq \varnothing$. Для любых двух элементов $\mathbf{g}, \mathbf{h} \in \mathbf{G} \cap \mathbf{N}_{\alpha-1}^{-}(A)$ выполнено $\mathbf{g}^{-1} \mathbf{h} \in \mathbf{N}_{\alpha-1}^{+}(A)$, и так как по условию имеем $\mathbf{G} \cap \mathbf{N}_{\alpha-1}^{+}(A) \subseteq \mathbf{N}_{\alpha-1}^{*}(A)$, то $\mathbf{g}^{-1} \mathbf{h} \in \mathbf{N}_{\alpha-1}^{*}(A)$. Тогда в силу предложений $7.3,7.8$ и следствия 7.11 имеем ЭСогл $\Omega_{(A)}(\mathbf{g E}, \mathbf{h E})$ для любых $\mathbf{g}, \mathbf{h} \in \mathbf{G} \cap \mathbf{N}_{\alpha-1}^{-}(A)$, и, как видно из доказательства (iii), элементы $\mathbf{g} \in \mathbf{G} \cap \mathbf{N}_{\alpha-1}^{-}(A)$ представляются словами искомого вида $A^{r} P w A^{-r}$, где $r$ - общее для всех g. Так как $[A]_{\alpha-1}^{r} \mathbf{N}_{\alpha-1}^{*}(A)[A]_{\alpha-1}^{-r}=\mathbf{N}_{\alpha-1}^{*}(A)$, то в силу (i) каждый элемент из $\mathbf{G} \cap \mathbf{N}_{\alpha-1}^{*}(A)$ представляется словом вида $A^{r} w A^{-r}$, где $w \in \mathcal{H}_{\alpha-1}$.

13.3. ПРЕДЛОЖЕНИЕ. Для любого $A \in \bar{\varepsilon}_{\beta}$ при $1 \leqslant \beta \leqslant \alpha$ любой әлемент $\mathbf{g} \in \mathbf{S}_{\alpha}(A)$ может бить представлен словом вида Р $w \in \mathcal{H}_{\beta}$, где $P$ - начало слова $A^{n}$ или $A^{-n}$ и $w \in \mathcal{H}_{\beta-1}$. Если при этом $\mathbf{g} \in \mathbf{S}_{\alpha}^{+}(A) \backslash \mathbf{S}_{\alpha}^{*}(A)$, то $\mathbf{g}$ представляется словом $A^{t} w \in \mathcal{H}_{\beta}$, где $0<t<n, \quad w \in \mathcal{H}_{\beta-1} u[w]_{\beta-1} \in$ $\mathbf{N}_{\beta-1}^{*}(A)$. 
ДОКАЗАТЕЛЬСТВО. Пусть $\mathbf{g}=\pi_{\beta-1, \alpha}(\tilde{\mathbf{g}})$, где $\tilde{\mathbf{g}} \in \mathbf{N}_{\beta-1}(A)$ и в случаях $\mathbf{g} \in \mathbf{S}_{\alpha}^{+}(A)$ или $\mathbf{g} \in \mathbf{S}_{\alpha}^{-}(A)$ элемент $\mathbf{g}$ лежит соответственно в $\mathbf{N}_{\beta-1}^{+}(A)$ или $\mathbf{N}_{\beta-1}^{-}(A)$. Если $\tilde{\mathbf{g}} \in \mathbf{N}_{\beta-1}^{-}(A)$, то по лемме 13.2 , (iii) имеем $\tilde{\mathbf{g}}=[P w]_{\beta-1}$, где $P$ - начало слова вида $A^{ \pm t}$ и $w \in \mathcal{H}_{\beta-1}$. Тогда $\mathbf{g}=[P w]_{\alpha}$, и так как $A^{n} \stackrel{\alpha}{=} 1$, то в качестве $P$ можно взять некоторое начало слова $A^{ \pm n}$. В этом случае в силу определения 5.8 имеем $P w \in \mathcal{H}_{\beta}$. Пусть $\tilde{\mathbf{g}} \in \mathbf{N}_{\beta-1}^{+}(A)$. По лемме 13.2, (ii) представим элемент $\tilde{\mathbf{g}}$ словом $A^{t} w$, где $w \in \mathcal{H}_{\beta-1}$ и $[w]_{\beta-1} \in \mathbf{N}_{\beta-1}^{*}(A)$. Тогда $\mathbf{g}=\left[A^{t} w\right]_{\alpha}$ и в силу равенства $A^{n} \stackrel{\alpha}{=} 1$ можно считать $0 \leqslant t<n$. Так как $[w]_{\alpha} \in \mathbf{S}_{\alpha}^{*}(A)$, то в случае $\mathbf{g} \in \mathbf{S}_{\alpha}^{+}(A) \backslash \mathbf{S}_{\alpha}^{*}(A)$ имеем $t \neq 0$. Остается заметить, что $A^{t} w \in \mathcal{H}_{\beta}$ в силу определения 5.8 .

13.4. ОПРЕДЕЛЕНИЕ. Слово вида $A^{t} w$ (формально - пару слов $A^{t}$ и $w$ ), где $A \in \overline{\mathcal{E}}_{\beta}, 1 \leqslant \beta \leqslant \alpha, 0<t<n, w \in \mathcal{H}_{\beta-1}$ и $[w]_{\beta-1} \in \mathbf{N}_{\beta-1}^{*}(A)$, будем называть нормальной формой ранга $\beta$ (участие ранга $\alpha$ в этом определении связано только с тем, что подгруппа $\mathbf{N}_{\beta-1}^{*}(A)$ формально определена только для $\beta \leqslant \alpha$, см. определения $7.12,7.9)$.

Из определения непосредственно вытекает, что любая нормальная форма $A^{t} w$ ранга $\beta \leqslant \alpha$ представляет в группе $\mathbf{B}_{\alpha}$ элемент из $\mathbf{S}_{\alpha}(A)$. В силу предложения 7.14 , (iii) любой такой элемент имеет конечньй порядок. Покажем, что с точностью до сопряженности любой элемент конечного порядка группы $\mathbf{B}_{\alpha}$ может быть представлен некоторой нормальной формой ранга $\beta \leqslant \alpha$.

13.5. ПРЕДЛОЖЕНИЕ. Всякий нетривиальный әлемент конечного порядка группы $\mathbf{B}_{\alpha}$ сопряжен әлементу вида $\left[A^{t} w\right]_{\alpha}$, где $A^{t} w-$ нормальная форма ранга $\beta \leqslant \alpha$.

ДокАЗАТЕЛЬСТВО. Пусть $1 \neq \mathbf{g} \in \mathbf{B}_{\alpha}$ и $\mathbf{g}$ имеет конечньй порядок. Пусть $\beta$ - наименьший ранг, для которого сушествует элемент $\tilde{\mathbf{g}} \in \mathbf{B}_{\beta}$ конечного порядка такой, что $\pi_{\beta, \alpha}(\tilde{\mathbf{g}})=\mathrm{g}$. Ясно, что $\beta \geqslant 1$. В силу следствия 12.25 элемент $\tilde{\mathbf{g}}$ сопряжен некоторому элементу $\mathbf{h} \in \mathbf{S}_{\beta}^{+}(A)$, где $A \in \mathcal{E}_{\gamma}$ и $\gamma \leqslant \beta$. В силу леммы 7.20 можно считать $A \in \bar{\varepsilon}_{\gamma}$. Если $\mathbf{h} \in \mathbf{S}_{\beta}^{*}(A)$, то согласно определению 7.13 для некоторого $\tilde{\mathbf{h}} \in \mathbf{N}_{\gamma-1}^{*}(A)$ выполнено $\pi_{\gamma-1, \beta}(\tilde{\mathbf{h}})=\mathbf{h}$, и в силу предложения 7.14, (ii) $\tilde{\mathbf{h}}$ - элемент конечного порядка, что противоречит выбору $\beta$. Следовательно, $\mathbf{h} \in \mathbf{S}_{\beta}^{+}(A) \backslash \mathbf{S}_{\beta}^{*}(A)$, и тогда утверждение вытекает из предложения 13.3.

13.6. ПрЕДЛОЖЕНИЕ. Если $A^{t} w$ - нормальная форма ранга $\beta \leqslant \alpha$, то $\left[A^{t} w\right]_{\alpha} \neq 1$.

ДоказАтельство. Допустим, $A^{t} w$ - нормальная форма ранга $\beta \leqslant \alpha$ и $A^{t} w \stackrel{\alpha}{=} 1$. Так как $A^{t} w \in \mathcal{H}_{\beta}$, то по предложению 11.3 имеем $A^{t} w \stackrel{\beta}{=} 1$. Применяя предложение 11.2 при $\alpha:=\beta, X:=u:=Y:=1$ и $v:=A^{t} w$, получаем $A^{t^{\prime}} w^{\beta-1}={ }^{1}$, где $t^{\prime}=t$ или $t^{\prime}=n-t$. Но это невозможно, так как в силу предложения 7.10 , (iii) $\operatorname{ind}_{A}\left(\left[A^{t^{\prime}} w\right]_{\beta-1}\right)=t^{\prime} \neq 0$, т.e. $\left[A^{t^{\prime}} w\right]_{\beta-1} \neq 1$. 
13.7. ПРЕДЛОЖЕНИЕ. (i) Если $\mathbf{G} \leqslant \mathbf{B}_{\beta}-$ конечная подгруппа группь $\mathbf{B}_{\beta}$ при $\beta \leqslant \alpha$, то эпиморфизм $\pi_{\beta, \alpha}$ индуцирует изоморфизм $\mathbf{G} \rightarrow \pi_{\beta, \alpha}(\mathbf{G}) . \quad B$ частности, для любого $A \in \mathcal{E}_{\beta}$ естественный эпиморфизм $\mathbf{S}_{\beta}(A) \rightarrow \mathbf{S}_{\alpha}(A)$ является изоморфизмом.

(ii) Для любого $A \in \mathcal{E}_{\beta}$ при $1 \leqslant \beta \leqslant \alpha$ ядро естественного әпиморфизма $\mathbf{N}_{\beta-1}(A) \rightarrow \mathbf{S}_{\alpha}(A)$ есть чиклическая подгруппа, породсденная әлемен$\operatorname{moм}[A]_{\beta-1}^{n}$.

ДокАЗАТЕЛЬСтво. Утверждение (i) непосредственно вытекает из предложений 13.5, 13.6 и 7.14, (iii). Докажем (ii). Очевидно, $[A]_{\beta-1}^{n} \in \operatorname{ker} \pi_{\beta-1, \alpha}$. Докажем, что $\operatorname{ker} \pi_{\beta-1, \alpha} \cap \mathbf{N}_{\beta-1}(A) \subseteq\left\langle[A]_{\beta-1}^{n}\right\rangle$. Ввиду (i) достаточно рассмотреть случай $\beta=\alpha$. В силу леммы 7.2 , (iii), заменяя слово $A$ на его циклический сдвиг, можно считать $A \in \bar{\varepsilon}_{\alpha}$. Если $\mathbf{g} \in \mathbf{N}_{\alpha-1}^{-}(A)$, то $\mathbf{g} \neq 1$ в силу леммы 7.14 , (ii) и $\mathbf{g}$ имеет конечный порядок в силу следствия 7.17 и предложения 7.14 , (ii). Тогда $\pi_{\alpha-1, \alpha}(\mathbf{g}) \neq 1$ в силу предложений 13.5 и 13.6 . Пусть $\mathbf{g} \in \mathbf{N}_{\alpha-1}^{+}(A)$. Предположим, $\pi_{\alpha-1, \alpha}(\mathbf{g})=1$. По предложению 13.2, (i) имеем $\mathbf{g}=\left[A^{t} w\right]_{\alpha-1}$, где $[w]_{\alpha-1} \in \mathbf{N}_{\alpha-1}^{*}(A)$. Пусть $t^{\prime} \equiv t(\bmod n)$ и $0 \leqslant t^{\prime}<n$. Так как $\left[A^{t^{\prime}} w\right]_{\alpha}=\left[A^{t} w\right]_{\alpha}=\pi_{\alpha-1, \alpha}(\mathbf{g})=1$, то по предложению 13.6 имеем $t^{\prime}=0$, иначе $A^{t^{\prime}} w$-нормальная форма ранга $\alpha$. Тогда в силу (i) и конечности $\mathbf{N}_{\alpha-1}^{*}(A)$ получаем $[w]_{\alpha-1}=1$. Следовательно, $\mathbf{g}=[A]_{\alpha-1}^{k n}$ для некоторого $k$, что и требовалось.

Перейдем к анализу соотношений сопряженности элементов конечного порядка группы $\mathbf{B}_{\alpha}$.

13.8. Лемма. Пусть $\mathrm{T}^{-1} \mathbf{u T}^{\prime}-$ nуть на графе $\Gamma_{\alpha}$ c $\mathcal{L}(\mathrm{T})$ 으 $\mathcal{L}\left(\mathrm{T}^{\prime}\right) \in \mathcal{R}_{\alpha}$ $u[\mathcal{L}(\mathrm{u})]_{\alpha} \in \mathbf{S}_{\alpha}(A)$ для некоторого $A \in \bigcup_{\beta \leqslant \alpha} \bar{\varepsilon}_{\beta}$. Пусть $\mathrm{E} \in$ Эл $_{\alpha}(\mathrm{T}, h+8)$,

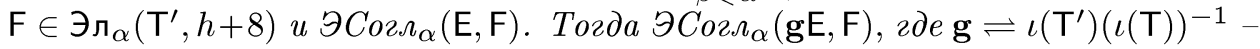
әлемент группи $\mathbf{B}_{\alpha}$, для которого $\mathrm{T}^{\prime}=\mathrm{g} \mathrm{T}$.

ДокАЗАТЕЛЬСТво. Допустим, $\neg$ ЭСогл $\Omega_{\alpha}(\mathrm{gE}, \mathrm{F})$. Так как $\mathrm{gE}$ и $\mathrm{F}$ - подпути пути $\mathrm{T}^{\prime}$, то по предложению 8.40 имеем либо $\mathrm{gE}<\mathrm{T}^{\prime} \mathrm{F}$, либо $\mathrm{F}<\boldsymbol{T}^{\prime} \mathrm{gE}$. Заметим, что утверждение леммы симметрично относительно замены $\mathrm{T}^{-1} \mathrm{uT}^{\prime} \rightarrow \mathrm{T}^{\prime-1} \mathrm{u}^{-1} \mathrm{~T}$, $\mathrm{E} \rightarrow \mathrm{F}, \mathrm{F} \rightarrow \mathrm{E}$ и $\mathbf{g} \rightarrow \mathbf{g}^{-1}$. Так как из $\mathrm{F}<_{\mathrm{T}^{\prime}} \mathbf{g E}$ следует $\mathbf{g}^{-1} \mathrm{~F}<_{\mathrm{T}} \mathrm{E}$, то достаточно ограничиться рассмотрением случая, когда $g E<_{T^{\prime}} F$. Мы получим противоречие, указав сколь угодно большое число попарно различных подпутей пути Т. Более точно, индукцией по $k \geqslant 0$ мы докажем следующее утверждение:

существуют подпути $\mathrm{E}_{0} \rightleftharpoons \mathrm{E}, \mathrm{E}_{1}, \mathrm{E}_{2}, \ldots, \mathrm{E}_{k} \in \ni r_{\alpha}(\mathrm{T})$ nути $\mathrm{T}$, обладающие следующими свойствами:

(i) $\mathrm{E}_{k} \ll_{\mathrm{T}} \mathrm{E}_{k-1} \ll_{\mathrm{T}} \cdots \ll_{\mathrm{T}} \mathrm{E}_{0}$;

(ii) $Э \operatorname{Coz}_{\alpha}\left(\mathrm{g}^{i} \mathrm{E}, \mathrm{E}_{i}\right)(i=0,1, \ldots, k)$.

При $k=0$ доказывать нечего. Докажем, что из (i) и (ii) вытекает существование пути $\mathrm{E}_{k+1} \in \ni_{\alpha}(\mathrm{T})$ такого, что $\mathrm{E}_{k+1} \ll \mathrm{T} \mathrm{E}_{k}$ и $Э$ Согл $\Omega_{\alpha}\left(\mathrm{g}^{k+1} \mathrm{E}, \mathrm{E}_{k+1}\right)$. Пусть $\mathrm{T}^{\prime}=\mathrm{UFV}$ и $\mathrm{T}=\mathrm{WE}_{k} \mathrm{Z}$. Мы хотим применить предложение 11.17 при $\mathrm{X}:=\mathrm{g}^{k}(\mathrm{UF})$, $\mathrm{Y}:=\mathrm{W}_{\text {и }} \mathrm{E}:=\mathbf{g}^{k+1} \mathrm{E}$. Так как $\mathbf{g E}<\mathrm{T}^{\prime} \mathrm{F}$, то $\mathbf{g}^{k+1} \mathrm{E}<\mathbf{g}^{k} \mathbf{T}^{\prime} \mathbf{g}^{k} \mathrm{~F}$, т.е. $\mathbf{g}^{k+1} \mathrm{E}$ 
содержится в $\mathbf{g}^{k}(\mathrm{UF})$. Далее, из ЭСогл $\Omega_{\alpha}(\mathrm{E}, \mathrm{F})$ и (ii) следует $Э С о г \Omega_{\alpha}\left(\mathrm{E}_{k}, \mathbf{g}^{k} \mathrm{~F}\right)$, а из $\neg$ ЭСог, $\Omega_{\alpha}(\mathrm{gE}, \mathrm{F})$ следует $\neg Э$ Сог $\Omega_{\alpha}\left(\mathrm{g}^{k+1} \mathrm{E}, \mathrm{g}^{k} \mathrm{~F}\right)$. Тогда по лемме 11.19 конечные вершины $\iota\left(\mathrm{E}_{k}\right)$ и $\tau\left(\mathrm{g}^{k} \mathrm{~F}\right)$ путей $\mathrm{W}$ и $\mathrm{g}^{k}(\mathrm{UF})$ могут быть соединены путем $\mathbf{v} \mathrm{c}$ $\mathcal{L}(\mathrm{v}) \in \mathcal{H}_{\alpha}$, относительно которого устойчив путь $\mathrm{g}^{k+1} \mathrm{E}$. Докажем, что вершины $\iota\left(\mathbf{g}^{k}(\mathrm{UF})\right)=\iota\left(\mathbf{g}^{k+1} \mathrm{~T}\right)$ и $\iota(\mathrm{W})=\iota(\mathrm{T})$ также могут быть соединены путем $\mathrm{x} \mathrm{c}$ $\mathcal{L}(\mathrm{x}) \in \mathcal{H}_{\alpha}$, относительно которого устойчив $\mathrm{g}^{k+1} \mathrm{E}$. Имеем

$$
\begin{aligned}
\mathbf{h} \rightleftharpoons\left(\iota\left(\mathbf{g}^{k+1} \mathbf{T}\right)\right)^{-1} \iota(\mathbf{T}) & =\left((\iota(\mathbf{T}))^{-1} \mathbf{g} \iota(\mathbf{T})\right)^{-k-1} \\
& =\left((\iota(\mathbf{T}))^{-1} \iota\left(\mathbf{T}^{\prime}\right)\right)^{-k-1}=[\mathcal{L}(\mathrm{u})]_{\alpha}^{-k-1} \in \mathbf{S}_{\alpha}(A) .
\end{aligned}
$$

Пусть $A \in \overline{\mathcal{E}}_{\beta}$, где $\beta \leqslant \alpha$. В силу предложения 13.3 представим $\mathbf{h}$ словом $P w \in \mathcal{H}_{\beta}$, где $P$ - начало слова $A^{ \pm n}$ и $w \in \mathcal{H}_{\beta-1}$. Тогда вершины $\iota\left(\mathbf{g}^{k+1} \mathbf{T}\right)$ и $\iota(\mathrm{T})$ соединены путем $\mathrm{x}=\mathrm{Pw} \mathrm{c} \mathcal{L}(\mathrm{P})$ 으 $P$ и $\mathcal{L}(\mathrm{w})$ 으 $w$. Если $\beta<\alpha$, то устойчивость $\mathrm{g}^{k+1} \mathrm{E}$ относительно $\mathrm{x}$ непосредственно вытекает из определения 11.11. Пусть $\beta=\alpha$. Допустим, $\mathrm{G} \rightleftharpoons \mathrm{g}^{k+1} \mathrm{E}$ не устойчив относительно х. Согласно определению 11.11 имеем $|\mathrm{P}| \geqslant 2|A|$ и для некоторых прообразов $\overline{\mathrm{G}}$ и $\overline{\mathrm{P}}$ на графе $\Gamma_{\alpha-1}$ путей $\mathrm{G}$ и $\mathrm{P}$ и некоторого $\epsilon= \pm 1$ выполнено $\left.\operatorname{Coгл(~} \mathrm{Q}, \overline{\mathrm{P}}^{\epsilon}\right)$, где $\mathrm{Q}$ - периодическая основа для $\overline{\mathrm{G}}$. Пусть $\mathbf{f} \in \mathbf{N}_{\alpha-1}(A)$ - элемент групшы $\mathbf{B}_{\alpha-1}$, для которого $\pi_{\alpha-1, \alpha}(\mathbf{f})=[\mathcal{L}(\mathbf{u})]_{\alpha}$. По предложению 7.3 имеем $\operatorname{Coгл~}(\overline{\mathrm{P}}, \overline{\mathbf{g}} \overline{\mathrm{P}})$ или $\operatorname{Coзл}\left(\overline{\mathrm{P}}, \overline{\mathbf{g}} \overline{\mathrm{P}}^{-1}\right)$, где $\overline{\mathbf{g}}=\iota(\overline{\mathrm{P}}) \mathbf{f}(\iota(\overline{\mathrm{P}}))^{-1}$. Отсюда следует $\operatorname{Cozл}\left(\mathrm{Q}, \overline{\mathbf{g}} \mathrm{Q}^{\delta}\right)$ для некоторого $\delta= \pm 1$. Так как $\pi_{\alpha-1, \alpha}(\iota(\overline{\mathrm{P}}))=$ $\iota(\mathrm{P})=\iota(\mathbf{T})$, то $\pi_{\alpha-1, \alpha}(\overline{\mathbf{g}})=\iota(\mathbf{T})[\mathcal{L}(\mathrm{u})]_{\alpha}(\iota(\mathbf{T}))^{-1}=\iota\left(\mathbf{T}^{\prime}\right)(\iota(\mathbf{T}))^{-1}=\mathbf{g}$. Тогда согласно определениям 5.18 и 5.20 получаем $Э \operatorname{Coz}_{\alpha}(\mathrm{G}, \mathrm{gG})$ и, следовательно, $Э С о г \Omega_{\alpha}(\mathrm{E}, \mathrm{gE})$. Но по условию $Э С о г \Omega_{\alpha}(\mathrm{E}, \mathrm{F})$, и мы получаем противоречие с допушением $\neg$ ЭСогл $\Omega_{\alpha}(\mathrm{gE}, \mathrm{F})$. Мы доказали, что $\mathrm{g}^{k+1} \mathrm{E}$ устойчив относительно $\mathrm{x}$, и тем самым проверили вьполнение всех условий посылки предложения 11.17 (при $\mathrm{X}:=\mathrm{g}^{k}(\mathrm{UF}), \mathrm{u} \mathrm{Yv}:=\mathrm{xWv}$ и $\left.\mathrm{E}:=\mathrm{g}^{k+1} \mathrm{E}\right)$. В силу этого предложения найдется подпуть $\mathrm{E}_{k+1} \in \ni_{\alpha}(\mathrm{T})$ пути $\mathrm{W}$ такой, что $Э$ Согл $\Omega_{\alpha}\left(\mathrm{g}^{k+1} \mathrm{E}, \mathrm{E}_{k+1}\right)$. Так как $\mathrm{E}_{k+1} \ll \mathrm{T} \mathrm{E}_{k}$, то тем самым завершен индуктивный переход от $k$ к $k+1$, и, таким образом, (13.1) установлено для всех значений $k$. В частности, согласно (i) для любого $k$ должно существовать $k$ различных подпутей пути Т. Полученное противоречие завершает доказательство леммы.

13.9. ПреДЛОЖЕнИЕ. Для любого слова Т существует слово $T^{*}$, обладающее следующим свойством: для любых ограниченных в ранге $\alpha$ слов $u, v \in \mathcal{H}_{\alpha}$ mаких, что $[u]_{\alpha} \in \mathbf{S}_{\alpha}(A), \quad[v]_{\alpha} \in \mathbf{S}_{\alpha}(B)$ для некоторых $A, B \in \bigcup_{\beta \leqslant \alpha} \bar{\varepsilon}_{\beta}$, из $u \stackrel{\alpha}{=} T v T^{-1}$ cледует $u \stackrel{\alpha=1}{=} T^{*} v T^{*-1}$.

ДоКАЗАТЕЛЬСТВо. В силу предложения 12.13 достаточно ограничиться рассмотрением случая $T \in \mathcal{R}_{\alpha}$. Если $T$ не содержит в качестве подслов элементарных $q_{4}$-степеней ранга $\alpha$, то в силу предложения 11.3 можно положить $T^{*} \rightleftharpoons T$. Пусть $T$ содержит хотя бы одну элементарную $q_{4}$-степень ранга $\alpha$. Рассмотрим путь $T$ на графе $\Gamma_{\alpha-1}$ с $\mathcal{L}(\mathrm{T})$ 으 $T$ и, используя предложение 8.38 , (ii), найдем подпути $\mathrm{E}_{1}, \mathrm{E}_{2}, \ldots, \mathrm{E}_{t} \in$ Эл $_{\alpha}\left(\mathrm{T}, q_{4}\right)$, удовлетворяющие следуюшим условиям:

(i) $\neg \Pi$ Согл $\Omega_{\alpha}\left(\mathrm{E}_{i}, \mathrm{E}_{j}\right)$ при $i \neq j$ и $\mathrm{E}_{1}<\mathrm{T} \mathrm{E}_{2}<\mathrm{T} \cdots<\mathrm{T}_{t}$;

(ii) если $\mathrm{E} \in$ Эл $_{\alpha}\left(\mathrm{T}, q_{4}\right)$, то ПСогл $\Omega_{\alpha}\left(\mathrm{E}_{i}, \mathrm{E}\right)$ для некоторого $i$. 
Выберем для каждого $\mathrm{E}_{i}$ периодическую основу $\mathrm{N}_{i} \in \prod_{\text {ер }}\left(\Gamma_{\alpha-1}, C_{i}, 2\right)$, где $C_{i} \in \mathcal{E}_{\alpha}$. Без ограничения обшности можно считать, что слово $\mathcal{L}\left(\mathrm{N}_{i}\right)$ начинается с $C_{i}$. Пусть $\mathrm{X}_{i} \mathrm{E}_{i}-$ начало пути $\mathrm{T}$, и пусть $\mathrm{f}_{i}-$ произвольный путь, соединяюший вершины $\iota\left(\mathrm{E}_{i}\right)$ и $\iota\left(\mathrm{N}_{i}\right)$. Обозначим $X_{i} \rightleftharpoons \mathcal{L}\left(\mathrm{X}_{i}\right)$ и $f_{i} \rightleftharpoons \mathcal{L}\left(\mathrm{f}_{i}\right)$. Мы докажем, что слово

$$
T^{*} \rightleftharpoons\left(\prod_{i=1}^{t} X_{i} f_{i} C_{i}^{n / 2} f_{i}^{-1} X_{i}^{-1}\right) T
$$

является искомым.

Пусть слова $u, v \in \mathcal{H}_{\alpha}$ таковы, как указано в условии предложения, т.е. $[u]_{\alpha} \in$ $\mathbf{S}_{\alpha}(A),[v]_{\alpha} \in \mathbf{S}_{\alpha}(B)$ для некоторых $A, B \in \bigcup_{\beta \leqslant \alpha} \bar{\varepsilon}_{\beta}, u$ и $v$ ограничены в ранге $\alpha$ и имеет место равенство $u \stackrel{\alpha}{=} T v T^{-1}$. Нам нужно доказать соотношение

$$
u \stackrel{\alpha=1}{=}\left(\prod_{i=1}^{t} X_{i} f_{i} C_{i}^{n / 2} f_{i}^{-1} X_{i}^{-1}\right) T v T^{-1}\left(\prod_{i=1}^{t} X_{i} f_{i} C_{i}^{n / 2} f_{i}^{-1} X_{i}^{-1}\right)^{-1} .
$$

По предложению 11.2 при $X:=Y:=T, u:=u$ и $v:=v^{-1}$ либо имеет место равенство

$$
T \stackrel{\alpha=1}{=} u T v^{-1}
$$

либо для некоторого $r \geqslant 1$ сушествуют слова $K_{i}, L_{i}(i=0,1, \ldots, r), G_{i}, H_{i}, S_{i}, U_{i}$, $T_{i}, V_{i}$, элементарные периоды $D_{i} \in \mathcal{E}_{\alpha}$ и слова $x_{i}, y_{i}, z_{i}, w_{i} \in \mathcal{H}_{\alpha-1}(i=1,2, \ldots, r)$ такие, что выполнены соотношения:

$$
\begin{gathered}
T \text { 으 } K_{0} G_{1} K_{1} \ldots G_{r} K_{r} \text { 으 } L_{0} H_{1} L_{1} \ldots H_{r} L_{r}, \\
S_{i}^{-1} U_{i} T_{i} V_{i} \approx D_{i}^{n} \quad(i=1,2, \ldots, r), \\
G_{i} \stackrel{\alpha=1}{=} x_{i} S_{i} y_{i}, \quad H_{i} \stackrel{\alpha=1}{=} z_{i} T_{i} w_{i} \quad(i=1,2, \ldots, r), \\
K_{0} \stackrel{\alpha=1}{=} u L_{0} z_{1} U_{1}^{-1} x_{1}^{-1}, \\
K_{i} \stackrel{\alpha=1}{=} y_{i}^{-1} V_{i}^{-1} w_{i} L_{i} z_{i+1} U_{i+1}^{-1} x_{i+1}^{-1} \quad(i=1,2, \ldots, r-1), \\
K_{r} \stackrel{\alpha=1}{=} y_{r}^{-1} V_{r}^{-1} w_{r} L_{r} v^{-1}, \\
\left|S_{i}\right|,\left|T_{i}\right|>(q-2 p-32)\left|D_{i}\right| \quad(i=1,2, \ldots, r) .
\end{gathered}
$$

Будем считать $r=0$ в случае, когда имеет место равенство (13.4). Согласно (13.5) представим Т в виде

$$
\mathrm{T}=\mathrm{K}_{0} \mathrm{G}_{1} \mathrm{~K}_{1} \ldots \mathrm{G}_{r} \mathrm{~K}_{r}, \quad \mathcal{L}\left(\mathrm{K}_{i}\right) \text { 으 } K_{i}, \quad \mathcal{L}\left(\mathrm{G}_{i}\right) \text { 으 } L_{i} .
$$

Согласно равенствам (13.7) для каждого $i$ выберем путь $\mathrm{x}_{i} \mathrm{~S}_{i} \mathrm{y}_{i}$ с теми же, что и у пути $\mathrm{G}_{i}$, начальной и конечной вершинами, где метки путей $\mathrm{x}_{i}, \mathrm{~S}_{i}$ и $\mathrm{y}_{i}$ равны соответственно $x_{i}, S_{i}$ и $y_{i}$. Так как пути $\mathrm{G}_{i}$ и $\mathrm{S}_{i}$ близки в ранге $\alpha-1$ и в силу (13.6), (13.11) имеем $\mathrm{S}_{i} \in \Pi_{\mathrm{ep}}\left(\Gamma_{\alpha-1}, D_{i}^{-1}, q-2 p-32\right)$, то по предложению 8.23 найдется подпуть $\mathrm{G}_{i}^{*} \in$ ППер $\alpha\left(D_{i}^{-1}, q_{4}\right)$ пути $\mathrm{G}_{i}$ с периодической основой 
$\mathrm{S}_{i}^{*} \in \Pi_{\mathrm{ep}}\left(\Gamma_{\alpha-1}, D_{i}^{-1}, 2\right)$, содержащейся в $\mathrm{S}_{i}$. В силу условия (ii) для каждого $i$ найдется индекс $k_{i}$ такой, что ПСог $\Omega_{\alpha}\left(\mathrm{G}_{i}^{*}, \mathrm{E}_{k_{i}}\right)$. Заметим, что $\neg \Pi C о г \Omega_{\alpha}\left(\mathrm{G}_{i}^{*}, \mathrm{G}_{j}^{*}\right)$ при $i \neq j$, так как иначе, воспользовавшись предложениями 8.35 и 8.11, мы получили бы противоречие с приведенностью слова $T$ в ранге $\alpha$ и леммой 12.12 . Тогда из (i) и предложения 8.39 следует $k_{1}<k_{2}<\cdots<k_{r}$. Обозначим

$$
\begin{gathered}
Z_{0} \rightleftharpoons u, \\
Z_{i} \rightleftharpoons K_{0} G_{1} \ldots K_{i-1} G_{i} y_{i}^{-1} V_{i}^{-1} w_{i} H_{i}^{-1} L_{i-1}^{-1} \ldots H_{1}^{-1} L_{0}^{-1} \quad(i=1,2, \ldots, r) .
\end{gathered}
$$

Докажем соотношения

$$
\begin{gathered}
X_{i} f_{i} C_{i}^{-n / 2} f_{i}^{-1} X_{i}^{-1} Z_{j} X_{i} f_{i} C_{i}^{n / 2} f_{i}^{-1} X_{i}^{-1} \stackrel{\alpha=1}{=} Z_{j}, \\
\left\{\begin{array}{c}
j=0, \quad i=1,2, \ldots, t, \\
j=0, \quad i=1,2, \ldots, k_{1}-1, \\
1 \leqslant j \leqslant r-1, \quad i=k_{j}+1, k_{j}+2, \ldots, k_{j+1}-1, \\
j=r, \quad i=k_{r}+1, k_{r}+2, \ldots, t
\end{array}\right\} \text { если } r>0, \\
X_{i} f_{i} C_{i}^{-n / 2} f_{i}^{-1} X_{i}^{-1} Z_{j} X_{i} f_{i} C_{i}^{n / 2} f_{i}^{-1} X_{i}^{-1} \stackrel{\alpha=1}{=} Z_{j+1} \\
\left(0 \leqslant j \leqslant r-1, i=k_{j+1}\right) .
\end{gathered}
$$

Заметим, что на основе (13.12) и (13.13) легко выводится искомое равенство (13.3). Действительно, из (13.12), (13.13) и $Z_{0}$ 드 $u$ следует

$$
\left(\prod_{i=1}^{t} X_{i} f_{i} C_{i}^{n / 2} f_{i}^{-1} X_{i}^{-1}\right)^{-1} u\left(\prod_{i=1}^{t} X_{i} f_{i} C_{i}^{n / 2} f_{i}^{-1} X_{i}^{-1}\right) \stackrel{\alpha=1}{=} Z_{r}
$$

и для вывода (13.3) нужно лишь заметить, что в случае $r=0$ из (13.4) следует $Z_{0} \stackrel{\alpha=1}{=} T v T^{-1}$, а в случае $r>0$ из (13.5), (13.10) следует $Z_{r} \stackrel{\alpha=1}{=} T v T^{-1}$.

В зависимости от условий на $j$ и $r$ возможны четыре случая:

(a) $j=0, r=0$,

(b) $j=0, r>0$,

(c) $1 \leqslant j \leqslant r-1, r>0$,

(d) $j=r, r>0$.

В этих случаях доказательство равенств (13.12) и (13.13) различается незначительно (а случаи (a) и (b) отпадают при доказательстве (13.13)), и мы проведем подробные рассуждения лишь в случае (c), имеюшем наиболее обший вид.

Итак, пусть $1 \leqslant j \leqslant r-1$ и $r>0$. В соответствии с равенствами (13.7), (13.9) рассмотрим пути на графе $\Gamma_{\alpha-1}$, изображенные на рис. 61 , обозначенные теми же буквами, что и их метки. Обозначим $\mathrm{T}^{\prime} \rightleftharpoons \mathrm{L}_{0} \mathrm{H}_{1} \mathrm{~L}_{1} \ldots \mathrm{H}_{r} \mathrm{~L}_{r}$. Заметим, что из (13.6)-(13.9) легко вытекает $Z_{j} \stackrel{\alpha}{=} u$. Отсюда следует, что начальные вершины путей $\pi_{\alpha-1, \alpha}(\mathrm{T})$ и $\pi_{\alpha-1, \alpha}\left(\mathbf{T}^{\prime}\right)$ соединены некоторым путем иิ с $\mathcal{L}(\hat{\mathrm{u}})$ 으 $u$ 


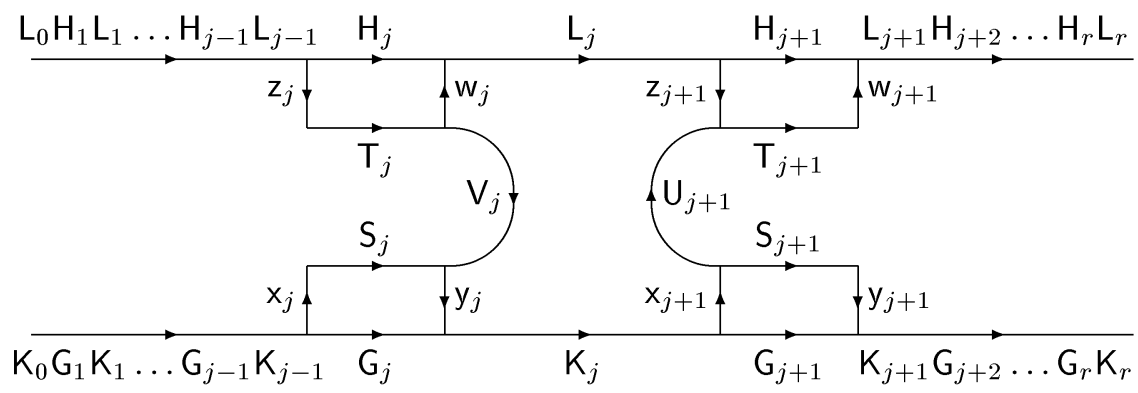

Рис. 61

и, таким образом, мы можем пользоваться леммой 13.8 при $\mathbf{T}:=\pi_{\alpha-1, \alpha}(\mathrm{T})$ и $\mathrm{T}^{\prime}:=\pi_{\alpha-1, \alpha}\left(\mathrm{T}^{\prime}\right)$.

Перейдем непосредственно к доказательству соотношений (13.12) и (13.13). Зафиксируем $i$ в интервале $k_{j}+1 \leqslant i \leqslant k_{j+1}-1$. Из ПСогл $\Omega_{\alpha}\left(\mathrm{G}_{j}^{*}, \mathrm{E}_{k_{j}}\right)$, ПСогл $\alpha\left(\mathrm{G}_{j+1}^{*}, \mathrm{E}_{k_{j+1}}\right)$, (i) и предложения 8.39 следует $\mathrm{G}_{j}^{*}<\mathrm{T} \mathrm{E}_{i}<\mathrm{T} \mathrm{G}_{j+1}^{*}$, т.е.

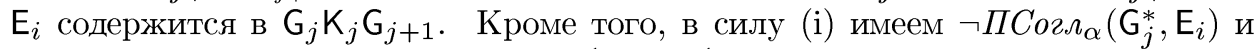
$\neg \Pi$ Согл $\Omega_{\alpha}\left(\mathrm{G}_{j+1}^{*}, \mathrm{E}_{i}\right)$, и так как $\mathrm{S}_{j} \mathrm{~V}_{j}^{-1}$ и $\mathrm{U}_{j+1}^{-1} \mathrm{~S}_{j+1}-$ периодические продолжения для $\mathrm{S}_{j}^{*}$ и $\mathrm{S}_{j+1}^{*}$, то отсюда следует $\neg \operatorname{Coгл~}\left(\mathrm{S}_{j} \mathrm{~V}_{j}^{-1}, \mathrm{~N}_{i}\right)$ и $\neg \operatorname{Coгл}\left(\mathrm{U}_{j+1}^{-1} \mathrm{~S}_{j+1}, \mathrm{~N}_{i}\right)$. По лемме 11.10 найдется подпуть $\mathrm{F} \in$ ППер $_{\alpha}\left(C_{i}, q_{4}-h\right)$ пути $\mathrm{L}_{j}$ такой, что ПСогл $\alpha\left(\mathrm{E}_{i}, \mathrm{~F}\right)$. Так как ЭСогл $\Omega_{\alpha}\left(\pi_{\alpha-1, \alpha}\left(\mathrm{E}_{i}\right), \pi_{\alpha-1, \alpha}(\mathrm{F})\right)$, то по лемме 13.8 имеем ЭСогл $\Omega_{\alpha}\left(\pi_{\alpha-1, \alpha}\left(\mathrm{gE}_{i}\right), \pi_{\alpha-1, \alpha}(\mathrm{F})\right)$, где $\mathbf{g} \rightleftharpoons \iota\left(\mathrm{T}^{\prime}\right)(\iota(\mathbf{T}))^{-1}$ - элемент группы $\mathbf{B}_{\alpha-1}$, для которого $\mathrm{T}^{\prime}=\mathrm{g} \mathrm{T}$. Так как $\mathrm{gE}_{i}$ и $\mathrm{F}_{i}-$ подпути пути $\mathrm{T}^{\prime}$, то в силу предложений 11.24 и 8.46 получаем ПСогл $\left(\mathrm{F}_{,} \mathrm{gE}_{i}\right)$ и, следовательно, ПСогл ${ }_{\alpha}\left(\mathrm{E}_{i}, \mathrm{gE}_{i}\right)$. Так как $\mathrm{gN}_{i}-$ периодическая основа для $\mathbf{g} \mathrm{E}_{i}$ и слово $\mathcal{L}\left(\mathrm{N}_{i}\right)$ начинается с $C_{i}$, то по предложению 7.3

$$
\left(\iota\left(\mathrm{N}_{i}\right)\right)^{-1} \iota\left(\mathrm{gN}_{i}\right) \in \mathbf{N}_{\alpha-1}^{+}\left(C_{i}\right) .
$$

Теперь заметим, что $\left(\iota\left(\mathrm{N}_{i}\right)\right)^{-1} \iota\left(\mathbf{g N}_{i}\right)=[\mathcal{L}(\mathrm{W})]_{\alpha-1}$, где $\mathrm{W}$ - произвольный путь, соединяюший вершины $\iota\left(\mathrm{N}_{i}\right)$ и $\iota\left(\mathrm{gN}_{i}\right)$. Пусть

$$
\mathrm{Z}_{j} \rightleftharpoons \mathrm{K}_{0} \mathrm{G}_{1} \ldots \mathrm{K}_{j-1} \mathrm{G}_{j} \mathrm{y}_{j}^{-1} \mathrm{~V}_{j}^{-1} \mathrm{w}_{j} \mathrm{H}_{j}^{-1} \mathrm{~L}_{j-1}^{-1} \ldots \mathrm{H}_{1}^{-1} \mathrm{~L}_{0}^{-1}
$$

Рассматривая в качестве $\mathrm{W}_{\text {путь }} \mathrm{f}_{i}^{-1} \mathrm{X}_{i}^{-1} \mathrm{Z}_{j}\left(\mathrm{gX}_{i}\right)\left(\mathrm{gf}_{i}\right)$ и учитывая, что $\mathcal{L}\left(\mathrm{Z}_{j}\right)$ 으 $Z_{j}$, получаем

$$
\left(\iota\left(\mathrm{N}_{i}\right)\right)^{-1} \iota\left(\mathrm{gN}_{i}\right)=\left[f_{i}^{-1} X_{i}^{-1} Z_{j} X_{i} f_{i}\right]_{\alpha-1} .
$$

Следовательно, $\left[f_{i}^{-1} X_{i}^{-1} Z_{j} X_{i} f_{i}\right]_{\alpha-1} \in \mathbf{N}_{\alpha-1}^{+}\left(C_{i}\right)$. По предложению 7.18 элемент $\left[C_{i}\right]_{\alpha-1}^{n / 2}$ лежит в центре подгруппы $\mathbf{N}_{\alpha-1}^{+}\left(C_{i}\right)$. Поэтому

$$
C_{i}^{-n / 2} f_{i}^{-1} X_{i}^{-1} Z_{j} X_{i} f_{i} C_{i}^{n / 2} \stackrel{\alpha=1}{=} f_{i}^{-1} X_{i}^{-1} Z_{j} X_{i} f_{i}
$$

что и доказывает (13.12). 
Докажем (13.13). Из соотношений (13.7) при $i:=j+1$ и (13.9) при $i:=j$ вытекает

$$
\begin{aligned}
Z_{j+1} Z_{j}^{-1} \stackrel{\alpha=1}{=} & K_{0} G_{1} K_{1} \ldots G_{j} K_{j} x_{j+1} \cdot S_{j+1} V_{j+1}^{-1} T_{j+1}^{-1} U_{j+1}^{-1} \\
& \times x_{j+1}^{-1} K_{j}^{-1} G_{j}^{-1} \ldots K_{1}^{-1} G_{1}^{-1} K_{0}^{-1}
\end{aligned}
$$

Следовательно, с учетом $(13.6)$, считая, что слово $S_{j+1}$ начинается с $D_{j+1}^{-1}$, имеем

$$
Z_{j+1} Z_{j}^{-1} \stackrel{\alpha=1}{=} K_{0} G_{1} K_{1} \ldots G_{j} K_{j} x_{j+1} D_{j+1}^{-n} x_{j+1}^{-1} K_{j}^{-1} G_{j}^{-1} \ldots K_{1}^{-1} G_{1}^{-1} K_{0}^{-1} .
$$

Обозначим $i:=k_{j+1}$. Из ПСогл $\Omega_{\alpha}\left(\mathrm{G}_{j+1}^{*}, \mathrm{E}_{i}\right)$ следует $\operatorname{Coгл}\left(\mathrm{S}_{j+1}^{*}, \mathrm{~N}_{i}\right)$, а значит, и $\operatorname{Coгл~}\left(\mathrm{S}_{j+1}, \mathrm{~N}_{i}\right)$. Пусть $\mathrm{U}$ - произвольный путь, соединяющий вершины $\iota\left(\mathrm{N}_{i}\right)$ и $\iota\left(\mathrm{S}_{j+1}\right)$. Так как слова $\mathcal{L}\left(\mathrm{N}_{i}\right)$ и $\mathcal{L}\left(\mathrm{S}_{j+1}\right)$ начинаются с $C_{i}$ и $D_{j+1}^{-1}$, то из Согл $\left(\mathrm{S}_{j+1}, \mathrm{~N}_{i}\right)$ следует, что для некоторого $t>0$

$$
\mathcal{L}(\mathrm{U}) D_{j+1}^{-t} \mathcal{L}(\mathrm{U})^{-1} \stackrel{\alpha=1}{=} C_{i}^{t} .
$$

Тогда имеем $\left[\mathcal{L}(\mathrm{U}) D_{j+1}^{-1} \mathcal{L}(\mathrm{U})^{-1}\right]_{\alpha-1} \in \mathrm{N}_{\alpha-1}^{+}\left(C_{i}\right)$, и в силу предложения 7.10, (iii), (iv) $\operatorname{ind}_{C_{i}}\left[\mathcal{L}(\mathrm{U}) D_{j+1}^{-1} \mathcal{L}(\mathrm{U})^{-1}\right]_{\alpha-1}=1$. По предложению 7.18, (i)

$$
\mathcal{L}(\mathrm{U}) D_{j+1}^{-n} \mathcal{L}(\mathrm{U})^{-1} \stackrel{\alpha=1}{=} C_{i}^{n}
$$

Рассматривая теперь в качестве $\mathrm{U}$ путь $\mathrm{f}_{i}^{-1} \mathrm{X}_{i}^{-1} \mathrm{~K}_{0} \mathrm{G}_{1} \mathrm{~K}_{1} \ldots \mathrm{G}_{j} \mathrm{~K}_{j} \mathrm{x}_{j+1}$, получаем

$$
f_{i}^{-1} X_{i}^{-1} K_{0} G_{1} K_{1} \ldots G_{j} K_{j} x_{j+1} D_{j+1}^{-n} x_{j+1}^{-1} K_{j}^{-1} G_{j}^{-1} \ldots K_{1}^{-1} G_{1}^{-1} K_{0}^{-1} X_{i} f_{i} \stackrel{\alpha=1}{=} C_{i}^{n},
$$

после чего, подставив в (13.15), находим, что

$$
Z_{j+1} Z_{j}^{-1} \stackrel{\alpha=1}{=} X_{i} f_{i} C_{i}^{n} f_{i}^{-1} X_{i}^{-1}
$$

По предложению 8.23 найдем подпуть $\mathrm{H}_{j+1}^{*} \in$ ППер $\alpha\left(D_{j+1}, q_{4}\right)$ пути $\mathrm{H}_{j+1}$ с периодической основой $\mathrm{T}_{j+1}^{*} \in \Pi е \mathrm{p}\left(\Gamma_{\alpha-1}, D_{j+1}, 2\right)$, содержащейся в $\mathrm{T}_{j+1}$. Так как периодические основы $\left(\mathrm{S}_{j+1}^{*}\right)^{-1}$ и $\mathrm{T}_{j+1}^{*}$ для $\left(\mathrm{G}_{j+1}^{*}\right)^{-1}$ и $\mathrm{F}_{j+1}^{*}$ имеют общее периодическое продолжение $\mathrm{S}_{j+1}^{-1} \mathrm{U}_{j+1} \mathrm{~T}_{j+1}$ (см. рис. 61), то ПСогл $\alpha\left(\left(\mathrm{G}_{j+1}^{*}\right)^{-1}, \mathrm{~F}_{j+1}^{*}\right)$, и, следовательно, ЭСогл $\left.\Omega_{\alpha-1, \alpha}\left(\mathrm{G}_{j+1}^{*}\right), \pi_{\alpha-1, \alpha}\left(\mathrm{F}_{j+1}^{*}\right)\right)$. Тогда по лемме 13.8 имеем $Э С о г \Omega_{\alpha}\left(\pi_{\alpha-1, \alpha}\left(\mathrm{gG}_{j+1}^{*}\right), \pi_{\alpha-1, \alpha}\left(\mathrm{F}_{j+1}^{*}\right)\right)$, откуда в силу предложений 11.24 и 8.46 вытекает ПСогл $\Omega_{\alpha}\left(\mathrm{gG}_{j+1}^{*}, \mathrm{~F}_{j+1}^{*}\right)$. Так как $П С о \Omega_{\alpha}\left(\left(\mathrm{G}_{j+1}^{*}\right)^{-1}, \mathrm{~F}_{j+1}^{*}\right)$ и $\Pi$ Погл $\Omega_{\alpha}\left(\mathrm{G}_{j+1}^{*}, \mathrm{E}_{i}\right)$, то отсюда следует ПСогл $\Omega_{\alpha}\left(\mathrm{gE}_{i}, \mathrm{E}_{i}^{-1}\right)$. Тогда по предложению 7.3

$$
\left(\iota\left(\mathrm{N}_{i}\right)\right)^{-1} \iota\left(\mathbf{g N}_{i}\right) \in \mathbf{N}_{\alpha-1}^{-}\left(C_{i}\right)
$$


Воспользовавшись полученным вьше равенством (13.14) (которое сохраняет силу и при $\left.i=k_{j+1}\right)$, получаем

$$
\left[f_{i}^{-1} X_{i}^{-1} Z_{j} X_{i} f_{i}\right]_{\alpha-1} \in \mathbf{N}_{\alpha-1}^{-}\left(C_{i}\right) .
$$

Отсюда по предложению 7.18, (iii) следует

$$
C_{i}^{n / 2} f_{i}^{-1} X_{i}^{-1} Z_{j} X_{i} f_{i} \stackrel{\alpha=1}{=} f_{i}^{-1} X_{i}^{-1} Z_{j} X_{i} f_{i} C_{i}^{-n / 2}
$$

или, вместе с (13.16),

$$
Z_{j+1} \stackrel{\alpha=1}{=} X_{i} f_{i} C_{i}^{n / 2} f_{i}^{-1} X_{i}^{-1} Z_{j} X_{i} f_{i} C_{i}^{-n / 2} f_{i}^{-1} X_{i}^{-1}
$$

что и требовалось. Мы доказали соотношения (13.12) и (13.13) в случае (c). В остальных случаях (a), (b) и (d) рассуждения повторяются почти дословно, с той лишь модификацией, что вместо одного или обоих путей $\mathrm{x}_{j} \mathrm{~S}_{j} \mathrm{~V}_{j}^{-1} \mathrm{w}_{j}$ и $\mathrm{z}_{j+1} \mathrm{U}_{j+1}^{-1} \mathrm{~S}_{j+1} \mathrm{y}_{j+1}$ на рис. 61 будут пути и или соответственно $\mathrm{v}^{-1}$, где $\mathcal{L}(\mathrm{u})$ 으 $u$ и $\mathcal{L}(\mathrm{v})$ 으 $v$, и при этом пути $\mathrm{G}_{j}, \mathrm{H}_{j}$ или соответственно $\mathrm{G}_{j+1}, \mathrm{H}_{j+1}$ будут отсутствовать.

13.10. ПРЕДЛОЖЕНИЕ. Пусть $A^{t} w$ u $B^{s} v$ - нормальные формы рангов $\alpha u$ $\beta \leqslant \alpha$ соответственно, $T \in \mathcal{R}_{\alpha} u A^{t} w \stackrel{\alpha}{=} T B^{s} v T^{-1}$. Тогда $\alpha=\beta u A^{t^{\prime} k} \stackrel{\alpha=1}{=}$ $T B^{s^{\prime} k} T^{-1}$ для некоторых $k>0 u t^{\prime}, s^{\prime}$, где $t^{\prime} \equiv t, s^{\prime} \equiv s(\bmod n) u\left|t^{\prime}\right|=\left|s^{\prime}\right|$.

ДокАЗАТЕЛЬСтво. Применим предложение 11.2 при $X:=Y:=T, u_{1}:=1$, $Q:=A^{t}, u_{2}:=w, v_{1}:=v^{-1}, R:=B^{-s}$ и $v_{2}:=1$. Согласно этому предложению либо $T \stackrel{\alpha=1}{=} A^{t^{\prime}} w T v^{-1} B^{-s^{\prime}}$, где $t^{\prime}=t-\epsilon n, s^{\prime}=s-\delta n$ для некоторых $\delta, \epsilon \in\{0,1\}$, либо имеет место утверждение 11.2 , (ii), т.е. для некоторого $r \geqslant 1$ существуют слова $K_{i}, L_{i}(i=0,1, \ldots, r), G_{i}, H_{i}, S_{i}, U_{i}, T_{i}, V_{i}$, элементарные периоды $D_{i} \in \mathcal{E}_{\alpha}$ и слова $x_{i}, y_{i}, z_{i}, w_{i} \in \mathcal{H}_{\alpha-1} \quad(i=1,2, \ldots, r)$, удовлетворяюшие соотношениям

$$
\begin{gathered}
T \text { 으 } K_{0} G_{1} K_{1} \ldots G_{r} K_{r} \text { 으 } L_{0} H_{1} L_{1} \ldots H_{r} L_{r}, \\
S_{i}^{-1} U_{i} T_{i} V_{i} \approx D_{i}^{n} \quad(i=1,2, \ldots, r), \\
G_{i} \stackrel{\alpha=1}{=} x_{i} S_{i} y_{i}, \quad H_{i} \stackrel{\alpha=1}{=} z_{i} T_{i} w_{i} \quad(i=1,2, \ldots, r), \\
K_{0} \stackrel{\alpha=1}{=} A^{t^{\prime}} w L_{0} z_{1} U_{1}^{-1} x_{1}^{-1}, \\
K_{i} \stackrel{\alpha=1}{=} y_{i}^{-1} V_{i}^{-1} w_{i} L_{i} z_{i+1} U_{i+1}^{-1} x_{i+1}^{-1} \quad(i=1,2, \ldots, r-1), \\
K_{r} \stackrel{\alpha=1}{=} y_{r}^{-1} V_{r}^{-1} w_{r} L_{r} v^{-1} B^{-s^{\prime}}, \\
\left|S_{i}\right|,\left|T_{i}\right|>(q-2 p-32)\left|D_{i}\right| \quad(i=1,2, \ldots, r),
\end{gathered}
$$

где $t^{\prime}$ и $s^{\prime}$ таковы, как указано выше. Предположим, имеет место первый случай $T \stackrel{\alpha=1}{=} A^{t^{\prime}} w T v^{-1} B^{-s^{\prime}}$, т.е. $A^{t^{\prime}} w^{\alpha=1}=T B^{s^{\prime}} v T^{-1}$. Так как $\left[A^{t^{\prime}} w\right]_{\alpha-1} \in \mathbf{N}_{\alpha-1}^{+}(A)$, 
$\operatorname{ind}_{A}\left(\left[A^{t^{\prime}} w\right]_{\alpha-1}\right)=t^{\prime},\left[B^{s^{\prime}} v\right]_{\beta-1} \in \mathbf{N}_{\beta-1}^{+}(B)$ и $\operatorname{ind}_{B}\left(\left[B^{s^{\prime}} v\right]_{\beta-1}\right)=s^{\prime}$ в силу определения 13.4 и предложения 7.10 , (iii), то в силу предложения 7.10 , (i) для некоторого $k>0$ имеем $A^{t^{\prime} k} \stackrel{\alpha=1}{=} T B^{s^{\prime} k} T^{-1}$. Тогда из бесконечности порядка элемента $[A]_{\alpha-1}$ и конечности порядка элемента $[B]_{\alpha-1}$ при $\beta \leqslant \alpha-1$ вытекает $\alpha=\beta$. Кроме того, так как $[A]_{\alpha-1} \in[T]_{\alpha-1} \mathbf{N}_{\alpha-1}^{+}(B)[T]_{\alpha-1}^{-1}$, то, используя предложение $7.10,(\mathrm{i})$, получаем $A^{i} \stackrel{\alpha=1}{=} T B^{i j} T^{-1}$ для некоторых $i, j \neq 0$, откуда легко выводим, что $s^{\prime}$ кратно $t^{\prime}$. Аналогично доказывается, что $t^{\prime}$ кратно $s^{\prime}$, т.е. $\left|t^{\prime}\right|=\left|s^{\prime}\right|$, и, следовательно, выполнены все условия заключения предложения 13.10.

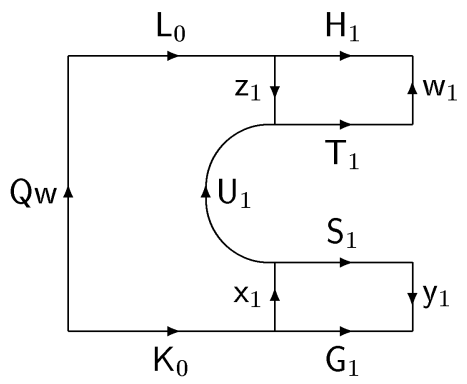

Рис. 62

Таким образом, остается доказать невозможность случая 11.2, (ii). Допустим, этот случай имеет место. В соответствии с равенствами (13.19) при $i=1$ и (13.20) рассмотрим пути $\mathrm{K}_{0}, \mathrm{G}_{1}, \mathrm{x}_{1}, \mathrm{~S}_{1}, \mathrm{y}_{1}, \mathrm{Q}$, w, $\mathrm{L}_{0}, \mathrm{z}_{1}, \mathrm{U}_{1}, \mathrm{H}_{1}, \mathrm{~T}_{1}, \mathrm{w}_{1}$ на графе $\Gamma_{\alpha-1}$ с метками соответственно $K_{0}, G_{1}, x_{1}, S_{1}, y_{1}, A^{t^{\prime}}, w, L_{0}, z_{1}, U_{1}, H_{1}$, $T_{1}$ и $w_{1}$, расположенные, как указано на рис. 62 . В силу (13.17) можно считать, что $\mathrm{K}_{0} \mathrm{G}_{1}$ есть начало пути $\mathrm{T}$ и $\mathrm{L}_{0} \mathrm{H}_{1}$ есть начало пути $\mathrm{T}^{\prime}$ с $\mathcal{L}(\mathrm{T})$ 으 $\mathcal{L}\left(\mathrm{T}^{\prime}\right)$ 으 $T$. Используя соотношения (13.18), (13.23) и предложение 8.23, найдем подпути $\mathrm{G}^{*}$ и $\mathrm{H}^{*}$ путей $\mathrm{G}_{1}$ и $\mathrm{H}_{1}$ такие, что $\mathrm{G}^{*} \in$ ППер $\alpha\left(D_{1}^{-1}, q_{4}\right), \mathrm{H}^{*} \in \Pi$ Пер $\alpha\left(D_{1}, q_{4}\right)$ и периодические основы $\mathrm{S}^{*}$ для $\mathrm{G}^{*}$ и $\mathrm{T}^{*}$ для $\mathrm{H}^{*}$ содержатся в $\mathrm{S}_{1}$ и в $\mathrm{T}_{1}$ соответственно. Заменяя слово $D_{1}$ на его циклический сдвиг, можно считать, что слово $\mathcal{L}\left(\mathrm{S}^{*}\right)$ начинается с $D_{1}^{-1}$. Так как в силу (13.18) при $i:=1$ пути $\mathrm{S}^{*-1}$ и $\mathrm{T}^{*}$ имеют общее периодическое продолжение $\mathrm{S}_{1}^{-1} \mathrm{U}_{1} \mathrm{~T}_{1}$, то ПСогл $\alpha\left(\mathrm{G}^{*-1}, \mathrm{H}^{*}\right)$ и, следовательно, ЭСогл $\alpha\left(\pi_{\alpha-1, \alpha}\left(\mathrm{G}^{*}\right), \pi_{\alpha-1, \alpha}\left(\mathrm{H}^{*}\right)\right)$. По лемме 13.8 при $\mathbf{T}^{-1} \mathbf{u T}^{\prime}:=$ $\pi_{\alpha-1, \alpha}\left(\mathbf{T}^{-1} \mathbf{Q} \mathbf{w} \mathbf{T}^{\prime}\right)$ получаем $Э \operatorname{Coz}_{\alpha}\left(\pi_{\alpha-1, \alpha}\left(\mathbf{g} \mathbf{G}^{*}\right), \pi_{\alpha-1, \alpha}\left(\mathbf{H}^{*}\right)\right)$, где

$$
\mathbf{g} \rightleftharpoons \iota\left(\mathbf{T}^{\prime}\right)(\iota(\mathbf{T}))^{-1}=\iota(\mathbf{T})\left[A^{t^{\prime}} w\right]_{\alpha-1}(\iota(\mathbf{T}))^{-1}
$$

- элемент группы $\mathbf{B}_{\alpha-1}$, для которого $\mathbf{T}^{\prime}=\mathbf{g} \mathbf{T}$. Тогда в силу предложений 11.24 и 8.46 имеем ПСогл $\Omega_{\alpha}\left(\mathbf{g G}^{*}, \mathrm{H}^{*}\right)$ и, следовательно, ПСогл $\Omega_{\alpha}\left(\mathbf{g G}^{*}, \mathrm{G}^{*-1}\right)$. По предложению 7.3

$$
\left(\iota\left(\mathrm{S}^{*}\right)\right)^{-1} \mathbf{g} \iota\left(\mathrm{S}^{*}\right) \in \mathbf{N}_{\alpha-1}^{-}\left(D_{1}\right),
$$

откуда в силу следствия 7.17 и предложения 7.14 , (ii) вытекает, что $\mathbf{g}$ имеет конечньй порядок. Но $\mathbf{g}$ сопряжен элементу $\left[A^{t^{\prime}} w\right]_{\alpha-1} \in \mathbf{N}_{\alpha-1}^{+}(A) \operatorname{cind}_{A}\left(\left[A^{t^{\prime}} w\right]_{\alpha-1}\right)=$ $t^{\prime} \neq 0$, который в силу предложения $7.10,(\mathrm{i})$ и леммы 6.7 имеет бесконечный порядок. Получили противоречие. Предложение доказано. 
13.11. СлЕДСТвИЕ. Если $A \in \mathcal{E}_{\alpha}, \mathbf{g}, \mathbf{h} \in \mathbf{S}_{\alpha}^{+}(A) \backslash \mathbf{S}_{\alpha}^{*}(A) u \mathbf{g}=\mathbf{f h f}^{-1}, m o$ $\mathbf{f} \in \mathbf{S}_{\alpha}(A)$.

ДокАЗАТЕЛЬСтво. Заменяя элементарный период $A$ на его циклический сдвиг и используя лемму 7.20 , можно считать $A \in \bar{\varepsilon}_{\alpha}$. Используя предложения 13.3 и 12.13 , представим элементы $\mathbf{g}$ и $\mathbf{h}$ нормальными формами $A^{t} w$ и $A^{s} v$ ранга $\alpha$, а элемент $\mathbf{f}$ - словом $T \in \mathcal{R}_{\alpha}$. Так как $A^{t} w \stackrel{\alpha}{=} T A^{s} v T^{-1}$, то по предложению 13.10 в группе $\mathbf{B}_{\alpha-1}$ имеет место равенство $A^{r} \stackrel{\alpha=1}{=} T A^{\epsilon r} T^{-1}$, где $r \neq 0$ и $\epsilon= \pm 1$. Тогда согласно определению 7.1 имеем $[T]_{\alpha-1} \in \mathbf{N}_{\alpha-1}(A)$, и, следовательно, $\mathbf{f} \in \mathbf{S}_{\alpha}(A)$.

13.12. СлЕДСТВИЕ. Если $A^{t} w u B^{s} v-$ нормальные формы рангов $\beta, \gamma \leqslant \alpha$ соответственно, представляющие сопряженные әлементы группы $\mathbf{B}_{\alpha}$, то $\beta=\gamma$ и верно одно из равенств $t=s$ или $t+s=n$.

ДоКАЗАТЕЛЬСТво. Без ограничения обшности можно считать $\gamma \leqslant \beta$. По условию имеем $A^{t} w \stackrel{\alpha}{=} T B^{s} v T^{-1}$ для некоторого $T$. Применяя последовательно предложение 13.9 при $\alpha:=\alpha, \alpha-1, \ldots, \beta+1$, найдем слово $T^{*}$ такое, что $A^{t} w \stackrel{\beta}{=} T^{*} B^{s} v T^{*-1}$. Тогда, выбрав слово $T^{*}$ приведенным в ранге $\beta$ в силу предложения 12.13 и воспользовавшись предложением 13.10 , получим $\beta=\gamma$ и $t \equiv \pm s$ $(\bmod n)$, что с учетом неравенств $0<t<n$ и $0<s<n$ влечет $t=s$ или $t+s=n$.

13.13. ОПРЕДЕЛЕНИЕ. Пусть $\mathbf{g} \in \mathbf{B}_{\alpha}-$ неединичный элемент конечного порядка, и пусть $A^{t} w$ - нормальная форма ранга $\beta \leqslant \alpha$, представляющая сопряженный ему элемент группы $\mathbf{B}_{\alpha}$. Число $\beta$ будем называть рангом әлемента $\mathbf{g}$ и обозначать ранг g. Число $t$ будем называть степенью әлемента $\mathbf{g}$.

По определению полагаем также ранг $1 \rightleftharpoons 0$.

Если $\mathbf{G} \leqslant \mathbf{B}_{\alpha}-$ конечная подгруппа группы $\mathbf{B}_{\alpha}$, то по определению

$$
\text { ранг } \mathbf{G} \rightleftharpoons \max \{\text { ранг } \mathbf{g} \mid \mathbf{g} \in \mathbf{G}\}
$$

Из следствия 13.12 и предложения 13.6 вытекает, что ранг любого элемента $\mathbf{g} \in$ $\mathbf{B}_{\alpha}$ конечного порядка определен однозначно, а если $\mathbf{g} \neq 1$, то степень $\mathbf{g}$ может принимать лишь два значения $t$ и $s$, связанные соотношением $t+s=n$. Если $\mathbf{g}$, $\mathbf{h}$ - сопряженные элементы конечного порядка групшы $\mathbf{B}_{\alpha}$, то, очевидно, ранг $\mathbf{g}=$ ранг $\mathbf{h}$ и степени $\mathbf{g}$ и $\mathbf{h}$ совпадают.

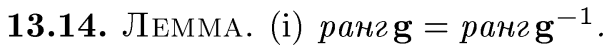

(ii) Если $\mathbf{g}$ имеет степень $t, k>0 u k t<n$, то ранг $\mathbf{g}^{k}=$ ранг $\mathbf{g} u \mathbf{g}^{k}$ имеет степень kt.

(iii) Если $\beta<\alpha u \mathbf{g} \in \mathbf{B}_{\beta}$ - әлемент конечного порядка, то ранг $\mathbf{g}=$

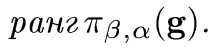

ДокаЗАтельство. (i), (ii): Пусть $\mathbf{g} \neq 1$ и элемент $\mathbf{g}$ сопряжен элементу $\mathbf{h}=\left[A^{t} w\right]_{\alpha}$, где $A^{t} w$ - нормальная форма ранга $\gamma \rightleftharpoons$ ранг $\mathbf{g}$. Так как согласно определению $7.12 \mathbf{N}_{\gamma-1}^{*}(A) \unlhd \mathbf{N}_{\gamma-1}^{+}(A)$, то $\left[A^{t} w^{-1} A^{-t}\right]_{\gamma-1} \in \mathbf{N}_{\gamma-1}^{*}(A)$ и для любого $k>0\left[A^{-k t}\left(A^{t} w\right)^{k}\right]_{\gamma-1} \in \mathbf{N}_{\gamma-1}^{*}(A)$. Тогда в силу леммы 13.2 , (i) элементы $\mathbf{h}^{-1}$ и $\mathbf{h}^{k}$ при $k t<n$ представляются нормальными формами $\left(A^{-1}\right)^{t} w_{1}$ 
и $A^{k t} w_{2}$ ранга $\gamma$ соответственно для некоторых $w_{1}$ и $w_{2}$. Отсюда следует, что ранг $\mathbf{g}^{-1}=\operatorname{paнг~}^{k}=\gamma$ и $\mathbf{g}^{k}$ имеет степень $k t$.

(iii): Если $\mathbf{g}=1$, то доказывать нечего. Если $\mathbf{g} \neq 1$ и $\mathbf{g}$ сопряжен элементу $\mathbf{h}=\left[A^{t} w\right]_{\beta}$, где $A^{t} w$ - нормальная форма ранга $\gamma=p a н г \mathbf{g}$, то элемент $\pi_{\beta, \alpha}(\mathbf{g})$ сопряжен элементу $\pi_{\beta, \alpha}(\mathbf{h})$, который также представляется нормальной формой $A^{t} w$ ранга $\gamma$.

13.15. ПРЕДЛОЖЕНИЕ. Пусть $A \in \mathcal{E}_{\beta}, \quad \beta \leqslant \alpha$. Тогда ранг[A] $]_{\alpha}^{t}=\beta$ для любого $0<t<n$, ранг $\mathbf{S}_{\alpha}(A)=\beta$ и для любого $\mathbf{g} \in \mathbf{S}_{\alpha}(A)$ выполнено

$$
\operatorname{paнг} \mathbf{g}=\beta \Leftrightarrow \mathbf{g} \in \mathbf{S}_{\alpha}^{+}(A) \backslash \mathbf{S}_{\alpha}^{*}(A)
$$

ДокАЗАТЕльство. Переходя к циклическому сдвигу слова $A$, можно считать $A \in \overline{\mathcal{E}}_{\beta}$. В силу предложения 13.3 для любого элемента $\mathbf{g} \in \mathbf{S}_{\alpha}^{+}(A) \backslash \mathbf{S}_{\alpha}^{*}(A)$ имеем $p a н \imath \mathbf{g}=\beta$, и так как согласно определению 13.4 слово $A^{t}$ при $0<t<n$ есть нормальная форма ранга $\beta$, то ранг $[A]_{\alpha}^{t}=\beta$. Для доказательства предложения остается проверить, что ранг $\mathbf{g}<\beta$ при $\mathbf{g} \in \mathbf{S}_{\alpha}^{*}(A) \cup \mathbf{S}_{\alpha}^{-}(A)$. В силу индуктивного предположения 13.5 и леммы 13.4 , (iii) для этого достаточно убедиться, что $\mathbf{g}=$ $\pi_{\alpha-1, \alpha}(\tilde{\mathbf{g}})$ для некоторого элемента $\tilde{\mathbf{g}} \in \mathbf{B}_{\alpha-1}$ конечного порядка. Для $\mathbf{g} \in \mathbf{S}_{\alpha}^{*}(A)$ это следует из предложения 7.14, (ii). Для $\mathbf{g} \in \mathbf{S}_{\alpha}^{-}(A)$ нужно воспользоваться еще следствием 7.17 .

13.16. ПРЕДЛОЖЕНИЕ. Пусть $A \in \mathcal{E}_{\beta}, \beta \leqslant \alpha, \mathbf{g}_{1}, \mathbf{g}_{2}, \ldots, \mathbf{g}_{r}, \mathbf{h}_{1}, \mathbf{h}_{2}, \ldots, \mathbf{h}_{r} \in$ $\mathbf{S}_{\alpha}(A) u \mathbf{g}_{i}=\mathbf{x h}_{i} \mathbf{x}^{-1} \quad(i=1,2, \ldots, r)$. Тогда если ранг хотя бы одного из элементов $\mathbf{g}_{i}$ или $\mathbf{h}_{i}$ равен $\beta$, то существует әлемент $\mathbf{y} \in \mathbf{S}_{\alpha}(A)$ такой, что $\mathbf{g}_{i}=\mathbf{y h}_{i} \mathbf{y}^{-1} \quad(i=1,2, \ldots, r)$.

ДокаЗАТЕЛЬСтво. Сопрягая элементы $\mathbf{g}_{i}, \mathbf{h}_{i}, \mathbf{x}$ и $\mathbf{y}$ одним и тем же подходящим элементом групшы $\mathbf{B}_{\alpha}$ и используя лемму 7.20 , можно считать $A \in \overline{\mathcal{E}}_{\beta}$. Без ограничения общности можно считать $р а н г \mathbf{g}_{1}=$ ранг $\mathbf{h}_{1}=\beta$. По предложению 13.3 представим каждый элемент $\mathbf{g}_{i}$ словом $x_{i} \in \mathcal{H}_{\beta}$, а каждый элемент $\mathbf{h}_{i}-$ словом $y_{i} \in \mathcal{H}_{\beta}$, где $\left[x_{i}\right]_{\beta},\left[y_{i}\right]_{\beta} \in \mathbf{S}_{\beta}(A)$ при всех $i$. Имеем $x_{i} \stackrel{\alpha}{=} T y_{i} T^{-1}$ при всех $i$, и по предложению 13.9 найдется слово $T^{*}$ такое, что $x_{i} \stackrel{\beta}{=} T^{*} y_{i} T^{*-1}$ при всех $i$. Так как в силу предложения $13.15\left[x_{1}\right]_{\beta},\left[y_{1}\right]_{\beta} \in \mathbf{S}_{\beta}^{+}(A) \backslash \mathbf{S}_{\beta}^{*}(A)$, то в силу следствия 13.11 имеем $\left[T^{*}\right]_{\beta} \in \mathbf{S}_{\beta}(A)$. Тогда можно положить $\mathbf{y} \rightleftharpoons\left[T^{*}\right]_{\alpha}$.

13.17. ПРЕДЛОЖЕНИЕ. Если $A, B \in \bigcup_{\beta \leqslant \alpha} \mathcal{E}_{\beta} u \mathbf{S}_{\alpha}(A)=\mathbf{x S}_{\alpha}(B) \mathbf{x}^{-1}, m o$ $\mathbf{S}_{\alpha}^{+}(A)=\mathbf{x} \mathbf{S}_{\alpha}^{+}(B) \mathbf{x}^{-1}, \mathbf{S}_{\alpha}^{-}(A)=\mathbf{x} \mathbf{S}_{\alpha}^{-}(B) \mathbf{x}^{-1} u \mathbf{S}_{\alpha}^{*}(A)=\mathbf{x} \mathbf{S}_{\alpha}^{*}(B) \mathbf{x}^{-1}$.

ДокАЗАТЕльство. Заметим, что в силу предложения 13.15 элементарные периоды $A$ и $B$ имеют один и тот же ранг $\beta$. В силу леммы 7.20 достаточно ограничиться рассмотрением случая, когда $A, B \in \bar{\varepsilon}_{\beta}$. Пусть $\mathbf{S}_{\alpha}(A)=\left\{\mathbf{g}_{0}, \mathbf{g}_{1}, \ldots, \mathbf{g}_{k}\right\}$ и $\mathbf{S}_{\alpha}(B)=\left\{\mathbf{h}_{0}, \mathbf{h}_{1}, \ldots, \mathbf{h}_{k}\right\}$, где $\mathbf{g}_{i}=\mathbf{x h}_{i} \mathbf{x}^{-1}$ и ранг $\mathbf{g}_{0}=$ ранг $\mathbf{h}_{0}=\beta$. По предложению 13.3 представим каждый элемент $\mathbf{g}_{i}$ словом $x_{i} \in \mathcal{H}_{\beta}$, а каждьй элемент $\mathbf{h}_{i}-$ словом $y_{i} \in \mathcal{H}_{\beta}$, причем $x_{0}$ 을 $A^{t} w$ и $y_{0}$ 을 $B^{r} v$-нормальные формы ранга $\beta$. Представим также элемент $\mathbf{x}$ произвольным словом $T$. Так как $x_{i} \stackrel{\alpha}{=} T y_{i} T^{-1}$ при всех $i$, 
то по предложению 13.9 найдется слово $T^{*}$ такое, что $x_{i} \stackrel{\beta}{=} T^{*} y_{i} T^{*-1}$ при всех $i$. Заменяя $T^{*}$ на приведенное в ранге $\beta$ слово и применяя предложение 13.10 и лемму 7.20, получаем, что все требуемые в предложении равенства выполнены, если $\mathbf{x}$ заменить на $\mathbf{y} \rightleftharpoons\left[T^{*}\right]_{\alpha}$. Но так как $\mathbf{y} \mathbf{h}_{i} \mathbf{y}^{-1}=\mathbf{x h}_{i} \mathbf{x}^{-1}$ при всех $i$, то эти равенства вьполнены и для первоначального $\mathbf{x}$.

\section{$\S 14$. Вложение конечных подгрупп} группы $\mathbf{B}_{\alpha}$ в полные конечные подгруппы

14.1. ОПРедЕлЕнИЕ. Конечную подгруппу $\mathbf{G}$ группы $\mathbf{B}_{\alpha}$ вида $\mathbf{G}=\mathbf{g S}_{\alpha}(A) \mathbf{g}^{-1}$ при $A \in \bigcup_{\beta \leqslant \alpha} \mathcal{E}_{\alpha}$ будем называть полной конечной подгруппой группы $\mathbf{B}_{\alpha}$ (не путать с понятием полной группы, т.е. группы $\mathbf{G}$, для которой уравнение $\mathbf{x}^{k}=\mathbf{g}$ в $\mathbf{G}$ разрешимо для любых $\mathbf{g} \in \mathbf{G}$ и $k>0$ ).

Если $\mathbf{H} \leqslant \mathbf{G}$, где $\mathbf{H}$ - конечная подгруппа группы $\mathbf{B}_{\alpha}, \mathbf{G}$ - полная конечная подгруппа группы $\mathbf{B}_{\alpha}$, и ранг $\mathbf{G}=$ ранг $\mathbf{H}$, то $\mathbf{G}$ будем называть пололнением $\mathbf{H}$.

14.2. ПРЕДЛОЖЕНИЕ. Если $\mathbf{G}_{1}, \mathbf{G}_{2}$ - два пополнения конечной подгруппы $\mathbf{H} \leqslant \mathbf{B}_{\alpha}$, то $\mathbf{G}_{1}=\mathbf{z G}_{2} \mathbf{z}^{-1}$ для некоторого $\mathbf{z} \in \mathbf{B}_{\alpha}$, коммутирующего со всеми әлементами из $\mathbf{H}$.

ДокаЗАТЕЛЬСТво. Сопрягая подгруппы $\mathbf{H}, \mathbf{G}_{1}$ и $\mathbf{G}_{2}$ с помошью подходящего элемента группы $\mathbf{B}_{\alpha}$ и используя лемму 7.20 , можно считать, что $\mathbf{G}_{1}=\mathbf{S}_{\alpha}(A)$ и $\mathbf{G}_{2}=\mathbf{g S}_{\alpha}(B) \mathbf{g}^{-1}$, где $A, B$ - нормализованные элементарные периоды, причем по предложению 13.15 имеем $A, B \in \bar{\varepsilon}_{\beta}$, где $\beta \rightleftharpoons$ ранг $\mathbf{H}$. Пусть $\mathbf{H}=$ $\left\{\mathbf{h}_{1}, \mathbf{h}_{2}, \ldots, \mathbf{h}_{r}\right\}$, и пусть ранг $\mathbf{h}_{1}=\beta$. В силу предложения 13.15 имеем также $\mathbf{h}_{1} \in \mathbf{S}_{\alpha}^{+}(A) \backslash \mathbf{S}_{\alpha}^{*}(A)$ и $\mathbf{g}^{-1} \mathbf{h}_{1} \mathbf{g} \in \mathbf{S}_{\alpha}^{+}(B) \backslash \mathbf{S}_{\alpha}^{*}(B)$. По предложению 13.3 представим каждый элемент $\mathbf{h}_{i}$ словом $u_{i}$ и каждый элемент $\mathbf{g}^{-1} \mathbf{h}_{i} \mathbf{g}$ словом $v_{i}$, где $u_{i}, v_{i} \in \mathcal{H}_{\beta}$ и $u_{1}$ 으 $A^{t} w, v_{1}$ 으 $B^{s} v$ - нормальные формы ранга $\beta$. В силу предложения 12.13 представим элемент $\mathbf{g}$ словом $T \in \mathcal{R}_{\alpha}$. Имеем

$$
u_{i} \stackrel{\alpha}{=} T v_{i} T^{-1} \quad(i=1,2, \ldots, r) .
$$

Применив предложение 13.9 последовательно при $\alpha:=\alpha, \alpha-1, \ldots, \beta+1$, найдем слово $T^{*}$ такое, что

$$
u_{i} \stackrel{\beta}{=} T^{*} v_{i} T^{*-1} \quad(i=1,2, \ldots, r) .
$$

В силу предложения 12.13 можно считать $T^{*} \in \mathcal{R}_{\beta}$, и тогда из (14.2) при $i:=$ 1 в силу предложения 13.10 и леммы 7.20 следует $\mathbf{S}_{\alpha}(A)=\left[T^{*}\right]_{\alpha} \mathbf{S}_{\alpha}(B)\left[T^{*}\right]_{\alpha}^{-1}$. Полагая $\mathbf{z} \rightleftharpoons\left[T^{*} T^{-1}\right]_{\alpha}$, получаем $\mathbf{G}_{1}=\mathbf{z G}_{2} \mathbf{z}^{-1}$, а из равенств (14.1) и (14.2) следует, что $\mathbf{z}$ коммутирует со всеми элементами $\mathbf{h}_{i}$.

Основная цель параграфа - доказательство следуюшего утверждения.

14.3. ПрЕДЛОЖЕНИЕ. Всякая неединичная конечная подгруппа группы $\mathbf{B}_{\alpha}$ обладает некоторым пополнением. 
14.4. ЛЕмма. Для любой нормальной формы $A^{t} w$ ранга $\beta \leqslant \alpha$ существуют период Х ранга $\beta$ и слово $U$, обладающие следующими свойствами:

(i) $A^{t} w \stackrel{\beta=1}{=} U^{-1} X U$;

(ii) никакой циклический сдвиг слова $X$ не содержит әлементарных 12 -степеней ранга $\gamma$ при $\beta+1 \leqslant \gamma \leqslant \alpha$;

(iii) если $t \leqslant n-q-75$, то $X-$ чиклически приведенное в ранге $\alpha$ слово;

(iv) пусть $\mathrm{X} u \mathrm{UR}$ - пути на графе $\Gamma_{\beta-1}$ с общей начальной вершиной, где $\mathcal{L}(\mathrm{X})$ 드 $X, \mathcal{L}(\mathrm{U})$ 드 $U u \mathcal{L}(\mathrm{R})$ 으 $A^{2} ;$ тогда существует путь $Z X Z^{\prime} \in \Pi \operatorname{pe}_{\beta}(A) \cap \Pi е р\left(\Gamma_{\beta-1}, X, 2\right)$ mакой, ито $|Z|,\left|Z^{\prime}\right| \geqslant|X|$ и некоторое периодическое продолжение для $\mathrm{R}$ является периодической основой для $\mathrm{ZXZ}^{\prime}$;

(v) если $t \geqslant 4$, то для любого ииклического сдвига $X^{\prime}$ слова $X$ выполнено $\ell_{\alpha, A}\left(X^{\prime}\right) \geqslant t-3$ (в смысле определения 8.13);

(vi) ecли $\mathrm{Y}-$ nymь на графе $\Gamma_{\beta-1} c \mathcal{L}(\mathrm{Y})$ 으 $X^{2} u \mathrm{E} \in \Pi_{\text {nep }}(B, p+34)$, $\mathrm{F} \in \Pi \Pi е \mathrm{p}_{\beta}(C, p+34) \quad\left(B, C \in \mathcal{E}_{\beta}\right)-$ nодnути nути $\mathrm{Y}$, mo $\Pi$ Согл $\beta(\mathrm{E}, \mathrm{F})$.

ДокАЗАТЕЛЬСтво. Так как $\left[A^{t} w\right]_{\beta-1}$ - элемент бесконечного порядка, то по индуктивному предположению 12.24 найдутся период $X$ ранга $\beta$ и слово $U$ такие, что $A^{t} w \stackrel{\beta=1}{=} U^{-1} X U$, т.е. имеет место утверждение (i) леммы. Заметим, что (ii) вытекает из (iv) в силу предложения 8.17 и леммы 8.16. Докажем утверждения (iii)-(vi) в следующей последовательности: (iv), (v), (iii) и (vi).

Пусть $\mathrm{X}$ и UR - пути на графе $\Gamma_{\beta-1}$ с общей начальной вершиной, где $\mathcal{L}(\mathrm{X})$ 으 $X$, $\mathcal{L}(\mathrm{U})$ 드 $U$ и $\mathcal{L}(\mathrm{R})$ 으 $A^{2}$. Рассмотрим путь $\mathrm{VXV}^{\prime}$, где $\mathcal{L}(\mathrm{V})$ 으 $\mathcal{L}\left(\mathrm{V}^{\prime}\right)$ 으 $X$. Так как $\left[A^{t} w\right]_{\beta-1} \in \mathbf{N}_{\beta-1}^{+}(A)$, то по предложению 7.10, (i) имеем $A^{t k} \stackrel{\beta-1}{=} U^{-1} X^{k} U$ для некоторого $k>0$, откуда следует $\mathbf{s}_{A, \mathrm{R}}^{t k}=\mathbf{s}_{X, \mathrm{VXV}}^{k}$. В силу предложения 7.7 найдем строго близкие в ранге $\rho(A)$ периодические продолжения $\overline{\mathrm{R}}$ для $\mathrm{R}$ и $\overline{\mathrm{X}}=$ $\mathrm{ZXZ}^{\prime}$ для $\mathrm{VXV}^{\prime}$, где для некоторых $\mathrm{K} \in$ Эл $_{\rho(A)}\left(\overline{\mathrm{R}}, q_{3}\right), \mathrm{L}, \mathrm{L}^{\prime} \in \ni$ л $_{\rho(A)}\left(\overline{\mathrm{X}}, q_{1}\right)$ и $r \geqslant 1$ выполнено $\overline{\mathrm{R}}=\mathrm{K} \cup \cup_{\overline{\mathrm{R}}} \mathrm{s}_{A, \overline{\mathrm{R}}}^{r} \mathrm{~K}, \overline{\mathrm{X}}=\mathrm{L} \cup_{\overline{\mathrm{X}}} \mathrm{L}^{\prime}, \vartheta \operatorname{Coг}_{\rho(A)}(\mathrm{K}, \mathrm{L})$ и $Э \operatorname{Coг}_{\rho(A)}\left(\mathrm{s}_{A, \overline{\mathrm{R}}}^{r} \mathrm{~K}, \mathrm{~L}^{\prime}\right)$. Тогда $\overline{\mathrm{X}} \in \Pi_{\text {Пер }}(A)$ и $\overline{\mathrm{R}}$ - периодическая основа для $\overline{\mathrm{X}}$. Тем самым доказано (iv).

Обозначим $\mathrm{L}_{0} \rightleftharpoons \mathrm{L}$ и $\mathrm{L}_{r} \rightleftharpoons \mathrm{L}^{\prime}$. Используя лемму 8.2, (iii) и предложение 8.41,

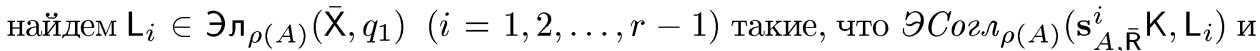
$\mathrm{L}_{0} \ll_{\mathrm{E}} \mathrm{L}_{1} \ll_{\mathrm{E}} \cdots \ll_{\mathrm{E}} \mathrm{L}_{r}$. По лемме 7.21 для любых $0 \leqslant i \leqslant r$ и $j \in \mathbb{Z}$ выполнено ЭСогл $_{\rho(A)}\left(\mathbf{s}_{A, \overline{\mathrm{R}}}^{j t+i} \mathrm{~K}, \mathbf{s}_{X, \overline{\mathrm{X}}}^{j} \mathrm{~L}_{i}\right)$. Отсюда следует

$$
{ } \operatorname{Coz}_{\rho(A)}\left(\mathbf{s}_{X, \overline{\mathrm{x}}} \mathrm{L}_{i}, \mathrm{~L}_{i+t}\right) \quad(0 \leqslant i \leqslant r-t) .
$$

Пусть $t \geqslant 4$ и $X^{\prime}-$ произвольный циклический сдвиг слова $X$. Так как $|\overline{\mathrm{X}}| \geqslant 3|X|$, то для некоторого подпути $\mathrm{X}^{\prime}$ пути $\overline{\mathrm{X}}$ имеем $\mathcal{L}\left(\mathrm{X}^{\prime}\right)$ 으 $X^{\prime}$. Пусть $i$ и $j$-соответственно максимальный и минимальный индексы такие, что $X^{\prime}$ содержится в $L_{i} \cup_{\overline{\mathrm{X}}} \mathrm{L}_{j}$. B силу (14.3) и индуктивного предположения 11.25 имеем $\mathrm{L}_{i+t-1}<\overline{\mathrm{x}} \mathbf{s}_{X, \overline{\mathrm{x}}} \mathrm{L}_{i}$. Так как $\left|X^{\prime}\right|=|X|$, то отсюда следует $j-i \geqslant t-1$. В силу выбора $i$ и $j$ пути $\mathrm{L}_{i+1}$ и $\mathrm{L}_{j-1}$ содержатся в $\mathrm{X}^{\prime}$. Так как $\mathrm{L}_{i+1} \cup_{\overline{\mathrm{X}}} \mathrm{L}_{j-1} \in \Pi$ Пер $\beta(A, t-3)$, то мы получили $\ell_{\beta, A}\left(X^{\prime}\right) \geqslant t-3$, что и доказывает (v).

Для доказательства (iii) в силу (ii) и леммы 12.12 достаточно убедиться, что при $t \leqslant n-q-75$ никакой циклический сдвиг слова $X$ не содержит элементарных 
$(n-q-36)$-степеней ранга $\beta$. Допустим, $t \leqslant n-q-75$ и в некоторый циклический сдвиг слова $X$ входит элементарная $(n-q-36)$-степень $E$ ранга $\beta$. Пусть $\mathrm{E}-$ подпуть пути $\overline{\mathrm{X}} \mathrm{c} \mathcal{L}(\mathrm{E})$ 으 $E$, т.е. $\mathrm{E} \in \Pi_{\text {Пер }}(B, n-q-36)$, где $B \in \mathcal{E}_{\beta}$. В силу леммы 8.2 , (ii) и предложения 8.36 имеем $C$ огл $(\overline{\mathrm{R}}, \mathrm{Q})$, где $\mathrm{Q}$ - периодическая основа для $\mathrm{E}$. Тогда в силу леммы 7.22 и предложения 8.35 для некоторого подпути $\mathrm{F}$ пути $\mathrm{E}$ имеем $\mathrm{F} \in \Pi_{\text {Пер }}(A, n-q-72)$. Пусть $\mathrm{S} \in$ Пер $\left(\Gamma_{\beta-1}, A, n-q-72\right)$ - периодическая основа для $\mathrm{F}$. В силу лемм 8.4 и 8.2 , (iii) найдем $\mathrm{M}, \mathrm{s}_{A, \mathrm{~S}} \mathrm{M}, \ldots, \mathrm{s}_{A, \mathrm{~S}}^{n-q-74} \mathrm{M} \in$ Эл $\rho_{\rho(A)}\left(\mathrm{S}, q_{2}\right), \mathrm{N}_{0}, \mathrm{~N}_{1}, \ldots, \mathrm{N}_{n-q-74} \in Э_{\rho(A)}\left(\mathrm{F}, q_{1}\right)$, где ЭСогл $\boldsymbol{\vartheta}_{\rho(A)}\left(\mathbf{s}_{A, S}^{i} \mathrm{M}, \mathrm{N}_{i}\right)$ при всех $i$ и $\mathrm{N}_{0}<_{\mathrm{F}} \mathrm{N}_{1}<_{\mathrm{F}} \cdots<_{\mathrm{F}} \mathrm{N}_{n-q-74}$. Аналогично соотношению (14.3) выводим $Э \operatorname{Coг}_{\rho(A)}\left(\mathbf{s}_{X, \overline{\mathrm{X}}} \mathrm{N}_{i}, \mathrm{~N}_{i+t}\right)(0 \leqslant i \leqslant n-q-74-t)$. Тогда с учетом допушения $t \leqslant n-q-75$ по индуктивному предположению 11.25 имеем $\mathbf{s}_{X}, \overline{\mathrm{X}} \mathrm{N}_{0}<_{\mathrm{F}} \mathrm{N}_{t+1}$ и, следовательно, $|\mathrm{F}| \geqslant\left|\mathrm{N}_{0} \cup_{\mathrm{F}} \mathrm{N}_{t+1}\right|>|X|$. Но это невозможно, так как в силу нашего допущения слово $\mathcal{L}(\mathrm{F})$ входит в некоторый циклический сдвиг слова $X$.

(vi) Очевидно, достаточно ограничиться рассмотрением случая, когда $\mathrm{Y}$ содержится в ZXZ'. В этом случае утверждение легко вытекает из предложения 8.38 , (ii).

14.5. Лемма. Пусть $x, y \in \mathcal{H}_{\alpha},[x]_{\alpha} \in \mathbf{S}_{\alpha}(A),[y]_{\alpha} \in \mathbf{S}_{\alpha}(B)$ для некоторых $A, B \in \bigcup_{\beta \leqslant \alpha} \bar{\varepsilon}_{\beta}$ и слова $x, y$ имеют вид $x$ 드 $x_{1} Q x_{2}$ и y 으 $y_{1} R y_{2}$, где $x_{i}, y_{i} \in$ $\mathcal{H}_{\alpha-1}(i=1,2), Q \in \Pi e p(A), \quad R \in \Pi е p(B),|Q|<2|A| u|R|<2|B|$. Пусть $T \in$ $\mathcal{R}_{\alpha}$, әлемент $\left[T^{-1} x T y\right]_{\alpha}$ имеет конечный порядок и ранг $\left[T^{-1} x T y\right]_{\alpha} \leqslant \alpha-1$. Тогда верны следующие утверждения:

(i) если слово $T$ не содержит әлементарных $q_{4}$-степеней ранга $\alpha$, то әлемент $\left[T^{-1} x T y\right]_{\alpha-1}$ имеет конечный порядок и ранг $\left[T^{-1} x T y\right]_{\alpha-1}=$ ранг $\left[T^{-1} x T y\right]_{\alpha}$

(ii) nусть $\mathrm{T}-$ nуть на графе $\Gamma_{\alpha} c \mathcal{L}(\mathrm{T})$ 으 $T$; положим $\mathbf{g} \rightleftharpoons \iota(\mathrm{T})[x]_{\alpha}^{-1}(\iota(\mathrm{T}))^{-1}$ $u \mathbf{h} \rightleftharpoons \tau(\mathrm{T})[y]_{\alpha}(\tau(\mathrm{T}))^{-1} ;$ тогда для любого $\mathrm{E} \in \ni л_{\alpha}(\mathrm{T}, 2 h+3 p+233)$ имеет место хотя бы одно из соотношений

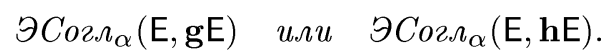

ДокАЗАТЕЛЬСтво. Сначала рассмотрим случай, когда $T^{-1} x T y \stackrel{\alpha}{=} 1$. Тогда (i) верно в силу предложения 11.3. Докажем (ii). Пусть $\mathrm{T}$ - путь на графе $\Gamma_{\alpha} \mathrm{c}$ $\mathcal{L}(\mathrm{T})$ 으 $T$. Рассмотрим замкнутый путь $\mathrm{T}^{\prime-1} \mathrm{x}$ Ту на $\Gamma_{\alpha}$, где $\mathcal{L}\left(\mathrm{T}^{\prime}\right)$ 으 $T, \mathcal{L}(\mathrm{x})$ 으 $x$ и $\mathcal{L}(\mathrm{y})$ 으 $y$. Заметим, что $\mathrm{T}^{\prime}=\mathbf{g} \mathbf{T}$, где $\mathbf{g}=\iota(\mathrm{T})[x]_{\alpha}^{-1}(\iota(\mathrm{T}))^{-1}-$ элемент, указанный в (ii). Из посылки леммы и определения 11.11 следует, что любой путь $\mathrm{E} \in \ni_{\alpha}\left(\Gamma_{\alpha}\right)$ устойчив относительно $\mathrm{x}^{-1}$ и $\mathrm{y}^{-1}$. Тогда по предложению 11.17 при $\mathrm{X}:=\mathrm{T}$ и uYv $:=\mathrm{x}^{-1} \mathrm{~T}^{\prime} \mathrm{y}^{-1}$ для любого $\mathrm{E} \in$ Эл $_{\alpha}(\mathrm{T}, 2 h+3 p+233)$ найдется $\mathrm{F} \in \ni л_{\alpha}\left(\mathrm{T}^{\prime}, h+3 p+233\right)$ такой, что $Э$ Согл $\Omega_{\alpha}(\mathrm{E}, \mathrm{F})$. По лемме 13.8 имеем $\exists$ Эогл $\Omega_{\alpha}(\mathrm{gE}, \mathrm{F})$, т.е. $Э$ Согл $_{\alpha}(\mathrm{E}, \mathrm{gE})$, что и доказывает (ii) в случае $T^{-1} x T y \stackrel{\alpha}{=} 1$.

Пусть $T^{-1} x T y \stackrel{\alpha}{\neq} 1$. Тогда по условию и в силу определения 13.13 слово $T^{-1} x T y$ сопряжено в группе $\mathbf{B}_{\alpha}$ некоторой нормальной форме $U$ ранга $\beta \leqslant \alpha-1$. 
По лемме 14.4 слово $U$ сопряжено в группе $\mathbf{B}_{\beta-1}$ циклически приведенному в ранге $\alpha$ слову $Z$ такому, что никакой циклический сдвиг $Z$ не содержит элементарных 12 -степеней ранга $\alpha$. Рассмотрим кольцевую $B$-диаграмму $\Delta$ ранга $\alpha$ с граничными циклами $\widetilde{\mathrm{T}}^{\prime-1} \tilde{\mathrm{x}}_{1} \widetilde{\mathrm{Q}}_{2} \widetilde{\mathrm{T}}_{1} \widetilde{\mathrm{R}}_{2}$ и $\mathrm{Z}^{-1}$, где метки путей $\widetilde{\mathrm{T}}^{\prime}, \tilde{\mathrm{x}}_{i}, \widetilde{\mathrm{Q}}, \widetilde{\mathrm{T}}, \tilde{\mathrm{y}}_{i}, \widetilde{\mathrm{R}}$ и Z равны словам $T, x_{i}, Q, T, y_{i}, R$ и $Z$ соответственно,

$$
x_{\alpha}(\Delta)=\left\{\widetilde{\mathrm{T}}^{\prime-1}, \widetilde{\mathrm{T}}, \mathrm{Z}^{-1}\right\}, \quad y_{\alpha-1}(\Delta)=\left\{\tilde{\mathrm{x}}_{1}, \tilde{\mathrm{x}}_{2}, \tilde{\mathrm{y}}_{1}, \tilde{\mathrm{y}_{2}}\right\} \quad \text { и } z_{\alpha}(\Delta)=\{\widetilde{\mathrm{Q}}, \widetilde{\mathrm{R}}\} .
$$

В силу предложений 9.9 и 9.19 можно считать, что $\Delta-$ приведенная $B$-диаграмма ранга $\alpha$ и $\mathcal{S}$ - некоторая карта примыканий ранга $\alpha-1$ на $\Delta^{\alpha-1}$.

Докажем утверждение (i). Если диаграмма $\Delta$ не имеет областей ранга $\alpha$, то слова $T^{-1} x T y$ и $Z$ сопряжены в группе $\mathbf{B}_{\alpha-1}$. Так как по выбору $Z$ элемент $[Z]_{\alpha-1}$ имеет конечный порядок, то в этом случае по лемме 13.14 , (iii) $\left[T^{-1} x T y\right]_{\alpha-1}-$ элемент конечного порядка и ранг $\left[T^{-1} x T y\right]_{\alpha-1}=$ ранг $\left[T^{-1} x T y\right]_{\alpha}$, что и требовалось. Поэтому достаточно доказать, что если $\Delta$ имеет хотя бы одну область ранга $\alpha$, то в слово $T$ входит элементарная $q_{4}$-степень ранга $\alpha$.

Пусть $\mathrm{A} \in \mathbf{R}_{\alpha}(\Delta)$ - произвольная область. Пусть $\mathcal{L}(\delta \mathrm{A}) \approx C^{n}$, где $C \in \mathcal{E}_{\alpha}$. Пусть $\Pi_{1}, \Pi_{2}, \ldots, \Pi_{r} \in \mathcal{S}$ - все поддиаграммы примыкания граничного цикла области А к участкам границы $\widetilde{\mathrm{T}}$ и $\widetilde{\mathrm{T}}^{\prime-1}$ диаграммы $\Delta^{\alpha-1}$. Пусть $\widetilde{\mathrm{P}}_{i}, \widetilde{\mathrm{G}}_{i}-$ участки примыкания поддиаграммы примыкания $\Pi_{i}$, входящие соответственно в $\delta$ А и в $\widetilde{T}$ или $\widetilde{\mathrm{T}}^{\prime-1}$. По предложению 10.9 , (ii) имеется не более одной поддиаграммы примыкания $\Pi \in \mathcal{S}$ граничного цикла области $\mathrm{A}$ к $\mathrm{Z}^{-1}$. Так как никакой циклический сдвиг слова $Z^{-1}$ не содержит элементарных 12 -степеней ранга $\alpha$, то в силу предложения 8.24 , если П - такая поддиаграмма примыкания и $\mathrm{P}$ - ее участок примыкания, входяший в $\delta \mathrm{A}$, то $|\mathcal{L}(\mathrm{P})|<48|C|$. Тогда по предложению 10.13 , (ii)

$$
\sum_{i=1}^{r}\left|\mathcal{L}\left(\widetilde{\mathrm{P}}_{i}\right)\right| \geqslant(n-6 p-112)|C| .
$$

Так как $T \in \mathcal{R}_{\alpha}$, то для каждого $i$

$$
\left|\mathcal{L}\left(\widetilde{\mathrm{P}}_{i}\right)\right|<(n-q)|C| .
$$

По предложению 10.9 , (ii) имеем $r \leqslant 3$. Так как $n-6 p-112>n-q+2(h+44)$, то из (14.5) и (14.6) следует, что имеется не более одной поддиаграммы $\Pi_{i}$, для которой $\left|\mathcal{L}\left(\widetilde{\mathrm{P}}_{i}\right)\right|<(h+44)|C|$. Исключив из рассмотрения такую поддиаграмму $\Pi_{i}$, если она существует, вместо неравенства (14.5) получим

$$
\sum_{i=1}^{r}\left|\mathcal{L}\left(\widetilde{\mathrm{P}}_{i}\right)\right| \geqslant(n-h-6 p-156)|C|,
$$

но теперь для всех $i$ будем иметь

$$
\left|\mathcal{L}\left(\widetilde{\mathrm{P}}_{i}\right)\right|>(h+44)|C| .
$$


Заметим, что для нового значения $r$ неравенство $r \leqslant 3$ по-прежнему имеет место. С другой стороны, из (14.6) и (14.7) следует $r \geqslant 2$.

Если $r=2$, то из $(14.8)$ следует $\mathcal{L}\left(\widetilde{\mathrm{P}}_{i}\right) \geqslant \frac{1}{2}(n-h-6 p-156)|C|$ при $i=1$ или $i=2$. Тогда по предложению 8.24 в слово $T$ входит элементарная $t$-степень ранга $\alpha$, где $t \geqslant \frac{1}{2}(n-h-6 p-156)-36>q_{4}$, что и требовалось.

Пусть $r=3$. С точностью до нумерации $\Pi_{i}$, возможны два случая:

1) $\widetilde{\mathrm{G}}_{1}, \widetilde{\mathrm{G}}_{2}$ входят в $\widetilde{\mathrm{T}}, \widetilde{\mathrm{G}}_{3}$ входит в в $\widetilde{\mathrm{T}}^{\prime-1}$;

2) $\widetilde{\mathrm{G}}_{1}, \widetilde{\mathrm{G}}_{2}$ входят в $\widetilde{\mathrm{T}}^{\prime-1}, \widetilde{\mathrm{G}}_{3}$ входит в $\widetilde{\mathrm{T}}$.

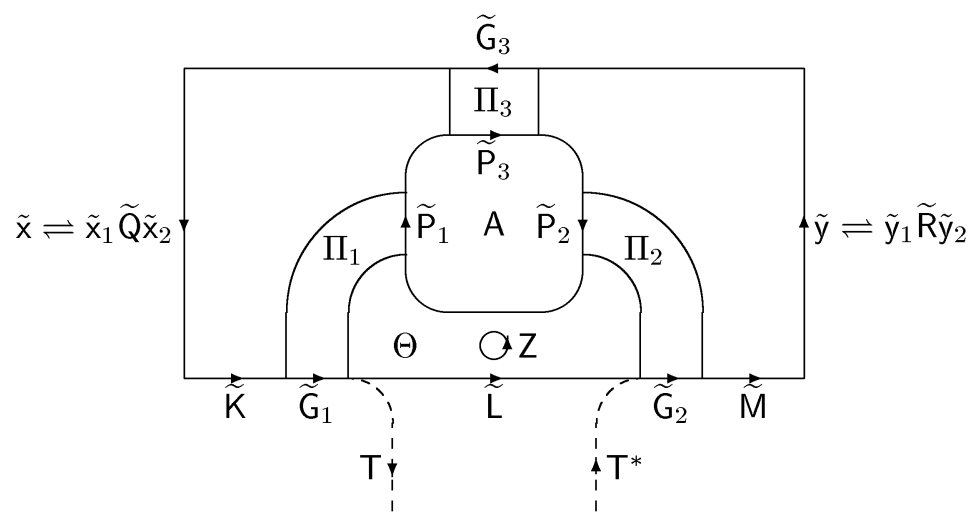

Рис. 63

Случай 1 ): $\widetilde{\mathrm{G}}_{1}, \widetilde{\mathrm{G}}_{2}$ входят в $\widetilde{\mathrm{T}}, \widetilde{\mathrm{G}}_{3}$ входит в $\widetilde{\mathrm{T}}^{\prime-1}$. Можно считать, что $\widetilde{\mathrm{T}}$ имеет вид $\widetilde{\mathrm{T}}=\widetilde{\mathrm{K}} \widetilde{\mathrm{G}}_{1} \widetilde{\mathrm{LG}}_{2} \widetilde{\mathrm{M}}$. В силу леммы 10.8 , (i) поддиаграмма диаграммы $\Delta$, обозначенная через $\Theta$ на рис. 63 , не может быть односвязной. Так как $\Delta-$ кольцевая диаграмма, то $\Theta$ - кольцевая диаграмма, а диаграмма $\Delta-\Theta$ односвязна. Рассмотрим отображение $\omega$ множества путей на диаграмме $\Delta-\Theta$ во множество путей на графе $\Gamma_{\alpha}$ $($ см. 4.12), где $\omega(\iota(\widetilde{\mathrm{K}}))=\iota(\mathrm{T})$. Если $\widetilde{\mathrm{X}}-$ некоторый путь на $\Delta-\Theta$, то обозначим $\mathrm{X} \rightleftharpoons \omega(\widetilde{\mathrm{X}})$. Очевидно, $\mathrm{KG}_{1}$ есть начало пути $\mathrm{T}, \mathrm{G}_{3}^{-1}$ содержится в $\mathrm{T}^{\prime}$ и $\mathrm{G}_{2} \mathrm{M}$ есть конец некоторого пути $\mathrm{T}^{*}$ с $\mathcal{L}(\mathrm{T})$ 드 $\mathcal{L}\left(\mathrm{T}^{*}\right)$ 으 $T$ (см. рис. 63). В силу (14.8) и леммы 11.15 каждый путь $\mathrm{G}_{i}$ содержит подпуть $\mathrm{F}_{i} \in \ni \boldsymbol{л}_{\alpha}\left(\Gamma_{\alpha}, h+8\right)$ с периодической основой, содержашейся в $\mathrm{P}_{i}^{-1}$, причем,

$$
\ell_{\alpha}\left(\mathrm{F}_{i}\right) \geqslant\left|\mathcal{L}\left(\widetilde{\mathrm{P}}_{i}\right)\right| /|C|-37 \quad(i=1,2,3) .
$$

По лемме 11.14 имеем ЭСогл $\alpha\left(\mathrm{F}_{1}, \mathrm{~F}_{3}^{-1}\right)$ и $Э С о г \Omega_{\alpha}\left(\mathrm{F}_{2}^{-1}, \mathrm{~F}_{3}\right)$. Тогда, применяя вначале лемму 13.8 при $\mathrm{T}^{-1} \mathrm{uT}^{\prime}:=\mathrm{T}^{-1} \mathrm{x}^{-1} \mathrm{~T}^{\prime}, \mathrm{E}:=\mathrm{F}_{1}$ и $\mathrm{F}:=\mathrm{F}_{3}^{-1}$, получаем ЭСогл $\Omega_{\alpha}\left(\mathrm{gF}_{1}, \mathrm{~F}_{3}^{-1}\right)$, а затем при $\mathrm{T}^{-1} \mathrm{uT}^{\prime}:=\mathrm{T}^{*} \mathrm{y}^{\prime-1}, \mathrm{E}:=\mathrm{F}_{2}^{-1}$ и $\mathrm{F}:=\mathrm{F}_{3}$, получаем ЭСогл $\alpha\left(\mathbf{f F}_{2}^{-1}, \mathrm{~F}_{3}\right)$, где $\mathbf{g}$ и $\mathbf{f}$ - элементы группы $\mathbf{B}_{\alpha}$, для которых $\mathbf{T}^{\prime}=\mathbf{g} \mathbf{T}$ и $\mathbf{T}^{\prime}=\mathbf{f T}^{*}$. Отсюда следует $Э$ богл $_{\alpha}\left(\mathrm{F}_{1}, \mathbf{g}^{-1} \mathbf{f} \mathrm{F}_{2}\right)$. Так как $\mathbf{g}^{-1} \mathbf{f} \mathbf{T}^{*}=\mathrm{T}$, то $\mathbf{g}^{-1} \mathbf{f} F_{2}$ - подпуть пути $\mathrm{T}$. Очевидно, $\mathrm{F}_{1} \ll \mathbf{T} \mathbf{g}^{-1} \mathbf{f} \mathrm{F}_{2}$. Тогда, используя предложения $11.24,8.46$ и 8.11, легко видеть, что $\overline{\mathrm{F}} \rightleftharpoons \mathrm{F}_{1} \cup \mathrm{T}_{\mathrm{T}} \mathbf{g}^{-1} \mathbf{f} \mathrm{F}_{2} \in \ni{ }_{\alpha}\left(\Gamma_{\alpha}\right)$ и

$$
\ell_{\alpha}(\overline{\mathrm{F}}) \geqslant \ell_{\alpha}\left(\mathrm{F}_{1}\right)+\ell_{\alpha}\left(\mathrm{F}_{2}\right)-1 \geqslant \frac{1}{|C|}\left(\left|\mathcal{L}\left(\widetilde{\mathrm{P}}_{1}\right)\right|+\left|\mathcal{L}\left(\widetilde{\mathrm{P}}_{2}\right)\right|\right)-75 .
$$


С учетом (14.9) и (14.7) имеем

$$
\ell_{\alpha}(\overline{\mathrm{F}})+\ell_{\alpha}\left(\mathrm{F}_{3}\right) \geqslant n-h-6 p-268>2 q_{4} .
$$

Отсюда следует, что слово $T$ содержит элементарную $q_{4}$-степень ранга $\alpha$, что и требовалось доказать.

Случай 2): $\widetilde{\mathrm{G}}_{1}, \widetilde{\mathrm{G}}_{2}$ входят в $\widetilde{\mathrm{T}}^{\prime-1}, \widetilde{\mathrm{G}}_{3}$ входит в $\widetilde{\mathrm{T}}$. Этот случай рассматривается аналогично случаю 1 ), с той лишь разнищей, что роли путей $\widetilde{T}$ и $\widetilde{T}^{\prime}$ меняются местами.

Утверждение (i) доказано.

Перейдем к доказательству (ii). Пусть $\mathrm{T}$ - путь на графе $\Gamma_{\alpha}$ с $\mathcal{L}(\mathrm{T})$ 으 $T$, и пусть $\mathrm{E} \in \ni л_{\alpha}\left(\Gamma_{\alpha}, 2 h+3 p+233\right)$ - подпуть пути $\mathrm{T}$. Рассмотрим путь $\mathrm{T}^{\prime-1} \mathbf{x} \mathrm{Ty}^{\prime \prime}-1$ на $\Gamma_{\alpha}$, где $\mathcal{L}\left(\mathrm{T}^{\prime}\right)$ 으 $\mathcal{L}\left(\mathrm{T}^{\prime \prime}\right)$ 으 $T, \mathcal{L}(\mathrm{x})$ 으 $x$ и $\mathcal{L}(\mathrm{y})$ 으 $y$. Заметим, что

$$
\mathbf{g} \mathbf{T}=\mathrm{T}^{\prime} \text { и } \mathbf{h} \mathbf{T}=\mathrm{T}^{\prime \prime},
$$

где $\mathbf{g}=\iota(\mathbf{T})[x]_{\alpha}^{-1}(\iota(\mathbf{T}))^{-1}$ и $\mathbf{h}=\tau(\mathbf{T})[y]_{\alpha}(\tau(\mathbf{T}))^{-1}$ - элементы группы $\mathbf{B}_{\alpha}$, указанные в (ii).

Предположим, граничный цикл некоторой области $\mathrm{A} \in \mathbf{R}_{\alpha}(\Delta)$ имеет две поддиаграммы примыкания $\Pi_{1}, \Pi_{2} \in \mathcal{S}$ к одному участку границы $\widetilde{\mathrm{T}}$ или $\widetilde{\mathrm{T}}^{\prime-1}$. Пусть $\delta \Pi_{i}=\widetilde{\mathrm{P}}_{i} \tilde{\mathrm{u}}_{i} \widetilde{\mathrm{G}}_{i} \tilde{\mathrm{v}}_{i}$, где $\mathcal{L}\left(\widetilde{\mathrm{u}}_{i}\right), \mathcal{L}\left(\widetilde{\mathrm{v}}_{i}\right) \in \mathcal{H}_{\alpha-1}$ и $\widetilde{\mathrm{P}}_{i}, \widetilde{\mathrm{G}}_{i}-$ участки примыкания, входящие соответственно в $\delta$ А и в $\widetilde{\mathrm{T}}$ или $\widetilde{\mathrm{T}}^{\prime-1}$.

Рассмотрим сначала более простой

Случай 1: $\widetilde{\mathrm{G}}_{1}$ и $\widetilde{\mathrm{G}}_{2}$ входят в $\widetilde{\mathrm{T}}^{\prime-1}$. Можно считать, что $\widetilde{\mathrm{T}}^{\prime}$ имеет вид $\widetilde{\mathrm{T}}^{\prime}=$ $\widetilde{\mathrm{K}} \widetilde{\mathrm{G}}_{1}^{-1} \widetilde{\mathrm{LG}}_{2}^{-1} \widetilde{\mathrm{M}}$. Пусть $\Theta$ - поддиаграмма диаграммы $\Delta$, "заключенная" между А, $\Pi_{1}, \Pi_{2}$ и $\widetilde{\top}$ (см. рис. 64 ). По лемме 10.8 , (i) $\Theta$ - кольцевая диаграмма. Следовательно, поддиаграмма $\Delta-\Theta$ диаграммы $\Delta$ односвязна. Рассмотрим отображение $\omega$ множества путей на диаграмме $\Delta-\Theta$ во множество путей на графе $\Gamma_{\alpha}$, где $\omega(\iota(\widetilde{\mathrm{T}}))=\iota(\mathrm{T})$. Для любого пути $\widetilde{\mathrm{X}}$ на $\Delta-\Theta$ обозначим $\widehat{\mathrm{X}} \rightleftharpoons \omega(\widetilde{\mathrm{X}})$. Очевидно, $\widehat{\mathrm{T}}=\mathrm{T}, \hat{\mathrm{x}}=\mathrm{x}, \hat{\mathrm{y}}=\mathrm{y}, \widehat{\mathrm{K}} \widehat{\mathrm{G}}_{1}^{-1}$ есть начало пути $\mathrm{T}^{\prime}$ и $\widehat{\mathrm{G}}_{2}^{-1} \widehat{\mathrm{M}}$ есть конец пути $\mathrm{T}^{\prime \prime}$. Из неравенств (14.5) и (14.6) вытекает $\left|\mathcal{L}\left(\widetilde{\mathrm{P}}_{1}\right)\right|+\left|\mathcal{L}\left(\widetilde{\mathrm{P}}_{2}\right)\right| \geqslant(q-6 p-112)|C|$, следовательно, для $i=1$ или $i=2$ имеем $\left|\mathcal{L}\left(\widetilde{\mathrm{P}}_{i}\right)\right| \geqslant \frac{1}{2}(q-6 p-112)|C|>h+44$. По лемме 11.5 путь $\widehat{\mathrm{G}}_{i}$ содержит подпуть $\mathrm{G}^{*} \in$ Эл $_{\alpha}\left(\Gamma_{\alpha}, h+8\right)$ с периодической основой, содержашейся в $\widehat{\mathrm{P}}_{i}^{-1}$.

Если ЭСогл $\alpha\left(\mathrm{E}, \mathrm{G}^{*}\right)$ и $i=1$, то, применив лемму 13.8 при $\mathrm{T}^{-1} \mathrm{uT}^{\prime}:=\mathrm{T}^{-1} \mathrm{x}^{-1} \mathrm{~T}^{\prime}$, $\mathrm{E}:=\mathrm{E}$ и $\mathrm{F}:=\mathrm{G}^{*}$, получим $Э \mathrm{Coz}_{\alpha}(\mathrm{E}, \mathrm{gE})$, т.е. имеет место первое из соотношений (14.4).

Если ЭСогл $\alpha\left(\mathrm{E}, \mathrm{G}^{*}\right)$ и $i=2$, то по лемме 13.8 при $\mathrm{T}^{-1} \mathrm{uT}^{\prime}:=\mathrm{Ty}^{\prime \prime-1}, \mathrm{E}:=\mathrm{E}^{-1}$ и $\mathrm{F}:=\mathrm{G}^{*-1}$ получаем $Э С о г \Omega_{\alpha}(\mathrm{E}, \mathrm{hE})$, т.е. второе соотношение (14.4).

Пусть $\neg$ Эогл $\Omega_{\alpha}\left(\mathrm{E}, \mathrm{G}^{*}\right)$. Пусть $\widetilde{\mathrm{W}}-$ подпуть $\delta \mathrm{A}$, соединяющий вершины $\tau\left(\widetilde{\mathrm{P}}_{2}\right)$ и $\iota\left(\widetilde{\mathrm{P}}_{1}\right)$. По лемме 11.16 путь Е устойчив относительно $\hat{\mathrm{v}}_{1} \widehat{\mathrm{W}}^{-1} \hat{\mathrm{u}}_{2}$. Так как согласно определению 11.11 Е устойчив относительно х и у и по условию

$$
\ell_{\alpha}(\mathrm{E}) \geqslant(3 p+217)+2(h+8)
$$




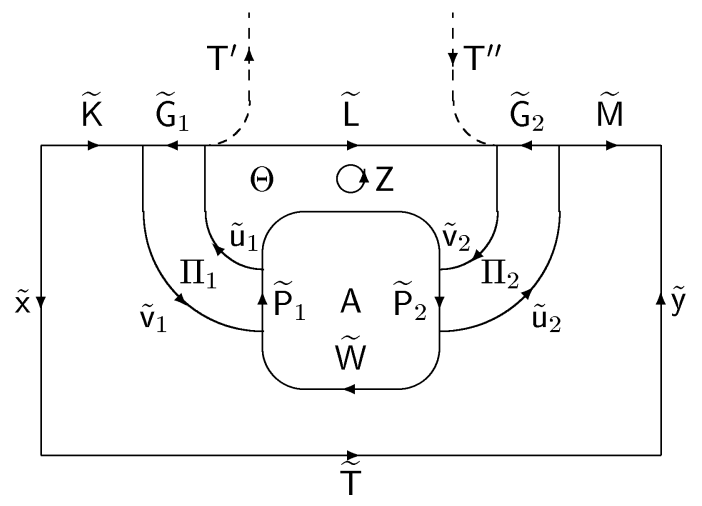

Рис. 64

то по предложению 11.30 найдется подпуть $\mathrm{F} \in$ Эл $_{\alpha}\left(\Gamma_{\alpha}, h+8\right)$ одного из путей $\widehat{\mathrm{K}}$ или $\widehat{\mathrm{M}}$ такой, что $Э С о г \Omega_{\alpha}(\mathrm{E}, \mathrm{H})$. Тогда аналогично предыдущему, используя лемму 13.8 , получаем $Э С о г \Omega_{\alpha}(\mathrm{E}, \mathrm{gE})$ в случае, если $\mathrm{F}$ содержится в $\widehat{\mathrm{K}}$, и $Э С о г \Omega_{\alpha}(\mathrm{E}, \mathrm{hE})$ в случае, если $\mathrm{F}$ содержится в $\widehat{\mathrm{M}}$.

Случай 2: $\widetilde{\mathrm{G}}_{1}$ и $\widetilde{\mathrm{G}}_{2}$ входят в $\widetilde{\mathrm{T}}$. Можно считать, что $\widetilde{\mathrm{T}}$ имеет вид $\widetilde{\mathrm{T}}=\widetilde{\mathrm{K}} \widetilde{\mathrm{G}}_{1} \widetilde{\mathrm{L}} \widetilde{\mathrm{G}}_{2} \widetilde{\mathrm{M}}$. Как и в случае 1 , подлиаграмма $\Theta$ диаграммы $\Delta$, "заключенная" между $\mathrm{A}, \Pi_{1}$, $\Pi_{2}$ и $\widetilde{\mathrm{T}}$, является кольцевой диаграммой и, следовательно, диаграмма $\Delta-\Theta$ односвязна. Рассмотрим отображение $\omega: \widetilde{\mathrm{X}} \mapsto \widehat{\mathrm{X}}$ множества путей на диаграмме $\Delta-\Theta$ во множество путей на графе $\Gamma_{\alpha}$ такое, что $\omega(\iota(\widetilde{\mathbf{T}}))=\iota(\mathrm{T})$. Очевидно, $\widehat{\mathrm{T}}^{\prime}=\mathrm{T}^{\prime}, \hat{\mathrm{x}}=\mathrm{x}, \widehat{\mathrm{K}} \widehat{\mathrm{G}}_{1}$ есть начало пути $\mathrm{T}$, a $\widehat{\mathrm{G}}_{2} \widehat{\mathrm{M}}$ есть конец некоторого пути $\mathrm{T}^{*}$ с $\mathcal{L}\left(\mathrm{T}^{*}\right)$ 으 $T$ (см. рис. 65). Рассуждая аналогично тому, как при рассмотрении случая 1 , рассматривая вместо подпути $\mathrm{E}$ пути $\mathrm{T}$ подпуть $\mathrm{gE}$ пути $\mathrm{T}^{\prime}$, получаем ЭСогл $\Omega_{\alpha}(\mathbf{g E}, \mathrm{E})$ или $Э С о г \Omega_{\alpha}\left(\mathrm{gE}, \mathbf{f}^{-1} \mathbf{g E}\right)$, где $\mathbf{f}$ - элемент группы $\mathbf{B}_{\alpha}$, для которого $\boldsymbol{T}^{\prime}=\mathbf{f} \mathbf{T}^{*}$. Если $Э$ Согл $_{\alpha}(\mathbf{g E}, \mathrm{E})$, то доказывать больше нечего. В случае ЭСог $\Omega_{\alpha}\left(\mathbf{g E}, \mathbf{f}^{-1} \mathbf{g E}\right)$ имеем $Э С о г \Omega_{\alpha}\left(\mathrm{E}, \mathbf{g}^{-1} \mathbf{f g E}\right)$, поэтому достаточно убедиться в том, что $\mathbf{g}^{-1} \mathbf{f} \mathbf{g}=\mathbf{h}$. Из определений $\mathbf{g}$ и $\mathbf{f}$ следует $\mathbf{f}^{-1} \mathbf{g} \boldsymbol{T}=\mathrm{T}^{*}$, откуда вытекает $\mathbf{f}^{-1} \mathbf{g}\left(\mathrm{Ty}^{\prime \prime} \mathbf{T}^{-1}\right)=\mathrm{T}^{*} \hat{\mathrm{y}} \mathrm{T}^{\prime-1}$ (см. рис. 65) и, следовательно, $\mathbf{f}^{-1} \mathbf{g} \mathrm{T}^{\prime \prime}=\mathrm{T}^{\prime}$. Последнее равенство можно переписать в виде $\mathbf{f}^{-1} \mathbf{g h T}=\mathbf{g} \mathbf{T}$, т.е. $\mathbf{f}^{-1} \mathbf{g h}=\mathbf{g}$, что и требовалось.

Таким образом, можно предполагать, что граничный цикл каждой области $\mathrm{A} \in \mathbf{R}_{\alpha}(\Delta)$ имеет не более одной поддиаграммы примыкания $\Pi \in \mathcal{S}$ к каждому из участков границы $\widetilde{\mathrm{T}}$ и $\widetilde{\mathrm{T}}^{\prime-1}$ диаграммы $\Delta^{\alpha-1}$. Из (14.5) и (14.6) следует, что для каждой области $\mathrm{A} \in \mathbf{R}_{\alpha}(\Delta)$ таких поддиаграмм примыкания ровно одна для $\widetilde{\top}$ и ровно одна для $\widetilde{\mathrm{T}}^{\prime-1}$. Пусть $\mathbf{R}_{\alpha}(\Delta)=\left\{\mathrm{A}_{1}, \mathrm{~A}_{2}, \ldots, \mathrm{A}_{t}\right\}$. Пусть $\Pi_{i}$ - поддиаграмма примыкания $\delta \mathrm{A} \mathrm{\kappa}^{\mathrm{T}}$ и $\Pi_{i}^{\prime}-$ поддиаграмма примыкания $\delta \mathrm{A} \mathrm{\kappa}^{\prime-1}$. Пусть $\widetilde{\mathrm{P}}_{i}$, $\widetilde{\mathrm{G}}_{i}$ - участки примыкания поддиаграммы примыкания $\Pi_{i}$, входяшие соответственно в $\delta$ А и в $\widetilde{T}$, и $\widetilde{S}_{i}, \widetilde{H}_{i}-$ участки примыкания поддиаграммы примыкания $\Pi_{i}^{\prime}$, входящие соответственно в $\delta$ и и в $\widetilde{T}^{\prime-1}$. Перенумеровав $\mathrm{A}_{i}$, можно считать, что $\widetilde{\mathrm{T}}_{\text {и }} \widetilde{\mathrm{T}}^{\prime}$ имеют вид $\widetilde{\mathrm{T}}=\widetilde{\mathrm{K}}_{0} \widetilde{\mathrm{G}}_{1} \widetilde{\mathrm{K}}_{1} \ldots \widetilde{\mathrm{G}}_{t} \widetilde{\mathrm{K}}_{t}$ и $\widetilde{\mathrm{T}}^{\prime}=\widetilde{\mathrm{L}}_{0} \widetilde{\mathrm{H}}_{1}^{-1} \widetilde{\mathrm{L}}_{1} \ldots \widetilde{\mathrm{H}}_{t}^{-1} \widetilde{\mathrm{L}}_{t}$. Пусть $\Theta_{0}, \Theta_{1}, \ldots, \Theta_{t}-$ связ- 


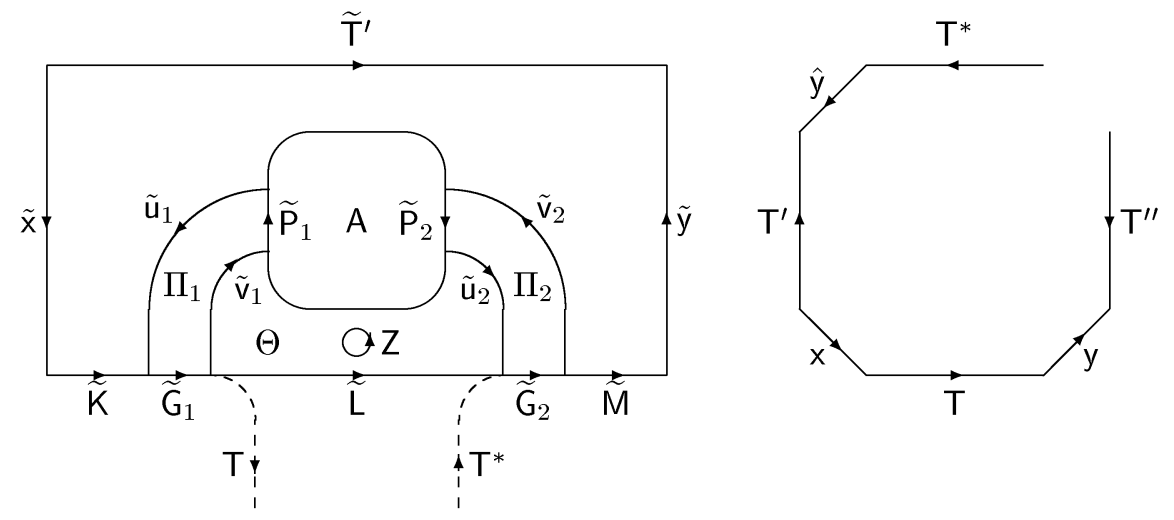

Рис. 65

ные компоненты поддиаграммы диаграммы $\Delta$, полученной удалением из $\Delta$ всех областей $A_{i}$ и поддиаграмм $\Pi_{i}, \Pi_{i}^{\prime}$ (см. рис. 66). Так как $\Delta-$ кольцевая диаграмма, то ровно одна из поддиаграмм $\Theta_{i}$ является кольцевой диаграммой, а остальные дисковыми диаграммами. Пусть $\Theta_{k}-$ кольцевая диаграмма. Пусть диаграмма $\bar{\Delta}$ получена из $\Delta$ удалением поддиаграммы $\Theta_{k}$, за исключением ребер, составляюших путь $\widetilde{\mathrm{K}}_{k}$. Рассмотрим отображение $\omega: \widetilde{\mathrm{X}} \mapsto \widehat{\mathrm{X}}$ множества путей на $\bar{\Delta}$ во множество путей на графе $\Gamma_{\alpha}$ такое, что $\omega(\iota(\widetilde{\mathrm{T}}))=\iota(\mathrm{T})$. Ясно, что $\widehat{\mathrm{T}}=\mathrm{T}$, путь $\widehat{\mathrm{L}}_{0} \widehat{\mathrm{H}}_{1}^{-1} \ldots \widehat{\mathrm{L}}_{k-1} \widehat{\mathrm{H}}_{k}^{-1}$ есть начало пути $\mathrm{T}^{\prime}$ и путь $\widehat{\mathrm{H}}_{k+1}^{-1} \widehat{\mathrm{L}}_{k+1} \ldots \widehat{\mathrm{H}}_{t}^{-1} \widehat{\mathrm{L}}_{t}$ есть конец пути $\mathrm{T}^{\prime \prime}$.

Используя леммы 11.15 и 11.14 , для каждого $i$ найдем подпути $\mathrm{G}_{i}^{*}, \mathrm{H}_{i}^{*} \in$ Эл $\alpha_{\alpha}\left(\Gamma_{\alpha}\right.$, $h+8)$ путей $\widehat{\mathrm{G}}_{i}, \widehat{\mathrm{H}}_{i}^{-1}$ соответственно такие, что $Э$ Согл $\alpha\left(\mathrm{G}_{i}^{*}, \mathrm{H}_{i}^{*}\right)$. Используя лемму 13.8 сперва при $\mathrm{T}^{-1} \mathrm{u}^{\prime}:=\mathrm{T}^{-1} \mathrm{x}^{-1} \mathrm{~T}^{\prime}, \mathrm{E}:=\mathrm{G}_{i}^{*}, \mathrm{~F}:=\mathrm{H}_{i}^{*}(1 \leqslant i \leqslant k)$, а затем при $\mathrm{T}^{-1} \mathrm{uT}^{\prime}:=\mathrm{Ty}^{\prime \prime-1}, \mathrm{E}:=\mathrm{G}_{i}^{*-1}, \mathrm{~F}:=\mathrm{H}_{i}^{*-1}(k+1 \leqslant i \leqslant t)$, получаем

$$
\ni \operatorname{Coг~} \Omega_{\alpha}\left(\mathbf{g G} \mathbf{G}_{i}^{*}, \mathrm{H}_{i}^{*}\right)(1 \leqslant i \leqslant k) \text { и } Э \operatorname{Coг\Omega _{\alpha }}\left(\mathbf{h} \mathrm{G}_{i}^{*}, \mathrm{H}_{i}^{*}\right)(k+1 \leqslant i \leqslant t) .
$$

Если $Э$ Согл $\Omega_{\alpha}\left(\mathrm{E}, \mathrm{G}_{i}^{*}\right)$ для некоторого $i$, то из $(14.10)$ и $Э С о г \Omega_{\alpha}\left(\mathrm{G}_{i}^{*}, \mathrm{H}_{i}^{*}\right)$ следует ЭСог $\Omega_{\alpha}(\mathrm{E}, \mathbf{g E})$ в случае $i \leqslant k$ и ЭСог $\Omega_{\alpha}(\mathrm{E}, \mathbf{h E})$ в случае $i \geqslant k+1$.

Пусть $\neg$ ЭСгл $\Omega_{\alpha}\left(\mathrm{E}, \mathrm{G}_{i}^{*}\right)$ ни для какого $i$. Если $k \geqslant 1$ и $\mathrm{E}<<_{\mathrm{T}} \mathrm{G}_{k}^{*}$, то, применяя лемму 11.16 и предложение 11.17 при $\mathrm{X}:=\widehat{\mathrm{K}}_{0} \widehat{\mathrm{G}}_{1} \ldots \widehat{\mathrm{K}}_{k-1} \widehat{\mathrm{G}}_{k}$ и $\mathrm{Y}:=\widehat{\mathrm{L}}_{0} \widehat{\mathrm{H}}_{1}^{-1} \ldots \widehat{\mathrm{L}}_{k-1} \widehat{\mathrm{H}}_{k}^{-1}$, найдем подпуть $\mathrm{F} \in$ Эл $_{\alpha}\left(\Gamma_{\alpha}, h+8\right)$ пути $\mathrm{T}^{\prime}$ такой, что $Э$ Согл $\alpha(\mathrm{E}, \mathrm{F})$, и, как мы уже видели вьше, из существования $\mathrm{F}$ в силу леммы 13.8 вытекает $Э C_{0} \Omega_{\alpha}(\mathrm{E}, \mathrm{gE})$. Аналогично, если $k \leqslant t-1$ и $\mathrm{G}_{k}^{*}<\mathrm{T}$, то в силу леммы 11.16 и предложения 11.17 найдется подпуть $\mathrm{F} \in \ni_{\alpha}\left(\Gamma_{\alpha}, h+8\right)$ пути $\mathrm{T}^{\prime}$ такой, что $Э С о г \Omega_{\alpha}(\mathrm{E}, \mathrm{F})$, откуда по лемме 13.8 будет следовать $Э С о г \Omega_{\alpha}(\mathrm{E}, \mathbf{h E})$. Поэтому достаточно ограничиться рассмотрением случая, когда $\mathrm{G}_{k}^{*}<_{\mathrm{T}} \mathrm{E}<_{\mathrm{T}} \mathrm{G}_{k+1}^{*}$ (соответственно $\mathrm{E}<\mathrm{T} \mathrm{G}_{1}^{*}$ при $k=0$ и $\mathrm{G}_{t}^{*}<\mathrm{T}$ Е при $k=t$ ). Так как из (14.10) в силу предложения 11.25 следует $\mathrm{E}<_{\mathrm{T}} \mathrm{G}_{i}^{*} \Leftrightarrow \mathrm{gE}<_{\mathrm{T}^{\prime}} \mathrm{H}_{i}^{*}$, то имеем также

$$
\mathrm{H}_{k}^{*}<_{\boldsymbol{T}^{\prime}} \mathbf{g E}<_{\boldsymbol{T}^{\prime}} \mathrm{H}_{k+1}^{*}\left(\mathbf{g E}<_{\mathrm{T}^{\prime}} \mathrm{H}_{1}^{*} \text { при } k=0 \text { и } \mathrm{H}_{t}^{*}<_{\mathrm{T}^{\prime}} \mathbf{g E} \text { при } k=t\right) .
$$




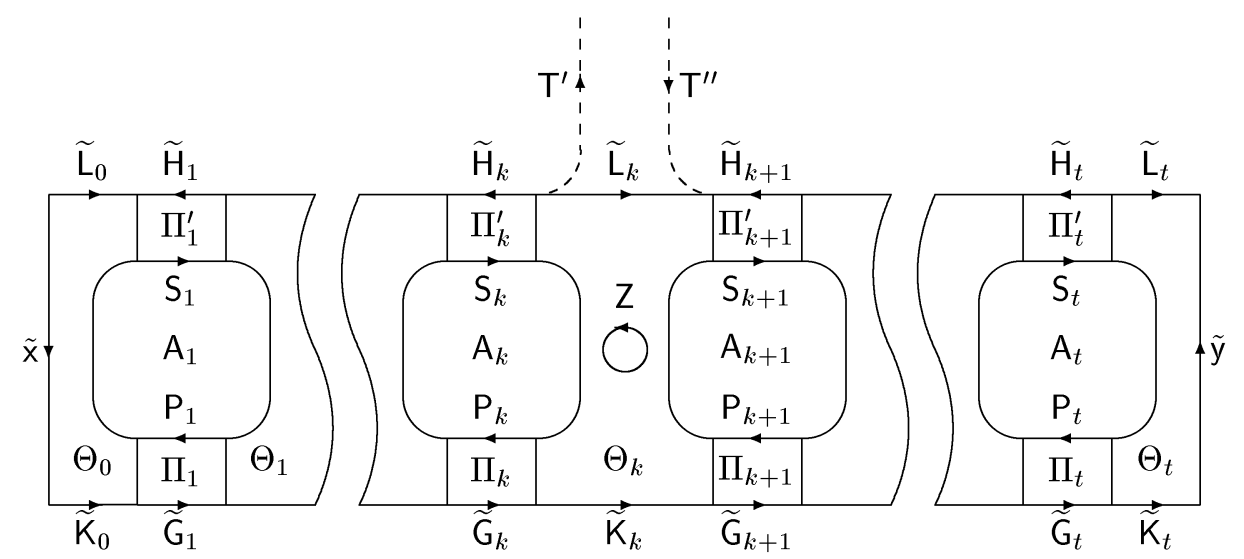

Рис. 66

Обозначим $E \rightleftharpoons \mathcal{L}(\mathrm{E})$, и пусть $E \in \Pi$ ППер $\alpha(D, 2 h+3 p+233)$, где $D \in \mathcal{E}_{\alpha}$. Предположим, $\Pi_{0} \in \mathcal{S}$ - поддиаграмма примыкания $\widetilde{\mathrm{T}}_{\mathrm{\kappa}} \widetilde{\mathrm{T}}^{\prime-1}, \delta \Pi_{0} \approx \widetilde{\mathrm{P}} \tilde{\mathrm{S}} \tilde{\mathrm{v}}$, где $\widetilde{\mathrm{P}}, \widetilde{\mathrm{S}}-$ участки примыкания, входяшие соответственно в $\widetilde{\mathrm{T}}$ и в $\widetilde{\mathrm{T}}^{\prime-1}$, и обшая часть вхождения слова $E$ в $T$ и одного из вхождений слова $\mathcal{L}(\mathrm{P})$ или $(\mathcal{L}(\mathrm{S}))^{-1}$ в $T$ содержит подслово $E^{\prime}$ слова $E$ такое, что $\ell_{\alpha, D}\left(E^{\prime}\right) \geqslant h+42$. Подлиаграмма $\Pi_{0}$ содержится в $\Theta_{k}$ и разбивает $\Theta_{k}$ на две связных компоненты $\Theta^{\prime}$ и $\Theta^{\prime \prime}$, одна из которых является кольцевой диаграммой, а другая односвязна (см. рис. 67). Пусть для определенности поддиаграмма $\Theta^{\prime}$ односвязна. Доопределим отображение $\omega$ на поддиаграммах $\Theta^{\prime}$ и $\Pi_{0}$, рассматривая вместо $\bar{\Delta}$ объединение $\bar{\Delta}$ и поддиаграмм $\Theta^{\prime}, \Pi_{0}$. Переходя к прообразу на графе $\Gamma_{\alpha-1}$ замкнутого пути $\widehat{P} \hat{\mathrm{u}} \widehat{\hat{v}}$ и используя предложение 8.26 , находим подпуть $\mathrm{F} \in \ni{ }_{\alpha}\left(\Gamma_{\alpha}, h+8\right)$ пути $\mathrm{T}^{\prime}$ такой, что $Э \mathrm{Coz}_{\alpha}(\mathrm{E}, \mathrm{F}) . \mathrm{Kak}$ мы уже видели вьше, отсюда в силу леммы 13.8 следует $Э С о г \Omega_{\alpha}(\mathrm{E}, \mathrm{gE})$. Аналогично, если $\Theta^{\prime \prime}$ односвязна, то найдется подпуть $\mathrm{F} \in$ Эл $_{\alpha}\left(\Gamma_{\alpha}, h+8\right)$ пути $\mathrm{T}^{\prime \prime}$ такой, что ЭСогл $\Omega_{\alpha}(\mathrm{E}, \mathrm{F})$, откуда вытекает $Э С о г \Omega_{\alpha}(\mathrm{E}, \mathrm{hE})$.

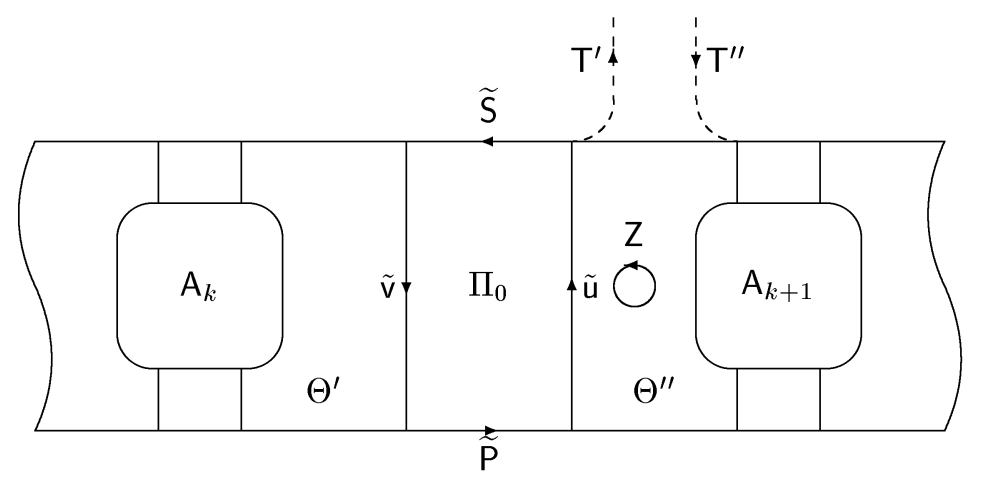

Рис. 67 
Итак, нам остается доказать сушествование поддиаграммы примыкания $\Pi_{0} \in$ $\mathcal{S}$, метка одного из участков примыкания которой покрывает подслово $E^{\prime}$ слова $E$, для которого $\ell_{\alpha, D}\left(E^{\prime}\right) \geqslant h+42$.

Предположим, имеется подлиаграмма примыкания $\Pi \in \mathcal{S}$ участка границы $\widetilde{\mathrm{T}}^{\prime-1}$ к самому себе. Диаграмма $\Delta-\Pi$ состоит из двух связных компонент $\Delta_{1}$ и $\Delta_{2}$ (см. рис. 68). Диаграмма $\Delta_{1}$ не может быть односвязной в силу леммы 10.8 , (ii), следовательно, $\Delta_{2}$ - односвязная диаграмма. В этом случае уже не существует поддиаграммы примыкания пути $\widetilde{\top}$ к самому себе. Таким образом, $\mathcal{S}$ не содержит одновременно поддиаграммы примыкания $\widetilde{\mathrm{T}}_{\mathrm{k}} \widetilde{\mathrm{T}}_{\text {и }} \widetilde{\mathrm{T}}^{\prime-1} \mathrm{\kappa}^{\prime-1}$. В силу полной аналогии можно ограничиться рассмотрением случая, когда $\mathcal{S}$ не содержит поддиаграммы примыкания пути Т к самому себе.

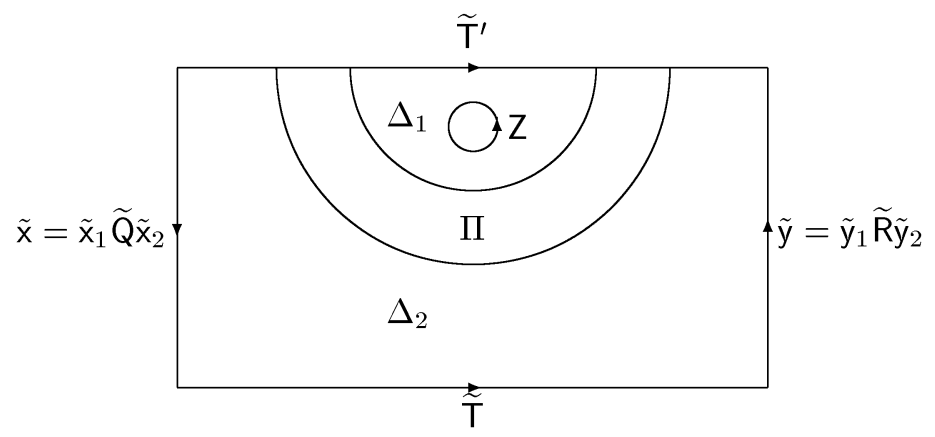

Рис. 68

Дальнейшие рассуждения будут слегка различаться в зависимости от того, является или не является поддиаграмма $\Theta_{k}$ крайней. Формально, мы рассмотрим четыре случая:

1. $0<k<t$;

2. $k=0, t>0$;

3. $k=t, t>0$;

4. $k=t=0$.

Случай 1: $0<k<t$. Пусть $\widetilde{\mathrm{K}}_{k}=\mathrm{w}_{0} \mathrm{~N}_{1} \mathrm{w}_{1} \ldots \mathrm{N}_{s} \mathrm{w}_{s}$, где $\mathrm{N}_{1}, \mathrm{~N}_{2}, \ldots, \mathrm{N}_{s}$ - участки примыкания поддиаграмм примыкания $\Sigma_{1}, \Sigma_{2}, \ldots, \Sigma_{s} \in \mathcal{S}$ и $\mathbf{w}_{i}-$ переходные участки диаграммы $\Delta^{\alpha-1}$ относительно $\mathcal{S}$. Обозначим через $\bar{G}_{k}, \bar{w}_{0}, \bar{N}_{1}, \bar{w}_{1}, \ldots$ $\ldots, \bar{N}_{s}, \bar{w}_{s}, \bar{G}_{k+1}$ подслова слова $E$, которые покрываются соответствуюшими вхождениями слов $\mathcal{L}\left(\widetilde{\mathrm{G}}_{k}\right), \mathcal{L}\left(\mathrm{w}_{0}\right), \mathcal{L}\left(\mathrm{N}_{1}\right), \mathcal{L}\left(\mathrm{w}_{1}\right), \ldots, \mathcal{L}\left(\mathrm{N}_{s}\right), \mathcal{L}\left(\mathrm{w}_{s}\right)$ и $\mathcal{L}\left(\widetilde{\mathrm{G}}_{k+1}\right)$ в $T$ (т.е. обшие части вхождений слова $E$ и указанных слов; если вхождения не пересекаются, то их общую часть будем считать пустым словом). Так как $E$ содержится в $\mathcal{L}\left(\widetilde{\mathrm{G}}_{k} \widetilde{\mathrm{K}}_{k} \widetilde{\mathrm{G}}_{k+1}\right)$, то

$$
E \text { 으 } \bar{G}_{k} \bar{w}_{0} \bar{N}_{1} \bar{w}_{1} \ldots \bar{N}_{s} \bar{w}_{s} \bar{G}_{k+1} \text {. }
$$

(При рассмотрении случая, когда нет поддиаграмм примыкания $\widetilde{\mathrm{T}}^{\prime-1} \mathrm{~K}^{\prime-1}$, нужно воспользоваться соотношением (14.11), из которого следует, что $E$ содержится в $\mathcal{L}\left(\widetilde{\mathrm{H}}_{k}^{-1} \widetilde{\mathrm{L}}_{k} \widetilde{\mathrm{H}}_{k+1}^{-1}\right)$.) Заметим, что все поддиаграммы примыкания $\Sigma_{i}$ содержатся 
в $\Theta_{k}$. По лемме 9.22 множество $\mathcal{S}_{1}$ поддиаграмм примыкания $\Pi \in \mathcal{S}$, содержащихся в $\Theta_{k}$, образует карту примыканий ранга $\alpha-1$ на $\Theta_{k}$. По лемме 9.23 , (ii) среди $\Sigma_{i}$ не более одной поддиаграммы примыкания $\widetilde{T}_{\text {к }} Z^{-1}$ и не более двух поддиаграмм примыкания $\widetilde{\mathrm{T}}_{\mathrm{k}} \widetilde{\mathrm{T}}^{\prime-1}$ (по предположению других поддиаграмм примыкания быть не может). В частности, $s \leqslant 3$. По предложению 10.4 имеем $\sum_{i=0}^{s} \mathcal{W}_{\alpha-1}\left(\mathcal{L}\left(\mathrm{w}_{i}\right)\right) \leqslant 4 \eta$. Тогда по предложению $8.22,(\mathrm{i})$

$$
\sum_{i=0}^{s} \ell_{\alpha, D}\left(\bar{w}_{i}\right) \leqslant 13 \cdot 4 \eta+4<25
$$

Так как никакой циклический сдвиг слова $Z$ не содержит элементарных 12 -степеней ранга $\alpha$, то в силу предложения 8.27 , если $\Sigma_{i}$ - поддиаграмма примыкания $\widetilde{T}$ к $Z^{-1}$, выполнено $\ell_{\alpha, D}\left(\bar{N}_{i}\right)<46$.

Если бы выполнялось $\ell_{\alpha, D}\left(\bar{G}_{k}\right) \geqslant p+34$, то, переходя к путям на графе $\Gamma_{\alpha-1}$ и используя предложение 8.38 , (ii), получили бы $Э С о г \Omega_{\alpha}\left(\mathrm{E}, \mathrm{G}_{k}^{*}\right)$ вопреки предположению. Следовательно, $\ell_{\alpha, D}\left(\bar{G}_{k}\right)<p+34$. Аналогично имеем $\ell_{\alpha, D}\left(\bar{G}_{k+1}\right)<$ $p+34$.

Так как

$$
\ell_{\alpha, D}(E) \geqslant 2 h+3 p+233>2(h+42)+2(p+34)+46+25+2 \cdot 6,
$$

то, используя предложение 8.14 , (ii), получаем, что по крайней мере одна из $\Sigma_{i}-$ подлиаграмма примыкания $\widetilde{\mathrm{T}}_{\mathrm{K}} \widetilde{\mathrm{T}}^{\prime-1}$, причем $\ell_{\alpha, D}\left(\bar{N}_{i}\right) \geqslant h+42$, что и требовалось доказать.

Случай $2: k=0, t>0$. В этом случае слово $E$ содержится в $\mathcal{L}\left(\widetilde{\mathrm{K}}_{0} \widetilde{\mathrm{G}}_{1}\right)$. В отличие от случая 1 , поддиаграмма $\Theta_{0}$ может содержать поддиаграммы примыкания $\widetilde{\mathrm{T}}_{\mathrm{K}} \widetilde{\mathrm{Q}}$. Пусть $\widetilde{\mathrm{K}}_{0}=\mathrm{w}_{0} \mathrm{~N}_{1} \mathrm{w}_{1} \ldots \mathrm{N}_{s} \mathrm{w}_{s}$, где $\mathrm{N}_{1}, \mathrm{~N}_{2}, \ldots, \mathrm{N}_{s}$ - участки примыкания поддиаграмм примыкания $\Sigma_{1}, \Sigma_{2}, \ldots, \Sigma_{s} \in \mathcal{S}$ и $\mathrm{w}_{i}$ - переходные участки диаграммы $\Delta^{\alpha-1}$ относительно $\mathcal{S}$, либо, возможно, $\mathbf{w}_{0}$ - пустой путь. Как и в случае 1 , через $\bar{w}_{0}, \bar{N}_{1}, \bar{w}_{1}, \ldots, \bar{N}_{s}, \bar{w}_{s}, \bar{G}_{1}$ обозначим обшие части вхождений слова $E$ и соответствуюших слов $\mathcal{L}\left(\mathrm{w}_{0}\right), \mathcal{L}\left(\mathrm{N}_{1}\right), \mathcal{L}\left(\mathrm{w}_{1}\right), \ldots, \mathcal{L}\left(\mathrm{N}_{s}\right), \mathcal{L}\left(\mathrm{w}_{s}\right)$ и $\mathcal{L}\left(\widetilde{\mathrm{G}}_{1}\right)$ в слово $T$. В силу лемм 9.22 и 9.23 , (ii) среди $\Sigma_{i}$ не более одной поддиаграммы примыкания $\widetilde{T}$

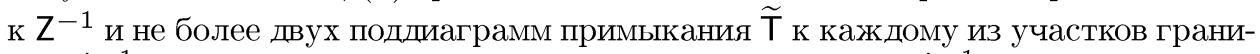
цы $\widetilde{T}^{\prime-1}$ и $\widetilde{Q}$, причем по две поддиаграммы примыкания к $\widetilde{T}^{\prime-1}$ и $\widetilde{Q}$ одновременно быть не может.

Так как по условию леммы $|\widetilde{\mathrm{Q}}|<2|A|$, где $\mathcal{L}(\widetilde{\mathrm{Q}}) \in \Pi$ Пер $(A)$ и $A \in \mathcal{E}_{\alpha}$, то в силу предложения 8.44, (ii), в случае, если $\Sigma_{i}$ - поддиаграмма примыкания $\widetilde{\mathrm{T}}$ к $\widetilde{\mathrm{Q}}$, имеем $\ell_{\alpha, D}\left(\bar{N}_{i}\right)<p+34$.

Далее можно повторить рассуждения из случая 1 , принимая во внимание, что в случае, если среди $\Sigma_{i}$ имеются две поддиаграммы примыкания $\widetilde{T}_{\mathrm{K}} \widetilde{\mathrm{T}}^{\prime-1}$, подлиаграмм примыкания $\widetilde{T}_{\mathrm{k}} \widetilde{\mathrm{Q}}$ не более одной (так как $h+42>p+34$, то отсюда следует, что для $\ell_{\alpha, D}(E)$ достаточно нижней оценки (14.12), с увеличением правой части неравенства (14.12) на 5 из-за новой оценки $s \leqslant 4$ вместо $s \leqslant 3)$. 
Случай 3: $k=t, t>0$. Этот случай симметричен случаю 2.

Случай 4: $k=t=0$. Рассматривается аналогично случаю 2 , с тем отличием, что $\Theta_{0}$ может содержать поддиаграммы примыкания $\widetilde{T}$ к двум участкам границы $\widetilde{Q}$ и $\widetilde{R}$ (см. замечание в конще случая 2 ).

14.6. Лемма. $Е с л и ~ 1 \leqslant \beta \leqslant \alpha, A \in \mathcal{E}_{\beta} u \mathbf{G} \subseteq \mathbf{S}_{\alpha}^{*}(A) \cup \mathbf{S}_{\alpha}^{-}(A)-$ nодгрупnа группы $\mathbf{B}_{\alpha}$, то существует конечная подгруппа $\widetilde{\mathbf{G}}$ группь $\mathbf{B}_{\beta-1}$ такая, что $\mathbf{G}=\pi_{\beta-1, \alpha}(\widetilde{\mathbf{G}}) u \widetilde{\mathbf{G}} \subseteq \mathbf{N}_{\beta-1}^{*}(A) \cup \mathbf{N}_{\beta-1}^{-}(A)$.

ДокАЗАТЕЛЬСТво. Если $\mathbf{G} \subseteq \mathbf{S}_{\alpha}^{*}(A)$, то в качестве $\widetilde{\mathbf{G}}$ можно взять подгруппу $\pi_{\beta-1, \alpha}^{-1}(\mathbf{G}) \cap \mathbf{N}_{\beta-1}^{*}(A)$, которая конечна в силу предложения 7.14 , (ii). Пусть найдется хотя бы один элемент $\mathbf{g}_{0} \in \mathbf{G} \cap \mathbf{S}_{\alpha}^{-}(A)$. Заметим, что в силу предложений 13.7 , (ii) и 7.2 , (ii) множества $\mathbf{S}_{\alpha}^{+}(A)$ и $\mathbf{S}_{\alpha}^{-}(A)$ образуют смежные классы групшы $\mathbf{S}_{\alpha}(A)$ по ее нормальной подгруппе $\mathbf{S}_{\alpha}^{+}(A)$ индекса 2 . Следовательно, $\mathbf{G}=\mathbf{H} \cup \mathbf{g}_{0} \mathbf{H}$, где $\mathbf{H} \rightleftharpoons \mathbf{G} \cap \mathbf{S}_{\alpha}^{+}(A)=\mathbf{G} \cap \mathbf{S}_{\alpha}^{*}(A)$. Выберем прообраз $\tilde{\mathbf{g}}_{0} \in \mathbf{N}_{\beta-1}^{-}(A)$ в группе $\mathbf{B}_{\beta-1}$ элемента $\mathbf{g}_{0}$ и положим $\widetilde{\mathbf{G}} \rightleftharpoons\left\langle\widetilde{\mathbf{H}}, \tilde{\mathbf{g}}_{0}\right\rangle$, где $\widetilde{\mathbf{H}} \rightleftharpoons \pi_{\beta-1, \alpha}^{-1}(\mathbf{H}) \cap \mathbf{N}_{\beta-1}^{*}(A)$. Тогда имеем $\pi_{\beta-1, \alpha}(\widetilde{\mathbf{G}})=\mathbf{G}$, а конечность подгруппы $\widetilde{\mathbf{G}}$ легко вытекает из предложения 7.14 , (ii) и следствий 7.16, 7.17.

14.7. Лемма. Пусть $1 \leqslant \beta \leqslant \alpha-1, \quad 1 \leqslant \gamma \leqslant \alpha-1, \quad x_{1}, x_{2}, \ldots, x_{k}-$ слова, представляющие все әлементы некоторой подгруппы $\mathbf{H} \subseteq \mathbf{S}_{\alpha}(A)$, где $A \in \overline{\mathcal{E}}_{\beta}$, и пусть у - нормальная форма ранга $\gamma$. Пусть дано слово T такое, что для каждого $i$ әлемент $\left[T^{-1} x_{i} T y\right]_{\alpha}$ группь $\mathbf{B}_{\alpha}$ имеет конечный порядок и ранг $\left[T^{-1} x_{i} T y\right]_{\alpha} \leqslant \alpha-1$. Тогда существуют слова $x_{1}^{*}, x_{2}^{*}, \ldots, x_{k}^{*}, T^{*} u$ нормальная форма $y^{*}$ ранга $\gamma$ такие, что для некоторых $U, V$ выполнены соотношения

$$
T \stackrel{\alpha}{=} U T^{*} V, \quad U^{-1} x_{i} U \stackrel{\alpha}{=} x_{i}^{*}(i=1,2, \ldots, k), \quad V y V^{-1} \stackrel{\alpha}{=} y^{*},
$$

для каждого $і$ әлемент $\left[T^{*-1} x_{i}^{*} T^{*} y^{*}\right]_{\alpha-1}$ группь $\mathbf{B}_{\alpha-1}$ имеет конечный порядок, ранг $\left[T^{*-1} x_{i}^{*} T^{*} y^{*}\right]_{\alpha-1}=$ ранг $\left[T^{-1} x_{i} T y\right]_{\alpha}, \mathbf{H}^{*} \rightleftharpoons\left\{\left[x_{i}^{*}\right]_{\alpha-1}\right\}_{i}-$ подгруппа группь $\mathbf{B}_{\alpha-1} u \mathbf{H}^{*} \subseteq \mathbf{S}_{\alpha-1}\left(A^{*}\right)$ для некоторого $A^{*} \in \overline{\mathcal{E}}_{\beta}$.

ДокаЗАТЕльство. Докажем сначала более слабое утверждение, изменив заключение леммы следуюшим образом: вместо условия, чтобы слово $y^{*}$ было нормальной формой ранга $\gamma$, потребуем только, чтобы элемент $\left[y^{*}\right]_{\alpha-1}$ имел конечньй порядок, а вместо условия $\mathbf{H}^{*} \subseteq \mathbf{S}_{\alpha-1}\left(A^{*}\right)\left(A^{*} \in \overline{\mathcal{\varepsilon}}_{\beta}\right)$ потребуем, чтобы $\mathbf{H}^{*}$ была конечной подгруппой группы $\mathbf{B}_{\alpha-1}$.

В силу предложения 12.13 можно считать $T \in \mathcal{R}_{\alpha}$. В силу условия $\mathbf{H} \subseteq \mathbf{S}_{\alpha}(A)$ $\left(A \in \bar{\varepsilon}_{\beta}\right)$, предложения 13.3 и замечания после определения 13.4 (при $\alpha:=\alpha-1$ ) можно считать $x_{i} \in \mathcal{H}_{\beta} \subseteq \mathcal{H}_{\alpha-1}$ при всех $i$, причем элементы $\left[x_{i}\right]_{\alpha-1}$ образуют некоторую конечную подгруппу группы $\mathbf{B}_{\alpha-1}$. Выберем произвольный путь $\mathbf{T}$ на $\Gamma_{\alpha-1}$ с $\mathcal{L}(\mathbf{T})$ 으 $T$. Положим

$$
\begin{aligned}
\widehat{\mathbf{T}} \rightleftharpoons \pi_{\alpha-1, \alpha}(\mathbf{T}), & \mathbf{g}_{i} \rightleftharpoons \iota(\widehat{\mathbf{T}})\left[x_{i}\right]_{\alpha}^{-1} \quad(\iota(\widehat{\mathbf{T}}))^{-1}(i=1,2, \ldots, k), \\
& \mathbf{h} \rightleftharpoons \tau(\widehat{\mathbf{T}})[y]_{\alpha}(\tau(\widehat{\mathbf{T}}))^{-1} .
\end{aligned}
$$


Пусть $\mathrm{E} \in$ ППер $\alpha(C, p)$ - подпуть пути Т, где $C \in \mathcal{E}_{\alpha}$. Назовем $\mathrm{E} x$-зависимым, если при всех $i$ вьполнено $Э$ Согл $\alpha\left(\pi_{\alpha-1, \alpha}(\mathrm{E}), \mathbf{g}_{i} \pi_{\alpha-1, \alpha}(\mathrm{E})\right)$, и $y$-зависимылм, если $\vartheta \operatorname{Coz}_{\alpha}\left(\pi_{\alpha-1, \alpha}(\mathrm{E}), \mathbf{h} \pi_{\alpha-1, \alpha}(\mathrm{E})\right)$. Очевидно,

если $\mathrm{E} \in \Pi \Pi е p_{\alpha}(C, p), \quad \mathrm{F} \in \Pi \Pi е p_{\alpha}(D, p) \quad\left(C, D \in \mathcal{E}_{\alpha}\right)-$ nодпути пути $\mathrm{T}$,

ПСогл $\alpha$ (Е, F) и Е $х$-зависим (у-зависим), то F $x$-зависим (у-зависим).

Кроме того, согласно лемме 14.5, (ii)

если $\mathrm{E} \in \Pi \Pi е p_{\alpha}(C, 2 h+3 p+233) \quad\left(C \in \mathcal{E}_{\alpha}\right)-$ nодnymь nymu $\mathrm{T}$,

то Е х-зависим или у-зависим.

Если Т содержит хотя бы один $x$-зависимый подпуть, представим $\mathrm{T}$ в виде $\mathrm{T}=\mathrm{XW}$, где $\mathrm{X}=\mathrm{X}_{1} \mathrm{E}, \mathrm{E}-x$-зависимый подпуть пути $\mathrm{T}$ и $\mathrm{W}$ не содержит $x$-зависимых подпутей $\mathrm{T}$. В противном случае, если Т не содержит $x$-зависимых подпутей, положим $\mathrm{W} \rightleftharpoons \mathrm{T}$. Если теперь $\mathrm{W}$ содержит хотя бы один $y$-зависимый подпуть, представим $\mathrm{W}$ в виде $\mathrm{W}=\mathrm{YZ}$, где $\mathrm{Z}=\mathrm{FZ}, \mathrm{F}-y$-зависимый подпуть $\mathrm{W}$ и $\mathrm{Y}$ не содержит таких подпутей. В противном случае положим $\mathrm{Y} \rightleftharpoons \mathrm{W}$. C учетом (14.14) получаем, что имеет место один из четырех случаев:

(i) Т не содержит $x$-зависимых или $y$-зависимых подпутей;

(ii) $\mathrm{T}=\mathrm{XY}$, где $\mathrm{X}=\mathrm{X}_{1} \mathrm{E}, \mathrm{E}-x$-зависимый подпуть и $\mathrm{Y}$ не содержит $x$-зависимых или $y$-зависимых подпутей;

(iii) $\mathrm{T}=\mathrm{YZ}$, где $\mathrm{Z}=\mathrm{FZ} \mathrm{Z}_{1}, \mathrm{~F}$ - $y$-зависимый подпуть и $\mathrm{Y}$ не содержит $x$-зависимых или $y$-зависимых подпутей;

(iv) $\mathrm{T}=\mathrm{XYZ}$, где $\mathrm{X}=\mathrm{X}_{1} \mathrm{E}, \mathrm{Z}=\mathrm{FZ} 1, \mathrm{E}-x$-зависимьй подпуть, $\mathrm{F}$ - $y$-зависимьй подпуть, $\neg \Pi$ Согл $\Omega_{\alpha}(\mathrm{E}, \mathrm{F})$ и $\mathrm{Y}$ не содержит $x$-зависимых или $y$-зависимых подпутей.

Случай (i) самый простой. В этом случае в силу (14.15) слово $T$ не содержит элементарных $(2 h+3 p+233)$-степеней ранга $\alpha$, и тогда в силу леммы 14.5, (i) можно положить $x_{i}^{*} \rightleftharpoons x_{i}, T^{*} \rightleftharpoons T, y^{*} \rightleftharpoons y$ и $U \rightleftharpoons V \rightleftharpoons 1$. Из трех оставшихся случаев подробно рассмотрим только (iv). Рассуждения в случаях (ii) и (iii) будут получаться несложной модификацией рассуждений для случая (iv).

Итак, пусть $\mathrm{T}=\mathrm{XYZ}, \quad \mathrm{X}=\mathrm{X}_{1} \mathrm{E}, \mathrm{Z}=\mathrm{FZ}_{1}, \mathrm{E} \in \Pi_{\text {пер }}(C, p), \quad \mathrm{F} \in$ ППер $\alpha(D, p) \quad\left(C, D \in \mathcal{E}_{\alpha}\right), \mathrm{E}-x$-зависимый подпуть, $\mathrm{F}$ - $y$-зависимый подпуть, $\neg \Pi C о \Omega_{\alpha}(\mathrm{E}, \mathrm{F})$ и $\mathrm{Y}$ не содержит $x$-зависимых или $y$-зависимых подпутей. Пусть $\mathrm{P} \in \Pi_{\mathrm{p}}\left(\Gamma_{\alpha-1}, C, 2\right)$ - периодическая основа для $\mathrm{E}$ и $\mathrm{Q} \in \Pi_{\mathrm{ep}}\left(\Gamma_{\alpha-1}, D, 2\right)$ - периодическая основа для $\mathrm{F}$. Будем считать, что слово $\mathcal{L}(\mathrm{P})$ оканчивается на $C$, а слово $\mathcal{L}(\mathrm{Q})$ начинается с $D$. Заметим, что в этом случае $C, D \in \bar{\varepsilon}_{\alpha}$ в силу определений $5.16,13.1$ и леммы 7.6. В силу леммы 8.2 , (ii) соединим вершины $\tau(\mathrm{P})$ и $\tau(\mathrm{E})$ путем и с $\mathcal{L}(\mathrm{u}) \in \mathcal{H}_{\alpha-1}$, а вершины $\iota(\mathrm{F})$ и $\iota(\mathrm{Q})$ путем v с $\mathcal{L}(\mathrm{v}) \in \mathcal{H}_{\alpha-1}$. Через $X, Y, Z, u$ и $v$ обозначим метки путей $\mathrm{X}, \mathrm{Y}, \mathrm{Z}, \mathrm{u}$ и $\mathbf{v}$ соответственно. Из $\Im \operatorname{Cогл}_{\alpha}\left(\pi_{\alpha-1, \alpha}(\mathrm{E}), \mathbf{g}_{i} \pi_{\alpha-1, \alpha}(\mathrm{E})\right)$ следует $\operatorname{Cогл}\left(\mathrm{P}, \tilde{\mathbf{g}}_{i} \mathrm{P}\right)$ или $\operatorname{Cогл~}\left(\mathrm{P}, \tilde{\mathbf{g}}_{i} \mathrm{P}^{-1}\right)$ для некоторого $\tilde{\mathbf{g}}_{i} \in \mathbf{B}_{\alpha-1}$, где $\pi_{\alpha-1, \alpha}\left(\tilde{\mathbf{g}}_{i}\right)=\mathbf{g}_{i}$. Тогда по предложению 7.3 при $\mathrm{P}:=\mathrm{P}^{-1}$ и $\mathbf{g}:=\tilde{\mathbf{g}}_{i}$

$$
(\tau(\mathrm{P}))^{-1} \tilde{\mathbf{g}}_{i} \tau(\mathrm{P}) \in \mathbf{N}_{\alpha-1}(C),
$$


откуда, применив к обеим частям $\pi_{\alpha-1, \alpha}$ и используя выражение для $\mathbf{g}_{i}$, выводим

$$
\pi_{\alpha-1, \alpha}\left((\tau(\mathrm{P}))^{-1} \iota(\mathrm{T})\right)\left[x_{i}\right]_{\alpha} \pi_{\alpha-1, \alpha}\left((\iota(\mathrm{T}))^{-1} \tau(\mathrm{P})\right) \in \mathbf{S}_{\alpha}(C) .
$$

Так как вершины $\tau(\mathrm{P})$ и $\iota(\mathrm{T})$ соединены путем $\mathrm{u}^{-1}$, то последнее включение можно переписать в виде

$$
\left[u X^{-1} x_{i} X u^{-1}\right]_{\alpha} \in \mathbf{S}_{\alpha}(C) \quad(i=1,2, \ldots, k)
$$

Аналогично, из $y$-зависимости пути $F$, применяя предложение 7.3 при $\mathrm{P}:=\mathrm{Q}$ и $\mathbf{g}:=\tilde{\mathbf{h}}$ для некоторого $\tilde{\mathbf{h}}$, где $\pi_{\alpha-1, \alpha}(\tilde{\mathbf{h}})=\mathbf{h}$, выводим

$$
\left[v^{-1} Z y Z^{-1} v\right]_{\alpha} \in \mathbf{S}_{\alpha}(D)
$$

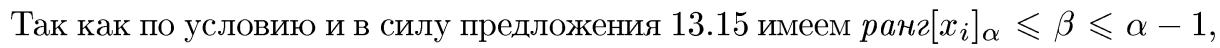
то снова в силу предложения $13.15\left\{\left[u X^{-1} x_{i} X u^{-1}\right]_{\alpha}\right\}_{i} \subseteq \mathbf{S}_{\alpha}^{*}(C) \cup \mathbf{S}_{\alpha}^{-}(C)$. Так как, кроме того, $\left\{\left[u X^{-1} x_{i} X u^{-1}\right]_{\alpha}\right\}_{i}$-подгруппа групшы $\mathbf{S}_{\alpha}(C)$, то, используя леммы 14.6 и 13.2 , (iv), найдем $r \in \mathbb{Z}$ и ограниченные в ранге $\alpha$ слова $x_{i}^{*} \in \mathcal{H}_{\alpha}$ такие, что

$$
u X^{-1} x_{i} X u^{-1} \stackrel{\alpha}{=} C^{-r} x_{i}^{*} C^{r} \quad(i=1,2, \ldots, k)
$$

и элементы $\left[x_{i}^{*}\right]_{\alpha-1}$ образуют конечную подгруппу $\mathbf{H}^{*}$ группы $\mathbf{B}_{\alpha-1}$. Аналогично, в силу предложения $13.15\left[v^{-1} Z y Z^{-1} v\right]_{\alpha} \in \mathbf{S}_{\alpha}^{*}(D) \cup \mathbf{S}_{\alpha}^{-}(D)$, и тогда в силу леммы 13.2 , (i), (iii) для некоторого $s \in \mathbb{Z}$ и некоторого ограниченного в ранге $\alpha$ слова $y^{*} \in \mathcal{H}_{\alpha}$ имеет место равенство

$$
v^{-1} Z y Z^{-1} v \stackrel{\alpha}{=} D^{-s} y^{*} D^{s}
$$

причем $\left[y^{*}\right]_{\alpha-1}$ - элемент конечного порядка (последнее вытекает из предложения 7.14 , (ii) и следствия 7.17). Так как в силу следствия 7.19 элементы $[C]_{\alpha}^{n / 2}$ и $[D]_{\alpha}^{n / 2}$ лежат в центрах подгрупп $\mathbf{S}_{\alpha}(C)$ и $\mathbf{S}_{\alpha}(D)$ соответственно, то можно считать $|r|,|s| \leqslant n / 4$.

Выберем слово $T^{*} \in \mathcal{R}_{\alpha-1}$ такое, что

$$
T^{*} \stackrel{\alpha=1}{=} C^{r} u Y v D^{-s}
$$

Заметим, что из равенств $T$ ㅇ $X Y Z$ и (14.16)-(14.18) следует

$$
\begin{aligned}
T^{-1} x_{i} T y & \stackrel{\alpha}{=} Z^{-1} Y^{-1} X^{-1} \cdot X u^{-1} C^{-r} x_{i}^{*} C^{r} u X^{-1} \cdot X Y Z \cdot Z^{-1} v D^{-s} y^{*} D^{s} v^{-1} Z \\
& \stackrel{\alpha}{=} Z^{-1} v D^{-s}\left(T^{*-1} x_{i}^{*} T^{*} y^{*}\right) D^{s} v^{-1} Z .
\end{aligned}
$$

Поэтому для каждого $i$ элемент $\left[T^{*-1} x_{i}^{*} T^{*} y^{*}\right]_{\alpha}$ имеет конечный порядок и ранги элементов $\left[T^{*-1} x_{i}^{*} T^{*} y^{*}\right]_{\alpha}$ и $\left[T^{-1} x_{i} T y\right]_{\alpha}$ совпадают. Кроме того, непосредственно проверяется выполнение соотношений (14.13) при $U:=X u^{-1} C^{-r}$ и 
$V:=D^{s} v^{-1} Z$. Остается доказать, что элемент $\left[T^{*-1} x_{i}^{*} T^{*} y^{*}\right]_{\alpha-1}$ группы $\mathbf{B}_{\alpha-1}$

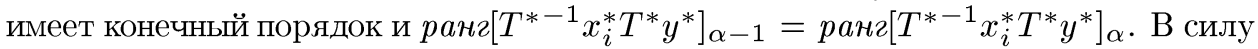
леммы 14.5 , (i) для этого достаточно проверить, что слово $T^{*}$ не содержит элементарных $q_{4}$-степеней ранга $\alpha$.

В силу равенства (14.18) на графе $\Gamma_{\alpha-1}$ имеется путь $\mathrm{P}_{1}^{-1} \mathrm{~T}^{*} \mathrm{Q}_{1}$ с началом в $\iota(\mathrm{uYv})$ и с конщом в $\tau(\mathrm{uYv})$, где метки $\mathrm{P}_{1}, \mathrm{~T}^{*}$ и $\mathrm{Q}_{1}$ равны $C^{r}, T^{*}$ и $D^{s}$ соответственно (см. рис. 69). Допустим, $\mathrm{G} \in$ ППер $\alpha\left(B, q_{4}\right)$ - подпуть пути $\mathrm{T}^{*}$ для некоторого $B \in \mathcal{E}_{\alpha}$. Так как $|r|,|s| \leqslant n / 4$, то в силу леммы 8.2, (ii) и предложения 8.44, (ii) для любого подпути $\mathrm{H} \in$ ППер $\alpha(B)$ пути $\mathrm{P}_{1}$ или $\mathrm{Q}_{1}^{-1}$ имеем $\ell_{\alpha, B}(\mathrm{H}) \leqslant n / 4+77$. Кроме того, в силу (14.15) путь $\mathrm{Y}_{\text {не содержит подпутей } \mathrm{H} \in \Pi \text { Пер } \alpha}(B, 2 h+3 p+233)$. Тогда в силу леммы 11.7 , (ii) при $\mathrm{X}:=\mathrm{Y}, \mathrm{Y}:=\mathrm{Y}, \mathrm{W}:=\mathrm{u}^{-1} \mathrm{P}_{1}^{-1} \mathrm{~T}^{*} \mathrm{Q}_{1} \mathrm{v}^{-1}, \mathrm{Z}_{1}:=\mathrm{P}_{1}^{-1}$, $\mathrm{Z}_{2}:=\mathrm{T}^{*}, \mathrm{Z}_{3}:=\mathrm{Q}_{1}$ и неравенства

$$
q_{4}-45 \cdot 3+5>\max \left\{\frac{n}{4}+77,2 h+3 p+233\right\}+p
$$

найдутся подпути $\mathrm{H}_{1}, \mathrm{H}_{2} \in$ ППер $\alpha(B, p)$ двух различных путей $\mathrm{P}_{1}, \mathrm{Y}$ или $\mathrm{Q}_{1}^{-1}$ такие, что ПСогл $\Omega_{\alpha}\left(\mathrm{H}_{1}, \mathrm{H}_{2}\right)$. Вывод искомого противоречия разбивается на три случая.

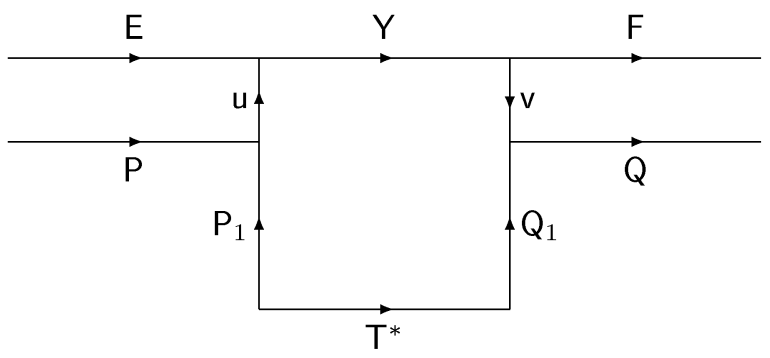

Рис. 69

Случай 1: $\mathrm{H}_{1}$ содержится в $\mathrm{P}_{1}, \mathrm{H}_{2}$ содержится в $\mathrm{Q}_{1}^{-1}$. По предложению 8.44, (i) имеем $\left|\mathrm{P}_{1}\right|>2|C|,\left|\mathrm{Q}_{1}\right|>2|D|$. По предложению 8.36 получаем $C о г л\left(\mathrm{P}_{1}, \mathrm{R}_{1}\right)$ и Согл $\left(\mathrm{Q}_{1}^{-1}, \mathrm{R}_{2}\right)$, где $\mathrm{R}_{i} \in$ Пер $\left(\Gamma_{\alpha-1}, B, 2\right)$ - периодическая основа для $\mathrm{H}_{i}$. Так как ПСогл $\Omega_{\alpha}\left(\mathrm{H}_{1}, \mathrm{H}_{2}\right)$, то отсюда следует Cогл $\left(\mathrm{P}_{1}, \mathrm{Q}_{1}^{-1}\right)$. Так как слово $\mathcal{L}(\mathrm{P})$ оканчивается на $C$ и $\mathcal{L}\left(\mathrm{P}_{1}\right)$ 으 $C^{r}$, то при некотором $\epsilon= \pm 1$ пути $\mathrm{P}$ и $\mathrm{P}_{1}^{\epsilon}$ имеют общее периодическое продолжение. По той же причине при некотором $\delta= \pm 1$ пути $\mathrm{Q}$ и $\mathrm{Q}_{1}^{\delta}$ имеют общее периодическое продолжение. Тогда из Согл $\left(\mathrm{P}_{1}, \mathrm{Q}_{1}^{-1}\right)$ следует $\operatorname{Coгл}(\mathrm{P}, \mathrm{Q})$ или $\operatorname{Coгл}\left(\mathrm{P}, \mathrm{Q}^{-1}\right)$, т.е. ПСогл $\alpha(\mathrm{E}, \mathrm{F})$ или $П С о г \Omega_{\alpha}\left(\mathrm{E}, \mathrm{F}^{-1}\right)$. Но первое соотношение невозможно в силу предположения случая (iv), а второе - в силу предложения 8.46 .

Случай 2: $\mathrm{H}_{1}$ содержится в $\mathrm{P}_{1}, \mathrm{H}_{2}$ содержится в $\mathrm{Y}$. Тогда, рассуждая аналогично случаю 1 , получаем ПСогл $\Omega_{\alpha}\left(\mathrm{E}, \mathrm{H}_{2}\right)$ или $\Pi$ Согл $\Omega_{\alpha}\left(\mathrm{E}, \mathrm{H}_{2}^{-1}\right)$. В первом случае получаем противоречие с (14.14) и условием на $\mathrm{Y}$ в (iv), а во втором - с предложением 8.46 . 
Случай 3: $\mathrm{H}_{1}$ содержится в $\mathrm{Y}, \mathrm{H}_{2}$ содержится в $\mathrm{Q}_{1}^{-1}$. В этом случае аналогично случаю 1 получаем ПСогл $\alpha\left(\mathrm{F}, \mathrm{H}_{1}\right)$ или ПСог $\Omega_{\alpha}\left(\mathrm{F}, \mathrm{H}_{1}^{-1}\right)$. Противоречие с (14.14), условием на $\mathrm{Y}_{\text {в (iv) и предложением } 8.46 .}$

Случай (iv) рассмотрен.

Аналогичные рассуждения проходят и в случаях (ii) и (iii). При этом в (ii) нужно положить $x_{i}^{*} \rightleftharpoons x_{i}$ при всех $i$, а в (iii) нужно положить $y^{*} \rightleftharpoons y$. Соответственно, в случае (ii) на рис. 69 будут отсутствовать пути $\mathrm{E}, \mathrm{P}, \mathrm{u}, \mathrm{P}_{1}$, и пути $\mathrm{T}^{*}$ и $\mathrm{Y}$ будут иметь общую начальную вершину, а в случае (iii) будут отсутствовать пути $F, Q$, $v, Q_{1}$ и пути $T^{*}$ и $\mathrm{Y}$ будут иметь обшую конечную вершину.

Тем самым мы доказали утверждение леммы в ослабленном варианте, т.е. с требованием, что $\left[y^{*}\right]_{\alpha-1}-$ элемент конечного порядка и $\mathbf{H}^{*} \rightleftharpoons\left\{\left[x_{i}^{*}\right]_{\alpha-1}\right\}_{i}-$ конечная подгупппа. Выберем теперь нормальную форму $y^{\prime}$ ранга $\gamma$, где $M^{-1} y^{*} M^{\alpha=1} y^{\prime}$ для некоторого $M$, и по индуктивному предположению 14.3 найдем подгруппу $\mathbf{H}^{\prime} \rightleftharpoons\left\{\left[x_{i}^{\prime}\right]_{\alpha-1}\right\}_{i} \subseteq \mathbf{S}_{\alpha-1}\left(A^{*}\right)\left(A^{*} \in \bar{\varepsilon}_{\beta}\right)$ такую, что $N x_{i}^{*} N^{-1} \stackrel{\alpha=1}{=} x_{i}^{\prime}$ для некоторого $N$ при всех $i$ (равенства ранг $\left[y^{*}\right]_{\alpha-1}=\gamma$ и ранг $\mathbf{H}^{*}=\beta$ выполнены ввиду леммы 13.14 , (iii); кроме того, чтобы найти пополнение подгруппы $\mathbf{H}^{*}$ вида $[N]_{\alpha-1}^{-1} \mathbf{S}_{\alpha-1}\left(A^{*}\right)[N]_{\alpha-1}$, где $A^{*}$ - нормализованный элементарный период ранга $\beta$, помимо индуктивного предположения 14.3 нужно воспользоваться еще леммой 7.20). Непосредственно проверяется, что все требуемые условия заключения леммы 14.7 сохраняют силу, если слова $x_{i}^{*}, y^{*}, T^{*}, U$ и $V$ заменить соответственно на $x_{i}^{\prime}, y^{\prime}, N T^{*} M, U N^{-1}$ и $M^{-1} V$. Лемма 14.7 доказана.

14.8. Лемма. Пусть $\mathbf{g} \in \mathbf{S}_{\alpha}^{+}(A) \backslash \mathbf{S}_{\alpha}^{*}(A)$, где $A \in \mathcal{E}_{\alpha}, u$ nусть $4 p+304 \leqslant$ степень $\mathbf{g} \leqslant n-4 p-304$. Тогда если $\mathbf{h}_{1}, \mathbf{h}_{2} \in \mathbf{B}_{\alpha}$ - элементы конечного порядка $и \mathbf{g}=\mathbf{h}_{1} \mathbf{h}_{2}$, mо $\mathbf{h}_{1}, \mathbf{h}_{2} \in \mathbf{S}_{\alpha}(A)$.

ДокАЗАТЕЛЬСтво. Без ограничения общности можно считать $A \in \bar{\varepsilon}_{\alpha}$ и $\mathbf{h}_{1}, \mathbf{h}_{2} \neq 1$. Пусть $\mathbf{g}=\left[A^{t} w\right]_{\alpha}$, где $A^{t} w-$ нормальная форма ранга $\alpha$ и $4 p+$ $304 \leqslant t \leqslant n-4 p-304$. Представим каждый элемент $\mathbf{h}_{i}$ в виде

$$
\mathbf{h}_{i}=\left[T_{i}^{-1} B_{i}^{s_{i}} z_{i} T_{i}\right]_{\alpha} \quad(i=1,2),
$$

где $B_{i}^{s_{i}} z_{i}$ - нормальная форма ранга $\beta_{i} \leqslant \alpha$. Заменяя $A^{t}$ и $B_{i}^{s_{i}}$ при необходимости на $\left(A^{-1}\right)^{n-t}$ и $\left(B_{i}^{-1}\right)^{n-s_{i}}$, можно считать $t, s_{i} \leqslant n / 2$. По лемме 14.4 найдем слова $U, X$ и $V_{i}, Y_{i}(i=1,2)$ такие, что выполнены соответствуюшие условия (i)-(v); в частности,

$$
\begin{gathered}
A^{t} w \stackrel{\alpha=1}{=} U^{-1} X U, \\
B_{i}^{s_{i}} z_{i} \stackrel{\alpha=1}{=} V_{i}^{-1} Y_{i} V_{i} \quad(i=1,2) .
\end{gathered}
$$

Равенство $\mathbf{g}=\mathbf{h}_{1} \mathbf{h}_{2}$ можно переписать в виде

$$
U^{-1} X U T_{2}^{-1} V_{2}^{-1} Y_{2}^{-1} V_{2} T_{2} T_{1}^{-1} V_{1}^{-1} T_{1} Y_{1}^{-1} V_{1} T_{1} \stackrel{\alpha}{=} 1 .
$$


Пусть $\widetilde{\Delta}$ - дисковая диаграмма ранга $\alpha$ с граничным циклом

$$
\delta \widetilde{\Delta} \approx \mathrm{U}^{\prime-1} \mathrm{XUT}_{2}^{\prime-1} \mathrm{~V}_{2}^{\prime-1} \mathrm{Y}_{2}^{-1} \mathrm{~V}_{2} \mathrm{~T}_{2} \mathrm{~T}_{1}^{\prime-1} \mathrm{~V}_{1}^{\prime-1} \mathrm{Y}_{1}^{-1} \mathrm{~V}_{1} \mathrm{~T}_{1}
$$

где метки соответствуюших подпутей равны словам из левой части равенства (14.22). Пусть диаграмма $\Delta$ с тремя граничными циклами $\mathrm{X}, \mathrm{Y}_{1}^{-1}$ и $\mathrm{Y}_{2}^{-1}$ получена из $\widetilde{\Delta}$ склеиванием участков границы вдоль пар путей $\mathrm{U}_{\text {и }} \mathrm{U}^{\prime}, \mathrm{V}_{2} \mathrm{~T}_{2}$ и $\mathrm{V}_{2}^{\prime} \mathrm{T}_{2}^{\prime}$, $\mathrm{V}_{1} \mathrm{~T}_{1}$ и $\mathrm{V}_{1}^{\prime} \mathrm{T}_{1}^{\prime}$, как описано в 4.6 (см. рис. 70$)$.

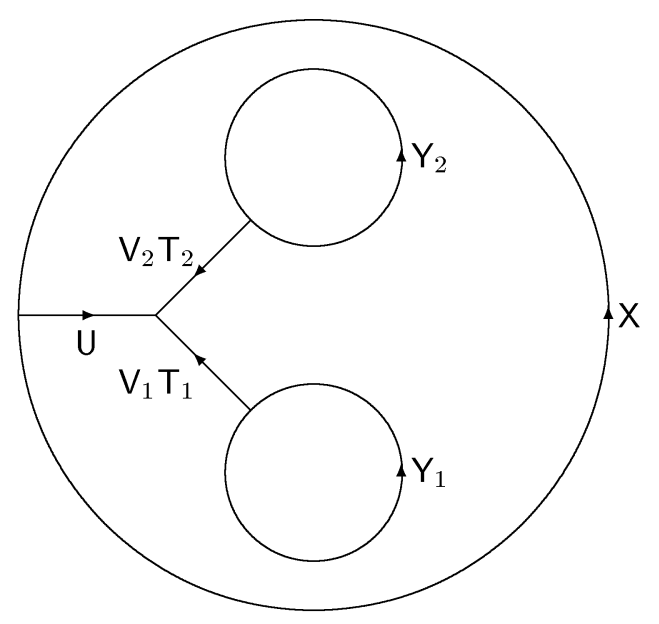

Рис. 70

Группа $\pi_{1}(\Delta, \iota(\mathrm{X}))$ порождается двумя классами гомотопных петель $\left[\mathrm{UT}_{i}^{-1} \mathrm{~V}_{i}^{-1} \mathrm{Y}_{i} \mathrm{~V}_{i} \mathrm{~T}_{i} \mathrm{U}^{-1}\right]_{\Delta}(i=1,2)$, и так как из (14.19), (14.21) следует

$$
\left[\mathcal{L}\left(\mathrm{UT}_{i}^{-1} \mathrm{~V}_{i}^{-1} \mathrm{Y}_{i} \mathrm{~V}_{i} \mathrm{~T}_{i} \mathrm{U}^{-1}\right)\right]_{\alpha}=\left[U T_{i}^{-1} V_{i}^{-1} Y_{i} V_{i} T_{i} U^{-1}\right]_{\alpha}=[U]_{\alpha} \mathbf{h}_{i}[U]_{\alpha}^{-1},
$$

то заключение леммы равносильно включению

$$
[\mathcal{L}]_{\alpha}\left(\pi_{1}(\Delta, \iota(\mathbf{X}))\right) \subseteq[U]_{\alpha} \mathbf{S}_{\alpha}(A)[U]_{\alpha}^{-1},
$$

где $[\mathcal{L}]_{\alpha}$ - отображение, сопоставляющее классу гомотопных путей $[\mathrm{P}]_{\Delta}$ элемент $[\mathcal{L}(\mathrm{P})]_{\alpha}$ группы $\mathbf{B}_{\alpha}$. В силу условия (iii) леммы 14.4 слова $X$ и $Y_{i}$ циклически приведены в ранге $\alpha$. Следовательно, диаграмму $\Delta$ можно рассматривать как $B$-диаграмму ранга $\alpha$, для которой

$$
x_{\alpha}(\Delta)=\left\{\mathrm{X}, \mathrm{Y}_{1}^{-1}, \mathrm{Y}_{2}^{-1}\right\} \text { и } y_{\alpha-1}(\Delta)=z_{\alpha}(\Delta)=\varnothing
$$

По предложению 9.9 найдется приведенная $B$-диаграмма $\Delta^{\prime}$ ранга $\alpha$, полученная из $\Delta$ некоторой последовательностью внутренних преобразований ранга $\alpha$ и 
добавлений областей ранга $\alpha$. Так как $z_{\alpha}(\Delta)=\varnothing$, то в последовательности преобразований, переводящей $\Delta$ в $\Delta^{\prime}$, могут встретиться только внутренние преобразования ранга $\alpha$, т.е. измельчения и замены дисковых или кольцевых поддиаграмм (см. определение 9.4). Тогда по лемме 4.11 для некоторого элемента $\mathbf{f} \in \mathbf{B}_{\alpha}$, коммутируюшего с $[\mathcal{L}(\mathbf{X})]_{\alpha}=[U]_{\alpha} \mathbf{g}[U]_{\alpha}^{-1}$, выполнено

$$
[\mathcal{L}]_{\alpha}\left(\pi_{1}\left(\Delta^{\prime}, \iota(\mathbf{X})\right)\right)=\mathbf{f}[\mathcal{L}]_{\alpha}\left(\pi_{1}(\Delta, \iota(\mathbf{X}))\right) \mathbf{f}^{-1}
$$

В силу следствия 13.11 имеем $\mathbf{f} \in[U]_{\alpha} \mathbf{S}_{\alpha}(A)[U]_{\alpha}^{-1}$. Тогда включение (14.23) равносильно тому же включению с заменой $\Delta$ на $\Delta^{\prime}$, и, следовательно, для доказательства (14.23) с самого начала можно считать, что $\Delta$ есть приведенная $B$-диаграмма ранга $\alpha$. По той же причине можно считать, что на $\Delta^{\alpha-1}$ задана карта примыканий $\mathcal{S}$ ранга $\alpha-1$. В зависимости от наличия или отсутствия областей ранга $\alpha$ у диаграммы $\Delta$ доказательство включения (14.23) разбивается на два основных случая. Предварительно заметим, что группа $\pi_{1}(\Delta, \iota(\mathrm{X}))$ порождается двумя классами гомотопных петель, в качестве одного из которых можно взять $[\mathrm{X}]_{\Delta}$ и для которого в силу $(14.20)$ уже имеем $[\mathcal{L}(\mathrm{X})]_{\alpha} \in[U]_{\alpha} \mathbf{S}_{\alpha}(A)[U]_{\alpha}^{-1}$. Наша задача будет состоять в том, чтобы подходящим образом указать второй порождаюший $[\mathrm{W}]_{\Delta}$ и доказать для него включение $[\mathcal{L}(\mathrm{W})]_{\alpha} \in[U]_{\alpha} \mathbf{S}_{\alpha}(A)[U]_{\alpha}^{-1}$.

Случай 1: $\mathbf{R}_{\alpha}(\Delta) \neq 0$. Выберем произвольную область $\mathrm{A} \in \mathbf{R}_{\alpha}(\Delta)$. Пусть $\mathcal{L}(\delta \mathrm{A}) \approx C^{n}, C \in \mathcal{E}_{\alpha}$, и пусть $\Pi_{1}, \Pi_{2}, \ldots, \Pi_{k} \in \mathcal{S}$ - поддиаграммы примыкания $\delta \mathrm{A}$ к граничным циклам $\mathrm{X}_{\text {и }} \mathrm{Y}_{i}^{-1}$. Пусть $\delta \Pi_{i} \approx \mathrm{P}_{i} \mathrm{x}_{i} \mathrm{Q}_{i} \mathrm{y}_{i}$, где $\mathrm{P}_{i}, \mathrm{Q}_{i}-$ участки примыкания, $\mathrm{P}_{i}$ входит в $\delta \mathrm{A}$ и $\mathcal{L}\left(\mathrm{x}_{i}\right), \mathcal{L}\left(\mathrm{y}_{i}\right) \in \mathcal{H}_{\alpha-1}$. По предложению 10.9 , (iii) имеем $k \leqslant 4$. По предложению 10.13 , (iii)

$$
\sum_{i=1}^{k}\left|\mathcal{L}\left(\mathrm{P}_{i}\right)\right| \geqslant(n-6 p-68)|C| .
$$

Покажем, что из поддиаграмм $\Pi_{1}, \Pi_{2}, \ldots, \Pi_{k}$ можно выбрать две $\Pi_{i_{0}}$ и $\Pi_{i_{1}}$ таким образом, что участки примыкания $\mathrm{Q}_{i_{0}}$ и $\mathrm{Q}_{i_{1}}$ входят в разные граничные циклы $\mathrm{X}$, $\mathrm{Y}_{1}^{-1}$ и $\mathrm{Y}_{2}^{-1}$ диаграммы $\Delta$ и при этом имеют место неравенства

$$
\left|\mathcal{L}\left(\mathrm{P}_{i_{0}}\right)\right|,\left|\mathcal{L}\left(\mathrm{P}_{i_{1}}\right)\right| \geqslant(p+70)|C| .
$$

Предположим, поддиаграммы $\Pi_{i}$ упорядочены по убыванию длин меток путей $\mathrm{P}_{i}$. В качестве $\Pi_{i_{0}}$ возьмем поддиаграмму $\Pi_{1}$, для которой в силу $(14.24)$

$$
\left|\mathcal{L}\left(\mathrm{P}_{1}\right)\right| \geqslant \frac{1}{4}(n-6 p-68)|C|>(p+70)|C| .
$$

Так как $\left|\mathcal{L}\left(\mathrm{P}_{1}\right)\right|<(n-q)|C|$ в силу циклической приведенности слова $X$ в ранге $\alpha$, то

$$
\left|\mathcal{L}\left(\mathrm{P}_{2}\right)\right| \geqslant \frac{1}{3}(q-6 p-68)|C|>(p+70)|C| .
$$


Если $\mathrm{Q}_{1}$ и $\mathrm{Q}_{2}$ входят в разные граничные циклы $\mathrm{X}, \mathrm{Y}_{1}^{-1}$ и $\mathrm{Y}_{2}^{-1}$, то можно взять $i_{1} \rightleftharpoons 2$. Пусть $\mathrm{Q}_{1}$ и $\mathrm{Q}_{2}$ входят в один граничный цикл $\mathrm{X}, \mathrm{Y}_{1}^{-1}$ или $\mathrm{Y}_{2}^{-1}$, для определенности, скажем, в $\mathrm{X}$. Из предложения 8.23 следует тогда, что при $i=1,2$ в подслово $\mathcal{L}\left(Q_{i}\right)$ некоторого циклического сдвига $X^{\prime}$ слова $X$ входит слово $E_{i} \in \Pi$ Пер ${ }_{\alpha}\left(C^{-1}, p+34\right)$, где $\ell_{\alpha, C^{-1}}\left(E_{i}\right) \geqslant\left|\mathcal{L}\left(\mathrm{P}_{i}\right)\right| /|C|-37$. Используя условие (vi) леммы 14.4 и предложение 8.11 , получаем, что в $X^{\prime}$ входит слово $E \in \Pi$ Пер ${ }_{\alpha}\left(C^{-1}\right)$ с $\ell_{\alpha, C^{-1}}(E) \geqslant\left(\left|\mathcal{L}\left(\mathrm{P}_{1}\right)\right|+\left|\mathcal{L}\left(\mathrm{P}_{2}\right)\right|\right) /|C|-75$, откуда по лемме 12.12 вытекает $\left|\mathcal{L}\left(\mathrm{P}_{1}\right)\right|+\left|\mathcal{L}\left(\mathrm{P}_{2}\right)\right| \leqslant(n-q+75)|C|$. Тогда, используя (14.24), получаем $\left|\mathcal{L}\left(\mathrm{P}_{3}\right)\right| \geqslant(p+70)|C|$, но теперь уже в силу предложения 10.9 , (iii) путь $\mathrm{Q}_{3}$ не может входить в $\mathrm{X}$ и можно положить $i_{1} \rightleftharpoons 3$.

Итак, перенумеровав $\Pi_{i}$, можно считать, что $Q_{1}$ и $Q_{2}$ входят в разные граничные циклы $X, Y_{1}^{-1}$ и $Y_{2}^{-1}$ (на самом деле выполнение этого условия не играет принципиальной роли в рассуждениях, а лишь позволяет уменьшить количество рассматриваемых подслучаев) и

$$
\left|\mathcal{L}\left(\mathrm{P}_{1}\right)\right|,\left|\mathcal{L}\left(\mathrm{P}_{2}\right)\right| \geqslant(p+70)|C|
$$

В силу полной аналогии достаточно ограничиться двумя подслучаями: $\mathrm{Q}_{1}, \mathrm{Q}_{2}$ входят в $\mathrm{X}, \mathrm{Y}_{1}^{-1}$ и $\mathrm{Q}_{1}, \mathrm{Q}_{2}$ входят в $\mathrm{Y}_{1}^{-1}$ и $\mathrm{Y}_{2}^{-1}$. Пусть

$$
\delta \mathrm{A} \approx \mathrm{P}_{1} \mathrm{~S}_{1} \mathrm{P}_{2} \mathrm{~S}_{2}
$$

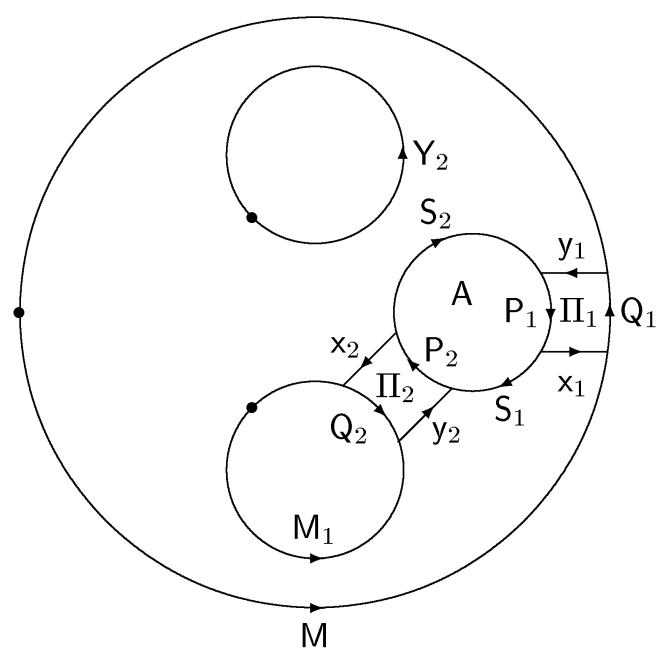

Рис. 71

Случай 1A: $\mathrm{Q}_{1}$ и $\mathrm{Q}_{2}$ входят в $\mathrm{X}$ и $\mathrm{Y}_{1}^{-1}$ соответственно. Заметим, что в этом случае из условия (ii) леммы 14.4 для $A^{t} w:=B_{1}^{s_{1}} z_{1}, X:=Y_{1}$ и следствия 8.24 вытекает $\beta_{1}=\alpha$. Пусть $\mathrm{M}$ - начало пути $\mathrm{X}$ с $\tau(\mathrm{M})=\iota\left(\mathrm{Q}_{1}\right)$ и $\mathrm{M}_{1}$ - начало пути $\mathrm{Y}_{1}$ с $\tau(\mathrm{M})=\iota\left(\mathrm{Q}_{2}^{-1}\right)$. Группа $\pi_{1}(\Delta, \iota(\mathrm{X}))$ порождается классами $[\mathrm{X}]_{\Delta}$ 
и $\left[\mathrm{Mx}_{1}^{-1} \mathrm{~S}_{1} \mathrm{y}_{2}^{-1} \mathrm{M}_{1}^{-1} \mathrm{Y}_{1} \mathrm{M}_{1} \mathrm{y}_{2} \mathrm{~S}_{1}^{-1} \mathrm{x}_{1} \mathrm{M}^{-1}\right]_{\Delta}$ (см. рис. 71), поэтому достаточно проверить включение

$$
\left[\mathcal{L}\left(\mathrm{Mx}_{1}^{-1} \mathrm{~S}_{1} \mathrm{y}_{2}^{-1} \mathrm{M}_{1}^{-1} \mathrm{Y}_{1} \mathrm{M}_{1} \mathrm{y}_{2} \mathrm{~S}_{1}^{-1} \mathrm{x}_{1} \mathrm{M}^{-1}\right)\right]_{\alpha} \in[U]_{\alpha} \mathrm{S}_{\alpha}(A)[U]_{\alpha}^{-1} .
$$

Для этого рассмотрим пути на графе $\Gamma_{\alpha-1}$, расположенные, как на рис. 72, где $\mathcal{L}(\mathrm{U})$ 드 $U, \mathcal{L}(\mathrm{R})$ 드 $A^{2}, \mathcal{L}\left(\mathrm{V}_{1}\right)$ 드 $V_{1}, \mathcal{L}\left(\mathrm{R}_{1}\right)$ 드 $B_{1}^{2}$ и метки остальных путей равны меткам соответствующих путей на диаграмме $\Delta$.

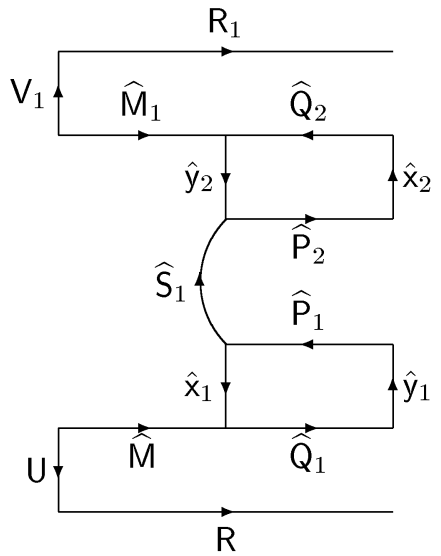

Рис. 72

Пусть $\widehat{\mathrm{X}}_{\text {и }} \widehat{\mathrm{Y}}_{1}$ - пути на $\Gamma_{\alpha-1}$ с $\mathcal{L}(\widehat{\mathrm{X}})$ 으 $X, \mathcal{L}\left(\widehat{\mathrm{Y}}_{1}\right)$ 으 $Y_{1}$ и начальными вершинами $\iota(\widehat{\mathrm{M}})$ и $\iota\left(\widehat{\mathrm{M}}_{1}\right)$ соответственно. В силу 14.4 , (iv) найдутся $Z \widehat{X} Z^{\prime} \in \Pi$ Пер $\alpha(A) \cap$ Пер $\left(\Gamma_{\alpha-1}, X, 2\right)$ и периодическая основа $\overline{\mathrm{R}} \in \Pi \operatorname{ep}\left(\Gamma_{\alpha-1}, A, 2\right)$ для $Z \widehat{X} Z^{\prime}$ такие, что $|\mathrm{Z}|,\left|\mathrm{Z}^{\prime}\right| \geqslant|X|$ и $\overline{\mathrm{R}}$ есть периодическое продолжение $\mathrm{R}$. В силу 14.4 , (iv) для $A^{t} w:=$ $B_{1}^{s_{1}} z_{1}, X:=Y_{1}$ и $U:=V_{1}$ найдутся $Z_{1} \widehat{Y}_{1} Z_{1}^{\prime} \in \Pi$ Пер $\alpha\left(B_{1}\right) \cap \Pi е р\left(\Gamma_{\alpha-1}, Y_{1}, 2\right)$ и периодическая основа $\overline{\mathrm{R}}_{1} \in$ Пер $\left(\Gamma_{\alpha-1}, B_{1}, 2\right)$ для $\mathrm{Z}_{1} \widehat{\mathrm{Y}}_{1} \mathrm{Z}_{1}^{\prime}$, где $\left|\mathrm{Z}_{1}\right|,\left|\mathrm{Z}_{1}^{\prime}\right| \geqslant\left|Y_{1}\right|$ и $\overline{\mathrm{R}}_{1}$ есть периодическое продолжение $\mathrm{R}_{1}$. Так как $\mathcal{L}\left(\widehat{\mathrm{M}} \widehat{Q}_{1}\right)$ есть начало слова $X^{2}$ и $\left|Z^{\prime}\right| \geqslant|X|$, то $\widehat{\mathrm{M}} \widehat{\mathrm{Q}}_{1}$ есть начало пути $\widehat{\mathrm{X}} \mathrm{Z}^{\prime}$. Так как $\mathcal{L}\left(\widehat{\mathrm{M}}_{1} \widehat{\mathrm{Q}}_{2}^{-1}\right)$ есть начало слова $Y_{1}^{2}$ и $\left|\mathrm{Z}_{1}^{\prime}\right| \geqslant\left|Y_{1}\right|$, то $\widehat{\mathrm{M}}_{1} \widehat{\mathrm{Q}}_{2}^{-1}$ есть начало пути $\widehat{\mathrm{Y}}_{1} \mathrm{Z}_{1}^{\prime}$. Так как $\left|\mathcal{L}\left(\widehat{\mathrm{P}}_{1}\right)\right| \geqslant(p+70)|C|$, то по предложению 8.23 в $\widehat{\mathrm{Q}}_{1}$ входит путь $\mathrm{G} \in$ ППер $\alpha\left(C^{-1}, p+34\right)$ с периодической основой, содержащейся в $\widehat{\mathrm{P}}_{1}^{-1}$. Тогда в силу предложения 8.38 , (i) получаем Солл $\left(\widehat{\mathrm{P}}_{1}^{-1}, \overline{\mathrm{R}}\right)$. Аналогично выводим Солл $\left(\widehat{\mathrm{P}}_{2}, \overline{\mathrm{R}}_{1}\right)$. Так как $\widehat{\mathrm{P}}_{1} \widehat{\mathrm{S}}_{1} \widehat{\mathrm{P}}_{2}$ - общее периодическое продолжение для $\widehat{\mathrm{P}}_{1}$ и $\widehat{\mathrm{P}}_{2}$, то отсюда следует Согл $\left(\overline{\mathrm{R}}, \overline{\mathrm{R}}_{1}^{-1}\right)$, и, таким образом, получаем $C о г л\left(\mathrm{R}, \mathrm{R}_{1}^{-1}\right)$. В силу определения 3.3 и леммы $3.2,(\mathrm{v})$ для некоторого $r>0$ имеет место равенство $\iota(\mathrm{R})[A]_{\alpha-1}^{r}(\iota(\mathrm{R}))^{-1}=\iota\left(\mathrm{R}_{1}\right)\left[B_{1}\right]_{\alpha-1}^{-r}\left(\iota\left(\mathrm{R}_{1}\right)\right)^{-1}$, т.e.

$$
[A]_{\alpha-1}^{r}=\left((\iota(\mathrm{R}))^{-1} \iota\left(\mathrm{R}_{1}\right)\right)\left[B_{1}\right]_{\alpha-1}^{-r}\left((\iota(\mathrm{R}))^{-1} \iota\left(\mathrm{R}_{1}\right)\right)^{-1} .
$$

Так как вершины $\iota(\mathrm{R})$ и $\iota\left(\mathrm{R}_{1}\right)$ соединены путем $\mathrm{U}^{-1} \widehat{\mathrm{M}} \hat{\mathrm{x}}_{1}^{-1} \widehat{\mathrm{S}}_{1} \hat{\mathrm{y}}_{2}^{-1} \widehat{\mathrm{M}}_{1}^{-1} \mathrm{~V}_{1}$, то последнее равенство можно переписать в виде

$$
A^{r} \stackrel{\alpha=1}{=} \mathcal{L}\left(\mathrm{U}^{-1} \widehat{\mathrm{M}} \hat{\mathrm{x}}_{1}^{-1} \widehat{\mathrm{S}}_{1} \hat{\mathrm{y}}_{2}^{-1} \widehat{\mathrm{M}}_{1}^{-1} \mathrm{~V}_{1}\right) B_{1}^{-r}\left(\mathcal{L}\left(\mathrm{U}^{-1} \widehat{\mathrm{M}} \hat{\mathrm{x}}_{1}^{-1} \widehat{\mathrm{S}}_{1} \hat{\mathrm{y}}_{2}^{-1} \widehat{\mathrm{M}}_{1}^{-1} \mathrm{~V}_{1}\right)\right)^{-1},
$$


откуда в силу леммы 7.20

$$
\mathbf{S}_{\alpha}(A)=\left[\mathcal{L}\left(\mathrm{U}^{-1} \widehat{\mathrm{M}}_{1}^{-1} \widehat{\mathrm{S}}_{1} \hat{\mathrm{y}}_{2}^{-1} \widehat{\mathrm{M}}_{1}^{-1} \mathrm{~V}_{1}\right)\right]_{\alpha} \mathbf{S}_{\alpha}\left(B_{1}\right)\left[\mathcal{L}\left(\mathrm{U}^{-1} \widehat{\mathrm{M}}_{1}^{-1} \widehat{\mathrm{S}}_{1} \hat{\mathrm{y}}_{2}^{-1} \widehat{\mathrm{M}}_{1}^{-1} \mathrm{~V}_{1}\right)\right]_{\alpha}^{-1}
$$

Тогда в силу (14.21) и включения $\left[B_{1}^{s_{1}} z_{1}\right]_{\alpha} \in \mathbf{S}_{\alpha}\left(B_{1}\right)$ получаем

$$
\left[\mathcal{L}\left(\mathrm{U}^{-1} \widehat{\mathrm{M}} \hat{\mathrm{x}}_{1}^{-1} \widehat{\mathrm{S}}_{1} \hat{\mathrm{y}}_{2}^{-1} \widehat{\mathrm{M}}_{1}^{-1}\right)\right]_{\alpha}\left[Y_{1}\right]_{\alpha}\left[\mathcal{L}\left(\mathrm{U}^{-1} \widehat{\mathrm{M}} \hat{\mathrm{x}}_{1}^{-1} \widehat{\mathrm{S}}_{1} \hat{\mathrm{y}}_{2}^{-1} \widehat{\mathrm{M}}_{1}^{-1}\right)\right]_{\alpha}^{-1} \in \mathbf{S}_{\alpha}(A)
$$

что и доказывает искомое включение (14.26).

Cлучай 1B: $\mathrm{Q}_{1}$ и $\mathrm{Q}_{2}$ входят в $\mathrm{Y}_{1}^{-1}$ и $\mathrm{Y}_{2}^{-1}$ соответственно. Соединим вершины $\iota(\mathrm{X})$ и $\iota\left(\mathrm{Y}_{1}\right)$ диаграммы $\Delta$ произвольным простым путем $\mathrm{W}$ (для этого, например, можно взять путь, соединяюший $\iota(\mathrm{X})$ и $\iota\left(\mathrm{Y}_{1}\right)$ и содержаший наименьшее возможное число ориентированных ребер). Группа $\pi_{1}(\Delta, \iota(\mathrm{X}))$ порождается классами $[\mathrm{X}]_{\Delta}$ и $\left[\mathrm{WY}_{1} \mathrm{~W}^{-1}\right]_{\Delta}$, поэтому достаточно установить включение

$$
\left[\mathcal{L}\left(\mathrm{WY}_{1} \mathrm{~W}^{-1}\right)\right]_{\alpha} \in[U]_{\alpha} \mathbf{S}_{\alpha}(A)[U]_{\alpha}^{-1}
$$

Для этого мы докажем равенство

$$
\mathbf{S}_{\alpha}\left(B_{1}\right)=\left[V_{1}^{-1}(\mathcal{L}(\mathrm{W}))^{-1} U\right]_{\alpha} \mathbf{S}_{\alpha}(A)\left[U^{-1} \mathcal{L}(\mathrm{W}) V_{1}\right]_{\alpha}
$$

откуда с учетом (14.21) будет непосредственно вытекать (14.27).

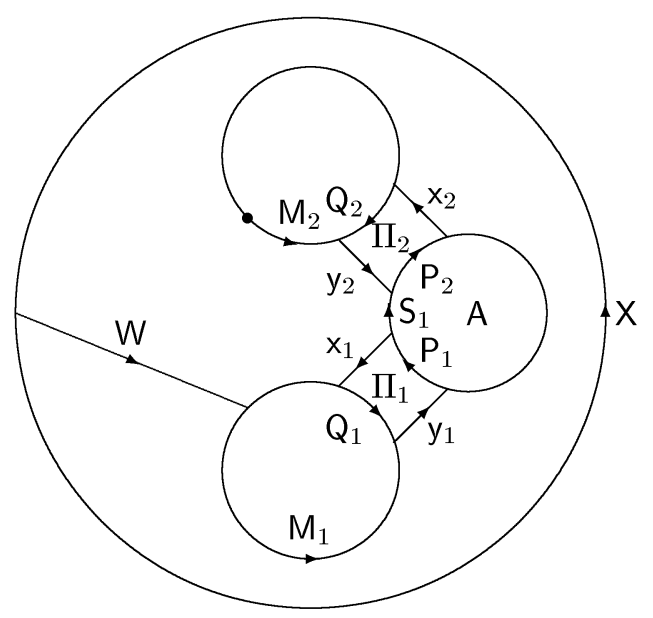

Рис. 73 


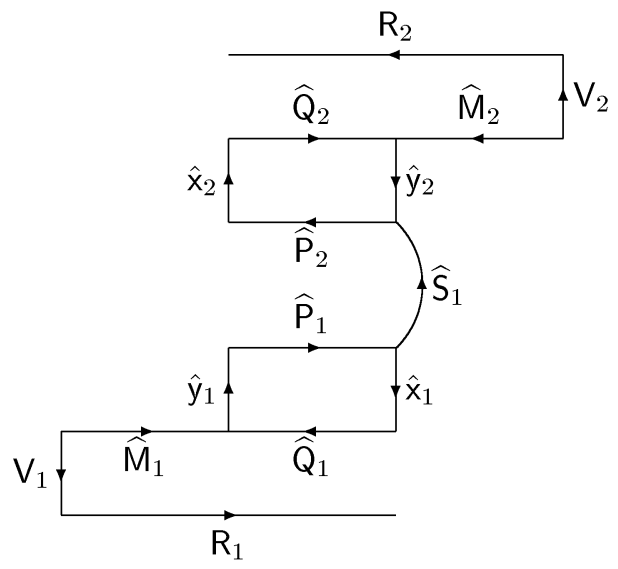

Рис. 74

Заметим, что из выполнения условия (ii) леммы 14.4 для $A^{t} w:=B_{i}^{s_{i}} z_{i}, X:=Y_{i}$ и следствия 8.24 вытекает $\beta_{1}=\beta_{2}=\alpha$. Обозначим через $\mathrm{M}_{i}(i=1,2)$ начало пути $\mathrm{Y}_{i} \mathrm{c} \tau\left(\mathrm{M}_{i}\right)=\tau\left(\mathrm{Q}_{i}\right)$. Аналогично случаю $1 \mathrm{~A}$ рассмотрим пути на графе $\Gamma_{\alpha-1}$, расположенные, как на рис. 74 , где $\mathcal{L}\left(\mathrm{V}_{i}\right)$ 으 $V_{i}, \mathcal{L}\left(\mathrm{R}_{i}\right)$ 으 $B_{i}^{2}(i=1,2)$ и метки остальных путей равны меткам соответствующих путей на диаграмме $\Delta$ (см. рис. 73).

Используя условие (iv) леммы 14.4 для $A^{t} w:=B_{i}^{s_{i}} z_{i}, X:=Y_{i}$ и $U:=V_{i}$ $(i=1,2)$, неравенства $\left|\mathcal{L}\left(\widehat{\mathrm{P}}_{i}\right)\right| \geqslant(p+70)|C|$, предложения 8.23 и 8.38 , (i), аналогично тому, как при рассмотрении случая $1 \mathrm{~A}$, получаем $C$ огл $\left(\mathrm{R}_{1}, \mathrm{R}_{2}\right)$. Так как вершины $\iota\left(\mathrm{R}_{1}\right)$ и $\iota\left(\mathrm{R}_{2}\right)$ соединены путем $\mathrm{V}_{1}^{-1} \widehat{\mathrm{M}}_{1} \hat{\mathrm{y}}_{1} \widehat{\mathrm{P}}_{1} \widehat{\mathrm{S}}_{1} \hat{\mathrm{y}}_{2}^{-1} \widehat{\mathrm{M}}_{2}^{-1} \mathrm{~V}_{2}$, то отсюда следует, что для некоторого $r>0$ имеет место равенство

$$
B_{1}^{r} \stackrel{\alpha=1}{=} \mathcal{L}\left(\mathrm{V}_{1}^{-1} \widehat{\mathrm{M}}_{1} \hat{\mathrm{y}}_{1} \widehat{\mathrm{P}}_{1} \widehat{\mathrm{S}}_{1} \hat{\mathrm{y}}_{2}^{-1} \widehat{\mathrm{M}}_{2}^{-1} \mathrm{~V}_{2}\right) B_{2}^{r}\left(\mathcal{L}\left(\mathrm{V}_{1}^{-1} \widehat{\mathrm{M}}_{1} \hat{\mathrm{y}}_{1} \widehat{\mathrm{P}}_{1} \widehat{\mathrm{S}}_{1} \hat{\mathrm{y}}_{2}^{-1} \widehat{\mathrm{M}}_{2}^{-1} \mathrm{~V}_{2}\right)\right)^{-1}
$$

Тогда по лемме 7.20

$\mathbf{S}_{\alpha}\left(B_{1}\right)=\left[\mathcal{L}\left(\mathrm{V}_{1}^{-1} \widehat{\mathrm{M}}_{1} \hat{\mathrm{y}}_{1} \widehat{\mathrm{P}}_{1} \widehat{\mathrm{S}}_{1} \hat{\mathrm{y}}_{2}^{-1} \widehat{\mathrm{M}}_{2}^{-1} \mathrm{~V}_{2}\right)\right]_{\alpha} \mathbf{S}_{\alpha}\left(B_{2}\right)\left[\mathcal{L}\left(\mathrm{V}_{1}^{-1} \widehat{\mathrm{M}}_{1} \hat{\mathrm{y}}_{1} \widehat{\mathrm{P}}_{1} \widehat{\mathrm{S}}_{1} \hat{\mathrm{y}}_{2}^{-1} \widehat{\mathrm{M}}_{2}^{-1} \mathrm{~V}_{2}\right)\right]_{\alpha}^{-1}$, откуда с учетом (14.21) и включения $\left[B_{2}^{s_{2}} z_{2}\right]_{\alpha} \in \mathbf{S}_{\alpha}\left(B_{2}\right)$ выводим

$$
\left[\mathcal{L}\left(\widehat{\mathrm{M}}_{1} \hat{\mathrm{y}}_{1} \widehat{\mathrm{P}}_{1} \widehat{\mathrm{S}}_{1} \hat{\mathrm{y}}_{2}^{-1} \widehat{\mathrm{M}}_{2}^{-1}\right)\right]_{\alpha}\left[Y_{2}\right]_{\alpha}\left[\mathcal{L}\left(\widehat{\mathrm{M}}_{1} \hat{\mathrm{y}}_{1} \widehat{\mathrm{P}}_{1} \widehat{\mathrm{S}}_{1} \hat{\mathrm{y}}_{2}^{-1} \widehat{\mathrm{M}}_{2}^{-1}\right)\right]_{\alpha}^{-1} \in\left[V_{1}\right]_{\alpha} \mathbf{S}_{\alpha}\left(B_{1}\right)\left[V_{1}\right]_{\alpha}^{-1}
$$

Заметим, что путь $\mathrm{X}$ на диаграмме $\Delta$ гомотопен пути

$$
W M_{1} y_{1} P_{1} S_{1} y_{2}^{-1} M_{2}^{-1} Y_{2} M_{2} y_{2} S_{1}^{-1} P_{1}^{-1} y_{1}^{-1} M_{1}^{-1} Y_{1} W^{-1}
$$

(этот факт легко усматривается, например, из того, что при разрезании диаграммы $\Delta$ вдоль путей $\mathrm{W}_{\text {и }} \mathrm{y}_{1} \mathrm{P}_{1} \mathrm{~S}_{1} \mathrm{y}_{2}^{-1}$ получается дисковая диаграмма и ее граничньй цикл стягиваем). Следовательно, в силу леммы 4.2

$$
X \stackrel{\alpha}{=} \mathcal{L}\left(\mathrm{WM}_{1} \mathrm{y}_{1} \mathrm{P}_{1} \mathrm{~S}_{1} \mathrm{y}_{2}^{-1} \mathrm{M}_{2}^{-1} \mathrm{Y}_{2} \mathrm{M}_{2} \mathrm{y}_{2} \mathrm{~S}_{1}^{-1} \mathrm{P}_{1}^{-1} \mathrm{y}_{1}^{-1} \mathrm{M}_{1}^{-1} \mathrm{Y}_{1} \mathrm{~W}^{-1}\right)
$$


Так как в силу $(14.21)\left[\mathcal{L}\left(\mathrm{Y}_{1}\right)\right]_{\alpha} \in\left[V_{1}\right]_{\alpha} \mathbf{S}_{\alpha}\left(B_{1}\right)\left[V_{1}\right]_{\alpha}^{-1}$ и в силу $(14.29)$

$$
\left[\mathcal{L}\left(\mathrm{M}_{1} \mathrm{y}_{1} \mathrm{P}_{1} \mathrm{~S}_{1} \mathrm{y}_{2}^{-1} \mathrm{M}_{2}^{-1} \mathrm{Y}_{2} \mathrm{M}_{2} \mathrm{y}_{1} \mathrm{P}_{1} \mathrm{~S}_{1} \mathrm{y}_{2}^{-1} \mathrm{M}_{1}^{-1}\right)\right]_{\alpha} \in\left[V_{1}\right]_{\alpha} \mathrm{S}_{\alpha}\left(B_{1}\right)\left[V_{1}\right]_{\alpha}^{-1},
$$

то отсюда следует

$$
[\mathcal{L}(\mathrm{W})]_{\alpha}^{-1}[X]_{\alpha}[\mathcal{L}(\mathrm{W})]_{\alpha} \in\left[V_{1}\right]_{\alpha} \mathbf{S}_{\alpha}\left(B_{1}\right)\left[V_{1}\right]_{\alpha}^{-1}
$$

Обозначим

$$
\mathbf{d} \rightleftharpoons\left[V_{1}^{-1}(\mathcal{L}(\mathrm{W}))^{-1} X \mathcal{L}(\mathrm{W}) V_{1}\right]_{\alpha}=\left[V_{1}^{-1}(\mathcal{L}(\mathrm{W}))^{-1} U A^{t} w U^{-1} \mathcal{L}(\mathrm{W}) V_{1}\right]_{\alpha}
$$

Так как в силу следствия 13.12 имеем ранг $\mathbf{d}=\operatorname{paн\imath }\left[A^{t} w\right]_{\alpha}=\alpha$, то в силу $(14.30)$ и предложения $13.15 \mathbf{d} \in \mathbf{S}_{\alpha}^{+}\left(B_{1}\right) \backslash \mathbf{S}_{\alpha}^{*}\left(B_{1}\right)$. Пусть $B_{1}^{r} v$ - нормальная форма ранга $\alpha$, представляющая элемент $\mathbf{d}$, существуюшая в силу предложения 13.3. Так как

$$
B_{1}^{r} v \stackrel{\alpha}{=} V_{1}^{-1}(\mathcal{L}(\mathrm{W}))^{-1} U A^{t} w U^{-1} \mathcal{L}(\mathrm{W}) V_{1}
$$

то, выбрав слово $N \in \mathcal{R}_{\alpha}$, равное в $\mathbf{B}_{\alpha}$ слову $V_{1}^{-1}(\mathcal{L}(\mathrm{W}))^{-1} U$, и применив предложение 13.10 и лемму 7.20, получим

$$
\mathbf{S}_{\alpha}\left(B_{1}\right)=\left[V_{1}^{-1}(\mathcal{L}(\mathrm{W}))^{-1} U\right]_{\alpha} \mathbf{S}_{\alpha}(A)\left[U^{-1} \mathcal{L}(\mathrm{W}) V_{1}\right]_{\alpha}
$$

т.е. искомое равенство (14.28). Рассмотрение случая 1В, а значит, и случая 1 , завершено.

Случай 2: $\Delta$ не имеет областей ранга $\alpha$. Тогда $\mathcal{S}$ - карта примыканий ранга $\alpha-1$ на диаграмме $\Delta=\Delta^{\alpha-1}$. Пусть $\mathrm{X} \approx \mathrm{P}_{1} \mathrm{~S}_{1} \mathrm{P}_{2} \mathrm{~S}_{2} \ldots \mathrm{P}_{k} \mathrm{~S}_{k}$, где $\mathrm{P}_{i}-$ участки примыкания поддиаграмм примыкания $\Pi \in \mathcal{S}$ и $\mathcal{S}_{i}-$ переходные участки диаграммы $\Delta$ относительно $\mathcal{S}$, входяшие в Х. Заметим, что в силу леммы 9.23 , (iv) $k \leqslant 4$. В силу $14.4,(\mathrm{v})$ имеем $\ell_{\alpha, A}\left(\mathcal{L}\left(\mathrm{P}_{1} \mathrm{~S}_{1} \mathrm{P}_{2} \mathrm{~S}_{2} \ldots \mathrm{P}_{k} \mathrm{~S}_{k}\right)\right) \geqslant t-3$. По предложению 10.4 имеем $\sum_{i=1}^{k} \mathcal{W}_{\alpha-1}\left(\mathcal{L}\left(\mathrm{S}_{i}\right)\right) \leqslant 2 \eta$, откуда в силу предложения 8.22 , (ii) следует $\sum_{i=1}^{k} \ell_{\alpha, A}\left(\mathcal{L}\left(\mathrm{S}_{i}\right)\right) \leqslant 13 \cdot 2 \eta+4<15$. Тогда в силу предложения 8.14 , (ii)

$$
\begin{aligned}
\ell_{\alpha, A}\left(\mathcal{L}\left(\mathrm{P}_{1} \mathrm{~S}_{1} \mathrm{P}_{2} \mathrm{~S}_{2} \ldots \mathrm{P}_{k} \mathrm{~S}_{k}\right)\right) & \leqslant \sum_{i=1}^{k} \ell_{\alpha, A}\left(\mathcal{L}\left(\mathrm{P}_{i}\right)\right)+\sum_{i=1}^{k} \ell_{\alpha, A}\left(\mathcal{L}\left(\mathrm{S}_{i}\right)\right)+14 \\
& <\sum_{i=1}^{k} \ell_{\alpha, A}\left(\mathcal{L}\left(\mathrm{P}_{i}\right)\right)+29
\end{aligned}
$$

Так как по условию $t-3 \geqslant 4(p+68)+29$, то отсюда следует, что для некоторого $i_{0}$ выполнено $\ell_{\alpha, A}\left(\mathcal{L}\left(\mathrm{P}_{i_{0}}\right)\right) \geqslant p+68$. 


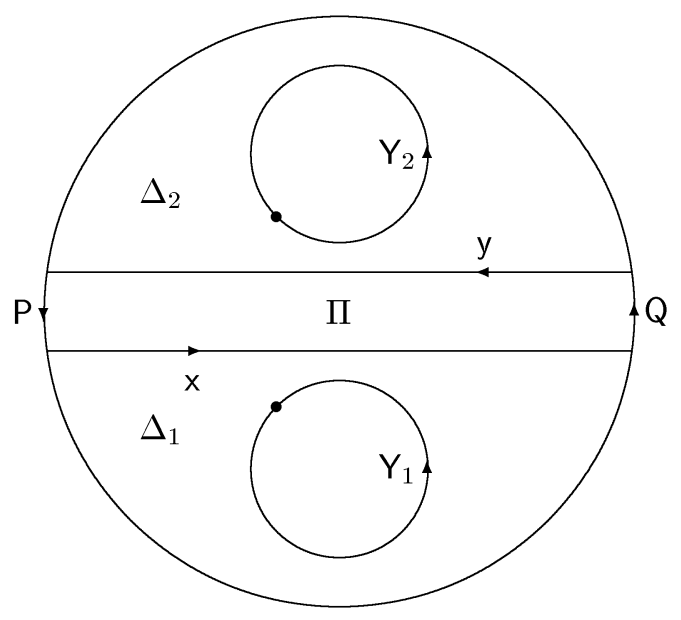

Рис. 75

Обозначим $\mathrm{P} \rightleftharpoons \mathrm{P}_{i_{0}}$, и пусть $\mathrm{P}$ входит в граничный цикл поддиаграммы примыкания $\Pi \in \mathcal{S}$. Пусть $\delta \Pi \approx \mathrm{PxQ}$, где $\mathrm{P}, \mathrm{Q}$ - участки примыкания и $\mathrm{x}, \mathrm{y}$ - невырожденные связки ранга $\alpha-1$ (см. определение 9.13$)$. В зависимости от того, в какой из граничных циклов $\mathrm{X}$ или $\mathrm{Y}_{i}^{-1}$ входит путь $\mathrm{Q}$, рассмотрим два подслучая.

Случай 2A: $\mathrm{Q}$ входит в $\mathrm{X}$ (см. рис. 75$)$. Пусть $\mathrm{M}$ и $\mathrm{N}$ - начала пути $\mathrm{X}$ с конечными вершинами $\iota(\mathrm{P})$ и $\iota(\mathrm{Q})$ соответственно. Диаграмма $\Delta-\Pi$ состоит из двух связных компонент $\Delta_{1}$ и $\Delta_{2}$, ни одна из которых не может быть односвязной в силу невырожденности связок х и у ранга $\alpha-1$ и определения 9.11. Следовательно, $\Delta_{1}$ и $\Delta_{2}-$ кольцевые диаграммы. В этом случае легко видеть, что группа $\pi_{1}(\Delta, \iota(\mathrm{X}))$ порождается классами $[\mathrm{X}]_{\Delta}$ и $\left[\mathrm{MPxN}^{-1}\right]_{\Delta}$. Поэтому достаточно установить включение

$$
\left[\mathcal{L}\left(\mathrm{MPxN}^{-1}\right)\right]_{\alpha} \in[U]_{\alpha} \mathbf{S}_{\alpha}(A)[U]_{\alpha}^{-1}
$$

Рассмотрим пути на графе $\Gamma_{\alpha-1}$, расположенные, как на рис. 76 , где $\mathcal{L}(\mathrm{U})$ 으 $\mathcal{L}\left(\mathrm{U}^{\prime}\right)$ 으 $U, \mathcal{L}(\mathrm{R})$ 으 $\mathcal{L}\left(\mathrm{R}^{\prime}\right)$ 으 $A^{2}$ и метки остальных путей совпадают с метками соответствуюших путей на диаграмме $\Delta$.

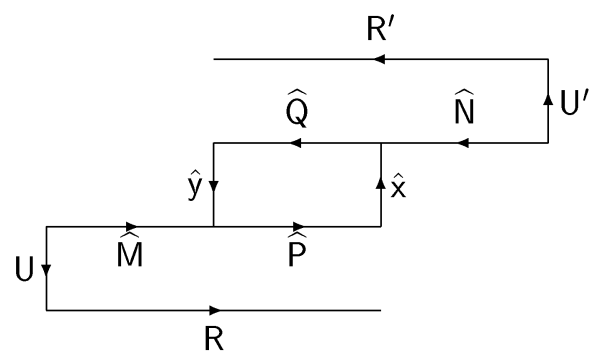

Рис. 76 
По выбору Р некоторый путь $\mathrm{E} \in$ ППер $\alpha(A, p+68)$ входит в $\widehat{\mathrm{P}}$. В силу 14.4 , (iv) и предложения 8.38, (i) имеем $\operatorname{Cozл}(\mathrm{R}, \mathrm{T})$, где $\mathrm{T} \in \Pi_{\mathrm{ep}}\left(\Gamma_{\alpha-1}, A, 2\right)$ - периодическая основа для Е. Так как пути $\widehat{\mathrm{P}}$ и $\widehat{\mathrm{Q}}^{-1}$ близки в ранге $\alpha-1$, то в силу предложения 8.26 для некоторого подпути $\mathrm{F} \in$ ППер $\alpha(A, p+34)$ пути $\widehat{\mathrm{Q}}^{-1}$ выполнено $\Pi$ Согл $\Omega_{\alpha}(\mathrm{E}, \mathrm{F})$. Тогда снова в силу 14.4 , (iv) и предложения 8.38 , (i) получим $\operatorname{Cozл}\left(\mathrm{R}^{\prime-1}, \mathrm{~T}^{\prime}\right)$, где $\mathrm{T}^{\prime} \in$ Пер $\left(\Gamma_{\alpha-1}, A, 2\right)$ - периодическая основа для $\mathrm{F}$. Отсюда следует $C о г л\left(\mathrm{R}, \mathrm{R}^{\prime-1}\right)$. Используя предложение 7.3 и тот факт, что вершины $\iota(\mathrm{R})$ и $\iota\left(\mathrm{R}^{\prime}\right)$ соединены путем $\mathrm{U}^{-1} \widehat{\mathrm{M}} \widehat{\mathrm{P}} \hat{\mathrm{x}} \widehat{\mathrm{N}}^{-1} \mathrm{U}^{\prime}$, получаем

$$
\left[\mathcal{L}\left(\mathrm{U}^{-1} \widehat{\mathrm{M}} \widehat{\mathrm{P}} \hat{\mathrm{x}} \widehat{\mathrm{N}}^{-1} \mathrm{U}^{\prime}\right)\right]_{\alpha-1} \in \mathbf{N}_{\alpha-1}(A)
$$

что равносильно искомому включению (14.31).

Случай 2B: $\mathrm{Q}$ входит в $\mathrm{Y}_{1}^{-1}$ или в $\mathrm{Y}_{2}^{-1}$. В силу симметрии ограничимся рассмотрением случая, когда $\mathrm{Q}$ входит в $\mathrm{Y}_{1}^{-1}$ (рис. 77 ). Пусть $\mathrm{M}$ - начало пути $\mathrm{X}$ с $\tau(\mathrm{M})=\iota(\mathrm{P})$ и $\mathrm{N}-$ начало пути $\mathrm{Y}_{1} \mathrm{c} \tau(\mathrm{N})=\tau(\mathrm{Q})$. Так как группа $\pi_{1}(\Delta, \iota(\mathrm{X}))$ порождается классами $[\mathrm{X}]_{\Delta}$ и $\left[\mathrm{My}^{-1} \mathrm{~N}^{-1} \mathrm{Y}_{1} \mathrm{NyM}^{-1}\right]_{\Delta}$, то достаточно убедиться в истинности включения

$$
\left[\mathcal{L}\left(\mathrm{My}^{-1} \mathrm{~N}^{-1} \mathrm{Y}_{1} \mathrm{NyM}^{-1}\right)\right]_{\alpha} \in[U]_{\alpha} \mathbf{S}_{\alpha}(A)[U]_{\alpha}^{-1}
$$

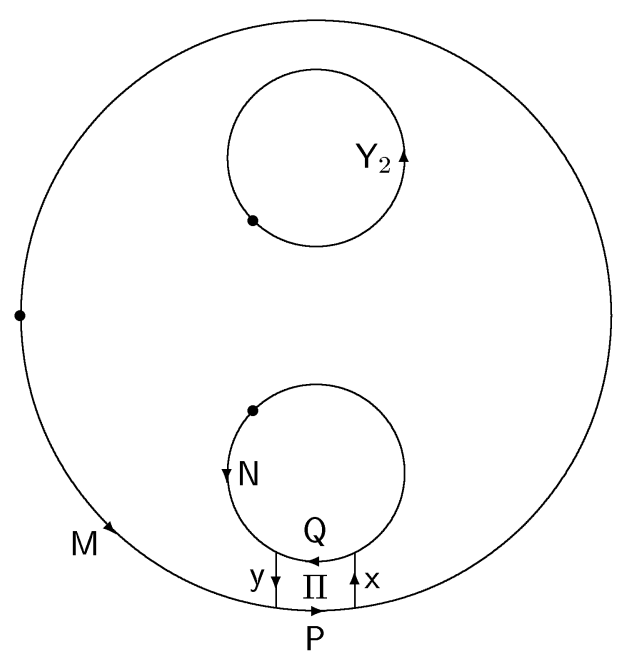

Рис. 77

Рассмотрим соответствующие пути на графе $\Gamma_{\alpha-1}$ (см. рис. 78), где $\mathcal{L}(\mathrm{U})$ 드 $U$, $\mathcal{L}(\mathrm{R})$ 으 $A^{2}, \mathcal{L}\left(\mathrm{V}_{1}\right)$ 으 $V_{1}$ и $\mathcal{L}\left(\mathrm{R}_{1}\right)$ 으 $B_{1}^{2}$. Как и в случае $2 \mathrm{~A}$, рассмотрим подпуть $\mathrm{E} \in$ ППер $\alpha(A, p+68)$ пути $\widehat{\mathrm{P}}$ и подпуть $\mathrm{F} \in \Pi$ Пер $\alpha(A, p+34)$ пути $\widehat{\mathrm{Q}}^{-1}$, где ПСогл $\Omega_{\alpha}(\mathrm{E}, \mathrm{F})$. Заметим, что из наличия $\mathrm{F}$ и выполнения условия 14.4 , (ii) для $A^{t} w:=B_{1}^{s_{1}} z_{1}, X:=Y_{1}$ и $U:=V_{1}$ вытекает $\beta_{1}=\alpha$. Далее, так как $\ell_{\alpha, A}(\mathrm{~F}) \geqslant$ 
$p+34$, то, используя 14.4 , (iv) для $A^{t} w:=B_{1}^{s_{1}} z_{1}, X:=Y_{1}, U:=V_{1}$ и предложение 8.38, (i), выводим Согл $\left(\mathrm{R}_{1}, \mathrm{~T}^{\prime}\right)$, где $\mathrm{T}^{\prime}$ - периодическая основа для $\mathrm{F}$. Отсюда следует $C о г л\left(\mathrm{R}, \mathrm{R}_{1}\right)$. Тогда, аналогично тому, как это делалось выше, для некоторого $r>0$ получаем

$$
A^{r} \stackrel{\alpha=1}{=} \mathcal{L}\left(\mathrm{U}^{-1} \widehat{\mathrm{M}}^{-1} \widehat{\mathrm{N}}^{-1} \mathrm{~V}_{1}\right) B_{1}^{r}\left(\mathcal{L}\left(\mathrm{U}^{-1} \widehat{\mathrm{M}} \hat{\mathrm{y}}^{-1} \widehat{\mathrm{N}}^{-1} \mathrm{~V}_{1}\right)^{-1}\right.
$$

и, следовательно, по лемме 7.20

$$
\mathbf{S}_{\alpha}(A)=\left[\mathcal{L}\left(\mathrm{U}^{-1} \widehat{\mathrm{M}} \hat{\mathrm{y}}^{-1} \widehat{\mathrm{N}}^{-1} \mathrm{~V}_{1}\right)\right]_{\alpha} \mathbf{S}_{\alpha}\left(B_{1}\right)\left[\left(\mathcal{L}\left(\mathrm{U}^{-1} \widehat{\mathrm{M}} \hat{\mathrm{y}}^{-1} \widehat{\mathrm{N}}^{-1} \mathrm{~V}_{1}\right)\right]_{\alpha}^{-1}\right.
$$

Тогда с учетом (14.21)

$$
\left[\mathcal{L}\left(\mathbf{U}^{-1} \widehat{\mathrm{M}}^{-1} \widehat{\mathrm{N}}^{-1}\right)\right]_{\alpha}\left[Y_{1}\right]_{\alpha}\left[\left(\mathcal{L}\left(\mathrm{U}^{-1} \widehat{\mathrm{M}} \hat{\mathrm{y}}^{-1} \widehat{\mathrm{N}}^{-1}\right)\right]_{\alpha}^{-1} \in \mathbf{S}_{\alpha}(A)\right.
$$

что влечет искомое включение (14.32).

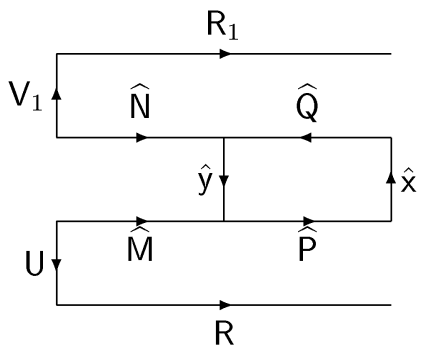

Рис. 78

Доказательство леммы 14.8 завершено.

ДОКАЗАТЕЛЬСТВО ПРЕДЛОЖЕНИЯ 14.3. Пусть $\mathbf{G} \neq 1$ - конечная подгруппа группы $\mathbf{B}_{\alpha}$ и $\beta \rightleftharpoons$ ранг $\mathbf{G} \geqslant 1$. Выберем произвольный элемент $\mathbf{g}_{0} \in \mathbf{G}$ такой, что $p a н z \mathbf{g}_{0}=\beta$. Заменяя $\mathbf{g}_{0}$ на некоторую его степень в силу леммы 13.14 , (ii), можно считать $n / 4 \leqslant$ степень $\mathbf{g}_{0} \leqslant 3 n / 4$. В силу определения 13.13 и предложения 13.15 элемент $\mathbf{g}_{0}$ сопряжен некоторому элементу из $\mathbf{S}_{\alpha}^{+}\left(A_{0}\right) \backslash \mathbf{S}_{\alpha}^{*}\left(A_{0}\right)$, где $A_{0} \in \overline{\mathcal{\varepsilon}}_{\beta}$. Докажем вспомогательный факт, из которого утверждение предложения 14.3 вытекает в силу очевидной индукции.

Пусть $\mathbf{H}$ - конечная подгруппа группы $\mathbf{B}_{\alpha}$, содержащая әлемент $\mathbf{g}_{0}$, $u \mathbf{H} \subseteq \mathbf{f}^{-1} \mathbf{S}_{\alpha}(A) \mathbf{f}$ для некоторых $\mathbf{f} \in \mathbf{B}_{\alpha} u A \in \overline{\mathcal{E}}_{\beta}$. Пусть $\mathbf{g} \in \mathbf{B}_{\alpha} u$ для любого $\mathbf{h} \in \mathbf{H}$ әлемент $\mathbf{g h}$ имеет конечный порядок и ранг $\mathbf{g h} \leqslant \beta$. Тогда $\langle\mathbf{H}, \mathbf{g}\rangle \subseteq \mathbf{f}^{*-1} \mathbf{S}_{\alpha}\left(A^{*}\right) \mathbf{f}^{*}$ для некоторых $\mathbf{f}^{*} \in \mathbf{B}_{\alpha} u A^{*} \in \bar{\varepsilon}_{\beta}$. 
Пусть верна посылка (14.33). В силу следствия 13.12 и предложения 13.15 имеем $\mathbf{h}_{0} \rightleftharpoons \mathbf{f g}_{0} \mathbf{f}^{-1} \in \mathbf{S}_{\alpha}^{+}(A) \backslash \mathbf{S}_{\alpha}^{*}(A)$. Если $\beta=\alpha$, то из соотношения $\mathbf{h}_{0}=\mathbf{f g}^{-1} \mathbf{f}^{-1} \times$ $\mathbf{f g g}_{0} \mathbf{f}^{-1}$, неравенства $n / 4 \geqslant 4 p+304$ и конечности порядка элементов $\mathbf{g}^{-1}$ и $\mathbf{g g}_{0}$ (которая вытекает из посылки (14.33) при $\mathbf{h}:=1$ и $\mathbf{h}:=\mathbf{g}_{0}$ ) по лемме 14.8 получаем $\mathbf{g}^{-1} \in \mathbf{f}^{-1} \mathbf{S}_{\alpha}(A) \mathbf{f}$. В этом случае $\langle\mathbf{H}, \mathbf{g}\rangle \subseteq \mathbf{f}^{-1} \mathbf{S}_{\alpha}(A) \mathbf{f}$ и можно положить $\mathbf{f}^{*} \rightleftharpoons \mathbf{f}$, $A^{*} \rightleftharpoons A$. Пусть $\beta<\alpha$. Пусть

$$
\mathbf{f} \mathbf{H} \mathbf{f}^{-1}=\left\{\mathbf{h}_{0}, \mathbf{h}_{1}, \ldots, \mathbf{h}_{k}\right\} \quad \text { и } \mathbf{h}_{i}=\left[x_{i}\right]_{\alpha} \text { для некоторых } x_{i} .
$$

Представим $\mathbf{g}$ в виде

$$
\mathbf{g}=\mathbf{e}^{-1}[y]_{\alpha} \mathbf{e},
$$

где $y$ - нормальная форма некоторого ранга $\gamma \leqslant \alpha-1$. Выберем произвольное слово $T$ такое, что

$$
\mathbf{f e}^{-1}=[T]_{\alpha} .
$$

Так как элемент $\mathbf{f}^{-1} \mathbf{h}_{i} \mathbf{f g}$ сопряжен элементу $\left[T^{-1} x_{i} T y\right]_{\alpha}$, то согласно посылке (14.33) для каждого $i$ элемент $\left[T^{-1} x_{i} T y\right]_{\alpha}$ имеет конечный порядок и ранг $\left[T^{-1} x_{i} T y\right]_{\alpha} \leqslant \beta$. Так как ранг $\mathbf{g} \leqslant$ ранг $\mathbf{H}=\beta$, то $\gamma \leqslant \beta$. Применяя лемму 14.7 последовательно при $\alpha:=\alpha, \alpha-1, \ldots, \beta+1$, найдем слова $U_{\gamma}, V_{\gamma}$ $(\gamma=\alpha, \alpha-1, \ldots, \beta+1), x_{i, \gamma}, T_{\gamma}$ и $y_{\gamma}(\gamma=\alpha, \alpha-1, \ldots, \beta)$ такие, что $x_{i}$ 으 $x_{i, \alpha}$, $T$ 흐 $T_{\alpha}, y$ 드 $y_{\alpha}$,

$$
\begin{gathered}
T_{\gamma} \stackrel{\gamma}{=} U_{\gamma} T_{\gamma-1} V_{\gamma} \quad(\gamma=\alpha, \alpha-1, \ldots, \beta+1) \\
U_{\gamma}^{-1} x_{i, \gamma} U_{\gamma} \stackrel{\gamma}{=} x_{i, \gamma-1}, \quad V_{\gamma} y_{\gamma} V_{\gamma}^{-1} \stackrel{\gamma}{=} y_{\gamma-1} \quad(\gamma=\alpha, \alpha-1, \ldots, \beta+1)
\end{gathered}
$$

для каждого $i$ элемент $\left[T_{\beta}^{-1} x_{i, \beta} T_{\beta} y_{\beta}\right]_{\beta}$ имеет конечный порядок, ранг $\left[T_{\beta}^{-1} x_{i, \beta} T_{\beta} y_{\beta}\right]_{\beta} \leqslant \beta$ и элементы $\left[x_{i, \beta}\right]_{\beta}$ образуют подгруппу группы $\mathbf{S}_{\beta}\left(A^{*}\right)$ для некоторого $A^{*} \in \overline{\mathcal{E}}_{\beta}$. Имеем

$$
x_{0, \beta} \stackrel{\beta}{=} T_{\beta}\left(T_{\beta}^{-1} x_{0, \beta} T_{\beta} y_{\beta}\right) T_{\beta}^{-1} \cdot T_{\beta} y_{\beta}^{-1} T_{\beta}^{-1},
$$

и так как с учетом леммы 13.14 , (i) $р а н г\left[T_{\beta} y_{\beta}^{-1} T_{\beta}^{-1}\right]_{\beta}=\operatorname{paн2}^{-1} \leqslant \beta$, то, применив индуктивное предположение 14.8, получим

$$
\left[T_{\beta} y_{\beta} T_{\beta}^{-1}\right]_{\beta} \in \mathbf{S}_{\beta}\left(A^{*}\right)
$$

Положим

$$
U \rightleftharpoons U_{\alpha} U_{\alpha-1} \ldots U_{\beta+1}, \quad V \rightleftharpoons V_{\beta+1} V_{\beta+2} \ldots V_{\alpha} \quad \text { и } \quad \mathbf{f}^{*} \rightleftharpoons[U]_{\alpha}^{-1} \mathbf{f} .
$$

Из равенств (14.36), (14.37) следует

$$
T \stackrel{\alpha}{=} U T_{\beta} V, \quad U^{-1} x_{i} U \stackrel{\alpha}{=} x_{i, \beta} \quad(i=0,1, \ldots, k), \quad V y V^{-1} \stackrel{\alpha}{=} y_{\beta} .
$$


Тогда для любого элемента $\mathbf{f}^{-1} \mathbf{h}_{i} \mathbf{f}$ подгрупшы $\mathbf{H}$ имеем

$$
\mathbf{f}^{*}\left(\mathbf{f}^{-1} \mathbf{h}_{i} \mathbf{f}\right) \mathbf{f}^{*-1}=[U]_{\alpha}^{-1} \mathbf{h}_{i}[U]_{\alpha}=\left[U^{-1} x_{i} U\right]_{\alpha}=\left[x_{i, \beta}\right]_{\alpha} \in \mathbf{S}_{\alpha}\left(A^{*}\right) .
$$

Кроме того, используя соотношения (14.34), (14.35), (14.38) и (14.39), получаем

$$
\begin{aligned}
\mathbf{f}^{*} \mathbf{g f}^{*-1} & =[U]_{\alpha}^{-1} \mathbf{f e}^{-1}[y]_{\alpha} \mathbf{e f}^{-1}[U]_{\alpha}=\left[U^{-1} T y T^{-1} U\right]_{\alpha} \\
& =\left[T_{\beta} V y V^{-1} T_{\beta}^{-1}\right]_{\alpha}=\left[T_{\beta} y_{\beta} T_{\beta}^{-1}\right]_{\alpha} \in \mathbf{S}_{\alpha}\left(A^{*}\right) .
\end{aligned}
$$

Таким образом, $\langle\mathbf{H}, \mathbf{g}\rangle \subseteq \mathbf{f}^{*-1} \mathbf{S}_{\alpha}\left(A^{*}\right) \mathbf{f}^{*}$, что и требовалось.

В оставшейся части параграфа мы докажем некоторое усиление предложения 14.3 для случая конечной подгруппы ранга, меньшего $\alpha$ (предложение 14.12).

14.9. Лемма. Пусть $\mathrm{T}^{-1} \mathbf{u T}^{\prime}-n y m b$ на графе $\Gamma_{\alpha-1} c \mathcal{L}(\mathrm{T})$ 으 $\mathcal{L}\left(\mathrm{T}^{\prime}\right) \in \mathcal{R}_{\alpha-1}$ $u[\mathcal{L}(\mathbf{u})]_{\alpha-1} \in \mathbf{S}_{\alpha-1}(D)$ для некоторого $D \in \bigcup_{\beta \leqslant \alpha-1} \overline{\mathcal{E}}_{\beta}$. Пусть $A \in \mathcal{E}_{\alpha} u$ $\mathrm{E}, \mathrm{F} \in \Pi$ Пер $\alpha(A, 42)$ - строго близкие в ранге $\rho(A)$ подпути путей $\mathrm{T}$ и $\mathrm{T}^{\prime}$ соответственно. Тогда ПСогл $(\mathrm{E}, \mathbf{g E})$, где $\mathbf{g} \rightleftharpoons \iota\left(\mathrm{T}^{\prime}\right)(\iota(\mathrm{T}))^{-1}$ - әлемент группы $\mathbf{B}_{\alpha-1}, \partial л я$ которого $\mathrm{T}^{\prime}=\mathbf{g T}$.

ДокАЗАТЕЛЬСТВо. Допустим, $\neg П С о \Omega_{\alpha}(\mathrm{E}, \mathrm{gE}) . \quad$ В силу леммы 8.12 ПСогл $\Omega_{\alpha}(\mathrm{E}, \mathrm{F})$, и, следовательно, имеем $\neg \Pi \operatorname{Coгл}_{\alpha}(\mathrm{gE}, \mathrm{F})$. Тогда по предложению 8.9 имеем либо $\mathrm{gE}<<_{\mathrm{T}^{\prime}} \mathrm{F}$, либо $\mathrm{F}<<_{\mathrm{T}^{\prime}} \mathrm{gE}$. Заметим, что утверждение леммы симметрично относительно замены $\mathrm{T}^{-1} \mathrm{uT}^{\prime} \rightarrow \mathrm{T}^{\prime-1} \mathrm{u}^{-1} \mathrm{~T}, \mathrm{E} \rightarrow \mathrm{F}, \mathrm{F} \rightarrow \mathrm{E}$ и $\mathbf{g} \rightarrow \mathbf{g}^{-1}$. Так как из $\mathrm{F}<_{\mathrm{T}^{\prime}} \mathbf{g E}$ следует $\mathbf{g}^{-1} \mathrm{~F}<_{\mathrm{T}} \mathrm{E}$, то достаточно ограничиться рассмотрением случая, когда $\mathrm{gE}<_{\mathrm{T}^{\prime}} \mathrm{F}$. Пусть $\overline{\mathrm{E}}$ - устойчивая часть пути $\mathrm{E}$ (см. 8.28). Мы получим противоречие, указав сколь угодно большое число попарно различных подпутей пути Т. Более точно, для любого $k \geqslant 0$ индукцией по $k$ мы докажем следующее утверждение:

существуют подпути $\mathrm{G}_{0}, \mathrm{G}_{1}, \ldots, \mathrm{G}_{k} \in \Pi_{\text {Пер }}(A, 8)$ пути $\mathrm{T}$, обладающие следующими свойствами:

(i) $\mathrm{G}_{k} \ll \mathrm{T} \mathrm{G}_{k-1} \ll \mathrm{T} \cdots \ll_{\mathrm{T}} \mathrm{G}_{0}$;

(ii) для каждого $i=0,1, \ldots, k$ пути $\mathbf{g}^{i} \overline{\mathrm{E}} u \mathrm{G}_{i}$ строго близки в ранге $\rho(A)$.

При $k=0$ можно положить $\mathrm{G}_{0} \rightleftharpoons \overline{\mathrm{E}}$ (тот факт, что путь $\mathrm{G}_{0}$ строго близок в ранге $\rho(A)$ к самому себе, вытекает из определений 6.5 и 5.16). Докажем, что из (i) и (ii) вытекает существование подпути $\mathrm{G}_{k+1} \in \Pi_{\text {Пер }}(A, 8)$ пути $\mathrm{T}$ такого, что $\mathrm{G}_{k+1} \ll \mathrm{T} \mathrm{G}_{k}$ и пути $\mathrm{G}_{k+1}$ и $\mathbf{g}^{k+1} \overline{\mathrm{E}}$ строго близки в ранге $\rho(A)$. В силу замечания из 8.28 найдется устойчивая часть $\overline{\mathrm{F}}$ для $\mathrm{F}$ такая, что пути $\overline{\mathrm{E}}$ и $\overline{\mathrm{F}}$ строго близки в ранге $\rho(A)$. Пусть $\mathbf{T}^{\prime}=\mathbf{U} \bar{F} V_{\text {и }} \mathbf{T}=\mathbf{W G}_{k} \mathrm{Z}$. Так как

$$
\begin{aligned}
\left(\iota\left(\mathbf{g}^{k} \mathbf{T}^{\prime}\right)\right)^{-1} \iota(\mathbf{T}) & =\left(\iota\left(\mathbf{g}^{k+1} \mathbf{T}\right)\right)^{-1} \iota(\mathbf{T})=\left((\iota(\mathbf{T}))^{-1} \mathbf{g} \iota(\mathbf{T})\right)^{-k-1} \\
& =\left((\iota(\mathbf{T}))^{-1} \iota\left(\mathbf{T}^{\prime}\right)\right)^{-k-1}=[\mathcal{L}(\mathbf{u})]_{\alpha-1}^{-k-1} \in \mathbf{S}_{\alpha-1}(D)
\end{aligned}
$$


и в силу индуктивного предположения 13.3 любой элемент из $\mathbf{S}_{\alpha-1}(D)$ представляется словом из $\mathcal{H}_{\alpha-1}$, то вершины $\iota\left(\mathbf{g}^{k} \mathrm{U}\right)=\iota\left(\mathbf{g}^{k} \mathrm{~T}^{\prime}\right)$ и $\iota(\mathrm{W})=\iota(\mathrm{T})$ близки в ранге $\alpha-1$. Заметим, что из $\mathrm{gE}<_{\mathrm{T}^{\prime}} \mathrm{F}$ и $\neg$ ПСог $\Omega_{\alpha}(\mathrm{gE}, \mathrm{F})$ следует $\mathrm{g}^{k+1} \mathrm{E}<_{\mathbf{g}^{k+1} \mathrm{~T}} \mathrm{~g}^{k} \mathrm{~F}$ и $\neg \Pi$ Согл $\Omega_{\alpha}\left(\mathbf{g}^{k+1} \mathrm{E}, \mathbf{g}^{k} \mathrm{~F}\right)$. Так как $\mathbf{g}^{k} \overline{\mathrm{F}}-$ устойчивая часть $\mathbf{g}^{k} \mathrm{~F}$, то верны утверждения (i) и (ii) леммы 8.25 для $\mathrm{E}:=\mathrm{g}^{k} \mathrm{~F}$ и $\overline{\mathrm{E}}:=\mathbf{g}^{k} \overline{\mathrm{F}}$. Из $\neg П С о \imath \Omega_{\alpha}\left(\mathbf{g}^{k+1} \mathrm{E}, \mathbf{g}^{k} \mathrm{~F}\right)$ в силу 8.25 , (i) и предложения 8.9 вытекает $\mathbf{g}^{k+1} \mathrm{E} \ll_{\mathbf{g}^{k+1}} \mathrm{~T} \mathbf{g}^{k} \overline{\mathrm{F}}$, т.е. $\mathbf{g}^{k+1} \mathrm{E}$ содержится в $\mathbf{g}^{k} \mathrm{U}$. Так как пути $\mathbf{g}^{k} \overline{\mathrm{F}}$ и $\mathbf{g}^{k} \overline{\mathrm{E}}$ строго близки в ранге $\rho(A)$, то в силу (ii) при $i:=k$ и леммы 8.2, (i), (ii) пути $\mathbf{g}^{k} \overline{\mathrm{F}}_{\text {и }} \mathrm{G}_{k}$ близки в ранге $\rho(A)$. Следовательно, конечные вершины $\tau\left(\mathbf{g}^{k} \mathbf{U}\right)=\iota\left(\mathbf{g}^{k} \overline{\mathrm{F}}\right)$ и $\tau(\mathrm{W})=\iota\left(\mathrm{G}_{k}\right)$ путей $\mathbf{g}^{k} \mathrm{U}$ и $\mathbf{W}$, как и их начальные вершины, близки в ранге $\alpha-1$. Тогда $\mathbf{g}^{k} \mathrm{U}$ и $\mathrm{W}$ - близкие в ранге $\alpha-1$

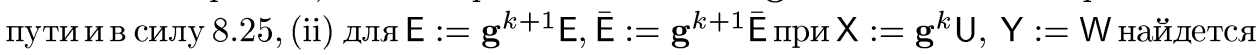
подпуть $\mathrm{G}_{k+1} \in$ ППер $\alpha(A, 8)$ пути $\mathrm{W}$ такой, что $\mathbf{g}^{k+1} \overline{\mathrm{E}}_{\text {и }} \mathrm{G}_{k+1}$ - строго близкие в ранге $\rho(A)$ пути. Тем самым завершен индуктивный переход от $k$ к $k+1$ и (14.40) установлено для всех значений $k$. В частности, согласно (14.40) (i) для любого $k$ должно существовать $k$ различных подпутей пути Т. Полученное противоречие завершает доказательство леммы.

14.10. Лемма. Пусть $\beta \geqslant \alpha u \mathrm{E} \in \ni_{\alpha}\left(\Gamma_{\beta}\right)$. Тогда существуют әлементарный период $A \in \overline{\mathcal{E}}_{\alpha}$ и слово $w \in \mathcal{H}_{\alpha-1}$ такие, что для любого $\mathbf{g} \in \mathbf{B}_{\beta}$ из ЭСог, $\Omega_{\alpha}(\mathrm{E}, \mathbf{g E})$ следует $(\tau(\mathrm{E}))^{-1} \mathbf{g} \tau(\mathrm{E}) \in[w]_{\beta}^{-1} \mathbf{S}_{\beta}(A)[w]_{\beta}$.

Доказательство. Пусть $\overline{\mathrm{E}} \in$ ППер $\alpha(A, 8)\left(A \in \mathcal{E}_{\alpha}\right)$ - прообраз $\mathrm{E}$ на графе $\Gamma_{\alpha-1}$ и $\mathrm{P} \in \Pi_{\mathrm{p}}\left(\Gamma_{\alpha-1}, A, 2\right)$ - периодическая основа для $\overline{\mathrm{E}}$. Можно считать, что слово $\mathcal{L}(\mathrm{P})$ оканчивается на $A$. Тогда из определения 5.16 и леммы 7.6 следует $A \in \overline{\mathcal{E}}_{\alpha}$. Пусть $\mathbf{g} \in \mathbf{B}_{\beta}$-произвольный элемент такой, что ЭСогл $\Omega_{\alpha}(\mathrm{E}, \mathbf{g E})$. Для некоторого $\overline{\mathbf{g}} \in \mathbf{B}_{\alpha-1}$ имеем $\pi_{\alpha-1, \alpha}(\overline{\mathbf{g}})=\mathbf{g}$ и $\Pi$ Сог $\Omega_{\alpha}(\overline{\mathrm{E}}, \overline{\mathbf{g}} \overline{\mathrm{E}})$ или $\Pi C о г \Omega_{\alpha}\left(\overline{\mathrm{E}}, \overline{\mathbf{g}} \overline{\mathrm{E}}^{-1}\right)$ и, следовательно, $С о г л(\mathrm{P}, \overline{\mathbf{g}} \mathrm{P})$ или $\operatorname{Coг} \Omega\left(\mathrm{P}, \overline{\mathbf{g}} \mathrm{P}^{-1}\right)$. Тогда в силу предложения 7.3 при $\mathrm{P}:=\mathrm{P}^{-1}$ и $A:=A^{-1}$ получаем $(\tau(\mathrm{P}))^{-1} \overline{\mathbf{g}} \tau(\mathrm{P}) \in \mathbf{N}_{\alpha-1}(A)$. Так как в силу леммы 8.2, (ii) пути Р и $\overline{\mathrm{E}}$ близки в ранге $\alpha-1$, то для некоторого $w \in \mathcal{H}_{\alpha-1}$ выполнено $(\tau(\mathrm{P}))^{-1} \tau(\overline{\mathrm{E}})=[w]_{\alpha-1}$. Отсюда следует $(\tau(\overline{\mathrm{E}}))^{-1} \overline{\mathbf{g}} \tau(\overline{\mathrm{E}}) \in[w]_{\alpha-1}^{-1} \mathbf{N}_{\alpha-1}(A)[w]_{\alpha-1}$. Применяя $\pi_{\alpha-1, \beta}$, получаем $(\tau(\mathrm{E}))^{-1} \mathbf{g} \tau(\mathrm{E}) \in[w]_{\beta}^{-1} \mathbf{S}_{\beta}(A)[w]_{\beta}$, что и требовалось.

14.11. Лемма $(\alpha \geqslant 2)$. Для любых $A \in \bar{\varepsilon}_{\alpha-1} u 1 \neq \mathbf{G} \leqslant \mathbf{S}_{\alpha-1}(A) c y$ ществуют әлементарный период $D \in \bar{\varepsilon}_{\beta}$ ранга $\beta \rightleftharpoons$ ранг $\mathbf{G}$, число $r \in \mathbb{Z} u$ слово $T \in \mathcal{R}_{\alpha}$, не содержащее әлементарных $(p+126)$-степеней ранга $\alpha-1$ и әлементарных 16-степеней ранга $\alpha$ такие, что

$$
\mathbf{G} \subseteq[A]_{\alpha-1}^{-r}[T]_{\alpha-1}^{-1} \mathbf{S}_{\alpha-1}(D)[T]_{\alpha-1}[A]_{\alpha-1}^{r}
$$

ДОКАЗАТЕЛЬСТВО. При $\beta=\alpha-1$ можно положить $D \rightleftharpoons A, r \rightleftharpoons 0$ и $T \rightleftharpoons 1$. Пусть $\beta<\alpha-1$. Тогда в силу индуктивных предположений 13.15 и 14.6 для некоторой конечной подгруппы $\widetilde{\mathbf{G}}$ группы $\mathbf{B}_{\alpha-2}$ имеем $\mathbf{G}=\pi_{\alpha-2, \alpha-1}(\widetilde{\mathbf{G}})$ и $\widetilde{\mathbf{G}} \subseteq \mathbf{N}_{\alpha-2}^{*}(A) \cup \mathbf{N}_{\alpha-2}^{-}(A)$. Пусть $\widetilde{\mathbf{G}}=\left\{\mathbf{g}_{1}, \mathbf{g}_{2}, \ldots, \mathbf{g}_{k}\right\}$. В силу индуктивного предположения 13.2, (iv) представим каждый элемент $\mathbf{g}_{i}$ в виде

$$
\mathbf{g}_{i}=\left[A^{-r} P_{i} u_{i} A^{r}\right]_{\alpha-2}
$$


где $P_{i}$ - начало слова $A^{ \pm 1}$ и $u_{i} \in \mathcal{H}_{\alpha-2}$. Обозначим

$$
\mathbf{h}_{i} \rightleftharpoons\left[P_{i} u_{i}\right]_{\alpha-2}=[A]_{\alpha-2}^{r} \mathbf{g}_{i}[A]_{\alpha-2}^{-r}
$$

Используя индуктивное предположение 14.3 , найдем пополнение $\left[T_{0}\right]_{\alpha-2}^{-1} \mathbf{S}_{\alpha-2}\left(D_{0}\right)\left[T_{0}\right]_{\alpha-2}$ подгруппы $\mathbf{H} \rightleftharpoons\left\{\mathbf{h}_{i}\right\}_{i}$. Заметим, что в силу леммы 13.14 , (iii) ранг $\mathbf{H}=$ ранг $\widetilde{\mathbf{G}}=$ ранг $\mathbf{G}=\beta$. Тогда в силу леммы 13.15 имеем $D_{0} \in \mathcal{E}_{\beta}$. В силу леммы 7.20 можно считать $D_{0} \in \overline{\mathcal{E}}_{\beta}$. Кроме того, можно считать $T_{0} \in \mathcal{R}_{\alpha-2}$.

Если в слово $T_{0}$ не входят элементарные $(p+126)$-степени ранга $\alpha-1$ и элементарные 16 -степени ранга $\alpha$, то по лемме 12.12 имеем $T_{0} \in \mathcal{R}_{\alpha}$ и можно положить $T \rightleftharpoons T_{0}, D \rightleftharpoons D_{0}$. Пусть в $T_{0}$ входит слово $E$, являюшееся элементарной $(p+126)$-степенью ранга $\alpha-1$ или элементарной 16-степенью ранга $\alpha$.

Пусть $\mathrm{F} \in \Pi_{\text {ер }}{ }_{\alpha-1}(B, 8)$, где $B \in \mathcal{E}_{\alpha-1}$. Путь $\mathrm{F}$ назовем плохим, если для каждого $i$ выполнено ПСогл $\Omega_{\alpha-1}\left(\mathrm{~F}, \mathbf{h}_{i} \mathrm{~F}\right)$. Пусть $\mathrm{T}_{0}-$ путь на $\Gamma_{\alpha-2}$ с $\tau\left(\mathrm{T}_{0}\right)=1$ и $\mathcal{L}\left(\mathrm{T}_{0}\right)$ 으 $T_{0}$. Докажем, что $\mathrm{T}_{0}$ содержит хотя бы один плохой подпуть $\mathrm{F} \in$ ППер $\alpha-1(B, 118)$ для некоторого $B \in \mathcal{E}_{\alpha-1}$.

Используя индуктивное предположение 13.3 , выберем слова $x_{i} \in \mathcal{H}_{\alpha-2}(i=$ $1,2, \ldots, k)$, представляюшие элементы подгруппы $\left[T_{0}\right]_{\alpha-2} \mathbf{H}\left[T_{0}\right]_{\alpha-2}^{-1} \subseteq \mathbf{S}_{\alpha-2}\left(D_{0}\right)$, т.е.

$$
x_{i} \stackrel{\alpha=2}{=} T_{0} P_{i} u_{i} T_{0}^{-1} \quad(i=1,2, \ldots, k) .
$$

Для каждого $i$ рассмотрим пути $\mathrm{P}_{i}, \mathrm{u}_{i}, \mathrm{x}_{i}$ и $\mathrm{T}_{0, i}$ с метками соответственно $P_{i}, u_{i}, x_{i}$ и $T_{0}$, расположенные, как на рис. 79 . Так как $\left(\tau\left(\mathrm{T}_{0}\right)\right)^{-1} \tau\left(\mathrm{T}_{0, i}\right)=\left[P_{i} u_{i}\right]_{\alpha-2}=\mathbf{h}_{i}$, то

$$
\mathrm{T}_{0, i}=\mathbf{h}_{i} \mathrm{~T}_{0}
$$

Пусть $\mathrm{E}$ - подпуть пути $\mathrm{T}_{0} \mathrm{c} \mathcal{L}(\mathrm{E})$ 으 $E$. Рассмотрим два случая.

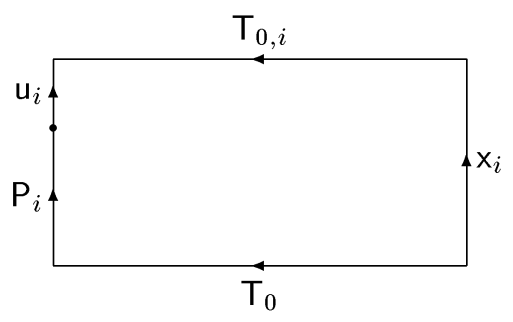

Рис. 79

Случай 1: $E$ - элементарная $(p+126)$-степень ранга $\alpha-1$, т.е. $\mathrm{E} \in \Pi$ Пер ${ }_{\alpha-1}(B$, $p+126)$ для некоторого $B \in \mathcal{E}_{\alpha-1}$. Так как $\left|P_{i}\right| \leqslant|A|$, то в силу индуктивных предположений 8.2 , (ii) и 8.44 , (i) для любого подпути $\mathrm{G} \in$ ППер $\alpha-1(B)$ пути $\mathrm{P}_{i}$ имеем $\ell_{\alpha, B}(\mathrm{G}) \leqslant p-1$. Так как $p+126-2 \cdot 45+5=p-1+42$, то в силу индуктивных предположений 11.7 , (ii) при $\mathrm{X}:=\mathrm{T}_{0}, \mathrm{~W}:=\mathrm{x}_{i} \mathrm{~T}_{0, i} \mathrm{u}_{i}^{-1} \mathrm{P}_{i}^{-1}, \mathrm{Z}_{1}:=\mathrm{T}_{0, i}$, $\mathrm{Z}_{2}:=\mathrm{P}_{i}^{-1}$ и 8.12 для некоторого подпути $\mathrm{H} \in$ ППер $\alpha-1(B, 42)$ пути $\mathrm{T}_{0, i}$ имеем 
ПСогл $\Omega_{\alpha-1}(\mathrm{E}, \mathrm{H})$, причем $\mathrm{H}$ и некоторый подпуть $\mathrm{H}^{\prime} \in \Pi_{\text {Пер }}{ }_{\alpha-1}(B, 42)$ пути $\mathrm{E} \mathrm{стро-}$ го близки в ранге $\rho(B)$. Тогда по лемме 14.9 (формально - по индуктивному предположению 14.9) при $\mathrm{T}^{-1} \mathbf{u T}^{\prime}:=\mathrm{T}_{0, i}^{-1} \mathrm{x}_{i}^{-1} \mathrm{~T}_{0}, \mathrm{E}:=\mathrm{H}$ и $\mathrm{F}:=\mathrm{H}^{\prime}$ для любого $i$ имеем ПСогл $\Omega_{\alpha-1}\left(\mathbf{H}, \mathbf{h}_{i}^{-1} \mathbf{H}\right)$, и, следовательно, ПСог $\Omega_{\alpha-1}\left(\mathrm{E}, \mathbf{h}_{i}^{-1} \mathrm{E}\right)$, т.е. путь Е является плохим.

Случай 2: $E$ - элементарная 16-степень ранга $\alpha$. По лемме 8.16 путь $Е$ можно разложить в произведение $\mathrm{E}=\mathrm{U}_{0} \mathrm{M}_{1} \mathrm{U}_{1} \ldots \mathrm{M}_{15} \mathrm{U}_{15}$, где $\mathrm{M}_{i} \in \Pi$ Пер $\alpha-1\left(B_{i}, 118\right)$ и никакой путь $\mathrm{M}_{k} \mathrm{U}_{k} \mathrm{M}_{k+1} \ldots \mathrm{U}_{k+9} \mathrm{M}_{k+10}$ не содержится в пути, близком в ранге $\alpha-2$ к пути $\mathrm{R} \in$ Пер $\left(\Gamma_{\alpha-2}, C\right)$ при $C \in \mathcal{E}_{\alpha-1}$. В частности, $\mathrm{M}_{4} \mathrm{U}_{4} \mathrm{M}_{5} \ldots \mathrm{U}_{13} \mathrm{M}_{14}$ не содержится ни в каком пути, близком в ранге $\alpha-2$ к подпути пути $\mathrm{P}_{i}^{-1}$. Применяя индуктивное предположение 11.7 , (i) при $\mathrm{X}:=\mathrm{Y}:=\mathrm{T}_{0}, \mathrm{~W}:=\mathrm{x}_{i} \mathrm{~T}_{0, i} \mathrm{u}_{i}^{-1} \mathrm{P}_{i}^{-1}$ и добавляя, если нужно, в полученное разложение пути $\mathrm{T}_{0}$ пустые пути, представим $\mathrm{T}_{0}$ в виде $\mathrm{T}_{0}=\mathrm{w}_{0} \mathrm{~S}_{1} \mathrm{w}_{1} \mathrm{~S}_{2} \mathrm{w}_{2}$, где $\sum_{j=0}^{2} \mathcal{W}_{\alpha-2}\left(\mathcal{L}\left(\mathrm{w}_{i}\right)\right) \leqslant \eta$ и каждый путь $\mathrm{S}_{j}$ либо пуст, либо входит в некоторый подпуть пути $\mathrm{T}_{0}$, близкий в ранге $\alpha-2$ к некоторому подпути пути $\mathrm{T}_{0, i}$ при $j=1$ или $\mathrm{P}_{i}^{-1}$ при $j=2$. В силу индуктивных предположений 8.22 , (i) и 8.20 , (iv) $\mathrm{M}_{1}$ не содержится в $\mathrm{w}_{0}$ и $\mathrm{M}_{15}$ не содержится в $w_{2}$. Следовательно, $\mathrm{M}_{2} \mathrm{U}_{2} \mathrm{M}_{3} \ldots \mathrm{U}_{13} \mathrm{M}_{14}$ содержится в $\mathrm{S}_{1} \mathrm{w}_{1} \mathrm{~S}_{2}$. В силу замечания, сделанного выше, $\mathrm{M}_{4} \mathrm{U}_{4} \mathrm{M}_{5} \ldots \mathrm{U}_{13} \mathrm{M}_{14}$ не содержится в $\mathrm{S}_{2}$, и так как в силу индуктивных предположений 8.22 , (i) и 8.20 , (iv) $\mathrm{M}_{3}$ не содержится в $\mathrm{w}_{1}$, получаем, что $\mathrm{M}_{2}$ содержится в $\mathrm{S}_{1}$. По индуктивному предположению 8.25 устойчивая часть $\overline{\mathrm{M}}_{2}$ для $\mathrm{M}_{2}$ и некоторый подпуть $\mathrm{N} \in$ ППер ${ }_{\alpha-1}(B, 84)$ пути $\mathrm{T}_{0, i}$ строго близки в ранге $\rho(B)$. Тогда в силу индуктивного предположения 14.9 путь $\overline{\mathrm{M}}_{2}$ является плохим.

В силу доказанного достаточно выбрать элементарный период $D \in \bar{\varepsilon}_{\beta}$ и слово $T \in \mathcal{R}_{\alpha-2}$ такие, что $\mathbf{H} \subseteq[T]_{\alpha-2}^{-1} \mathbf{S}_{\alpha-2}(D)[T]_{\alpha-2}$ и соответствуюший путь $\mathrm{T}$ на графе $\Gamma_{\alpha-2}$ с $\tau(\mathrm{T})=1$ и $\mathcal{L}(\mathrm{T})$ 으 $T$ не содержит плохих подпутей $\mathrm{F} \in \Pi \Pi е p_{\alpha-1}(B, 118)\left(B \in \mathcal{E}_{\alpha-1}\right)$.

Выберем плохой подпуть $\mathrm{F} \in \Pi_{\text {Пер }}{ }_{\alpha-1}(B, 8) \quad\left(B \in \mathcal{E}_{\alpha-1}\right)$ пути $\mathrm{T}_{0}$ с тем условием, что $\mathrm{T}_{0}=\mathrm{XFY}$, где $\mathrm{Y}$ не содержит плохих подпутей. Пусть $\mathrm{Q} \in$ Пер $\left(\Gamma_{\alpha-2}, B, 2\right)$ - периодическая основа для $\mathrm{F}$. Без ограничения обшности можно считать, что слово $\mathcal{L}(\mathrm{Q})$ оканчивается на $B$. Тогда из определения 5.16 и леммы 7.6 следует $B \in \bar{\varepsilon}_{\alpha-1}$. Так как $\mathbf{h}_{i} \mathrm{Q}$ - периодическая основа для $\mathbf{h}_{i} \mathrm{~F}$, то из ПСогл $\Omega_{\alpha-1}\left(\mathrm{~F}, \mathbf{h}_{i} \mathrm{~F}\right)$ следует $\operatorname{Coгл}\left(\mathrm{Q}, \mathbf{h}_{i} \mathrm{Q}\right)$. По индуктивному предположению 7.3 каждый элемент $\mathbf{h}_{i}$ сопряжен некоторому $\mathbf{f}_{i} \in \mathbf{N}_{\alpha-2}^{+}(B)$. Так как в силу индуктивных предположений 7.10 , (i) и 6.7 любой элемент из $\mathbf{N}_{\alpha-2}^{+}(B) \backslash$ $\mathbf{N}_{\alpha-2}^{*}(B)$ имеет бесконечный порядок, то $\mathbf{f}_{i} \in \mathbf{N}_{\alpha-2}^{*}(B)$. Так как по условию $\mathbf{G} \neq 1$, то в силу индуктивного предположения 7.14 , (i) имеем $\rho(B)>0$. Пусть

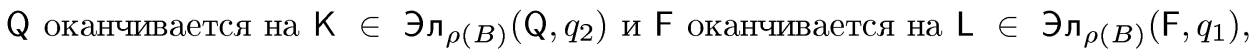
где $Э \operatorname{Coгл}_{\rho(B)}(\mathrm{K}, \mathrm{L})$. Из $\operatorname{Cогл}\left(\mathrm{Q}, \mathbf{h}_{i} \mathrm{Q}\right)$ и конечности порядка $\mathbf{h}_{i}$ в силу индук-

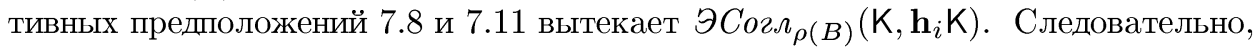
для каждого $i$ имеем ЭСогл $\rho_{\rho(B)}\left(\mathrm{L}, \mathbf{h}_{i} \mathrm{~L}\right)$. По лемме 14.10 выберем $C \in \bar{\varepsilon}_{\rho(B)}$ и $w \in \mathcal{H}_{\rho(B)-1}$ такие, что $(\tau(\mathrm{L}))^{-1} \mathbf{H} \tau(\mathrm{L}) \subseteq[w]_{\alpha-2}^{-1} \mathbf{S}_{\alpha-2}(C)[w]_{\alpha-2}$. Так как $\tau(\mathrm{L})=\iota(\mathrm{Y})=[Y]_{\alpha-2}^{-1}$, где $Y \rightleftharpoons \mathcal{L}(\mathrm{Y})$, то отсюда следует

$$
[w Y]_{\alpha-2} \mathbf{H}\left[Y^{-1} w^{-1}\right]_{\alpha-2} \subseteq \mathbf{S}_{\alpha-2}(C) .
$$


По индуктивному предположению леммы найдутся элементарный период $D \in \overline{\mathcal{\varepsilon}}_{\beta}$, число $s$ и слово $Z \in \mathcal{R}_{\alpha-1}$, не содержащее элементарных 16-степеней ранга $\alpha-1$, такие, что

$$
[w Y]_{\alpha-2} \mathbf{H}\left[Y^{-1} w^{-1}\right]_{\alpha-2} \subseteq[C]_{\alpha-2}^{-s}[Z]_{\alpha-2}^{-1} \mathbf{S}_{\alpha-2}(D)[Z]_{\alpha-2}[C]_{\alpha-2}^{s}
$$

Выберем слово $T \in \mathcal{R}_{\alpha-2}$ такое, что

$$
T \stackrel{\alpha=2}{=} Z C^{s} w Y
$$

Тогда из (14.41) следует

$$
\mathbf{H} \subseteq[T]_{\alpha-2}^{-1} \mathbf{S}_{\alpha-2}(D)[T]_{\alpha-2}
$$

Докажем, что $D$ и $T$ искомые, т.е. что путь $\mathrm{T}$ на $\Gamma_{\alpha-2}$ с $\tau(\mathrm{T})=1$ и $\mathcal{L}(\mathrm{T})$ 으 $T$ не содержит плохих подпутей $\mathrm{G} \in$ ППер ${ }_{\alpha-1}\left(B^{\prime}, 118\right)\left(B^{\prime} \in \mathcal{E}_{\alpha-1}\right)$.

Допустим, такой подпуть $\mathrm{G}$ пути $\mathrm{T}$ найдется, т.е. для всех $i$ выполнено $\Pi$ Согл $\Omega_{\alpha-1}\left(\mathrm{G}, \mathbf{h}_{i} \mathrm{G}\right)$. Рассмотрим пути $Z$ и $\vee$ на графе $\Gamma_{\alpha-2}$ с метками $\mathcal{L}(\mathbf{Z})$ 으 $Z$ и $\mathcal{L}(\mathrm{v})$ 으 $C^{s} w \in \mathcal{H}_{\rho(B)} \subseteq \mathcal{H}_{\alpha-2}$, как изображено на рис. 80. Так как $\ell_{\alpha-1, B^{\prime}}(\mathrm{G})>16+2 \cdot 45-5+8$, то по индуктивному предположению 11.7 , (ii) для некоторого подпути $\mathrm{H} \in$ ППер ${ }_{\alpha-1}\left(B^{\prime}, 8\right)$ пути $\mathrm{Y}_{\text {выполнено } П С о г \Omega_{\alpha-1}}(\mathrm{G}, \mathrm{H})$. Тогда для всех $i$ имеем ПСогл $\Omega_{\alpha-1}\left(\mathrm{H}, \mathbf{h}_{i} \mathrm{H}\right)$ и, следовательно, $\mathrm{H}$ - плохой путь. Но это противоречит выбору Ү. Лемма доказана.

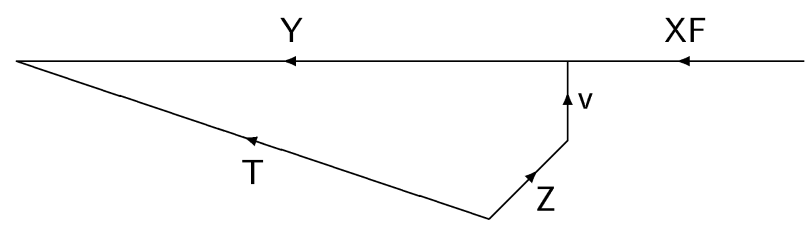

Рис. 80

14.12. ПРЕДЛОЖЕНИЕ. Пусть $\mathbf{G}, \mathbf{H} \leqslant \mathbf{B}_{\alpha}$ - конечные подгруппы, $1 \neq \mathbf{G} \subseteq$ $\mathbf{H}$ и ранг $\mathbf{H} \leqslant \alpha-1$. Тогда существует такое пополнение $\overline{\mathbf{G}}$ подгруппь $\mathbf{G}$, что для любых $\mathbf{g} \in \overline{\mathbf{G}} u \mathbf{h} \in \mathbf{H}$ әлемент $\mathbf{g h}$ имеет конечный порядок $и$ ранг $\mathbf{g h} \leqslant$ ранг $\mathbf{H}+1$.

ДокАЗАТЕЛЬСТво. Обозначим $\beta \rightleftharpoons р а н г \mathbf{H}$ и $\gamma \rightleftharpoons р а н г \mathbf{G}$. Если $\gamma=\beta$, то в качестве $\overline{\mathbf{G}}$ можно взять любое пополнение подгруппы $\mathbf{H}$. Пусть $\gamma<\beta$. Рассматривая вместо подгрупшы $\mathbf{H}$ ее пополнение и сопрягая $\mathbf{G}$ и $\mathbf{H c}$ помощью подходящего элемента группы $\mathbf{B}_{\alpha}$, можно считать, что $\mathbf{H}=\mathbf{S}_{\alpha}(A)$ для некоторого $A \in \overline{\mathcal{E}}_{\beta}$. По лемме 14.11 при $\alpha:=\beta+1$ и $\mathbf{G}:=\pi_{\beta, \alpha}^{-1}(\mathbf{G})$ найдутся элементарный период $D \in \overline{\mathcal{E}}_{\gamma}$, число $r \in \mathbb{Z}$ и слово $T$, не содержащее элементарных 16-степеней ранга $\beta$ и элементарных $(p+126)$-степеней ранга $\beta-1$, такие, что $\pi_{\beta, \alpha}^{-1}(\mathbf{G}) \subseteq$ 
$[A]_{\beta}^{-r}[T]_{\beta}^{-1} \mathbf{S}_{\beta}(D)[T]_{\beta}[A]_{\beta}^{r}$. Докажем, что $\overline{\mathbf{G}} \rightleftharpoons[A]_{\alpha}^{-r}[T]_{\alpha}^{-1} \mathbf{S}_{\alpha}(D)[T]_{\alpha}[A]_{\alpha}^{r}-$ искомое пополнение $\mathbf{G}$.

Пусть $\mathbf{g} \in \overline{\mathbf{G}}$ и $\mathbf{h} \in \mathbf{S}_{\alpha}(A)$ - произвольные элементы. Допустим, gh имеет бесконечный порядок или gh имеет конечный порядок, но ранг gh $\geqslant \beta+2$. По предложению 13.3 представим элементы $[T]_{\alpha}[A]_{\alpha}^{r} \mathbf{g}[A]_{\alpha}^{-r}[T]_{\alpha}^{-1} \in \mathbf{S}_{\alpha}(D)$ и $[A]_{\alpha}^{r} \mathbf{h}[A]_{\alpha}^{-r} \in \mathbf{S}_{\alpha}(A)$ словами $x \in \mathcal{H}_{\gamma} \subseteq \mathcal{H}_{\beta-1}$ и $y$ 으 $P w \in \mathcal{H}_{\beta}$, где $P$ - подслово слова $A^{n}$ и $w \in \mathcal{H}_{\beta-1}$. Заметим, что gh и $\left[T^{-1} x T y\right]_{\alpha}$ сопряжены. Если $\left[T^{-1} x T y\right]_{\alpha}$ имеет бесконечный порядок, то по предложению 12.24 слово $T^{-1} x T y$ сопряжено в группе $\mathbf{B}_{\alpha}$ некоторому периоду $C$ ранга $\alpha+1$. Если $\left[T^{-1} x T y\right]_{\alpha}$ имеет конечный порядок и ранг $\left[T^{-1} x T y\right]_{\alpha}=\delta$ при $\beta+2 \leqslant \delta \leqslant \alpha$, то, используя предложение 13.5 и лемму 14.4 (в нормальной форме $B^{s} v$ ранга $\delta$, представляющей элемент, сопряженный $\left[T^{-1} x T y\right]_{\alpha}$, можно считать $\left.s \leqslant \frac{n}{2}\right)$, найдем период $C$ ранга $\delta$ такой, что слова $T^{-1} x T y$ и $C$ сопряжены в $\mathbf{B}_{\alpha}$ и $C$ - циклически приведенное в ранге $\alpha$ слово. В обоих случаях слово $C$ циклически приведено в ранге $\alpha$ и по лемме 5.10 является периодом ранга $\beta+2$. Тогда по предложению 12.27 некоторый циклический сдвиг слова $C$ содержит элементарную 109-степень ранга $\beta+1$.

По предложению 11.34 , заменяя, возможно, слово $y$ на дополнительное (при этом вместо $P \in \Pi е р(A)$ будем иметь $\left.P \in \Pi е p(A) \cup \Pi е p\left(A^{-1}\right)\right)$, получаем, что слова $T^{-1} x T y$ и $C$ сопряжены уже в группе $\mathbf{B}_{\beta-1}$. Рассмотрим кольцевую А-диаграмму $\Delta$ ранга $\beta-1$ с двумя граничными циклами $\mathrm{T}^{\prime-1} \mathrm{xTvPw}$ и $\mathrm{C}^{-1}$, где

$$
\begin{gathered}
\mathcal{L}\left(\mathrm{T}^{\prime}\right) \text { 으 } \mathcal{L}(\mathrm{T}) \text { 으 } T, \quad \mathcal{L}(\mathrm{x}) \text { 으 } x, \quad \mathcal{L}(\mathrm{v}) \text { 으 } 1, \quad \mathcal{L}(\mathrm{P}) \text { 으 } P, \quad \mathcal{L}(\mathrm{w}) \text { 으 } w \\
x_{\beta-1}(\Delta)=\left\{\mathrm{T}^{\prime-1}, \mathrm{~T}, \mathrm{P}, \mathrm{C}^{-1}\right\} \text { и } y_{\beta-1}(\Delta)=\{\mathrm{x}, \mathrm{v}, \mathrm{w}\} .
\end{gathered}
$$

По предложению 9.19 можно считать, что на $\Delta$ задана карта примыканий $\mathcal{S}$ ранга $\beta-1$. Пусть $\mathrm{C} \approx \mathrm{R}_{1} \mathrm{~S}_{1} \mathrm{R}_{2} \mathrm{~S}_{2} \ldots \mathrm{R}_{\ell} \mathrm{S}_{\ell}$, где $\mathrm{R}_{i}^{-1}$ - содержашиеся в $\mathrm{C}^{-1}$ участки примыкания подлиаграмм примыкания $\Pi \in \mathcal{S}_{\text {и }} \mathrm{S}_{i}^{-1}$ - переходные участки границы диаграммы $\Delta$ относительно $\mathcal{S}$. По лемме 9.23 , (iii) имеется не более одной поддиаграммы примыкания $\Pi \in \mathcal{S}$ граничного цикла $C^{-1}$ к каждому из участков границы $\mathrm{T}^{\prime-1}, \mathrm{~T}$ и $\mathrm{P}$ и не существует поддиаграмм примыкания $\mathrm{C}^{-1} \mathrm{~K}^{-1}$. В частности, $\ell \leqslant 3$. Перенумеровав $\mathrm{R}_{i}$, будем считать, что $\mathrm{R}_{1}$ - участок примыкания поддиграммы примыкания $\mathrm{C}^{-1}$ к $\mathrm{T}^{\prime-1}, \mathrm{R}_{2}-\mathrm{C}^{-1}$ к $\mathrm{T}$ и $\mathrm{R}_{3}-\mathrm{C}^{-1}$ к $\mathrm{P}$ (некоторые из $\mathrm{R}_{i}$ теперь могут отсутствовать). Согласно лемме 9.14 каждое слово $\mathcal{L}\left(\mathrm{R}_{i}\right)$ и некоторое подслово соответствуюшего слова $T^{-1}, T$ или $P$ близки в ранге $\beta-1$.

Пусть $E \in \Pi$ Пер $p_{\beta+1}(B, 109)\left(B \in \mathcal{E}_{\beta+1}\right)$ - слово, входящее в циклический сдвиг слова $C$. Тогда $E$ имеет вид $w_{0} Q_{1} w_{1} \ldots Q_{t} w_{t}$, где $t \leqslant 4, Q_{i}$ - подслово некоторого слова $\mathcal{L}\left(\mathrm{R}_{j_{i}}\right)$ и $w_{i}$ либо пусто, либо входит в некоторое слово $\mathcal{L}\left(\mathrm{S}_{k_{i}}\right)$, причем все $j_{i}$ попарно различны, за исключением, возможно, пары $j_{1}$ и $j_{t}$. В силу индуктивных предположений 10.4 и 8.20 , (ii) для каждого $i$ имеем $\mathcal{W}_{\beta}\left(w_{i}\right) \leqslant 3 \eta$, откуда в силу индуктивного предположения 8.22 , (i) вытекает $\ell_{\beta+1, B}\left(w_{i}\right) \leqslant 1$. Далее, с учетом индуктивного предположения 8.30 имеем $\ell_{\beta+1, B}\left(Q_{i}\right) \leqslant 23$, если $j_{i}=1$ или $j_{i}=2$, а так как в силу индуктивных предположений 8.15 и 8.2, (ii) слово $P$ не содержит

\footnotetext{
7 Серия математическая, №3
} 
элементарных 10 -степеней ранга $\beta+1$, то $\ell_{\beta+1, B}\left(Q_{i}\right) \leqslant 17$, если $j_{i}=3$. Тогда, используя индуктивное предположение 8.14 , (ii), получаем $\ell_{\beta+1, B}(E) \leqslant 3 \cdot 23+$ $17+5+2 \cdot 8=107$. Противоречие.

Таким образом, для любых $\mathbf{g} \in \overline{\mathbf{G}}$ и $\mathbf{h} \in \mathbf{H}$ элемент $\mathbf{g h}$ имеет конечный порядок и $р а н г \mathbf{g h} \leqslant \beta+1$.

\section{$\S 15$. Строение конечных подгрупп группы $\mathrm{B}_{\alpha}$}

15.1. ОПРЕДЕЛЕНИЕ. Пусть $\mathbf{G}=\mathbf{x}^{-1} \mathbf{S}_{\alpha}(A) \mathbf{x}\left(A \in \bigcup_{\beta \leqslant \alpha} \mathcal{E}_{\beta}\right)$ - полная конечная подгруппа группы $\mathbf{B}_{\alpha}$.

Через $\mathbf{G}^{+}, \mathbf{G}^{*}$ и $\mathbf{G}^{-}$будем обозначать соответственно подгруппы $\mathbf{x}^{-1} \mathbf{S}_{\alpha}^{+}(A) \mathbf{x}$, $\mathbf{x}^{-1} \mathbf{S}_{\alpha}^{*}(A) \mathbf{x}$ и множество $\mathbf{x}^{-1} \mathbf{S}_{\alpha}^{-}(A) \mathbf{x}$.

Элемент $\mathbf{x}^{-1}[A]_{\alpha} \mathbf{x}$ будем называть базисным әлементом подгруппы $\mathbf{G}$.

Из предложения 13.17 вытекает, что обозначения $\mathbf{G}^{+}, \mathbf{G}^{*}$ и $\mathbf{G}^{-}$корректны, т.е. не зависят от выбора представления группы $\mathbf{G}$ в виде $\mathbf{x}^{-1} \mathbf{S}_{\alpha}(A) \mathbf{x}$ при $A \in \bigcup_{\beta \leqslant \alpha} \mathcal{E}_{\beta}$. Сформулируем еще два непосредственных следствия из утверждений, доказанных в $\S 13$ и $\S 14$, которые понадобятся нам в дальнейшем.

15.2. ПРЕДЛОЖЕНИЕ. Если $\mathbf{G}_{1} u \mathbf{G}_{2}$ - два пополнения конечной подгруппь $\mathbf{H} \leqslant \mathbf{B}_{\alpha}, m o \mathbf{H} \cap \mathbf{G}_{1}^{+}=\mathbf{H} \cap \mathbf{G}_{2}^{+}, \mathbf{H} \cap \mathbf{G}_{1}^{*}=\mathbf{H} \cap \mathbf{G}_{2}^{*} u \mathbf{H} \cap \mathbf{G}_{1}^{-}=\mathbf{H} \cap \mathbf{G}_{2}^{-}$.

ДокАЗАТЕльСТво. Вытекает из предложений 13.17 и 14.2.

15.3. ПРЕДЛОЖЕНИЕ. Если $\mathbf{G}$ - пополнение конечной подгруппь $\mathbf{H} \leqslant \mathbf{B}_{\alpha}$, $\mathbf{x} \in \mathbf{B}_{\alpha} u \mathbf{x}^{-1} \mathbf{H} \mathbf{x} \subseteq \mathbf{G}$, mо найдется $\mathbf{y} \in \mathbf{G}$ такой, что $\mathbf{x}^{-1} \mathbf{h x}=\mathbf{y}^{-1} \mathbf{h y}$ для всех $\mathbf{h} \in \mathbf{H}$.

ДокАЗАТЕЛЬСТво. Вытекает из предложения 13.16.

Наша цель сейчас - получить некоторое описание конечных подгрупп группы $\mathbf{B}_{\alpha}$ (предложение 15.9) и одновременно подготовить техническую базу для $\S 16$, в доказательстве основного результата которого (предложение 16.1) свойства конечных подгрупп групп $\mathbf{B}_{\alpha}$ будут играть главную роль. Фактически мы уже сделали первый шаг в этом направлении: благодаря предложению 14.3 задача описания конечных подгрупп групшы $\mathbf{B}_{\alpha}$ сводится к задаче описания ее полных конечных подгрупп. Ряд фактов о строении полных конечных подгрупп $\mathbf{B}_{\alpha}$ по существу был получен ранее, в предыдущих параграфах. Соберем эти факты вместе.

Прежде всего заметим, что в силу определения 7.13 , предложения 13.7 , (ii) и предложения 7.2 , (ii) для любой полной конечной подгруппы $\mathbf{G} \leqslant \mathbf{B}_{\alpha}$ имеем либо $\mathbf{G}=\mathbf{G}^{+}$, либо $\left|\mathbf{G}: \mathbf{G}^{+}\right|=2$ и во втором случае $\mathbf{G} / \mathbf{G}^{+}=\left\{\mathbf{G}^{+}, \mathbf{G}^{-}\right\}$. Более существенную информацию о строении $\mathbf{G}$ можно получить, рассмотрев ее подгруппу $\mathbf{G}^{*}$, которая в силу следствия 7.16 нормальна в $\mathbf{G}$. В силу определения 7.12, предложения 7.10, (iii), (iv) и предложения 13.7, (ii) факторгруппа $\mathbf{G}^{+} / \mathbf{G}^{*}$ - циклическая группа порядка $n$, порожденная элементом $\mathbf{c G}^{*}$, где 
$\mathbf{c}-$ базисный элемент $\mathbf{G}$. Более того, так как $\mathbf{c}^{n}=1$, то $\mathbf{G}^{+}$, а значит, и сама группа $\mathbf{G}$ в случае $\mathbf{G}^{-}=\varnothing$, есть полупрямое произведение нормальной подгруппы $\mathbf{G}^{*}$ и циклической подгруппы $\langle\mathbf{c}\rangle$. Теперь пусть $\mathbf{G}^{-} \neq \varnothing$. В этом случае $\left|\mathbf{G}: \mathbf{G}^{*}\right|=\left|\mathbf{G}: \mathbf{G}^{+}\right| \cdot\left|\mathbf{G}^{+}: \mathbf{G}^{*}\right|=2 n$. В силу следствия 7.17 для любого $\mathbf{a} \in \mathbf{G}^{-}$имеем $\mathbf{a}^{2} \in \mathbf{G}^{*}$, и, в частности, $\left|\left\langle\mathbf{G}^{*}, \mathbf{a}\right\rangle: \mathbf{G}^{*}\right|=2$. Так как $\mathbf{G}=\left\langle\mathbf{G}^{+}, \mathbf{a}\right\rangle=\left\langle\mathbf{G}^{*}, \mathbf{c}, \mathbf{a}\right\rangle$ и $\mathbf{a c} \in \mathbf{G}^{-}$, то факторгруппа $\mathbf{G} / \mathbf{G}^{*}$ порождается двумя инволюциями $\mathbf{a G}^{*}$ и $\mathbf{a}^{-1} \mathbf{c G}^{*}$, где $\mathbf{a} \in \mathbf{G}^{-}$- произвольный элемент. Отсюда следует, что $\mathbf{G} / \mathbf{G}^{*}$ - диэдральная группа порядка $2 n$. Из сказанного следует также, что $\mathbf{G} / \mathbf{G}^{*}$ имеет задание $\left\langle\overline{\mathbf{a}}, \overline{\mathbf{c}} \mid \overline{\mathbf{a}}^{2}=(\overline{\mathbf{a}} \overline{\mathbf{c}})^{2}=\overline{\mathbf{c}}^{n}=1\right\rangle$, где $\overline{\mathbf{a}} \rightleftharpoons \mathbf{a G}^{*}$ и $\overline{\mathbf{c}} \rightleftharpoons \mathbf{c G}^{*}$. Мы будем пользоваться этим фактом для вычислений в группе $\mathbf{G} / \mathbf{G}^{*}$.

Таким образом, нам известно строение факторгруппы $\mathbf{G} / \mathbf{G}^{*}$. Наша задача сейчас - свести соотношения в группе $\mathbf{G}$ к соотношениям в конечной подгруппе $\mathbf{H} \leqslant \mathbf{B}_{\alpha}$ меньшего ранга, не теряя при этом информацию о соотношениях в $\mathbf{G}^{*}$. Более точно, для любой полной конечной подгруппы $\mathbf{G} \leqslant \mathbf{B}_{\alpha}$ мы построим гомоморфизм $\mathbf{G}$ на некоторую подгруппу $\mathbf{H} \leqslant \mathbf{B}_{\alpha}$ с ранг $\mathbf{H}<$ ранг $\mathbf{G}$, тождественный на $\mathbf{G}^{*}$. Заметим, что если $\mathbf{H} \leqslant \mathbf{B}_{\alpha}-$ конечная подгруппа ранга $\beta<\alpha$, то в силу предложений 14.3 и 13.7 , (i) в группе $\mathbf{B}_{\beta}$ найдется подгруппа $\widetilde{\mathbf{H}}$, изоморфная $\mathbf{H}$. Так как мы действуем в рамках индукции по рангу $\alpha$, то строение подгруппы $\mathbf{H}$ можно считать известным.

Нам понадобится описание элементов максимального ранга полной конечной подгруппы $\mathbf{G} \in \mathbf{B}_{\alpha}$, данное в предложении 13.15 . Согласно этому предложению

$$
\forall \mathbf{g} \in \mathbf{G}\left\{\begin{array}{l}
\text { ранг } \mathbf{g}=\operatorname{ранг} \mathbf{G} \Leftrightarrow \mathbf{g} \in \mathbf{G}^{+} \backslash \mathbf{G}^{*} \\
\text { ранг } \mathbf{g}<\operatorname{ранг} \mathbf{G} \Leftrightarrow \mathbf{g} \in \mathbf{G}^{*} \cup \mathbf{G}^{-}
\end{array}\right.
$$

Попутно заметим, что из (15.1) и сделанных выше замечаний легко вытекает следуюшая лемма, которая будет использоваться в $\S 16$.

15.4. ЛЕмма. Пусть $\mathbf{G} \leqslant \mathbf{B}_{\alpha}-$ полная конечная подгруппа, $\mathbf{H} \leqslant \mathbf{G} u$ ранг $\mathbf{H}<$ ранг $\mathbf{G}$. Тогда

(i) $р а н г \mathbf{H G} \mathbf{G}^{*}<$ ранг $\mathbf{G}$;

(ii) $е с л u \mathbf{h} \in \mathbf{H} \cap \mathbf{G}^{-}$, mo $\mathbf{H} \subseteq\left\langle\mathbf{G}^{*}, \mathbf{h}\right\rangle$;

(iii) $\left|\mathbf{H G}^{*}: \mathbf{G}^{*}\right| \leqslant 2 u\left|\mathbf{H}: \mathbf{H} \cap \mathbf{G}^{*}\right| \leqslant 2$.

Перейдем к построению указанного гомоморфизма полной конечной подгруппы $\mathbf{G} \in \mathbf{B}_{\alpha}$ на конечную подгруппу меньшего ранга.

15.5. Лемма. Пусть $\mathbf{G}, \mathbf{H}-$ конечные подгруппь группь $\mathbf{B}_{\alpha}, \mathbf{H} \subseteq \mathbf{G} u$ $|\mathbf{G}: \mathbf{H}| \leqslant 2$. Пусть $\overline{\mathbf{H}}$ - пополнение подгруппы $\mathbf{H}$. Тогда существует гомоморфизм $\phi: \mathbf{G} \rightarrow \overline{\mathbf{H}}$, тождественный на $\mathbf{H}$.

ДоКАЗАТЕЛЬСТВО. Если $р а н г \mathbf{G}=$ ранг $\mathbf{H}$, то в силу предложений 14.3 и 14.2 имеем $\mathbf{G} \subseteq \mathbf{z}^{-1} \overline{\mathbf{H}} \mathbf{z}$ для некоторого элемента $\mathbf{z} \in \mathbf{B}_{\alpha}$, коммутирующего со всеми элементами из $\mathbf{H}$. В этом случае в качестве $\phi$ можно взять вложение $\mathbf{G} \rightarrow \overline{\mathbf{H}}$, 
определяемое равенством $\phi(\mathbf{g})=\mathbf{z g z}^{-1}$. Пусть ранг $\mathbf{G}>$ ранг $\mathbf{H}$. В силу очевидной индукции достаточно указать тождественный на $\mathbf{H}$ гомоморфизм $\psi: \mathbf{G} \rightarrow \mathbf{B}_{\alpha}$

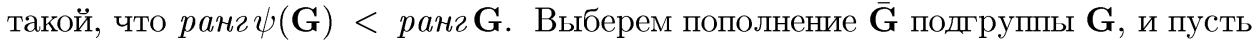
$\mathbf{c}$ - базисньй элемент $\mathbf{G}$. Очевидно, $\mathbf{c}^{n / 2}$ - инволюция, и в силу следствия 7.19 элемент $\mathbf{c}^{n / 2}$ лежит в центре групш $\overline{\mathbf{G}}$. Так как $|\mathbf{G}: \mathbf{H}| \leqslant 2$, то отображение $\psi: \mathbf{G} \rightarrow \mathbf{B}_{\alpha}$, задаваемое равенствами

$$
\psi(\mathbf{g})= \begin{cases}\mathbf{g}, & \text { если } \mathbf{g} \in \mathbf{H}, \\ \mathbf{c}^{n / 2} \mathbf{g}, & \text { если } \mathbf{g} \in \mathbf{G} \backslash \mathbf{H},\end{cases}
$$

является гомоморфизмом групп. Докажем, что ранг $\psi(\mathbf{g})<$ ранг $\mathbf{G}$ для любого $\mathbf{g} \in \mathbf{G}$. Если $\mathbf{g} \in \mathbf{G} \cap \overline{\mathbf{G}}^{-}$, то $\psi(\mathbf{g}) \in \overline{\mathbf{G}}^{-}$, и тогда ранг $\psi(\mathbf{g})<$ ранг $\mathbf{G}$ согласно (15.1). Пусть $\mathbf{g} \in \mathbf{G} \cap \overline{\mathbf{G}}^{+}$. Так как $\mathbf{g}^{2} \in \mathbf{H}$, то ранг $\mathbf{g}^{2}<$ ранг $\mathbf{G}$, откуда в силу (15.1) имеем либо $\mathbf{g} \in \overline{\mathbf{G}}^{*}$, либо $\mathbf{g} \in \mathbf{c}^{n / 2} \overline{\mathbf{G}}^{*}$. В случае $\mathbf{g} \in \overline{\mathbf{G}}^{*}$ имеем $\mathbf{g} \in \mathbf{H}$, так как иначе взяв элемент $\mathbf{g}^{\prime} \in \mathbf{G} \backslash \mathbf{H}$ с ранг $\mathbf{g}^{\prime}=$ ранг $\mathbf{G}$, получим $\mathbf{g g}^{\prime} \in \mathbf{H}$ и в силу (15.1) ранг $\mathbf{g g}^{\prime}=$ ранг $\mathbf{G}$ вопреки предположению. Следовательно, $\psi(\mathbf{g})=\mathbf{g} \in \overline{\mathbf{G}}^{*}$, и тогда ранг $\psi(\mathbf{g})<$ ранг $\mathbf{G}$. Наконец, если $\mathbf{g} \in \mathbf{c}^{n / 2} \overline{\mathbf{G}}^{*}$, то в этом случае $\mathbf{g} \in \mathbf{G} \backslash \mathbf{H}$ ввиду ранг $\mathbf{g}=$ ранг $\mathbf{G}$, и, следовательно, $\psi(\mathbf{g}) \in \overline{\mathbf{G}}^{*}$, откуда вытекает ранг $\psi(\mathbf{g})<$ ранг $\mathbf{G}$.

15.6. Лемма. Пусть $\mathbf{G}-$ произвольная группа $u \mathbf{G}=\langle\mathbf{K}, \mathbf{a}, \mathbf{b}\rangle$, где $\mathbf{K} \unlhd \mathbf{G}$, $|\langle\mathbf{K}, \mathbf{a}\rangle: \mathbf{K}| \leqslant 2, \quad|\langle\mathbf{K}, \mathbf{b}\rangle: \mathbf{K}| \leqslant 2$ и әлемент $\mathbf{a b}$ имеет порядок $n$ или $\infty$. Пусть $\mathbf{H}$ - некоторая группа периода $n$, и пусть гомоморфизмы $\phi:\langle\mathbf{K}, \mathbf{a}\rangle \rightarrow \mathbf{H}$ $u \psi:\langle\mathbf{K}, \mathbf{b}\rangle \rightarrow \mathbf{H}$ таковы, что $\left.\phi\right|_{\mathbf{K}}=\left.\psi\right|_{\mathbf{K}}$. Тогда $\phi$ и $\psi$ продолжаются до гомоморфизма группь $\mathbf{G}$ в $\mathbf{H}$, т.е. существует гомоморфизм $\omega: \mathbf{G} \rightarrow \mathbf{H}$ maкой, что $\left.\omega\right|_{\langle\mathbf{K}, \mathbf{a}\rangle}=\left.\phi u \omega\right|_{\langle\mathbf{K}, \mathbf{b}\rangle}=\psi$.

ДоКАЗАТЕЛЬСТво. Достаточно рассмотреть случай, когда $|\langle\mathbf{K}, \mathbf{a}\rangle: \mathbf{K}|=2$ и $|\langle\mathbf{K}, \mathbf{b}\rangle: \mathbf{K}|=2$. Предположим сначала, что $\mathbf{a b}$ имеет порядок $n$. Пусть $\mathbf{F}=\langle\overline{\mathbf{a}}, \overline{\mathbf{b}}\rangle$ - свободная группа ранга 2. Рассмотрим эпиморфизм $\pi$ свободного произведения $\mathbf{K} * \mathbf{F}$ на $\mathbf{G}$, определенньй равенствами $\left.\pi\right|_{\mathbf{K}}=\mathrm{id}, \pi(\overline{\mathbf{a}})=\mathbf{a}$ и $\pi(\overline{\mathbf{b}})=\mathbf{b}$. Мы утверждаем, что $\operatorname{ker} \pi$ есть нормальное замыкание в $\mathbf{K} * \mathbf{F}$ элементов

$$
\begin{gathered}
\overline{\mathbf{a}}^{2} \mathbf{a}^{-2}, \quad \overline{\mathbf{b}}^{2} \mathbf{b}^{-2}, \quad(\overline{\mathbf{a}} \overline{\mathbf{b}})^{n}, \quad \overline{\mathbf{a}}^{-1} \mathbf{g} \overline{\mathbf{a}}^{-1} \mathbf{g}^{-1} \mathbf{a} \quad(\mathbf{g} \in \mathbf{K}) \\
\text { и } \overline{\mathbf{b}}^{-1} \mathbf{g} \overline{\mathbf{b}} \mathbf{b}^{-1} \mathbf{g}^{-1} \mathbf{b} \quad(\mathbf{g} \in \mathbf{K}) .
\end{gathered}
$$

Для доказательства этого факта обозначим через $\mathbf{N}$ нормальное замыкание элементов (15.2) в $\mathbf{K} * \mathbf{F}$. Очевидно, $\mathbf{N} \subseteq \operatorname{ker} \pi$. С учетом $\mathbf{K} \unlhd \mathbf{G}$ для любого $\mathbf{g} \in\left\{\mathbf{a}, \mathbf{b}, \mathbf{a}^{-1}, \mathbf{b}^{-1}\right\}$ имеем $\mathbf{g}^{-1} \mathbf{K g} \subseteq(\pi(\mathbf{g}))^{-1} \mathbf{K} \pi(\mathbf{g}) \mathbf{N}=\mathbf{K N}$, следовательно, $\mathbf{K N} \unlhd \mathbf{K} * \mathbf{F}$. Более того, из вида элементов (15.2) следует, что $\mathbf{K} * \mathbf{F} / \mathbf{K N}$ есть диэдральная группа порядка $2 n$. С другой стороны, из условия леммы следует, что $\mathbf{G} / \mathbf{K} \simeq \mathbf{K} * \mathbf{F} / \mathbf{K} \operatorname{ker} \pi$ - также диэдральная группа порядка $2 n$. Поэтому $\mathbf{K N}=\mathbf{K} \operatorname{ker} \pi$. Так как $\mathbf{K} \cap \operatorname{ker} \pi=1$, то отсюда следует $\mathbf{N}=\operatorname{ker} \pi$.

Теперь определим гомоморфизм $\bar{\omega}: \mathbf{K} * \mathbf{F} \rightarrow \mathbf{H}$ равенствами

$$
\left.\bar{\omega}\right|_{\mathbf{K}}=\left.\phi\right|_{\mathbf{K}}=\left.\psi\right|_{\mathbf{K}}, \quad \bar{\omega}(\overline{\mathbf{a}})=\phi(\mathbf{a}) \text { и } \bar{\omega}(\overline{\mathbf{b}})=\psi(\mathbf{b}) .
$$


Так как $\bar{\omega}(\mathbf{g})=1$ для любого элемента $\mathbf{g}$ из списка (15.2), то $\operatorname{ker} \pi \subseteq \operatorname{ker} \bar{\omega}$ и, таким образом, $\bar{\omega}$ индуцирует гомоморфизм $\omega: \mathbf{G} \rightarrow \mathbf{H}$, который, очевидно, является искомым.

В случае, когда $\mathbf{a b}$ имеет бесконечный порядок, рассуждения отличаются лишь тем, что в списке (15.2) будет отсутствовать элемент $(\overline{\mathbf{a}} \overline{\mathbf{b}})^{n}$ и в доказательстве равенства $\mathbf{K N}=\mathbf{K} \operatorname{ker} \pi$ роль диэдральной группы порядка $2 n$ играет бесконечная диэдральная группа $\mathbf{D}_{\infty}$ (при этом нужно использовать тот факт, что никакая нетривиальная факторгруппа группы $\mathbf{D}_{\infty}$ не изоморфна $\left.\mathbf{D}_{\infty}\right)$.

Теперь мы готовы доказать основную лемму.

15.7. Лемма. Пусть $\mathbf{G}$ - полная конечная подгруппа группь $\mathbf{B}_{\alpha}$, и пусть $\mathbf{H}$ - пополнение $\mathbf{G}^{*}$. Тогда существует тождественный на $\mathbf{G}^{*}$ гомоморфизм $\phi: \mathbf{G} \rightarrow \mathbf{H}$.

ДокаЗАТЕЛЬСтво. Так как ранг $\mathbf{H}<\alpha$ ввиду (15.1), то в силу предложения 13.7, (i) и индуктивного предположения 15.10 , (i) $\mathbf{H}$ - группа периода $n$. Пусть $\mathbf{c}-$ базисный элемент $\mathbf{G}$. Рассмотрим два случая.

Случай $1: \mathbf{G}^{-}=\varnothing$. Так как $\mathbf{G}=\mathbf{G}^{*} \lambda\langle\mathbf{c}\rangle$, то нам достаточно указать элемент $\phi(\mathbf{c}) \in \mathbf{H}$ такой, что $\mathbf{c}^{-1} \mathbf{g} \mathbf{c}=(\phi(\mathbf{c}))^{-1} \mathbf{g} \phi(\mathbf{c})$ для любого $\mathbf{g} \in \mathbf{G}^{*}$. Сушествование такого элемента $\phi(\mathbf{c})$ утверждается в предложении 15.3 .

Случай 2: $\mathbf{G}^{-} \neq \varnothing$. Выберем произвольньй $\mathbf{a} \in \mathbf{G}^{-}$. Тогда сушествование искомого гомоморфизма $\phi$ вытекает из леммы 15.5 и леммы 15.6 при $\mathbf{K}:=\mathbf{G}^{*}$, $\mathbf{a}:=\mathbf{a}$ и $\mathbf{b}:=\mathbf{a}^{-1} \mathbf{c}$.

15.8. ЛЕмма. Для любой полной конечной подгруппы $\mathbf{G} \subseteq \mathbf{B}_{\alpha}$ группа $\mathbf{G}^{*}$ является 2-группой.

ДоКАЗАТЕЛЬСТВО. Ввиду индукции и определения 7.13 достаточно проверить, что при $A \in \mathcal{E}_{\alpha}$ группа $\mathbf{N}_{\alpha-1}^{*}(A)$ является 2 -группой, а в силу леммы 7.2 , (iii) достаточно ограничиться рассмотрением случая $A \in \bar{\varepsilon}_{\alpha}$. Кроме того, в силу предложения 7.14 , (i) можно считать $\rho(A)>0$.

Сначала докажем, что

$$
\mathbf{N}_{\alpha-1}^{*}(A) \subseteq \pi_{\rho(A), \alpha-1}\left(\mathbf{N}_{\rho(A)}^{*}(A)\right)
$$

Пусть $\mathbf{g} \in \mathbf{N}_{\alpha-1}^{*}(A)$ - произвольньй элемент. Представим $\mathbf{g}$ словом $w \in \mathcal{H}_{\rho(A)}$ согласно лемме 13.2 , (i). Согласно определению 7.1 для некоторого $t>0$ выполнено $A^{t} \stackrel{\alpha=1}{=} w^{-1} A^{t} w$. По индуктивному предположению 11.3 имеем $A^{t} \stackrel{\rho(A)}{=} w^{-1} A^{t} w$ и, следовательно, $[w]_{\rho(A)} \in \mathbf{N}_{\rho(A)}(A)$. Если $[w]_{\rho(A)} \notin \mathbf{N}_{\rho(A)}^{*}(A)$, то по предложению 7.10 , (i) выполнено $w^{k} \stackrel{\rho(A)}{=} A^{r}$ для некоторых $k, r \neq 0$, откуда вытекает $w^{k} \stackrel{\alpha=1}{=} A^{r}$. Но $\mathbf{g}=[w]_{\alpha-1}-$ элемент конечного порядка в силу предложения 7.14 , (ii), в то время как $[A]_{\alpha-1}$ имеет бесконечньй порядок. Следовательно, $[w]_{\rho(A)} \in \mathbf{N}_{\rho(A)}^{*}(A)$ и тем самым для любого $\mathbf{g} \in \mathbf{N}_{\alpha-1}^{*}(A)$ мы указали элемент $\tilde{\mathbf{g}} \rightleftharpoons[w]_{\rho(A)} \in \mathbf{N}_{\rho(A)}^{*}(A)$, для которого $\pi_{\rho(A), \alpha-1}(\tilde{\mathbf{g}})=\mathbf{g}$, что и доказывает (15.3). 
В силу (15.3) достаточно проверить, что $\mathbf{N}_{\rho(A)}^{*}(A)-2$-группа. По предложению 7.14, (i) имеем $\mathbf{N}_{\rho(A)}(A) \subseteq \mathbf{x}^{-1} \mathbf{S}_{\rho(A)}(B) \mathbf{x}$ для некоторых $\mathbf{x} \in \mathbf{B}_{\rho(A)}$ и $B \in \mathcal{E}_{\rho(A)}$. Пусть $\mathbf{g} \in \mathbf{N}_{\rho(A)}^{*}(A)$ - произвольный элемент, и пусть $[A]_{\rho(A)}^{t}=$ $\mathbf{g}^{-1}[A]_{\rho(A)}^{t} \mathbf{g}$, где $t>0$. Если $\mathbf{x g} \mathbf{x}^{-1} \in \mathbf{S}_{\rho(A)}^{+}(B) \backslash \mathbf{S}_{\rho(A)}^{*}(B)$, то, применив индуктивное предположение 13.11 при $A:=B, \mathbf{g}:=\mathbf{h}:=\mathbf{x g x}^{-1}$ и $\mathbf{f}:=\mathbf{x}[A]_{\rho(A)}^{t} \mathbf{x}^{-1}$, получим $\mathbf{x}[A]_{\rho(A)}^{t} \mathbf{x}^{-1} \in \mathbf{S}_{\rho(A)}(B)$ вопреки бесконечности порядка $[A]_{\rho(A)}$. Следовательно, $\mathbf{x g x} \mathbf{x}^{-1} \in \mathbf{S}_{\rho(A)}^{*}(B) \cup \mathbf{S}_{\rho(A)}^{-}(B)$, и тогда $\mathbf{x g}^{2} \mathbf{x}^{-1} \in \mathbf{S}_{\rho(A)}^{*}(B)$. Так как по индуктивному предположению леммы $\mathbf{S}_{\rho(A)}^{*}(B)-2$-группа, то $\mathbf{g}^{2^{k}}=1$ для некоторого $k$, что и требовалось доказать.

15.9. ПРЕДЛОЖЕНИЕ. Любая конечная подгруппа группь $\mathbf{B}_{\alpha}$ изоморфно вкладывается в конечное прямое произведение вида $\mathbf{D}_{n} \times \mathbf{D}_{\bar{n}} \times \mathbf{D}_{\bar{n}} \times \cdots \times \mathbf{D}_{\bar{n}}$, əде $\bar{n}$ - наибольший делитель $n$ вида $2^{k}$.

ДокАЗАТЕльСтво. Пусть $\mathbf{G} \leqslant \mathbf{B}_{\alpha}-$ конечная подгруппа. В силу предложения 14.3 можно считать, что $\mathbf{G}$ - полная конечная подгруппа. Как было замечено в начале параграфа, факторгруппа $\mathbf{G} / \mathbf{G}^{*}$ является либо циклической группой $\mathbf{C}_{n}=\left\langle\mathbf{c} \mid \mathbf{c}^{n}=1\right\rangle$, либо диэдральной группой $\mathbf{D}_{n}=\left\langle\mathbf{a}, \mathbf{c} \mid \mathbf{a}^{2}=(\mathbf{a c})^{2}=\mathbf{c}^{n}=1\right\rangle$. Считая группу $\mathbf{C}_{n}$ вложенной в $\mathbf{D}_{n}$, получаем гомоморфизм $\pi: \mathbf{G} \rightarrow \mathbf{D}_{n} \mathrm{c}$ $\operatorname{ker} \pi=\mathbf{G}^{*}$. Выберем пополнение $\mathbf{H}$ подгруппы $\mathbf{G}^{*}$, и пусть $\phi: \mathbf{G} \rightarrow \mathbf{H}$ - тождественный на $\mathbf{G}^{*}$ гомоморфизм, построенньй по лемме 15.7. Мы имеем, таким образом, вложение

$$
\pi \times \phi: \mathbf{G} \rightarrow \mathbf{D}_{n} \times \mathbf{H}
$$

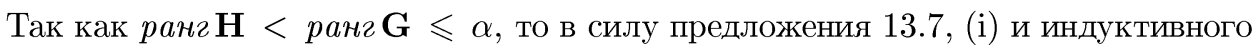
предположения существует вложение

$$
\lambda_{1} \times \lambda_{2} \times \cdots \times \lambda_{r}: \mathbf{H} \rightarrow \underbrace{\mathbf{D}_{n} \times \mathbf{D}_{\bar{n}} \times \mathbf{D}_{\bar{n}} \times \cdots \times \mathbf{D}_{\bar{n}}}_{r} .
$$

Пусть $n=\bar{n} t$ и $\rho: \mathbf{D}_{n} \rightarrow \mathbf{D}_{\bar{n}}$ - эпиморфизм с $\operatorname{ker} \rho=\left\langle\mathbf{c}^{t}\right\rangle$. Докажем, что гомоморфизм

$$
\pi \times \rho \lambda_{1} \phi \times \lambda_{2} \phi \times \cdots \times \lambda_{r} \phi: \mathbf{G} \rightarrow \underbrace{\mathbf{D}_{n} \times \mathbf{D}_{\bar{n}} \times \mathbf{D}_{\bar{n}} \times \cdots \times \mathbf{D}_{\bar{n}}}_{r+1}
$$

является вложением. Пусть

$$
\mathbf{g} \in \operatorname{ker}\left(\pi \times \rho \lambda_{1} \phi \times \lambda_{2} \phi \times \cdots \times \lambda_{r} \phi\right)=\operatorname{ker} \pi \cap \operatorname{ker} \rho \lambda_{1} \phi \cap \bigcap_{i=2}^{r} \operatorname{ker} \lambda_{i} \phi .
$$

Так как $\mathbf{g} \in \operatorname{ker} \pi=\mathbf{G}^{*}$, то по лемме 15.8 порядок элемента $\mathbf{g}$, а значит, и элемента $\lambda_{1} \phi(\mathbf{g})$, есть число вида $2^{k}$. С другой стороны, порядок любого элемента из ker $\rho$ есть нечетное число. Следовательно, $\lambda_{1} \phi(\mathbf{g})=1$, и, значит,

$$
\phi(\mathbf{g}) \in \bigcap_{i=1}^{r} \operatorname{ker} \lambda_{i}=\{1\}
$$

Отсюда вытекает $\mathbf{g}=1$, что и требовалось. 
15.10. ПРЕДЛОЖЕНИЕ. Любая конечная подгруппа группы $\mathbf{B}_{\alpha}$ удовлетворяет тождествам:

(i) $\mathbf{x}^{n}=1$;

(ii) $\left[\mathbf{x}^{n / 2}, \mathbf{y}\right]=1$;

(iii) $\left[\mathbf{x}^{2}, \mathbf{y}^{2}\right]=1$;

(iv) $\left[\mathrm{x}^{n / 4}, \mathbf{y}, \mathbf{z}\right]=1$;

(v) $\mathbf{x}^{-3} \mathbf{w} \mathbf{x}^{3}=\mathbf{x}^{-1} \mathbf{w} \mathbf{x} \cdot \mathbf{w}^{-1} \cdot \mathbf{x}^{-2} \mathbf{w} \mathbf{x}^{2}$.

ДоказАТеЛЬСтво. Так как группа $\mathbf{D}_{\bar{n}}$ есть гомоморфный образ групшы $\mathbf{D}_{n}$, то в силу предложения 15.9 достаточно проверить выполнение указанных тождеств на группе $\mathbf{D}_{n}=\left\langle\mathbf{a}, \mathbf{c} \mid \mathbf{a}^{2}=(\mathbf{a c})^{2}=\mathbf{c}^{n}=1\right\rangle$, что является несложным упражнением (тождество (iv) выполнено при $n$, кратном 8 , для остальных тождеств достаточно потребовать, чтобы $n$ было четным). Покажем, например, что для любых $\mathbf{x}, \mathbf{w} \in \mathbf{D}_{n}$ имеет место равенство (v). Если $\mathbf{x} \in \mathbf{D}_{n} \backslash\langle\mathbf{c}\rangle$, то $\mathbf{x}^{2}=1$, и в этом случае (v) преврашается в тривиальное равенство. Пусть $\mathbf{x} \in\langle\mathbf{c}\rangle$. Если $\mathbf{w} \in\langle\mathbf{c}\rangle$, то $\mathbf{x}^{-1} \mathbf{w} \mathbf{x}=\mathbf{w}$, и тогда равенство (v) снова становится тривиальным. Если же $\mathbf{w} \in \mathbf{D}_{n} \backslash\langle\mathbf{c}\rangle$, то имеет место равенство $\mathbf{w}^{-1} \mathbf{x w}=\mathbf{x}^{-1}$, и, следовательно, $\left[\mathbf{w}, \mathbf{x}^{t}\right]=\mathbf{w}^{-1} \mathbf{x}^{-t} \mathbf{w} \mathbf{x}^{t}=\mathbf{x}^{2 t}$. В частности, $\left[\mathbf{w}, \mathbf{x}^{3}\right]=[\mathbf{w}, \mathbf{x}]\left[\mathbf{w}, \mathbf{x}^{2}\right]$, откуда сокращением слева на $\mathbf{w}^{-1}$ получаем требуемое соотношение (v).

15.11. ПРЕДЛОЖЕНИЕ. Пусть $\mathbf{G} \leqslant \mathbf{B}_{\alpha}-$ конечная подгруппа $u \mathbf{x} \in \mathbf{B}_{\alpha}-$ әлемент бесконечного порядка, лежащий в нормализаторе $\mathbf{N}_{\mathbf{B}_{\alpha}}(\mathbf{G})$ подгруппь G. Тогда верны следующие утверждения:

(i) $(\mathbf{x g})^{n}=\mathbf{x}^{n} u \mathbf{x}^{n / 2} \mathbf{g}=\mathbf{g x}^{n / 2}$ для любого $\mathbf{g} \in \mathbf{G}$;

(ii) $e c \wedge u \mathbf{h} \in \mathbf{N}_{\mathbf{B}_{\alpha}}(\mathbf{G}) u \mathbf{h}^{2},(\mathbf{x h})^{2} \in \mathbf{G}, m o \mathbf{h}^{-1} \mathbf{x}^{n / 2} \mathbf{h}=\mathbf{x}^{-n / 2}$.

ДокАЗАТЕЛЬСтво. (i) Пусть $\overline{\mathbf{G}}$ - пополнение подгруппы G. По предложению 15.3 найдется $\mathbf{y} \in \overline{\mathbf{G}}$ такой, что $\mathbf{x}^{-1} \mathbf{g x}=\mathbf{y}^{-1} \mathbf{g y}$ для любого $\mathbf{g} \in \mathbf{G}$. Так как, очевидно, группа $\langle\mathbf{G}, \mathbf{x}\rangle$ является полупрямым произведением своей нормальной подгруппы $\mathbf{G}$ и бесконечной циклической группы $\langle\mathbf{x}\rangle$, то можно определить гомоморфизм $\phi:\langle\mathbf{G}, \mathbf{x}\rangle \rightarrow \overline{\mathbf{G}}$, полагая $\left.\phi\right|_{\mathbf{G}}=\mathrm{id}$ и $\phi(\mathbf{x})=\mathbf{y}$. Для любого $\mathbf{g} \in \mathbf{G}$ имеем $(\mathbf{x g})^{n} \mathbf{x}^{-n} \in \mathbf{G}$ и $\left[\mathbf{x}^{n / 2}, \mathbf{g}\right] \in \mathbf{G}$, следовательно, используя предложение 15.10, (i), (ii), получаем

$$
(\mathbf{x g})^{n} \mathbf{x}^{-n}=\phi\left((\mathbf{x g})^{n} \mathbf{x}^{-n}\right)=(\phi(\mathbf{x}) \mathbf{g})^{n}(\phi(\mathbf{x}))^{-n}=1
$$

и

$$
\left[\mathbf{x}^{n / 2}, \mathbf{g}\right]=\phi\left(\left[\mathbf{x}^{n / 2}, \mathbf{g}\right]\right)=\left[(\phi(\mathbf{x}))^{n / 2}, \mathbf{g}\right]=1 .
$$

(ii) Из посылки следует $|\langle\mathbf{G}, \mathbf{h}\rangle: \mathbf{G}| \leqslant 2$ и $|\langle\mathbf{G}, \mathbf{x h}\rangle: \mathbf{G}| \leqslant 2$. Тогда в силу бесконечности порядка элемента $\mathbf{x}^{-1}=\mathbf{h}(\mathbf{x h})^{-1}$, лемм $15.5,15.6$ и предложения 15.10 , (i) существует тождественный на $\mathbf{G}$ гомоморфизм $\omega:\langle\mathbf{G}, \mathbf{x}, \mathbf{h}\rangle \rightarrow \overline{\mathbf{G}}$, где $\overline{\mathbf{G}}$ - пополнение $\mathbf{G}$. Из $\mathbf{h}^{-1} \mathbf{x h \mathbf { x }}=\mathbf{h}^{-2}(\mathbf{h} \mathbf{x})^{2} \in \mathbf{G}$ и $\mathbf{G} \unlhd\langle\mathbf{G}, \mathbf{x}, \mathbf{h}\rangle$ следует $\mathbf{h}^{-1} \mathbf{x}^{n / 2} \mathbf{h} \mathbf{x}^{n / 2} \in \mathbf{G}$, поэтому, с учетом предложения 15.10 , (i), (ii),

$$
\mathbf{h}^{-1} \mathbf{x}^{n / 2} \mathbf{h} \mathbf{x}^{n / 2}=\omega\left(\mathbf{h}^{-1} \mathbf{x}^{n / 2} \mathbf{h} \mathbf{x}^{n / 2}\right)=\left[\omega(\mathbf{h}),(\omega(\mathbf{x}))^{-n / 2}\right](\omega(\mathbf{x}))^{n}=1 .
$$




\section{$\S 16$. Цепи сопряженных элементов в группе $\mathbf{B}_{\alpha}$}

Цель этого параграфа - доказательство следующего утверждения:

16.1. ПРЕДЛОЖЕНИЕ. Если конечная подгруппа $\mathbf{G} \leqslant \mathbf{B}_{\alpha}$ и элементы $\mathbf{x}, \mathbf{w} \in \mathbf{B}_{\alpha}$ таковы, что $\mathbf{x}^{-i} \mathbf{w} \mathbf{x}^{i} \in \mathbf{G}$ при $i=0,1, \ldots, 5$, mо $\mathbf{x}^{-i} \mathbf{w} \mathbf{x}^{i} \in \mathbf{G}$ при любом $i$.

16.2. ОПРЕДЕЛЕНИЕ. Пусть $\overline{\mathbf{w}}=\left(\mathbf{w}_{1}, \mathbf{w}_{2}, \ldots, \mathbf{w}_{\ell}\right)-$ конечная последовательность элементов группы $\mathbf{B}_{\alpha}$. Свяжем с $\overline{\mathbf{w}}$ следующие подгрупшы:

$$
\langle\overline{\mathbf{w}}\rangle \rightleftharpoons\left\langle\mathbf{w}_{1}, \mathbf{w}_{2}, \ldots, \mathbf{w}_{\ell}\right\rangle,
$$

$\mathbf{M}(\overline{\mathbf{w}}) \rightleftharpoons$ нормальное замыкание $\mathbf{w}_{1}^{2}, \mathbf{w}_{2}, \mathbf{w}_{3}, \ldots, \mathbf{w}_{\ell-1}, \mathbf{w}_{\ell}^{2} \mathbf{\text { в }}\langle\overline{\mathbf{w}}\rangle$,

$$
\begin{aligned}
& \mathbf{L}(\overline{\mathbf{w}}) \rightleftharpoons\left\langle\mathbf{M}(\overline{\mathbf{w}}), \mathbf{w}_{1}\right\rangle, \\
& \mathbf{R}(\overline{\mathbf{w}}) \rightleftharpoons\left\langle\mathbf{M}(\overline{\mathbf{w}}), \mathbf{w}_{\ell}\right\rangle .
\end{aligned}
$$

Очевидно, $|\mathbf{L}(\overline{\mathbf{w}}): \mathbf{M}(\overline{\mathbf{w}})| \leqslant 2,|\mathbf{R}(\overline{\mathbf{w}}): \mathbf{M}(\overline{\mathbf{w}})| \leqslant 2$ и $\langle\overline{\mathbf{w}}\rangle=\langle\mathbf{L}(\overline{\mathbf{w}}), \mathbf{R}(\overline{\mathbf{w}})\rangle=$ $\left\langle\mathbf{M}(\overline{\mathbf{w}}), \mathbf{w}_{1}, \mathbf{w}_{\ell}\right\rangle$.

16.3. ОПРЕДЕЛЕНИЕ. Конечную последовательность $\overline{\mathbf{w}}=\left(\mathbf{w}_{1}, \mathbf{w}_{2}, \ldots, \mathbf{w}_{\ell}\right)$ элементов группы $\mathbf{B}_{\alpha}$ назовем иепью, если для некоторого $\mathbf{x} \in \mathbf{B}_{\alpha}$ выполнено

$$
\mathbf{x}^{-1} \mathbf{w}_{i} \mathbf{x}=\mathbf{w}_{i+1} \quad(i=1,2, \ldots, \ell-1)
$$

и подгруппа $\mathbf{M}(\overline{\mathbf{w}})$ конечна. Элемент $\mathbf{x}$, удовлетворяюший равенствам (16.1), будем называть сопрягающим әлементом цепи $\overline{\mathbf{w}}$.

Если элементы $\mathbf{x}$ и $\mathbf{w}$ удовлетворяют посылке предложения 16.1, то последовательность $\overline{\mathbf{w}}=\left(\mathbf{w}, \mathbf{x}^{-1} \mathbf{w} \mathbf{x}, \ldots, \mathbf{x}^{-5} \mathbf{w} \mathbf{x}^{5}\right)$ является цепью длины 6 . На первый взгляд, использование этого понятия для доказательства предложения 16.1 кажется неудобным, так как в нем не участвует явно элемент х. Однако мы сведем сейчас условие заключения предложения 16.1 к некоторому свойству элементов из последовательности $\overline{\mathbf{w}}$, не зависящему от $\mathbf{x}$.

16.4. Лемма. Пусть $\overline{\mathbf{w}}=\left(\mathbf{w}_{1}, \mathbf{w}_{2}, \ldots, \mathbf{w}_{\ell}\right)$ - цепь длиньь $\ell \geqslant 4 u \mathbf{x}-$ conрягающий әлемент $\overline{\mathbf{w}}$. Тогда следующие утверждения әквивалентны:

(i) группа $\langle\overline{\mathbf{w}}\rangle$ конечна $u \mathbf{x}^{-i} \mathbf{w} \mathbf{x}^{i} \in\langle\overline{\mathbf{w}}\rangle$ при любом $i$;

(ii) $\mathbf{w}_{i+3}=\mathbf{w}_{i+1} \mathbf{w}_{i}^{-1} \mathbf{w}_{i+2}$ для некоторого (а значит, и для любого) $i$ при $1 \leqslant i \leqslant \ell-3$.

ДоказАтельство. (i) $\Rightarrow$ (ii): Выберем пополнение $\mathbf{G}$ подгруппы $\langle\overline{\mathbf{w}}\rangle$. Так как $\mathbf{x}^{-1}\langle\overline{\mathbf{w}}\rangle \mathbf{x}=\langle\overline{\mathbf{w}}\rangle$, то по предложению 15.3 найдется $\mathbf{y} \in \mathbf{G}$ такой, что $\mathbf{x}^{-1} \mathbf{g} \mathbf{x}=$ $\mathbf{y}^{-1} \mathbf{g y}$ для любого $\mathbf{g} \in\langle\overline{\mathbf{w}}\rangle$. Тогда по предложению $15.10,(\mathrm{v})$ имеем $\mathbf{y}^{-3} \mathbf{w}_{1} \mathbf{y}^{3}=$ $\mathbf{y}^{-1} \mathbf{w}_{1} \mathbf{y} \cdot \mathbf{w}_{1}^{-1} \cdot \mathbf{y}^{-2} \mathbf{w}_{1} \mathbf{y}^{2}$, т.е. $\mathbf{w}_{4}=\mathbf{w}_{2} \mathbf{w}_{1}^{-1} \mathbf{w}_{3}$.

(ii) $\Rightarrow$ (i): Обозначим $\mathbf{w}_{i} \rightleftharpoons \mathbf{x}^{-i+1} \mathbf{w}_{1} \mathbf{x}^{i-1}$ для любого $i \in \mathbb{Z}$. Сопрягая равенство $\mathbf{w}_{i+3}=\mathbf{w}_{i+1} \mathbf{w}_{i}^{-1} \mathbf{w}_{i+2}$ всевозможными степенями $\mathbf{x}$, получаем, что при любом $i \in \mathbb{Z}$ подгруппа $\left\langle\mathbf{w}_{i}, \mathbf{w}_{i+1}, \mathbf{w}_{i+2}\right\rangle$ содержит $\mathbf{w}_{i-1} \mathbf{u}_{i+3}$. Отсюда следует

$$
\left\langle\mathbf{w}_{i}\right\rangle_{i \in \mathbb{Z}} \subseteq\left\langle\mathbf{w}_{2}, \mathbf{w}_{3}, \mathbf{w}_{4}\right\rangle \subseteq \mathbf{R}(\overline{\mathbf{w}}) .
$$

Остается заметить, что из конечности $\mathbf{M}(\overline{\mathbf{w}})$ вытекает конечность $\mathbf{R}(\overline{\mathbf{w}})$. 
16.5. ОПРЕДЕЛЕНИЕ. Цепь $\overline{\mathbf{w}}=\left(\mathbf{w}_{1}, \mathbf{w}_{2}, \ldots, \mathbf{w}_{\ell}\right)$ длины $\ell \geqslant 4$ назовем $p e-$ гулярной, если для некоторого (или, эквивалентно, для любого) $1 \leqslant i \leqslant \ell-3$ выполнено $\mathbf{w}_{i+3}=\mathbf{w}_{i+1} \mathbf{w}_{i}^{-1} \mathbf{w}_{i+2}$.

Ввиду леммы 16.4 предложение 16.1 вытекает из следующего утверждения.

16.6. ПРЕДЛОЖЕНИЕ. Любая цепь $\overline{\mathbf{w}}=\left(\mathbf{w}_{1}, \mathbf{w}_{2}, \ldots, \mathbf{w}_{\ell}\right)$ длины $\ell \geqslant 6$ регулярна.

Доказательству предложения 16.6 посвяшена оставшаяся часть параграфа. Мы будем использовать индукцию, шаг которой состоит в сведении контрпримера к предложению 16.6 к более простому контрпримеру. Роль параметра индукции будет играть число ранг $\mathbf{L}(\overline{\mathbf{w}})+$ ранг $\mathbf{R}(\overline{\mathbf{w}})$. Заметим, что если последовательность $\overline{\mathbf{w}}=\left(\mathbf{w}_{1}, \mathbf{w}_{2}, \ldots, \mathbf{w}_{\ell}\right)$ является цепю, то любая ее подпоследовательность $\overline{\mathbf{v}}=\left(\mathbf{w}_{r}, \mathbf{w}_{r+1}, \ldots, \mathbf{w}_{r+t}\right)$ также является цепью, причем если $\overline{\mathbf{v}}$ имеет длину $t+1 \geqslant 4$, то $\overline{\mathbf{w}}$ регулярна тогда и только тогда, когда регулярна $\overline{\mathbf{v}}$. Наша задача будет состоять в том, чтобы для нерегулярной цепи $\overline{\mathbf{w}}$ длины $\ell \geqslant 6$ построить новую цепь $\overline{\mathbf{w}}^{\prime}$ той же длины, отличаюшуюся от $\overline{\mathbf{w}}$ лишь элементами $\mathbf{w}_{1}$ и $\mathbf{w}_{\ell}$, но с меньшим параметром ранг $\mathbf{L}(\overline{\mathbf{w}})+$ ранг $\mathbf{R}(\overline{\mathbf{w}})$.

Заметим, что при выводе импликации (i) $\Rightarrow$ (ii) в доказательстве леммы 16.4 фактически доказана

16.7. Лемма. Если $\overline{\mathbf{w}}=\left(\mathbf{w}_{1}, \mathbf{w}_{2}, \ldots, \mathbf{w}_{\ell}\right)$ - цепь длиньь $\ell \geqslant 4$ и для некоторого сопрягающего әлемента $\mathbf{x}$ цепи $\overline{\mathbf{w}}$ подгруппа $\langle\overline{\mathbf{w}}, \mathbf{x}\rangle$ конечна, то щепь $\overline{\mathbf{w}}$ регулярна.

16.8. Лемма. Пусть $\overline{\mathbf{w}}=\left(\mathbf{w}_{1}, \mathbf{w}_{2}, \ldots, \mathbf{w}_{\ell}\right)-$ цепь длиньь $\ell \geqslant 4$

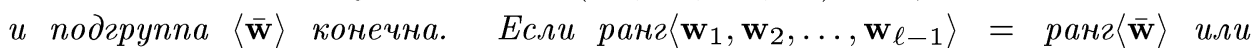
ранг $\left\langle\mathbf{w}_{2}, \mathbf{w}_{3}, \ldots, \mathbf{w}_{\ell}\right\rangle=$ ранг $\langle\overline{\mathbf{w}}\rangle$, то $\overline{\mathbf{w}}-$ регулярная цепь.

ДоКАЗАТЕЛЬСТво. Обозначим $\mathbf{H} \rightleftharpoons\left\langle\mathbf{w}_{1}, \mathbf{w}_{2}, \ldots, \mathbf{w}_{\ell-1}\right\rangle$ и $\mathbf{K} \rightleftharpoons\left\langle\mathbf{w}_{2}, \mathbf{w}_{3}, \ldots\right.$ $\left.\ldots, \mathbf{w}_{\ell}\right\rangle$. Предположим, ранг $\mathbf{H}=\operatorname{paн}\langle\overline{\mathbf{w}}\rangle$ или $р а н г \mathbf{K}=$ ранг $\langle\overline{\mathbf{w}}\rangle$. Выберем пополнение $\mathbf{G}$ подгруппы $\langle\overline{\mathbf{w}}\rangle$, и пусть $\mathbf{x}$ - сопрягаюший элемент цепи $\overline{\mathbf{w}}$. Так как $\mathbf{x}^{-1} \mathbf{H x}=\mathbf{K}$, то по предложению 15.3 найдется $\mathbf{y} \in \mathbf{G}$ такой, что $\mathbf{x}^{-1} \mathbf{g x}=\mathbf{y}^{-1} \mathbf{g y}$ для любого $\mathbf{g} \in \mathbf{H}$. Тогда $\mathbf{y}$ - также сопрягающий элемент цепи $\overline{\mathbf{w}}$, и нам остается воспользоваться леммой 16.7.

16.9. Лемма. Пусть $\overline{\mathbf{w}}=\left(\mathbf{w}_{1}, \mathbf{w}_{2}, \ldots, \mathbf{w}_{\ell}\right)$ - нерегулярная чепь длинь $\ell \geqslant$ 4 , подгруппа $\langle\overline{\mathbf{w}}\rangle$ конечна $и \mathbf{G}-$ ее пополнение. Тогда $\mathbf{w}_{1}, \mathbf{w}_{\ell} \in \mathbf{G}^{-}, \mathbf{w}_{1} \mathbf{w}_{\ell} \in$ $\mathbf{G}^{+} \backslash \mathbf{G}^{*} u \mathbf{w}_{2}, \mathbf{w}_{3}, \ldots, \mathbf{w}_{\ell-1} \in \mathbf{G}^{*}$.

ДокАЗАТЕЛЬСТво. По лемме 16.8

$$
\operatorname{paн2}\left\langle\mathbf{w}_{1}, \mathbf{w}_{2}, \ldots, \mathbf{w}_{\ell-1}\right\rangle\left\langle\operatorname{paн}\langle\overline{\mathbf{w}}\rangle \text { и } \quad \operatorname{paнг}\left\langle\mathbf{w}_{2}, \mathbf{w}_{3}, \ldots, \mathbf{w}_{\ell}\right\rangle\langle\operatorname{paнг}\langle\overline{\mathbf{w}}\rangle .\right.
$$

В частности, для любого $i$ имеем ранг $\mathbf{w}_{i}<$ ранг $\langle\overline{\mathbf{w}}\rangle$, ив силу (15.1) $\mathbf{w}_{i} \in \mathbf{G}^{*} \cup \mathbf{G}^{-}$. Если $\mathbf{w}_{1} \in \mathbf{G}^{*}$, то $\langle\overline{\mathbf{w}}\rangle \subseteq\left\langle\mathbf{w}_{2}, \mathbf{w}_{3}, \ldots, \mathbf{w}_{\ell}\right\rangle \mathbf{G}^{*}$ вопреки второму неравенству (16.2) 
и лемме $15.4,(\mathrm{i})$. Следовательно, $\mathbf{w}_{1} \in \mathbf{G}^{-}$. Аналогично, $\mathbf{w}_{\ell} \in \mathbf{G}^{-}$. Тогда по лемме 15.4, (ii)

$$
\left\langle\mathbf{w}_{1}, \mathbf{w}_{2}, \ldots, \mathbf{w}_{\ell-1}\right\rangle \subseteq\left\langle\mathbf{G}^{*}, \mathbf{w}_{1}\right\rangle \text { и }\left\langle\mathbf{w}_{2}, \mathbf{w}_{3}, \ldots, \mathbf{w}_{\ell}\right\rangle \subseteq\left\langle\mathbf{G}^{*}, \mathbf{w}_{\ell}\right\rangle,
$$

и, следовательно, $\langle\overline{\mathbf{w}}\rangle \subseteq\left\langle\mathbf{G}^{*}, \mathbf{w}_{1}, \mathbf{w}_{\ell}\right\rangle$. Так как в силу (15.1) подгруппа $\langle\overline{\mathbf{w}}\rangle$ содержит элемент из $\mathbf{G}^{+} \backslash \mathbf{G}^{*}$, то $\mathbf{w}_{1} \mathbf{w}_{\ell} \notin \mathbf{G}^{*}$, т.е. $\mathbf{w}_{1} \mathbf{w}_{\ell} \in \mathbf{G}^{+} \backslash \mathbf{G}^{*}$. Наконец, если бы при каком-нибудь $2 \leqslant i \leqslant \ell-1$ вьполнялось $\mathbf{w}_{i} \in \mathbf{G}^{-}$, то один из элементов $\mathbf{w}_{1} \mathbf{w}_{i}$ или $\mathbf{w}_{\ell} \mathbf{w}_{i}$ лежал бы в $\mathbf{G}^{+} \backslash \mathbf{G}^{*}$ вопреки (16.2).

16.10. ЛЕМма. Пусть $\overline{\mathbf{w}}=\left(\mathbf{w}_{1}, \mathbf{w}_{2}, \ldots, \mathbf{w}_{\ell}\right)-$ uenb.

(i) Пусть $\phi: \mathbf{L}(\overline{\mathbf{w}}) \rightarrow \mathbf{B}_{\alpha}-$ гомоморфизм такой, ито $\left.\phi\right|_{\mathbf{M}(\overline{\mathbf{w}})}=\mathrm{id} u \phi\left(\mathbf{w}_{1}\right)=$ $\mathbf{y}^{-1} \mathbf{w}_{1} \mathbf{y}$ для некоторого $\mathbf{y} \in \mathbf{B}_{\alpha}$, коммутирующего $c \mathbf{w}_{2}, \mathbf{w}_{3}, \ldots, \mathbf{w}_{\ell-1}$. Тогда последовательность $\overline{\mathbf{w}}^{\prime} \rightleftharpoons\left(\phi\left(\mathbf{w}_{1}\right), \mathbf{w}_{2}, \ldots, \mathbf{w}_{\ell}\right)$ является цепью, причем $\mathbf{M}\left(\overline{\mathbf{w}}^{\prime}\right)=\mathbf{M}(\overline{\mathbf{w}}), \mathbf{L}\left(\overline{\mathbf{w}}^{\prime}\right)=\phi(\mathbf{L}(\overline{\mathbf{w}})) u \mathbf{R}\left(\overline{\mathbf{w}}^{\prime}\right)=\mathbf{R}(\overline{\mathbf{w}})$.

(ii) Пусть $\psi: \mathbf{R}(\overline{\mathbf{w}}) \rightarrow \mathbf{B}_{\alpha}-$ гомоморфизм такой, что $\psi||_{\mathbf{M}(\overline{\mathbf{w}})}=$ id $u \psi\left(\mathbf{w}_{\ell}\right)=\mathbf{y}^{-1} \mathbf{w}_{\ell} \mathbf{y}$ для некоторого $\mathbf{y} \in \mathbf{B}_{\alpha}$, коммутирующего $c$ $\mathbf{w}_{2}, \mathbf{w}_{3}, \ldots, \mathbf{w}_{\ell-1}$. Тогда последовательность $\overline{\mathbf{w}}^{\prime \prime} \rightleftharpoons\left(\mathbf{w}_{1}, \mathbf{w}_{2}, \ldots, \psi\left(\mathbf{w}_{\ell}\right)\right)$ является цепью, причем $\mathbf{M}\left(\overline{\mathbf{w}}^{\prime \prime}\right)=\mathbf{M}(\overline{\mathbf{w}}), \mathbf{L}\left(\overline{\mathbf{w}}^{\prime \prime}\right)=\mathbf{L}(\overline{\mathbf{w}})$ u $\mathbf{R}\left(\overline{\mathbf{w}}^{\prime \prime}\right)=\psi(\mathbf{R}(\overline{\mathbf{w}}))$.

ДокАЗАТЕЛЬСТво. Утверждения (i) и (ii) симметричны относительно замены порядка элементов $\mathbf{w}_{i}$ на обратный. Ограничимся доказательством утверждения (i).

Так как $\left.\phi\right|_{\mathbf{M}(\overline{\mathbf{w}})}=\mathrm{id}$ и $\mathbf{w}_{1}^{2} \in \mathbf{M}(\overline{\mathbf{w}})$, то

$$
\left(\phi\left(\mathbf{w}_{1}\right)\right)^{2}=\phi\left(\mathbf{w}_{1}^{2}\right)=\mathbf{w}_{1}^{2},
$$

и так как $\mathbf{w}_{1}^{-1} \mathbf{M}(\overline{\mathbf{w}}) \mathbf{w}_{1}=\mathbf{M}(\overline{\mathbf{w}})$, то для любого $\mathbf{g} \in \mathbf{M}(\overline{\mathbf{w}})$

$$
\left(\phi\left(\mathbf{w}_{1}\right)\right)^{-\epsilon} \mathbf{g}\left(\phi\left(\mathbf{w}_{1}\right)\right)^{\epsilon}=\phi\left(\mathbf{w}_{1}^{-\epsilon} \mathbf{g} \mathbf{w}_{1}^{\epsilon}\right)=\mathbf{w}_{1}^{-\epsilon} \mathbf{g} \mathbf{w}_{1}^{\epsilon} \quad(\epsilon= \pm 1) .
$$

Тогда из $\mathbf{M}(\overline{\mathbf{w}}) \unlhd\langle\overline{\mathbf{w}}\rangle$ вытекает $\mathbf{M}(\overline{\mathbf{w}}) \unlhd\left\langle\overline{\mathbf{w}}^{\prime}\right\rangle$, и так как элементы $\left(\phi\left(\mathbf{w}_{1}\right)\right)^{2}$, $\mathbf{w}_{2}, \mathbf{w}_{3}, \ldots, \mathbf{w}_{\ell-1}, \mathbf{w}_{\ell}^{2}$ лежат в $\mathbf{M}(\overline{\mathbf{w}})$, то $\mathbf{M}\left(\overline{\mathbf{w}}^{\prime}\right) \subseteq \mathbf{M}(\overline{\mathbf{w}})$. С другой стороны, из (16.3) и $\mathbf{M}\left(\overline{\mathbf{w}}^{\prime}\right) \unlhd\left\langle\overline{\mathbf{w}}^{\prime}\right\rangle$ вытекает $\mathbf{M}\left(\overline{\mathbf{w}}^{\prime}\right) \unlhd\langle\overline{\mathbf{w}}\rangle$, и так как элементы $\mathbf{w}_{1}^{2}, \mathbf{w}_{2}, \mathbf{w}_{3}, \ldots, \mathbf{w}_{\ell-1}, \mathbf{w}_{\ell}^{2}$ лежат в $\mathbf{M}\left(\overline{\mathbf{w}}^{\prime}\right)$, то $\mathbf{M}(\overline{\mathbf{w}}) \subseteq \mathbf{M}\left(\overline{\mathbf{w}}^{\prime}\right)$. Следовательно, $\mathbf{M}(\overline{\mathbf{w}})=\mathbf{M}\left(\overline{\mathbf{w}}^{\prime}\right)$. Отсюда вытекает $\mathbf{R}\left(\overline{\mathbf{w}}^{\prime}\right)=\mathbf{R}(\overline{\mathbf{w}})$ и $\mathbf{L}\left(\overline{\mathbf{w}}^{\prime}\right)=$ $\left\langle\mathbf{M}\left(\overline{\mathbf{w}}^{\prime}\right), \phi\left(\mathbf{w}_{1}\right)\right\rangle=\phi\left(\left\langle\mathbf{M}\left(\overline{\mathbf{w}}^{\prime}\right), \mathbf{w}_{1}\right\rangle\right)=\phi(\mathbf{L}(\overline{\mathbf{w}}))$. Остается заметить, что из равенств $\phi\left(\mathbf{w}_{1}\right)=\mathbf{y}^{-1} \mathbf{w}_{1} \mathbf{y}, \mathbf{y}^{-1} \mathbf{w}_{i} \mathbf{y}=\mathbf{w}_{i}(i=2,3, \ldots, \ell-1)$ и $\mathbf{x}^{-1} \mathbf{w}_{i} \mathbf{x}=\mathbf{w}_{i+1}$ $(i=1,2, \ldots, \ell-1)$ следует $\left(\mathbf{y}^{-1} \mathbf{x}\right)^{-1} \phi\left(\mathbf{w}_{1}\right) \mathbf{y}^{-1} \mathbf{x}=\mathbf{w}_{2}$ и $\left(\mathbf{y}^{-1} \mathbf{x}\right)^{-1} \mathbf{w}_{i} \mathbf{y}^{-1} \mathbf{x}=\mathbf{w}_{i+1}$ $(i=2,3, \ldots, \ell-1)$, и, таким образом, согласно определению 16.3 последовательность $\overline{\mathbf{w}}^{\prime}$ является цепью.

16.11. Лемма. Цепь $\overline{\mathbf{w}}=\left(\mathbf{w}_{1}, \mathbf{w}_{2}, \ldots, \mathbf{w}_{\ell}\right)$ длины $\ell \geqslant 5$ регулярна тогда и только тогда, когда ранг $\mathbf{L}(\overline{\mathbf{w}})=$ ранг $\mathbf{R}(\overline{\mathbf{w}})=\operatorname{ранг~} \mathbf{M}(\overline{\mathbf{w}})$. 
ДоКАЗАТЕЛЬСТВО. Если $\overline{\mathbf{w}}-$ регулярная цепь длины $\ell \geqslant 5$, то согласно определению 16.5 имеем $\langle\overline{\mathbf{w}}\rangle=\left\langle\mathbf{w}_{2}, \mathbf{w}_{3}, \mathbf{w}_{4}\right\rangle$ и, следовательно, $\mathbf{L}(\overline{\mathbf{w}})=\mathbf{R}(\overline{\mathbf{w}})=\mathbf{M}(\overline{\mathbf{w}})$.

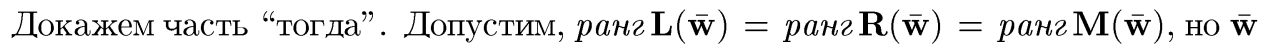
не является регулярной. Выберем пополнения $\mathbf{G}$ и $\mathbf{H}$ подгрупп $\mathbf{L}(\overline{\mathbf{w}})$ и $\mathbf{R}(\overline{\mathbf{w}})$ соответственно. Так как $\mathbf{G}$ и $\mathbf{H}$ являются также пополнениями $\mathbf{M}(\overline{\mathbf{w}})$, то по предложению 14.2 имеем $\mathbf{H}=\mathbf{z}^{-1} \mathbf{G z}$ для некоторого $\mathbf{z}$, коммутирующего со всеми элементами из $\mathbf{M}(\overline{\mathbf{w}})$. Рассмотрим вложение $\phi: \mathbf{L}(\overline{\mathbf{w}}) \rightarrow \mathbf{B}_{\alpha}$, определенное равенством $\phi(\mathbf{g})=\mathbf{z}^{-1} \mathbf{g z}$. По лемме 16.10 последовательность $\overline{\mathbf{w}}^{\prime} \rightleftharpoons\left(\phi\left(\mathbf{w}_{1}\right), \mathbf{w}_{2}, \ldots, \mathbf{w}_{\ell}\right)$ является цепю, для которой $\mathbf{M}\left(\overline{\mathbf{w}}^{\prime}\right)=\mathbf{M}(\overline{\mathbf{w}}), \mathbf{L}\left(\overline{\mathbf{w}}^{\prime}\right)=\mathbf{z}^{-1} \mathbf{L}(\overline{\mathbf{w}}) \mathbf{z} \subseteq \mathbf{H}$ и $\mathbf{R}\left(\overline{\mathbf{w}}^{\prime}\right)=\mathbf{R}(\overline{\mathbf{w}}) \subseteq \mathbf{H}$. Так как $\langle\overline{\mathbf{w}}\rangle=\langle\mathbf{L}(\overline{\mathbf{w}}), \mathbf{R}(\overline{\mathbf{w}})\rangle$, то подгруппа $\langle\overline{\mathbf{w}}\rangle$ конечна и $\mathbf{H}$ - ее пополнение. Тогда по лемме 16.9 с учетом включения $\left(\mathbf{H}^{-}\right)^{2} \subseteq \mathbf{H}^{*}$ получаем $\left(\phi\left(\mathbf{w}_{1}\right)\right)^{2}, \mathbf{w}_{2}, \ldots, \mathbf{w}_{\ell-1}, \mathbf{w}_{\ell}^{2} \in \mathbf{H}^{*}$, откуда ввиду $\mathbf{H}^{*} \unlhd \mathbf{H}$ следует $\mathbf{M}\left(\overline{\mathbf{w}}^{\prime}\right) \subseteq \mathbf{H}^{*}$. Но это противоречит равенству ранг $\mathbf{M}\left(\overline{\mathbf{w}}^{\prime}\right)=$ ранг $\mathbf{M}(\overline{\mathbf{w}})=$ ранг $\mathbf{H}$. Следовательно, $\overline{\mathbf{w}}$ - регулярная цепь.

16.12. ЛЕмма. Пусть $\overline{\mathbf{w}}-$ цепь длины $\ell \geqslant 5$. Тогда:

(i) если ранг $\mathbf{L}(\overline{\mathbf{w}})>$ ранг $\mathbf{M}(\overline{\mathbf{w}})$ и $\mathbf{G}$ есть пополнение погруппь $\mathbf{L}(\overline{\mathbf{w}})$, то $\mathbf{M}(\overline{\mathbf{w}}) \cap \mathbf{G}^{-} \neq \varnothing ;$

(i) если ранг $\mathbf{R}(\overline{\mathbf{w}})>$ ранг $\mathbf{M}(\overline{\mathbf{w}})$ и $\mathbf{H}$ есть пополнение погруппы $\mathbf{R}(\overline{\mathbf{w}})$, то $\mathbf{M}(\overline{\mathbf{w}}) \cap \mathbf{H}^{-} \neq \varnothing$.

ДокАЗАТЕЛЬСТво. В силу симметрии ограничимся доказательством утверждения (i). Пусть $\overline{\mathbf{w}}=\left(\mathbf{w}_{1}, \mathbf{w}_{2}, \ldots, \mathbf{w}_{\ell}\right)$ - цепь длины $\ell \geqslant 5, \operatorname{paн\imath ~} \mathbf{L}(\overline{\mathbf{w}})>$ ранг $\mathbf{M}(\overline{\mathbf{w}})$ и $\mathbf{G}-$ пополнение подгруппы $\mathbf{L}(\overline{\mathbf{w}})$. Допустим, $\mathbf{M}(\overline{\mathbf{w}}) \cap \mathbf{G}^{-}=\varnothing$. Тогда $\mathbf{M}(\overline{\mathbf{w}}) \leqslant \mathbf{G}^{*}$ в силу (15.1), и, следовательно, $\mathbf{L}(\overline{\mathbf{w}}) \subseteq\left\langle\mathbf{G}^{*}, \mathbf{w}_{1}\right\rangle$. Так как при $\mathbf{w}_{1} \in \mathbf{G}^{*} \cup \mathbf{G}^{-}$имели бы ранг $\mathbf{L}(\overline{\mathbf{w}}) \leqslant$ ранг $\left\langle\mathbf{G}^{*}, \mathbf{w}_{1}\right\rangle<$ ранг $\mathbf{G}$, то $\mathbf{w}_{1} \in \mathbf{G}^{+} \backslash \mathbf{G}^{*}$. Учитывая, что $\mathbf{w}_{i} \in \mathbf{L}(\overline{\mathbf{w}})$ при $1 \leqslant i \leqslant \ell-1$, получаем

$$
\operatorname{paн2}\left\langle\mathbf{w}_{1}, \mathbf{w}_{2}, \ldots, \mathbf{w}_{\ell-2}\right\rangle=\operatorname{paн2}\left\langle\mathbf{w}_{1}, \mathbf{w}_{2}, \ldots, \mathbf{w}_{\ell-1}\right\rangle=\operatorname{paн2} \mathbf{G} .
$$

Тогда по лемме 16.8 цепь $\left(\mathbf{w}_{1}, \mathbf{w}_{2}, \ldots, \mathbf{w}_{\ell-1}\right)$, а значит, и цепь $\left(\mathbf{w}_{1}, \mathbf{w}_{2}, \ldots, \mathbf{w}_{\ell}\right)$ peгулярны. Но в этом случае по лемме 16.11 имеем $\operatorname{paнг} \mathbf{L}(\overline{\mathbf{w}})=$ ранг $\mathbf{M}(\overline{\mathbf{w}})$ вопреки предположению. Следовательно, $\mathbf{M}(\overline{\mathbf{w}}) \cap \mathbf{G}^{-} \neq \varnothing$.

Теперь займемся некоторое время изучением общей ситуации, когда дана полная конечная подгруппа $\mathbf{G} \leqslant \mathbf{B}_{\alpha}$ и ее подгруппа $\mathbf{M}$.

16.13. ЛЕмма. Пусть $\mathbf{G}_{0} \leqslant \mathbf{B}_{\alpha}-$ полная конечная подгруппа, $\mathbf{M} \leqslant \mathbf{G}_{0} u$ ранг $\mathbf{M}<$ ранг $\mathbf{G}_{0}$. Тогда для некоторого $k \geqslant 1$ существует последовательность $\mathbf{G}_{0}, \mathbf{G}_{1}, \ldots, \mathbf{G}_{k}$ полных конечных подгрупп $\mathbf{G}_{i} \leqslant \mathbf{B}_{\alpha}$, удовлетворяющая следующим условиям:

(i) $\operatorname{paн2} \mathbf{G}_{0}>\operatorname{paн2} \mathbf{G}_{1}>\cdots>\operatorname{paн2} \mathbf{G}_{k}$;

(ii) $\mathbf{M} \leqslant \bigcap_{i=0}^{k} \mathbf{G}_{i}$;

(iii) $\mathbf{G}_{i+1}$ есть пополнение подгруппы $\mathbf{M G}_{i}^{*}$ при всех $i=0,1, \ldots, k-1$;

(iv) $р а н г \mathbf{G}_{i}^{*}<$ ранг $\mathbf{G}_{i+1}$ nри $i=0,1, \ldots, k-2$;

(v) $р$ анг $\mathbf{M}=$ ранг $\mathbf{G}_{k}$ или ранг $\mathbf{G}_{k-1}^{*}=\operatorname{paнг} \mathbf{G}_{k}$. 
ДоКАЗАТЕЛЬСТво. Построим индуктивно последовательность $\mathbf{G}_{0}, \mathbf{G}_{1}, \ldots$ полных конечных подгрупп групшы $\mathbf{B}_{\alpha}$, беря в качестве $\mathbf{G}_{i+1}$ произвольное пополнение подгруппы $\mathbf{M G}_{i}^{*}$ (последняя конечна ввиду $\mathbf{M} \subseteq \mathbf{G}_{i}$ ). Если ранг $\mathbf{M}<$ ранг $\mathbf{G}_{i}$, то по лемме 15.4 , (i) имеем ранг $\mathbf{M G}_{i}^{*}<$ ранг $\mathbf{G}_{i}$, и, следовательно, ранг $\mathbf{G}_{i+1}<$ ранг $\mathbf{G}_{i}$. Таким образом, на некотором шаге $i=k$ выполнится условие (v), на котором мы и оборвем процесс построения подгрупп $\mathbf{G}_{i}$. Выполнение остальных условий (i)-(iv) очевидно.

16.14. ОПРЕДЕЛЕнИЕ. Последовательность $\mathbf{G}_{0}, \mathbf{G}_{1}, \ldots, \mathbf{G}_{k}$ полных конечных подгрупп $\mathbf{G}_{i}$ группы $\mathbf{B}_{\alpha}$, удовлетворяюшую условиям $16.13,(\mathrm{i})-(\mathrm{v})$, будем называть лестничей над $\mathbf{M}$.

16.15. ЛЕмма. Пусть $\left(\mathbf{G}_{0}, \mathbf{G}_{1}, \ldots, \mathbf{G}_{k}\right)$ - лестница над конечной подгруппой $\mathbf{M} \leqslant \mathbf{B}_{\alpha}$. Пусть для кажсдого $i \mathbf{c}_{i}-$ базисный әлемент $\mathbf{G}_{i}$. Тогда верны следующие утверждения:

(i) При любом $1 \leqslant i \leqslant k-1$ последовательность $\left(\mathbf{G}_{i}, \mathbf{G}_{i+1}, \ldots, \mathbf{G}_{k}\right)$ есть лестница над $\mathbf{M}$.

(ii) $E c л u k \geqslant 2$, mo $\mathbf{M} \cap \mathbf{G}_{i}^{-} \neq \varnothing$ nрu $i=0,1, \ldots, k-1 u \mathbf{G}_{i}^{*} \cap \mathbf{G}_{i+1}^{-} \neq \varnothing n p u$ $i=0,1, \ldots, k-2$.

(iii) $\mathbf{M} \cap \mathbf{G}_{i}^{*}=\mathbf{M} \cap \mathbf{G}_{i+1}^{*} u \mathbf{M} \cap \mathbf{G}_{i}^{-}=\mathbf{M} \cap \mathbf{G}_{i+1}^{-}$при любом $0 \leqslant i \leqslant k-2$.

(iv) Пусть $0 \leqslant i \leqslant k-2 u \mathbf{f} \in \mathbf{M} \cap \mathbf{G}_{i}^{-}$. Тогда

$$
\mathbf{G}_{i}^{*} \mathbf{G}_{i+1}^{*} / \mathbf{G}_{i+1}^{*}=\left\{\mathbf{G}_{i+1}^{*}, \mathbf{c}_{i+1}^{n / 2} \mathbf{f G}_{i+1}^{*}\right\}
$$

$u$

$$
\mathbf{M G}_{i}^{*} \mathbf{G}_{i+1}^{*} / \mathbf{G}_{i+1}^{*}=\left\{\mathbf{G}_{i+1}^{*}, \mathbf{f G}_{i+1}^{*}, \mathbf{c}_{i+1}^{n / 2} \mathbf{G}_{i+1}^{*}, \mathbf{c}_{i+1}^{n / 2} \mathbf{f G}_{i+1}^{*}\right\} .
$$

(v) Пусть $0 \leqslant i \leqslant k-2$. Тогда для любого $\mathbf{g} \in \mathbf{M G}_{i}^{*} \backslash \mathbf{G}_{i}^{*}$ из ранг $\mathbf{g}<$ ранг $\mathbf{G}_{i+1}$ следует $\mathbf{g} \in \mathbf{M G}_{i+1}^{*} \backslash \mathbf{G}_{i+1}^{*} u$ для любой подгруппь $\mathbf{H} \subseteq \mathbf{M G}_{i}^{*}$ из $\mathbf{H} \nsubseteq \mathbf{G}_{i}^{*}$ и ранг $\mathbf{H}<\operatorname{ранг~} \mathbf{G}_{i+1}$ следует $\mathbf{H} \subseteq \mathbf{M G}_{i+1}^{*} u \mathbf{H} \nsubseteq \mathbf{G}_{i+1}^{*}$.

(vi) Если $k \geqslant 2$, то либо для некоторого $r$ в интервале $2 \leqslant r \leqslant k-1$ имеем ранг $\mathbf{G}_{0}^{*}=$ ранг $\mathbf{G}_{r}$ и $\mathbf{c}_{1}^{-n / 4} \mathbf{G}_{r} \mathbf{c}_{1}^{n / 4}$ есть пополнение $\mathbf{G}_{0}^{*}$, либо $\mathbf{G}_{0}^{*} \subseteq$ $\mathbf{c}_{1}^{-n / 4} \mathbf{G}_{k} \mathbf{c}_{1}^{n / 4}$.

(vii) $E c л и ~ \mathbf{M} \cap \mathbf{G}_{0}^{-} \neq \varnothing, \mathbf{u} \in \mathbf{G}_{0}, \quad$ ранг $\langle\mathbf{M}, \mathbf{u}\rangle=\operatorname{ранг~} \mathbf{G}_{0} u|\langle\mathbf{M}, \mathbf{u}\rangle: \mathbf{M}| \leqslant 2$, mo

$$
\mathbf{c}_{0}^{n / 2} \mathbf{u} \in \mathbf{M G}_{0}^{*}
$$

и либо для некоторого $r \leqslant k-1$ выполнено ранг $\mathbf{u}=\operatorname{paнг} \mathbf{G}_{r} u$

$$
\mathbf{c}_{0}^{-n / 4} \mathbf{u c}_{0}^{n / 4} \in \mathbf{c}_{r}^{n / 2} \mathbf{G}_{r}^{*}
$$

либо ранг $\mathbf{u} \leqslant$ ранг $\mathbf{G}_{k} u$

$$
\mathbf{c}_{0}^{-n / 4} \mathbf{u c}_{0}^{n / 4} \in \bigcap_{i=0}^{k-1}\left(\mathbf{M G}_{i}^{*} \backslash \mathbf{G}_{i}^{*}\right) .
$$


ДоКАЗАТЕЛЬСТВО. (i) вытекает непосредственно из определения 16.14.

(ii): Имеем ранг $\mathbf{M}<$ ранг $\mathbf{G}_{i}$ при $i=0,1, \ldots, k-1$ в силу 16.13 , (i), (ii). Далее, $\mathbf{M} \nsubseteq \mathbf{G}_{i}^{*}$ при $i=0,1, \ldots, k-2$ в силу 16.13 , (iii), (iv), а если допустить $\mathbf{M} \subseteq \mathbf{G}_{k-1}^{*}$, то из 16.13 , (iv) при $i:=k-2$ по лемме 15.4 , (i) следовало бы ранг $\mathbf{M G}_{k-2}^{*}<$ ранг $\mathbf{G}_{k-1}$ вопреки 16.13 , (iii). Следовательно, с учетом (15.1) имеем $\mathbf{M} \cap \mathbf{G}_{i}^{-} \neq \varnothing$ при всех $i=0,1, \ldots, k-1$.

Теперь пусть $0 \leqslant i \leqslant k-2$. Так как ранг $\mathbf{M} \leqslant$ ранг $\mathbf{G}_{k}<$ ранг $\mathbf{G}_{i+1}$ и согласно 16.13 , (iii) ранг $\mathbf{M} \mathbf{G}_{i}^{*}=$ ранг $\mathbf{G}_{i+1}$, то по лемме 15.4 , (i) имеем $\mathbf{G}_{i}^{*} \nsubseteq \mathbf{G}_{i+1}^{*}$, откуда в силу 16.13, (iv) следует $\mathbf{G}_{i}^{*} \cap \mathbf{G}_{i+1}^{-} \neq \varnothing$.

(iii): Так как при $i \leqslant k-2$ имеем ранг $\mathbf{M}<\operatorname{paнг} \mathbf{G}_{i+1}<\operatorname{paн2}_{i} \mathbf{G}_{i}$, то $\mathbf{M} \subseteq \mathbf{G}_{i}^{*} \cup \mathbf{G}_{i}^{-}$и $\mathbf{M} \subseteq \mathbf{G}_{i+1}^{*} \cup \mathbf{G}_{i+1}^{-}$. Поэтому достаточно доказать, что $\mathbf{M} \cap \mathbf{G}_{i}^{*}=\mathbf{M} \cap \mathbf{G}_{i+1}^{*}$. Если бы сушествовал элемент $\mathbf{g} \in\left(\mathbf{M} \cap \mathbf{G}_{i}^{*}\right) \backslash \mathbf{G}_{i+1}^{*}$, то из ранг $\mathbf{M}<$ ранг $\mathbf{G}_{i+1}$ и ранг $\mathbf{G}_{i}^{*}<$ ранг $\mathbf{G}_{i+1}$ по лемме 15.4 , (ii) следовало бы $\mathbf{M} \subseteq\left\langle\mathbf{G}_{i+1}^{*}, \mathbf{g}\right\rangle$ и $\mathbf{G}_{i}^{*} \subseteq\left\langle\mathbf{G}_{i+1}^{*}, \mathbf{g}\right\rangle$, т.е. $\mathbf{M G}_{i}^{*} \subseteq\left\langle\mathbf{G}_{i+1}^{*}, \mathbf{g}\right\rangle$. Но в этом случае ранг $\mathbf{M G}_{i}^{*}<$ ранг $\mathbf{G}_{i+1}$ вопреки 16.13 , (iii). Следовательно, $\mathbf{M} \cap \mathbf{G}_{i}^{*} \subseteq \mathbf{G}_{i+1}^{*}$. Так как из (ii) в силу леммы 15.4 , (iii) следует $\left|\mathbf{M}: \mathbf{M} \cap \mathbf{G}_{i}^{*}\right|=\left|\mathbf{M}: \mathbf{M} \cap \mathbf{G}_{i+1}^{*}\right|=2$, то $\mathbf{M} \cap \mathbf{G}_{i}^{*}=\mathbf{M} \cap \mathbf{G}_{i+1}^{*}$.

(iv): Выберем элемент $\mathbf{h} \in \mathbf{G}_{i}^{*} \cap \mathbf{G}_{i+1}^{-}$, существуюший согласно (ii). По лемме 15.4 , (ii) имеем $\mathbf{G}_{i}^{*} \mathbf{G}_{i+1}^{*}=\left\langle\mathbf{G}_{i+1}^{*}, \mathbf{h}\right\rangle$ и $\mathbf{M G}_{i+1}^{*}=\left\langle\mathbf{G}_{i+1}^{*}, \mathbf{f}\right\rangle$, следовательно, $\mathbf{h} \mathbf{f}^{-1} \notin \mathbf{G}_{i+1}^{*}$, так как иначе получили бы $\mathbf{M G}_{i}^{*} \subseteq \mathbf{M G}_{i+1}^{*}$ вопреки 16.13, (i), (iii). Так как ранг $\mathbf{h f} \mathbf{f}^{-1} \leqslant$ ранг $\mathbf{M G}_{i}^{*}=$ ранг $\mathbf{G}_{i+1}<$ ранг $\mathbf{G}_{i}$, то $\left(\mathbf{h} \mathbf{f}^{-1}\right)^{2} \in \mathbf{G}_{i}^{*}$. Ввиду $\mathbf{G}_{i}^{*} \subseteq \mathbf{G}_{i+1}^{*} \cup \mathbf{G}_{i+1}^{-}$и $\left(\mathbf{h f}^{-1}\right)^{2} \in \mathbf{G}_{i+1}^{+}$имеем $\left(\mathbf{h f}^{-1}\right)^{2} \in \mathbf{G}_{i+1}^{*}$. Но $\mathbf{h} \mathbf{f}^{-1} \in \mathbf{G}_{i+1}^{+}$, следовательно, $\mathbf{h} \mathbf{f}^{-1} \in \mathbf{c}_{i+1}^{n / 2} \mathbf{G}_{i+1}^{*}$, т.е. $\mathbf{h} \in \mathbf{c}_{i+1}^{n / 2} \mathbf{f} \mathbf{G}_{i+1}^{*}$. Тогда $\mathbf{G}_{i}^{*} \mathbf{G}_{i+1}^{*} / \mathbf{G}_{i+1}^{*}=$ $\left\{\mathbf{G}_{i+1}^{*}, \mathbf{h G}_{i+1}^{*}\right\}=\left\{\mathbf{G}_{i+1}^{*}, \mathbf{c}_{i+1}^{n / 2} \mathbf{f G}_{i+1}^{*}\right\}$. Так как $\mathbf{h}^{2}, \mathbf{f}^{2},\left(\mathbf{h} \mathbf{f}^{-1}\right)^{2} \in \mathbf{G}_{i+1}^{*}$, то $\mathbf{M G}_{i}^{*} \mathbf{G}_{i+1}^{*} / \mathbf{G}_{i+1}^{*}=\left\{\mathbf{G}_{i+1}^{*}, \mathbf{f G}_{i+1}^{*}, \mathbf{c}_{i+1}^{n / 2} \mathbf{G}_{i+1}^{*}, \mathbf{c}_{i+1}^{n / 2} \mathbf{f G}_{i+1}^{*}\right\}$.

(v): Пусть $\mathbf{g} \in \mathbf{M G}_{i}^{*} \backslash \mathbf{G}_{i}^{*}$ и ранг $\mathbf{g}<$ ранг $\mathbf{G}_{i+1}$. Выберем элемент $\mathbf{f} \in \mathbf{M} \cap$ $\bigcap_{i=0}^{k-1} \mathbf{G}_{i}^{-}$, существуюший в силу (ii) и (iii). Так как в силу (ii) и леммы 15.4 (iii) $\left|\mathbf{M G}_{i}^{*}: \mathbf{G}_{i}^{*}\right|=2$ и в силу (iv) $\left|\mathbf{M G}_{i}^{*} / \mathbf{M G}_{i}^{*} \cap \mathbf{G}_{i+1}^{*}: \mathbf{G}_{i}^{*} / \mathbf{G}_{i}^{*} \cap \mathbf{G}_{i+1}^{*}\right|=\mid \mathbf{M G}_{i}^{*} \mathbf{G}_{i+1}^{*}$ : $\mathbf{G}_{i}^{*} \mathbf{G}_{i+1}^{*} \mid=2$, то $\mathbf{M G}_{i}^{*} \cap \mathbf{G}_{i+1}^{*}=\mathbf{G}_{i}^{*} \cap \mathbf{G}_{i+1}^{*}$. Отсюда и из (iv) следует $\mathbf{M G}_{i}^{*} \backslash \mathbf{G}_{i}^{*} \subseteq$ $\mathbf{f G}_{i+1}^{*} \cup \mathbf{c}_{i+1}^{n / 2} \mathbf{G}_{i+1}^{*}$. Так как, кроме того, в силу леммы 15.4 , (ii) $\mathbf{M G}_{i+1}^{*} \backslash \mathbf{G}_{i+1}^{*}=$ $\mathbf{f G}_{i+1}^{*}$, то $\mathbf{g} \in \mathbf{f G}_{i+1}^{*}=\mathbf{M G}_{i+1}^{*} \backslash \mathbf{G}_{i+1}^{*}$, что доказывает первое утверждение. Если $\mathbf{H} \leqslant \mathbf{M G}_{i}^{*}, \mathbf{H} \nsubseteq \mathbf{G}_{i}^{*}$ и ранг $\mathbf{H}<$ ранг $\mathbf{G}_{i+1}$, то, выбрав элемент $\mathbf{g} \in \mathbf{H} \backslash \mathbf{G}_{i}^{*}$, получим по доказанному $\mathbf{g} \in \mathbf{M G}_{i+1}^{*} \backslash \mathbf{G}_{i+1}^{*}$. Так как $р а н г \mathbf{g}<$ ранг $\mathbf{G}_{i+1}$, то $\mathbf{g} \in \mathbf{G}_{i+1}^{-}$и по лемме 15.4, (i), (ii) $\mathbf{H} \subseteq\left\langle\mathbf{G}_{i+1}^{*}, \mathbf{g}\right\rangle=\mathbf{M G}_{i+1}^{*}$. Наличие элемента $\mathbf{g} \in \mathbf{H} \cap \mathbf{G}_{i+1}^{-}$влечет также $\mathbf{H} \nsubseteq \mathbf{G}_{i+1}^{*}$.

(vi): Выберем элемент $\mathbf{f} \in \mathbf{M} \cap \bigcap_{i=0}^{k-1} \mathbf{G}_{i}^{-}$в силу (ii) и (iii). Согласно (iv) имеем $\mathbf{G}_{0}^{*} \subseteq \mathbf{G}_{1}^{*} \cup \mathbf{c}_{1}^{n / 2} \mathbf{f} \mathbf{G}_{1}^{*}$. Заметим, что $\mathbf{c}_{1}^{n / 4} \mathbf{G}_{1}^{*} \mathbf{c}_{1}^{-n / 4}=\mathbf{G}_{1}^{*}$, и так как в диэдральной группе $\mathbf{D}_{n}=\left\langle\mathbf{a}, \mathbf{c} \mid \mathbf{a}^{2}=(\mathbf{a c})^{2}=\mathbf{c}^{n}=1\right\rangle$ для любых $\mathbf{x} \in \mathbf{D}_{n} \backslash\langle\mathbf{c}\rangle$ и $t$ имеет место равенство $\mathbf{c}^{t} \mathbf{x c}^{t}=\mathbf{x}$, то $\mathbf{c}_{1}^{n / 4}\left(\mathbf{c}_{1}^{-n / 2} \mathbf{f G}_{1}^{*}\right) \mathbf{c}_{1}^{-n / 4}=\mathbf{f G}_{1}^{*}$. Следовательно,

$$
\mathbf{c}_{1}^{n / 4} \mathbf{G}_{0}^{*} \mathbf{c}_{1}^{-n / 4} \subseteq \mathbf{G}_{1}^{*} \cup \mathbf{f G}_{1}^{*}=\mathbf{M G}_{1}^{*} .
$$

Кроме того, в силу (ii)

$$
\mathbf{c}_{1}^{n / 4} \mathbf{G}_{0}^{*} \mathbf{c}_{1}^{-n / 4} \nsubseteq \mathbf{G}_{1}^{*}
$$


Теперь предположим, что для некоторого $1 \leqslant i \leqslant k-1$ выполнено

$$
\mathbf{c}_{1}^{n / 4} \mathbf{G}_{0}^{*} \mathbf{c}_{1}^{-n / 4} \subseteq \mathbf{M G}_{i}^{*} \text { и } \mathbf{c}_{1}^{n / 4} \mathbf{G}_{0}^{*} \mathbf{c}_{1}^{-n / 4} \not \mathbf{G}_{i}^{*} .
$$

Если $i=k-1$ или ранг $\mathbf{G}_{0}^{*}=$ ранг $\mathbf{G}_{i+1}$, то имеет место заключение утверждения (vi). В противном случае в силу (v)

$$
\mathbf{c}_{1}^{n / 4} \mathbf{G}_{0}^{*} \mathbf{c}_{1}^{-n / 4} \subseteq \mathbf{M G}_{i+1}^{*} \quad \text { и } \mathbf{c}_{1}^{n / 4} \mathbf{G}_{0}^{*} \mathbf{c}_{1}^{-n / 4} \not \subset \mathbf{G}_{i+1}^{*}
$$

и работает очевидная индукция.

(vii): Заметим, что из ранг $\langle\mathbf{M}, \mathbf{u}\rangle=$ ранг $\mathbf{G}_{0}$ и ранг $\mathbf{M} \mathbf{G}_{0}^{*}=$ ранг $\mathbf{G}_{1}<$ ранг $\mathbf{G}_{0}$ следует $\mathbf{u} \notin \mathbf{M G}_{0}^{*}$. Так как $\mathbf{u}^{2} \in \mathbf{M}$, то ранг $\mathbf{u}^{2} \leqslant$ ранг $\mathbf{M}<$ ранг $\mathbf{G}_{0}$. Следовательно, $\mathbf{u} \in \mathbf{c}_{0}^{n / 2} \mathbf{G}_{0}^{*} \cup \mathbf{G}_{0}^{-}$. Если $\mathbf{u} \in \mathbf{c}_{0}^{n / 2} \mathbf{G}_{0}^{*}$, то утверждение (vii) верно при $r=0$. Пусть $\mathbf{u} \in \mathbf{G}_{0}^{-}$. Выберем элемент $\mathbf{f} \in \mathbf{M} \cap \mathbf{G}_{0}^{-}$. Имеем $\mathbf{u} \mathbf{f}^{-1} \in \mathbf{G}_{0}^{+} \backslash \mathbf{G}_{0}^{*}$, и так как $\left(\mathbf{u f}^{-1}\right)^{2} \in \mathbf{M}$, то ранг $\left(\mathbf{u} \mathbf{f}^{-1}\right)^{2}<$ ранг $\mathbf{G}_{0}$, откуда вытекает $\mathbf{u f}^{-1} \in \mathbf{c}_{0}^{n / 2} \mathbf{G}_{0}^{*}$. Тем самым доказана первая часть утверждения. Докажем вторую часть. Как было замечено в доказательстве (vi), в группе $\mathbf{G}_{0} / \mathbf{G}_{0}^{*} \simeq \mathbf{D}_{n}$ имеет место равенство $\mathbf{c}_{0}^{n / 4}\left(\mathbf{f G}_{0}^{*}\right) \mathbf{c}_{0}^{n / 4}=\mathbf{f G}_{0}^{*}$. Поэтому

$$
\mathbf{c}_{0}^{-n / 4} \mathbf{u c}_{0}^{n / 4} \in \mathbf{f G}_{0}^{*}=\mathbf{M G}_{0}^{*} \backslash \mathbf{G}_{0}^{*} .
$$

Теперь предположим, что при каком-нибудь $i \leqslant k-2$

$$
\mathbf{c}_{0}^{-n / 4} \mathbf{u c}_{0}^{n / 4} \in \mathbf{M G}_{i}^{*} \backslash \mathbf{G}_{i}^{*}
$$

Если ранг $\mathbf{u}=\operatorname{paнг}_{\mathbf{2}} \mathbf{G}_{i+1}$, то в силу 16.13 , (iii) искомое утверждение верно при $r:=i+1$. Если же ранг $\mathbf{u}<$ ранг $\mathbf{G}_{i+1}$, то в силу (v)

$$
\mathbf{c}_{0}^{-n / 4} \mathbf{u c}_{0}^{n / 4} \in \mathbf{M G}_{i+1}^{*} \backslash \mathbf{G}_{i+1}^{*}
$$

и проходит индукция.

16.16. Лемма. Если $\mathbf{G}, \mathbf{H} \in \mathbf{B}_{\alpha}$ - конечные подгруппь, $\mathbf{G} \subseteq \mathbf{H} u|\mathbf{H}: \mathbf{G}| \leqslant$ 2 , то $\left(\mathbf{N}_{\mathbf{B}_{\alpha}}(\mathbf{H})\right)^{4} \subseteq \mathbf{N}_{\mathbf{B}_{\alpha}}(\mathbf{G})$ (әде, напомним, $\mathbf{X}^{t}$ обозначает $\left.\left\{\mathbf{x}^{t} \mid \mathbf{x} \in \mathbf{X}\right\}\right)$.

ДокаЗАТЕЛЬСтво. Пусть $\mathbf{x} \in \mathbf{N}_{\mathbf{B}_{\alpha}}(\mathbf{H})$. Докажем, что $\mathbf{x}^{4} \in \mathbf{N}_{\mathbf{B}_{\alpha}}(\mathbf{G})$. Выберем пополнение $\overline{\mathbf{H}}$ подгруппы $\mathbf{H}$ и по предложению 15.3 найдем $\mathbf{y} \in \overline{\mathbf{H}}$ такой, что $\mathbf{y}^{-1} \mathbf{g y}=\mathbf{x}^{-1} \mathbf{g x}$ для любого $\mathbf{g} \in \mathbf{H}$. Достаточно проверить, что $\mathbf{y}^{4} \in \mathbf{N}_{\mathbf{B}_{\alpha}}(\mathbf{G})$. Используя тождество $15.10,(\mathrm{v})$, для любого $\mathbf{g} \in \mathbf{G}$ выводим

$$
\begin{aligned}
\mathbf{y}^{-4} \mathbf{g y} & =\mathbf{y}^{-2} \mathbf{g y} \mathbf{y}^{2} \cdot \mathbf{y}^{-1} \mathbf{g}^{-1} \mathbf{y} \cdot \mathbf{y}^{-3} \mathbf{g y} \\
& =\mathbf{y}^{-2} \mathbf{g} \mathbf{y}^{2} \cdot \mathbf{y}^{-1} \mathbf{g}^{-1} \mathbf{y} \cdot\left(\mathbf{y}^{-1} \mathbf{g y} \cdot \mathbf{g}^{-1} \cdot \mathbf{y}^{-2} \mathbf{g}^{-1} \mathbf{y}^{2}\right) \\
& =\mathbf{y}^{-2} \mathbf{g} \mathbf{y}^{2} \cdot \mathbf{g}^{-1} \cdot \mathbf{y}^{-2} \mathbf{g} \mathbf{y}^{2} .
\end{aligned}
$$

Так как по условию $|\mathbf{H}: \mathbf{G}| \leqslant 2$, то из $\mathbf{g} \in \mathbf{G}$ следует $\mathbf{h g h} \in \mathbf{G}$ для любого $\mathbf{h} \in \mathbf{H}$. Следовательно, $\mathbf{y}^{-4} \mathbf{g} \mathbf{y}^{4} \in \mathbf{G}$, и, таким образом, $\mathbf{y}^{4} \in \mathbf{N}_{\mathbf{B}_{\alpha}}(\mathbf{G})$. 
16.17. Лемма. Пусть $\left(\mathbf{G}_{0}, \mathbf{G}_{1}, \ldots, \mathbf{G}_{k}\right)$-лестница над конечной подгруппой $\mathbf{M} \leqslant \mathbf{B}_{\alpha}$ и ранг $\mathbf{G}_{k-1}^{*}<$ ранг $\mathbf{G}_{k}$. Тогда верны следующие утверждения:

(i) $\mathbf{G}_{0} \cap \mathbf{G}_{k}^{*}=\bigcap_{i=0}^{k} \mathbf{G}_{i}^{*}$;

(ii) $е с л u \mathbf{u} \in \mathbf{G}_{0} u|\langle\mathbf{M}, \mathbf{u}\rangle: \mathbf{M}| \leqslant 2, m o \mathbf{u}^{2} \in \mathbf{M} \cap \mathbf{G}_{k}^{*} u[\mathbf{u}, \mathbf{g}] \in \mathbf{M} \cap \mathbf{G}_{k}^{*} \partial \Omega s$ любого $\mathbf{g} \in \mathbf{M}$;

(iii) $\mathbf{G}_{0}^{4} \subseteq \mathbf{N}_{\mathbf{B}_{\alpha}}\left(\mathbf{G}_{0}^{*} \cap \mathbf{G}_{k}^{*}\right)$.

ДоказАтельСтво. (i): Достаточно доказать включение $\mathbf{G}_{0} \cap \mathbf{G}_{k}^{*} \subseteq \bigcap_{i=0}^{k} \mathbf{G}_{i}^{*}$. Воспользуемся ндукцией по $k$. Сначала рассмотрим случай $k=1$. Имеем $\mathbf{M} \nsubseteq \mathbf{G}_{0}^{*}$, так как иначе $\mathbf{M G}_{0}^{*}=\mathbf{G}_{0}^{*}$ и $р а н г \mathbf{G}_{0}^{*}=$ ранг $\mathbf{G}_{1}$ вопреки условию. Так как ранг $\mathbf{M}<$ ранг $\mathbf{G}_{0}$, то отсюда следует $\mathbf{M} \cap \mathbf{G}_{0}^{-} \neq \varnothing$. Пусть $\mathbf{f} \in \mathbf{M} \cap \mathbf{G}_{0}^{-}$. Так как $\mathbf{f} \in \mathbf{G}_{1} \cap \mathbf{G}_{0}^{-}$и ранг $\left(\mathbf{G}_{0} \cap \mathbf{G}_{1}\right)<$ ранг $\mathbf{G}_{0}$, то по лемме 15.4 , (ii) имеем $\mathbf{G}_{0} \cap \mathbf{G}_{1} \subseteq\left\langle\mathbf{G}_{0}^{*}, \mathbf{f}\right\rangle=\mathbf{M G}_{0}^{*}$. Поэтому для доказательства искомого включения $\mathbf{G}_{0} \cap \mathbf{G}_{1}^{*} \subseteq \mathbf{G}_{0}^{*}$ достаточно проверить, что $\mathbf{M G}_{0}^{*} \cap \mathbf{G}_{1}^{*}=\mathbf{G}_{0}^{*} \cap \mathbf{G}_{1}^{*}$. Имеем ранг $\mathbf{M G}_{0}^{*}=$ ранг $\mathbf{G}_{1}<$ ранг $\mathbf{G}_{0}$, следовательно, по лемме 15.4, (iii) $\left|\mathbf{M G}_{0}^{*}: \mathbf{G}_{0}^{*}\right|=2$. Из ранг $\mathbf{G}_{0}^{*}<$ ранг $\mathbf{G}_{1}$ в силу леммы 15.4, (i) вытекает ранг $\mathbf{G}_{0}^{*} \mathbf{G}_{1}^{*}<$ ранг $\mathbf{G}_{1}=$ ранг $\mathbf{M} \mathbf{G}_{0}^{*} \mathbf{G}_{1}^{*}$, следовательно, $\mathbf{G}_{0}^{*} \mathbf{G}_{1}^{*} \subsetneq \mathbf{M} \mathbf{G}_{0}^{*} \mathbf{G}_{1}^{*}$. Тогда $\left|\mathbf{M G}_{0}^{*} / \mathbf{M G}_{0}^{*} \cap \mathbf{G}_{1}^{*}: \mathbf{G}_{0}^{*} / \mathbf{G}_{0}^{*} \cap \mathbf{G}_{1}^{*}\right|=\left|\mathbf{M G}_{0}^{*} \mathbf{G}_{1}^{*}: \mathbf{G}_{0}^{*} \mathbf{G}_{1}^{*}\right| \geqslant 2$, откуда вытекает невозможность собственного включения $\mathbf{G}_{0}^{*} \cap \mathbf{G}_{1}^{*} \subsetneq \mathbf{M G}_{0}^{*} \cap \mathbf{G}_{1}^{*}$, что и доказывает равенство $\mathbf{M G}_{0}^{*} \cap \mathbf{G}_{1}^{*}=\mathbf{G}_{0}^{*} \cap \mathbf{G}_{1}^{*}$. Тем самым при $k=1$ утверждение доказано.

Теперь пусть $k \geqslant 2$. Выберем элемент $\mathbf{f} \in \mathbf{M} \cap \mathbf{G}_{0}^{-} \cap \mathbf{G}_{1}^{-}$согласно лемме 16.15 , (ii), (iii). Имеем $\mathbf{f} \in \mathbf{G}_{0}^{-} \cap \mathbf{G}_{k}$ и ранг $\left(\mathbf{G}_{0} \cap \mathbf{G}_{k}\right)<$ ранг $\mathbf{G}_{0}$, поэтому по лемме 15.4 , (ii) получаем $\mathbf{G}_{0} \cap \mathbf{G}_{k} \subseteq\left\langle\mathbf{G}_{0}^{*}, \mathbf{f}\right\rangle=\mathbf{M G}_{0}^{*}$. Так как $\mathbf{M G}_{0}^{*} \subseteq \mathbf{G}_{1}$, то, применив индуктивное предположение при $k:=k-1$ с учетом леммы 16.15 , (i), выводим $\mathbf{G}_{0} \cap \mathbf{G}_{k}^{*}=\bigcap_{i=1}^{k} \mathbf{G}_{i}^{*}$. Остается заметить, что из $\mathbf{G}_{0} \cap \mathbf{G}_{k}^{*} \subseteq \mathbf{M G}_{0}^{*} \cap \mathbf{G}_{1}^{*}$ и доказанного вьше включения $\mathbf{M G}_{0}^{*} \cap \mathbf{G}_{1}^{*} \subseteq \mathbf{G}_{0}^{*}$ следует $\mathbf{G}_{0} \cap \mathbf{G}_{k}^{*} \subseteq \mathbf{G}_{0}^{*}$.

(ii): Из $|\langle\mathbf{M}, \mathbf{u}\rangle: \mathbf{M}| \leqslant 2$ следует $\mathbf{u}^{2} \in \mathbf{M}$ и $[\mathbf{u}, \mathbf{M}] \subseteq \mathbf{M}$ (где, по определению, $[\mathbf{x}, \mathbf{Y}] \rightleftharpoons\{[\mathbf{x}, \mathbf{y}] \mid \mathbf{y} \in \mathbf{Y}\})$, поэтому достаточно убедиться в истинности включений $\mathbf{u}^{2} \in \mathbf{G}_{k}^{*}$ и $[\mathbf{u}, \mathbf{g}] \in \mathbf{G}_{k}^{*}$ для любого $\mathbf{g} \in \mathbf{M}$. Воспользуемся индукцией по $k$. Пусть сначала ранг $\langle\mathbf{M}, \mathbf{u}\rangle=$ ранг $\mathbf{G}_{0}$. Как было установлено в доказательстве (i), $\mathbf{M} \cap \mathbf{G}_{0}^{-} \neq \varnothing$. Тогда по лемме 16.15 , (vii) имеем $\mathbf{c}_{0}^{n / 2} \mathbf{u} \in \mathbf{M G}_{0}^{*}$. Полагая $\mathbf{u}^{\prime} \rightleftharpoons \mathbf{c}_{0}^{n / 2} \mathbf{u}$ и замечая, что $\mathbf{c}_{0}^{n / 2}$ - центральная инволюция в $\mathbf{G}_{0}$ в силу предложения 15.10 , (i), (ii), получаем $\left|\left\langle\mathbf{M}, \mathbf{u}^{\prime}\right\rangle: \mathbf{M}\right| \leqslant 2, \mathbf{u}^{2}=\mathbf{u}^{\prime 2}$ и $[\mathbf{u}, \mathbf{g}]=\left[\mathbf{u}^{\prime}, \mathbf{g}\right]$ для любого $\mathbf{g} \in \mathbf{M}$, но теперь $\left\langle\mathbf{M}, \mathbf{u}^{\prime}\right\rangle \subseteq \mathbf{M G}_{0}^{*} \subseteq \mathbf{G}_{1}$. Тем самым случай $р а н г\langle\mathbf{M}, \mathbf{u}\rangle=\operatorname{pанг~} \mathbf{G}_{0}$ сводится к случаю $р а н г\langle\mathbf{M}, \mathbf{u}\rangle<\operatorname{pанг~} \mathbf{G}_{0}$. Пусть ранг $\langle\mathbf{M}, \mathbf{u}\rangle<$ ранг $\mathbf{G}_{0}$. Выбрав элемент $\mathbf{f} \in \mathbf{M} \cap \mathbf{G}_{0}^{-}$и воспользовавшись леммой 15.4, (ii), получим

$$
\langle\mathbf{M}, \mathbf{u}\rangle \subseteq\left\langle\mathbf{G}_{0}^{*}, \mathbf{f}\right\rangle=\mathbf{M G}_{0}^{*}
$$

Для любого $\mathbf{g} \in \mathbf{M G}_{0}^{*}$ выполнено ранг $\mathbf{g} \leqslant$ ранг $\mathbf{G}_{1}<$ ранг $\mathbf{G}_{0}$, следовательно, с учетом (15.1) и включения $\left(\mathbf{G}_{0}^{-}\right)^{2} \subseteq \mathbf{G}_{0}^{*}$ имеем $\mathbf{g}^{2} \in \mathbf{G}_{0}^{*}$. Тогда ранг $\mathbf{g}^{2} \leqslant$ ранг $\mathbf{G}_{0}^{*}<$ ранг $\mathbf{G}_{1}$, и так как $\mathbf{g}^{2} \in \mathbf{G}_{1}^{+}$, то $\mathbf{g}^{2} \in \mathbf{G}_{1}^{*}$. Таким образом, $\mathbf{M G}_{0}^{*} \mathbf{G}_{1}^{*} / \mathbf{G}_{1}^{*}$ - группа периода 2 , и поэтому $\mathbf{u}^{2} \in \mathbf{G}_{1}^{*}$ и $[\mathbf{u}, \mathbf{g}] \in \mathbf{G}_{1}^{*}$ для любого $\mathbf{g} \in \mathbf{M}$. Тем самым мы рассмотрели случай $k=1$. Так как из (16.4) следует 
$\mathbf{u} \in \mathbf{G}_{1}$, то в случае $k \geqslant 2$ можно воспользоваться леммой 16.15 , (i) и индуктивным предположением.

(iii): Индукция по $k$. Если $k=1$, то из ранг $\mathbf{G}_{0}^{*}<$ ранг $\mathbf{G}_{1}$ по лемме 15.4 , (iii) следует $\left|\mathbf{G}_{0}^{*}: \mathbf{G}_{0}^{*} \cap \mathbf{G}_{1}^{*}\right|=2$. Так как $\mathbf{G}_{0} \subseteq \mathbf{N}_{\mathbf{B}_{\alpha}}\left(\mathbf{G}_{0}^{*}\right)$, то по лемме 16.16 получаем $\mathbf{G}_{0}^{4} \subseteq \mathbf{N}_{\mathbf{B}_{\alpha}}\left(\mathbf{G}_{0}^{*} \cap \mathbf{G}_{1}^{*}\right)$.

Пусть $k \geqslant 2$. Пусть $\mathbf{c}_{1}$ - базисный элемент подгруппы $\mathbf{G}_{1}$. Так как $n$ кратно 16 , то по индуктивному предположению $\mathbf{c}_{1}^{n / 4} \in \mathbf{N}_{\mathbf{B}_{\alpha}}\left(\mathbf{G}_{1}^{*} \cap \mathbf{G}_{k}^{*}\right)$. Тогда, используя (i) и лемму $16.15,(\mathrm{i})$, выводим

$$
\mathbf{G}_{0}^{*} \cap \mathbf{G}_{k}^{*} \subseteq \mathbf{G}_{1}^{*} \cap \mathbf{G}_{k}^{*}=\mathbf{c}_{1}^{-n / 4}\left(\mathbf{G}_{1}^{*} \cap \mathbf{G}_{k}^{*}\right) \mathbf{c}_{1}^{n / 4}=\bigcap_{i=1}^{k} \mathbf{c}_{1}^{-n / 4} \mathbf{G}_{i}^{*} \mathbf{c}_{1}^{n / 4}
$$

и

$$
\begin{aligned}
\mathbf{G}_{0}^{*} \cap \mathbf{c}_{1}^{-n / 4} \mathbf{G}_{k}^{*} \mathbf{c}_{1}^{n / 4} \subseteq \mathbf{G}_{1} \cap \mathbf{c}_{1}^{-n / 4} \mathbf{G}_{k}^{*} \mathbf{c}_{1}^{n / 4} & =\mathbf{c}_{1}^{-n / 4}\left(\mathbf{G}_{1} \cap \mathbf{G}_{k}^{*}\right) \mathbf{c}_{1}^{n / 4} \\
& =\mathbf{c}_{1}^{-n / 4}\left(\mathbf{G}_{1}^{*} \cap \mathbf{G}_{k}^{*}\right) \mathbf{c}_{1}^{n / 4} \subseteq \mathbf{G}_{k}^{*}
\end{aligned}
$$

Отсюда следует

$$
\mathbf{G}_{0}^{*} \cap \mathbf{G}_{k}^{*}=\mathbf{G}_{0}^{*} \cap \mathbf{c}_{1}^{-n / 4} \mathbf{G}_{k}^{*} \mathbf{c}_{1}^{n / 4}
$$

и для любого $1 \leqslant r \leqslant k$

$$
\mathbf{G}_{0}^{*} \cap \mathbf{G}_{k}^{*}=\mathbf{G}_{0}^{*} \cap \mathbf{c}_{1}^{-n / 4}\left(\mathbf{G}_{r}^{*} \cap \mathbf{G}_{k}^{*}\right) \mathbf{c}_{1}^{n / 4}
$$

Если ранг $\mathbf{G}_{0}^{*}<$ ранг $\mathbf{G}_{k}$, то по лемме 16.15 , (vi) имеем $\mathbf{G}_{0}^{*} \subseteq \mathbf{c}_{1}^{-n / 4} \mathbf{G}_{k} \mathbf{c}_{1}^{n / 4}$, а по лемме 15.4 , (iii) $\left|\mathbf{G}_{0}^{*}: \mathbf{G}_{0}^{*} \cap \mathbf{c}_{1}^{-n / 4} \mathbf{G}_{k}^{*} \mathbf{c}_{1}^{n / 4}\right| \leqslant 2$. В этом случае по лемме 16.16 с учетом (16.5) получаем $\mathbf{G}_{0}^{4} \subseteq \mathbf{N}_{\mathbf{B}_{\alpha}}\left(\mathbf{G}_{0}^{*} \cap \mathbf{G}_{k}^{*}\right)$. Пусть ранг $\mathbf{G}_{0}^{*} \geqslant$ ранг $\mathbf{G}_{k}$. Тогда по лемме 16.15 , (vi), принимая во внимание, что в случае $\mathbf{G}_{0}^{*} \subseteq \mathbf{c}_{1}^{-n / 4} \mathbf{G}_{k} \mathbf{c}_{1}^{n / 4}$ выполнено ранг $\mathbf{G}_{0}^{*}=$ ранг $\mathbf{G}_{k}$, получаем, что для некоторого $2 \leqslant r \leqslant k$ подгруппа $\mathbf{c}_{1}^{-n / 4} \mathbf{G}_{r} \mathbf{c}_{1}^{n / 4}$ есть пополнение $\mathbf{G}_{0}^{*}$. Пусть $\mathbf{g} \in \mathbf{G}_{0}-$ произвольный элемент. Если $r=k$, то $\mathbf{c}_{1}^{-n / 4} \mathbf{G}_{k} \mathbf{c}_{1}^{n / 4}$ и $\mathbf{g}^{-1} \mathbf{c}_{1}^{-n / 4} \mathbf{G}_{k} \mathbf{c}_{1}^{n / 4} \mathbf{g}-$ два пополнения подгруппы $\mathbf{G}_{0}^{*}$ и в силу предложения 15.2

$$
\mathbf{g}^{-1}\left(\mathbf{G}_{0}^{*} \cap \mathbf{c}_{1}^{-n / 4} \mathbf{G}_{k}^{*} \mathbf{c}_{1}^{n / 4}\right) \mathbf{g}=\mathbf{G}_{0}^{*} \cap \mathbf{c}_{1}^{-n / 4} \mathbf{G}_{k}^{*} \mathbf{c}_{1}^{n / 4}
$$

т.е. ввиду (16.5) $\mathbf{g} \in \mathbf{N}_{\mathbf{B}_{\alpha}}\left(\mathbf{G}_{0}^{*} \cap \mathbf{G}_{k}^{*}\right)$. Пусть $r<k$. По предложению 15.3 найдется $\mathbf{y} \in \mathbf{c}_{1}^{-n / 4} \mathbf{G}_{r} \mathbf{c}_{1}^{n / 4}$ такой, что $\mathbf{g}^{-1} \mathbf{h g}=\mathbf{y}^{-1} \mathbf{h y}$ для любого $\mathbf{h} \in \mathbf{G}_{0}^{*}$. По индуктивному предположению $\mathbf{y}^{4} \in \mathbf{N}_{\mathbf{B}_{\alpha}}\left(\mathbf{c}_{1}^{-n / 4}\left(\mathbf{G}_{r}^{*} \cap \mathbf{G}_{k}^{*}\right) \mathbf{c}_{1}^{n / 4}\right)$. Так как $\mathbf{y} \in \mathbf{N}_{\mathbf{B}_{\alpha}}\left(\mathbf{G}_{0}^{*}\right)$, то ввиду (16.6) $\mathbf{y}^{4} \in \mathbf{N}_{\mathbf{B}_{\alpha}}\left(\mathbf{G}_{0}^{*} \cap \mathbf{G}_{k}^{*}\right)$, а значит, и $\mathbf{g}^{4} \in \mathbf{N}_{\mathbf{B}_{\alpha}}\left(\mathbf{G}_{0}^{*} \cap \mathbf{G}_{k}^{*}\right)$.

Следующее утверждение играет роль базы индукции для доказательства предложения 16.6. 
16.18. Лемма. Пусть $\overline{\mathbf{w}}=\left(\mathbf{w}_{1}, \mathbf{w}_{2}, \ldots, \mathbf{w}_{\ell}\right)-$ цепь длины $\ell \geqslant 6, \mathbf{G}_{0} u$ $\mathbf{H}_{0}$ - пополнения подгрупп $\mathbf{L}(\overline{\mathbf{w}})$ u $\mathbf{R}(\overline{\mathbf{w}})$ соответственно. Предположим, что выполнены следующие условия:

(i) либо $\quad$ ранг $\mathbf{L}(\overline{\mathbf{w}})=$ ранг $\mathbf{M}(\overline{\mathbf{w}}), \quad$ либо $\quad$ ранг $\mathbf{L}(\overline{\mathbf{w}})>$ ранг $\mathbf{M}(\overline{\mathbf{w}})$, $\left(\mathbf{G}_{0}, \mathbf{G}_{1}, \ldots, \mathbf{G}_{k}\right)$ - лестница над $\mathbf{M}(\overline{\mathbf{w}})$ и ранг $\mathbf{G}_{k-1}^{*}<$ ранг $\mathbf{G}_{k} ;$

(ii) либо $\quad$ ранг $\mathbf{R}(\overline{\mathbf{w}})=$ ранг $\mathbf{M}(\overline{\mathbf{w}})$, либо $\quad$ ранг $\mathbf{R}(\overline{\mathbf{w}})>\operatorname{pанг~} \mathbf{M}(\overline{\mathbf{w}})$, $\left(\mathbf{H}_{0}, \mathbf{H}_{1}, \ldots, \mathbf{H}_{t}\right)$ - лестница над $\mathbf{M}(\overline{\mathbf{w}})$ и ранг $\mathbf{H}_{t-1}^{*}<$ ранг $\mathbf{H}_{t}$.

Тогда $\overline{\mathbf{w}}-$ регулярная цепь.

ДокАЗАТЕльСтво. Допустим, $\overline{\mathbf{w}}$ не является регулярной. В этом случае в силу леммы 16.11 ранг $\mathbf{L}(\overline{\mathbf{w}})>$ ранг $\mathbf{M}(\overline{\mathbf{w}})$ или $р$ ранг $\mathbf{R}(\overline{\mathbf{w}})>$ ранг $\mathbf{M}(\overline{\mathbf{w}})$. Эти два случая симметричны, поэтому ограничимся рассмотрением случая, когда $\operatorname{paнг} \mathbf{L}(\overline{\mathbf{w}})>\operatorname{pанг~} \mathbf{M}(\overline{\mathbf{w}})$. Тогда по условию имеем ранг $\mathbf{G}_{k-1}^{*}<\operatorname{paнг}_{\mathbf{G}} \mathbf{G}_{k}$, где $\left(\mathbf{G}_{0}, \mathbf{G}_{1}, \ldots, \mathbf{G}_{k}\right)$ - лестница над $\mathbf{M}(\overline{\mathbf{w}})$ и в силу 16.13 , (v) ранг $\mathbf{M}(\overline{\mathbf{w}})=$ ранг $\mathbf{G}_{k}$. Мы утверждаем, что

$$
\mathbf{M}(\overline{\mathbf{w}})=\left\langle\mathbf{w}_{2}, \mathbf{w}_{3}, \ldots, \mathbf{w}_{\ell-1}\right\rangle\left(\mathbf{G}_{k}^{*} \cap \mathbf{M}(\overline{\mathbf{w}})\right) .
$$

Так как $\mathbf{w}_{i} \in \mathbf{M}(\overline{\mathbf{w}}) \subseteq \mathbf{G}_{k}(i=2,3, \ldots, \ell-1)$, то $\mathbf{w}_{2}, \mathbf{w}_{3}, \ldots, \mathbf{w}_{\ell-1} \in \mathbf{N}_{\mathbf{B}_{\alpha}}\left(\mathbf{G}_{k}^{*} \cap\right.$ $\mathbf{M}(\overline{\mathbf{w}}))$, поэтому правая часть равенства (16.7) является группой. Обозначим ее через $\mathbf{P}$. Очевидно, $\mathbf{P} \subseteq \mathbf{M}(\overline{\mathbf{w}})$. Докажем обратное включение. Из определения 16.2 видно, что для этого достаточно доказать включения $\mathbf{w}_{1}^{2} \in \mathbf{P}$, $\left[\mathbf{w}_{1}, \mathbf{P}\right] \subseteq \mathbf{P}, \mathbf{w}_{\ell}^{2} \in \mathbf{P}$ и $\left[\mathbf{w}_{\ell}, \mathbf{P}\right] \subseteq \mathbf{P}$. По лемме 16.17 , (iі) имеем $\mathbf{w}_{1}^{2} \in \mathbf{G}_{k}^{*} \cap$ $\mathbf{M}(\overline{\mathbf{w}}) \subseteq \mathbf{P}$ и $\left[\mathbf{w}_{1}, \mathbf{P}\right] \subseteq\left[\mathbf{w}_{1}, \mathbf{M}(\overline{\mathbf{w}})\right] \subseteq \mathbf{G}_{k}^{*} \cap \mathbf{M}(\overline{\mathbf{w}}) \subseteq \mathbf{P}$. Для доказательства включений $\mathbf{w}_{\ell}^{2} \in \mathbf{P}$ и $\left[\mathbf{w}_{\ell}, \mathbf{P}\right] \subseteq \mathbf{P}$ рассмотрим два случая.

Случай 1: ранг $\mathbf{R}(\overline{\mathbf{w}})>$ ранг $\mathbf{M}(\overline{\mathbf{w}})$.

Тогда по условию для лестницы $\left(\mathbf{H}_{0}, \mathbf{H}_{1}, \ldots, \mathbf{H}_{t}\right)$ над $\mathbf{M}(\overline{\mathbf{w}})$ имеем ранг $\mathbf{H}_{t-1}^{*}<$ ранг $\mathbf{H}_{t}$ и в силу 16.13 , (v) ранг $\mathbf{M}(\overline{\mathbf{w}})=p a н г \mathbf{H}_{t}$. В этом случае в силу леммы 16.17 , (ii) и предложения 15.2

$$
\begin{gathered}
\mathbf{w}_{\ell}^{2} \in \mathbf{H}_{t}^{*} \cap \mathbf{M}(\overline{\mathbf{w}})=\mathbf{G}_{k}^{*} \cap \mathbf{M}(\overline{\mathbf{w}}) \subseteq \mathbf{P}, \\
{\left[\mathbf{w}_{\ell}, \mathbf{P}\right] \subseteq\left[\mathbf{w}_{\ell}, \mathbf{M}(\overline{\mathbf{w}})\right] \subseteq \mathbf{H}_{t}^{*} \cap \mathbf{M}(\overline{\mathbf{w}})=\mathbf{G}_{k}^{*} \cap \mathbf{M}(\overline{\mathbf{w}}) \subseteq \mathbf{P} .}
\end{gathered}
$$

Случай 2: ранг $\mathbf{R}(\overline{\mathbf{w}})=$ ранг $\mathbf{M}(\overline{\mathbf{w}})$.

Тогда по предложению 15.2

$$
\mathbf{G}_{k}^{*} \cap \mathbf{M}(\overline{\mathbf{w}})=\mathbf{H}_{0}^{*} \cap \mathbf{M}(\overline{\mathbf{w}}) .
$$

Так как по лемме 16.8 при $\overline{\mathbf{w}}:=\left(\mathbf{w}_{2}, \mathbf{w}_{3}, \ldots, \mathbf{w}_{\ell}\right)$ имеем

$$
\operatorname{pанг~} \mathbf{w}_{\ell}<\operatorname{ранг}\left\langle\mathbf{w}_{2}, \mathbf{w}_{3}, \ldots, \mathbf{w}_{\ell}\right\rangle \leqslant \text { ранг } \mathbf{H}_{0},
$$

то $\mathbf{w}_{\ell} \in \mathbf{H}_{0}^{*} \cup \mathbf{H}_{0}^{-}$, и, следовательно, $\mathbf{w}_{\ell}^{2} \in \mathbf{H}_{0}^{*}$, т.е.

$$
\mathbf{w}_{\ell}^{2} \in \mathbf{H}_{0}^{*} \cap \mathbf{M}(\overline{\mathbf{w}})=\mathbf{G}_{k}^{*} \cap \mathbf{M}(\overline{\mathbf{w}}) \subseteq \mathbf{P} .
$$


Ввиду $\mathbf{w}_{\ell} \in \mathbf{N}_{\mathbf{B}_{\alpha}}\left(\mathbf{H}_{0}^{*} \cap \mathbf{M}(\overline{\mathbf{w}})\right)$ и $(16.8)$ для доказательства включения $\left[\mathbf{w}_{\ell}, \mathbf{P}\right] \subseteq \mathbf{P}$ достаточно проверить, что

$$
\left[\mathbf{w}_{\ell}, \mathbf{w}_{i}\right] \in \mathbf{H}_{0}^{*} \quad(i=2,3, \ldots, \ell-1) .
$$

Обозначим $\mathbf{F} \rightleftharpoons\left\langle\mathbf{w}_{2}, \mathbf{w}_{3}, \ldots, \mathbf{w}_{\ell}\right\rangle$. Если ранг $\mathbf{F}<$ ранг $\mathbf{H}_{0}$, то по лемме 15.4 , (iii) имеем $\left|\mathbf{F}: \mathbf{F} \cap \mathbf{H}_{0}^{*}\right| \leqslant 2$, что, очевидно, влечет выполнение (16.9). Пусть ранг $\mathbf{F}=$ ранг $\mathbf{H}_{0}$. Тогда по лемме 16.9 при $\overline{\mathbf{w}}:=\left(\mathbf{w}_{2}, \mathbf{w}_{3}, \ldots, \mathbf{w}_{\ell}\right)$ имеем $\mathbf{w}_{2}, \mathbf{w}_{\ell} \in \mathbf{H}_{0}^{-}$и $\mathbf{w}_{3}, \mathbf{w}_{4}, \ldots, \mathbf{w}_{\ell-1} \in \mathbf{H}_{0}^{*}$, что влечет выполнение (16.9) при $i=3,4, \ldots, \ell-1$. Если $\left[\mathbf{w}_{2}, \mathbf{w}_{\ell}\right] \notin \mathbf{H}_{0}^{*}$, то $\left[\mathbf{w}_{2}, \mathbf{w}_{\ell}\right] \in \mathbf{H}_{0}^{+} \backslash \mathbf{H}_{0}^{*}$, т.е. ранг $\left[\mathbf{w}_{2}, \mathbf{w}_{\ell}\right]=$ ранг $\mathbf{H}_{0}$. Но тогда

$$
\operatorname{pанг}\left[\mathbf{w}_{1}, \mathbf{w}_{\ell-1}\right]=\operatorname{pан2}\left[\mathbf{w}_{2}, \mathbf{w}_{\ell}\right]=\operatorname{paн2} \mathbf{H}_{0}=\operatorname{pанг} \mathbf{G}_{k}
$$

вопреки доказанному выше включению $\left[\mathbf{w}_{1}, \mathbf{w}_{\ell-1}\right] \in\left[\mathbf{w}_{1}, \mathbf{P}\right] \subseteq \mathbf{G}_{k}^{*}$. Следовательно, $\left[\mathbf{w}_{2}, \mathbf{w}_{\ell}\right] \in \mathbf{H}_{0}^{*}$, что завершает доказательство (16.7).

Теперь уже несложно вывести противоречие для доказательства леммы 16.18. Из (16.7) в силу леммы 15.4, (i) вытекает

$$
\operatorname{pанг}\left\langle\mathbf{w}_{2}, \mathbf{w}_{3}, \ldots, \mathbf{w}_{\ell-1}\right\rangle=\operatorname{paнг} \mathbf{M}(\overline{\mathbf{w}})=\operatorname{paнг}_{\mathbf{G}} .
$$

Тогда по лемме 16.9 при $\overline{\mathbf{w}}:=\left(\mathbf{w}_{2}, \mathbf{w}_{3}, \ldots, \mathbf{w}_{\ell-1}\right)$ получаем $\mathbf{w}_{\ell-2} \in \mathbf{G}_{k}^{*}$ и

$$
\operatorname{paнг} \mathbf{w}_{2} \mathbf{w}_{\ell-1}=\operatorname{paн2} \mathbf{G}_{k} .
$$

По лемме 16.17 , (i) имеем $\mathbf{G}_{0} \cap \mathbf{G}_{k}^{*} \subseteq \mathbf{G}_{0}^{*}$, следовательно, $\mathbf{w}_{\ell-2} \in \mathbf{G}_{0}^{*} \cap \mathbf{G}_{k}^{*}$. Обозначив через $\mathbf{c}_{0}$ базисный элемент подгруппы $\mathbf{G}_{0}$, по лемме 16.17 , (iii) получаем $\mathbf{c}_{0}^{n / 4} \in \mathbf{N}_{\mathbf{B}_{\alpha}}\left(\mathbf{G}_{0}^{*} \cap \mathbf{G}_{k}^{*}\right)$, следовательно,

$$
\mathbf{c}_{0}^{-n / 4} \mathbf{w}_{\ell-2} \mathbf{c}_{0}^{n / 4} \in \mathbf{G}_{k}^{*} .
$$

Так как ранг $\mathbf{w}_{1}=$ ранг $\mathbf{w}_{2} \leqslant$ ранг $\mathbf{G}_{k}$, то в силу лемм 16.12 , (i) и 16.15 , (vii) при $\mathbf{M}:=\mathbf{M}(\overline{\mathbf{w}})$ и $\mathbf{u}:=\mathbf{w}_{1}$

$$
\mathbf{c}_{0}^{-n / 4} \mathbf{w}_{1} \mathbf{c}_{0}^{n / 4} \in \mathbf{G}_{k} .
$$

Так как элементы $\mathbf{c}_{0}^{-n / 4} \mathbf{w}_{1} \mathbf{c}_{0}^{n / 4}$ и $\mathbf{w}_{\ell-2}$ сопряжены, то

$$
\operatorname{paнг} \mathbf{c}_{0}^{-n / 4} \mathbf{w}_{1} \mathbf{c}_{0}^{n / 4}=\operatorname{paн2} \mathbf{w}_{\ell-2}<\operatorname{paн2} \mathbf{G}_{k} .
$$

Следовательно, $\mathbf{c}_{0}^{-n / 4} \mathbf{w}_{1} \mathbf{c}_{0}^{n / 4} \in \mathbf{G}_{k}^{*} \cup \mathbf{G}_{k}^{-}$. Тогда $\mathbf{c}_{0}^{-n / 4} \mathbf{w}_{1} \mathbf{w}_{\ell-2} \mathbf{c}_{0}^{n / 4} \in \mathbf{G}_{k}^{*} \cup \mathbf{G}_{k}^{-}$ и

$$
\operatorname{paн2} \mathbf{c}_{0}^{-n / 4} \mathbf{w}_{1} \mathbf{w}_{\ell-2} \mathbf{c}_{0}^{n / 4}<\operatorname{paн2} \mathbf{G}_{k} .
$$

Но с другой стороны, элементы $\mathbf{w}_{2} \mathbf{w}_{\ell-1}$ и $\mathbf{c}_{0}^{-n / 4} \mathbf{w}_{1} \mathbf{w}_{\ell-2} \mathbf{c}_{0}^{n / 4}$ сопряжены и должно быть

$$
\operatorname{paнг} \mathbf{c}_{0}^{-n / 4} \mathbf{w}_{1} \mathbf{w}_{\ell-2} \mathbf{c}_{0}^{n / 4}=\operatorname{pa\mu \imath } \mathbf{w}_{2} \mathbf{w}_{\ell-1}=\operatorname{pa\mu \imath } \mathbf{G}_{k} .
$$

Лемма доказана. 
16.19. Лемма. Пусть $\mathbf{F}, \mathbf{G}, \mathbf{H} \leqslant \mathbf{B}_{\alpha}$ - конечные подгруппы, $\mathbf{G} \subseteq \mathbf{F} \cap \mathbf{H}$,

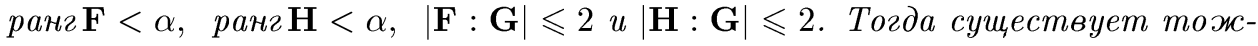
дественный на $\mathbf{G}$ гомоморфизм $\phi: \mathbf{F} \rightarrow \mathbf{B}_{\alpha}$ такой, что $\langle\phi(\mathbf{F}), \mathbf{H}\rangle$ - конечная подгруппа ранга, не превосходящего ранг $\mathbf{H}+1$.

ДОКАЗАТЕЛЬСТВО. При $\mathbf{G}=1$ можно взять тривиальный гомоморфизм $\phi: \mathbf{F} \rightarrow 1 \leqslant \mathbf{B}_{\alpha}$. Пусть $\mathbf{G} \neq 1$. По предложению 14.12 сушествует пополнение $\overline{\mathbf{G}}$ подгруппы $\mathbf{G}$ такое, что

$$
\forall \mathbf{h} \in \mathbf{H} \quad \forall \mathbf{g} \in \overline{\mathbf{G}} \quad \text { ранг } \mathbf{g h} \leqslant \text { ранг } \mathbf{H}+1 .
$$

По лемме 15.5 найдем гомоморфизм $\phi: \mathbf{F} \rightarrow \overline{\mathbf{G}}$, тождественный на $\mathbf{G}$. Так как $|\phi(\mathbf{F}): \mathbf{G}| \leqslant 2$ и $|\mathbf{H}: \mathbf{G}| \leqslant 2$, то $\mathbf{G} \unlhd\langle\phi(\mathbf{F}), \mathbf{H}\rangle$ и факторгруппа $\langle\phi(\mathbf{F}), \mathbf{H}\rangle / \mathbf{G}$ порождается двумя инволюциями $\mathbf{g}_{0} \mathbf{G}$ и $\mathbf{h}_{0} \mathbf{G}$ для некоторых $\mathbf{g}_{0} \in \phi(\mathbf{F}) \subseteq \overline{\mathbf{G}}$ и $\mathbf{h}_{0} \in \mathbf{H}$. Так как $\left(\mathbf{g}_{0} \mathbf{h}_{0}\right)^{n}=1$ в силу $(16.10)$, то $\langle\phi(\mathbf{F}), \mathbf{H}\rangle / \mathbf{G}$ есть гомоморфный образ диэдральной группы $\mathbf{D}_{n}$. В частности, подгруппа $\langle\phi(\mathbf{F}), \mathbf{H}\rangle$ конечна. Нам остается доказать, что ранг $\langle\phi(\mathbf{F}), \mathbf{H}\rangle \leqslant$ ранг $\mathbf{H}+1$.

Пусть $\mathbf{K}$ - пополнение подгруппы $\langle\phi(\mathbf{F}), \mathbf{H}\rangle . \quad$ Если $\mathbf{h}_{0} \mathbf{g}_{0} \in \mathbf{K}^{+} \backslash \mathbf{K}^{*}$, то

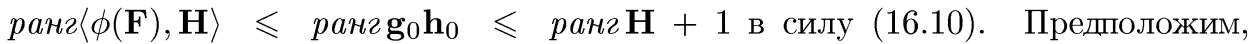
$\mathbf{h}_{0} \mathbf{g}_{0} \in \mathbf{K}^{*} \cap \mathbf{K}^{-}$. Тогда $\left(\mathbf{h}_{0} \mathbf{g}_{0}\right)^{2} \in \mathbf{K}^{*}$. Любой элемент группы $\langle\phi(\mathbf{F}), \mathbf{H}\rangle / \mathbf{G}$ имеет вид $\left(\mathbf{h}_{0} \mathbf{g}_{0}\right)^{2 t} \mathbf{z G}$, где $\mathbf{z} \in\left\{1, \mathbf{h}_{0}, \mathbf{g}_{0}, \mathbf{h}_{0} \mathbf{g}_{0}\right\}$. Другими словами, для любого $\mathbf{x} \in\langle\phi(\mathbf{F}), \mathbf{H}\rangle$ имеем $\left(\mathbf{h}_{0} \mathbf{g}_{0}\right)^{-2 t} \mathbf{x}=\mathbf{z g}$ для некоторых $\mathbf{g} \in \mathbf{G}, t$ и некоторого $\mathbf{z}$ указанного вида. Тогда один из элементов zg должен иметь ранг, равньй рангу подгруппы $\langle\phi(\mathbf{F}), \mathbf{H}\rangle$. Но все они имеют ранг, не превосходящий ранг $\mathbf{H}+1$ в силу (16.10). Следовательно, ранг $\langle\phi(\mathbf{F}), \mathbf{H}\rangle \leqslant$ ранг $\mathbf{H}+1$.

16.20. ОПРЕДЕЛЕНИЕ. Пусть $\overline{\mathbf{G}}=\left(\mathbf{G}_{0}, \mathbf{G}_{1}, \ldots, \mathbf{G}_{k}\right)$ - лестница наднекоторой конечной подгруппой $\mathbf{M}$ группы $\mathbf{B}_{\alpha}$. Набор $\bar{\phi}=\left(\phi_{0}, \phi_{1}, \ldots, \phi_{k}\right)$ гомоморфизмов $\phi_{i}: \mathbf{G}_{i} \rightarrow \mathbf{B}_{\alpha}$ назовем понижсающим отображением для $\overline{\mathbf{G}}$, если выполнены следующие условия:

(i) $\phi_{i}\left|\mathbf{G}_{i} \cap \mathbf{G}_{j}=\phi_{j}\right| \mathbf{G}_{i} \cap \mathbf{G}_{j}$ при любых $0 \leqslant i<j \leqslant k$;

(ii) $\phi_{k}=\mathrm{id}$;

(iii) $\operatorname{pанг~}\left(\phi_{i}\left(\mathbf{G}_{i}\right)\right)<$ ранг $\mathbf{G}_{i}$ при $i=0,1, \ldots, k-1$.

16.21. ЛЕммА. Пусть $\overline{\mathbf{G}}=\left(\mathbf{G}_{0}, \mathbf{G}_{1}, \ldots, \mathbf{G}_{k}\right)$ - лестница над некоторой конечной подгруппой $\mathbf{M} \leqslant \mathbf{B}_{\alpha}, k \geqslant 2 u\left(\phi_{1}, \phi_{2}, \ldots, \phi_{k}\right)$ - понижающее отображение для лестницы $\left(\mathbf{G}_{1}, \mathbf{G}_{2}, \ldots, \mathbf{G}_{k}\right)$ над $\mathbf{M}$. Тогда существует гомомормизм $\phi_{0}: \mathbf{G}_{0} \rightarrow \mathbf{B}_{\alpha}$ такой, что $\left(\phi_{0}, \phi_{1}, \ldots, \phi_{k}\right)$ - понижающее отображение $\partial_{\Omega s} \overline{\mathbf{G}}$.

ДоказАТЕЛЬСтво. Выберем элемент $\mathbf{f} \in \mathbf{M} \cap \bigcap_{i=1}^{k-1} \mathbf{G}_{0}^{-}$по лемме 16.15, (ii), (iii). Для любого $1 \leqslant i \leqslant k$ имеем ранг $\mathbf{G}_{0} \cap \mathbf{G}_{i}<$ ранг $\mathbf{G}_{0}$, следовательно, по лемме 15.4(ii)

$$
\mathbf{G}_{0} \cap \mathbf{G}_{i} \subseteq\left\langle\mathbf{G}_{0}^{*}, \mathbf{f}\right\rangle=\mathbf{M G}_{0}^{*} \subseteq \mathbf{G}_{1} .
$$

Отсюда и из определения 16.20 видно, что нам достаточно указать гомоморфизм $\phi_{0}: \mathbf{G}_{0} \rightarrow \mathbf{B}_{\alpha}$, удовлетворяюший условиям

$$
\left.\phi_{0}\right|_{\left\langle\mathbf{G}_{0}^{*}, \mathbf{f}\right\rangle}=\left.\phi_{1}\right|_{\left\langle\mathbf{G}_{0}^{*}, \mathbf{f}\right\rangle} \text { и } \quad \text { ранг } \phi_{0}\left(\mathbf{G}_{0}\right)<\operatorname{pанг~} \mathbf{G}_{0} .
$$


Пусть $\mathbf{c}_{0}$ и $\mathbf{c}_{1}$ - базисные элементы подгрупп $\mathbf{G}_{0}$ и $\mathbf{G}_{1}$. Обозначим $\mathbf{e} \rightleftharpoons \mathbf{f}^{-1} \mathbf{c}_{0}$. По лемме 16.15 , (vi) либо для некоторого $r$ при $2 \leqslant r \leqslant k-1$ подгруппа $\mathbf{c}_{1}^{-n / 4} \mathbf{G}_{r} \mathbf{c}_{1}^{n / 4}$ есть пополнение $\mathbf{G}_{0}^{*}$, либо $\mathbf{G}_{0}^{*} \subseteq \mathbf{c}_{1}^{-n / 4} \mathbf{G}_{k} \mathbf{c}_{1}^{n / 4}$. Рассмотрим эти два случая.

Случай 1: Для некоторого $r$ при $2 \leqslant r \leqslant k-1$ подгруппа $\mathbf{c}_{1}^{-n / 4} \mathbf{G}_{r} \mathbf{c}_{1}^{n / 4}$ есть пополнение $\mathbf{G}_{0}^{*}$. Так как $\mathbf{e} \in \mathbf{G}_{0}^{-}$, то по лемме 15.5 найдется гомоморфизм

$$
\omega:\left\langle\mathbf{G}_{0}^{*}, \mathbf{e}\right\rangle \rightarrow \mathbf{c}_{1}^{-n / 4} \mathbf{G}_{r} \mathbf{c}_{1}^{n / 4}
$$

такой, что $\left.\omega\right|_{\mathbf{G}_{0}^{*}}=\mathrm{id}$. Определим, далее, гомоморфизм

$$
\xi: \mathbf{c}_{1}^{-n / 4} \mathbf{G}_{r} \mathbf{c}_{1}^{n / 4} \rightarrow \mathbf{B}_{\alpha}
$$

равенством

$$
\xi(\mathbf{g}) \rightleftharpoons \phi_{1}\left(\mathbf{c}_{1}^{-n / 4}\right) \phi_{r}\left(\mathbf{c}_{1}^{n / 4} \mathbf{g c}_{1}^{-n / 4}\right) \phi_{1}\left(\mathbf{c}_{1}^{n / 4}\right) .
$$

Из условия (i) определения 16.20 следует $\left.\xi\right|_{\mathbf{G}_{0}^{*}}=\left.\phi_{1}\right|_{\mathbf{G}_{0}^{*}}$. Далее, по лемме 16.19 при $\mathbf{F}:=\xi \omega\left(\left\langle\mathbf{G}_{0}^{*}, \mathbf{e}\right\rangle\right), \mathbf{G}:=\phi_{1}\left(\mathbf{G}_{0}^{*}\right)$ и $\mathbf{H}:=\phi_{1}\left(\left\langle\mathbf{G}_{0}^{*}, \mathbf{f}\right\rangle\right)$ сушествует гомоморфизм

$$
\psi: \xi \omega\left(\left\langle\mathbf{G}_{0}^{*}, \mathbf{e}\right\rangle\right) \rightarrow \mathbf{B}_{\alpha}
$$

такой, что $\left.\psi\right|_{\phi_{1}\left(\mathbf{G}_{0}^{*}\right)}=\mathrm{id}$, подгруппа

$$
\mathbf{P} \rightleftharpoons\left\langle\phi_{1}\left(\left\langle\mathbf{G}_{0}^{*}, \mathbf{f}\right\rangle\right), \psi \xi \omega\left(\left\langle\mathbf{G}_{0}^{*}, \mathbf{e}\right\rangle\right)\right\rangle
$$

конечна и

$$
\operatorname{paнг} \mathbf{P} \leqslant \operatorname{paнг} \phi_{1}\left(\left\langle\mathbf{G}_{0}^{*}, \mathbf{f}\right\rangle\right)+1 \leqslant \operatorname{paнг} \phi_{1}\left(\mathbf{G}_{1}\right)+1 \leqslant \operatorname{paнг} \mathbf{G}_{1}
$$

Так как $\mathbf{G}_{0}=\left\langle\mathbf{G}_{0}^{*}, \mathbf{e}, \mathbf{f}\right\rangle$, то по лемме 15.6 гомоморфизмы $\left.\phi_{1}\right|_{\left\langle\mathbf{G}_{0}^{*}, \mathbf{f}\right\rangle}$ и $\psi \xi \omega$ продолжаются до гомоморфизма $\phi_{0}: \mathbf{G}_{0} \rightarrow \mathbf{B}_{\alpha}$, который, очевидно, является искомым.

Случай $2: \mathbf{G}_{0}^{*} \subseteq \mathbf{c}_{1}^{-n / 4} \mathbf{G}_{k} \mathbf{c}_{1}^{n / 4}$. Используя условия (i) и (ii) определения 16.20 , для любого $\mathbf{g} \in \mathbf{G}_{0}^{*}$ получаем

$$
\begin{aligned}
\phi_{1}(\mathbf{g}) & =\phi_{1}\left(\mathbf{c}_{1}^{-n / 4}\right) \phi_{1}\left(\mathbf{c}_{1}^{n / 4} \mathbf{g c}_{1}^{-n / 4}\right) \phi_{1}\left(\mathbf{c}_{1}^{n / 4}\right)= \\
& =\phi_{1}\left(\mathbf{c}_{1}^{-n / 4}\right) \phi_{k}\left(\mathbf{c}_{1}^{n / 4} \mathbf{g c}_{1}^{-n / 4}\right) \phi_{1}\left(\mathbf{c}_{1}^{n / 4}\right)= \\
& =\phi_{1}\left(\mathbf{c}_{1}^{-n / 4}\right) \mathbf{c}_{1}^{n / 4} \mathbf{g c}_{1}^{-n / 4} \phi_{1}\left(\mathbf{c}_{1}^{n / 4}\right),
\end{aligned}
$$

т.е.

$$
\phi_{1}(\mathbf{g})=\mathbf{y}^{-1} \mathbf{g y}, \quad \text { где } \mathbf{y} \rightleftharpoons \mathbf{c}_{1}^{-n / 4} \phi_{1}\left(\mathbf{c}_{1}^{n / 4}\right)
$$

Пусть

$$
\xi:\left\langle\mathbf{G}_{0}^{*}, \mathbf{e}\right\rangle \rightarrow \mathbf{B}_{\alpha}
$$


- вложение, определенное равенством

$$
\xi(\mathrm{g}) \rightleftharpoons \mathbf{y}^{-1} \mathbf{g y} .
$$

По лемме 16.19 при $\mathbf{F}:=\xi\left(\left\langle\mathbf{G}_{0}^{*}, \mathbf{e}\right\rangle\right), \mathbf{G}:=\phi_{1}\left(\mathbf{G}_{0}^{*}\right)$ и $\mathbf{H}:=\phi_{1}\left(\left\langle\mathbf{G}_{0}^{*}, \mathbf{f}\right\rangle\right)$ найдем гомоморфизм

$$
\psi: \xi\left(\left\langle\mathbf{G}_{0}^{*}, \mathbf{e}\right\rangle\right) \rightarrow \mathbf{B}_{\alpha}
$$

такой, что $\left.\psi\right|_{\phi_{1}\left(\mathbf{G}_{0}^{*}\right)}=\mathrm{id}$, подгруппа

$$
\mathbf{P} \rightleftharpoons\left\langle\phi_{1}\left(\left\langle\mathbf{G}_{0}^{*}, \mathbf{f}\right\rangle\right), \psi \xi\left(\left\langle\mathbf{G}_{0}^{*}, \mathbf{e}\right\rangle\right)\right\rangle
$$

конечна и

$$
\operatorname{pанг} \mathbf{P} \leqslant \operatorname{paнг} \phi_{1}\left(\left\langle\mathbf{G}_{0}^{*}, \mathbf{f}\right\rangle\right)+1 \leqslant \operatorname{paнг} \phi_{1}\left(\mathbf{G}_{1}\right)+1 \leqslant \operatorname{paнг} \mathbf{G}_{1} .
$$

Так как $\mathbf{G}_{0}=\left\langle\mathbf{G}_{0}^{*}, \mathbf{e}, \mathbf{f}\right\rangle$ и $\left.\psi \xi\right|_{\mathbf{G}_{0}^{*}}=\left.\phi_{1}\right|_{\mathbf{G}_{0}^{*}}$, то по лемме 15.6 гомоморфизмы $\left.\phi_{1}\right|_{\left\langle\mathbf{G}_{0}^{*}, \mathbf{f}\right\rangle}$ и $\psi \xi$ продолжаются до искомого гомоморфизма $\phi_{0}: \mathbf{G}_{0} \rightarrow \mathbf{B}_{\alpha}$.

16.22. Лемма. Пусть $\overline{\mathbf{G}}=\left(\mathbf{G}_{0}, \mathbf{G}_{1}, \ldots, \mathbf{G}_{k}\right)$ - лестница над некоторой конечной подгруппой $\mathbf{M} \leqslant \mathbf{B}_{\alpha}$. Если ранг $\mathbf{G}_{k-1}^{*}=$ ранг $\mathbf{G}_{k}$, то существует некоторое понижающее отображение для $\overline{\mathbf{G}}$.

ДокАЗАТЕльство. В силу лемм 16.15, (i), 16.21 и очевидной индукции достаточно рассмотреть случай $k=1$. Из равенства $р а н г \mathbf{G}_{0}^{*}=$ ранг $\mathbf{G}_{1}$ и условия (iii) определения 16.14 следует, что в этом случае $\mathbf{G}_{1}$ есть пополнение подгруппы $\mathbf{G}_{0}^{*}$. Рассмотрим два случая.

Случай 1: $\mathbf{G}_{1} \cap \mathbf{G}_{0}^{-}=\varnothing$. Тогда из ранг $\mathbf{G}_{1}<$ ранг $\mathbf{G}_{0}$ следует $\mathbf{G}_{0} \cap \mathbf{G}_{1} \subseteq \mathbf{G}_{0}^{*}$. По лемме 15.7 сушествует гомоморфизм $\phi_{0}: \mathbf{G}_{0} \rightarrow \mathbf{G}_{1}$ такой, что $\left.\phi_{0}\right|_{\mathbf{G}_{0}^{*}}=\mathrm{id}$. В таком случае $\left(\phi_{0}, \mathrm{id}_{\mathbf{G}_{1}}\right)$ - искомое понижающее отображение.

Случай 2: $\mathbf{G}_{1} \cap \mathbf{G}_{0}^{-} \neq \varnothing$. Выберем элемент $\mathbf{f} \in \mathbf{G}_{1} \cap \mathbf{G}_{0}^{-}$и пусть $\mathbf{c}_{0}-$ базисньй элемент $\mathbf{G}_{0}$. По лемме 15.4 , (ii)

$$
\mathbf{G}_{0} \cap \mathbf{G}_{1} \subseteq\left\langle\mathbf{G}_{0}^{*}, \mathbf{f}\right\rangle .
$$

Обозначив $\mathbf{e} \rightleftharpoons \mathbf{f}^{-1} \mathbf{c}_{0}$, по лемме 15.5 найдем гомоморфизм $\psi:\left\langle\mathbf{G}_{0}^{*}, \mathbf{e}\right\rangle \rightarrow \mathbf{G}_{1}$ такой, что $\left.\psi\right|_{\mathbf{G}_{0}^{*}}=\mathrm{id}$. По лемме 15.6 гомоморфизмы $\mathrm{id}_{\left\langle\mathbf{G}_{0}^{*}, \mathbf{f}\right\rangle}$ и $\psi$ продолжаются до гомоморфизма $\phi_{0}: \mathbf{G}_{0} \rightarrow \mathbf{G}_{1}$. Тогда $\left(\phi_{0}, \mathrm{id}_{\mathbf{G}_{1}}\right)$ - искомое понижаюшее отображение.

Следующая лемма - индуктивный шаг доказательства предложения 16.6.

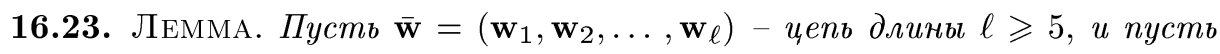
выполнено хотя бы одно из двух следующий условий:

(i) $\quad$ ранг $\mathbf{L}(\overline{\mathbf{w}})>$ ранг $\mathbf{M}(\overline{\mathbf{w}}), \quad \mathbf{G}_{0}-$ nополнение подгруппь $\mathbf{L}(\overline{\mathbf{w}})$, $\left(\mathbf{G}_{0}, \mathbf{G}_{1}, \ldots, \mathbf{G}_{k}\right)-$ лестнича над $\mathbf{M}(\overline{\mathbf{w}})$ и ранг $\mathbf{G}_{k-1}^{*}=$ ранг $\mathbf{G}_{k} ;$ или

(ii) $\quad$ анг $\mathbf{R}(\overline{\mathbf{w}})>$ ранг $\mathbf{M}(\overline{\mathbf{w}}), \quad \mathbf{H}_{0}-$ пополнение подгруппь $\mathbf{R}(\overline{\mathbf{w}})$, $\left(\mathbf{H}_{0}, \mathbf{H}_{1}, \ldots, \mathbf{H}_{t}\right)$ - лестница над $\mathbf{M}(\overline{\mathbf{w}})$ и ранг $\mathbf{H}_{t-1}^{*}=$ ранг $\mathbf{G}_{t} ;$

Тогда существует иепь $\overline{\mathbf{w}}^{\prime}=\left(\mathbf{w}_{1}^{\prime}, \mathbf{w}_{2}, \mathbf{w}_{3}, \ldots, \mathbf{w}_{\ell-1}, \mathbf{w}_{\ell}^{\prime}\right)$, для которой

$$
\operatorname{paн2} \mathbf{L}\left(\overline{\mathbf{w}}^{\prime}\right)+\operatorname{paн2} \mathbf{R}\left(\overline{\mathbf{w}}^{\prime}\right)<\operatorname{paн2} \mathbf{L}(\overline{\mathbf{w}})+\operatorname{paн2} \mathbf{R}(\overline{\mathbf{w}}) .
$$


ДоКАЗАТЕЛЬСТВО. Условия (i) и (ii) симметричны относительно замены порядка элементов $\mathbf{w}_{i}$ на обратный. Поэтому без ограничения обшности предположим, что выполнено условие (i). Выберем базисный элемент $\mathbf{c}_{i}$ каждой подгруппы $\mathbf{G}_{i}$. Так как $\mathbf{w}_{2} \in \mathbf{M}(\overline{\mathbf{w}})$, то

$$
\text { ранг } \mathbf{w}_{1}=\operatorname{pанг\mathbf {w}} 2 \leqslant \operatorname{pанг~} \mathbf{M}(\overline{\mathbf{w}}) \leqslant \operatorname{paнг} \mathbf{G}_{k} .
$$

Тогда в силу лемм 16.12 , (i) и 16.15, (vii) при $\mathbf{M}:=\mathbf{M}(\overline{\mathbf{w}})$ и $\mathbf{u}:=\mathbf{w}_{1}$

$$
\mathbf{c}_{0}^{-n / 4} \mathbf{w}_{1} \mathbf{c}_{0}^{n / 4} \in \bigcap_{i=0}^{k-1}\left(\mathbf{M}(\overline{\mathbf{w}}) \mathbf{G}_{i}^{*} \backslash \mathbf{G}_{i}^{*}\right) \subseteq \bigcap_{i=0}^{k} \mathbf{G}_{i} .
$$

При любом $2 \leqslant j \leqslant \ell-1$ имеем $\mathbf{L}(\overline{\mathbf{w}})=\left\langle\mathbf{M}(\overline{\mathbf{w}}), \mathbf{w}_{1} \mathbf{w}_{j}\right\rangle$, и так как при $2 \leqslant j \leqslant \ell-2$

$$
\operatorname{paнг} \mathbf{w}_{1} \mathbf{w}_{j}=\operatorname{paн2} \mathbf{w}_{2} \mathbf{w}_{j+1} \leqslant \operatorname{paн\imath } \mathbf{M}(\overline{\mathbf{w}}) \leqslant \operatorname{paн2} \mathbf{G}_{k},
$$

то снова по лемме 16.15, (vii)

$$
\mathbf{c}_{0}^{-n / 4} \mathbf{w}_{1} \mathbf{w}_{j} \mathbf{c}_{0}^{n / 4} \in \bigcap_{i=0}^{k} \mathbf{G}_{i}(j=2,3, \ldots, \ell-2) .
$$

Следовательно,

$$
\mathbf{c}_{0}^{-n / 4} \mathbf{w}_{j} \mathbf{c}_{0}^{n / 4} \in \bigcap_{i=0}^{k} \mathbf{G}_{i} \quad(j=1,2, \ldots, \ell-2) .
$$

По лемме 16.22 найдем понижаюшее отображение $\left(\phi_{0}, \phi_{1}, \ldots, \phi_{k}\right)$ для $\left(\mathbf{G}_{0}, \mathbf{G}_{1}, \ldots, \mathbf{G}_{k}\right)$. Имеем

$$
\phi_{0}\left(\mathbf{c}_{0}^{-n / 4} \mathbf{w}_{j} \mathbf{c}_{0}^{n / 4}\right)=\phi_{k}\left(\mathbf{c}_{0}^{-n / 4} \mathbf{w}_{j} \mathbf{c}_{0}^{n / 4}\right)=\mathbf{c}_{0}^{-n / 4} \mathbf{w}_{j} \mathbf{c}_{0}^{n / 4} \quad(j=1,2, \ldots, \ell-2),
$$

т.e.

$$
\phi_{0}\left(\mathbf{w}_{j}\right)=\mathbf{y}^{-1} \mathbf{w}_{j} \mathbf{y}, \quad \text { где } \mathbf{y} \rightleftharpoons \mathbf{c}_{0}^{n / 4} \phi_{0}\left(\mathbf{c}_{0}^{-n / 4}\right) \quad(j=1,2, \ldots, \ell-2) .
$$

$\mathrm{C}$ другой стороны, $\mathbf{w}_{2}, \mathbf{w}_{3}, \ldots, \mathbf{w}_{\ell-2} \in \mathbf{M}(\overline{\mathbf{w}}) \subseteq \mathbf{G}_{k}$, поэтому

$$
\phi_{0}\left(\mathbf{w}_{j}\right)=\mathbf{w}_{j} \quad(j=2,3, \ldots, \ell-2) .
$$

Таким образом, у коммутирует с $\mathbf{w}_{j}$ при $j=2,3, \ldots, \ell-2$. Если ранг $\mathbf{w}_{1} \mathbf{w}_{\ell-1} \leqslant$ ранг $\mathbf{G}_{k}$, то точно так же получим, что $\mathbf{y}$ коммутирует с $\mathbf{w}_{\ell-1}$, и тогда по лемме 16.10 в качестве искомой цепи $\overline{\mathbf{w}}^{\prime}$ можно взять последовательность $\left(\phi_{0}\left(\mathbf{w}_{1}\right), \mathbf{w}_{2}\right.$, $\left.\mathbf{w}_{3}, \ldots, \mathbf{w}_{\ell}\right)$. Поэтому будем предполагать, что

$$
\operatorname{paнг2}_{1} \mathbf{w}_{\ell-1}>\operatorname{paнг} \mathbf{G}_{k} .
$$


Тогда в силу лемм 16.12 , (i) и 16.15 , (vii) для некоторого $0 \leqslant r \leqslant k-1$ имеем ранг $\mathbf{w}_{1} \mathbf{w}_{\ell-1}=\operatorname{pанг~}_{\mathbf{G}_{r}}$ и

$$
\mathbf{c}_{0}^{-n / 4} \mathbf{w}_{1} \mathbf{w}_{\ell-1} \mathbf{c}_{0}^{n / 4} \in \mathbf{c}_{r}^{n / 2} \mathbf{G}_{r}^{*}
$$

Так как $\mathbf{w}_{1}, \mathbf{w}_{2}, \ldots, \mathbf{w}_{\ell-2} \in \mathbf{c}_{0}^{n / 4} \mathbf{G}_{r} \mathbf{c}_{0}^{-n / 4}$ в силу (16.12), то $\mathbf{c}_{0}^{n / 4} \mathbf{G}_{r} \mathbf{c}_{0}^{-n / 4}$ - пополнение подгрупшы $\left\langle\mathbf{w}_{1}, \mathbf{w}_{2}, \ldots, \mathbf{w}_{\ell-2}\right\rangle$.

Заметим, что

$$
\text { ранг } \mathbf{w}_{2} \mathbf{w}_{\ell}=\operatorname{paнг} \mathbf{w}_{1} \mathbf{w}_{\ell-1}>\operatorname{paнг} \mathbf{G}_{k} \geqslant \operatorname{pанг~} \mathbf{M}(\overline{\mathbf{w}}) .
$$

Отсюда следует ранг $\mathbf{R}(\overline{\mathbf{w}})>$ ранг $\mathbf{M}(\overline{\mathbf{w}})$. Выберем пополнение $\mathbf{H}_{0}$ подгруппы $\mathbf{R}(\overline{\mathbf{w}})$ и по лемме 16.13 построим лестницу $\left(\mathbf{H}_{0}, \mathbf{H}_{1}, \ldots, \mathbf{H}_{t}\right)$ над $\mathbf{M}(\overline{\mathbf{w}})$. Выберем, далее, базисные элементы $\mathbf{d}_{0}, \mathbf{d}_{1}, \ldots, \mathbf{d}_{t}$ подгрупп $\mathbf{H}_{0}, \mathbf{H}_{1}, \ldots, \mathbf{H}_{t}$. Если предположить ранг $\mathbf{w}_{2} \mathbf{w}_{\ell} \leqslant$ ранг $\mathbf{H}_{t}$, то отсюда будет следовать ранг $\mathbf{M}(\overline{\mathbf{w}})<$ ранг $\mathbf{H}_{t}$, и в силу условия $16.13,(\mathrm{v})$ определения 16.14 ранг $\mathbf{H}_{t-1}^{*}=p_{\text {aнг }} \mathbf{H}_{t}$. Но тогда симметрично рассмотренному выше случаю $р а н г \mathbf{w}_{1} \mathbf{w}_{\ell-1} \leqslant$ ранг $\mathbf{G}_{k}$ мы можем рассмотреть понижающее отображение $\left(\psi_{0}, \psi_{1}, \ldots, \psi_{t}\right)$ для $\left(\mathbf{H}_{0}, \mathbf{H}_{1}, \ldots, \mathbf{H}_{t}\right)$ и в качестве $\overline{\mathbf{w}}^{\prime}$ взять последовательность $\left(\mathbf{w}_{1}, \mathbf{w}_{2}, \ldots, \mathbf{w}_{\ell-1}, \psi_{0}\left(\mathbf{w}_{\ell}\right)\right)$. Поэтому будем считать

$$
\operatorname{paн2} \mathbf{w}_{2} \mathbf{w}_{\ell}>\operatorname{par} 2 \mathbf{H}_{t} .
$$

В силу лемм 16.12 , (ii) и 16.15 , (vii) для некоторого $0 \leqslant s \leqslant t-1$

$$
\mathbf{d}_{0}^{-n / 4} \mathbf{w}_{2} \mathbf{w}_{\ell} \mathbf{d}_{0}^{n / 4} \in \mathbf{d}_{s}^{n / 2} \mathbf{H}_{s}^{*}
$$

Симметрично включениям (16.12) получаем

$$
\mathbf{d}_{0}^{-n / 4} \mathbf{w}_{j} \mathbf{d}_{0}^{n / 4} \in \bigcap_{i=0}^{t} \mathbf{H}_{i} \quad(j=3,4, \ldots, \ell)
$$

откуда с учетом (16.14) следует, что $\mathbf{d}_{0}^{n / 4} \mathbf{H}_{s} \mathbf{d}_{0}^{-n / 4}-$ пополнение подгрупшы $\left\langle\mathbf{w}_{2}, \mathbf{w}_{3}, \ldots, \mathbf{w}_{\ell}\right\rangle$.

Пусть $\mathbf{x}$ - сопрягающий элемент цепи $\overline{\mathbf{w}}$. Имеем

$$
\mathbf{x}^{-1}\left\langle\mathbf{w}_{1}, \mathbf{w}_{2}, \ldots, \mathbf{w}_{\ell-1}\right\rangle \mathbf{x}=\left\langle\mathbf{w}_{2}, \mathbf{w}_{3}, \ldots, \mathbf{w}_{\ell}\right\rangle,
$$

и, следовательно, $\mathbf{c}_{0}^{n / 4} \mathbf{G}_{r} \mathbf{c}_{0}^{-n / 4}$ и $\mathbf{x} \mathbf{d}_{0}^{n / 4} \mathbf{H}_{s} \mathbf{d}_{0}^{-n / 4} \mathbf{x}^{-1}$ - два пополнения одной подгруппы $\left\langle\mathbf{w}_{1}, \mathbf{w}_{2}, \ldots, \mathbf{w}_{\ell-1}\right\rangle$. Тогда по предложению 14.2 найдется элемент $\mathbf{z} \in \mathbf{B}_{\alpha}$, коммутируюший с $\mathbf{w}_{1}, \mathbf{w}_{2}, \ldots, \mathbf{w}_{\ell-1}$, для которого

$$
\mathbf{z}^{-1} \mathbf{c}_{0}^{n / 4} \mathbf{G}_{r} \mathbf{c}_{0}^{-n / 4} \mathbf{z}=\mathbf{x d}_{0}^{n / 4} \mathbf{H}_{s} \mathbf{d}_{0}^{-n / 4} \mathbf{x}^{-1}
$$


Очевидно, zx - также сопрягающий элемент цепи $\overline{\mathbf{w}}$, и, заменяя $\mathbf{x}$ на $\mathbf{z x}$, можно предполагать

$$
\left(\mathbf{c}_{0}^{-n / 4} \mathbf{x d}_{0}^{n / 4}\right)^{-1} \mathbf{G}_{r} \mathbf{c}_{0}^{-n / 4} \mathbf{x d}_{0}^{n / 4}=\mathbf{H}_{s} .
$$

Теперь мы покажем, что, “подправив" подгруппы $\mathbf{H}_{s+j}$ при $j>0$, их можно выбрать сопряженными соответствуюшим подгруппам $\mathbf{G}_{r+j}$, причем с помощью одного и того же элемента $\mathbf{h}$. Идея рассуждений состоит в следуюшем. Как видно из условия 16.13 , (iii) определения 16.14 , каждая подгруппа $\mathbf{G}_{i+1}$ определяется подгруппой $\mathbf{G}_{i}$ и некоторым элементом из $\mathbf{M}(\overline{\mathbf{w}}) \mathbf{G}_{i}^{*} \backslash \mathbf{G}_{i}^{*}$, а каждая подгруппа $\mathbf{H}_{i+1}$ определяется подгруппой $\mathbf{H}_{i}$ и некоторым элементом из $\mathbf{M}(\overline{\mathbf{w}}) \mathbf{H}_{i}^{*} \backslash \mathbf{H}_{i}^{*}$. Наша задача будет состоять в том, чтобы выбрать сопряженные элементы, лежащие во множествах $\mathbf{M}(\overline{\mathbf{w}}) \mathbf{G}_{i}^{*} \backslash \mathbf{G}_{i}^{*}$ и $\mathbf{M}(\overline{\mathbf{w}}) \mathbf{H}_{i}^{*} \backslash \mathbf{H}_{i}^{*}$. В качестве таких элементов будут рассмотрены $\mathbf{w}_{1}$ и $\mathbf{d}_{s}^{-n / 4} \mathbf{d}_{0}^{-n / 4} \mathbf{w}_{2} \mathbf{d}_{0}^{n / 4} \mathbf{d}_{s}^{n / 4}$.

Мы хотим теперь применить лемму 16.15 , (vii) при $\overline{\mathbf{G}}:=\left(\mathbf{H}_{s}, \mathbf{H}_{s+1}, \ldots, \mathbf{H}_{t}\right)$, $\mathbf{M}:=\mathbf{M}(\overline{\mathbf{w}})$ и $\mathbf{u}:=\mathbf{d}_{0}^{-n / 4} \mathbf{w}_{2} \mathbf{d}_{0}^{n / 4}$. Заметим, что $\mathbf{M}(\overline{\mathbf{w}}) \cap \mathbf{H}_{s}^{-} \neq \varnothing \mathrm{в}$ силу лемм 16.12 , (ii) и 16.15 , (iii). Проверим выполнение остальных условий посылки леммы 16.15 , (vii):

$$
\begin{aligned}
& \mathbf{d}_{0}^{-n / 4} \mathbf{w}_{2} \mathbf{d}_{0}^{n / 4} \in \mathbf{H}_{s}, \\
& \left|\left\langle\mathbf{M}(\overline{\mathbf{w}}), \mathbf{d}_{0}^{-n / 4} \mathbf{w}_{2} \mathbf{d}_{0}^{n / 4}\right\rangle: \mathbf{M}(\overline{\mathbf{w}})\right| \leqslant 2, \\
& \operatorname{paн\imath }\left\langle\mathbf{M}(\overline{\mathbf{w}}), \mathbf{d}_{0}^{-n / 4} \mathbf{w}_{2} \mathbf{d}_{0}^{n / 4}\right\rangle=\operatorname{paн\imath } \mathbf{H}_{s} \text {. }
\end{aligned}
$$

Включение (16.17) вытекает из (16.14) и (16.15). Из тождеств $\left[\mathbf{x}^{n / 4}, \mathbf{y}^{2}\right]=1$ и $\left[\mathbf{x}^{n / 4}, \mathbf{y}, \mathbf{z}\right]=1$, выполненных на $\mathbf{H}_{0}$ в силу предложения 15.10 , (iii), (iv), следует

$$
\mathbf{d}_{0}^{-n / 4} \mathbf{w}_{2}^{2} \mathbf{d}_{0}^{n / 4}=\mathbf{w}_{2}^{2} \in \mathbf{M}(\overline{\mathbf{w}})
$$

и для любого $\mathbf{g} \in \mathbf{M}(\overline{\mathbf{w}})$

$$
\mathbf{d}_{0}^{-n / 4} \mathbf{w}_{2}^{-1} \mathbf{d}_{0}^{n / 4} \mathbf{g d}_{0}^{-n / 4} \mathbf{w}_{2} \mathbf{d}_{0}^{n / 4}=\mathbf{w}_{2}^{-1} \mathbf{g} \mathbf{w}_{2} \in \mathbf{M}(\overline{\mathbf{w}})
$$

Отсюда вытекает (16.18). Далее, по лемме $16.15,\left(\right.$ vii) при $\overline{\mathbf{G}}:=\left(\mathbf{H}_{0}, \mathbf{H}_{1}, \ldots, \mathbf{H}_{t}\right)$,

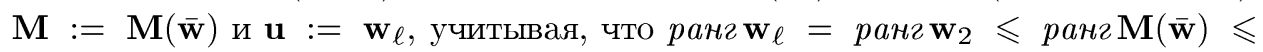
ранг $\mathbf{H}_{t}$, имеем

$$
\mathbf{d}_{0}^{-n / 4} \mathbf{w}_{\ell} \mathbf{d}_{0}^{n / 4} \in \mathbf{M}(\overline{\mathbf{w}}) \mathbf{H}_{s}^{*} \backslash \mathbf{H}_{s}^{*}=\mathbf{g} \mathbf{H}_{s}^{*} \quad \text { для некоторого } \mathbf{g} \in \mathbf{M}(\overline{\mathbf{w}}) \cap \mathbf{H}_{s}^{-},
$$

откуда с учетом (16.14) следует $\mathbf{d}_{0}^{-n / 4} \mathbf{w}_{2} \mathbf{d}_{0}^{n / 4} \in \mathbf{d}_{s}^{n / 2} \mathbf{g} \mathbf{H}_{s}^{*}$, т.е.

$$
\mathbf{d}_{0}^{-n / 4} \mathbf{w}_{2} \mathbf{d}_{0}^{n / 4} \mathbf{g}^{-1} \in \mathbf{d}_{s}^{n / 2} \mathbf{H}_{s}^{*}
$$

Тем самым мы указали элемент подгруппы $\left\langle\mathbf{M}(\overline{\mathbf{w}}), \mathbf{d}_{0}^{-n / 4} \mathbf{w}_{2} \mathbf{d}_{0}^{n / 4}\right\rangle$, имеющий ранг, равный $р а н г \mathbf{H}_{s}$. Следовательно, имеет место равенство (16.19). 
Итак, по лемме 16.15, (vii)

$$
\mathbf{d}_{s}^{-n / 4} \mathbf{d}_{0}^{-n / 4} \mathbf{w}_{2} \mathbf{d}_{0}^{n / 4} \mathbf{d}_{s}^{n / 4} \in \bigcap_{j=s}^{t-1}\left(\mathbf{M}(\overline{\mathbf{w}}) \mathbf{H}_{j}^{*} \backslash \mathbf{H}_{j}^{*}\right) .
$$

Обозначим

$$
\mathbf{h} \rightleftharpoons \mathbf{c}_{0}^{-n / 4} \mathbf{x d}_{0}^{n / 4} \mathbf{d}_{s}^{n / 4}
$$

Из (16.16) следует

$$
\mathbf{h}^{-1} \mathbf{G}_{r} \mathbf{h}=\mathbf{H}_{s}
$$

откуда по предложению 13.17

$$
\mathbf{h}^{-1} \mathbf{G}_{r}^{*} \mathbf{h}=\mathbf{H}_{s}^{*}
$$

Тогда, используя (16.20), (16.11) и лемму 15.4, (ii), получаем

$$
\begin{aligned}
\mathbf{M}(\overline{\mathbf{w}}) \mathbf{H}_{s}^{*} & =\left\langle\mathbf{H}_{s}^{*}, \mathbf{d}_{s}^{-n / 4} \mathbf{d}_{0}^{-n / 4} \mathbf{w}_{2} \mathbf{d}_{0}^{n / 4} \mathbf{d}_{s}^{n / 4}\right\rangle \\
& =\left\langle\mathbf{h}^{-1} \mathbf{G}_{r}^{*} \mathbf{h}, \mathbf{h}^{-1} \mathbf{c}_{0}^{-n / 4} \mathbf{w}_{1} \mathbf{c}_{0}^{n / 4} \mathbf{h}\right\rangle \\
& =\mathbf{h}^{-1}\left\langle\mathbf{G}_{r}^{*}, \mathbf{c}_{0}^{-n / 4} \mathbf{w}_{1} \mathbf{c}_{0}^{n / 4}\right\rangle \mathbf{h} \\
& =\mathbf{h}^{-1}\left(\mathbf{M}(\overline{\mathbf{w}}) \mathbf{G}_{r}^{*}\right) \mathbf{h} .
\end{aligned}
$$

Так как в процессе построения лестницы $\left(\mathbf{H}_{0}, \mathbf{H}_{1}, \ldots, \mathbf{H}_{t}\right)$ над $\mathbf{M}(\overline{\mathbf{w}})$ в качестве $\mathbf{H}_{s+1}$ можно взять любое пополнение подгруппы $\mathbf{M}(\overline{\mathbf{w}}) \mathbf{H}_{s}^{*}$ (см. доказательство леммы 16.13), то можно считать

$$
\mathbf{H}_{s+1}=\mathbf{h}^{-1} \mathbf{G}_{r+1} \mathbf{h} .
$$

Из предложения 15.2 и 16.13 , (v) следует $t=s+1 \Leftrightarrow k=r+1$. Если $k>r+1$, то аналогично предыдушему из (16.20), (16.11) и соотношения $\mathbf{H}_{s+1}=\mathbf{h}^{-1} \mathbf{G}_{r+1} \mathbf{h}$ получим, что в качестве $\mathbf{H}_{s+2}$ можно взять $\mathbf{h}^{-1} \mathbf{G}_{r+2} \mathbf{h}$. Продолжая этот процесс, в результате получим $t-s=k-r$ и

$$
\mathbf{H}_{s+i}=\mathbf{h}^{-1} \mathbf{G}_{r+i} \mathbf{h} \quad(i=0,1, \ldots, k-r) .
$$

Теперь определим гомоморфизмы $\psi_{s+i}: \mathbf{H}_{s+i} \rightarrow \mathbf{B}_{\alpha}$ при $i=0,1, \ldots, k-r$ равенствами

$$
\psi_{s+i}\left(\mathbf{h}^{-1} \mathbf{g h}\right) \rightleftharpoons \mathbf{h}^{-1} \phi_{r+i}(\mathbf{g}) \mathbf{h} \quad(i=0,1, \ldots, k-r) .
$$

Так как $\left(\phi_{0}, \phi_{1}, \ldots, \phi_{k}\right)$ есть понижающее отображение для $\left(\mathbf{G}_{0}, \mathbf{G}_{1}, \ldots, \mathbf{G}_{k}\right)$, то из определения 16.20 видно, что $\left(\psi_{s}, \psi_{s+1}, \ldots, \psi_{t}\right)$ есть понижающее отображение для $\left(\mathbf{H}_{s}, \mathbf{H}_{s+1}, \ldots, \mathbf{H}_{t}\right)$. Применяя лемму 16.21 в случае $s \geqslant 1$ (так как $s \leqslant t-1$ в силу выбора $s$, то при $s \geqslant 1$ выполнено условие $k \geqslant 2$ леммы 16.21), найдем 
понижающее отображение $\left(\psi_{0}, \psi_{1}, \ldots, \psi_{t}\right)$ для $\left(\mathbf{H}_{0}, \mathbf{H}_{1}, \ldots, \mathbf{H}_{t}\right)$. Мы докажем, что последовательность

$$
\overline{\mathbf{w}}^{\prime} \rightleftharpoons\left(\phi_{0}\left(\mathbf{w}_{1}\right), \mathbf{w}_{2}, \mathbf{w}_{3}, \ldots, \mathbf{w}_{\ell-1}, \psi_{0}\left(\mathbf{w}_{\ell}\right)\right)
$$

является искомой цепью.

Из (16.21) следует

$$
\mathbf{h}^{-1} \mathbf{c}_{0}^{-n / 4} \mathbf{w}_{j} \mathbf{c}_{0}^{n / 4} \mathbf{h}=\mathbf{d}_{s}^{-n / 4} \mathbf{d}_{0}^{-n / 4} \mathbf{w}_{j+1} \mathbf{d}_{0}^{n / 4} \mathbf{d}_{s}^{n / 4} \quad(j=1,2, \ldots, \ell-1) .
$$

При этом в силу (16.12), (16.13)

$$
\mathbf{c}_{0}^{-n / 4} \mathbf{w}_{j} \mathbf{c}_{0}^{n / 4} \in \mathbf{G}_{r}
$$

и в силу (16.15) и (16.17)

$$
\mathbf{d}_{s}^{-n / 4} \mathbf{d}_{0}^{-n / 4} \mathbf{w}_{j+1} \mathbf{d}_{0}^{n / 4} \mathbf{d}_{s}^{n / 4} \in \mathbf{H}_{s} .
$$

Применяя $\psi_{s}$ к левой и правой частям соотношений (16.23), с учетом (16.22) получаем

$$
\mathbf{h}^{-1} \phi_{r}\left(\mathbf{c}_{0}^{-n / 4} \mathbf{w}_{j} \mathbf{c}_{0}^{n / 4}\right) \mathbf{h}=\psi_{s}\left(\mathbf{d}_{s}^{-n / 4} \mathbf{d}_{0}^{-n / 4} \mathbf{w}_{j+1} \mathbf{d}_{0}^{n / 4} \mathbf{d}_{s}^{n / 4}\right) \quad(j=1,2, \ldots, \ell-1)
$$

откуда. учитывая, что $\phi_{r}\left|\mathbf{G}_{0} \cap \mathbf{G}_{r}=\phi_{0}\right| \mathbf{G}_{0} \cap \mathbf{G}_{r},\left.\psi_{s}\right|_{\mathbf{H}_{0} \cap \mathbf{H}_{s}}=\left.\psi_{0}\right|_{\mathbf{H}_{0} \cap \mathbf{H}_{s}}, \mathbf{w}_{j} \in$ $\mathbf{L}(\overline{\mathbf{w}}) \subseteq \mathbf{G}_{0}$ и $\mathbf{w}_{j+1} \in \mathbf{R}(\overline{\mathbf{w}}) \subseteq \mathbf{H}_{0}$, вьводим

$$
\begin{aligned}
\mathbf{h}^{-1} \phi_{0}\left(\mathbf{c}_{0}^{-n / 4}\right) \phi_{r}\left(\mathbf{w}_{j}\right) \phi_{0}\left(\mathbf{c}_{0}^{n / 4}\right) \mathbf{h}= & \psi_{s}\left(\mathbf{d}_{s}^{-n / 4}\right) \psi_{0}\left(\mathbf{d}_{0}^{-n / 4}\right) \\
& \times \psi_{0}\left(\mathbf{w}_{j+1}\right) \psi_{0}\left(\mathbf{d}_{0}^{n / 4}\right) \psi_{s}\left(\mathbf{d}_{s}^{n / 4}\right) .
\end{aligned}
$$

Так как $\mathbf{w}_{2}, \mathbf{w}_{3}, \ldots, \mathbf{w}_{\ell-1} \in \mathbf{M}(\overline{\mathbf{w}}) \subseteq \mathbf{G}_{k} \cap \mathbf{H}_{t}$, то

$$
\phi_{0}\left(\mathbf{w}_{j}\right)=\psi_{0}\left(\mathbf{w}_{j}\right)=\mathbf{w}_{j} \text { при } j=2,3, \ldots, \ell-1 .
$$

Следовательно, обозначив

$$
\tilde{\mathbf{x}} \rightleftharpoons \phi_{0}\left(\mathbf{c}_{0}^{n / 4}\right) \mathbf{h} \psi_{s}\left(\mathbf{d}_{s}^{-n / 4}\right) \psi_{0}\left(\mathbf{d}_{0}^{-n / 4}\right)
$$

получим

$$
\begin{gathered}
\tilde{\mathbf{x}}^{-1} \phi_{0}\left(\mathbf{w}_{1}\right) \tilde{\mathbf{x}}=\mathbf{w}_{2} \\
\tilde{\mathbf{x}}^{-1} \mathbf{w}_{j} \tilde{\mathbf{x}}=\mathbf{w}_{j+1}(j=2,3, \ldots, \ell-2), \\
\tilde{\mathbf{x}}^{-1} \mathbf{w}_{\ell-1} \tilde{\mathbf{x}}=\psi_{0}\left(\mathbf{w}_{\ell}\right)
\end{gathered}
$$


Далее, учитьвая, что $\left.\phi_{0}\right|_{\mathbf{M}(\overline{\mathbf{w}})}=\left.\phi_{k}\right|_{\mathbf{M}(\overline{\mathbf{w}})}=\mathrm{id}$ и $\left.\psi_{0}\right|_{\mathbf{M}(\overline{\mathbf{w}})}=\left.\psi_{t}\right|_{\mathbf{M}(\overline{\mathbf{w}})}=\mathrm{id}$, имеем

$$
\left(\phi_{0}\left(\mathbf{w}_{1}\right)\right)^{2}=\phi_{0}\left(\mathbf{w}_{1}^{2}\right)=\mathbf{w}_{1}^{2}, \quad\left(\psi_{0}\left(\mathbf{w}_{\ell}\right)\right)^{2}=\psi_{0}\left(\mathbf{w}_{\ell}^{2}\right)=\mathbf{w}_{\ell}^{2}
$$

и для любого $\mathbf{g} \in \mathbf{M}(\overline{\mathbf{w}})$ при $\epsilon= \pm 1$

$$
\begin{aligned}
& \left(\phi_{0}\left(\mathbf{w}_{1}\right)\right)^{-\epsilon} \mathbf{g}\left(\phi_{0}\left(\mathbf{w}_{1}\right)\right)^{\epsilon}=\phi_{0}\left(\mathbf{w}_{1}^{-\epsilon} \mathbf{g w}_{1}^{\epsilon}\right)=\mathbf{w}_{1}^{-\epsilon} \mathbf{g w}_{1}^{\epsilon}, \\
& \left(\psi_{0}\left(\mathbf{w}_{\ell}\right)\right)^{-\epsilon} \mathbf{g}\left(\psi_{0}\left(\mathbf{w}_{\ell}\right)\right)^{\epsilon}=\psi_{0}\left(\mathbf{w}_{\ell}^{-\epsilon} \mathbf{g w}_{\ell}^{\epsilon}\right)=\mathbf{w}_{\ell}^{-\epsilon} \mathbf{g w}_{\ell}^{\epsilon}
\end{aligned}
$$

Отсюда легко видеть (см. доказательство леммы 16.10), что $\mathbf{M}\left(\overline{\mathbf{w}}^{\prime}\right)=\mathbf{M}(\overline{\mathbf{w}})$. Тогда согласно определению $16.3 \overline{\mathbf{w}}^{\prime}$ является цепю с сопрягающим элементом $\tilde{\mathbf{x}}$. Наконец, заметим, что

$$
\mathbf{L}\left(\overline{\mathbf{w}}^{\prime}\right)=\left\langle\mathbf{M}(\overline{\mathbf{w}}), \phi_{0}\left(\mathbf{w}_{1}\right)\right\rangle=\left\langle\phi_{0}(\mathbf{M}(\overline{\mathbf{w}})), \phi_{0}\left(\mathbf{w}_{1}\right)\right\rangle=\phi_{0}(\mathbf{L}(\overline{\mathbf{w}}))
$$

и

$$
\mathbf{R}\left(\overline{\mathbf{w}}^{\prime}\right)=\left\langle\mathbf{M}(\overline{\mathbf{w}}), \psi_{0}\left(\mathbf{w}_{\ell}\right)\right\rangle=\left\langle\psi_{0}(\mathbf{M}(\overline{\mathbf{w}})), \psi_{0}\left(\mathbf{w}_{\ell}\right)\right\rangle=\psi_{0}(\mathbf{R}(\overline{\mathbf{w}}))
$$

откуда в силу условия (ii) определения 16.20 следует

$$
\begin{aligned}
\operatorname{pанг~} \mathbf{L}\left(\overline{\mathbf{w}}^{\prime}\right)+\operatorname{pанг} \mathbf{R}\left(\overline{\mathbf{w}}^{\prime}\right) & \leqslant \operatorname{pанг~} \phi_{0}\left(\mathbf{G}_{0}\right)+\operatorname{paнг} \psi_{0}\left(\mathbf{H}_{0}\right) \\
& <\operatorname{paн2} \mathbf{G}_{0}+\operatorname{paн2} \mathbf{H}_{0} \\
& =\operatorname{paн2} \mathbf{L}(\overline{\mathbf{w}})+\operatorname{paн2} \mathbf{R}(\overline{\mathbf{w}})
\end{aligned}
$$

Доказательство леммы завершено.

ДоКАЗАТЕЛЬСТВО ПРЕДЛОЖЕНИЯ 16.6. Допустим, найдется нерегулярная цепь $\overline{\mathbf{w}}=\left(\mathbf{w}_{1}, \mathbf{w}_{2}, \ldots, \mathbf{w}_{\ell}\right)$ длины $\ell \geqslant 6$. В соответствии с индукцией будем считать, что $\overline{\mathbf{w}}$ выбрана с минимальным значением ранг $\mathbf{L}(\overline{\mathbf{w}})+\operatorname{paнг} \mathbf{R}(\overline{\mathbf{w}})$. Пусть $\mathbf{G}_{0}$ - пополнение подгрупы $\mathbf{L}(\overline{\mathbf{w}})$ и $\mathbf{H}_{0}$ - пополнение подгруппы $\mathbf{R}(\overline{\mathbf{w}})$. Согласно лемме 16.13 построим лестницу $\left(\mathbf{G}_{0}, \mathbf{G}_{1}, \ldots, \mathbf{G}_{k}\right)$ над $\mathbf{M}(\overline{\mathbf{w}})$ в слу-

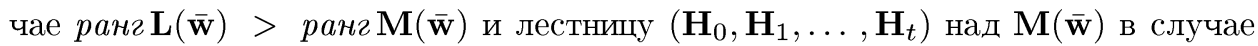
ранг $\mathbf{R}(\overline{\mathbf{w}})>$ ранг $\mathbf{M}(\overline{\mathbf{w}})$. Лемма 16.18 в точности утверждает выполнение одного из условий (i) или (ii) леммы 16.23. Тогда по лемме 16.23 существует цепь $\overline{\mathbf{w}}^{\prime}$ той же длины $\ell$, отличающаяся от $\overline{\mathbf{w}}$ лишь элементами $\mathbf{w}_{1}$ и $\mathbf{w}_{\ell}$, с тем условием, что ранг $\mathbf{L}\left(\overline{\mathbf{w}}^{\prime}\right)+$ ранг $\mathbf{R}\left(\overline{\mathbf{w}}^{\prime}\right)<$ ранг $\mathbf{L}(\overline{\mathbf{w}})+$ ранг $\mathbf{R}(\overline{\mathbf{w}})$. Но поскольку элементы $\mathbf{w}_{2}$, $\mathbf{w}_{3}, \ldots, \mathbf{w}_{\ell-1}$ общие для $\overline{\mathbf{w}}$ и $\overline{\mathbf{w}}^{\prime}$, то из нерегулярности $\overline{\mathbf{w}}$ вытекает нерегулярность $\overline{\mathbf{w}}^{\prime}$, и мы получаем противоречие с выбором $\overline{\mathbf{w}}$. Предложение 16.6 доказано.

Доказательство предложения 16.6 завершает совместную индукиию по рангу $\alpha$, начатую в $\S 6$. Тем самым все утверждения $\S 6-16$ доказаны для всех значений ранга $\alpha$. 


\section{$\S 17$. Доказательство теорем}

17.1. ЛЕммА. Для любого слова $X$ существует приведенное в ранге $\alpha \rightleftharpoons$ $|X|+1$ слово $Y$ такое, что $X \stackrel{\alpha=1}{=} Y$ и $Y$ не содержсит в качестве подслов элементарных слов ранга $\alpha$.

ДоказАТЕльСтво. Индукция по длине слова $X$. Если $X$ 으 1 , то в силу предложения 8.18 можно положить $Y \rightleftharpoons X$. Пусть $|X| \geqslant 1$ и $X$ 으 $X^{\prime} a^{\epsilon}$, где $a^{\epsilon} \in \mathcal{A}^{ \pm 1}$. По индуктивному предположению найдется слово $Y^{\prime} \in \mathcal{R}_{\alpha-1}$ такое, что $X^{\prime} \stackrel{\alpha=2}{=} Y^{\prime}$ и $Y^{\prime}$ не содержит элементарных слов ранга $\alpha-1$. Согласно предложению 12.13 при $\alpha:=\alpha-2$ выберем слово $Y \in \mathcal{R}_{\alpha-2}$ такое, что $Y^{\prime} a^{\epsilon} \stackrel{\alpha=2}{=} Y$. Тогда имеем $X \stackrel{\alpha=1}{=} Y$. В силу предложения 8.18 слово $a^{\epsilon}$ не содержит элементарных слов ранга $\alpha-1$. Выбрав пути $Y^{\prime} Z$ и $Y$ на $\Gamma_{\alpha-2}$ с общими начальными и конечными вершинами, где $\mathcal{L}\left(\mathrm{Y}^{\prime}\right)$ 으 $Y^{\prime}, \mathcal{L}(\mathrm{Z})$ 으 $a^{\epsilon}$ и $\mathcal{L}(\mathrm{Y})$ 으 $Y$, и применив лемму 11.7, (ii) при $\alpha:=\alpha-1, \mathrm{X}:=\mathrm{Y}, \mathrm{Z}_{1}:=\mathrm{Y}^{\prime}$ и $\mathrm{Z}_{2}:=\mathrm{Z}$, получим, что слово $Y$ не содержит элементарных 101-степеней ранга $\alpha-1$. Тогда в силу леммы 12.12 имеем $Y \in \mathcal{R}_{\alpha}$ и в силу леммы $8.16 Y$ не содержит элементарных слов ранга $\alpha$, т.е. $Y$ является искомым.

17.2. ПРЕДЛОЖЕНИЕ. Группа $\mathbf{B}(m, n)$ имеет задание

$$
\left\langle\mathcal{A} \mid A^{n}=1\left(A \in \bigcup_{\alpha} \mathcal{E}_{\alpha}\right)\right\rangle,
$$

или, другими словами, равенство слов $X$ и $Y$ в $\mathbf{B}(m, n)$ имеет место тогда и только тогда, когда $X \stackrel{\alpha}{=} Y$ для некоторого $\alpha$.

ДокАЗАТЕльСтво. Достаточно проверить, что для любого слова $X$ в групе с заданием (17.1) имеет место соотношение $X^{n}=1$, т.е. $X^{n} \stackrel{\alpha}{=} 1$ для некоторого $\alpha$. По лемме 17.1 найдем $\alpha \geqslant 1$ и слово $Y \in \mathcal{R}_{\alpha}$, такие, что $X \stackrel{\alpha=1}{=} Y$ и $Y$ не содержит элементарных слов ранга $\alpha$. Допустим, $X^{n} \stackrel{\alpha}{\neq} 1$. Тогда в силу предложения $15.10,(\mathrm{i})$ элемент $[X]_{\alpha}$ имеет бесконечный порядок и в силу предложения 12.24 элемент $[X]_{\alpha}$ сопряжен элементу $[Z]_{\alpha}$, где $Z-$ период ранга $\alpha+1$. В силу леммы 11.34 при $X:=Y, u:=Y:=v:=1$ и $Z:=Z$ слова $Y$ и $Z$ сопряжены в группе $\mathbf{B}_{\alpha-1}$.

Рассмотрим диаграмму $\Delta$ ранга $\alpha-1$ с двумя граничными циклами $\mathrm{Yu}_{\text {и }} \mathrm{Z}^{-1}$, где $\mathcal{L}(\mathrm{Y})$ 으 $Y, \mathcal{L}(\mathrm{u})$ 으 1 и $\mathcal{L}(\mathrm{Z})$ 으 $Z$. Можно считать, что $\Delta$ есть $A$-диаграмма ранга $\alpha-1$ с $X_{\alpha-1}(\Delta)=\left\{\mathrm{Y}, \mathrm{Z}^{-1}\right\}$ и $\mathcal{Y}_{\alpha-1}(\Delta)=\{\mathrm{u}\}$. Кроме того, в силу предложения 9.19 можно считать, что на $\Delta$ задана карта примыканий $\mathcal{S}$ ранга $\alpha-1$. В силу предложения 12.27 в некоторый циклический сдвиг слова $Z$ входит элементарная 109-степень ранга $\alpha$. В силу предложения 8.22 , (i) имеем $\mathcal{W}_{\alpha-1}\left(Z^{-1}\right) \geqslant \frac{108}{13}>\eta$. Тогда в силу предложения $10.4 Z^{-1}$ не является переходным участком границы диаграммы $\Delta$ относительно $\mathcal{S}$, т.е. $Z=S w$, где $\mathrm{S}^{-1}-$ участок примыкания некоторой поддиаграммы примыкания $\Pi \in \mathcal{S}$. Так как в силу предложения 9.23, (iii) в Sсодержится не более одной поддиаграммы примыкания $Z^{-1}$ к $\mathrm{Y}$ и нет поддиаграмм примыкания $\mathbf{Z}^{-1}$ к $Z^{-1}$, то П - поддиаграмма 
примыкания $Z^{-1}$ к $Y_{\text {и }} w^{-1}$ - переходный участок границы диаграммы $\Delta$ относительно $\mathcal{S}$. Обозначив $S \rightleftharpoons \mathcal{L}(\mathrm{S})$ и $w \rightleftharpoons \mathcal{L}(\mathrm{w})$, получаем, что слово $S$ и некоторое подслово слова $Y$ близки в ранге $\alpha-1$ и по предложению $10.4 \mathcal{W}_{\alpha-1}(w) \leqslant \eta$.

Пусть $E \in \Pi$ Пер $\alpha(A, 109)\left(A \in \mathcal{E}_{\alpha}\right)$ - элементарная 109-степень ранга $\alpha$, входяшая в некоторый циклический сдвиг слова $Z$. Тогда $E$ имеет один из видов $S^{\prime} w^{\prime} S^{\prime \prime}$ или $w^{\prime} S^{\prime} w^{\prime \prime}$, где $S^{\prime}, S^{\prime \prime}$ - непересекающиеся подслова слова $S$ и $w^{\prime}, w^{\prime \prime}$ - подслова слова $w$. Так как в силу предложения 8.22 , (i) имеем $\ell_{\alpha, A}(w)<7$, то, используя предложение 8.14, (ii), получаем, что в одно из слов $S^{\prime}$ или $S^{\prime \prime}$ входит некоторое слово $F \in \Pi \Pi е p_{\alpha}(A, 50)$. Тогда в силу предложения 8.27 в $Y$ должна входить некоторая элементарная 16-степень ранга $\alpha$. Но это противоречит выбору $Y$. Следовательно, $X^{n} \stackrel{\alpha}{=} 1$.

17.3. ЛЕмма. Любая әлементарная $q_{4}$-степень ранга $\alpha \geqslant 1$ содержит непустое подслово вида $A^{q_{4}-32}$.

ДокаЗАтельство. Пусть $E \in \Pi$ Пер $\alpha\left(A, q_{4}\right)$, где $A \in \mathcal{E}_{\alpha}$. Без ограничения обшности можно считать, что $E$ не содержит в качестве подслов элементарных $q_{4}$-степеней ранга $\beta$ при $1 \leqslant \beta \leqslant \alpha-1$. В силу леммы 8.2 , (ii) слово $E$ и некоторое слово $P \in \Pi е p\left(A, q_{4}\right)$ близки в ранге $\alpha-1$. Тогда в силу следствия 11.9 и предложения 8.22 , (ii) некоторое подслово $P^{\prime}$ слова $P$ длины $\left|P^{\prime}\right| \geqslant\left(q_{4}-32\right)|A|$ и некоторое подслово $E^{\prime}$ слова $E$ близки в ранге 0 , т.е. $P^{\prime}$ 으 $E^{\prime}$. Таким образом, $E$ содержит подслово $B^{q_{4}-32}$, где $B$ - некоторый циклический сдвиг слова $A$.

Заметим, что теорема 1 непосредственно вытекает из п. (i) теоремы 2 и существования бесконечного слова в двухбуквенном алфавите $\left\{a_{1}, a_{2}\right\}$, не содержашего непустых подслов вида $A^{3}$ (см., например, $[1, \S$ I.3]).

ДОКАЗАТЕЛЬСТВо ТЕОРЕМЫ 2. (i): Пусть $X$ - непустое несократимое слово, равное 1 в группе $\mathbf{B}(m, n)$. По предложению 17.2 для некоторого $\alpha$ имеем $X \stackrel{\alpha}{=} 1$. Тогда по предложению 11.4 получаем $X \notin \mathcal{R}_{\alpha}$, и так как $X \in \mathcal{R}_{0}$, то $X \in \mathcal{R}_{\beta-1} \backslash \mathcal{R}_{\beta}$ для некоторого $1 \leqslant \beta \leqslant \alpha$. По лемме 12.12 в этом случае слово $X$ содержит в качестве подслова некоторую элементарную $(n-q-36)$-степень ранга $\alpha$ и нам остается воспользоваться леммой 17.3.

Пункт (ii) теоремы 2 по существу вытекает из п. (i) и следуюшего факта, доказанного в [1, VI.2.15]: число всех несократимых слов длины $s$ в 2-буквенном групповом алфавите $\left\{a_{1}^{ \pm 1}, a_{2}^{ \pm 1}\right\}$, не содержаших непустых подслов вида $A^{8}$, не меньше $4\left(\frac{29}{10}\right)^{s-1}$. Действительно, из теоремы 2 , (i) следует, что любые два графически неравных слова указанного вида представляют разные элементы группы $\mathbf{B}(m, n)$. Поэтому функция роста $\gamma(s)$ групшы $\mathbf{B}(m, n)$ относительно канонического множества порождающих $\left\{a_{1}, a_{2}, \ldots, a_{m}\right\}$ при $m \geqslant 2$ удовлетворяет неравенству $\gamma(s) \geqslant 4\left(\frac{29}{10}\right)^{s-1}$, т.е. является экспоненциальной.

(iii): Если бы группа $\mathbf{B}(m, n)$ была конечно определена, то для ее задания было бы достаточно конечного числа определяюших соотношений из (17.1), т.е. имели бы $\mathbf{B}(m, n) \simeq \mathbf{B}_{\alpha}$ для некоторого $\alpha$. Поэтому достаточно для каждого $\alpha$ указать элемент бесконечного порядка в группе $\mathbf{B}_{\alpha}$. Для этого мы построим бесконечную последовательность $A_{1}, A_{2}, \ldots$ элементарных периодов $A_{\alpha}$ ранга $\alpha$. Тогда из лем- 
мы 6.7 будет следовать, что группа $\mathbf{B}_{\alpha}$ содержит элемент $\left[A_{\alpha+1}\right]_{\alpha}$ бесконечного порядка.

Положим

$$
A_{1} \rightleftharpoons a_{1} \quad \text { и } A_{\alpha} \rightleftharpoons \theta^{\alpha-1}\left(A_{1}\right) \quad(\alpha \geqslant 2),
$$

где $\theta(X)$ обозначает результат подстановки $a_{1} \rightarrow a_{1}^{p} a_{2}, a_{2} \rightarrow a_{2} a_{1}^{p}$ в слово $X$. Индукцией по $\alpha$ докажем, что

(i) если $B$ - непустое простое слово и слово $B^{r}$ входит в слово $A_{\alpha}^{t}$ при $r \geqslant 4$, то $B \approx A_{\beta}$ для некоторого $\beta \leqslant \alpha$, а если $r \geqslant 2 p+1$, то $B \approx A_{\alpha}$;

(ii) $A_{\alpha}$ - простое слово.

Оба утверждения тривиальны при $\alpha=1$. Пусть $\alpha>1$. Предположим,

$$
A_{\alpha}^{t} \text { 으 } X B^{r} Y
$$

где $r \geqslant 4$ и $B$ - непустое простое слово. Если $|B|=1$, то, очевидно, $B$ 드 $a_{1}$ 드 $A_{1}$, причем $A_{\alpha}^{t}$ не содержит вхождений слова $a_{1}^{2 p+1}$. Пусть $|B|>1$. Обозначим

$$
w_{1} \rightleftharpoons a_{1}^{p} a_{2} \quad \text { и } \quad w_{2} \rightleftharpoons a_{2} a_{1}^{p}
$$

Заметим, что $A_{\alpha}^{t}$ имеет вид

$$
A_{\alpha}^{t} \text { 으 } w_{i_{1}} w_{i_{2}} \ldots w_{i_{\ell}}
$$

где все слова $w_{i_{j}}$ имеют длину $p+1$. Если $|B|<p+1$, то $B$ - подслово слова вида $w_{i}$ или $w_{i} w_{j}$. Как легко видеть, в этом случае $B$ может иметь только один из видов $a_{1}^{k} a_{2} a_{1}^{s}(1 \leqslant k+s \leqslant p-1)$ или $a_{1}^{k} a_{2}^{2} a_{1}^{s}(0 \leqslant k+s \leqslant p-2)$. Тогда либо слово $a_{2} a_{1}^{k+s} a_{2}(1 \leqslant k+s \leqslant p-1)$, либо слово $a_{2}^{4}$ входит в $A_{\alpha}^{t}$, что невозможно. Следовательно, $|B| \geqslant p+1$. Тогда $A_{\alpha}^{t}$ 으 $X^{\prime} B^{\prime 3} Y^{\prime}$, где $\left|X^{\prime}\right| \equiv 0(\bmod p+1), B^{\prime}-$ циклический сдвиг слова $B$ и $B^{\prime}$ начинается с некоторого $w_{i}$.

Допустим, $|B| \not \equiv 0(\bmod p+1)$. Пусть, например, $B^{\prime}$ 으 $w_{1} B_{1}$ 으 $a_{1}^{p} a_{2} B_{1}$. Вхождение $X^{\prime} B^{\prime} a_{1}^{p} * a_{2} * B_{1} B^{\prime} Y^{\prime}$ буквы $a_{2}$ в слово $A_{\alpha}^{t}$ является либо концом некоторого вхождения $w_{1}$ в $(17.2)$, либо началом некоторого вхождения $w_{2}$. В первом случае $|B|$ кратно $p+1$ вопреки допущению. Следовательно, указанное вхождение буквы $a_{2}$ есть начало некоторого $w_{2}$ в $(17.2)$ и тогда, очевидно, $|B| \equiv 1$ $(\bmod p+1)$. Но, рассмотрев вместо вхождения $X^{\prime} B a_{1}^{p} * a_{2} * B_{1} B Y^{\prime}$ вхождение $X^{\prime} B^{2} a_{1}^{p} * a_{2} * B_{1} Y^{\prime}$, точно так же получим $2|B| \equiv 1(\bmod p+1)$. Противоречие. Аналогично, в случае, если $B^{\prime}$ начинается с $w_{2}$, получим противоречие $|B| \equiv-1$ и $2|B| \equiv-1(\bmod p+1)$. Следовательно, $|B| \equiv 0(\bmod p+1)$.

Если теперь $|X| \not \equiv 0(\bmod p+1)$, то для некоторого $w_{i}$ имеем $w_{i}$ 드 $w_{i}^{\prime} w_{i}^{\prime \prime}$, где $w_{i}^{\prime}, w_{i}^{\prime \prime}$ непусты, $X$ 으 $X_{1} w_{i}^{\prime}, B$ 으 $w_{i}^{\prime \prime} B_{1}$ и $\left|X_{1}\right| \equiv 0(\bmod p+1)$. Так как непустые конщы у слов $w_{1}$ и $w_{2}$ различны, то вхождение $X * B * w_{i}^{\prime \prime} B_{1} B^{r-2} Y$ оканчивается на $w_{i}^{\prime}$, т.е. $B$ 드 $B_{2} w_{i}^{\prime}$. Тогда $A_{\alpha}^{t}$ 으 $X_{1}\left(w_{i}^{\prime} B_{2}\right)^{r} w_{i}^{\prime} Y$ и, заменяя слово $B$ на его циклический сдвиг $w_{i}^{\prime} B_{2}$ и слова $X, Y$ соответственно на $X_{1}$ и $w_{i}^{\prime} Y$, можно считать $|X| \equiv 0(\bmod p+1)$. В таком случае $A_{\alpha-1}^{t}$ 으 $\widetilde{X} \widetilde{B}^{r} \widetilde{Y}$, где $\theta(\widetilde{X})$ 으 $X, \theta(\widetilde{B})$ 으 $B$ и $\theta(\widetilde{Y})$ 으 $Y$, и утверждение (i) сводится к индуктивному предположению. 
Используя те же рассуждения, легко свести к индуктивному предположению и (ii). Если $A_{\alpha}$ ㅇ $B^{r}$ при $r \geqslant 3$, то, как мы видели выше, $|B| \equiv 0(\bmod p+1)$. Тогда $A_{\alpha-1}$ 으 $\widetilde{B}^{r}$, где $\theta(\widetilde{B})$ 으 $B$, и работает индукция. Если $r=2$, то невозможен случай $|B| \equiv \pm 1(\bmod p+1)$. Тогда, используя предыдущие рассуждения, снова получаем $|B| \equiv 0(\bmod p+1)$. Снова противоречие с индуктивным предположением.

Используя индукцию по $\alpha$, докажем теперь, что $A_{\alpha}$ - элементарный период ранга $\alpha$. Для $\alpha=1$ это непосредственно вытекает из определений $\S 5$. Пусть $\alpha>1$.

Допустим, в слово $A_{\alpha}^{t}$ при $t>0$ входит элементарная $q_{1}$-степень $E$ ранга $1 \leqslant \beta \leqslant \alpha-1$, порожденная некоторым периодом $B \in \mathcal{E}_{\beta}$. Выберем минимальное такое $\beta$. Так как согласно определению 5.16 слово $E$ содержит некоторую элементарную $q_{1}$-степень ранга $\rho(B)$, то $\rho(B)=0$. В силу леммы 8.2 , (ii) и следствия 11.3 слово $E$ и некоторое слово $P \in \Pi е p\left(B, q_{1}\right)$ близки в ранге 0 , т.е. $E$ ㅇ $P$. Тогда в силу (i) $B \approx A_{\alpha}$. Но так как $A_{\beta}$ - подслово слова $A_{\alpha}$, то согласно определению 5.5 имеем $B \in \mathcal{N}_{\beta, 0}$, и, следовательно, $B \notin \mathcal{E}_{\beta}$. Получили противоречие. Следовательно, в слово $A_{\alpha}^{t}$ не входят элементарные $q_{1}$-степени рангов $1 \leqslant \beta \leqslant \alpha-1$. Отсюда в силу леммы 12.12 вытекает $A_{\alpha}^{t} \in \mathcal{R}_{\alpha-1}$ и, таким образом, согласно определению 5.2 слово $A_{\alpha}$ есть период ранга $\alpha$.

Допустим, $A_{\alpha}$ не является минимальным периодом ранга $\alpha$. Тогда в силу определения 5.3 и предложения 12.24 для некоторого периода $B$ ранга $\alpha$ и некоторых $t \geqslant 1$ и $r \geqslant 2$ слова $A^{t}$ и $B^{t r}$ сопряжены в группе $\mathbf{B}_{\alpha-1}$. В силу предложения 11.33 слова $A^{t}$ и $B^{t r}$ сопряжены в свободной группе $\mathbf{B}_{0}$. Но это невозможно в силу (ii). Следовательно, $A_{\alpha} \in \mathcal{M}_{\alpha}$.

Наконец, допустим, $A_{\alpha} \notin \mathcal{E}_{\alpha}$. Тогда $A_{\alpha} \in \bigcup_{i \geqslant 0} \mathcal{N}_{\alpha, i}$ и в силу предложения 8.34 слово $A_{\alpha}^{2}$ содержит элементарную 152 -степень ранга $\alpha$. Как показьвают предыдущие рассуждения, в этом случае в $A_{\alpha}^{2}$ входит некоторое слово $P \in \Pi е p(B, 152)$, где $B \in \mathcal{E}_{\alpha}$. В силу (i) $B \approx A_{\beta}$ для некоторого $\beta<\alpha$. Но по индуктивному предположению $A_{\beta}$ - элементарный период ранга $\beta$, и мы вновь приходим к противоречию, так как $\mathcal{E}_{\beta} \cap \mathcal{E}_{\alpha}=\varnothing$ (последнее вытекает, например, из того, что для любого $C \in \mathcal{E}_{\beta}$ порядок элемента $[C]_{\alpha-1}$ конечен, а для любого $C \in \mathcal{E}_{\alpha}$ элемент $[C]_{\alpha-1}$ имеет бесконечный порядок).

Таким образом, мы доказали, что при любом $\alpha$ слово $A_{\alpha}$ есть элементарный период ранга $\alpha$. Тем самым завершено доказательства п. (iii) теоремы 2.

Для доказательствап. (iv) теоремы 2 заметим, что в силу предложения 17.2 для любой конечной подгруппы $\mathbf{G}$ группы $\mathbf{B}(m, n)$ существует изоморфная ей подгруппа группы $\mathbf{B}_{\alpha}$ при некотором $\alpha$. Тогда в силу предложения 15.9 G вкладывается в конечное прямое произведение $\mathbf{D}_{n} \times \mathbf{D}_{\bar{n}} \times \cdots \times \mathbf{D}_{\bar{n}}$, где $\bar{n}-$ наибольший делитель $n$ вида $2^{k}$. Теорема доказана.

\section{Список литературы}

1. Адян С.И. Проблема Бернсайда и тождества в группах. М.: Наука, 1975.

2. Адян С.И. Исследования по проблеме Бернсайда и связанным с ней вопросам // Тр. Матем. ин-та им. В.А.Стеклова. 1984. Т. 168. С. 179-205.

3. Линдон P., Шупn П. Комбинаторная теория групп. М.: Мир, 1977. 
4. Льсенок И.Г. Бесконечность бернсайдовых групп периода $2^{k}$ при $k \geqslant 13 / /$ УМН. 1992. Т. 47. № 2. C. 201-202.

5. Новиков П.С., Адян С.И. Бесконечные периодические группы. I-III // Изв. АН СССР. Сер. матем. 1968. Т. 32. С. 212-244, 251-524, 709-731.

6. Ольшанский А.Ю. О теореме Новикова-Адяна // Матем. сб. 1982. Т. 118. № 2. C. 203-235.

7. Ольшанский А.Ю. Геометрия определяющих соотношений в группах. М.: Наука, 1989.

8. Санов И.Н. Решение проблемы Бернсайда для показателя 4 // Учен. Записки Ленингр. Унив. Сер. матем. 1940. Т. 10. С. 166-170.

9. Adian S.I. and Lysionok I.G. The method of classification of periodic words and the Burnside problem // Contemporary Math. Part 1. 1992. V. 131. P. 13-28.

10. Burnside $W$. On an unsettled question in the theory of discontinuous groups // Quart. J. Math. 1902. V. 33. P. 230-238.

11. Hall M. Solution of the Burnside problem for exponent six // Illinois J. Math. 1958. V. 2. P. 764-786.

12. Ivanov S. V. On the Burnside problem on periodic groups // Bull. of Amer. Math. Soc. 1992. V. 27. P. 257-260.

13. Ivanov S. V. The free Burnside groups of sufficiently large exponents // Int. J. of Algebra and Computation. 1994. V. 4. P. 1-307.

Математический институт РАН

Поступило в редакцию

им. В.А.Стеклова

28.IV.1995 